\title{
Elastic-Plastic Fracture Mechanics Analyses of 2D and 3D Test Specimens under Uniaxial and Biaxial Loading
}

\author{
By \\ Ping Ding \\ B.E., M.E. \\ A thesis submitted to \\ the Faculty of Graduate Studies and Research \\ in partial fulfillment of the requirements for the degree of \\ Doctor of Philosophy \\ in Mechanical Engineering \\ Department of Mechanical and Aerospace Engineering \\ Carleton University \\ Ottawa, Ontario, Canada
}

October, 2012

(C) Copyright

2012, Ping Ding 
Library and Archives

Canada

Published Heritage

Branch

395 Wellington Street

Ottawa ON K1A ON4

Canada
Bibliothèque et

Archives Canada

Direction du

Patrimoine de l'édition

395 , rue Wellington

Ottawa ON K1A ON4

Canada
Your file Votre référence

ISBN: 978-0-494-94218-5

Our file Notre référence

ISBN: $978-0-494-94218-5$
NOTICE:

The author has granted a nonexclusive license allowing Library and Archives Canada to reproduce, publish, archive, preserve, conserve, communicate to the public by telecommunication or on the Internet, loan, distrbute and sell theses worldwide, for commercial or noncommercial purposes, in microform, paper, electronic and/or any other formats.

The author retains copyright ownership and moral rights in this thesis. Neither the thesis nor substantial extracts from it may be printed or otherwise reproduced without the author's permission.
AVIS:

L'auteur a accordé une licence non exclusive permettant à la Bibliothèque et Archives Canada de reproduire, publier, archiver, sauvegarder, conserver, transmettre au public par télécommunication ou par l'Internet, prêter, distribuer et vendre des thèses partout dans le monde, à des fins commerciales ou autres, sur support microforme, papier, électronique et/ou autres formats.

L'auteur conserve la propriété du droit d'auteur et des droits moraux qui protege cette thèse. $\mathrm{Ni}$ la thèse ni des extraits substantiels de celle-ci ne doivent être imprimés ou autrement reproduits sans son autorisation.
In compliance with the Canadian Privacy Act some supporting forms may have been removed from this thesis.

While these forms may be included in the document page count, their removal does not represent any loss of content from the thesis.
Conformément à la loi canadienne sur la protection de la vie privée, quelques formulaires secondaires ont été enlevés de cette thèse.

Bien que ces formulaires aient inclus dans la pagination, il n'y aura aucun contenu manquant. 


\begin{abstract}
Extensive finite element analyses are performed to obtain numerical solutions of constraint parameter $A$ for two-dimensional (2D) and three-dimensional (3D) crack geometries under both uniaxial and biaxial loading condition through a least-square fitting method. Based on the determined numerical solutions of constraint parameter $A$, constraint effect at crack-tip (-front) of 2D and 3D cracked specimens are analyzed under both uniaxial and biaxial loading condition. Three sets of methodologies for estimating constraint parameter $A$ of elastic-plastic fracture mechanics are developed in the present research. They are: (1) estimating constraint parameter $A$ by curve shape similarity, (2) predicting $A$ values directly from the $T$-stress, and (3) determining parameter $A$ based on the fully plastic solutions of $A$. With the obtained numerical solutions of constraint parameter $A$, estimate formulas for $A$ values corresponding to the three sets of newlydeveloped estimate methodologies are developed for 2D and 3D cracked structures under both uniaxial and biaxial loading. It is shown that all three sets of methods can be used to predict $A$ values with good accuracy. In the present research, it has been validated that, the obtained solutions of constraint parameter $A$ (whether estimate methods / formulas or numerical solutions) can be utilized to predict other two commonly-used constraint parameters $Q$ and $A_{2}$ (a different normalized form of $A$ ) through the relationships between $A$ and $Q$ as well as $A$ and $A_{2}$.
\end{abstract}


In the loving memory of my maternal grandmother, whose love lighted up the beginning of my life.

\section{Dedicated to}

my parents, Ding, Hui-liang and Fan, Fa-fen, and my wife, Liu, Xiao-jing Without their love and support, this dissertation will be an impossible task. 


\section{ACKNOWLEDGEMENTS}

Author owes sincere gratitude to supervisor, Professor Xin Wang, for the suggesting of research problem as well as his advice, encouragement and excellent guidance through the whole investigation.

Sincere gratitude also goes to the Chair, Professor Metin I. Yaras, and all staffs in the Department of Mechanical and Aerospace Engineering for all their supports and helps.

The author gratefully acknowledges the financial supports from the Natural Sciences and Engineering Research Council (NSERC) of Canada and Ontario Centres of Excellence (OCE). 


\section{TABLE OF CONTENTS}

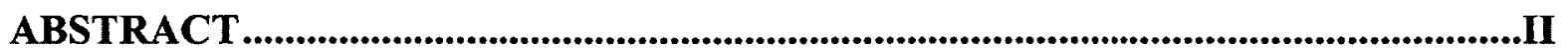

ACKNOWLEDGEMENTS _.......................................................................................... IV

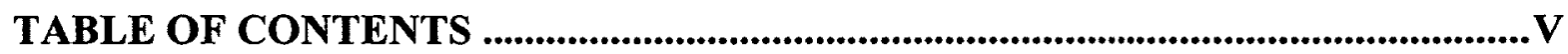

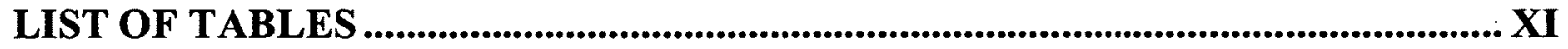

LIST OF FIGURES ....................................................................................................... XVI

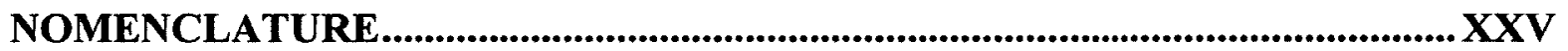

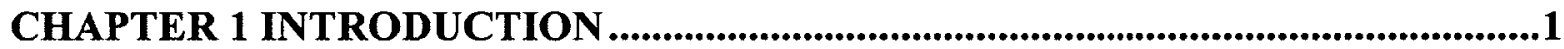

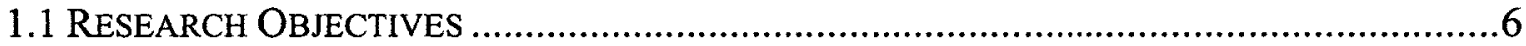

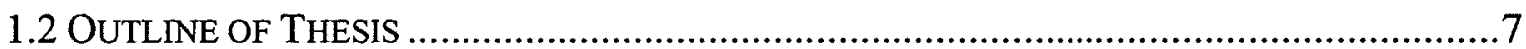

CHAPTER 2 THEORETICAL BACKGROUND AND LITERATURE REVIEW .10

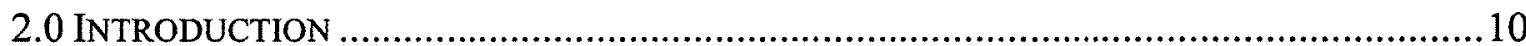

2.1 ChaRACTERIZATIONS OF CRACK-TIP (-Front) FIELDS.............................................10

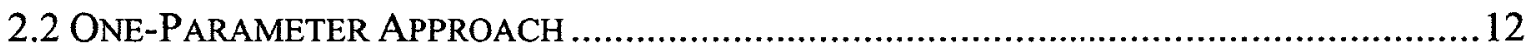

2.2.1 One-parameter approach of linear elastic fracture mechanics (LEFM) .............12

2.2.2 One-parameter approach of elastic-plastic fracture mechanics (EPFM) ...........12

2.3 Two-Parameter Approach of Linear Elastic Fracture Mechanics (LEFM) 13

2.3.1 K-T Two-Parameter Approach ........................................................................13

2.4 Two-Parameter Approach of Elastic-Plastic Fracture Mechanics (EPFM)14

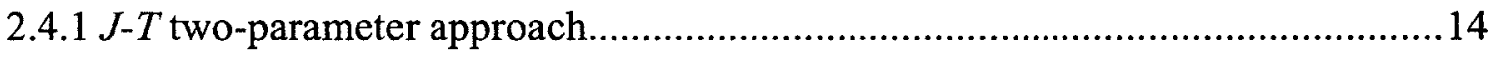

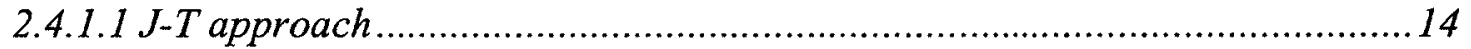

2.4.1.2 Modified boundary layer (MBL) problem............................................ 14

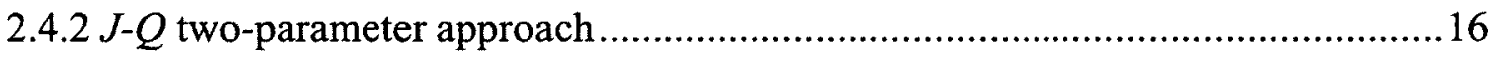

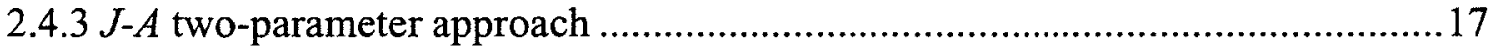

2.4.4 Application of two-parameter elastic-plastic fracture mechanics .......................19

2.5 METHODS FOR THE DETERMINATION OF DOMINANT FRACTURE MECHANICS

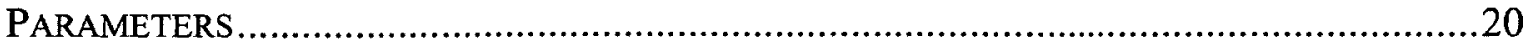




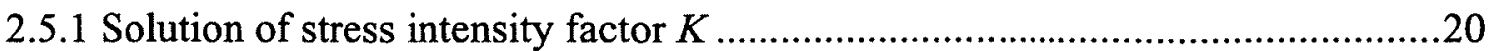

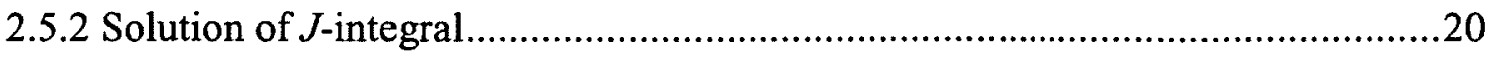

2.6 Methods For the Determination of CONSTRAINT Fracture MECHANICS

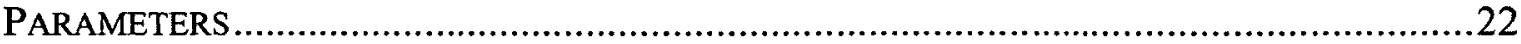

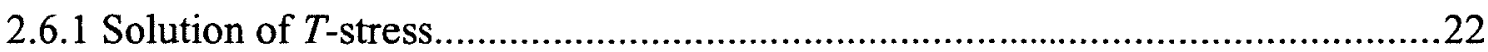

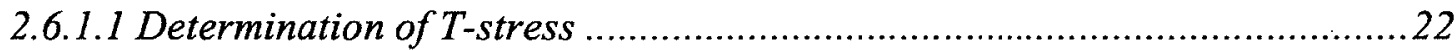

2.6.1.2 Prediction of T-stress by weight function method...................................22

2.6.2 Solution for the $Q$ parameter .........................................................23

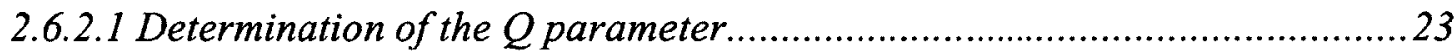

2.6.2.2 Prediction of $Q$ factor by $Q-T$ relationship...........................................23

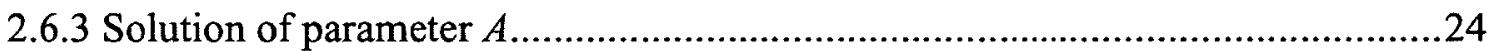

2.6.3.1 Numerical determination of parameter $A$...........................................25

2.6.3.2 Possibility of predicting parameter A by curve shape similarity................26

2.6.4 Relationships among constraint parameters .............................................27

2.6.4.1 Relationship between the constraint parameters $Q$ and $T$.......................27

2.6.4.2 Relationship between the constraint parameters $A$ and $A_{2} \ldots \ldots \ldots \ldots \ldots \ldots . . . . . .27$

2.6.4.3 Relationship between the constraint parameters $A$ and $Q$.....................28

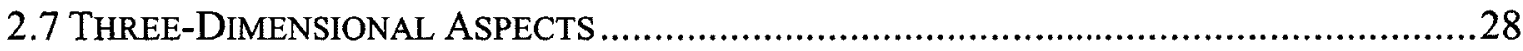

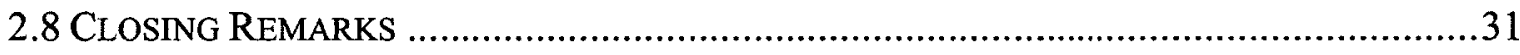

CHAPTER 3 THE DEVELOPMENT OF ESTIMATION METHODS ...................43

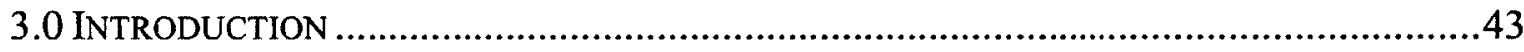

3.1 Prediction of Constraint Parameter $A$ Based on Curve Shape Similarity ..43

3.1.1 Formulating curve shape similarity of parameter $A$....................................43

3.1.2 Predicting parameter $A$ by curve shape similarity .....................................45

3.2 Prediction of Constraint Parameter $A$ Based on $T$-Stress .........................45

3.2.1 Determining parameter $A$ under small-scale yielding ..................................45

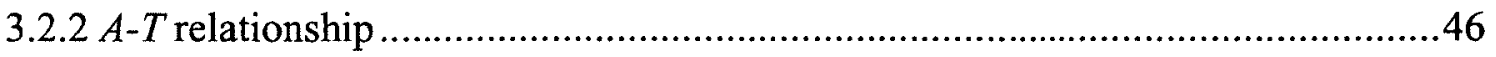

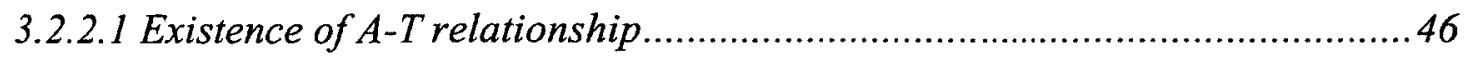

3.2.2.2 Modified boundary layer formulation for parameter $A$.......................... 47 
3.2.3 Predicting parameter $A$ by $T$-stress

3.3 Prediction of CONSTRaint Parameter $A$ Based on Fully-Plastic Solution ..50

3.3.1 Parameter $A$ under fully-plastic state .50

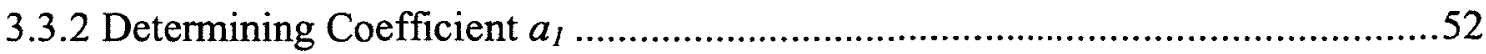

3.3.3 Determining parameter $A$ by fully plastic solution ........................................52

3.4 ApPlication of Estimation Methods on THREe-Dimensional Cracked

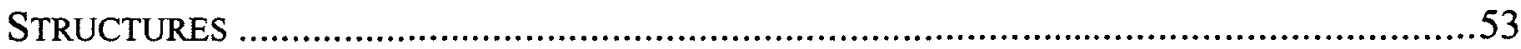

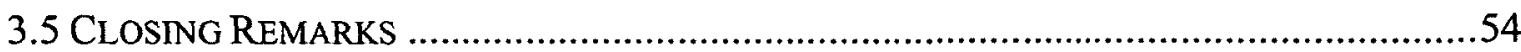

\section{CHAPTER 4 SOLUTION OF CONSTRAINT PARAMETER $\boldsymbol{A}$}

FOR 2D SPECIMENS UNDER UNIIAXIAL LOADING...............................................58

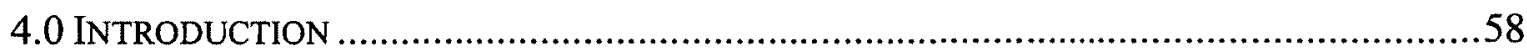

4.1 FINITE ELEMENT ANALYSIS OF MODIFIEd BOUNDARY LAYER FORMULATION ..........59

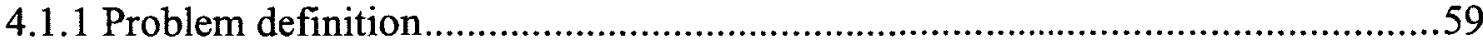

4.1.2 Finite element analysis and parameter $A$ determination...................................60

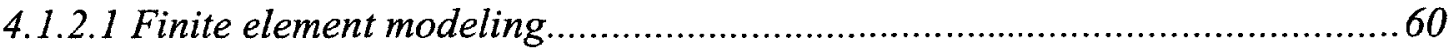

4.1.2.2 Determining parameter $A$ based on finite element analysis .......................61

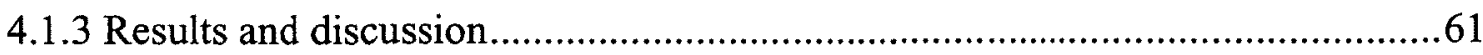

4.1.3.1 Verification of FEA model and analysis procedure....................................61

4.1.3.2 Numerical solution of parameter A...........................................................62

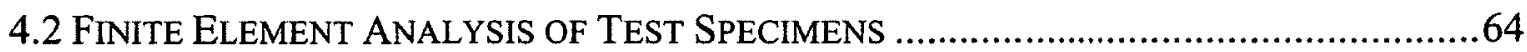

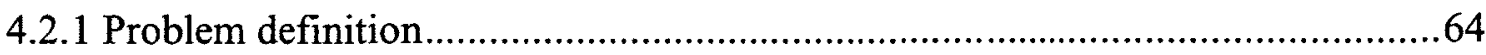

4.2.2 Finite element analysis and parameter $A$ determination....................................65

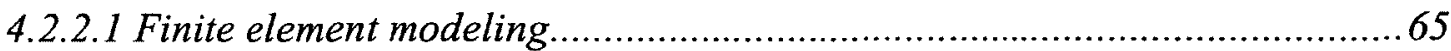

4.2.2.2 Determining parameter $A$ based on finite element analysis .........................66

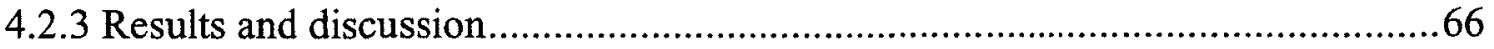

4.2.3.1 Verification of FEA model and analysis procedure..................................66

4.2.3.2 Numerical solution of parameter $A$ and discussion for constraint effect ....68

4.3 SOlUTION OF PARAMETER $A$ BY CURVE SHAPE SIMILARITY ......................................69

4.4 SOLUTION OF PARAMETER $A$ BY $T$-STRESS ...........................................................

vii 


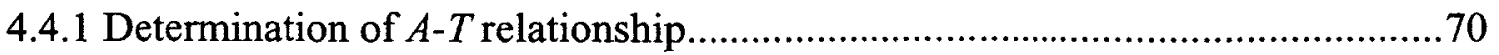

4.4.2 $T$-stress solution by weight function method .................................................72

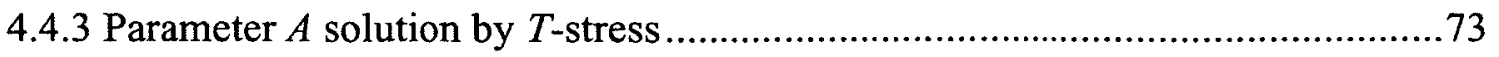

4.5 SOLUTION OF PARAMETER $A$ BY FUlly PLASTIC ANALYSIS .....................................75

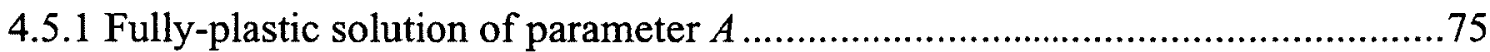

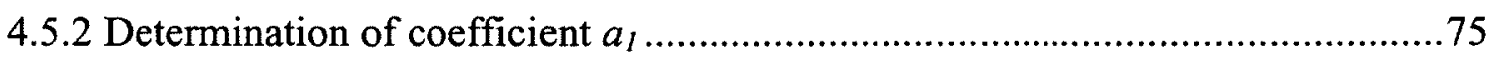

4.5.3 Parameter $A$ solution by fully-plastic analysis ................................................76

4.6 ESTIMATING OtHer CONSTRAINT PARAMETERS BY A SOLUTIONS..............................76

4.6.1 Estimating constraint parameter $A_{2}$ from parameter $A \ldots \ldots \ldots \ldots \ldots \ldots \ldots \ldots \ldots \ldots \ldots \ldots . . .77$

4.6.2 Estimating constraint parameter $Q$ from parameter $A$....................................78

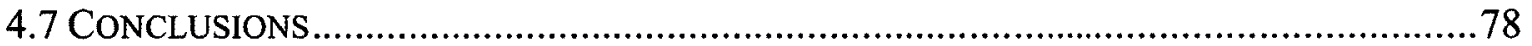

\section{CHAPTER 5 SOLUTION OF CONSTRAINT PARAMETER $\boldsymbol{A}$}

FOR 2D SPECIMENS UNDER BIAXIAL LOADING ….......................................116

5.0 INTRODUCTION

5.1 Finite ElEMENT ANALYSIS OF TeSt SPECIMENS ……........................................117

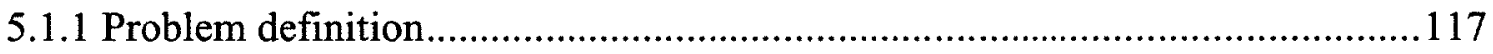

5.1.2 Finite element analysis and parameter $A$ determination.................................119

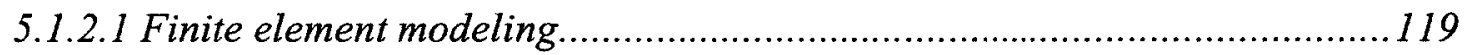

5.1.2.2 Determining parameter $A$ based on finite element analysis ......................119

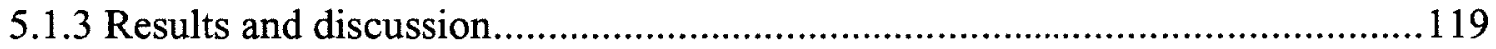

5.1.3.1 Verification of FEA model and analysis procedure.................................119

5.1.3.2 Numerical solution of parameter A and discussion for constraint effect ..120

5.2 SOLUTION OF PARAMETER $A$ BY CURVE-SHAPE SimILARITY ....................................122

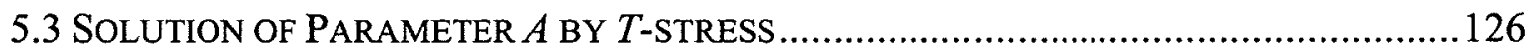

5.3.1 $T$-stress solution by weight function method ...............................................127

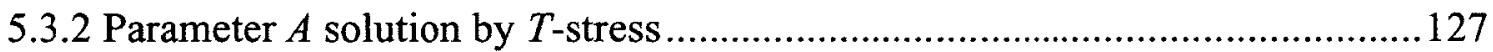

5.4 SOLUTION OF PARAMETER $A$ By FUlLy PLASTIC ANALYSIS .....................................130

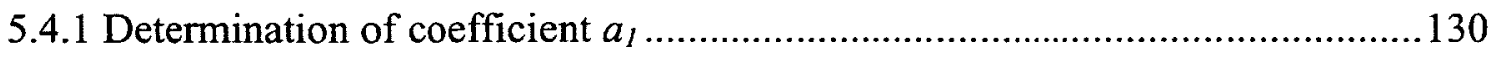

5.4.2 Parameter $A$ solution by fully plastic analysis .............................................131

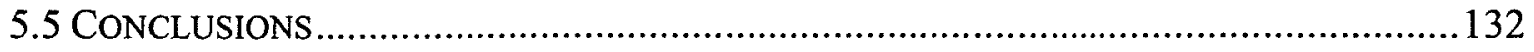




\section{CHAPTER 6 SOLUTION OF CONSTRAINT PARAMETER $\boldsymbol{A}$}

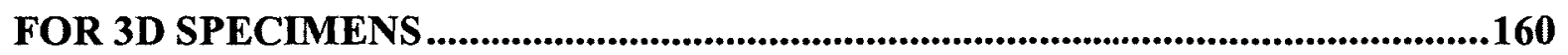

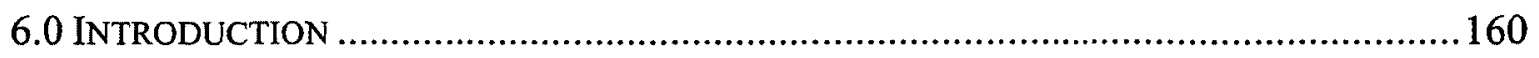

6.1 FINITE ELEMENT ANALYSIS OF 3D MODIFIED BOUNDARY LAYER ForMULATION..161

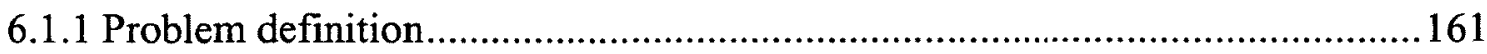

6.1.2 Finite element analysis and parameter $A$ determination..................................162

6.1.2.1 Finite element modeling..................................................................162

6.1.2.2 Determining parameter A based on finite element analysis .....................163

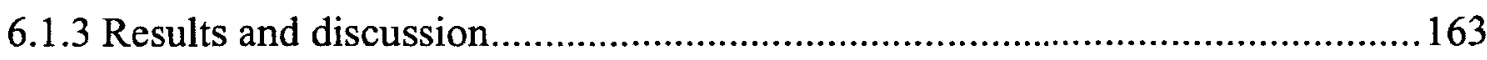

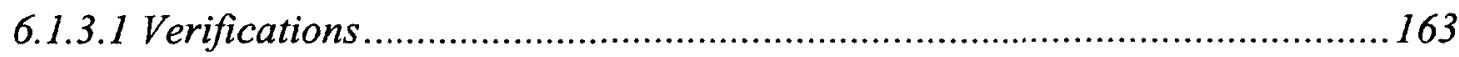

6.1.3.2 Numerical solutions of parameter A ….................................................. 164

6.2 FINITE ELEMENT ANALYSIS OF 3D TEST SPECIMENS..............................................165

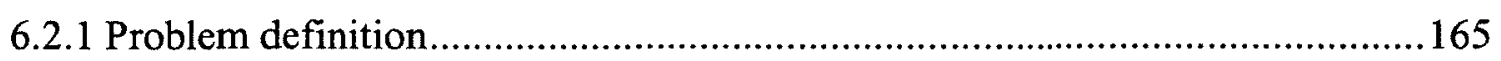

6.2.2 Finite element analysis and parameter $A$ determination................................166

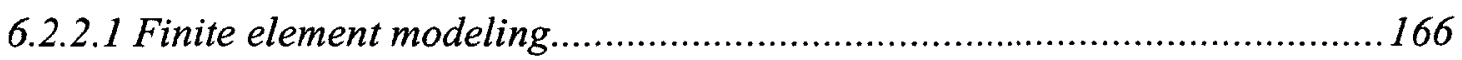

6.2.2.2 Determining parameter $A$ based on finite element analysis .....................167

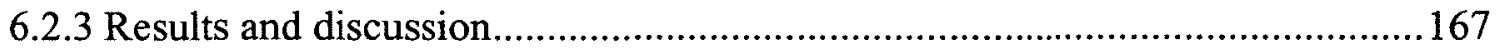

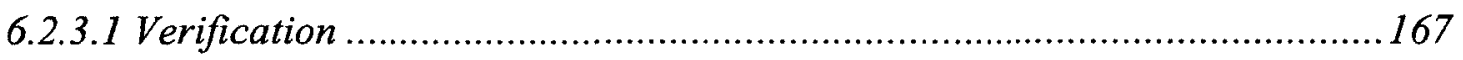

6.2.3.2 Numerical solutions of parameter $A$ and discussion for constraint effect.168

6.3 SOLUTION OF PARAMETER $A$ BY CURVE SHAPE SIMILARITY ...................................171

6.4 SOLUTION OF PARAMETER $A$ BY T-STRESS ............................................................ 173

6.4.1 Determination of $A-T$ relationship for 3D cases.........................................173

6.4.2 $T$-stress solution by finite element method ................................................. 174

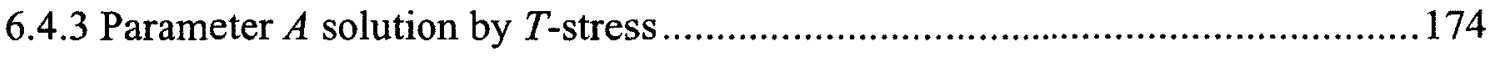

6.5 SOlution of Parameter $A$ By Fully Plastic ANALYSIS ...................................176

6.5.1 Fully plastic solution of parameter $A$........................................................176

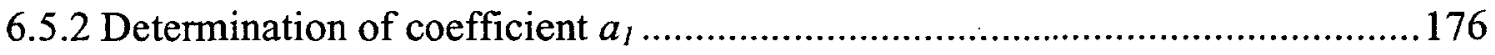

6.5.3 Parameter $A$ solution by fully plastic analysis ...............................................177

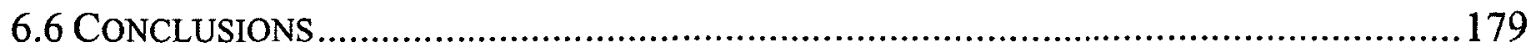

CHAPTER 7 CONCLUSIONS AND RECOMMENDATIONS .................................222 


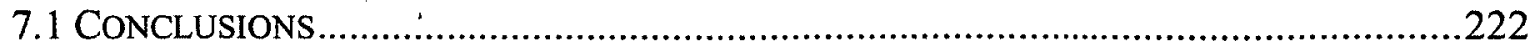

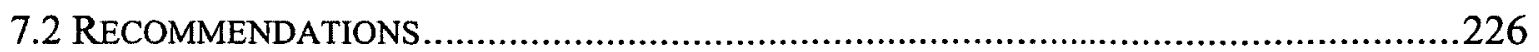

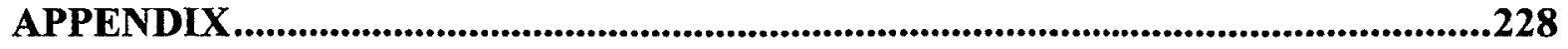

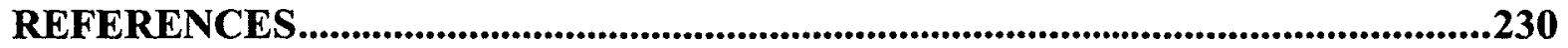

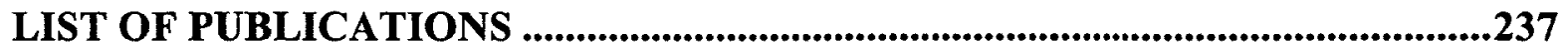




\section{LIST OF TABLES}

Table 4.1 Comparison of $A$ obtained from present and refined finite element models (MBL, $T=0)$ 80

Table 4.2 Comparison of $A$ values from present FEA and solutions from Nikishkov et al. (1995a), SSY problem 80

Table 4.3 $A$ values from FEA results for MBL problem (plane strain) 81

Table 4.4 Comparisons of the remote applied stress intensity factors, $K_{f a r}$ with those obtained from contour integral, $K_{\text {local }}$, for $n=10$ in the present MBL analyses (2D plane strain) 81

Table 4.5 Comparison of $A$ obtained from present and refined finite element models (SECP, $a / W=0.5$ ).

Table 4.6 Comparison of $A$ obtained from present and refined finite element models (SECP, $a / W=0.5$, further refinement in transition region)

Table 4.7 Comparison of values from present FEA and solutions from Nikishkov et al. (1995a), (SECP specimen, $n=5$ )

Table 4.8 Comparison of values from present FEA and solutions from Nikishkov et al. (1995a), (CCP specimen, $n=5)$

Table 4.9 Comparison of values from present FEA and solutions from Nikishkov et al. (1995a), (SECP specimen, $n=10$ ) 84

Table 4.10 Comparison of values from present FEA and solutions from Nikishkov et al.

$$
\text { (1995a), (CCP specimen, } n=10 \text { ) }
$$


Table 4.11 FEA results of $A$ for SECP specimens (plane strain under uniaxial tension load)

Table 4.12 FEA results of $A$ for CCP specimens (plane strain under uniaxial tension load)

Table 4.13 FEA results of $A$ for DECP specimens (plane strain under uniaxial tension load)

Table 4.14 The polynomial coefficients $e_{i j}$ for SECP specimens (plane strain under uniaxial tension load) 88

Table 4.15 The polynomial coefficients $e_{i j}$ for CCP specimens (plane strain under uniaxial tension load) 88

Table 4.16 The polynomial coefficients $e_{i j}$ for DECP specimens (plane strain under uniaxial tension load) 88

Table 4.17 The values of parameter $h$ for SECP, CCP and DECP specimens (plane strain under uniaxial tension load)

Table 4.18 Values of coefficients for one-to-one $A-T$ relationship polynomials (plane strain under uniaxial tension load) 89

Table 4.19 Coefficient $a_{l}$ values for SECP specimen (plane strain under uniaxial tension load) 90

Table 4.20 Coefficient $a_{l}$ values for CCP specimen (plane strain under uniaxial tension load) 90

Table 4.21 Coefficient $a_{l}$ values for DECP specimen (plane strain under uniaxial tension load) 90 
Table 4.22 The prediction of parameter $A_{2}$ for SECP specimen, $a / W=0.5$, compared with

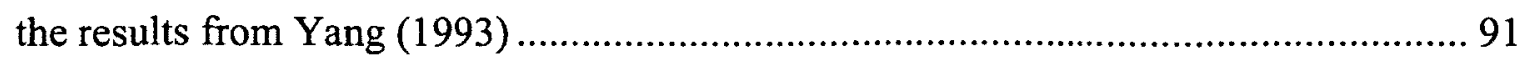

Table 4.23 The prediction of parameter $Q$ for DECP specimen, $a / W=0.5$, compared with

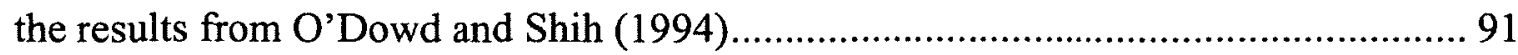

Table 5.1 Comparison of constraint parameter $Q$ for CCP under $a / W=0.5, \mathrm{n}=10, \lambda=0.5$, compared with results from O'Dowd et al. (1999).............................................. 134

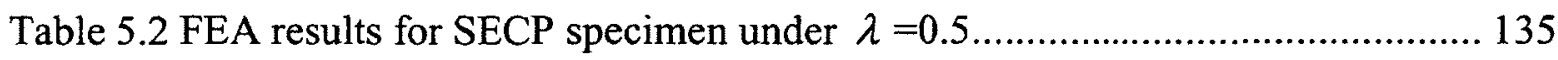

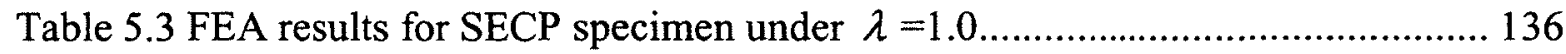

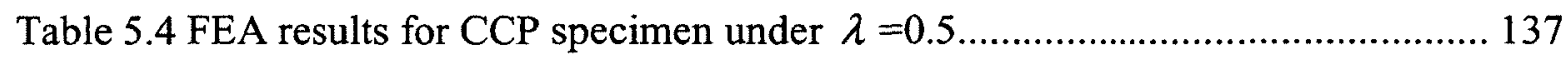

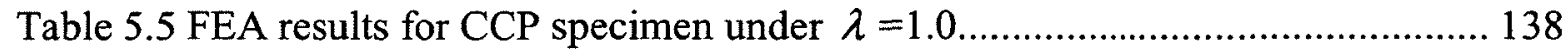

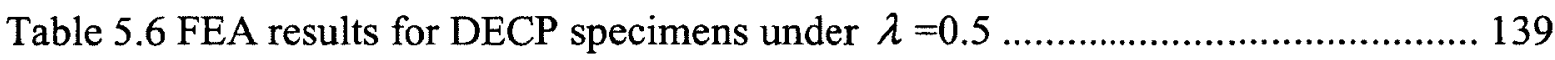

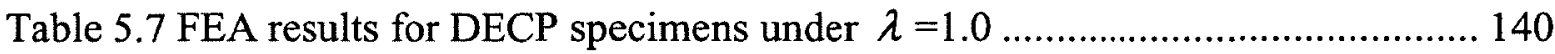

Table 5.8 The polynomial coefficients $e_{i j}$ for SECP specimens under $\lambda=0.5 \ldots \ldots \ldots \ldots 141$

Table 5.9 The polynomial coefficients $e_{i j}$ for CCP specimens under $\lambda=0.5 \ldots \ldots \ldots \ldots 141$

Table 5.10 The polynomial coefficients $e_{i j}$ for DECP specimens under $\lambda=0.5 \ldots \ldots \ldots 141$

Table 5.11 The polynomial coefficients $e_{i j}$ for SECP specimens under $\lambda=1.0 \ldots \ldots \ldots . .142$

Table 5.12 The polynomial coefficients $e_{i j}$ for CCP specimens under $\lambda=1.0 \ldots \ldots \ldots \ldots 142$

Table 5.13 The polynomial coefficients $e_{i j}$ for DECP specimens under $\lambda=1.0 \ldots \ldots \ldots 142$

Table 5.14 Limits of applicability in terms of normalized load, $\sigma / \sigma_{0}$, of the $A-T$

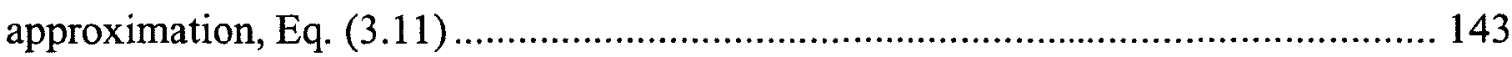

Table 5.15 Limits of applicability in terms of normalized load, $\sigma / \sigma_{0}$, of the simplified xiii 
Table 5.16 Coefficient $a_{1}$ values for SECP specimen .............................................. 144

Table 5.17 Coefficient $a_{1}$ values for CCP specimen .................................................. 144

Table 5.18 Coefficient $a_{1}$ values for DECP specimen ............................................ 144

Table 6.1 $A$ values from FEA results for the 3D MBL problem, plane I...................... 182

Table $6.2 A$ values from FEA results for the 3D MBL problem, plane II .................... 182

Table $6.3 A$ values from FEA results for the 3D MBL problem, plane III ................... 182

Table 6.4 Comparison of parameter $A_{2}$ between present results and those from Zhu et al. 183

Table 6.5 Variation of parameter $A$ along the crack-front of the 3D SECP, $a / W=0.3, n=5$

Table 6.6 Variation of $J$-integral along the crack-front of the 3D SECP, $a / W=0.3, n=5184$

Table $6.7 A$ values from FEA results for the 3D SECP specimen, $\lambda=0.0, a / W=0.1 \ldots \ldots .185$

Table $6.8 A$ values from FEA results for the 3D SECP specimen, $\lambda=0.0, a / W=0.3 \ldots \ldots .185$

Table $6.9 A$ values from FEA results for the 3D SECP specimen, $\lambda=0.0, a / W=0.7 \ldots \ldots .186$

Table $6.10 \mathrm{~A}$ values from FEA results for the 3D SECP specimen, $\lambda=1.0, a / W=0.1 \ldots .186$

Table 6.11 $A$ values from FEA results for the 3D SECP specimen, $\lambda=1.0, a / W=0.3 \ldots . .187$

Table 6.12 $\mathrm{A}$ values from FEA results for the 3D SECP specimen, $\lambda=1.0, a / W=0.7 \ldots . .187$

Table 6.13 The polynomial coefficients $e_{i j}$ for the 3D SECP specimen, $\lambda=0.0$, plane I 188

Table 6.14 The polynomial coefficients $e_{i j}$ for the 3D SECP specimen, $\lambda=0.0$, plane II 
Table 6.15 The polynomial coefficients $e_{i j}$ for the 3D SECP specimen, $\lambda=1.0$, plane I 189 Table 6.16 The polynomial coefficients $e_{i j}$ for the 3D SECP specimen, $\lambda=1.0$, plane II

Table 6.17 Values of coefficients for polynomial $A-T$ relationship, plane I.................. 190

Table 6.18 Values of coefficients for polynomial $A-T$ relationship, plane II ............... 190

Table 6.19 Numerical solutions of normalized $T$-stress $(V)$ for the 3D SECP specimen, $\lambda=0.0,1.0$, planes I and II

Table 6.20 Maximum load range of applicability of $A-T$ relation, $\sigma / \sigma_{0}$, for the 3D

SECP

Table 6.21 Coefficient $a_{1}$ values for the 3D SECP specimen, $\lambda=0.0$, planes I and II.... 192

Table 6.22 Coefficient $a_{1}$ values for the 3D SECP specimen, $\lambda=1.0$, planes I and II.... 192 


\section{LIST OF FIGURES}

Figure 2.1 Typical crack-tip (-front) stress field (Anderson (2005)) ......................... 33

Figure 2.2 Stress-strain curve for Ramberg-Osgood power-law strain hardening relation

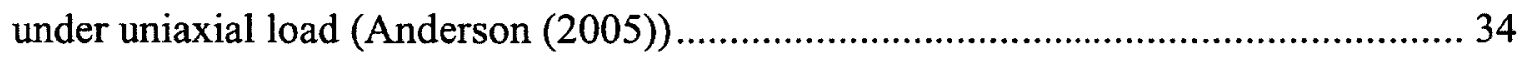

Figure 2.3 Modified boundary layer (MBL) problem model ................................... 35

Figure 2.4 The dependence of critical values of $J$-integral, $J_{c}$, on constraint level, $Q$,

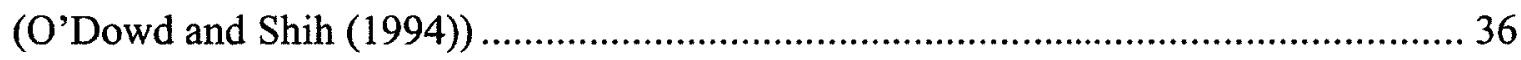

Figure 2.5 Illustration of the superposability of $J$-integral (Kumar et al. (1981)).......... 37

Figure 2.6 2D CCP model under biaxial loading condition................................. 38

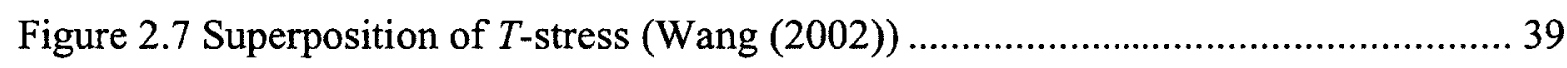

Figure 2.8 Illustration of one-to-one $Q-T$ correspondence for various hardening exponent

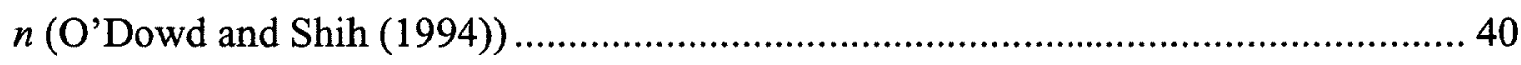

Figure 2.9 Curve shape similarity of parameter $A$ for SECP specimen with $\alpha=0.5$,

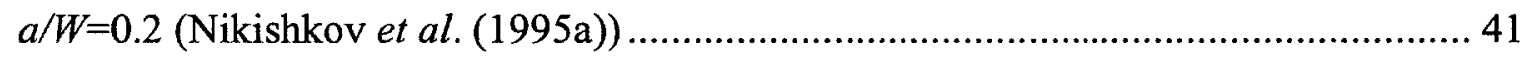

Figure 2.10 Illustration of terms "in-plane" and "out-of-plane" at $3 \mathrm{D}$ crack-front ........ 42

Figure 3.1 Shape similarity for curves of constraint parameter $A$ vs. remote load ratio

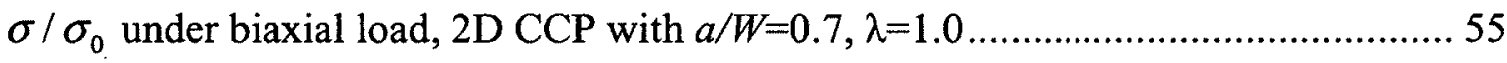

Figure 3.2 Curves of $A$ values from FEA data vs. normalized $T$-stress, $T / \sigma_{0}$, for $2 \mathrm{D}$

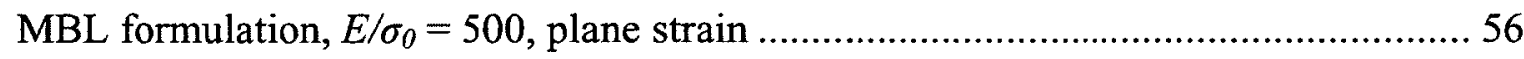

Figure 3.3 The superposition of small-scale yielding and fully plastic parts of constraint 
parameter $A$ for 2D CCP model with $a / W=0.4$ under uniaxial load 57

Figure 4.1 The modified boundary layer model …................................................... 92

Figure 4.2 FEA mesh for the modified boundary layer problem.................................. 93

Figure 4.3 Curves of $A$ values from FEA data vs. normalized $T$-stress, $T / \sigma_{0}$, for MBL,

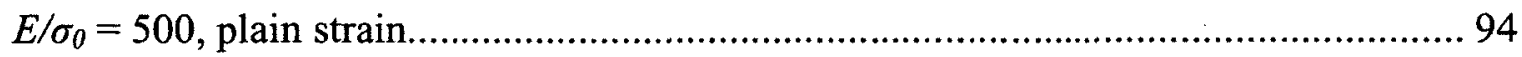

Figure 4.4 Comparison of $A_{T}\left(T / \sigma_{0}, n\right)$ for two different $E / \sigma_{0}$ ratios.............................. 94

Figure 4.5 Single edge cracked plate (SECP) model ................................................... 95

Figure 4.6 Centre cracked plate (CCP) model ................................................................ 96

Figure 4.7 Double edge cracked plate (DECP) model................................................... 97

Figure 4.8 Typical FEA mesh for plane strain models under uniaxial load, $a / W=0.3 \ldots 98$

Figure 4.9 Comparisons of predicted $A$ values by curve shape similarity with FEA data for SECP, $a / W=0.2$, plane strain under uniaxial tension load................................... 99

Figure 4.10 Comparisons of predicted $A$ values by curve shape similarity with FEA data

for SECP, $a / W=0.4$, plane strain under uniaxial tension load................................... 99

Figure 4.11 Comparisons of predicted $A$ values by curve shape similarity with FEA data for SECP, $a / W=0.6$, plane strain under uniaxial tension load............................... 100

Figure 4.12 Comparisons of predicted $A$ values by curve shape similarity with FEA data for SECP, $a / W=0.8$, plane strain under uniaxial tension load.............................. 100

Figure 4.13 Comparisons of predicted $A$ values by curve shape similarity with FEA data for CCP, $a / W=0.2$, plane strain under uniaxial tension load................................. 101

Figure 4.14 Comparisons of predicted $A$ values by curve shape similarity with FEA data for CCP, $a / W=0.4$, plane strain under uniaxial tension load. 101 
Figure 4.15 Comparisons of predicted $A$ values by curve shape similarity with FEA data for CCP, $a / W=0.6$, plane strain under uniaxial tension load

Figure 4.16 Comparisons of predicted $A$ values by curve shape similarity with FEA data for CCP, $a / W=0.8$, plane strain under uniaxial tension load 102

Figure 4.17 Comparisons of predicted $A$ values by curve shape similarity with FEA data for DECP, $a / W=0.2$, plane strain under uniaxial tension load. 103

Figure 4.18 Comparisons of predicted $A$ values by curve shape similarity with FEA data for DECP, $a / W=0.4$, plane strain under uniaxial tension load. 103

Figure 4.19 Comparisons of predicted $A$ values by curve shape similarity with FEA data for DECP, $a / W=0.6$, plane strain under uniaxial tension load. 104

Figure 4.20 Comparisons of predicted $A$ values by curve shape similarity with FEA data for DECP, $a / W=0.8$, plane strain under uniaxial tension load. 104

Figure 4.21 Comparisons of predicted $A$ values from $T$-stress with FEA data for SECP, $a / W=0.2$ 105

Figure 4.22 Comparisons of predicted $A$ values from $T$-stress with FEA data for CCP, $a / W=0.2$ 105

Figure 4.23 Comparisons of predicted $A$ values from $T$-stress with FEA data for DECP, $a / W=0.2$ 106

Figure 4.24 Results of $A, A_{T}$ and $\triangle A$ for SECP, $n=5$ and $a / W=0.4$, plane strain under uniaxial load. 107

Figure 4.25 Results of $A, A_{T}$ and $\Delta A$ for CCP, $n=5$ and $a / W=0.4$, plane strain under uniaxial load 
Figure 4.26 Results of $A, A_{T}$ and $\Delta A$ for DECP, $n=5$ and $a / W=0.4$, plane strain under uniaxial load

Figure 4.27 Differences between $A$ solutions from $T$-stress and from FEA, $\triangle A$, for SECP, $a / W=0.5$ with varying $n$

Figure 4.28 Comparisons of predicted $A$ values with FEA data for SECP, $a / W=0.1$, plane strain under uniaxial load

Figure 4.29 Comparisons of predicted $A$ values with FEA data for SECP, $a / W=0.5$, plane strain under uniaxial load

Figure 4.30 Comparisons of predicted $A$ values with FEA data for SECP, $a / W=0.8$, plane strain under uniaxial load

Figure 4.31 Comparisons of predicted $A$ values with FEA data for CCP, $a / W=0.1$, plane strain under uniaxial load

Figure 4.32 Comparisons of predicted $A$ values with FEA data for CCP, $a / W=0.5$, plane strain under uniaxial load

Figure 4.33 Comparisons of predicted $A$ values with FEA data for CCP, $a / W=0.8$, plane strain under uniaxial load 113

Figure 4.34 Comparisons of predicted $A$ values with FEA data for DECP, $a / W=0.1$, plane strain under uniaxial load 114

Figure 4.35 Comparisons of predicted $A$ values with FEA data for DECP, $a / W=0.5$, plane strain under uniaxial load 114

Figure 4.36 Comparisons of predicted $A$ values with FEA data for DECP, $a / W=0.8$, plane strain under uniaxial load 
Figure 5.1 Single edge cracked plate (SECP) model 145

Figure 5.2 Centre cracked plate (CCP) model ........................................................... 146

Figure 5.3 Double edge cracked plate (DECP) model............................................ 147

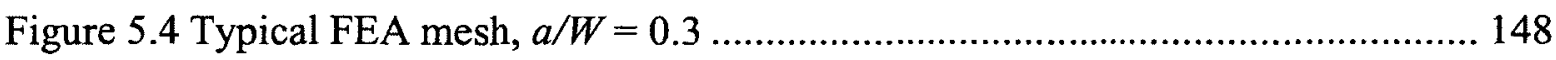

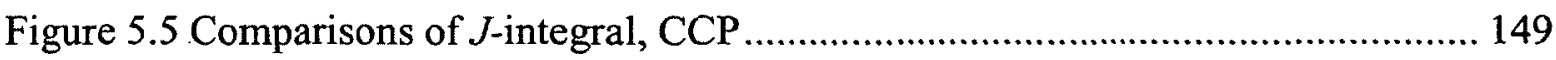

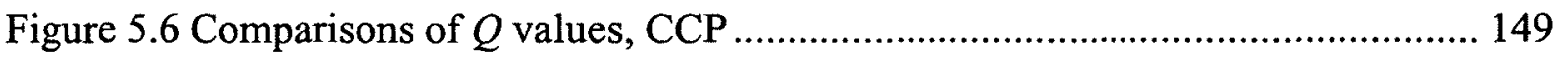

Figure 5.7 Comparisons of predicted $A$ values by curve shape similarity with FEA data

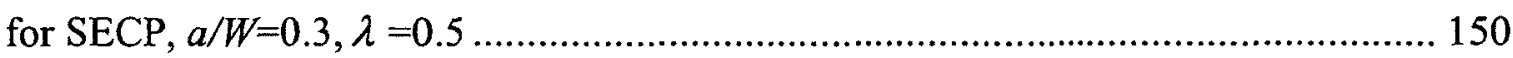

Figure 5.8 Comparisons of predicted $A$ values by curve shape similarity with FEA data for SECP, $\mathrm{a} / \mathrm{W}=0.7, \lambda=0.5$ 150

Figure 5.9 Comparisons of predicted $A$ values by curve shape similarity with FEA data for CCP, $a / W=0.3, \lambda=0.5$ 151

Figure 5.10 Comparisons of predicted $A$ values by curve shape similarity with FEA data for CCP, $a / W=0.7, \lambda=0.5$ 151

Figure 5.11 Comparisons of predicted $A$ values by curve shape similarity with FEA data for DECP, $a / W=0.3, \lambda=0.5$. 152

Figure 5.12 Comparisons of predicted $A$ values by curve shape similarity with FEA data for DECP, $a / W=0.7, \lambda=0.5$ 152

Figure 5.13 Comparisons of predicted $A$ values by curve shape similarity with FEA data for SECP, $a / W=0.3, \lambda=1.0$ 153

Figure 5.14 Comparisons of predicted $A$ values by curve shape similarity with FEA data for SECP, $a / W=0.7, \lambda=1.0$ 153 
Figure 5.15 Comparisons of predicted $A$ values by curve shape similarity with FEA data for CCP $, a / W=0.3, \lambda=1.0$

Figure 5.16 Comparisons of predicted $A$ values by curve shape similarity with FEA data for $\mathrm{CCP}, a / W=0.7, \lambda=1.0$ 154

Figure 5.17 Comparisons of predicted $A$ values by curve shape similarity with FEA data for DECP, $a / W=0.3, \lambda=1.0$. 155

Figure 5.18 Comparisons of predicted $A$ values by curve shape similarity with FEA data for DECP, $a / W=0.7,=1.0$ 155

Figure 5.19 Comparisons of predicted $A$ values from $T$-stress with FEA data for CCP, $a / W=0.1, \lambda=0.5$

Figure 5.20 Comparisons of predicted $A$ values from $T$-stress with FEA data for CCP, $a / W=0.5, \lambda=0.5$

Figure 5.21 Comparisons of predicted $A$ values from $T$-stress with FEA data for CCP, $a / W=0.1, \lambda=1.0$ 157

Figure 5.22 Comparisons of predicted $A$ values from $T$-stress with FEA data for CCP, $\mathrm{a} / \mathrm{W}=0.5, \lambda=1.0$ 157

Figure 5.23 Comparisons of predicted $A$ values by superposition with FEA data for CCP, $a / W=0.1, \lambda=0.5$ 158

Figure 5.24 Comparisons of predicted $A$ values by superposition with FEA data for CCP, $a / W=0.5, \lambda=0.5$ 158

Figure 5.25 Comparisons of predicted $A$ values by superposition with FEA data for CCP, $a / W=0.1, \lambda=1.0$ 159 
Figure 5.26 Comparisons of predicted $A$ values by superposition with FEA data for CCP,

$$
a / W=0.5, \lambda=1.0
$$

Figure 6.1 The modified boundary layer model

Figure 6.2 FEA mesh for the modified boundary layer problem 194

Figure 6.3 $A-T$ relationship curves from 3D MBL formulation, plane I 195

Figure 6.4 A-T relationship curves from 3D MBL formulation, plane II 195

Figure 6.5 $A-T$ relationship curves from 3D MBL formulation, plane III. 196

Figure 6.6 3D single edge cracked plate (SECP) model.

Figure 6.7 Typical FEA mesh for 3D SECP models, $a / W=0.3$

Figure 6.8 Comparison of normalized $T$-stress $(V)$ between FEA results and those from Nakamura and Parks (1992).

Figure 6.9 Comparison of parameter $A_{2}$ between present results and those from Zhu et al. (2002) 199

Figure 6.10 Variation of parameter $A$ along the crack-front of the 3D SECP, $a / W=0.3$, $n=5$ 200

Figure 6.11 Variation of $J$-integral along the crack-front of the 3D SECP, $a / W=0.3, n=5$ 201

Figure 6.12 Comparisons of $A$ values predicted by curve shape similarity with FEA data for the 3D SECP, $\lambda=0.0, a / W=0.1$, (a) plane I and (b) plane II. 202

Figure 6.14 Comparisons of $A$ values predicted by curve shape similarity with FEA data for the 3D SECP, $\lambda=0.0, a / W=0.7$, (a) plane I and (b) plane II. 204

Figure 6.15 Comparisons of $A$ values predicted by curve shape similarity with FEA data 
for the 3D SECP, $\lambda=1.0, a / W=0.1$, (a) plane I and (b) plane II. 205

Figure 6.16 Comparisons of $A$ values predicted by curve shape similarity with FEA data for the 3D SECP, $\lambda=1.0, a / W=0.3$, (a) plane I and (b) plane II. 206

Figure 6.17 Comparisons of $A$ values predicted by curve shape similarity with FEA data for the 3D SECP, $\lambda=1.0, a / W=0.7$, (a) plane I and (b) plane II. 207

Figure 6.18 Comparisons of $A$ values predicted from $T$-stress with FEA data for the 3D SECP, $\lambda=0.0, a / W=0.1$, (a) plane I and (b) plane II 208

Figure 6.19 Comparisons of $A$ values predicted from $T$-stress with FEA data for the 3D SECP, $\lambda=0.0, a / W=0.3$, (a) plane I and (b) plane II. 209

Figure 6.20 Comparisons of $A$ values predicted from $T$-stress with FEA data for the 3D

SECP, $\lambda=0.0, a / W=0.7$, (a) plane I and (b) plane II 210

Figure 6.21 Comparisons of $A$ values predicted from $T$-stress with FEA data for the 3D

SECP, $\lambda=1.0, a / W=0.1$, (a) plane I and (b) plane II 211

Figure 6.22 Comparisons of $A$ values predicted from $T$-stress with FEA data for the 3D SECP, $\lambda=1.0, a / W=0.3$, (a) plane I and (b) plane II. 212

Figure 6.23 Comparisons of $A$ values predicted from $T$-stress with FEA data for the 3D SECP, $\lambda=1.0, a / W=0.7$, (a) plane I and (b) plane II 213

Figure 6.24 Comparisons of $A$ values predicted by superposition with FEA data for the 3D SECP, $\lambda=0.0, a / W=0.1$, (a) plane I and (b) plane II. 214

Figure 6.25 Comparisons of $A$ values predicted by superposition with FEA data for the 3D SECP, $\lambda=0.0, a / W=0.3$, (a) plane I and (b) plane II. 215

Figure 6.26 Comparisons of $A$ values predicted by superposition with FEA data for the 
3D SECP, $\lambda=0.0, a / W=0.7$, (a) plane I and (b) plane II........................................ 216

Figure 6.27 Comparisons of $A$ values predicted by superposition with FEA data for the

3D SECP, $\lambda=1.0, a / W=0.1$, (a) plane I and (b) plane II 217

Figure 6.28 Comparisons of $A$ values predicted by superposition with FEA data for the 3D SECP, $\lambda=1.0, a / W=0.3$, (a) plane I and (b) plane II. 218

Figure 6.29 Comparisons of $A$ values predicted by superposition with FEA data for the 3D SECP, $\lambda=1.0, a / W=0.7$, (a) plane I and (b) plane II. 219

Figure 6.30 Results of $A, A_{T}$ and $\Delta A$ for the 3D SECP, $n=5, \lambda=0.0$, and $a / W=0.1 \ldots 220$ Figure 6.31 Results of $A, A_{T}$ and $\Delta A$ for the 3D SECP, $n=5, \lambda=1.0$, and $a / W=0.1 \ldots 221$ 


\section{NOMENCLATURE}

$a$

$a_{1}$

$a, b, c, d$

$a_{i}, b_{i}, c_{i}, d_{i}$

A

$A_{T}$

$A_{0}, A_{l}, A_{2}$

$A_{2}$

$A_{S S Y}$

$b_{1}, b_{2}, b_{3}$

$D_{1}, D_{2}$

$d_{1}, d_{2}, d_{3}$

$e_{i j}$

E

fadj

$f_{i j}$

$g_{i}$

$g_{1,} g_{2,} g_{3}$

$h$

$h_{1}$ depth of crack in the test specimen

coefficient for equations determining fully plastic solutions of constraint parameter $A$

coefficients of cubic equation for calculating constraint parameter $A$ from FEA results

coefficients of expression on deviation of the asymptotic stress fields from the FEA stress solution for $i$ th fitting point constraint parameter (second fracture parameter) in $J$ - $A$ crack-tip fields

$T$-stress-based solution of constraint parameter $A$ amplitudes of three-term asymptotic expansion for $J-A$ or $J-A_{2}$ crack-tip fields constraint parameter (second fracture parameter) in $J-A_{2}$ crack-tip fields small-scale yielding FEA solution of constraint parameter $A$ polynomial coefficients for $Q-T$ relation equation coefficients of formulas for predicting $T$-stress by weight function method coefficients of empirical formulas for predicting constraint parameter $A$ coefficients of empirical expressions for calculating coefficients $d_{1}, d_{2}, d_{3}$ Young's modulus adjusting factor in empirical formulas for predicting constraint parameter $A$

non-dimensional angular function in Williams' series expansion for stress angular functions for displacement boundary conditions in small-scale yielding model

coefficients in equations for predicting constraint parameter $A$ coefficient of empirical expression for calculating adjusting factor $f_{a d j}$ function in expression of fully plastic solution of $J$-integral 
half length of the test specimens

$I_{n}$

scaling integral depending on hardening exponent $n$

$J$

$J$-integral

$J_{c}$

critical value of $J$-integral

$J^{\text {center }}$

$J$-integral in the middle plane of 3D cracked model

$J_{E}$

elastic solution of $J$-integral

$J_{P}$

fully plastic solution of $J$-integral

$K_{I}$

stress intensity factor for mode I

$L$

crack characteristic dimension in $J-A_{2}$ crack tip fields

$m_{1}, m_{2}, m_{3}$

polynomial coefficients for $A-T$ relation equation

$n$

material hardening exponent

$n_{r}$

number of elements in radial direction at crack tip core region

$n_{\theta}$

number of elements in angular direction at crack tip core region

$Q$

constraint parameter (second fracture parameter) in $J-Q$ crack tip fields

$r$

radius in polar coordinates at crack tip

$\bar{r}$

dimensionless radius in polar coordinates at crack tip

$\bar{r}_{i}$

dimensionless radius in polar coordinates at crack tip for $i$ th fitting point

$r_{\mathrm{p}}$

radius of crack tip plastic zone

$\mathrm{R}$

maximum radius of the small-scale yielding model

$s, t$

powers in $J-A$ crack-tip fields

$s_{1}, s_{2}, s_{3}$

powers in $J-A_{2}$ crack-tip fields

$T$

$T$-stress

$u_{x}, u_{y}$

boundary displacement components in $x$ and $y$ directions of cartesian

coordinates

V

normalized $T$-stress

$V_{0}, V_{I}$

coefficients of formulas for predicting $T$-stress by weight function method

$w_{i}$ weight for the $i$ th fitting point

W

width of test specimen

$x, y, z$

Cartesian coordinates 


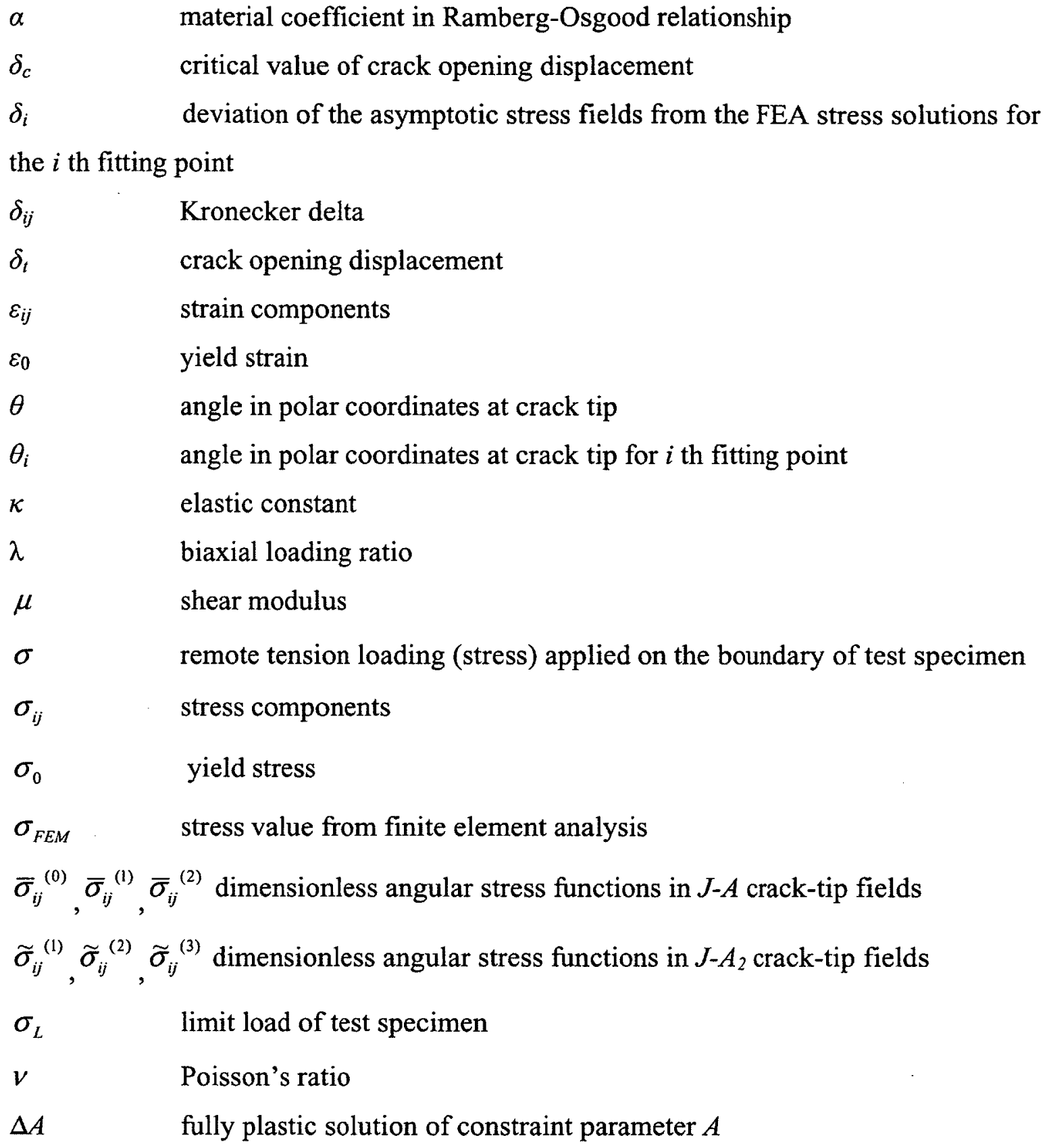




\section{CHAPTER 1 INTRODUCTION}

Two main divisions of fracture mechanics are: linear elastic fracture mechanics (LEFM) and elastic-plastic fracture mechanics (EPFM). In the earlier period of fracture mechanics development, characterization methods for material fracture were mainly based on a oneparameter approach for either elastic or elastic-plastic fracture. Since the 1980's, twoparameter methods have become more and more widely used in characterizing fracture for elastic-plastic fracture mechanics.

Irwin (1957) proposed and named the stress intensity factor $K$ to describe the stress state (stress intensity) near the tip (front) of a crack in a linear elastic material. Later, Hutchinson (1968) and Rice and Rosengren (1968) developed a one-parameter asymptotic solution of fields (HRR field) to define the one-parameter fracture criterion for elastic-plastic fracture cases. The base of the one-parameter criterion is $J$-integral (a path independent integral), which is proposed by Rice (1968).

For elastic-plastic fracture, it is well known that in many cases which are of practical significance, a one-parameter approach is not sufficient to describe the near-tip fields. For mode I plane-strain loading, for example, it is now known that the near-tip stress and displacement fields depend on crack geometry, type of loading and strain hardening of the material. Obviously, a single parameter $J$-integral, which represents the applied loading, is not enough to characterize the near-tip crack fields. Generally, one-parameter $J$-integral approach only works well for elastic-plastic conditions with high constraint. Therefore, to overcome the limitation of one-parameter $J$-based fracture mechanics approach, a second parameter needs to be induced to describe the effect from crack geometry, loading type and material hardening, etc.

Under linear elastic fracture state, Williams (1957) has suggested a series solution for $2 \mathrm{D}$ crack tip fields. In the series solution, applied loading on crack is represented by stress intensity factor $K_{I}$ (mode I fracture); meanwhile constraint effect from specimen geometry and loading configuration is described by $T$-stress, a uniform stress parallel to 
the crack face. The Williams' series solution is basis of the so-called $K-T$ two-parameter approach for linear elastic fracture mechanics. Based on finite element analysis, Betegon and Hancock (1991) as well as Al-Ani and Hancock (1991) extended the $K-T$ twoparameter approach from elastic material to elastic-plastic material, where $K$ is substituted by the $J$-integral as appropriate for an elastic-plastic state, i.e. $J-T$ approach. However, generally the $J-T$ two-parameter approach is only suitable for small-scale yielding (SSY), as $T$-stress is a parameter valid in the linear elastic domain.

In fact, several two-parameter approaches for elastic-plastic crack-tip field have been proposed for overcoming the limitation of one-parameter $J$-based approach from 1980's. Li and Wang (1986) and Sharma and Aravas (1991) first proposed the second term of the asymptotic expansion for the mode I plane-strain condition of a power-law hardening material. In the investigation of $\mathrm{Li}$ and Wang, the amplitude of the second term in the asymptotic expansion of the crack field $\left(K_{2}\right)$ is used as the second parameter of the two-parameter approach. They determined the value of $K_{2}$ by matching the two-term expansion with a numerical solution in the region of interest.

O'Dowd and Shih $(1991,1992)$ suggested the $J-Q$ approach based on the main feature of second order terms in asymptotic expansion. The second fracture parameter $Q$ which arises in the plasticity analysis is defined as the difference between the stresses in the crack-tip region determined by numerical analysis and by the HRR or small-scale yielding stress fields.

Following the steps of Li and Wang (1986) and Sharna and Aravas (1991), Yang, Chao and Sutton (Yang (1993), Yang et al. (1993)) conducted a more complete and sophisticated analysis for higher order terms of the asymptotic expansion of stress and displacement fields in the near crack-tip region. They derived a three-term expansion of the crack-tip field with two fracture parameters: the $J$-integral and a second fracture parameter, $A_{2}$, which is the dimensionless amplitude of the second order term of the expansion. This approach is the well-known $J-A_{2}$ two-parameter approach. Yang, Chao and Sutton have confirmed that in general a two-term expansion is not enough to predict accurately the field in the interested area near crack-tip, but using more than three terms 
in the asymptotic expansion of the field is redundant for plane-strain mode I cracks. Subsequently, Nikishkov et al. (Nikishkov (1995), Nikishkov et al. (1995a)) presented the same three-term expansion in another format and also suggested to use the magnitude of the second term in the series expansion, $A$, together with $J$-integral, to characterize the crack-tip fields ( $J-A$ approach). The $A$ used by Nikishkov et al. (1995a) is just a different form of normalizing the magnitude of the second term, thus $A_{2}$ and $A$ are directly related, which will be discussed in detail later in chapter 2 .

Overall, it has been shown that those two-parameter approaches $\left(J-T, J-Q\right.$ and $\left.J-A_{2}\right)$ provide effective characterization of plane strain elastic-plastic crack-tip fields in a variety of crack configurations and loading conditions. Current defect assessments and structural integrity procedures have incorporated the constraint effects, see the paper of Ainsworth et al. (2000) for example.

In order to use the constraint-based structural integrity procedures, both the first parameter ( $J$-integral) and the second parameter (constraint parameter) need to be obtained for the cracked components under consideration. Much of the effort had been focused on the $J$-integral solutions in the early development of EPFM; significant research efforts are made more recently to obtain the constraint parameters. In particular, the numerical and analytical methods to obtain $T$-stress for two-dimensional (2D) and three-dimensional (3D) crack geometries were well developed (see Kfouri (1986), Nakamura and Parks (1992) as well as Wang (2002)). Solutions of $T$-stress were developed for wide range of $2 \mathrm{D}$ and $3 \mathrm{D}$ crack geometries and loading conditions, for example, see the papers of Wang et al. (Wang (2002, 2003, 2004), Wang and Yu (2005)).

However, unlike the $T$-stress (second elastic parameter), the determination of the second elastic-plastic parameters, such as $Q$ and $A_{2}(A)$ is not as well established. Since elastic-plastic analysis involves material nonlinearities, these parameters will depend on the material hardening characteristics, specimen/crack geometry and external loading conditions.

Basically, the value of $Q$ parameter can be determined through its original definition based on finite element results. Obviously it makes the determination of $Q$ factor a laborious work for a wide range of loading, geometric and material properties and is not 
appropriate for engineering application or even theoretical research. To obtain the $Q$ factors more conveniently, based on the $J-Q$ and the $J-T$ two-parameter approach theories, O'Dowd (1995) has suggested the one-to-one relationships between the $Q$ factor and the $T$-stress to calculate the $Q$ factor from the elastic $T$-stress under small scale to contained yielding conditions. O'Dowd (1995) also suggests using pure power law hardening solutions to estimate $Q$ factors under fully plastic conditions. Finally, by interpolating between the small-scale yielding solutions based on $T$-stress and the fully plastic solutions, he proposed an overall empirical formula to estimate $Q$ factors from small- to large-scale yielding. Nonetheless, the amount of available solutions for the $Q$ parameter is very limited, whether for $2 \mathrm{D}$ or for $3 \mathrm{D}$ crack geometries. Several solutions for $Q$ parameter can be found for 2D (Gullerud and Dodds (1995), Wu et al. (1995)) and 3D crack geometries (Wang (2009)).

As for the parameter $A_{2}$ (or $A$ ), Yang et al. (Yang (1993), Yang et al. (1993)) determine the $A_{2}$ values by matching the three-term expansion on one stress component with the finite element solutions at one or several locations (points) within the plastic zone; Nikishkov et al. (1995a) suggested an algorithm to obtain the $A$ values by fitting the three-term expansion using one typical stress component with the finite element results in the region which is significant for the fracture process based on a least squares procedure. Both of these two methods for determining $A$ are based on finite element analysis (FEA) results. Just as mentioned above for the $Q$ factor determination, it is difficult to determine the $A_{2}(A)$ values for a wide range of loading, geometric and material properties by FEA. In addition, no empirical/estimation method for parameter $A_{2}$ (or $A$ ) determination is found in the literature. Therefore, for both $2 \mathrm{D}$ and $3 \mathrm{D}$ crack geometries, few solutions for $A_{2}(A)$ are available; and all of these solutions are obtained based on FEA results, either for 2D cracked specimens (Yang et al. (1993), Nikishkov et al. (1995a)) or for 3D cracked specimens (Nikishkov et al. (1995b), Zhu et al. (2002)).

It can therefore be concluded that, for the convenience of engineering application and theoretical investigation, further systematic developments of estimation methodologies for both constraint parameters $Q$ and $A_{2}(A)$ are desirable. 
Although many investigations have obtained crack constraint parameters during last two or three decades, the knowledge of constraint effects in crack region of cracked bodies is still limited. In particular, investigations for constraint effects based on constraint parameters $Q$ and $A_{2}(A)$, which were proposed in the 1990's together with $J-Q$ and $J-A_{2}(J-A)$ two-parameter approaches, are quite scarce. Most of the studies on $J-Q$ and $J-A_{2}(J-A)$ approaches are focused on two-dimensional (2D) crack geometries under uniaxial loading condition. For 2D cracked bodies under biaxial loading, few investigations of the $J-Q$ approach are carried out (see the paper of O'Dowd et al. (1999) as an example); and only one study of the $J-A_{2}$ approach (Wang et al. (2010)) is reported; no paper based on the $J-A$ approach is found in the literature. However, biaxial loading cases are of equal theoretical and engineering practical significance as uniaxial loading cases.

Up to now, most elastic-plastic fracture mechanics studies based on two-parameter approaches (J-T, J-Q or $J-A_{2}(A)$ approach) were focused on two-dimensional (2D) plane strain models. A real cracked structure is generally three-dimensional (3D). The difficulty of $3 \mathrm{D}$ crack problems lies in the $3 \mathrm{D}$ characteristics of the stress and strain fields at the crack front. Furthermore, the mechanism of fracture for 3D cracks is more complex than that for 2D cracks. For 2D (plane-strain and plane-stress) cracked components, only inplane constraints near the crack tip need to be considered. On the other hand, a real (3D) crack structure has both in-plane (perpendicular to the crack-front) and out-of plane (parallel to the crack front) constraints at the crack-front. Therefore, the fracture constraints in 3D cracks may be quite different from $2 \mathrm{D}$ cases.

For three-dimensional (3D) crack configurations, which are of engineering practical significant, the understanding of constraint effects and the corresponding two-parameter characterization is still quite limited. Several studies on 3D crack geometries through the $J-Q$ two-parameter approach have been reported in the literature. With most of them based on the uniaxial loading condition, few of these mentioned studies are carried out for 3D crack geometries under biaxial loading (e.g. papers of Dodds et al. (1993) and Wang (2009)).

As for investigations for 3D cracked bodies by $J-A_{2}$ (or $J-A$ ) approach, to the 
author's knowledge, only four papers have been found in the open literature. By $J-A$ twoparameter approach, Nikishkov et al. (1995b) conducted a crack-front field investigation of a semi-elliptical surface crack in a uniaxial tension plate. Kim et al. (2001) reported research on a 3D modified boundary layer problem based on the $J-A_{2}$ approach. Also based on the $J-A_{2}$ approach, Zhu et al. (2002) completed an analysis for two 3D specimens: through center cracked plate (CCP) under uniaxial loading and single edge notched bend specimen (SENB). Kim et al. (2003) carried out an investigation of the effect of specimen size and crack depth on 3D crack-front constraint for SENB specimens by $J-A_{2}$ approach. No implemented investigation on 3D crack structure under biaxial case is reported in any of these four papers. However, actually, most real (3D) crack structures, whether surface cracked or through cracked structures, undergo biaxial loading instead of simple uniaxial loading condition, such as, the surface cracks in nuclear pressure vessels possess complex biaxial stress states under pressurized thermal shock condition.

\subsection{Research Objectives}

Based on the description and analysis in above paragraphs, several aspects of necessary further researches on two-parameter approaches of elastic-plastic fracture mechanics are found. They are:

(1) estimation method development and more solutions for value of constraint parameter $A_{2}(A)$ to facilitate the theoretical research and engineering application of $J-A_{2}$ $\left(J-A_{2}\right)$ approach, for both 2D and 3D cracked bodies;

(2) more estimation methods and more solutions for value of constraint parameter $Q$ to facilitate the theoretical research and engineering application of $J-Q$ approach, for both 2D and 3D cracked bodies;

(3) understanding of constraint effect (in particular, described by constraint parameter $\left.A_{2}(A)\right)$ on 2D crack geometries under uniaxial loading and, in particular, under biaxial loading;

(4) understanding of constraint effect (in particular, described by constraint parameter $\left.A_{2}(A)\right)$ on 3D crack geometries under uniaxial loading and, in particular, under 
biaxial loading.

Therefore, the objectives of the present thesis are:

(1) Detailed finite element analysis will be conducted to study the crack-tip (-front) fields and determine the numerical solutions of constraint parameter $A$ for $2 \mathrm{D}$ and $3 \mathrm{D}$ cracked test specimens under uniaxial and biaxial loading for wide range of geometries, material properties and loading conditions (from small- to large-scale yielding);

(2) based on the numerical solutions of parameter $A$, constraint effect under uniaxial and biaxial loading on 2D and 3D specimens will be studied;

(3) three estimating methodologies will be proposed to estimate the constraint parameter $A$, which are based on the shape similarity of parameter $A$ curves, $A-T$ relationship and fully plastic solution of parameter $A$, respectively;

(4) it will be demonstrated that the obtained solution of constraint parameter $A$ can be used to predict other two common-used constraint parameter $Q$ and $A_{2}$ through the relationships among $A, Q$ and $A_{2}$.

\subsection{Outline of Thesis}

The thesis is organized as follows according to the implementation of research objectives mentioned above. Based on the theoretical back ground and literature review (chapter 2), three sets of methodologies to estimate values of constraint parameter $A$ conveniently and accurately have been developed in chapter 3 . They are: estimating parameter $A$ by curve shape similarity, predicting parameter $A$ values from $T$-stress directly and determining parameter $A$ based on fully plastic solutions of $A$.

The extensive finite element analyses (FEA) for 2D specimens under uniaxial loading, 2D specimens under biaxial loading, 3D specimens under uniaxial and biaxial loading, are presented in chapter 4,5 and 6, respectively. Obtained FEA results are used to determine numerical solutions of constraint parameter $A$ by a least square fitting method suggested by Nikishkov et al. (1995a). Determined numerical solutions of parameter $A$ are tabulated in chapter 4,5 and 6 , respectively. Based on the numerical solution of constraint parameter $A$, constraint effects are discussed in chapters 4,5 and 6 . 
Through the numerical solution of $A$, estimating formulas of $A$ corresponding to the three sets of methodologies are proposed and validated in chapter 4, 5 and 6, for $2 \mathrm{D}$ models under uniaxial loading, 2D models under biaxial loading, 3D ones under uniaxial and biaxial loading, respectively.

In addition, through several practical examples, a finding is demonstrated in chapter 4. It is, through the relationships between constraint parameter $A$ and other two commonused parameters, $Q$ and $A_{2}$, which have been summarized in chapter 2, the obtained parameter $A$ solutions can be utilized to predict $Q$ and $A_{2}$.

In chapter 4, first, extensive finite element analyses are conducted to obtain numerical solutions of $A$ term for three mode I crack plane-strain specimens, i.e. single edge cracked plate (SECP), center cracked plate (CCP) and double edge cracked plate (DECP), for wide range of geometries, material properties and loading conditions (uniaxial loading covering from small-scale to large-scale yielding). Obtained numerical solutions of constraint parameter $A$ for 2D cracked bodies under uniaxial loading are tabled systematically. Then, through the numerical solutions of parameter $A$, constraint effects on 2D cracks under uniaxial loading are analyzed. Thirdly, based on the numerical solutions and the three estimate methodologies developed in chapter 3 for parameter $A$, predicting formulas of parameter $A$ corresponding to the three estimate methodologies for 2D crack geometries under uniaxial loading are proposed and verified by comparing predicted $A$ solutions with their numerical solutions. Fourthly, to show and verify the method determining $Q$ and $A_{2}$ directly from $A$, predicted parameter $A$ values for several cases are used to obtain corresponding parameter $Q$ or $A_{2}$ by the relationships between $A$ and $Q$ as well as $A$ and $A_{2}$.

In chapter 5, to obtain numerical solutions of parameter $A$ for three 2D specimens, SECP, CCP and DECP under biaxial loading, for wide range of geometries, material properties and loading conditions (biaxial loading covering from small-scale to largescale yielding), extensive finite element analyses are carried out. Obtained numerical solutions of parameter $A$ for $2 \mathrm{D}$ cracked specimens under biaxial loading are tabled systematically. Constraint effects for 2D cracked specimens under biaxial loading are discussed. Estimating formulas corresponding to the three estimate methodologies are 
developed for the 2D specimens under biaxial loading. They are validated by comparing the predicted values of $A$ with the numerical solutions.

In chapter 6 , with wide range of geometries, material properties and loading conditions (uniaxial and biaxial loading covering from small-scale to large-scale yielding), extensive finite element analyses are carried out for 3D SECP specimens. From the FEA results, the numerical solutions of constraint parameter $A$ for 3D SECP specimens under both uniaxial and biaxial loading are determined and tabled. Obtained numerical solutions of $A$ are utilized to study the constraint effects for 3D cracked specimens under uniaxial and biaxial loading. Predicting formulas for parameter $A$ corresponding to the three sets of developed methodologies are proposed for 3D models. The developed formulas are verified by the comparison between the estimated $A$ values and their numerical solutions.

Finally, the conclusions for current research and recommendations for future work are summarized in chapter 7 . 


\section{CHAPTER 2 THEORETICAL BACKGROUND AND LITERATURE}

\section{REVIEW}

\subsection{Introduction}

In this chapter, one-parameter approaches for linear elastic fracture mechanics and elastic-plastic fracture mechanics are reviewed first. Then the two-parameter fracture mechanics approach for linear elastic fracture mechanics and several typical two-parameter approaches for elastic-plastic fracture mechanics are discussed. Next, analytical, numerical and approximate determination methods reported in the literature for both dominant fracture parameters and constraint fracture parameters are reviewed. Finally, application of typical elastic-plastic two-parameter approaches on crack-front field of three-dimensional (3D) cracked structures is reviewed and the need for further development of two-parameter approaches is discussed.

\subsection{Characterizations of Crack-Tip (-Front) Fields}

For the justification of fracture mechanics criteria, knowledge of stress and displacement (strain) fields in the microstructurally significant region near the crack-tip (front) of material is an important aspect. Note here, usually, term "crack-front" is utilized for three-dimension (3D) structure models by some researchers while term "crack-tip" is used for two-dimension (2D) cases. Stress and strain fields are related by deformation theories and corresponding stress-strain relationships (constitutive relations). Figure 2.1 shows a typical crack-tip (-front) stress field, where radius size $r$ and angle $\theta$ build the polar coordinates and axes $x, y$ and $z$ set up the Cartesian coordinate system.

Constitutive relations depend on material models. In linear elastic fracture mechanics (LEFM), the stress-strain relationship is described by the famous Hooke's law which comes from a linear elastic material model: 


$$
\varepsilon=\frac{\sigma}{E}
$$

where $\sigma$ is applied stress, $\varepsilon$ corresponding to strain; $E$ is Young's modulus.

In elastic-plastic fracture mechanics (EPFM), one of the usually applied material model is the deformation theory of plasticity. One of the most frequently used stressstrain relationships is the Ramberg-Osgood power-law strain hardening relation. Uniaxial tension stress-strain curve from Ramberg-Osgood relation is described as:

$$
\varepsilon=\frac{\sigma}{E}+\beta\left(\frac{\sigma}{E}\right)^{n}
$$

where $\beta$ are material coefficient and $n$ hardening exponent that depend on the material being considered. The first term on the right side, $\sigma / E$, equates to the elastic part of the strain while the second term, $\beta(\sigma / E)^{n}$, accounts for the plastic part. A material model in which the first term of Eq. (2.2), $\sigma / E$, is not present is called as "pure power-law material" by some researchers.

By introducing a new parameter, $\alpha$, related to $\beta$ as $\alpha=\beta\left(\sigma_{0} / E\right)^{n-1}$, Eq. (2.2) can be rewritten as:

$$
\frac{\varepsilon}{\varepsilon_{0}}=\frac{\sigma}{\sigma_{0}}+\alpha\left(\frac{\sigma}{\sigma_{0}}\right)^{n}
$$

where $\sigma_{0}$ and $\varepsilon_{0}$ is yield stress and yield strain respectively. The stress-strain curve for Ramberg-Osgood power-law strain hardening under uniaxial tension/compression is shown in Figure 2.2.

By deformation plasticity theory, the uniaxial tensile relation that follows the Ramberg-Osgood relationship can be generalized to the multi-axial state as:

$$
\frac{\varepsilon_{i j}}{\varepsilon_{0}}=(1+v) \frac{\sigma_{i j}}{\sigma_{0}}-v \frac{\sigma_{k k}}{\sigma_{0}} \delta_{i j}+\frac{3}{2} \alpha\left(\frac{\sigma_{e}}{\sigma_{0}}\right)^{n-1} \frac{s_{i j}}{\sigma_{0}}
$$

where $\sigma_{i j}$ is stress components; $\delta_{i j}$ is the Kronecker delta; $s_{i j}$ are the deviatoric stress components; and $\sigma_{e}=\sqrt{3 s_{i j} s_{i j} / 2}$ the von Mises effective stress.

With the knowledge of stress / strain fields of structure crack-tip (-front), 
characterization of crack-tip (-front) zone can be carried out and correspondingly structure failure criterion can be built. Originally, only one parameter directly relating the external load condition is utilized to characterize the crack-tip (-front) fields. However, in many cases, specifically for elastic-plastic material, one-parameter approaches are not sufficient for the description of crack-tip (-front) fields (Anderson (2005)). Second fracture mechanics parameters are therefore introduced to overcome the application limitation of one-parameter approaches. In the characterization of crack-tip (-front) zone, first fracture mechanics parameters are dominant parameters relative to those second and higher order ones, where the second order ones describe the constraint effect of crack-tip (-front) fields. Both one-parameter and two-parameter approaches will be reviewed in the following sections.

\subsection{One-Parameter Approach}

\subsubsection{One-parameter approach of linear elastic fracture mechanics (LEFM)}

In linear elastic fracture mechanics, the stress intensity factor $K$, which was originally named by Irwin (1957) ( $K$ for Kies, one of Irwin's collaborators), is used to predict the stress state (stress intensity) near the tip (front) of a crack caused by a remote load or residual stresses. It is a theory applicable to the homogeneous elastic material. It is useful for providing a failure criterion for brittle materials. The magnitude of $K$ depends on the sample geometry, size and location of the crack, as well as the magnitude, the mode and distribution of loads on the material. The stress distribution implied by $K$ will be invalid in the region very close to the crack-tip (-front) of an elastic-plastic material where plastic distortion typically occurs.

\subsubsection{One-parameter approach of elastic-plastic fracture mechanics (EPFM)}

In elastic-plastic fracture mechanics, a one-parameter fracture criterion is supported by a one-parameter asymptotic solution of stress and strain fields which was developed 
by Hutchinson (1968) and Rice and Rosengren (1968) (so-called HRR field). A path independent integral, the $J$-integral, which was originally proposed by Rice (1968), is the base of one-parameter criterion of local fracture. For an elastic-plastic material, if the plastic zone size is small compared to other geometric dimensions of the problem, such as the crack length, linear elastic fracture mechanics considerations (stress intensity factor $K$ ) can still hold. This is so-called "small-scale yielding" (SSY) approximation. Therefore, for plane-strain and plain-stress deformation field under small-scale yielding condition, $J$-integral could be expressed respectively as:

Plane strain: $\quad J=\frac{1-v^{2}}{E} K_{I}^{2}$

Plane stress:

$$
J=\frac{1}{E} K_{l}^{2}
$$

where $E$ is the Young's modulus, $K_{I}$ is stress intensity factor for fracture mode I and $v$ is Poisson's ratio.

\subsection{Two-Parameter Approach of Linear Elastic Fracture Mechanics}

\section{(LEFM)}

\subsubsection{K-T Two-Parameter Approach}

In linear elastic fracture mechanics, it has been shown that applied loading could be represented by the stress intensity factor $K_{I}$ (mode I), while the constraint effect from specimen geometry and loading configuration could be represented by a $T$-stress. According to Williams' (1957) series solution for 2D crack tip fields, one gets:

$$
\sigma_{i j}(r, \theta)=\frac{K_{I}}{\sqrt{2 \pi \cdot r}} f_{i j}(\theta)+T \delta_{1 i} \delta_{1 j}
$$

where $(r, \theta)$ are polar coordinates with the origin located at the crack tip. $K_{I}$ is the mode I stress intensity factor; $f_{i j}(\theta)$ are the non-dimensional angular functions; $T$ is a uniform stress parallel to the crack face, commonly referred to as $T$-stress. $\delta_{1 i}$ and $\delta_{1 j}$ are 
Kronecker deltas, indices $i, j$ have a range from 1-2. This expression is considered as the $K$ - $T$ two-parameter approach for linear elastic material.

\subsection{Two-Parameter Approach of Elastic-Plastic Fracture Mechanics}

\section{(EPFM)}

\subsection{1 $J-T$ two-parameter approach}

\subsubsection{J-T approach}

Similar to the two-parameter approach ( $K-T$ approach) for the linear elastic case, in the elastic-plastic regime, the $J$-integral is used to characterize the loading level instead of $K_{I}$ while the constraint effect is still described by $T$-stress. Betegon and Hancock (1991) and Al-Ani and Hancock (1991) extended the two-parameter $K_{I}-T$ approach for elastic material to elastic-plastic material (i.e. $J-T$ approach) through finite element analysis. Since $T$-stress is a parameter of linear elastic fracture mechanics, the $J-T$ approach generally is only suitable for small-scale yielding (SSY).

\subsubsection{Modified boundary layer (MBL) problem}

The modified boundary layer (MBL) problem is a practical investigating application which combines $J-T$ and $K-T$ two-parameter approaches. The modified boundary layer formulation is an elastic-plastic (near crack-tip or -front) problem with elastic boundary conditions: an asymptotic stress field characterized by far-field stress intensity factor $K_{I}$ and far-field $T$-stress, as shown in Figure 2.3. For this problem formulation, loadings are applied by displacement boundary conditions through the stress intensity factor $K_{I}$ and $T$-stress. The loadings are applied uniformly on the finite element model of the MBL problem across the model thickness at the far-field boundary, $r=r_{\max }$. From the $K_{I}-T$ stress fields expressed by Eq. (2.7), for the 2D case of plane-strain and plane-stress, the values of displacement components $u_{x}$ and $u_{y}$ can be respectively calculated as 
following:

Plane strain: $\quad u_{x}=\frac{K_{I}}{2 \mu} \sqrt{\frac{r}{2 \pi}} \cos \left(\frac{\theta}{2}\right)\left[\kappa-1+2 \sin ^{2}\left(\frac{\theta}{2}\right)\right]+\frac{1-v}{2 \mu} \operatorname{Tr} \cos \theta$

$$
u_{y}=\frac{K_{I}}{2 \mu} \sqrt{\frac{r}{2 \pi}} \sin \left(\frac{\theta}{2}\right)\left[\kappa+1-2 \cos ^{2}\left(\frac{\theta}{2}\right)\right]+\frac{(-v)}{2 \mu} \operatorname{Tr} \sin \theta
$$

Plane stress: $u_{x}=\frac{K_{I}}{2 \mu} \sqrt{\frac{r}{2 \pi}} \cos \left(\frac{\theta}{2}\right)\left[\kappa-1+2 \sin ^{2}\left(\frac{\theta}{2}\right)\right]+\frac{1}{2 \mu(1+v)} \operatorname{Tr} \cos \theta$

$$
u_{y}=\frac{K_{I}}{2 \mu} \sqrt{\frac{r}{2 \pi}} \sin \left(\frac{\theta}{2}\right)\left[\kappa+1-2 \cos ^{2}\left(\frac{\theta}{2}\right)\right]+\frac{(-v)}{2 \mu(1+v)} \operatorname{Tr} \sin \theta
$$

where $\mu$ is the shear modulus; $v$ is Poisson's ratio, $\kappa=3-4 v . K_{I}$ is the far-field stress intensity factor and $T$ is the far-field $T$-stress. By the relationships between far-field stress intensity $K_{I}$ and far-field $J$-integral expressed by Eqs. (2.5) and (2.6), the far-field stress intensity factor $K_{I}$ for plane strain and plane stress can be determined respectively from the far-field $J$-integral by:

$$
\begin{gathered}
K_{I}=\sqrt{\frac{J E}{1-v^{2}}} \\
K_{I}=\sqrt{J E}
\end{gathered}
$$

As utilized by Yuan and Brocks (1998), for the plane stress case, besides the inplane displacement components $u_{x}$ and $u_{y}$, the out-of-plane displacement component $u_{z}$ should be applied linearly across the MBL model thickness on its boundary so as to more accurately simulate the remote boundary condition. The values of $u_{z}$ can be calculated from the stress-strain relationships under plane-stress conditions as:

$$
u_{z}=-\frac{v}{E} \int\left(\sigma_{x x}+\sigma_{y y}\right) d z
$$

where

$\sigma_{x x}(r, \theta)=\frac{K_{I}}{\sqrt{2 \pi r}} \cos \frac{\theta}{2}\left(1-\sin \frac{\theta}{2} \sin \frac{3 \theta}{2}\right)+T$ 


$$
\sigma_{y y}(r, \theta)=\frac{K_{i}}{\sqrt{2 \pi r}} \cos \frac{\theta}{2}\left(1+\sin \frac{\theta}{2} \sin \frac{3 \theta}{2}\right)
$$

Usually the modified boundary layer formulation (MBL) is used to characterize a small-scale yielding condition of the model. A specific small-scale yielding condition with $T=0$ is called "standard small-scale yielding" condition by some researchers.

\subsubsection{J-Q two-parameter approach}

Based on the deformation theory of plasticity, Sharma and Aravas (1991) solved the plane-strain mode I crack problem, and obtained two-term expansions of crack tip fields in a power-law hardening material. Specially, stress field can be expressed as:

$$
\frac{\sigma_{i j}(r, \theta)}{\sigma_{0}}=\left(\frac{J}{\alpha \varepsilon_{0} \sigma_{0} I_{n} r}\right)^{1 /(n+1)} \bar{\sigma}_{i j}^{(0)}(\theta, n)+Q\left(\frac{r}{J / \sigma_{0}}\right)^{p} \bar{\sigma}_{i j}^{(l)}(\theta, n)
$$

where $\sigma_{0}\left(\varepsilon_{0}\right)$ is the reference stress (strain) which is generally equal to the yield stress (strain), and $\varepsilon_{0}=\sigma_{0} / E ; \alpha$ is a material hardening constant, $n$ is the hardening exponent; and $I_{n}$ is an integral constant which depends on $n . \bar{\sigma}_{i j}^{(0)}(\theta, n)$ and $\bar{\sigma}_{i j}^{(1)}(\theta, n)$ are normalized angular functions which depend on $\theta$ and $n$. The exponent $p$ is an undetermined parameter. The first term of the two-term expression is the HRR solution. Based on finite element calculations, O'Dowd and Shih $(1991,1992)$ developed a simple form of Eq. (2.14) in which the power exponent $p$ is set to zero:

or

$$
\begin{aligned}
& \sigma_{i j}(r, \theta)=\left(\sigma_{i j}(r, \theta)\right)_{H R R}+Q \sigma_{0} \delta_{i j} \\
& \sigma_{i j}(r, \theta)=\left(\sigma_{i j}(r, \theta)\right)_{S S Y ; T=0}+Q \sigma_{0} \delta_{i j}
\end{aligned}
$$

O'Dowd and Shih $(1991,1992)$ considered two fields: the HRR field and the standard small-scale yielding (SSY) solution $(T=0)$ respectively, as reference fields. Therefore there are two forms of expressions for the stress field (Eqs. (2.15a) and (2.15b)). Correspondingly, two forms of the $Q$ factor definition were obtained. Then one can get: 


$$
\begin{aligned}
& Q=\frac{\sigma_{i j}(\bar{r}, \theta)}{\sigma_{0}}-\frac{\left(\sigma_{i j}(\bar{r}, \theta)\right)_{H R R}}{\sigma_{0}} \text { at } \theta=0, r=2 J / \sigma_{0} \\
& Q=\frac{\sigma_{i j}(\bar{r}, \theta)}{\sigma_{0}}-\frac{\left(\sigma_{i j}(\bar{r}, \theta)\right)_{S S Y ; T=0}}{\sigma_{0}} \text { at } \theta=0, r=2 J / \sigma_{0}
\end{aligned}
$$

where $\bar{r}=r /\left(J / \sigma_{0}\right)$ is a dimensionless radius. The value of the $Q$ factor is proposed to be determined at $\bar{r}=2, \theta=0$. This two-parameter approach of fracture mechanics is the so-called $J-Q$ approach.

\subsubsection{J-A two-parameter approach}

Another two-parameter approach of elastic-plastic fracture mechanics, which is based on a three-term asymptotic expansion but only controlled by two fracture parameters: $J$ and $A_{2}$, was proposed by Yang (1993) and Yang et al. (1993). This twoparameter approach is called $J-A_{2}$ approach.

Yang, Chao and Sutton (Yang (1993), Yang et al. (1993) and Chao et al. (1994)) carried out a complete asymptotic analysis of higher-order crack tip fields in a power law hardening material. It has been claimed by Yang et al. that a two-term asymptotic expansion is not sufficient to characterize the crack near-tip field, while more than three terms are redundant for plane strain mode I cracks. Therefore they proposed a three-term expansion which is controlled only by two parameters, i.e. $J$ and $A_{2}$.

Following the format in paper of Chao et al. (1994), the stress expression of fracture near tip field by $J-A_{2}$ two-parameter approach can be presented as:

$$
\frac{\sigma_{i j}}{\sigma_{0}}=A_{1}\left[\left(\frac{r}{L}\right)^{S_{1}} \widetilde{\sigma}_{i j}^{(1)}(\theta)+A_{2}\left(\frac{r}{L}\right)^{S_{2}} \widetilde{\sigma}_{i j}^{(2)}(\theta)+A_{2}^{2}\left(\frac{r}{L}\right)^{S_{3}} \widetilde{\sigma}_{i j}^{(3)}(\theta)\right]
$$

where $\widetilde{\sigma}_{i j}^{(k)}(k=1,2,3)$ are dimensionless angular functions and $s_{k}\left(s_{1}<s_{2}<s_{3}\right)$ are powers only depending on the hardening exponent $n, L$ is crack characteristic length chosen as crack length, specimen sizes or unity. The parameter $A_{1}$ and exponents $s_{1}$ and $s_{3}$ are given as: 


$$
\begin{gathered}
A_{1}=\left(\frac{J}{\alpha \varepsilon_{0} \sigma_{0} I_{n} L}\right)^{-s_{1}} \\
s_{1}=-\frac{1}{n+1}, s_{3}=2 s_{2}-s_{1}
\end{gathered}
$$

where $I_{n}$ is a scaling integral only depending on hardening exponent $n$.

Nikishkov et al. (Nikishkov (1995), Nikishkov et al (1995a)) derived the same series expansion and also suggested that the magnitude of the second term in the series can be used together with $J$ to characterize the crack tip stress fields. Instead of $A_{2}$, it was proposed by Nikishkov et al. (Nikishkov (1995), Nikishkov et al (1995a)) to use $A$, which is in fact a different normalized form of $A_{2}$. Therefore, with this set of formula expressions and variable terminologies, the term " $J-A$ approach" is used instead of " $J-A_{2}$ approach". Nikishkov et al. (1995a) also demonstrate that the values of the parameter $A\left(A_{2}\right)$ are almost independent of the location where they are computed and therefore the distance from crack tip.

Following the formula expressions and the terminologies of variables used by Nikishkov et al. (Nikishkov (1995), Nikishkov et al. (1995a)), the three-term asymptotic solution for stress field near the crack tip in elastic-plastic material can be written as:

$$
\frac{\sigma_{i j}}{\sigma_{0}}=A_{0} \bar{r}^{s} \bar{\sigma}_{i j}^{(0)}(\theta)+A_{1} \bar{r}^{t} \bar{\sigma}_{i j}^{(1)}(\theta)+A_{2} \bar{r}^{2 t-s} \bar{\sigma}_{i j}^{(2)}(\theta)
$$

where $A_{0}=\left(\alpha \varepsilon_{0} I_{n}\right)^{-1 /(n+1)}$; dimensionless radius $\bar{r}$ is defined as $\bar{r}=r /\left(J / \sigma_{0}\right)$, power $t$ is an eigenvalue depending on the hardening exponent $n$; and $s=-1 /(n+1)$; $\bar{\sigma}_{i j}^{(0)}(\theta), \bar{\sigma}_{i j}^{(1)}(\theta)$ and $\bar{\sigma}_{i j}^{(2)}(\theta)$ are normalized angular functions. For the hardening exponent $n>3$ and plane strain conditions, the amplitude for the second order term $A_{2}$ is related to the values of $A_{0}$ and $A_{1}$ by: $A_{2}=A_{1}^{2} / A_{0}$. Note that " $A_{1}$ " and " $A_{2}$ " are not the same amplitudes as those in the $J-A_{2}$ approach (Eq. (2.17)) proposed by Yang (1993) and Yang et al. (1993). It is convenient to introduce the amplitude parameter $A=-A_{1}$. Then the three terms asymptotic expansion can be expressed as: 


$$
\frac{\sigma_{i j}}{\sigma_{0}}=A_{0} \bar{r}^{s} \bar{\sigma}_{i j}^{(0)}(\theta)-A \bar{r}^{t} \bar{\sigma}_{i j}^{(1)}(\theta)+\frac{A^{2}}{A_{0}} \bar{r}^{2 t-s} \bar{\sigma}_{i j}^{(2)}(\theta)
$$

In this thesis, the $J-A$ approach will be used to study the elastic-plastic crack-tip (-front) fields.

\subsubsection{Application of two-parameter elastic-plastic fracture mechanics}

In one-parameter approach of elastic-plastic fracture mechanics (EPFM), stress or displacement fields of near crack-tip (-front) are described by a single parameter $J$ integral. However, in two-parameter approach, the fields are described by two fracture parameters, load-related parameter, $J$-integral, and constraint parameter $\left(T, Q\right.$ or $\left.A_{2}(A)\right)$, see Eqs. (2.15) and (2.21) for example. In EPFM, fracture criterion can be presented by a critical $J$-integral, $J_{c}$, or a critical crack opening displacement, $\delta_{c}$, where $J_{c}$ (or $\delta_{c}$ ) is the value of $J$-integral (or crack opening displacement, $\delta_{t}$ ) at the onset of cleave fracture or ductile tearing. Crack opening displacement, $\delta_{t}$, is a function of $J$-integral.

The values of $J_{c}$ (or $\delta_{c}$ ) (fracture toughness) can be predicted through expressions for stress (displacement) fields of near crack-tip region which are from one-parameter approach of EPFM. Generally, for cases of low constraint of near crack-tip region, a predicted fracture toughness from $J$-based one-parameter approach, which does not include effect of constraint, will be smaller than actual toughness, namely, overconservative assessments for fracture toughness occur. In these cases, values of $J_{c}$ (or $\delta_{c}$ ) obtained from two-parameter approaches (e.g. J-Q, $J-A_{2}(A)$ approach), which calculate constraint effect through constraint parameter, $Q, A_{2}(A)$, will be closer to actual values of $J_{c}$ (or $\delta_{c}$ ) (fracture toughness). The critical values of $J$-integral (or crack opening displacement), $J_{c}$ (or $\delta_{c}$ ), can be obtained through asymptotic expansions for stress (displacement) fields of near crack-tip region based on two-parameter approach, e.g., Eqs. (2.15) and (2.21). In two-parameter approach, $J_{c}$ or $\delta_{c}$ is the function of constraint parameter $Q, A_{2}(A)$, for example, $J_{c}(Q), J_{c}(A)$ and $\delta_{c}\left(A_{2}\right)$. O'Dowd and Shih (1994) illustrated a comparison between cleavage toughness data measured from experiment, $\left(J_{c}\right)$, and those predicted through $J-Q$ two-parameter approach, $J_{c}(Q)$, see Figure 2.4 . In 
Figure 2.4, experimental data are indicated by the symbols and the dashed line is the predicted variation of $J_{c}(Q)$. More details on application of two-parameter approach of EPFM can be found in papers of several researchers, for example, O'Dowd and Shih (1994) and Nikishkov et al. (1995b).

\subsection{Methods for the Determination of Dominant Fracture Mechanics}

\section{Parameters}

For the two fracture mechanics parameters describing crack-tip (-front) fields, the parameter related to load level, i.e. $K$ or $J$-integral, is the dominant one. In this section, the methods for the determination of $K$ and $J$ are reviewed.

\subsubsection{Solution of stress intensity factor $K$}

Solutions of stress intensity factor $K$ have been well established since the 1950's when it was originally proposed by Irwin (1957). Many methods to determine the $K$ values have been proposed. These methods could be classified into several types: analytical method, numerical solution or estimating method, etc. (In fact, they are common methods of engineering parameter calculation, which are not only suitable for determining $K$ values but also for obtaining other fracture parameters). For convenience in engineering applications, some solutions of $K$ have been tabled in handbooks or papers (reports), for example the handbook of Tada et al. (2000). As for numerical determining methods, a typical tool is the use of finite element analysis.

\subsubsection{Solution of $J$-integral}

A great number of methods are proposed in the literature to calculate the $J$-integral. Besides analytical methods, the domain integral method proposed by Moran and Shih (1987) is a typical example of $J$-integral numerical solution, which has been adopted in the commercial code ABAQUS (2006). 
In addition, approximation (estimate) is another import way to obtain the value of the fracture parameter, $J$-integral. As an example of a $J$-integral estimating method, the EPRI (Electric Power Research Institute) solution of $J$-integral for 2D models under uniaxial loading is reviewed here. In the EPRI solution, Kumar et al. (1981) proposed the superposition property of the $\dot{J}$-integral, i.e.:

$$
J=J_{E}+J_{P}
$$

where $J_{E}$ is the elastic solution of $J$-integral and $J_{P}$ is the fully plastic solution. Eq. (2.22) means that the $J$-integral solution can be expressed as the summation of elastic part and fully plastic part. The suggested superposition of the $J$-integral is illustrated in Figure 2.5. Kumar et al. (1981) also suggested the fully plastic solutions of the $J$-integral for some typical test specimens. For example, fully plastic solution of the $J$-integral for center cracked plate (CCP) specimens is given by:

CCP:

$$
J_{P}=\alpha \sigma_{0} \varepsilon_{0}\left(1-\frac{a}{W}\right) a h_{1}(n, a / W)\left(\frac{\sigma}{\sigma_{L}}\right)^{(n+1)}
$$

where the parameter $h_{l}$ is a function of the hardening exponent $n$ and the ratio of crack depth $a$ to specimen width $W, a / W$, expressed as $h_{I}(a / W, n)$ in detail; and $\sigma_{L}$ is limit stress of specimen.

In addition, for the fully plastic solution of the $J$-integral for 2D models under biaxial loading, O'Dowd et al. (1999) proposed an expression for the CCP specimen as following:

$\mathrm{CCP}: \quad J_{P}=\alpha \sigma_{0} \varepsilon_{0}\left(1-\frac{a}{W}\right) a h_{1}(n, a / W, \lambda)\left(\frac{\sigma}{\sigma_{L}}\right)^{(n+1)}$

where the load biaxiality ratio $\lambda=\sigma_{x} / \sigma_{y}$, see Figure 2.6. This expression is very close in form to the EPRI (Electric Power Research Institute) solution (Eq. (2.23)). The difference between the Eq. (2.24) and Eq. (2.23) is the dependence of the parameter $h_{l}$ on the biaxiality ratio $\lambda$.

In the papers of both Kumar et al. (1981) and O'Dowd et al. (1999), the $h_{1}$ factors are tabled systematically. 


\subsection{Methods for the Determination of Constraint Fracture Mechanics}

\section{Parameters}

\subsubsection{Solution of $T$-stress}

\subsubsection{Determination of T-stress}

As an elastic constraint parameter, similar to the elastic loading-related parameter $K$, analytical solutions of $T$-stress for some cases are available in literature. Some numerical solutions are also reported by some researchers, e.g. Nakamura and Parks (1992) suggested a computational method for $T$-stress solution along three-dimensional (3D) crack fronts, which has been applied in the commercial code ABAQUS (2006). Numerical evaluation, for example based on finite element analysis, can be an accurate but inconvenient approach for fracture parameter determination in engineering applications. Many researchers therefore developed approximate methods of $T$-stress solutions for $2 \mathrm{D}$ and $3 \mathrm{D}$ models, such as Wang (2002), Yu and Wang (2005) and Wang and Bell (2004).

\subsubsection{Prediction of T-stress by weight function method}

As a typical example of an estimation method for determining $T$-stress, a weight function method suggested by Wang (2002) is reviewed here. The estimate proposed by Wang (2002) is based on the superposition of $T$-stress. According to the superposition theory, for a mode I crack problems, the $T$-stress in a crack body loaded by a stress field $Q$ (see Figure 2.7(a)) is the superposition of the $T$-stress for two cases. The $T$-stress of the first case is the one for the same cracked body loaded by a crack face pressure, $\sigma(x)$, which is induced by the remote stress field $Q$ in the uncracked body (Figure 2.7(b)). In the second case, the $T$-stress is the one in the uncracked body under remote stress field $Q$ (Figure 2.7(c)). Here, unlike the convention in other sections of this work, the letter ' $Q$ ' is used to represent the stress field instead of the constraint parameter.

Following Wang's paper, the $T$-stress for plane strain models can be obtained 
directly by the following equation:

$$
T=\int_{0}^{a} \sigma(x) w(x, a) d x+\left.\left(\sigma_{x}-\sigma_{y}\right)\right|_{x=a}
$$

where $\sigma(x)$ is the stress distribution on the crack face and $w(x, a)$ is the weight function for the $T$-stress which can be expressed as following:

$$
w(x, a)=\frac{2}{\pi a}\left[D_{1}\left(1-\frac{x}{a}\right)^{1 / 2}+D_{2}\left(1-\frac{x}{a}\right)^{3 / 2}\right]
$$

The first term on right side of Eq. (2.25) is the $T$-stress for the first case in superposition theory, and the second term is that for the second case. Expressions of coefficients $D_{1}$ and $D_{2}$ can be found in Wang's paper (Wang (2002)) for various 2D test specimens.

\subsubsection{Solution for the $Q$ parameter}

\subsubsection{Determination of the $Q$ parameter}

Based on finite element analysis, the constraint parameter $Q$ could be determined directly from Eq. (2.16a) or (2.16b) numerically, as shown in the papers of O'Dowd and Shih (1991, 1992). However, for the convenience of engineering application and theoretical research, estimation methods for $Q$ parameter solutions need to be established widely.

\subsubsection{Prediction of $Q$ factor by $Q-T$ relationship}

One of typical example of estimation methods for $Q$ is predicting $Q$ values from the $T$-stress using the one-to-one $Q-T$ relationship.

O'Dowd and Shih $(1992,1994)$ as well as Shih and O'Dowd (1992) have shown that: under small-scale yielding (SSY), $Q$ depends on $T$-stress alone, i.e. for a power-law hardening material with hardening exponent $n$ :

$$
Q=Q\left(\frac{T}{\sigma_{0}}, n\right)
$$

By least-square fitting, detailed expressions of the relationship between parameters $Q$ and $T$ can be obtained to a good approximate based on HRR and SSY reference fields 
respectively:

HRR reference field: $Q=b_{0}(n)+b_{1}(n)\left(\frac{T}{\sigma_{0}}\right)+b_{2}(n)\left(\frac{T}{\sigma_{0}}\right)^{2}+b_{3}(n)\left(\frac{T}{\sigma_{0}}\right)^{3}$

SSY reference field: $\quad Q=b_{1}(n)\left(\frac{T}{\sigma_{0}}\right)+b_{2}(n)\left(\frac{T}{\sigma_{0}}\right)^{2}+b_{3}(n)\left(\frac{T}{\sigma_{0}}\right)^{3}$

where $b_{1}, b_{2}$ and $b_{3}$ are polynomial coefficients. Eq. (2.28b) is illustrated in Figure 2.8 .

The $Q-T$ relationship is an effective estimation tool for $Q$ values from the $T$-stress, considering the fact that the $T$-stress solutions for a wide range of crack geometries in finite thickness plates have been developed from 2D and 3D finite element analyses, such as in the papers of Wang (2002), Wang and Bell (2004) as well as Wang and Yu (2005). In their investigations, O'Dowd (1995) and Wang (2008) have successfully utilized the one-to-one $Q-T$ relationship (Eq. (2.28b)) to predict parameter $Q$ values directly from $T$ stress for $2 \mathrm{D}$ and $3 \mathrm{D}$ models respectively.

\subsubsection{Prediction of $Q$ parameter under large-scale yielding}

The estimate of $Q$ based on $T$-stress is only appropriate for small-scale to contained yielding conditions as $T$-stress is an elastic fracture parameter.

Therefore, O'Dowd (1995) also suggests a method to estimate the $Q$ factor under large-scale yielding. He suggests that pure power law hardening solutions can be utilized to estimate $Q$ factors under fully plastic conditions. Then a method for predicting $Q$ under fully plastic conditions based on a pure power law hardening solution is proposed.

Finally, O'Dowd (1995) proposed an overall empirical formula to predict the values of $Q$ factor from small- to large-scale yielding by interpolating between the small-scale yielding solutions from $T$-stress and the fully plastic solutions.

\subsubsection{Solution of parameter $\boldsymbol{A}$}

To the author's knowledge, except for numerical solutions of the constraint parameter $A$, no analytical or estimating method for parameter $A$ determination has been reported in the literature (for either $2 \mathrm{D}$ or $3 \mathrm{D}$ cases). For engineering application and 
theoretical analysis, the development of estimation methods of $A$ solutions for various geometric and loading cases is necessary. Here numerical methods of obtaining parameter $A$ are first reviewed, then a possible estimation method is discussed.

\subsubsection{Numerical determination of parameter $A$}

To determine the amplitude $A$ numerically, a straight forward approach is to solve the quadratic equation (2.21) for one or several particular point(s) near the crack-tip using finite element data for one or several stress (or displacement) component(s). It is also called the "point match" method. For example, Yang (1993) and Yang et al. (1993) calculate $A_{2}(A)$ value by averaging calculated values at points $\bar{r}=2, \theta=0^{\circ}$ and $45^{\circ}$ for stress components $\sigma_{r}$ and/or $\sigma_{\theta}$.

Another possible method to numerically determine the value of amplitude $A$, which is proposed by Nikishkov et al. (1995a), is to find the value of $A$ by fitting expression (2.21) using finite element data in the region which is significant for the fracture process. For the $i$ th point, equation (2.21) can be expressed as:

$$
a_{i} A^{2}+b_{i} A+c_{i}=\delta_{i},
$$

and

$$
\begin{aligned}
& a_{i}=\frac{\bar{r}_{i}^{2 t-s}}{A_{0}} \bar{\sigma}^{(2)}\left(\theta_{i}\right), \\
& b_{i}=-\bar{r}_{i}^{t} \bar{\sigma}^{(1)}\left(\theta_{i}\right), \\
& c_{i}=A_{0} r_{i}^{s} \bar{\sigma}^{(0)}\left(\theta_{i}\right)-\frac{\sigma^{F E M}\left(\bar{r}_{i}, \theta_{i}\right)}{\sigma_{0}}
\end{aligned}
$$

where $\delta_{i}$ is the deviation of the asymptotic stress field from the finite element stress $\sigma^{\text {FEM }}$ at the $i$ th point with polar coordinates $\bar{r}_{i}, \theta_{i}$. Stress components $\sigma_{r}, \sigma_{\theta}$ or some combinations of them, e.g. $\left(\sigma_{r}+\sigma_{\theta}\right)$, can be used for fitting the stresses. Therefore, in Eq. (2.30) $\sigma$ means any stress component or combination used for fitting.

Minimizing the sum of squares of the deviations $\sum \delta_{i}{ }^{2}$ with weights leads to a cubic equation for amplitude $A$ as follows: 


$$
a A^{3}+b A^{2}+c A+d=0
$$

and

$$
\begin{aligned}
& a=2 \sum\left(a_{i}^{2} w_{i}^{2}\right), \\
& b=3 \sum\left(a_{i} b_{i} w_{i}^{2}\right), \\
& c=\sum\left(2 a_{i} c_{i} w_{i}^{2}+b_{i}^{2} w_{i}^{2}\right), \\
& d=\sum\left(b_{i} c_{i} w_{i}^{2}\right)
\end{aligned}
$$

where $w_{i}$ is the weight for the $i$ th point. Weights for the points can be proportional to the area which is represented by this fitting point. Numerical solutions of constraint parameter $A$ can be determined by solving Eq. (2.31).

In his previous paper (Nikishkov (1995), referring Eq. 2.20 or 2.21), Nikishkov has

proposed an algorithm to determine the normalized angular functions $\sigma_{i j}^{(0)}(\theta), \sigma_{i j}^{(1)}(\theta)$ and $\sigma_{i j}^{(2)}(\theta)$ as well as parameters $I_{n}, t$. In terms of stress and strain formulation, governing equations for the problem can be obtained. From the governing equations, some ordinary differential equations (systems) on stress and displacement components can be introduced. Using a fourth order Runge-Kutta method, these ordinary differential equations systems can be solved directly to get the values of stress and strain components.

\subsubsection{Possibility of predicting parameter $A$ by curve shape similarity}

Based on the finite element analysis for several 2D test specimen models under uniaxial tension, Nikishkov et al. (1995a) observed the phenomenon that when parameters $A$ are plotted vs. external loading ratio $\sigma / \sigma_{L}$, the curves for different values of hardening exponent $n$ have a similar shape (similarity) and only differ from each other by an additive constants (parameter $A$ value under zero load) that can be obtained from the standard small-scale yielding calculations. Figure 2.9 shows the $A$ curve shape similarity for SECP specimen with $\alpha=0.5, a / W=0.2$. Nikishkov et al. (1995a) suggested that this behavior could be utilized to develop approximate formulas for the estimation of the parameter $A$ for typical specimens. In the later part of this thesis, this suggestion will 
be adopted to develop an estimating methodology for the prediction of constraint parameter $A$.

\subsubsection{Relationships among constraint parameters}

The relationships among constraint parameters enable one constraint parameter to be obtained directly from another constraint parameter as long as the latter has been determined. It in fact enables increasing the availability of solutions for various constraint parameters greatly.

\subsubsection{Relationship between the constraint parameters $Q$ and $T$}

As mentioned in section 2.6.2.2, a one-to-one $Q-T$ relationship has been found by O'Dowd and Shih $(1992,1994)$ and Shih and O'Dowd (1992), see Eqs. (2.27) and (2.28). O'Dowd (1995) as well as Wang (2008) applied this relationship to predict $Q$ values from $T$-stress. In fact, this $Q-T$ relationship shows the equivalence of $J-T$ and $J-Q$ approaches under small-scale yielding.

\subsubsection{Relationship between the constraint parameters $A$ and $A_{2}$}

Comparing the crack-tip stress fields described in $J-A_{2}$ approach (Eq. (2.17)) and $J-A$ approach (Eq. (2.21)), one can find:

$$
s_{1}=s, s_{2}=t, s_{3}=2 t-s
$$

Furthermore, in fact, the angular functions $\widetilde{\sigma}_{i j}^{(1)}, \widetilde{\sigma}_{i j}^{(2)}$ and $\widetilde{\sigma}_{i j}^{(3)}$ in Eq.(2.17) are the same variables as the $\bar{\sigma}_{i j}^{(0)}, \bar{\sigma}_{i j}^{(1)}$ and $\bar{\sigma}_{i j}^{(2)}$ in Eq. (2.21). Then equalizing the Eq. (2.17) and Eq. (2.21), one can obtain the relationship between the parameter $A_{2}$ in the expression of Chao et al. (1994) and parameter $A$ in that of Nikishkov et al. (1995a) as:

$$
A_{2}=-\left(\alpha \varepsilon_{0} I_{n}\right)^{-s}\left(\frac{J}{\sigma_{0} L}\right)^{s-t} A
$$

where the power $s=-1 /(n+1)$. Equation (2.34) can be used to calculate $A_{2}$ from $A$ 
directly.

\subsubsection{Relationship between the constraint parameters $A$ and $Q$}

As demonstrated by Nikishkov et al. (1995a), if one assumes that the three-term expansion of elastic-plastic fracture mechanics, which is presented by Eq. (2.21), approximates the near-tip stress field quite well, Eq. (2.21) can be used to replace the $\sigma_{i j}(\vec{r}, \theta) / \sigma_{0}$ term in Eqs. (2.16a) and (2.16b). Meanwhile, the second term of the Eq. (2.16b) $\left(\sigma_{i j}(\bar{r}, \theta)\right)_{S S Y ; T=0} / \sigma_{0}$ also could be expressed by Eq. $(2.21)$ with $A=A_{S S Y}$, where $A_{S S Y}$ is value of parameter $A$ under small-scale yielding with $T=0$ (standard small-scale yielding condition). Furthermore, in fact, the zero order term of Eq. (2.17) $A_{0} \bar{r}^{s} \bar{\sigma}_{i j}^{(0)}(\theta)$ is the HRR stress field, i.e. the second term of Eq. $(2.16 \mathrm{a})\left(\sigma_{i j}(\bar{r}, \theta)\right)_{H R R} / \sigma_{0}$. With the recognition of these conditions, one can obtain the relationship between the two second fracture parameters $A$ and $Q$ :

$$
\begin{array}{ll}
Q=-A \bar{r}^{t} \bar{\sigma}_{i j}^{(1)}(\theta)+\frac{A^{2}}{A_{0}} \bar{r}^{2 t-s} \bar{\sigma}_{i j}^{(2)}(\theta) & \text { at } \theta=0, \bar{r}=2 \\
Q=-\left(A-A_{S S Y}\right) \bar{r}^{t} \bar{\sigma}_{i j}^{(1)}(\theta)+\frac{\left(A^{2}-A_{S S Y}^{2}\right)}{A_{0}} \bar{r}^{2 t-s} \bar{\sigma}_{i j}^{(2)}(\theta) & \text { at } \theta=0, \bar{r}=2
\end{array}
$$

Equations (2.35a) and (2.35b) can be used to calculate the $Q$ from parameter $A$.

\subsection{Three-Dimensional Aspects}

Almost all two-parameter elastic-plastic fracture mechanics approaches mentioned previously, including $J-T, J-Q$ and $J-A\left(A_{2}\right)$ approaches, are developed based on the plane strain condition. And correspondingly, up to now, most investigations of elastic-plastic crack-tip (-front) fields based on either $J-T, J-Q$ or $J-A\left(A_{2}\right)$ two-parameter approaches are focused on plane (2D model) problems, such as, the research of Nikishkov et al. (1995a) and Chao et al. (1994) based on the $J-A\left(A_{2}\right)$ approach. However, cracks (flaws) in practical structures are usually three-dimensional whose 
stress (strain) status varies along the $3 \mathrm{D}$ crack-front, and generally deviate from the plane conditions.

In $3 \mathrm{D}$ crack investigation, comparing with $2 \mathrm{D}$ crack cases, the difficulty lies in the 3D characteristics of the stress and strain fields at the crack-front of 3D crack specimens. Moreover, the fracture mechanism of 3D crack structures is more complex than that of 2D crack structures. In 3D crack cases, both in-plane (plane perpendicular to the crack front, $x-y$ plane in Figure 2.10) and out-of-plane (plane parallel to the crack front, $x-z$ plane in Figure 2.10) conditions will play important roles at the near-tip fields. Therefore, for fracture mechanics analysis in 3D crack structures, not only the in-plane load and geometry effects, but also the load and geometry effects from out-of-plane should be considered.

For the $J-Q$ two-parameter approach,' Shih et al. (1993) argued that when radial $r$ approaches zero, the out-of-plane strain components in $3 \mathrm{D}$ fields, $\varepsilon_{z}$, will be small compared to the in-plane singular terms and plane-strain conditions should be still dominant. Therefore it can be expected that 3D near-front crack fields converge to plane strain $J-Q$ fields. On the other hand, due to the nature of $3 \mathrm{D}$ cracks it is also expected that fracture parameters will not only depend on in-plane load and geometry conditions but also the out-of-plane load and geometry. In their paper, Shih et al. (1993) point out full scale 3D elastic-plastic finite element analyses are required to validate this suggestion. As an example of application for the suggestion, the $J-Q$ characterization of Wang (2008) on the $3 \mathrm{D}$ elastic-plastic crack-front field for surface cracked plates has verified the assertion of Shih et al. (1993).

Using the $J-A$ approach, Nikishkov et al. (1995b) carried out an investigation of a surface cracked plate under uniaxial loading. By using the $J-A_{2}$ approach, Kim et al. (2001) reported a crack-front solution for a 3D modified boundary layer problem of a thin plate; Zhu et al. (2002) completed a crack-front analysis for two 3D through-cracked thin plates (center cracked plate (CCP) and single edge notched bend specimen (SENB)); Kim et al. (2003) carried out research on the effect of specimen size and crack depth on 3D crack-front constraint for SENB through-cracked specimens.

All of four studies mentioned above on $3 \mathrm{D}$ crack-front fields by the $J-A\left(A_{2}\right)$ 
approach in fact are implemented based on the same method as that suggested by Shih et al. (1993) for the $J-Q$ approach of 3D crack-front fields. For the in-plane aspect, in brief, Nikishkov et al. (1995b), Kim et al. (2001) and Zhu et al. (2002) conclude that the $J-A$ $\left(A_{2}\right)$ approach (developed based on plane strain condition) gives a good description of the stress fields around the $3 \mathrm{D}$ crack-front. Based on results of finite element analyses for thin model thickness, Kim et al. (2001) and Zhu et al. (2002) find two phenomena in the planes perpendicular to the crack front (in-plane): (1) stress fields in the crack-front area are in plane strain state while the far field is dominated by plane stress state; (2) through almost the whole thickness of the model, in-plane stress fields at the crack-front are plane strain, except for the region near the free surface where plane stress is dominant. In their papers, these two findings are demonstrated by iso-contours of effective stress and plane strain constraint parameters. In fact, the two phenomena verify the suggestion given by Shih et al. (1993) about the 3D crack-front fields. Based on these two facts, Kim et al. (2001) and Zhu et al. (2002) therefore conclude that: the $J-A_{2}$ approach (developed based on the plane strain condition) can effectively characterize $3 \mathrm{D}$ crack-front stress fields through the thickness except for the region near the free surface.

As for the out-of-plane aspect, all of the four investigations (Nikishkov et al. (1995b), Kim et al. (2001), Zhu et al. (2002) and Kim et al. (2003)) present a monotonic variation of two fracture parameters (constraint parameter $A\left(A_{2}\right)$ and load-related parameter $J$-integral) along the crack-front curve (surface-cracked plate) or through the thickness of the specimen (through-cracked specimen).

Besides the applicability exploration of the $J-A\left(A_{2}\right)$ approach developed under plane strain conditions on 3D crack-fronts, investigations of characterization of $3 \mathrm{D}$ crack-front fields and then fracture mechanics criteria through the $J-A\left(A_{2}\right)$ approach also have been carried out by some researchers. By using the $J-A$ approach in conjunction with the weakest link model, Nikishkov et al. (1995b) estimate the geometry dependence of the fracture toughness for a three-dimensional crack. Through the $J-A_{2}$ two-parameter approach, the effect of specimen size and crack depth on $3 \mathrm{D}$ crack-front constraint for a SENB through-cracked specimens is investigated by Kim et al. (2003). In the study, they find that there is a distinct relationship between the crack-front constraint and the 
cleavage fracture toughness. By introducing failure curves, the minimum fracture toughness and scatter band can be well captured by using the asymptotic $J-A_{2}$ approach.

To the author's knowledge, except for those four papers mentioned in the previous paragraphs, there is no any other research on $3 \mathrm{D}$ crack-fronts using the $J-A\left(A_{2}\right)$ approach in the literature. With the validation of the applicability of the $J-A\left(A_{2}\right)$ approach on 3D crack-front fields, it can be expected that a great amount of investigation about applying the $J-A\left(A_{2}\right)$ two-parameter approach in 3D crack structures analysis will be implemented. The determination of the constraint parameter $A\left(A_{2}\right)$ is the precondition for applying the $J-A\left(A_{2}\right)$ approach. However, as mentioned in section 2.6.3, even for $2 \mathrm{D}$ models, not to mention 3D cases, there is almost no analytical or estimation method for obtaining the parameter $A\left(A_{2}\right)$ suggested in the literature except direct numerical solutions from the definition of $A\left(A_{2}\right)$. Obviously, it is a very time-consuming work to obtain numerical solution for the parameter $A\left(A_{2}\right)$ following the numerical methods illustrated in section 2.6.3.1. A "bottleneck" appears here which blocks the application of the $J-A\left(A_{2}\right)$ approach on $3 \mathrm{D}$ or even $2 \mathrm{D}$ crack models. Estimation methods for the constraint parameter $A\left(A_{2}\right)$ which predict $A\left(A_{2}\right)$ values quickly and accurately have to be developed systematically for the convenience of applying the $J-A\left(A_{2}\right)$ approach.

\subsection{Closing Remarks}

One-parameter and two-parameter approaches for linear elastic and elastic-plastic fracture mechanics have been reviewed. The determining methods for two kinds of fracture mechanics parameters, dominant and constraint parameters, are illustrated and discussed through literature review. Based on these reviews and discussions, applications of several typical two-parameter approaches of elastic-plastic fracture mechanics on twodimensional (2D) and three-dimensional (3D) cracked structures have been summarized and analyzed. Specifically, for the $J-A\left(A_{2}\right)$ two-parameter approach, it is evident that no estimation methods for the constraint parameter $A$ has been reported in the literature even for 2D cracked structures, not to mention 3D cases. Therefore, a systematical development of estimation methods for the constraint parameter $A$ is necessary for the 
convenience of engineering application and theoretical research.

Observing the shape similarity on curves of parameter $A$ vs. external loading ratio $\sigma / \sigma_{L}$, Nikishkov et al. (1995a) have suggested to develop approximate formulas for predicting the parameter $A$ based on the curve shape similarity, see section 2.6.3.2. O'Dowd and Shih $(1992,1994)$ have proved that the constraint parameter $Q$ can be predicted directly from the $T$-stress under low external load (see section 2.6.2.2). Therefore, it should also be possible to predict the constraint parameter $A$ from $T$-stress. As shown in section 2.5.2, Kumar et al. (1981) proposed the superposition of elastic and plastic parts of the $J$-integral, see Eq. (2.22). It is natural to think of the possibility of estimating the constraint parameter $A$ by expressing the $A$ solution as the superposition of small-scale yielding and plastic portions.

The details on developing estimation methods for constraint parameter $A$ will be presented in following chapters. In chapter 3 , three sets of methodologies for estimating the constraint parameter $A$ will be developed based on the three possibilities of predicting parameter $A$ that were mentioned above. In chapters 3, 4 and 5, extensive finite element analyses will be conducted to obtain parameter $A$ solutions. Based on these numerical solutions, the three developed estimation methodologies will be applied to develop approximate formulas of parameter $A$ for 2D and 3D cracked structures under uniaxial and biaxial loadings. 


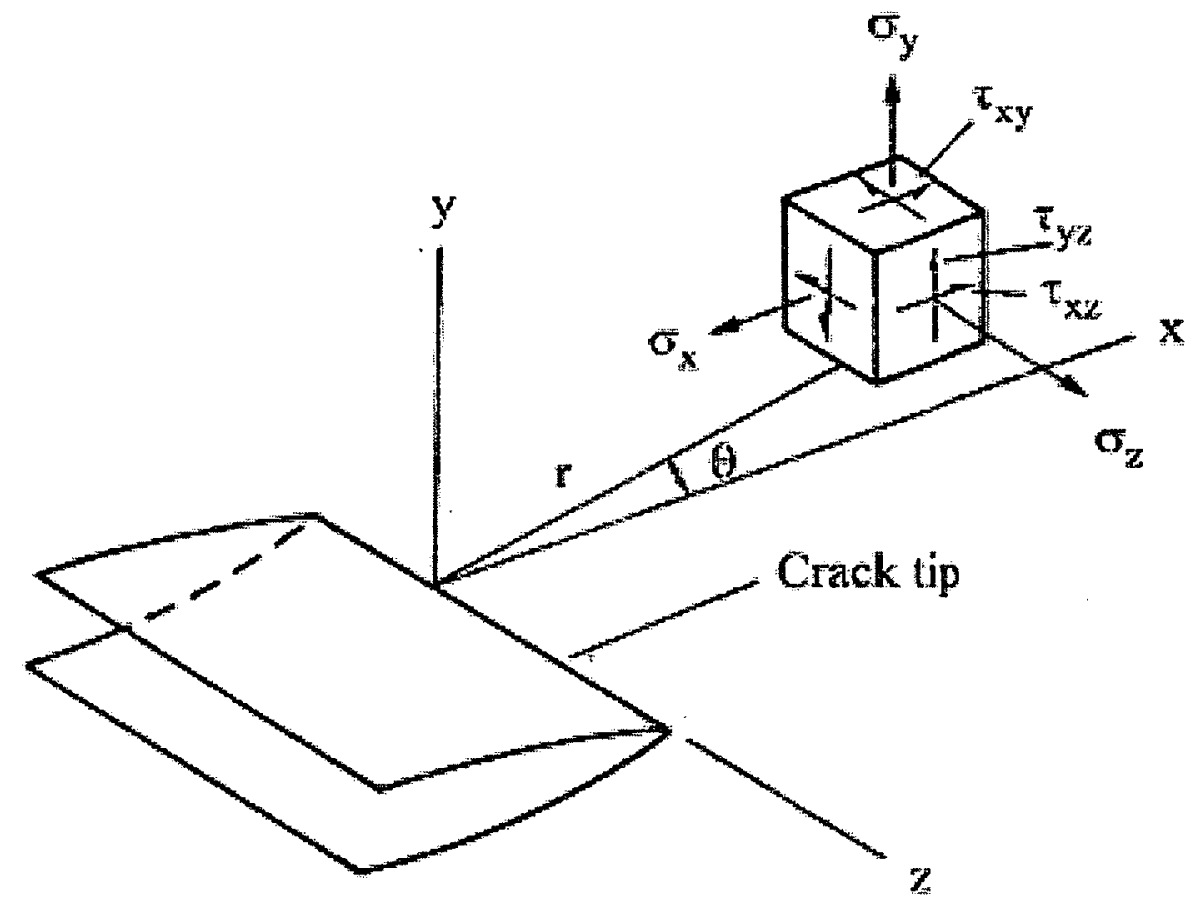

Figure 2.1 Typical crack-tip (-front) stress field (Anderson (2005)) 


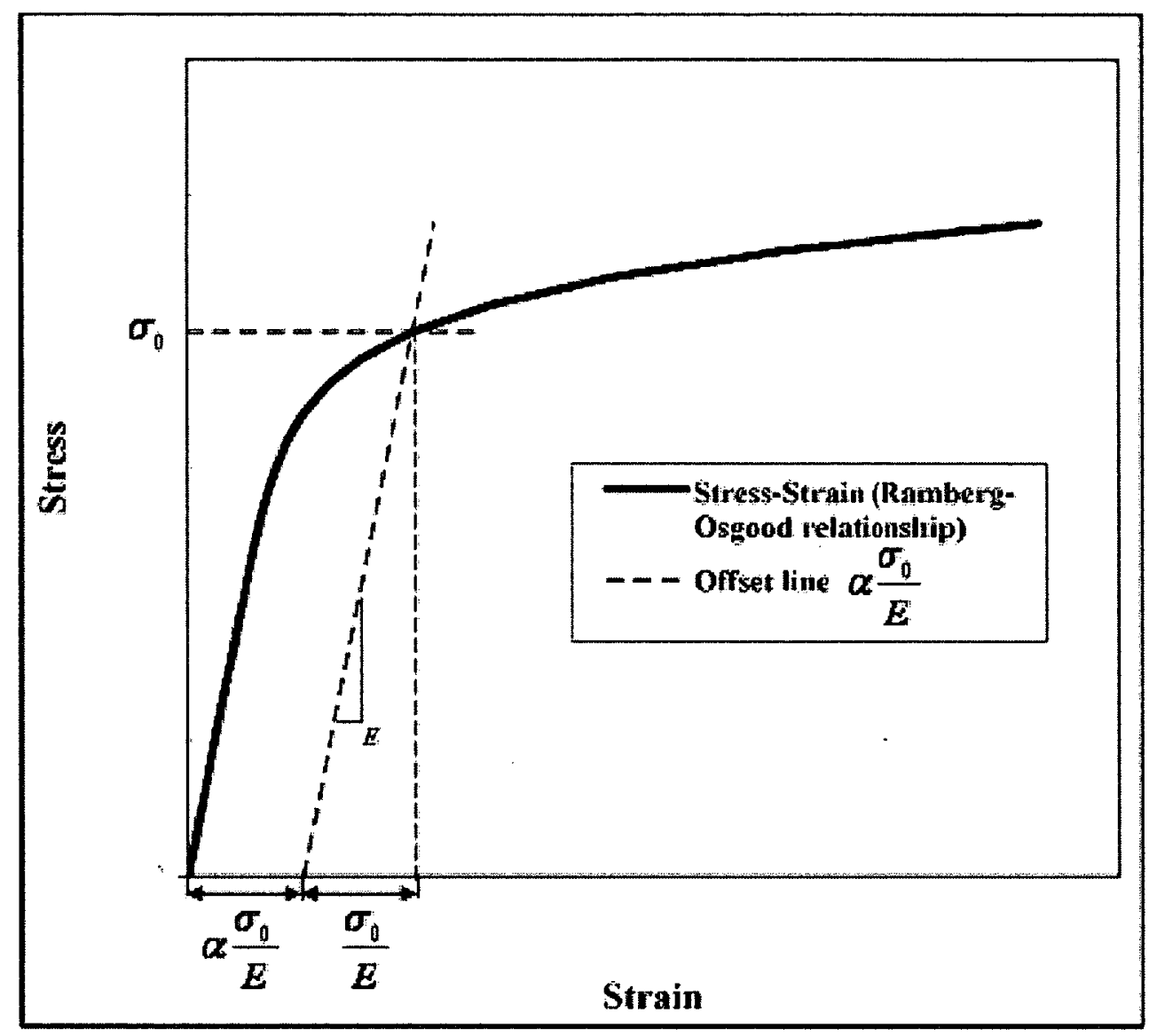

Figure 2.2 Stress-strain curve for Ramberg-Osgood power-law strain hardening relation under uniaxial load (Anderson (2005)) 


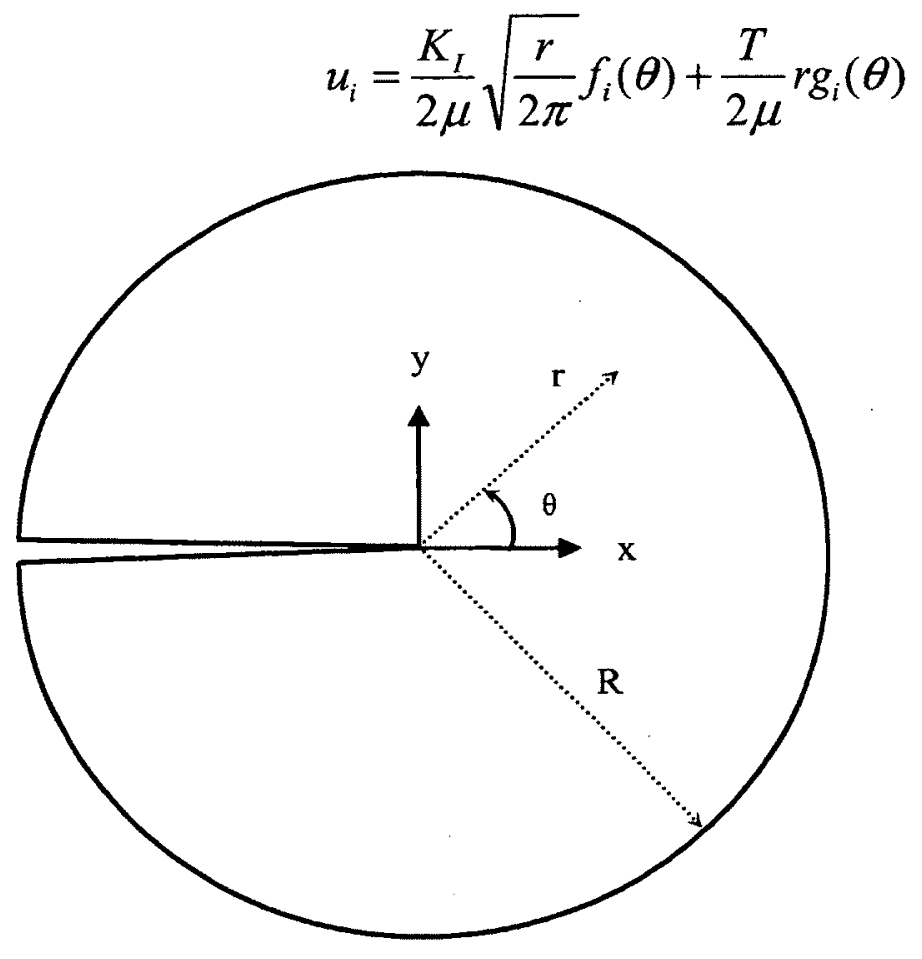

Figure 2.3 Modified boundary layer (MBL) problem model 


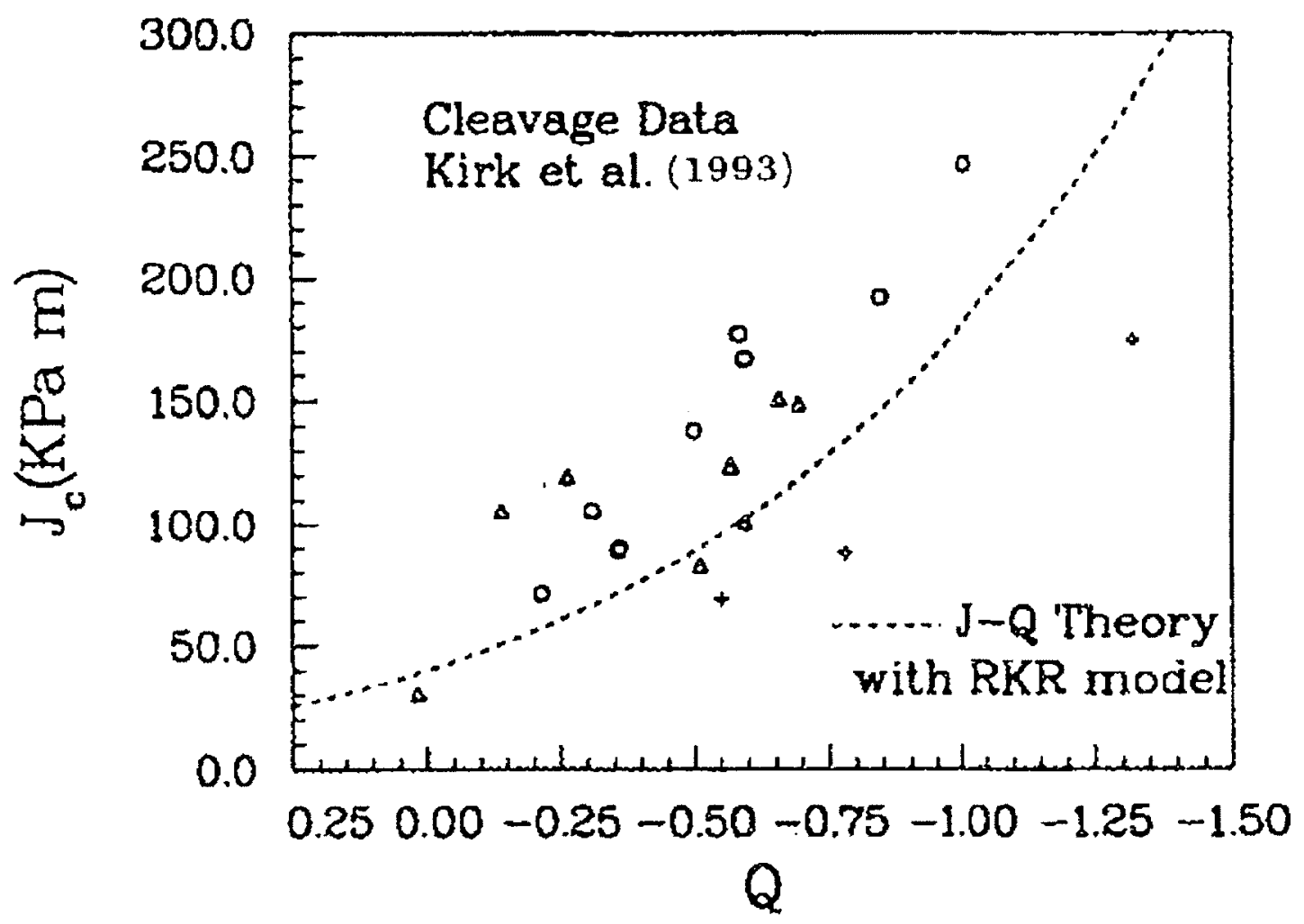

Figure 2.4 The dependence of critical values of $J$-integral, $J_{c}$, on constraint level, $Q$, (O'Dowd and Shih (1994)) 


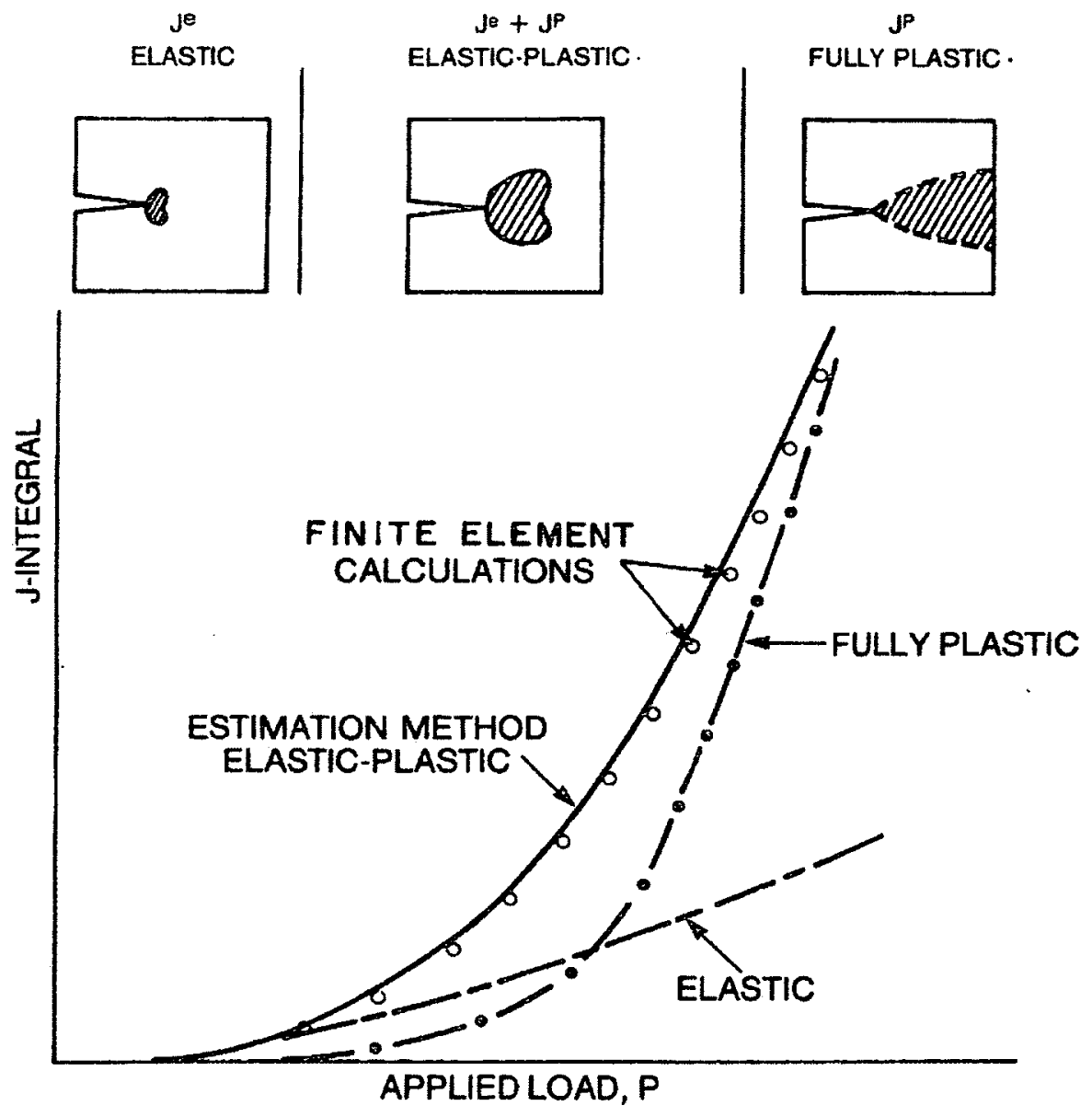

Figure 2.5 Illustration of the superposability of $J$-integral (Kumar et al. (1981)) 


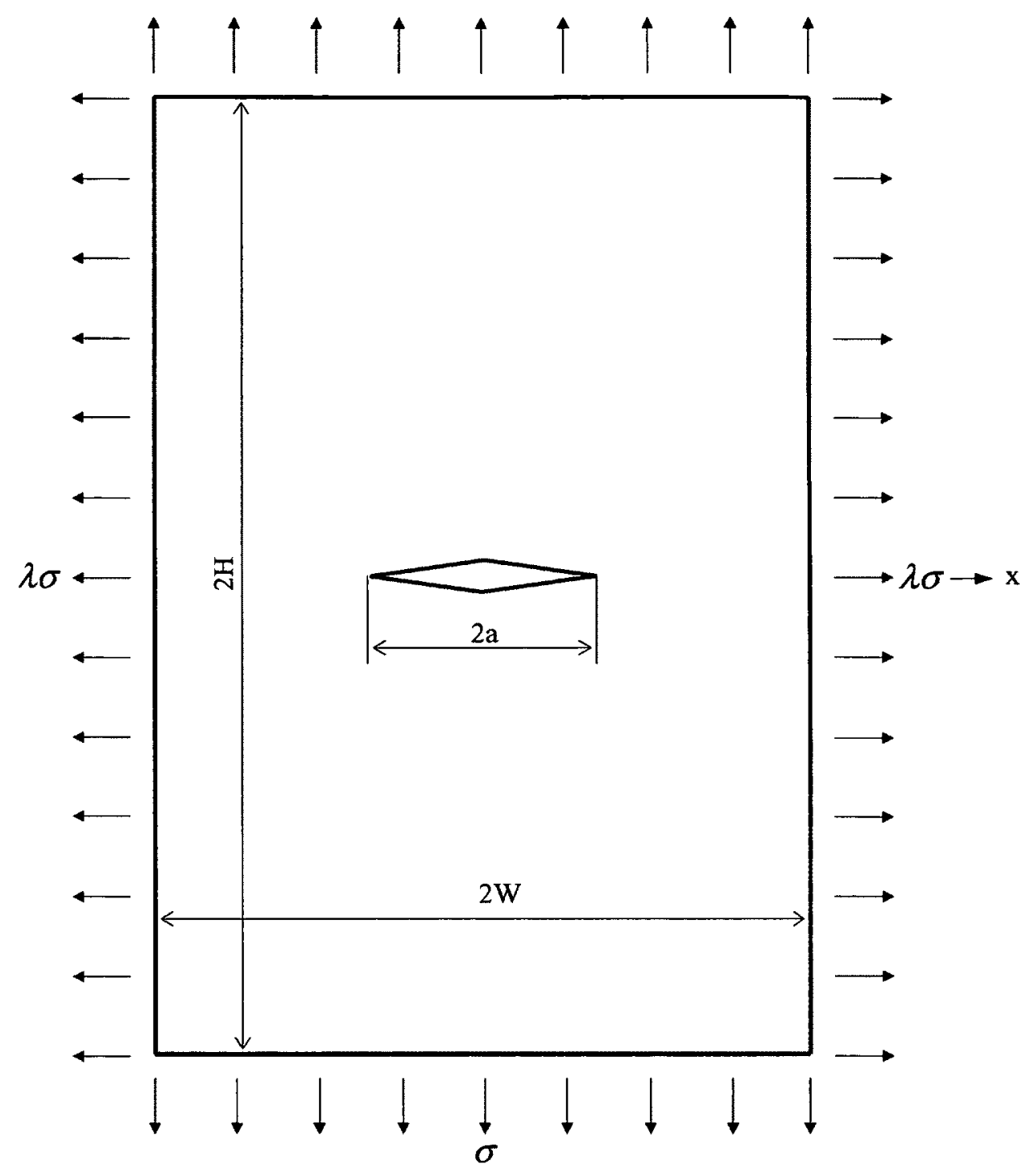

Figure 2.6 2D CCP model under biaxial loading condition 


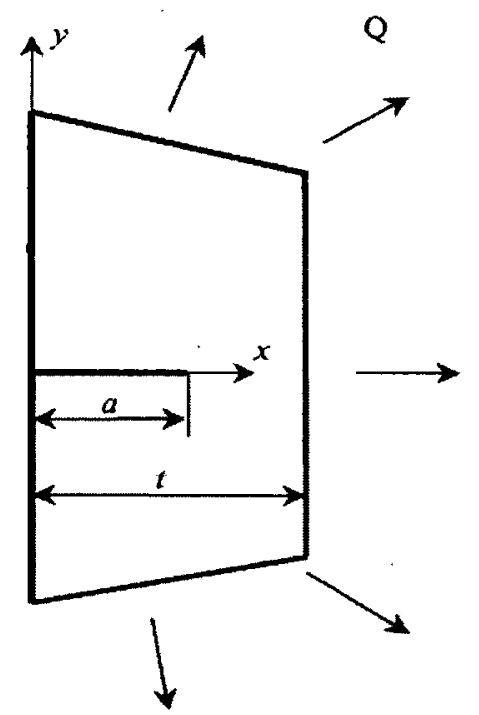

(a)

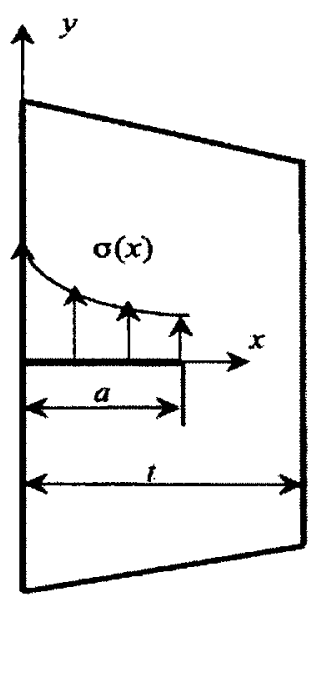

(b)

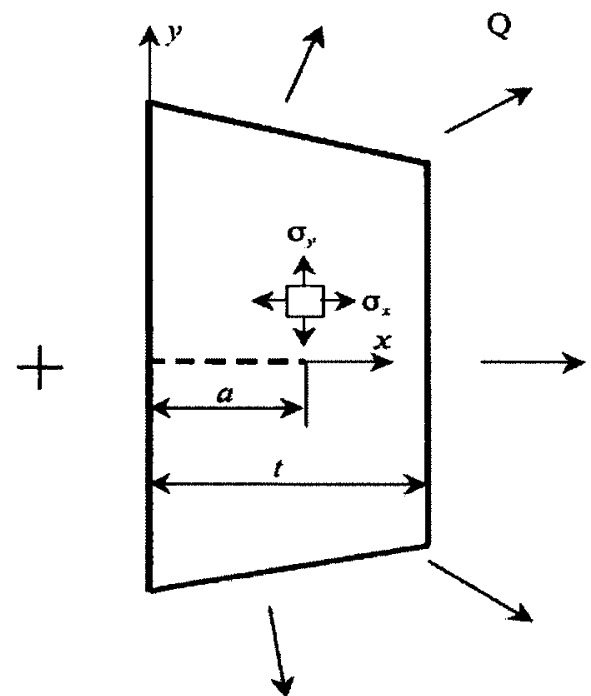

(c)

Figure 2.7 Superposition of $T$-stress (Wang (2002)) 


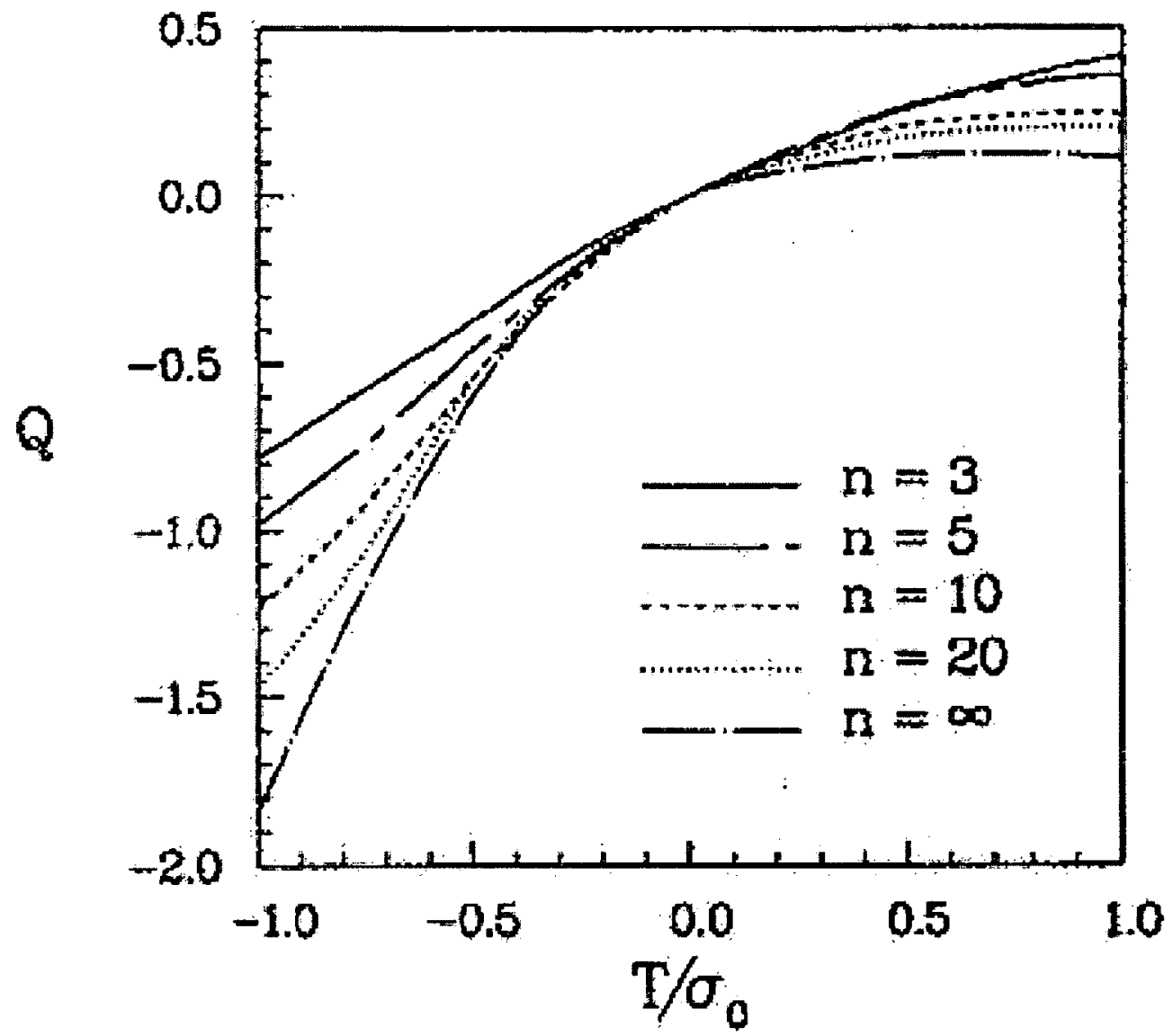

Figure 2.8 Illustration of one-to-one $Q-T$ correspondence for various hardening exponent $n$ (O’Dowd and Shih (1994)) 


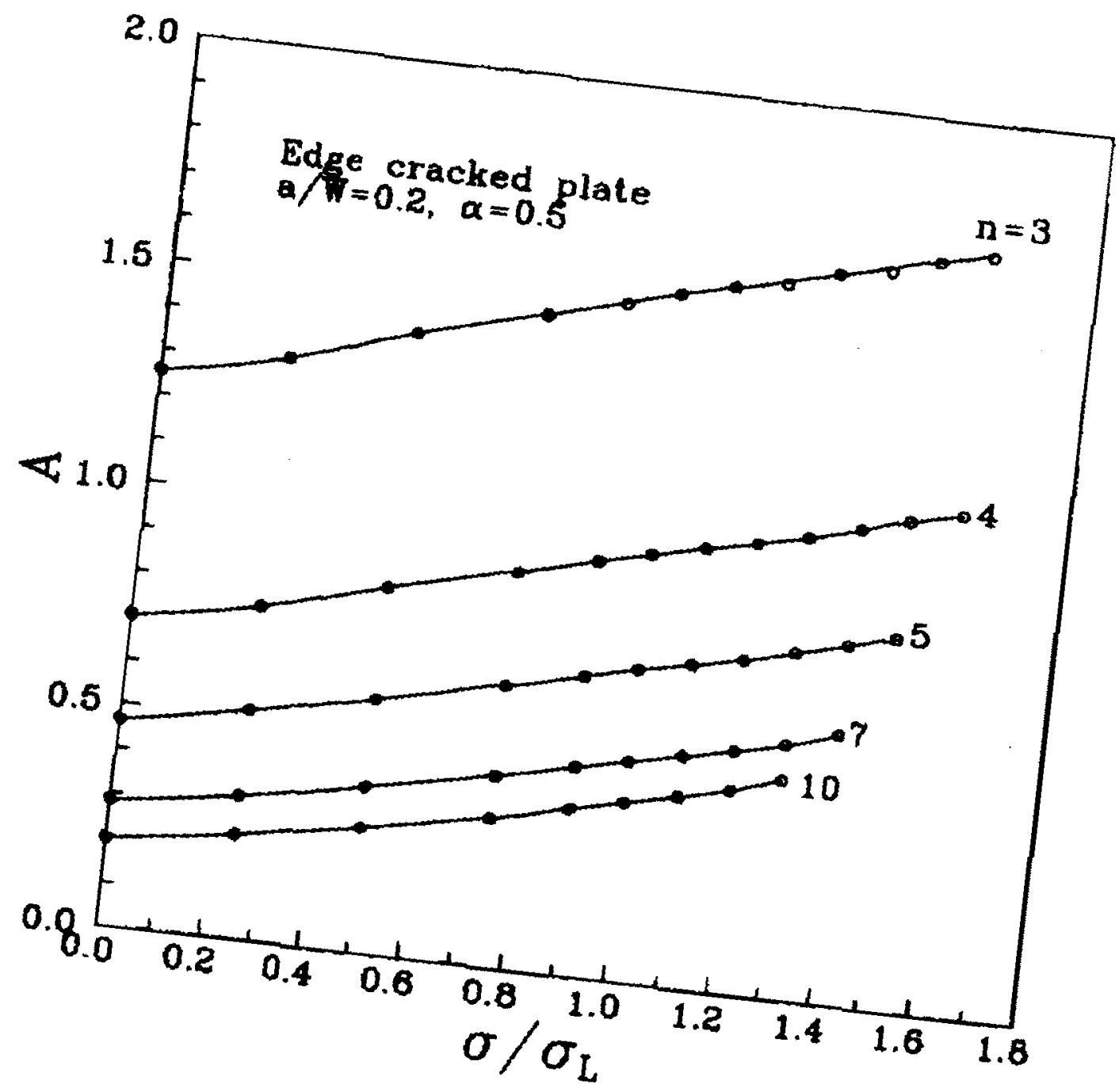

Figure 2.9 Curve shape similarity of parameter $A$ for SECP specimen with $\alpha=0.5$,
a/W=0.2 (Nikishkov et al. $(1995 \mathrm{a})$ ) 


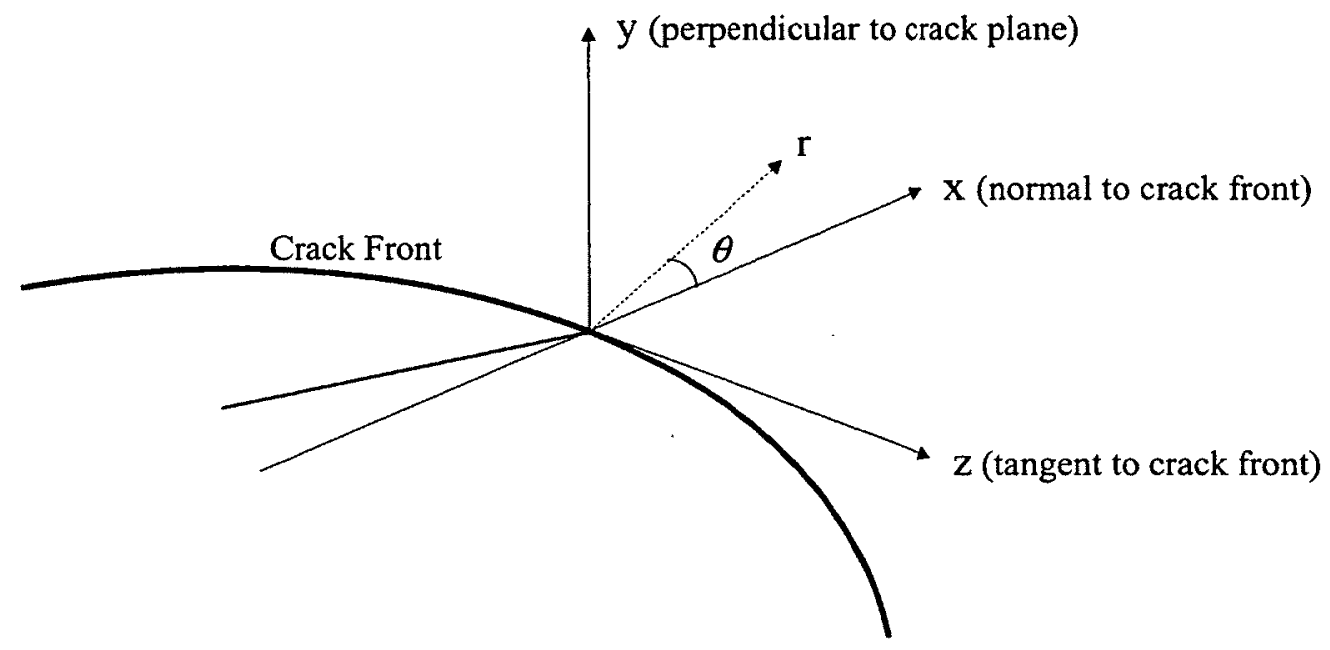

Figure 2.10 Illustration of terms "in-plane" and "out-of-plane" at 3D crack-front 


\section{CHAPTER 3 THE DEVELOPMENT OF ESTIMATION METHODS}

\subsection{Introduction}

In this chapter, several estimation methods for the determination of constraint parameter $A$ are developed based on those theories, laws and equations that were reviewed in chapter 2 . The method of predicting parameter $A$ values by the $A$ curve shape similarity property is proposed first. Next, the one-to-one $A-T$ relationship under smallscale yielding (SSY) condition is discussed, and the method for estimating the constraint parameter $A$ directly from the $T$-stress is formulated based on the relationship that is valid for SSY. Under large-scale yielding (LSY) conditions, the fully-plastic $A$ solution is shown to relate with external load by a scale coefficient. Constraint parameter $A$ solutions under LSY can be obtained when the scale coefficient is determined. Finally, combining parameter $A$ solutions for the SSY condition obtained from $T$-stress and fully plastic $A$ solutions, formulas predicting the $A$ values over the entire range from small-scale yielding to large-scale yielding condition are proposed. Applicability of all three developed estimate methods to three-dimensional cracked structures is also discussed.

\subsection{Prediction of Constraint Parameter $\boldsymbol{A}$ Based on Curve Shape}

\section{Similarity}

\subsubsection{Formulating curve shape similarity of parameter $\boldsymbol{A}$}

As mentioned in section 2.6.3.2, Nikishkov et al. (1995a) found the similarity of parameter $A$ curve shape based on numerical solutions of parameter $A$ for some planestrain cases (as shown in Figure 2.9). They suggested that the similarity could be utilized to develop approximate formulas for parameter $A$ determination for typical specimens. The parameter $A$ curve shape similarity is also observed in numerical solutions of parameter $A$ for all specimen models and loading conditions (both uniaxial and biaxial 
load) investigated in the present work (the later chapters will provide more details). Therefore, the similarity is used to develop empirical equations of $A$ for a wide range of loading, geometry and material hardening exponents.

According to the $A$ curve shape similarity, it can be said, for one particular specimen geometry, if the $A$ vs. $\sigma / \sigma_{L}$ curve for one particular hardening exponent $n$ has been obtained, it can be used to predict the $A$ vs. $\sigma / \sigma_{L}$ curves for other $n$ values. For example, if the results for $n=10$ have been obtained, the $A$ curves for other $n$ can be obtained simply from the following formulation:

$$
A\left(\frac{a}{W}, \frac{\sigma}{\sigma_{L}}, n\right)=A_{S S Y}(n)+\left\{A\left(\frac{a}{W}, \frac{\sigma}{\sigma_{L}}, n=10\right)-A_{S S Y}(n=10)\right\}
$$

where $A_{S S Y}(n)$ is value of parameter $A$ under small-scale yielding with $T=0$ (standard small-scale yielding condition) for various hardening exponent $n$. Here $A_{S S Y}$ is utilized to present parameter $A$ values under zero load. Nikishkov et al. (1995a) demonstrate that parameter $A$ solutions under zero load can be closely approximated by $A$ values under standard SSY with low enough external load.

Besides the similarity of the $A$ vs. $\sigma / \sigma_{L}$ curves under uniaxial load found by Nikishkov et al. (1995a), in fact, the $A$ vs. $\sigma / \sigma_{0}$ ( $\sigma_{0}$, yield stress) curves also follow the same shape similarity under biaxial load based on the observation for the numerical solutions of parameter $A$ obtained in present investigation (see details in chapter 5). As an example, Figure 3.1 illustrates the curve shape similarity for the 2D CCP model with $a / W=0.7$ under biaxial loading $(\lambda=1.0)$, which is a result from chapter 5 . The result in Figure 3.1 is for the curves with $\sigma / \sigma_{0}$ as remote load ratio. It can be argued that the parameter $A$ curve shape similarity is independent of the loading condition and the amplitude utilized to normalize the external load $\sigma$. For cases where the external load $\sigma$ is normalized by other reference strength parameters, e.g. yield stress $\sigma_{0}$, just replace the term $\sigma / \sigma_{L}$ in Eq. (3.1) by the term $\sigma / \sigma_{0}$. 


\subsubsection{Predicting parameter $\boldsymbol{A}$ by curve shape similarity}

Eq. (3.1), which simulates the parameter $A$ curve shape similarity, can be utilized to predict parameter $A$ solutions.

The first term of Eq. (3.1), $A_{S S Y}(n)$, is the zero load parameter $A$ solution. Just as described in section 3.1.1, $A_{S S Y}(n)$ can be approximately determined by standard smallscale yielding (SSY, $T=0$ ) simulation with extremely-small external loading.

After determination of parameter $A$ solutions for a specified hardening exponent $n$, the second term of Eq. (3.1) can be obtained by a least squares fitting method. For example, if the FEA solutions of parameter $A$ for $n=10$ are available, then parameters $A\left(a / W, \sigma / \sigma_{L}, n=10\right)$ for the specimen and $A_{S S Y}(n=10)$ for SSY with $n=10$ are used to carry out the curve fitting. Based on the least squares fitting, a polynomial expression of the second term in Eq. (3.1) can be determined. Then, for any other $n$ values, only the first term, $A_{S S Y}(n)$, changes as a function of $n$.

Having determined parameter $A$ solutions for one specified hardening exponent $n$, using the present method, the constraint parameter $A$ under arbitrary loading can be predicted by Eq. (3.1) for any other $n$ values based on its own zero load solution.

\subsection{Prediction of Constraint Parameter $A$ Based on $T$-stress}

\subsubsection{Determining parameter $A$ under small-scale yielding}

The small-scale yielding (SSY) condition corresponds to a load level with a small crack tip plastic zone, compared to the other dimensions of the cracked body. Under this condition, it has been demonstrated that the stress and strain fields are controlled by the first two terms of the Williams series expansion, with the first term relating to the stress intensity factor $K$ (or equivalently the $J$-integral) and the second being the elastic $T$-stress. Therefore elastic-plastic crack tip stress fields can be parameterized by two fracture parameters $J$ and $T$ (the so-called $J-T$ characterizations) (Betegon and Hancock (1991), Al-Ani and Hancock (1991) and Du and Hancock (1991)). 
O'Dowd and Shih $(1991,1992)$ have shown that there is a one-to-one relationship between $T$-stress and another constraint parameter $Q$ under SSY condition (see section 2.6.2.2). Since the $Q$ parameter is directly related to $A$ (Nikishkov et al. (1995a), Ding and Wang (2010)), it is expected that there is a one-to-one relationship between $T$-stress and $A$ under the small-scale yielding condition, which will enable the determination of $A$ for cracked specimens under SSY conditions through the $T$-stress. The $A-T$ relationship will be discussed and derived in the next section.

\subsection{2 $A-T$ relationship}

\subsubsection{Existence of A-T relationship}

In $J-Q$ two-parameter approach (O'Dowd and Shih $(1991,1992)$ ), the second fracture parameter $Q$ is defined by the difference between the crack tip stress field under consideration and a reference stress field. There are two reference fields that are commonly used, the HRR field and the standard small-scale yielding (SSY) solution (with $T=0$ ). In the following derivations, the $Q$ based on the SSY reference field is used (similar derivations can also be made using $Q$ based on the HRR reference field, see the Appendix for reference). The definition of parameter $Q$ based on the SSY reference stress fields is expressed by Eq. (2.16b).

O'Dowd and Shih (1991, 1992 and 1994) have shown that within the modified boundary layer (MBL) formulation (which will be discussed in the following paragraphs), $Q$ is directly related to $T$-stress. For a power-law hardening material with hardening exponent $n$, it follows that:

$$
Q=Q\left(\frac{T}{\sigma_{0}}, n\right)
$$

By least-squares fitting, a close approximate to the relationships between $Q$ (standard SSY reference field) and $T$ can be expressed as:

$$
Q=b_{1}(n)\left(\frac{T}{\sigma_{0}}\right)+b_{2}(n)\left(\frac{T}{\sigma_{0}}\right)^{2}+b_{3}(n)\left(\frac{T}{\sigma_{0}}\right)^{3}
$$


The coefficients $b_{1}(n)$ to $b_{3}(n)$ can be found in the papers of O'Dowd and Shih (1994). On the other hand, for the case of a standard SSY reference field, the relationship between $Q$ and $A$ is expressed by Eq. (2.35b). By comparing Eq. (3.3) and Eq. (2.35b) one can get relationship between $A$ and $T$. At $\theta=0, r=2 J / \sigma_{0}$ :

$$
\begin{gathered}
\frac{A^{2}}{A_{0}} \bar{r}^{2 t-s} \bar{\sigma}_{i j}^{(2)}(\theta)-A \bar{r}^{t} \bar{\sigma}_{i j}^{(1)}(\theta)-\left[\left(-A_{S S Y}(n) \bar{r}^{t} \bar{\sigma}_{i j}^{(l)}(\theta)+\frac{A_{S S Y}^{2}(n)}{A_{0}} \bar{r}^{2 t-s} \bar{\sigma}_{i j}^{(2)}(\theta)\right)\right. \\
\left.+b_{1}(n)\left(\frac{T}{\sigma_{0}}\right)+b_{2}(n)\left(\frac{T}{\sigma_{0}}\right)^{2}+b_{3}(n)\left(\frac{T}{\sigma_{0}}\right)^{3}\right]=0
\end{gathered}
$$

Here a quadratic equation for $A$ is established, which can be solved for the following $T-A$ relationship relating $T / \sigma_{0}$ and $n$ with $A$ :

$$
A=A_{T}\left(\frac{T}{\sigma_{0}}, n\right)
$$

That is, there is a one-to-one relationship between $A$ and $T$-stress. Here the term $A_{T}$ is used to indicate the $A$ values calculated through $T$-stress. This relationship can be obtained by solving Eq. (3.4), or by direct finite element analyses of modified boundary layer model.

\subsubsection{Modified boundary layer formulation for parameter $A$}

With the demonstration of existence of a one-to-one $A-T$ relationship, the detailed expression of the relationship needs to be determined. Modified boundary layer (MBL) simulation can be used to formulate the $A-T$ relationship.

In the MBL formulation, different crack-tip stress fields can be induced by applying different combinations of $K$ and $T$ values in the boundary analysis based on an elasticplastic finite element analysis (see Eq. (2.8) or (2.9)). In other words, a wide range of analysis for loading of the same $J(K)$ and varying $T$ can be conducted. Through these calculations, the crack-tip stress distribution of the MBL model characterized by two fracture parameters $J$ and $T$ then can be investigated. Then the parameter $A$ values corresponding to varying $T$-stress can be obtained from the calculated crack-tip stress fields by the fitting method suggested by Nikishkov et al. (1995a). 


\subsubsection{Determination of A-T relationship}

Using parameter $A$ solutions from FEA results for varying $T$-stress, the formulation of a one-to-one $A-T$ relationship can be obtained by least-square fitting (details will be discussed in chapter 4). Generally, it can be numerically expressed as:

$$
A_{T}\left(\frac{T}{\sigma_{0}}, n\right)=A_{S S Y}(n)+m_{1}(n)\left(\frac{T}{\sigma_{0}}\right)+m_{2}(n)\left(\frac{T}{\sigma_{0}}\right)^{2}+m_{3}(n)\left(\frac{T}{\sigma_{0}}\right)^{3}
$$

It means that the one-to-one $A-T$ relationship can be described in a third order polynomial format under low load conditions for each hardening exponent $n$ value. The values of coefficients $m_{1}(n), m_{2}(n)$ and $m_{3}(n)$ for each hardening exponent $n$ are determined in a fitting procedure. Note zero load solution of parameter $A, A_{S S Y}(n)$, are in fact the $A$ values for zero $T$-stress conditions.

Based on the finite element analysis of MBL problem carried out in the present work, a phenomenon is observed: the $A$ vs. $T / \sigma_{0}$ curves for different hardening exponents $n$ have a similar shape and generally they only differ from each other by the constants, $A_{S S Y}(n)$. Figure 3.2 shows the shape similarity of $A$ vs. $T / \sigma_{0}$ curves for $2 \mathrm{D} \mathrm{MBL}$ formulation, which is the result from the investigation in chapter 4. The mentioned phenomenon in the MBL model is similar to that observed by Nikishkov et al. (1995a) in several cracked specimens. Both for the MBL formulation and specific cracked specimens, once the $A$ vs. $T / \sigma_{0}\left(\sigma / \sigma_{L}\right.$ or $\sigma / \sigma_{0}$ for specific specimens) curve for one specified $n$ has been obtained, it also can be used to predict the $A$ vs. $T / \sigma_{0}\left(\sigma / \sigma_{L}\right.$ or $\left.\sigma / \sigma_{0}\right)$ curves for other $n$ values. Therefore, for example based on the parameter $A$ values for $n=10, A$ solutions in the MBL formulation can be obtained from the $T$-stress in a similar format as the equation for specific specimens (Eq. (3.1)):

$$
A_{T}\left(\frac{T}{\sigma_{0}}, n\right)=A_{S S Y}(n)+\left\{A_{T}\left(\frac{T}{\sigma_{0}}, n=10\right)-A_{S S Y}(n=10)\right\}
$$

With condition $n=10$, combining Eq. (3.6) and Eq. (3.7), a simplified format of Eq. (3.6) 
can be expressed as:

$$
A_{T}\left(\frac{T}{\sigma_{0}}, n\right)=A_{S S Y}(n)+m_{1}(n=10)\left(\frac{T}{\sigma_{0}}\right)+m_{2}(n=10)\left(\frac{T}{\sigma_{0}}\right)^{2}+m_{3}(n=10)\left(\frac{T}{\sigma_{0}}\right)^{3}
$$

Coefficients $m_{1}(n=10), m_{2}(n=10)$ and $m_{3}(n=10)$ are coefficients $m_{1}, m_{2}$ and $m_{3}$ for $n=10$ case.

After obtained from the analysis of MBL formulation, the one-to-one $A-T$ relationships described in Eq. (3.6) (or Eq. (3.8)) can be applied to various specimen geometries and loading types under small-scale yielding conditions as long as the $T$-stress solutions are available.

\subsubsection{Predicting parameter $\boldsymbol{A}$ by $\boldsymbol{T}$-stress}

To predict $A$ solutions of cracked specimens such as SECP, CCP or DECP investigated in the present thesis using the $A-T$ relationship, $T$-stress solutions are required. Once the $T$-stress values are obtained, Eq. (3.6) or (3.8) can be utilized to predict the parameter $A$ under small-scale yielding conditions. Comprehensive investigations of $T$-stress for a wide range of $2 \mathrm{D}$ and $3 \mathrm{D}$ crack configurations are reported in the literature, for example, the papers of Kfouri (1986), Nakamura and Parks (1992), Wang (2002, 2003 and 2004) as well as Yu and Wang (2005).These method enable predict $T$-stress conveniently and accurately. For the cases in which neither approximate nor analytical solutions for $T$-stress are available in the literature, $T$-stress can be determined numerically. For example, Nakamura and Park (1992) proposed an interaction integral method to calculate $T$-stress along a 3D crack-front by finite element analysis.

The term $T / \sigma_{0}$, the $T$-stress normalized by yield stress $\sigma_{0}$, as required in Eq. (3.6) or (3.8), can be expressed by the external load ratio $\sigma / \sigma_{L}$, which is normalized by limit stress $\sigma_{L}$, through the following:

$$
\frac{T}{\sigma_{0}}=\frac{T}{\sigma} \cdot \frac{\sigma}{\sigma_{0}}=V\left(\frac{a}{W}\right) \cdot \frac{\sigma}{\sigma_{0}}=V\left(\frac{a}{W}\right) \cdot\left(\frac{\sigma_{L}}{\sigma_{0}}\right) \cdot\left(\frac{\sigma}{\sigma_{L}}\right)
$$


where $V=T / \sigma$ is the normalized $T$-stress.

For the cases when the remote load applied on specimen $\sigma$ is normalized by the limit stress $\sigma_{L}$, substituting Eq. (3.9) into (3.6), one can get an estimate of $A$ in terms of the external load ratio $\sigma / \sigma_{L}$ as follows:

$$
A_{T}\left(\frac{\sigma}{\sigma_{L}}, \frac{a}{W}, n\right)=A_{S S Y}(n)+\left(\frac{\sigma}{\sigma_{L}}\right) g_{1}\left(\frac{a}{W}, n\right)+\left(\frac{\sigma}{\sigma_{L}}\right)^{2} g_{2}\left(\frac{a}{W}, n\right)+\left(\frac{\sigma}{\sigma_{L}}\right)^{3} g_{3}\left(\frac{a}{W}, n\right)
$$

where $g_{i}(a / W, n)=\left[V(a / W)\left(\sigma_{L}(a / W) / \sigma_{0}\right)\right]^{i} m_{i}(n), i=1,2,3$.

In other cases, if the remote load applied on specimen $\sigma$ is normalized by the material yield stress $\sigma_{0}$ instead of the specimen limit stress $\sigma_{L}$. Eq. (3.6) also can be expressed as:

$$
A_{T}\left(\frac{\sigma}{\sigma_{0}}, \frac{a}{W}, n\right)=A_{S S Y}(n)+\left(\frac{\sigma}{\sigma_{0}}\right) g_{1}\left(\frac{a}{W}, n\right)+\left(\frac{\sigma}{\sigma_{0}}\right)^{2} g_{2}\left(\frac{a}{W}, n\right)+\left(\frac{\sigma}{\sigma_{0}}\right)^{3} g_{3}\left(\frac{a}{W}, n\right)
$$

where $g_{i}(a / W, n)=[V(a / W)]^{i} m_{i}(n), i=1,2,3$. Note that the $g_{i}$ are different for loading normalized by $\sigma_{L}$ or $\sigma_{0}$.

For the application of the simplified form of the $A-T$ relationship (Eq. (3.8)), two equations which are similar to Eq. (3.10) and Eq. (3.11) can be obtained easily.

After all of the functions are determined in Eqs. (3.10) and (3.11), they are then ready to be used to predict parameter $A$ values of specimens from normalized $T$-stress, $V$.

\subsection{Prediction of Constraint Parameter $\boldsymbol{A}$ Based on Fully-Plastic Solution}

\subsubsection{Parameter $A$ under fully-plastic state}

The $A-T$ relationship (Eq. (3.6) or Eq. (3.8)) is only applicable for the estimation of $A$ for lower loading levels, because as the load increases, the plastic zone size increases and the stress fields are not sufficiently contained in the surrounding elastic field. In this section, the parameter $A$ solution for a pure power law material (fully plastic condition) is discussed. It forms the basis of estimating $A$ solutions under large-scale yielding condition. 
As discussed in chapter 2, the parameter $A$ and another parameter $A_{2}$ used in the $J-A_{2}$ characterization (Yang (1993), Yang et al. (1993) and Chao et al. (1994)) are directly related. They are related through equation (2.34). One can rewrite Eq. (2.34) as:

$$
A=-\left(\alpha \varepsilon_{0} I_{n}\right)^{s}\left(\frac{J}{\sigma_{0} L}\right)^{t-s} A_{2}
$$

Yang et al. (Yang (1993), Yang et al. (1993)) and Chao et al. (1994) have demonstrated that, under higher loading (large $J$-integral), $A_{2}$ approaches a constant which depends on the hardening exponent $n$. From Eq. (3.12), it can be seen that the parameter $A$ scales with $(J)^{t-s}$ for pure power law materials (fully plastic condition).

Furthermore, for fully-plastic condition, Kumar et al. (1981) (for uniaxial load, e.g. Eq. (2.23)) and O'Dowd et al. (1999) (for biaxial load, e.g. Eq. (2.24)) have shown that:

$$
J \propto\left(\frac{\sigma}{\sigma_{L}}\right)^{(n+1)}
$$

where $\sigma_{L}$ is the limit load for a given crack configuration. Substituting Eq. (3.13) into Eq. (3.12), it can be concluded that:

$$
A \propto\left(\frac{\sigma}{\sigma_{L}}\right)^{(n+1)(t-s)}
$$

That is, for pure power law materials, parameter $A$ is proportional to $\left(\sigma / \sigma_{L}\right)^{(n+1) /(t-s)}$. Therefore, for any specified geometry, equation (3.14) can be rewritten as:

$$
A=a_{1}\left(\frac{\sigma}{\sigma_{L}}\right)^{(n+1)(t-s)}
$$

where $a_{l}$ is a dimensionless constant depending only on the geometry configuration and the material hardening exponent $n$. Note that $s$ and $t$ are constants, depending on $n$.

For the cases in which the yield stress $\sigma_{0}$ (or another reference stress) instead of the limit stress $\sigma_{L}$ is utilized to normalize the remote stress $\sigma$, Eq. (3.15) can be rewritten as:

$$
A=a_{1}\left(\frac{\sigma}{\sigma_{0}}\right)^{(n+1)(t-s)}
$$


Here the value of $a_{l}$ is different from that in Eq. (3.15) by $\left(\sigma_{0} / \sigma_{L}\right)^{(n+1)(t-s)}$.

\subsubsection{Determining Coefficient $\boldsymbol{a}_{1}$}

According to Eq. (3.15) or (3.16), once the value of coefficient $a_{1}$ is determined, $A$ solutions of pure power law materials under arbitrary loading condition can be predicted.

Theoretically, the coefficient $a_{1}$ can be determined based on the constant $A_{2}$ under high loading condition by Eqs. (3.12) and (3.13). For example, for a CCP specimen under uniaxial load, substituting Eq. (2.23) into Eq. (3.12), and then comparing the result with Eq. (3.15), one can obtain the following expression of coefficient $a_{1}$ :

CCP:

$$
a_{1}(a / W, n)=-\left(\alpha \varepsilon_{0}\right)^{t} I_{n}{ }^{s}\left[\left(\frac{(1-a / W) a}{L}\right)^{(t-s)} h_{1}{ }^{(t-s)}\right] A_{2}
$$

Correspondingly, for cases when the remote stress $\sigma$ normalized by the yield stress $\sigma_{0}$, Eq. (3.17) can be rewritten as;

$\mathrm{CCP}: \quad a_{1}(a / W, n)=-\left(\alpha \varepsilon_{0}\right)^{t} I_{n}^{s}\left[\left(\frac{(1-a / W) a}{L}\right)^{(t-s)} h_{1}^{(t-s)}\left(\frac{\sigma_{0}}{\sigma_{L}}\right)^{(n+1)(t-s)}\right] A_{2}$

The difference between Eq. (3.17) and Eq. (3.18) is the term $\left(\sigma_{0} / \sigma_{L}\right)^{(n+1)(t-s)}$.

For practical applications, unfortunately, systemic solutions for the constraint parameter $A_{2}$ are not available in the literature which would be needed to obtain coefficient $a_{1}$ directly from $A_{2}$, e.g. by Eq. (3.17) or (3.18). Instead, $a_{1}$ is determined by directly solving Eq. (3.15) or (3.16) under several specified high loading conditions where the constraint parameter $A_{2}$ approaches a constant. In the present work, this straight-forward approach is applied, see next section for details.

\subsubsection{Determining parameter $\boldsymbol{A}$ by fully plastic solution}

Following the derivations in the previous sections, for a Ramberg-Osgood material represented by Eq. (2.3), it is natural to propose that the total $A$ can be obtained by the summation of two parts, one is for the small-scale yielding portion, $A_{T}$, and another is for 
the pure power law portion (or fully plastic condition), $\Delta A$ :

$$
A=A_{T}+\Delta A
$$

where $A_{T}=A_{T}\left(\mathrm{~T} / \sigma_{0}, n\right)$ as per Eq. (3.5), and $\Delta A=a_{1}\left(\sigma / \sigma_{\mathrm{L}}\right)^{(n+1)(t-s)}$ (Eq. (3.15)). So we have:

$$
A=A_{T}\left(\frac{T}{\sigma_{0}}, n\right)+a_{1}\left(\frac{\sigma}{\sigma_{L}}\right)^{(n+1)(t-s)}
$$

Here $\Delta A$ represents the $A$ solution for a pure power law materials (or fully plastic condition). Figure 3.3 shows an example of parameter $A$ superposition, which is from chapter 4 for the case of a 2D CCP model with $a / W=0.4$ under uniaxial tension load.

It can be seen here that for any cracked component, if we know the $T$-stress solution, together with the $A_{T}$ function, and the geometry parameter $a_{1}$, Eq. (3.20) provides estimates of parameter $A$ from small-scale yielding to large-scale yielding. Similarly, substituting Eq. (3.16) and Eq. (3.5) into Eq. (3.19), an equation similar to Eq. (3.20) can be obtained for cases of external load ratio $\sigma / \sigma_{0}$ :

$$
A=A_{T}\left(\frac{T}{\sigma_{0}}, n\right)+a_{1}\left(\frac{\sigma}{\sigma_{0}}\right)^{(n+1)(t-s)}
$$

For solving $a_{1}$ from Eq. (3.15) or (3.16), accurate $\Delta A$ solutions under several specified high loading conditions have to be determined first. By Eq. (3.19), $\Delta A$ solutions can be obtained based on numerical solutions for $A$ (from FEA) and the solutions for $A$ under small-scale yielding, $A_{T}$, which are obtained from the $T$-stress under lower load.

\subsection{Application of Estimation Methods on Three-Dimensional Cracked}

\section{Structures}

As mentioned in chapter $2, J-T, J-Q$ and $J-A\left(A_{2}\right)$ two-parameter approaches utilized to develop estimation methods for the parameter $A$ in this chapter are proposed based on plane strain conditions. Other theories, laws and equations used to develop the estimation 
methods are all for two-dimensional (2D) plane-strain and plane-stress or only planestrain cases, such as the fully plastic solution of $J$-integral for CCP specimen, Eq. (2.23) or (2.24). Therefore, all of the three estimation methods for parameter $A$ values developed in this chapter, namely curve shape similarity method, $T$-stress-based method and fully plastic solution, are in fact developed based on a plane strain assumption.

Although developed for a plane strain condition, the three newly-developed estimation methods for parameter $A$ also can be utilized for three-dimensional (3D) cracked structures, which are of practical engineering significance. As illustrated in section 2.7, in the plane perpendicular to the crack-front (in-plane) of a 3D crack model, the stress fields near the crack-front possess plane strain nature while the stress fields far from the crack-front are in the plane stress state. Therefore the newly-developed estimation methods for parameter $A$ can characterize the in-plane fields of 3D crack structures since fracture mechanics focuses on the near crack-front area. Along almost the whole crack-front (thickness) of a through-cracked specimen or almost the whole crackfront curve of a surface-cracked specimen, as mentioned in section 2.7, except for the region near the free surface, a plane strain state of stress prevails near the crack-front. Therefore, along almost the whole crack front except for the region near the free surface, in planes perpendicular to the 3D crack-front, the three newly-developed estimation methods can be applied. The out-of-plane effect along the crack-front, i.e. the variation of the field character and fracture parameters $J, A$ for each different plane perpendicular to the crack-front, however, needs to be investigated.

\subsection{Closing Remarks}

Three estimation methods for the determination of the constraint parameter $A$ have been developed. Their applicability to both two-dimensional (2D) and three-dimensional (3D) crack bodies has been rationalized. In the following chapters, the newly-developed estimation methods will be applied to both 2D and 3D crack models under uniaxial and biaxial loadings. 


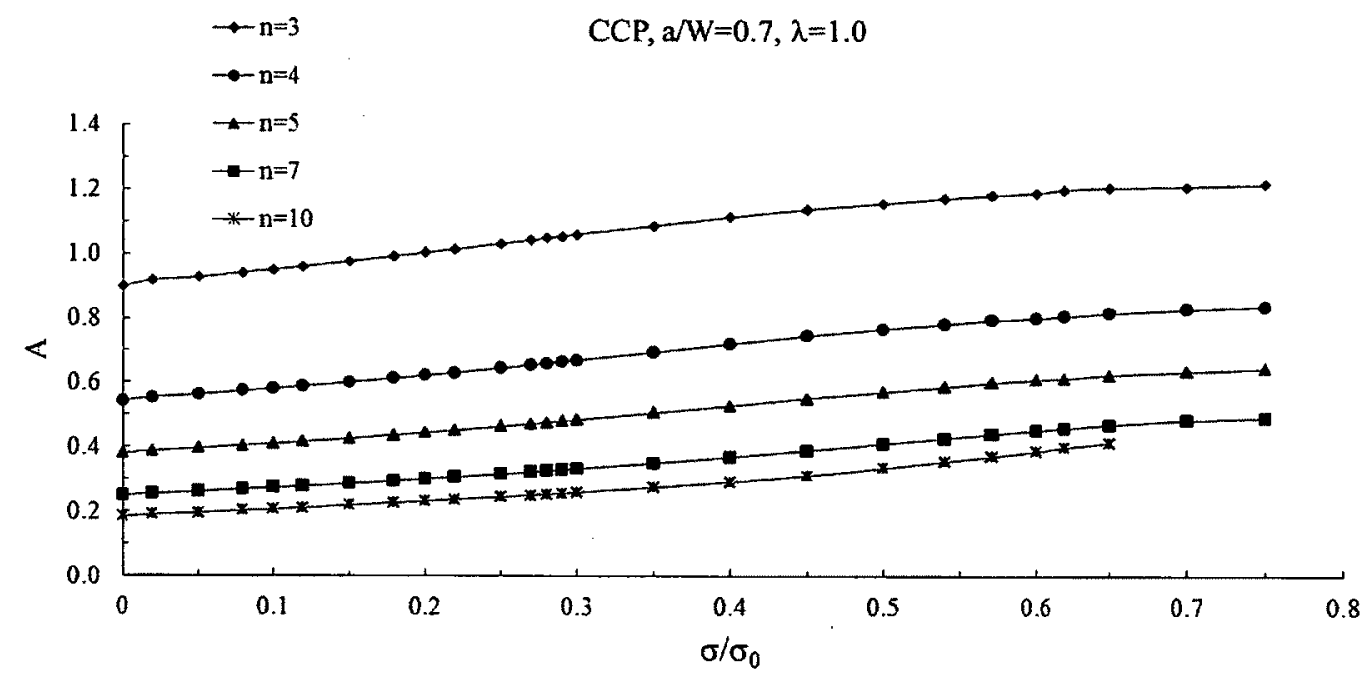

Figure 3.1 Shape similarity for curves of constraint parameter $A$ vs. remote load ratio $\sigma / \sigma_{0}$ under biaxial load, 2D CCP with $a / W=0.7, \lambda=1.0$ 


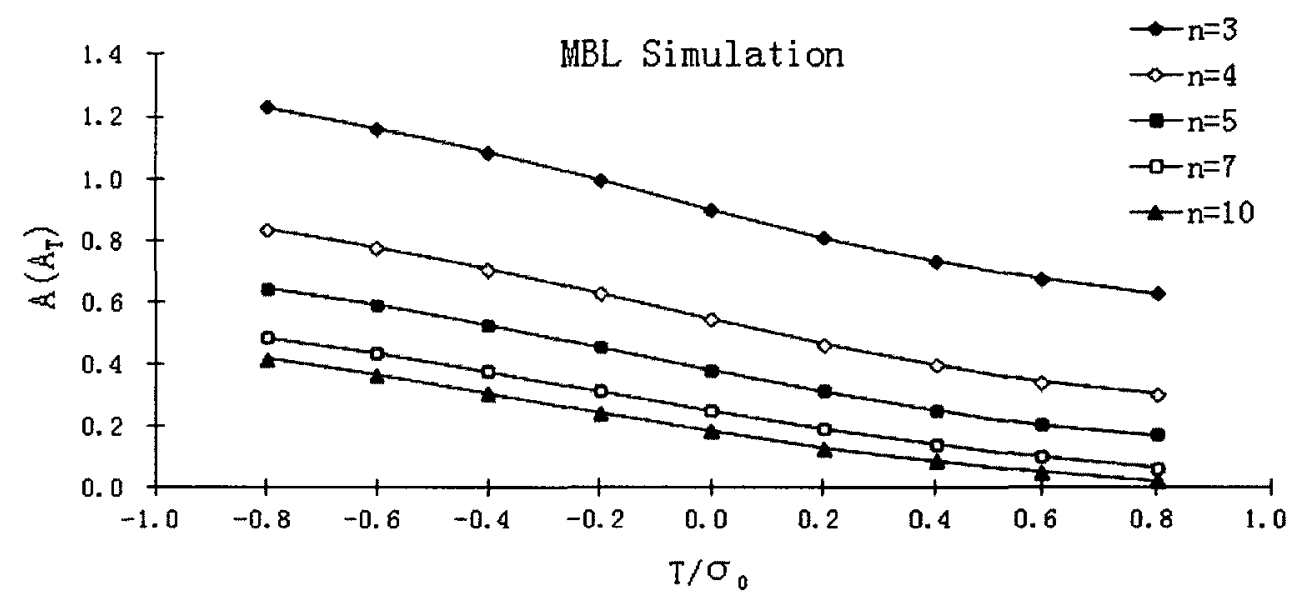

Figure 3.2 Curves of $A$ values from FEA data vs. normalized $T$-stress, $T / \sigma_{0}$, for $2 \mathrm{D}$ MBL formulation, $E / \sigma_{0}=500$, plane strain 


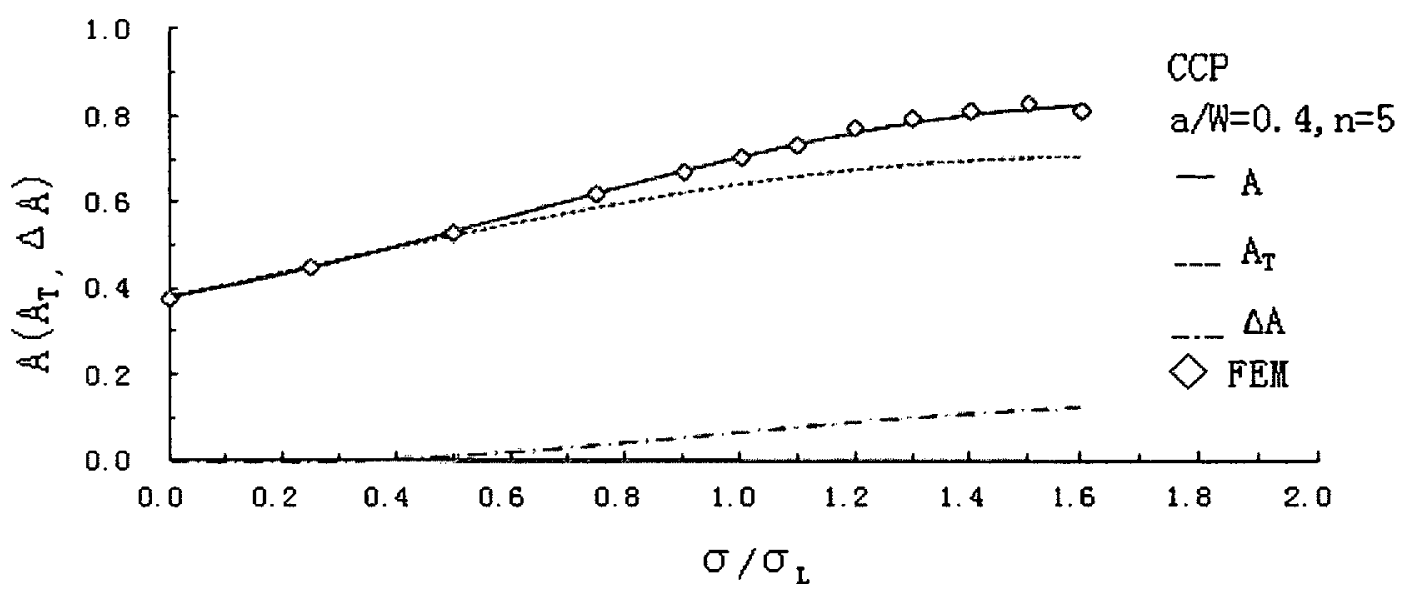

Figure 3.3 The superposition of small-scale yielding and fully plastic parts of constraint parameter $A$ for 2D CCP model with $a / W=0.4$ under uniaxial load 


\section{CHAPTER 4 SOLUTION OF CONSTRAINT PARAMETER $\boldsymbol{A}$}

\section{FOR 2D SPECIMENS UNDER UNIIAXIAL LOADING}

\subsection{Introduction}

In this chapter, extensive finite element analyses (FEA) are conducted to obtain the crack-tip stress fields for the modified boundary layer (MBL) problem and various test specimen models (SECP, CCP and DECP) under uniaxial loading. The details of the finite element models are outlined, including material properties. Within a specified fitting region, numerical solutions of the constraint parameter $A$ for MBL and SECP, CCP and DECP are determined by the fitting method proposed by Nikishkov et al. (1995a).

Through the obtained numerical solution of $A$, constraint levels of the three $2 \mathrm{D}$ specimens under uniaxial loading are discussed.

Based on the numerical solutions for constraint parameter $A$ for SECP, CCP and DECP, detailed expressions to estimate parameter $A$ values by $A$ curve shape similarity are obtained. With numerical solutions of parameter $A$ for the MBL problem, the $A-T$ relationship is determined, then formulas predicting the parameter $A$ from $T$-stress for specimens under small-scale yielding are proposed. Estimation equations for the parameter $A$ for specimens from low to high load (small-scale to large-scale yielding) are developed based on $A$ values from $T$-stress and numerical solutions of parameter $A$ with the help of a coefficient $a_{l}$.

Predicted parameter $A$ solutions from the three estimation formulas developed in this chapter are compared with those obtained from FEA results to discuss the applicability of suggested estimation formulas for constraint parameter $A$ on 2D cracked specimens under uniaxial loading.

By several practical examples, it is validated in this chapter that when the alternative constraint parameters $Q$ and $A_{2}$ are calculated from the obtained solutions of constraint parameter $A$ through the relationships among $Q, A_{2}$ and $A$, there is agreement with results 
reported in the literature.

\subsection{Finite Element Analysis of Modified Boundary Layer Formulation}

\subsubsection{Problem definition}

The geometry and coordinate systems (polar and Cartesian coordinates) of the twodimensional modified boundary layer (MBL) problem are illustrated in Figure 4.1, where $R$ is the maximum radius of MBL model.

The material model used in the finite element analysis (FEA) for the 2D modified boundary layer problem is the deformation theory of plasticity. A Ramberg-Osgood power-law strain hardening relation is provided in the finite element code ABAQUS (2006). The uniaxial stress-strain curve from the Ramberg-Osgood relation is described by Eq. (2.3).

$$
\frac{\varepsilon}{\varepsilon_{0}}=\frac{\sigma}{\sigma_{0}}+\alpha\left(\frac{\sigma}{\sigma_{0}}\right)^{n}
$$

Note that, in all the finite element analyses (FEA) in this thesis (this chapter and chapters 5 and 6), deformation plasticity model in ABAQUS are used. In all loading cases analyzed in this thesis, the loadings are proportional and monotonically increasing. Under this condition, deformation plasticity and incremental plasticity are essentially equivalent and their results of FEA should be almost same.

For the investigation in the current chapter, the following material properties are used for the FEA of MBL problem: yield stress $\sigma_{0}=4.0 \times 10^{8} \mathrm{~Pa}$; elasticity modulus $E=2.0 \times 10^{11} \mathrm{~Pa}$; Poisson ratio $v=0.3$; hardening exponent $n=3,4,5,7$, and 10 ; and material coefficient $\alpha=1.0$. These material properties cover the wide range of both high and low strain hardening behaviors.

For the purpose of comparing with the parameter $A$ results reported by Nikishkov $e t$ al. (1995a), with $n=3,4,5,7,10$, finite element analyses are also carried out for case of $\alpha=0.5$.

Note that the used material properties that are used here correspond to a $E / \sigma_{0}$ ratio 
of 500. Specially, for the MBL formulation in this chapter, to investigate the effect of varying $E / \sigma_{0}$ ratio, additional analyses with a different $\sigma_{0}=6.667 \times 10^{8} \mathrm{~Pa}\left(E / \sigma_{0}\right.$ ratio of 300 ) are also implemented for $n=3$ and 10 .

In this chapter, the MBL formulation under plane strain condition is analyzed. Through the stress intensity factor $K_{I}$ and $T$-stress, Eq. (2.8) expresses the displacement boundary conditions, which is used to describe load condition of the MBL problem.

Plane strain: $\begin{aligned} u_{x} & =\frac{K_{I}}{2 \mu} \sqrt{\frac{r}{2 \pi}} \cos \left(\frac{\theta}{2}\right)\left[\kappa-1+2 \sin ^{2}\left(\frac{\theta}{2}\right)\right]+\frac{1-v}{2 \mu} \operatorname{Tr} \cos \theta \\ u_{y} & =\frac{K_{I}}{2 \mu} \sqrt{\frac{r}{2 \pi}} \sin \left(\frac{\theta}{2}\right)\left[\kappa+1-2 \cos ^{2}\left(\frac{\theta}{2}\right)\right]+\frac{(-v)}{2 \mu} \operatorname{Tr} \sin \theta\end{aligned}$

To simulate the small-sale yielding (low load) condition, the value of far-field $J$ integral is fixed as $1.0 \times 10^{4} \mathrm{~J} / \mathrm{m}^{2}$, which is related with $K_{I}$ by Eq. (2.10). Finite element analyses are carried out with various values of $T$-stress, $T / \sigma_{0}=-0.8,-0.6,-0.4,-0.2,0.0$, $0.2,0.4,0.6,0.8$, for different hardening exponents $n=3,4,5,7,10$, respectively.

\subsubsection{Finite element analysis and parameter $\boldsymbol{A}$ determination}

\subsubsection{Finite element modeling}

Due to the geometrical symmetry, only half of the MBL model is modeled for finite element analysis (see Figure 4.1). Figure 4.2 illustrates a three-dimensional (3D) finite element mesh used for the MBL simulation. The mesh includes 960 elements and is assigned element type of 20-node quadratic hybrid brick with linear pressure, reduced integration (ABAQUS (2006)). Element radial sizes of finite element mesh are varied according to the geometric progression. Along the uncracked ligament of the model, the elements are constrained in $y$ direction but are free to displace in the $x$ direction (see Figures 4.2 and 4.1 ). In order to simulate the $2 \mathrm{D}$ plane strain conditions using the $3 \mathrm{D}$ mesh, an additional boundary condition, the displacement in the model thickness direction, $u_{z}=0$, is applied on this $3 \mathrm{D}$ mesh.

In addition, a mesh convergence study is conducted to ensure the finite element 
mesh is properly refined for computing the values of $A$. Different finite element models with different crack tip mesh refinements are used to calculate the $A$ values. The main varying modeling control parameters are the number of elements in the radial direction $(r), n_{r}$, and the number of elements in the angular direction $(\theta), n_{\theta}$ (see Figures 4.2 and 4.1). In the present MBL model (Figure 4.2), $n_{r}=40$ and $n_{\theta}=24$ (i.e. 960 elements) are used. A much more refined mesh with $n_{r}=100$ and $n_{\theta}=40$ (i.e. 4000 elements) is also used to calculate $A$ under standard small-scale yielding conditions ( $T=0)$.

\subsubsection{Determining parameter $A$ based on finite element analysis}

The fitting method to calculate constraint parameter $A$ from FEA results that was suggested by Nikishkov et al. (1995a) (Eqs. (2.29-2.32)) is used to obtain numerical $A$ solutions.

For the 2D MBL problem model in the present work, the fitting range to calculate the $A$ values from the FEA results by Eq. (2.29) is defined by $1.5 \leq \bar{r} \leq 3$ and $0^{\circ} \leq \theta \leq 45^{\circ}$. The stress component used for the fitting procedure following the method suggested by Nikishkov et al. (1995a) is set to be the opening stress: $\sigma_{\theta}$.

\subsubsection{Results and discussion}

\subsubsection{Verification of FEA model and analysis procedure}

Based on the finite element analysis for the 2D modified boundary layer (MBL) problem in this chapter, it has been shown that the far-field $J$-integral $\left(=1.0 \times 10^{4} \mathrm{~J} / \mathrm{m}^{2}\right)$, which is applied on the boundary of the MBL model, generates a plastic zone $r_{\mathrm{p}}$ in the finite element model which is less than $5 \%$ of the outside radius $r_{\max }=R$ of the model. This plastic zone is small enough to simulate small-scale yielding (SSY) conditions. Therefore, the choice of this $J$-integral value is appropriate. As an example, for standard small-scale yielding $(T=0)$ and for any hardening exponent $n$ analyzed, the specified farfield $J$-integral $\left(1.0 \times 10^{4} \mathrm{~J} / \mathrm{m}^{2}\right)$ generates a plastic zone $r_{\mathrm{p}}$ in the finite element model which is less than $2.2 \%$ of the outside radius $r_{\max }=R$ of the model. Of the 960 elements 
of the entire SSY model, typically 440 elements are within the plastic zone, since the element sizes decrease geometrically as $r \rightarrow 0$ to focus the greatest refinement near the crack tip.

Again, using standard small-scale yielding status $(T=0)$ as an example: with the specified fitting area and stress component to calculate the $A$ values from the FEA results by Eq. (2.29), the number of points used in the fitting of $A$ is at least 120 points, and the standard deviations in fitting are less than 0.063 for all standard SSY calculations.

The comparison between the parameter $A$ determined from the present mesh and those from the more refined mesh is shown in Table 4.1. The maximum difference of the compared $A$ values for the two meshes at different hardening exponent $n$ is $2.06 \%$. Therefore, the convergence of currently used finite element mesh for parameter $A$ determination is verified.

In order to further verify the finite element models for $2 \mathrm{D} \mathrm{MBL}$ problem and the corresponding analysis procedure, parameter $A$ values obtained from FEA are compared with the results presented in the paper of Nikishkov et al. (1995a). As the investigations of Nikishkov et al. (1995a) are based on the material coefficient $\alpha=0.5$, the analysis and calculation for comparing procedure are also carried out based on $\alpha=0.5$. Comparisons of $A$ values are made for standard SSY $(T=0)$ of the MBL problem for a wide range of values of the hardening exponent $n=3,4,5,7,10$. The results of comparing $A$ values are listed in Table 4.2. The results show that the differences in $A$ values are less than $3.5 \%$.

Through the above analyses and discussion, the present finite element models and the corresponding analysis procedures are considered appropriate to analyze the parameter $A$ values for the current MBL formulation.

\subsubsection{Numerical solution of parameter $A$}

Based on the analysis/calculation procedure and finite element meshes illustrated above, the constraint parameter $A$ solutions from FEA results for the 2D MBL problem with $T / \sigma_{0}=-0.8,-0.6,-0.4,-0.2,0.0,0.2,0.4,0.6,0.8$ and hardening exponent $n=3,4,5$, 7,10 have been obtained, which are summarized in Table 4.3. Besides results for a ratio $E / \sigma_{0}=500$, those for a ratio $E / \sigma_{0}=300$ with $n=3,10$ are also reported here for further 
discussion in a later section of this chapter.

As discussed in section 3.1.1, standard SSY status that can be utilized to simulate the zero load status of specimens corresponds to the $T=0$ case of the MBL problem. Therefore, parameter $A$ numerical solutions for standard SSY status, $A_{S S Y}$, are the $A$ numerical solutions shown in column $T / \sigma_{0}=0$ of Table 4.3.

Determined numerical solutions of the constraint parameter $A$ for the 2D MBL formulation with $E / \sigma_{0}=500$ (see Table 4.3) are presented in Figure 4.3; Figure 4.4 shows the comparison between the results of the $A-T$ relation for two $\sigma_{0} / E$ ratios, 500 and 300 , with $n=3$ and 10 .

It has been noticed when conducting MBL simulations using the Ramberg-Osgood material model, in the presence of $T$-stress, the applied far-field stress intensity factors $K$ (through Eq. (2.8), or equivalently $J$ from Eq. (2.10)), are not always the same as the ones obtained from $K$ values calculation using contour integral from the resulting crack tip stress fields. In the present work, the comparisons of the applied far-field $K\left(K_{\mathrm{far}}\right)$ and the $K$ calculated from contour integral $\left(K_{\text {local }}\right)$ obtained from finite element analyses for $n=$ 10 are shown in Table 4.4. This effect was reported in the literature by Betegon and Hancock (1991) and Wang (1993). It is due to the nonlinear portion of the RambergOsgood material model (Eq. (2.3)). Wang (1993), Wang and Parks (1995), as well as Nevalainen and Dodds (1995) have since used a modified stress-strain curve to remove this effect. For the cases studied in the present work, it can be seen the effect is relatively small except for high negative $T$-stress ratios, with a maximum $9.93 \%$ difference. In addition, in the data reduction process of calculating $A$, the local values of $K_{\text {local }}\left(J_{\text {local }}\right)$ are always used. Therefore, this effect should not have a significant impact on the outcome of the $A-T$ relations obtained from the present MBL analysis, which will be determined in section 4.4.1. 


\subsection{Finite Element Analysis of Test Specimens}

\subsubsection{Problem definition}

Three typical mode I plane strain test specimens, i.e. single edge cracked plate (SECP), center cracked plate (CCP) and double edge cracked plate (DECP) under uniaxial load are studied by finite element method. Their two-dimensional models are illustrated with geometry, coordinate systems and load condition in Figure 4.5, Figure 4.6 and Figure 4.7 respectively. The geometries of three specimen models are defined by the ratio of height to width, $H / W$, and the relative crack length $a / W$. For all three models, the ratio $H / W$ is fixed as 1.875 . Models with various relative crack length $a / W=0.1,0.2,0.3$, $0.4,0.5,0.6,0.7,0.8$ are investigated.

Same as described in section 4.1.1 for the MBL formulation, deformation theory of plasticity is used for the finite element analysis of the test specimens under uniaxial tension loading. The commercial code ABAQUS (2006) utilized in the present work provides a Ramberg-Osgood power-law strain hardening plasticity model. The details of the Ramberg-Osgood relation are described in section 2.1 .

For finite element analysis of the test specimens investigated in this chapter (SECP, $\mathrm{CCP}$ and DECP), the same material properties as those for FEA of the MBL problem are used, i.e.: yield stress $\sigma_{0}=4.0 \times 10^{8} \mathrm{~Pa}$; elasticity modulus $E=2.0 \times 10^{11} \mathrm{~Pa}$; Poisson ratio $v=0.3$; material coefficient $\alpha=1.0$; and hardening exponent $n=3,4,5,7$, and 10 . The utilized material properties cover a wide range of both high and low strain hardening behaviors.

Same as for the MBL formulation, to compare with the parameter $A$ results obtained by Nikishkov et al. (1995a), finite element analyses for SECP and CCEP specimens are also carried out for $\alpha=0.5$ with $a / W=01,0.3,0.5$ and $n=5,10$.

In the finite element analyses of three specimens (SECP, CCP and DECP), uniaxial remote tension loading is applied on the models. The limit loads (stress) $\sigma_{L}$ for rigidplastic bodies are used to normalize the applied loads for three models (Anderson (2005)): 
SECP:

$$
\sigma_{L}=1.455 \frac{a}{W}\left[-1+\sqrt{1+\left(\frac{W-a}{a}\right)^{2}}\right] \sigma_{0}
$$

$\mathrm{CCP}: \quad \sigma_{L}=\frac{2}{\sqrt{3}}\left(\frac{W-a}{W}\right) \sigma_{0}$

DECP:

$$
\sigma_{L}=\left[0.36+0.91\left(\frac{W-a}{W}\right)\right]\left(\frac{W-a}{W}\right) \sigma_{0}
$$

where $a$ is the crack depth and $W$ is the cracked plate width, as shown in Figures 4.5-4.7.

\subsubsection{Finite element analysis and parameter $\boldsymbol{A}$ determination}

\subsubsection{Finite element modeling}

In all three two-dimensional (2D) specimen models, only a half of SECP or a quarter of CCP or DECP structure is modeled in the finite element analysis due to the component symmetries (see Figures 4.5, 4.6 and 4.7).

Figure 4.8 illustrates a typical three-dimensional (3D) finite element mesh used for modeling those three two-dimensional (2D) specimen models, SECP, CCP and DECP. For all three models, the elements at the uncreacked ligaments are constrained in the direction normal to the section plane ( $y$ direction) but are free to displace along the section direction ( $x$ direction). There are totally 346 elements in each mesh (see Figure 4.8). The element type used is a 20 -node quadratic hybrid brick with linear pressure and reduced integration (ABAQUS (2006)). There are 11 wedge shaped elements that are used in the area of transition from crack tip core elements to the global elements, which are degenerated 20 -node elements.

Due to the geometrical symmetries, in both CCP and DECP models, the elements along the vertical symmetry plane are constrained perpendicular to the symmetry plane ( $x$ direction) but are able to displace freely in that plane ( $y$ direction). For CCP models, the vertical symmetry plane is at $x=0$ (see Figures 4.6 and 4.8 ), while for DECP models it is at $x=W$ (see Figures 4.7 and 4.8). To simulate 2D plane-strain conditions, additional boundary condition $\left(u_{z}=0\right)$ is used to constrain the displacements in the thickness 
direction.

As for the MBL problem, to ensure the finite element meshes are properly refined for the parameter $A$ calculation, a mesh convergence study was conducted. Single edge cracked plates (SECP) are analyzed to determine the $A$ values using the present mesh design (Figure 4.8) with $n_{r}=16$ and $n_{\theta}=15$ (i.e. 240 elements at the crack tip core region), and using the more refined mesh with $n_{r}=36$ and $n_{\theta}=15$ (i.e. 540 elements at the crack tip core). Additional analyses were also carried out using a mesh that has further refinement within the crack tip to global elements transition region.

\subsubsection{Determining parameter $A$ based on finite element analysis}

Same as for the MBL problem, to determine numerical solutions of constraint parameter $A$, the fitting method to calculate $A$ from FEA results suggested by Nikishkov et al. (1995a) (Eqs. (2.29-2.32)) is utilized.

As before, the fitting area used for determining the $A$ values for the three specimens from the FEA results using the method suggested by Nikishkov et al. (1995a) is also set as $1.5 \leq \bar{r} \leq 3$ and $0^{\circ} \leq \theta \leq 45^{\circ}$, and the stress component used in the fitting is the opening stress: $\sigma_{\theta}$.

\subsubsection{Results and discussion}

\subsubsection{Verification of FEA model and analysis procedure}

When calculating the constraint parameter $A$ from the FEA results by Eq. (2.29) with the specified area and stress component, the number of data points within the fitting area is generally between 12 and 56 . The standard deviation of the fitting is generally less than 0.1 for all specimens, material properties considered.

In Table 4.5, parameter $A$ determined from the current SECP mesh and those from the refined SECP mesh for $a / W=0.5$ with increasing $\sigma / \sigma_{L}$ are compared. The maximum difference of $A$ between meshes for all compared $n$ values and load levels is within $1.30 \%$. In addition, a similar comparison of parameter $A$ solutions between the 
current SECP mesh and the SECP mesh with further refinement within the transition region from crack tip to global element region is also performed. The maximum difference in $A$ values is within $0.95 \%$ of the results obtained using the present finite element (FE) mesh, as shown in Table 4.6. It can be concluded that the present finite element models are properly designed and sufficiently refined to obtain convergence for the parameter $A$ calculation.

The finite element models for the three test specimens together with the corresponding analysis procedure are then verified by comparing the $A$ values obtained from the current FEA with the results presented in the paper of Nikishkov et al. (1995a). The investigations of Nikishkov et al. (1995a) are based on the material coefficient $\alpha=0.5$, therefore the calculation for comparing procedure is also implemented based on condition $\alpha=0.5$. Comparisons of $A$ values are made for the SECP and CCP specimens with values of the hardening exponent $n=5,10$ and with a ratio of crack length to specimen width $a / W=0.1,0.3,0.5$.

The comparisons between parameter $A$ solutions from the current FEA and those reported by Nikishkov et al. (1995a) for SECP and CCP with $n=5$ are completed first. Tables 4.7 and 4.8 present the comparison with $n=5$ for SECP and CCP. It is shown that, for SECP and CCP cracked plates under a wide range of loading conditions, the differences are less than 5\%, except for one case with $5.0632 \%$ difference (CCP, $n=5$, $\left.a / W=0.1, \sigma / \sigma_{L}=1.5\right)$. Comparisons of SECP and CCP models were also carried out for $n=10$, and similar agreement was found, see Tables 4.9 and 4.10. For both the SECP and DECP models, almost all differences are less than $5 \%$, and the maximum difference is $5.64 \%$. Note that the finite element meshes used in the investigation of Nikishkov et al. (1995a) have a height to width ratio $H / W=3$. For meshes utilized for current 2D SECP, $\mathrm{CCP}$ and DECP models, the ratio $H / W=1.875$ is used. It is found from comparisons with FEA that the results of $A$ from the paper of Nikishkov et al. (1995a) with larger $H / W$ ratio $(H / W=3)$ show insignificant differences to the present results. Therefore, the present models are considered long enough to simulate the long plate specimens.

Based on the above analyses and discussions, it can be concluded the present finite 
element meshes and the corresponding analysis procedure are appropriate to determine the parameter $A$ values for the current 2D specimen models.

\subsubsection{Numerical solution of parameter $A$ and discussion for constraint effect}

The finite element analyses (FEA) of two-dimensional SECP, CCP and DECP specimen models are carried out to determine the $A$ values. Complete numerical solutions of the constraint parameter $A$ for SECP, CCP and DECP cracked specimens are obtained. The results of parameter $A$ are computed for a Ramberg-Osgood material of hardening exponent $n=3,4,5,7$ and 10. The calculations are carried out for a range of the ratio of crack length to specimen width $a / W=0.1,0.2,0.3,0.4,0.5,0.6,0.7,0.8$, under remote tension loading increasing from small-scale to large-scale yielding. The values of limit stress $\sigma_{L}$ for normalizing the remote load $\sigma$ are calculated by the formulas expressed in Eqs. (4.1a), (4.1b) and (4.1c). A complete list of numerically obtained $A$ values is in Tables 4.11-4.13, and typical results are plotted in Figures 4.9-4.20. Figures 4.9-4.12 show the results for SECP specimens; Figures 4.13-4.16 illustrate the results for CCP specimens; and the results for DECP specimens are plotted in Figures 4.17-4.20. Note that in these Tables and Figures for test specimens presented here, the $A$ values corresponding to zero load are the corresponding $A$ values obtained from standard SSY calculations of the MBL problem (column $T / \sigma_{0}=0$ of Table 4.3), see sections 4.1.3 and

\subsection{1.}

Based on the numerical solutions of parameter $A$ for test specimens models, referring Tables 4.11-4.13 and Figures 4.9-4.20, it can be observed generally that the $A$ value gradually increases with external load. Depending on the test specimen geometry (type of specimen, and $a / W$ ratio), for some situations, the increase of $A$ is quite significant, indicating loss of constraint. As expected, this is observed for shallow cracks in SECP and DECP and for all crack depths for CCP specimens, since these are wellknown low constraint specimens. For some other cases, the increase in $A$ value is relatively small as load increases, indicating that high crack tip constraint conditions are maintained. This is the case for deep cracks in SECP and DECP specimens.

Although the Tables for parameter $A$ numerical solutions presented here can be used 
directly, for engineering applications, it is more desirable to obtain empirical equations for $A$ of each specimen for different material properties, crack depths and load levels. This will be discussed in following sections.

\subsection{Solution of Parameter $\boldsymbol{A}$ by Curve Shape Similarity}

According to Eq. (3.1), to calculate the constraint parameter $A$ solutions by curve shape similarity, $A$ solutions under zero load have to be determined. As shown in section 4.1.3, the finite element analyses (FEA) of the modified boundary layer (MBL) problem have been carried out to obtain the $A$ values for various $T$-stress values, and numerical $A$ solutions under zero load, $A_{S S Y}$, have been tabulated in column $T / \sigma_{0}=0$ of Table 4.3.

The equation (3.1) and the fitting procedure developed in section 3.1.2 are used to predict the $A$ values for other $n$ values based on the numerical solutions of parameter $A$ for $n=10$ (Table 4.3 and Tables 4.11-4.13). Through the fitting procedure, the second term of the right side in Eq. (3.1) can be determined. Then a set of empirical formulas is obtained from Eq. (3.1) as following:

$$
A\left(\frac{a}{W}, \frac{\sigma}{\sigma_{L}}, n\right)=A_{S S Y}(n)+d_{1}\left(\frac{\sigma}{\sigma_{L}}\right)+d_{2}\left(\frac{\sigma}{\sigma_{L}}\right)^{2}+d_{3}\left(\frac{\sigma}{\sigma_{L}}\right)^{3}
$$

where

$$
\begin{aligned}
& d_{1}=e_{10}+e_{11}\left(\frac{a}{W}\right)+e_{12}\left(\frac{a}{W}\right)^{2}+e_{13}\left(\frac{a}{W}\right)^{3} \\
& d_{2}=e_{20}+e_{21}\left(\frac{a}{W}\right)+e_{22}\left(\frac{a}{W}\right)^{2}+e_{23}\left(\frac{a}{W}\right)^{3} \\
& d_{3}=e_{30}+e_{31}\left(\frac{a}{W}\right)+e_{32}\left(\frac{a}{W}\right)^{2}+e_{33}\left(\frac{a}{W}\right)^{3}
\end{aligned}
$$

The values of polynomial coefficients $e_{i j}$ are listed in Tables $4.14,4.15$ and 4.16 for SECP, CCP and DECP respectively.

The parameter $A$ values predicted from the developed empirical formula (Eq. (4.2)) are compared with those obtained from finite element analysis results (Tables 4.11-4.13). 
Good agreements are found between the predictions and finite element data. Generally, the differences are less than $10 \%$ for all the SECP, CCP and DECP specimens. The maximum differences between them are $10.06 \%, 18.50 \%, 10.77 \%$ for SECP, CCP and DECP, respectively.

It is found that if a factor $f_{a d j}(n)$ is introduced to further adjust the predicted $A$ values, the accuracy of the predictions will be even better. Therefore, an empirical fitting factor $f_{a d j}(n)$ is added in Eq. (4.2). Note that $f_{a d j}(n)$ is chosen by engineering judgment during the fitting process, it does not have any physical meaning. The values of the factor $f_{\text {adj }}(n)$ are very close to 1 .

By adding the adjustment factor, Eq. (4.2) is rewritten as:

$$
A\left(\frac{a}{W}, \frac{\sigma}{\sigma_{L}}, n\right)=\left[A_{S S Y}(n)+d_{1}\left(\frac{\sigma}{\sigma_{L}}\right)+d_{2}\left(\frac{\sigma}{\sigma_{L}}\right)^{2}+d_{3}\left(\frac{\sigma}{\sigma_{L}}\right)^{3}\right] f_{a d j}(n)
$$

where

$$
f_{a d j}(n)=(8.6-n) h+1
$$

The values of coefficient $h$ used for calculating the adjustment factor $f_{a d j}$, which is expressed in Eq. (4.5), are listed in Table 4.17.

Again, comparing the predicted parameter $A$ values from the developed empirical formula (Eq. (4.4)) with those obtained from finite element analysis results, very good agreements are achieved. Figures 4.9-4.20 illustrate the comparison between the predicted parameter $A$ values and those obtained from FEA results for SECP, CCP and DECP test specimens. Generally, the differences are less than $5 \%$ for all the SECP, CCP and DECP specimens. The maximum differences between them are $6.95 \%, 10.19 \%$, $6.59 \%$ for SECP, CCP and DECP, respectively.

\subsection{Solution of Parameter $\boldsymbol{A}$ by $\boldsymbol{T}$-stress}

\subsubsection{Determination of $\boldsymbol{A}-\boldsymbol{T}$ relationship}

In chapter 3 , the existence of a one-to-one $A-T$ relationship for small-scale yielding 
(SSY) has been demonstrated and a general expression of the relationship (Eq. (3.6)) has been established. In this section, the details (coefficients) of the $A-T$ relationship will be determined.

Extensive finite element analyses with varying $T$-stress values have been conducted in section 4.1 to determine the crack-tip stress fields using the modified boundary layer (MBL) model. Then, besides the $J$-integral, $A$ values were also obtained from these stress fields by the fitting method suggested by Nikishkov et al. (1995a). Based on the FEA results of $A$ under varying $T$-stress, which are shown in Table 4.3, the coefficients of $A-T$ relationship equation can be obtained.

According to the numerical solutions of parameter $A$ listed in Table 4.3, the results for $E / \sigma_{0}=500$ are illustrated in Figure 4.3. It can be found the $A$ vs. $T / \sigma_{0}$ curves for different case of hardening exponent $n$ have the similar shape and generally they only differ from each other by the constants, $A_{S S Y}(n)$. The resulting $A-T$ relations for two $\sigma_{0} / E$ ratios (500 and 300) are compared in Figure 4.4. It can be seen that there is a weak dependence of $A_{T}\left(T / \sigma_{0}, n\right)$ on $E / \sigma_{0}$. For engineering applications, the $A_{T}$ function obtained for $E / \sigma_{0}=500$ can be used to approximate the function for $E / \sigma_{0}=300$ with reasonable accuracy. In the following sections, only results for $E / \sigma_{0}=500$ will be further discussed.

Based on numerical solutions of $A$ from the MBL simulation, the values of the coefficients $m_{1}(n), m_{2}(n)$ and $m_{3}(n)$ in Eq. (3.6) for each case of hardening exponent $n$ were determined by the least square fitting procedure.

$$
A_{T}\left(\frac{T}{\sigma_{0}}, n\right)=A_{S S Y}(n)+m_{1}(n)\left(\frac{T}{\sigma_{0}}\right)+m_{2}(n)\left(\frac{T}{\sigma_{0}}\right)^{2}+m_{3}(n)\left(\frac{T}{\sigma_{0}}\right)^{3}
$$

Their values are given in Table 4.18. The $A-T$ relationship curves for various $n$ values expressed by Eq. (3.6) are presented in Figures 4.3. 


\subsection{2 $T$-stress solution by weight function method}

For the present cases of plane strain models under uniaxial load, $T$-stress solutions for all three specimens (SECP, CCP and DECP) can be directly obtained by the weight function method suggested by Wang (2002), referring to Eqs. (2.25) and (2.26). According to the paper of Wang (2002), the coefficients $D_{1}$ and $D_{2}$ in Eq. (2.26) can be determined by:

$$
\begin{aligned}
& D_{1}=\frac{15}{16} \pi\left(5 V_{0}-7 V_{1}\right) \\
& D_{2}=\frac{5}{16} \pi\left(35 V_{1}-21 V_{0}\right)
\end{aligned}
$$

where, for the SECP case:

$$
\begin{aligned}
& V_{0}=0.4012+38.5340\left(\frac{a}{W}\right)^{8.0252} \\
& V_{1}=0.2813+26.1749\left(\frac{a}{W}\right)^{8.0877}
\end{aligned}
$$

for the CCP case:

$$
\begin{aligned}
& V_{0}=\frac{1}{2.7206-0.4774 \exp \left(\frac{a}{W}\right)-1.4690\left(\frac{a}{W}\right)^{-2}} \\
& V_{1}=\frac{1}{4.5984-0.8062 \exp \left(\frac{a}{W}\right)-2.5343\left(\frac{a}{W}\right)^{-2}}
\end{aligned}
$$

for the DECP case:

$$
\begin{aligned}
& V_{0}=0.4729-0.09814\left(\frac{a}{W}\right)^{2}-0.02501\left(\frac{a}{W}\right)^{4} \\
& V_{1}=0.3239-0.05101\left(\frac{a}{W}\right)^{2}-0.01011\left(\frac{a}{W}\right)^{4}
\end{aligned}
$$

In addition, for plane strain models under uniaxial tension load that were examined here, the stress distribution and stress components in Eq. (2.25) are determined as: (a) under uniform loading condition: $\sigma(x)=\sigma_{\infty}$, where $\sigma_{\infty}$ is the applied far field load, and (b) uniaxial loading: $\sigma_{x}=0, \sigma_{y}=\sigma_{\infty}$. 


\subsubsection{Parameter $\boldsymbol{A}$ solution by $\boldsymbol{T}$-stress}

With the $A_{S S Y}(n)$ solutions listed in Table 4.3, coefficients $m_{i}(n)$ values from Table 4.18 and normalized $T$-stress, $V(V=T / \sigma)$, Eq. (3.10) is used to predict the parameter $A$ directly from $T$-stress under low load conditions for SECP, CCP and DECP.

$$
A_{T}\left(\frac{\sigma}{\sigma_{L}}, \frac{a}{W}, n\right)=A_{S S Y}(n)+\left(\frac{\sigma}{\sigma_{L}}\right) g_{1}\left(\frac{a}{W}, n\right)+\left(\frac{\sigma}{\sigma_{L}}\right)^{2} g_{2}\left(\frac{a}{W}, n\right)+\left(\frac{\sigma}{\sigma_{L}}\right)^{3} g_{3}\left(\frac{a}{W}, n\right)
$$

Then these $T$-based $A$ solutions from Eq. (3.10) are compared with those determined from FEA results of specimens (Tables 4.11-4.13), which vary with normalized external load, $\sigma / \sigma_{L}$. For SECP, CCP and DECP, with ratios of crack length to specimen width $a / W=0.1,0.2,0.3,0.4,0.5,0.6,0.7,0.8$, extensive comparisons between the $A$ solutions from $T$-stress and those from FEA results have been made for hardening exponents $n=3$, 4, 5, 7 and 10. Overall, good agreement is found. As examples, the comparison of results with $a / W=0.2$ are plotted in Figure 4.21 for SECP, Figure 4.22 for CCP and Figure 4.23 for DECP, respectively. Up to a load $\sigma / \sigma_{\mathrm{L}}=0.75$ for SECP, $\sigma / \sigma_{\mathrm{L}}=0.90$ for CCP and $\sigma / \sigma_{\mathrm{L}}=1.00$ for DECP, the differences are generally less than $5 \%$ for all three specimens, with maximum differences of $10.96 \%, 10.53 \%$ and $10.11 \%$ for SECP, CCP and DECP, respectively. For the wide range of material characteristics analyzed in the present work, it can be concluded that $T$-stress can be used to predict $A$ with acceptable accuracy up to an external load ratio $\sigma / \sigma_{L}=0.75,0.90,1.0$ for SECP, CCP and DECP, respectively.

For the simplified format of the one-to-one $A-T$ relationship, Eq. (3.7) or (3.8),

$$
\begin{gathered}
A_{T}\left(\frac{T}{\sigma_{0}}, n\right)=A_{S S Y}(n)+\left\{A_{T}\left(\frac{T}{\sigma_{0}}, n=10\right)-A_{S S Y}(n=10)\right\} \\
A_{T}\left(\frac{T}{\sigma_{0}}, n\right)=A_{S S Y}(n)+m_{1}(n=10)\left(\frac{T}{\sigma_{0}}\right)+m_{2}(n=10)\left(\frac{T}{\sigma_{0}}\right)^{2}+m_{3}(n=10)\left(\frac{T}{\sigma_{0}}\right)^{3}
\end{gathered}
$$

if substituting Eq. (3.9),

$$
\frac{T}{\sigma_{0}}=\frac{T}{\sigma} \cdot \frac{\sigma}{\sigma_{0}}=V\left(\frac{a}{W}\right) \cdot \frac{\sigma}{\sigma_{0}}=V\left(\frac{a}{W}\right) \cdot\left(\frac{\sigma_{L}}{\sigma_{0}}\right) \cdot\left(\frac{\sigma}{\sigma_{L}}\right)
$$


into Eq. (3.8), another form of Eq. (3.10) with normalized external load $\sigma / \sigma_{L}$ as variable can be rewritten as:

$$
\begin{aligned}
& A_{T}\left(\frac{\sigma}{\sigma_{L}}, \frac{a}{W}, n\right)=A_{S S Y}(n) \\
& +\left(\frac{\sigma}{\sigma_{L}}\right) g_{1}\left(\frac{a}{W}, n=10\right)+\left(\frac{\sigma}{\sigma_{L}}\right)^{2} g_{2}\left(\frac{a}{W}, n=10\right)+\left(\frac{\sigma}{\sigma_{L}}\right)^{3} g_{3}\left(\frac{a}{W}, n=10\right)
\end{aligned}
$$

where $g_{i}(a / W)=\left[V(a / W)\left(\sigma_{L}(a / W) / \sigma_{0}\right)\right]^{i} m_{i}(n=10), i=1,2,3$.

Parameter $A$ solutions obtained directly from the normalized $T$-stress, $V$ by Eq. (4.10) for SECP, CCP and DECP specimens are compared with those obtained from FEA models. Similarly, comparisons for SECP, CCP and DECP are made based on the ratios of crack length to specimen width $a / W=0.1,0.2,0.3,0.4,0.5,0.6,0.7,0.8$ and hardening exponents $n=3,4,5,7$ and 10. For all three specimens, up to a load $\sigma / \sigma_{L}=0.75$ for SECP, $\sigma / \sigma_{L}=0.5$ for $\mathrm{CCP}$ and $\sigma / \sigma_{L}=0.75$ for DECP, most differences are less than 5\%. Maximum differences are $11.66 \%, 11.68 \%$ and $11.63 \%$ for SECP, CCP and DECP respectively. Good agreement is shown.

Through the comparisons of predicted parameter $A$ from $T$-stress with results from FEA for SECP, CCP and DECP specimens, it is found that the load range with acceptable predictions of $A$ is smaller for the simplified $A-T$ relationship, Eq. (4.10) or Eq. (3.8), than for the original format, Eq. (3.10) or Eq. (3.6). The reason is that the curves of $A$ values vs. normalized $T$-stress, $T / \sigma_{0}$, in Figure 4.19 only have a "similar" shape; but in fact, these curves are not identical. The simplified format of the $A-T$ relationship, Eq. (4.10) or Eq. (3.8), utilizes the curve shape for a specified hardening exponent $n$, for example $n=10$, to predict $A$ solutions for other $n$ values.

Although it has smaller predicting range than the original format, the simplified $A-T$ relationship can be used to predict the parameter $A$ for other $n$ values only based on finite element analysis of the MBL problem for one single value of $n$. 


\subsection{Solution of Parameter $\boldsymbol{A}$ by Fully Plastic Analysis}

\subsubsection{Fully-plastic solution of parameter $A$}

As shown in section 4.4.3, the parameter $A$ solutions derived from $T$-stress, $A_{T}$, can provide a good estimate of $A$ up to a relatively low external loads. For SECP, CCP and DECP specimens, as the load increases, the difference term $\triangle A$, i.e. the differences between $A$ solutions from $T$-stress $\left(A_{T}\right)$ and those from FEA results, increases. Through Eq. (3.19), the $\Delta A$ term (fully-plastic solution of parameter $A$ ) for $a / W=0.1,0.2,0.3$, $0.4,0.5,0.6,0.7,0.8$ and $n=3,4,5,7$ and 10 under increasing loading is calculated based on $A_{T}$ and the finite element results (Tables 4.11-4.13). Typical results of $\Delta A$ are plotted in Figures 4.24-4.27. Figures 4.24-4.26 show the term $\Delta A$ together with the total $A$ and $A_{T}$ for SECP, DECP and CCP specimens with $a / W=0.4$ and $n=5$. Figure 4.27 shows the $\triangle A$ for varying $n$ for SECP specimen with $a / W=0.5$.

\subsubsection{Determination of coefficient $a_{1}$}

To obtain the difference $\Delta A$ under any (arbitrary) loading condition, Eq. (3.15) can be used, in which the coefficient $a_{1}$ has to be determined first. Here $a_{1}$ is determined by directly solving Eq. (3.15) with known $\Delta A$ for several specified high loading cases.

From Eq. (3.19), the $\Delta A$ values for several specified high loading cases are determined based on the $A$ solutions given by Eq. (3.6) or (3.10) $\left(A_{T}\right)$ and those obtained from FEA results for SECP, CCP and DECP (Tables 4.11-4.13).

$$
A=A_{T}+\triangle A
$$

In Tables 4.11-4.13, $A$ solutions from FEA analysis are given up to $\sigma / \sigma_{L}=1.3$ for SECP and $\sigma / \sigma_{L}=1.6$ for CCP, DECP, respectively. In the present work, the three highest loading cases for each specimens, i.e. $\sigma / \sigma_{L}=1.1,1.2,1.3$ for SECP and $\sigma / \sigma_{L}=1.4,1.5,1.6$ for CCP, DECP, are chosen to calculate $a_{1}$. The $a_{1}$ values calculated from the three highest loading cases are averaged to obtain the final coefficient $a_{1}$. 
As mentioned in section 3.3 , the constant $a_{1}$ depends on the hardening exponent $n$ and relative crack depth $a / W$, i.e. $a_{1}=a_{1}(a / W, n)$. The values of coefficient $a_{1}$ for SECP, CCP and DECP specimens are obtained and listed following a handbook format in Tables $4.19,4.20$ and 4.21 , respectively for $n=3,4,5,7,10$ and $a / W=0.1$ to 0.8 .

\subsubsection{Parameter $A$ solution by fully-plastic analysis}

With coefficient $a_{1}$ determined, the second term on Eq. (3.20) can be predicted for varying external load ratios. Now, substituting the expression of $A_{T}$ (Eq. (3.10) for the present cases) into Eq. (3.20), the final expression for predicting $A$ values for cracked specimens can be written as:

$$
A=A_{S S Y}(n)+\left(\frac{\sigma}{\sigma_{L}}\right) g_{1}+\left(\frac{\sigma}{\sigma_{L}}\right)^{2} g_{2}+\left(\frac{\sigma}{\sigma_{L}}\right)^{3} g_{3}+a_{1}\left(\frac{\sigma}{\sigma_{L}}\right)^{(n+1)(t-s)}
$$

where $g_{i}(a / W, n)=\left[V(a / W)\left(\sigma_{L}(a / W) / \sigma_{0}\right)\right]^{i} m_{i}(n), \mathrm{i}=1,2,3$, and $a_{1}(a / W, n)$ are given in Tables 4.19, 4.20 and 4.21.

Eq. (4.11) is utilized to predict the $A$ solutions for SECP, CCP and DECP cracked specimens from small-scale yielding to large-scale yielding conditions. The predicted $A$ solutions are compared with those from FEA results which are given in Tables 4.11-4.13. A complete range of comparisons $(a / W=0.1,0.2,0.3,0.4,0.5,0.6,0.7,0.8$ under $n=3$, 4, 5, 7 and 10, respectively) for SECP, CCP and DECP is carried out. Typical comparisons for $a / W=0.1,0.5,0.8$ and varying $n$ values are illustrated in Figures 4.284.36. Figures 4.28-4.30 show the results for SECP, Figures 4.31-4.33 are the comparisons for CCP and Figures 4.34-4.36 illustrate the results for DECP. Very good agreement is found for all cases. Up to a load ratio $\sigma / \sigma_{L}=1.30$ for SECP, and $\sigma / \sigma_{L}=1.60$ for CCP and DECP, the differences are generally less than $5 \%$ for all three specimens.

\subsection{Estimating Other Constraint Parameters by $\boldsymbol{A}$ Solutions}

The relationships between the second fracture mechanics parameters $A$ and $A_{2}$ as 
well as $A$ and $Q$, (i.e. Eqs. (2.34) and (2.35)), have been summarized in the chapter 2 . Using the relationships between the parameters $A, A_{2}$ and $Q$, not only numerical solutions of parameter $A$ but also the presently developed empirical formulas for predicting parameter $A$ can be utilized to predict $A_{2}$ and $Q$ values. As an example, the empirical formulas derived by $A$ curve shape similarity is used to demonstrate the possibility of predicting the constraint parameters $Q$ and $A_{2}$ from the estimated $A$ values. In other words, the parameter $A$ solutions estimated from the empirical formulas derived by curve shape similarity are used to predict the values of $Q$ and $A_{2}$.

\subsubsection{Estimating constraint parameter $A_{2}$ from parameter $A$}

The dissertation of Yang (1993) shows FEA results of parameter $A_{2}$ for one SECP model $(a / W=0.5)$ based on the following material constants $\sigma_{0}=296.5 \mathrm{MPa}$ $\varepsilon_{0}=0.001433, v=0.3$ and $\alpha=1.0$ and two hardening exponents ( $n=4$ and 7). The $A_{2}$ results were plotted as a function of the $J$-integral in the dissertation of Yang (1993). As emphasized by Yang (1993) and Chao et al. (1994), under fully plastic conditions, $A_{2}$ is independent of the material constants $\alpha, \sigma_{0}$ and $\varepsilon_{0}$. It depends only on the loading and hardening exponent $n$. Therefore, the FEA results of $A_{2}$ reported by Yang (1993) are used to compare with those predicted by $A$ curve shape similarity under fully plastic conditions. First, the $A$ values for the same SECP specimen are calculated using the developed empirical formula Eq. (4.4) for $n=4$ and 7. Eq. (2.34) is then used to convert the obtained $A$ solutions to $A_{2}$ values.

$$
A_{2}=-\left(\alpha \varepsilon_{0} I_{n}\right)^{-s}\left(\frac{J}{\sigma_{0} L}\right)^{s-t} A
$$

Note that in Eq. (2.34), the following material parameters are used for consistency, i.e., $\sigma_{0}=4.0 \times 10^{8} \mathrm{~Pa} \quad \varepsilon_{0}=\sigma_{0} / E=0.002$ and $\alpha=1.0$. The characteristic length $L$ is set as the model width, which is same as the one used by Yang (1993). The values of parameters $s, t$ and $I_{n}$ can be obtained following the algorithm suggested by Nikishkov 
(1995). To relate the $J$-integral to the external load ratio $\sigma / \sigma_{L}$, results from the present finite element analyses shown in section 4.2 are used. The comparison results are listed in Table 4.22, and very good agreement is found for both $n=4$ and 7 .

\subsubsection{Estimating constraint parameter $Q$ from parameter $A$}

The paper of O'Dowd and Shih (1994) provided parameter $Q$ results for a DECP model with the same material parameters as those used in the investigation presented in this chapter, i.e. $v=0.3, \varepsilon_{0}=\sigma_{0} / E=0.002$ and $\alpha=1.0$. In the paper of O'Dowd and Shih (1994), the FEA results of $Q$ factor for $n=10$ are plotted as the function of $\log \left(J /\left(a \sigma_{0}\right)\right)$. Here, the $A$ values for this specimen geometry are calculated using Eq. (4.4) first. This is then converted to $Q$. Since the parameter $Q$ results of O'Dowd and Shih (1994) are calculated based on a SSY reference field, Eq. (2.35b) is utilized to predict the corresponding $Q$ from parameter $A$.

$$
Q=-\left(A-A_{S S Y}\right) \bar{r}^{t} \bar{\sigma}_{i j}^{(1)}(\theta)+\frac{\left(A^{2}-A_{S S Y}^{2}\right)}{A_{0}} \bar{r}^{2 t-s} \bar{\sigma}_{i j}^{(2)}(\theta) \text { at } \theta=0, \bar{r}=2
$$

The present FEA results (in section 4.2) are also used for this specimen to relate the $J$-integral and external load ratio. The comparison of constraint parameter $Q$ is shown in Table 4.23. It is shown that the agreement is good.

In summary, it has been shown that the present results for the $A$ parameter can be used to predict other second elastic-plastic fracture mechanics parameters such as $A_{2}$ and $Q$.

\subsection{Conclusions}

Through extensive finite element analyses (FEA) of three plane strain mode I specimen: SECP, CCP and DECP, finite element (numerical) solutions of the constraint parameter $A$ for the three specimens under uniaxial tension load have been obtained in the current chapter. 
Based on the numerical solutions of the constraint parameter $A$, constraint levels of 2D cracked specimens under uniaxial loading are discussed.

In chapter 3 , three prediction methods for constraint parameter $A$ have been proposed. They are, estimating parameter $A$ based on curve-shape similarity, based on $T$ stress solutions for $A$ and parameter $A$ solutions based on fully plastic analysis. In the current chapter, with determined numerical solutions of $A$, under uniaxial loading condition, these three estimation methods are applied to three plane strain mode I specimens, SECP, CCP and DECP.

For each of the three estimation methods, predicted parameter $A$ solutions are compared with those from FEA results (numerical solutions of parameter $A$ ). Based on all of the comparing results, it is found that the developed three estimation methods for parameter $A$ are appropriate to 2D SECP, CCP and DECP models under uniaxial loading.

For 2D cracked specimens under uniaxial loading, generally, when external loading is lower (small-scale yielding), approximating constraint parameter $A$ directly from $T$ stress is a better choice among the three estimation methods. The reason is that the solutions of $T$-stress have been well established in the literature. For cases of external loadings varying from low to high (small-scale to large-scale yielding deformation), parameter $A$ can be estimated using curve shape similarity method. If loading varies from low to high and solutions of $T$-stress are available, method based on fully plastic solution should be more convenient.

By several examples, it has been validated in this chapter, the obtained solutions of constraint parameter $A$ can be utilized to determine other two commonly-used constraint parameters $Q$ and $A_{2}$ by the relationships among $A, Q$ and $A_{2}$ 


\begin{tabular}{ccccccccc}
\hline$n$ & 3 & 4 & 5 & 6 & 7 & 8 & 9 & 10 \\
\hline$A_{\text {present }}$ & 0.8984 & 0.5432 & 0.3803 & 0.2965 & 0.2489 & 0.2191 & 0.1988 & 0.1838 \\
\hline$A_{\text {refined }}$ & 0.8920 & 0.5368 & 0.3756 & 0.2923 & 0.2449 & 0.2153 & 0.1950 & 0.1801 \\
\hline Diff. (\%) & 0.71 & 1.19 & 1.24 & 1.43 & 1.63 & 1.78 & 1.94 & 2.06 \\
\hline
\end{tabular}

Table 4.1 Comparison of $A$ obtained from present and refined finite element models (MBL, $T=0$ )

\begin{tabular}{cccccc}
\hline & $\mathrm{n}=3$ & $\mathrm{n}=4$ & $\mathrm{n}=5$ & $\mathrm{n}=7$ & $\mathrm{n}=10$ \\
\hline$A_{\text {Present }}$ & 1.2525 & 0.7063 & 0.4716 & 0.2916 & 0.2063 \\
$A_{\text {[Niki.] }}$ & 1.2500 & 0.7000 & 0.4610 & 0.2818 & 0.2000 \\
\hline Diff. (\%) & 0.2022 & 0.9015 & 2.2903 & 3.4744 & 3.1654 \\
\hline
\end{tabular}

Table 4.2 Comparison of $A$ values from present FEA and solutions from Nikishkov et al. (1995a), SSY problem 


\begin{tabular}{|c|c|c|c|c|c|c|c|c|c|c|}
\hline $\mathrm{E} / \sigma_{0}$ & $n$ & $T / \sigma_{0}=-0.8$ & $T / \sigma_{0}=-0.6$ & $T / \sigma_{0}=-0.4$ & $T / \sigma_{0}=-0.2$ & $T / \sigma_{0}=0.0$ & $T / \sigma_{0}=0.2$ & $T / \sigma_{0}=0.4$ & $T / \sigma_{0}=0.6$ & $T / \sigma_{0}=0.8$ \\
\hline \multirow{5}{*}{500} & 3 & 1.2297 & 1.1626 & 1.0866 & 0.9959 & 0.8984 & 0.8077 & 0.7330 & 0.6767 & 0.6257 \\
\hline & 4 & 0.8371 & 0.7772 & 0.7074 & 0.6264 & 0.5432 & 0.4639 & 0.3957 & 0.3405 & 0.3011 \\
\hline & 5 & 0.6433 & 0.5913 & 0.5254 & 0.4529 & 0.3803 & 0.3114 & 0.2516 & 0.2032 & 0.1701 \\
\hline & 7 & 0.4857 & 0.4365 & 0.3757 & 0.3114 & 0.2489 & 0.1906 & 0.1409 & 0.0998 & 0.0652 \\
\hline & 10 & 0.4189 & 0.3659 & 0.3050 & 0.2429 & 0.1838 & 0.1298 & 0.0867 & 0.0522 & 0.0213 \\
\hline \multirow{2}{*}{300} & 3 & 1.1553 & 1.1003 & 1.0363 & 0.9617 & 0.8823 & 0.8090 & 0.7569 & 0.7205 & 0.7016 \\
\hline & 10 & 0.4229 & 0.3773 & 0.3258 & 0.2749 & 0.2272 & 0.1840 & 0.1488 & 0.1250 & 0.1226 \\
\hline
\end{tabular}

Table 4.3 $A$ values from FEA results for MBL problem (plane strain)

\begin{tabular}{cccccccccc}
\hline$T / \sigma_{0}$ & -0.8 & -0.6 & -0.4 & -0.2 & 0.0 & 0.2 & 0.4 & 0.6 & 0.8 \\
\hline$K_{\text {far }}$ & $4.688 \mathrm{E} 7$ & $4.688 \mathrm{E} 7$ & $4.688 \mathrm{E} 7$ & $4.688 \mathrm{E} 7$ & $4.688 \mathrm{E} 7$ & $4.688 \mathrm{E} 7$ & $4.688 \mathrm{E} 7$ & $4.688 \mathrm{E} 7$ & $4.688 \mathrm{E} 7$ \\
\hline$K_{\text {local }}$ & $4.223 \mathrm{E} 7$ & $4.507 \mathrm{E} 7$ & $4.613 \mathrm{E} 7$ & $4.642 \mathrm{E} 7$ & $4.654 \mathrm{E} 7$ & $4.659 \mathrm{E} 7$ & $4.660 \mathrm{E} 7$ & $4.661 \mathrm{E} 7$ & $4.700 \mathrm{E} 7$ \\
\hline diff. $(\%)$ & -9.93 & -3.86 & -1.61 & -0.97 & -0.73 & -0.63 & -0.60 & -0.58 & 0.24 \\
\hline
\end{tabular}

Table 4.4 Comparisons of the remote applied stress intensity factors, $K_{f a r}$ with those obtained from contour integral, $K_{\text {local }}$, for $n=10$ in the present MBL analyses (2D plane strain) 


\begin{tabular}{ccccc}
\hline & $\sigma / \sigma_{\mathrm{L}}$ & $A_{\text {present }}$ & $A_{\text {refined }}$ & Diff. (\%) \\
\hline$n=3$ & 0.5 & 0.9561 & 0.9559 & 0.03 \\
& 0.9 & 1.0213 & 1.0203 & 0.09 \\
& 1.1 & 1.0619 & 1.0618 & 0.01 \\
& 1.3 & 1.1089 & 1.1099 & -0.09 \\
\hline$n=5$ & 0.5 & 0.4133 & 0.4130 & 0.06 \\
& 0.9 & 0.4543 & 0.4543 & 0.00 \\
& 1.1 & 0.4843 & 0.4844 & -0.02 \\
& 1.3 & 0.5226 & 0.5220 & 0.11 \\
\hline$n=10$ & 0.5 & 0.2084 & 0.2057 & 1.30 \\
& 0.9 & 0.2357 & 0.2340 & 0.71 \\
& 1.1 & 0.2579 & 0.2585 & -0.24 \\
& 1.3 & 0.2958 & 0.2964 & -0.20 \\
\hline
\end{tabular}

Table 4.5 Comparison of $A$ obtained from present and refined finite element models (SECP, $a / W=0.5$ )

\begin{tabular}{ccccc}
\hline & $\sigma / \sigma_{\mathrm{L}}$ & $A_{\text {present }}$ & $A_{\text {refined }}$ & Diff. (\%) \\
\hline$n=3$ & 0.5 & 0.9561 & 0.9561 & 0.00 \\
& 0.9 & 1.0213 & 1.0204 & 0.09 \\
& 1.1 & 1.0619 & 1.0615 & 0.04 \\
\hline$n=5$ & 1.3 & 1.1089 & 1.1094 & -0.05 \\
\hline$n=10$ & 0.5 & 0.4133 & 0.4131 & 0.05 \\
& 0.9 & 0.4543 & 0.4543 & 0.01 \\
& 1.1 & 0.4843 & 0.4844 & -0.02 \\
& 1.3 & 0.5226 & 0.5216 & 0.20 \\
\hline & 0.5 & 0.2084 & 0.2064 & 0.95 \\
& 0.9 & 0.2357 & 0.2349 & 0.34 \\
& 1.1 & 0.2579 & 0.2588 & -0.34 \\
& 1.3 & 0.2958 & 0.2950 & 0.26 \\
\hline
\end{tabular}

Table 4.6 Comparison of $A$ obtained from present and refined finite element models (SECP, $a / W=0.5$, further refinement in transition region) 


\begin{tabular}{|c|c|c|c|c|c|c|c|c|c|}
\hline \multirow[b]{2}{*}{$\sigma / \sigma_{L}$} & \multicolumn{3}{|c|}{$a / W=0.1$} & \multicolumn{3}{|c|}{$a / W=0.3$} & \multicolumn{3}{|c|}{$a / W=0.5$} \\
\hline & $A_{\text {Present }}$ & $A_{\text {[Ref.] }}$ & $\begin{array}{l}\text { Diff. } \\
(\%)\end{array}$ & $A_{\text {Present }}$ & $A_{\text {[Ref] }}$ & $\begin{array}{l}\text { Diff. } \\
(\%)\end{array}$ & $A_{\text {Present }}$ & $A_{\text {[Ref.] }}$ & $\begin{array}{c}\text { Diff. } \\
(\%)\end{array}$ \\
\hline 0.0 & 0.4716 & 0.4610 & 2.29 & 0.4716 & 0.4610 & 2.29 & 0.4716 & 0.4610 & 2.29 \\
\hline 0.25 & 0.5270 & 0.5250 & 0.37 & 0.5063 & 0.5005 & 1.16 & 0.4838 & 0.4794 & 0.91 \\
\hline 0.50 & 0.5895 & 0.5940 & -0.76 & 0.5469 & 0.5444 & 0.46 & 0.5018 & 0.4936 & 1.66 \\
\hline 0.75 & 0.6640 & 0.6720 & -1.20 & 0.5912 & 0.5933 & -0.35 & 0.5202 & 0.5128 & 1.44 \\
\hline 0.90 & 0.7095 & 0.7183 & -1.22 & 0.6210 & 0.6244 & -0.55 & 0.5342 & 0.5278 & 1.21 \\
\hline 1.00 & 0.7289 & 0.7428 & -1.86 & 0.6403 & 0.6439 & -0.56 & 0.5476 & 0.5416 & 1.10 \\
\hline 1.10 & 0.7571 & 0.7806 & -3.02 & 0.6629 & 0.6639 & -0.15 & 0.5599 & 0.5523 & 1.38 \\
\hline 1.20 & 0.7867 & 0.8028 & -2.01 & 0.6819 & 0.6883 & -0.93 & 0.5735 & 0.5683 & 0.91 \\
\hline 1.30 & 0.8219 & 0.8345 & -1.51 & 0.7064 & 0.7050 & 0.20 & 0.5916 & 0.5872 & 0.75 \\
\hline 1.40 & 0.8601 & 0.8739 & -1.58 & 0.7256 & 0.7288 & -0.44 & 0.6099 & 0.6010 & 1.48 \\
\hline 1.50 & 0.9070 & 0.9167 & -1.06 & 0.7473 & 0.7496 & -0.31 & 0.6290 & 0.6272 & 0.28 \\
\hline
\end{tabular}

Table 4.7 Comparison of values from present FEA and solutions from Nikishkov et al. (1995a), (SECP specimen, $n=5$ )

\begin{tabular}{|c|c|c|c|c|c|c|c|c|c|}
\hline \multirow[b]{2}{*}{$\sigma / \sigma_{L}$} & \multicolumn{3}{|c|}{$a / W=0.1$} & \multicolumn{3}{|c|}{$a / W=0.3$} & \multicolumn{3}{|c|}{$a / W=0.5$} \\
\hline & $A_{\text {Present }}$ & $A_{\text {[Ref] }}$ & $\begin{array}{l}\text { Diff. } \\
(\%)\end{array}$ & $A_{\text {Present }}$ & $A_{\text {[Ref] }}$ & $\begin{array}{l}\text { Diff. } \\
(\%)\end{array}$ & $A_{\text {Present }}$ & $A_{\text {[Ref.] }}$ & $\begin{array}{l}\text { Diff. } \\
(\%)\end{array}$ \\
\hline 0.0 & 0.4716 & 0.4610 & 2.29 & 0.4716 & 0.4610 & 2.29 & 0.4716 & 0.4610 & 2.29 \\
\hline 0.25 & 0.5444 & 0.5611 & -2.99 & 0.5455 & 0.5456 & -0.01 & 0.5356 & 0.5317 & 0.73 \\
\hline 0.50 & 0.6567 & 0.6606 & -0.59 & 0.6261 & 0.6278 & -0.28 & 0.6021 & 0.6039 & -0.29 \\
\hline 0.75 & 0.7464 & 0.7600 & -1.79 & 0.7076 & 0.7167 & -1.26 & 0.6764 & 0.6811 & -0.69 \\
\hline 0.90 & 0.7935 & 0.8186 & -3.07 & 0.7531 & 0.7711 & -2.34 & 0.7200 & 0.7333 & -1.82 \\
\hline 1.00 & 0.8339 & 0.8469 & -1.54 & 0.7922 & 0.8053 & -1.63 & 0.7565 & 0.7708 & -1.86 \\
\hline 1.10 & 0.8583 & 0.8772 & -2.16 & 0.8199 & 0.8406 & -2.45 & 0.7841 & 0.8033 & -2.39 \\
\hline 1.20 & 0.8784 & 0.9056 & -2.99 & 0.8427 & 0.8775 & -3.96 & 0.8204 & 0.8361 & -1.88 \\
\hline 1.30 & 0.8962 & 0.9233 & -2.93 & 0.8782 & 0.9050 & -2.96 & 0.8432 & 0.8689 & -2.96 \\
\hline 1.40 & 0.9071 & 0.9406 & -3.56 & 0.8961 & 0.9236 & -2.98 & 0.8783 & 0.9000 & -2.41 \\
\hline 1.50 & 0.9130 & 0.9617 & -5.06 & 0.9056 & 0.9456 & -4.23 & 0.8963 & 0.9278 & -3.40 \\
\hline
\end{tabular}

Table 4.8 Comparison of values from present FEA and solutions from Nikishkov et al. (1995a), (CCP specimen, $n=5$ ) 


\begin{tabular}{|c|c|c|c|c|c|c|c|c|c|}
\hline \multirow[b]{2}{*}{$\sigma / \sigma_{L}$} & \multicolumn{3}{|c|}{$a / W=0.1$} & \multicolumn{3}{|c|}{$a / W=0.3$} & \multicolumn{3}{|c|}{$a / W=0.5$} \\
\hline & $A_{\text {Present }}$ & $A_{[\text {Ref.] }}$ & $\begin{array}{c}\text { Diff. } \\
(\%)\end{array}$ & $A_{\text {Present }}$ & $A_{\text {[Ref] }}$ & $\begin{array}{l}\text { Diff. } \\
(\%)\end{array}$ & $A_{\text {Present }}$ & $A_{\text {[Ref] }}$ & $\begin{array}{l}\text { Diff. } \\
(\%)\end{array}$ \\
\hline 0.00 & 0.2063 & 0.2000 & 3.17 & 0.2063 & 0.2000 & 3.17 & 0.2063 & 0.2000 & 3.17 \\
\hline 0.25 & 0.2507 & 0.2480 & 1.09 & 0.2330 & 0.2274 & 2.48 & 0.2144 & 0.2100 & 2.12 \\
\hline 0.50 & 0.3006 & 0.3000 & 0.20 & 0.2670 & 0.2591 & 3.06 & 0.2300 & 0.2210 & 4.08 \\
\hline 0.75 & 0.3676 & 0.3720 & -1.19 & 0.3041 & 0.2990 & 1.72 & 0.2421 & 0.2332 & 3.83 \\
\hline 0.90 & 0.4135 & 0.4200 & -1.55 & 0.3306 & 0.3273 & 1.01 & 0.2513 & 0.2436 & 3.16 \\
\hline 1.00 & 0.4479 & 0.4550 & -1.56 & 0.3512 & 0.3500 & 0.34 & 0.2631 & 0.2530 & 3.99 \\
\hline 1.10 & 0.4842 & 0.4890 & -0.98 & 0.3717 & 0.3720 & -0.09 & 0.2727 & 0.2654 & 2.76 \\
\hline 1.20 & 0.5325 & 0.5420 & -1.75 & 0.3935 & 0.3954 & -0.47 & 0.2885 & 0.2782 & 3.71 \\
\hline 1.30 & -..- & --. & -- & 0.4144 & 0.4173 & -0.69 & 0.3079 & 0.2950 & 4.38 \\
\hline 1.40 & --- & --. & -.- & 0.4407 & 0.4435 & -0.62 & 0.3309 & 0.3185 & 3.90 \\
\hline 1.50 & $\ldots$ & $\ldots$ & $\cdots$ & -- & $\ldots$ & $\ldots$ & 0.3571 & 0.3420 & 4.42 \\
\hline
\end{tabular}

Table 4.9 Comparison of values from present FEA and solutions from Nikishkov et al. (1995a), (SECP specimen, $n=10$ )

\begin{tabular}{|c|c|c|c|c|c|c|c|c|c|}
\hline \multirow[b]{2}{*}{$\sigma / \sigma_{L}$} & \multicolumn{3}{|c|}{$\mathrm{a} / \mathrm{W}=0.1$} & \multicolumn{3}{|c|}{$\mathrm{a} / \mathrm{W}=0.3$} & \multicolumn{3}{|c|}{$\mathrm{a} / \mathrm{W}=0.5$} \\
\hline & $A_{\text {Present }}$ & $A_{\text {[Ref] }}$ & $\begin{array}{l}\text { Diff. } \\
(\%)\end{array}$ & $A_{\text {Present }}$ & $A_{[\text {[Ref] }]}$ & $\begin{array}{l}\text { Diff. } \\
(\%)\end{array}$ & $A_{\text {Present }}$ & $A_{[\text {Ref. }]}$ & $\begin{array}{l}\text { Diff. } \\
(\%)\end{array}$ \\
\hline 0.00 & 0.2063 & 0.2000 & 3.17 & 0.2063 & 0.2000 & 3.17 & 0.2063 & 0.2000 & 3.17 \\
\hline 0.25 & 0.2740 & 0.2810 & -2.49 & 0.2657 & 0.2636 & 0.80 & 0.2601 & 0.2505 & 3.86 \\
\hline 0.50 & 0.3564 & 0.3600 & -1.01 & 0.3318 & 0.3318 & 0.00 & 0.3117 & 0.3089 & 0.91 \\
\hline 0.75 & 0.4227 & 0.4380 & -3.50 & 0.3962 & 0.3995 & -0.85 & 0.3698 & 0.3723 & -0.66 \\
\hline 0.90 & 0.4602 & 0.4720 & -2.51 & 0.4352 & 0.4382 & -0.68 & 0.4095 & 0.4136 & -0.99 \\
\hline 1.00 & 0.4868 & 0.4990 & -2.44 & 0.4642 & 0.4686 & -0.95 & 0.4388 & 0.4430 & -0.94 \\
\hline 1.10 & 0.5097 & 0.5130 & -0.65 & 0.4898 & 0.4966 & -1.36 & 0.4725 & 0.4748 & -0.48 \\
\hline 1.20 & 0.5261 & 0.5450 & -3.46 & 0.5159 & 0.5232 & -1.39 & 0.5028 & 0.5100 & -1.40 \\
\hline 1.30 & 0.5423 & 0.5600 & -3.15 & 0.5343 & 0.5507 & -2.97 & 0.5341 & 0.5443 & -1.88 \\
\hline 1.40 & 0.5482 & 0.5810 & -5.64 & 0.5535 & 0.5771 & -4.09 & 0.5546 & 0.5664 & -2.07 \\
\hline
\end{tabular}

Table 4.10 Comparison of values from present FEA and solutions from Nikishkov et al. (1995a), (CCP specimen, $n=10$ ) 


\begin{tabular}{|c|c|c|c|c|c|c|c|c|c|c|}
\hline & \multirow[b]{2}{*}{$a / W$} & \multicolumn{9}{|c|}{$\sigma / \sigma_{L}$} \\
\hline & & 0.00 & 0.25 & 0.50 & 0.75 & 0.90 & 1.00 & 1.10 & 1.20 & 1.30 \\
\hline \multirow[t]{8}{*}{$n=3$} & 0.1 & 0.8984 & 0.9957 & 1.0980 & 1.1855 & 1.2282 & 1.2713 & 1.2971 & 1.3360 & 1.3618 \\
\hline & 0.2 & 0.8984 & 0.9791 & 1.0647 & 1.1529 & 1.1938 & 1.2197 & 1.2545 & 1.2740 & 1.3082 \\
\hline & 0.3 & 0.8984 & 0.9608 & 1.0267 & 1.0949 & 1.1386 & 1.1623 & 1.1920 & 1.2110 & 1.2426 \\
\hline & 0.4 & 0.8984 & 0.9431 & 0.9901 & 1.0407 & 1.0745 & 1.1015 & 1.1209 & 1.1459 & 1.1746 \\
\hline & 0.5 & 0.8984 & 0.9269 & 0.9561 & 0.9940 & 1.0213 & 1.0399 & 1.0619 & 1.0852 & 1.1089 \\
\hline & 0.6 & 0.8984 & 0.9095 & 0.9260 & 0.9512 & 0.9733 & 0.9905 & 1.0110 & 1.0329 & 1.0567 \\
\hline & 0.7 & 0.8984 & 0.8946 & 0.8996 & 0.9178 & 0.9352 & 0.9491 & 0.9658 & 0.9931 & 1.0189 \\
\hline & 0.8 & 0.8984 & 0.8883 & 0.8780 & 0.8899 & 0.9030 & 0.9138 & 0.9296 & 0.9584 & 0.9816 \\
\hline \multirow[t]{8}{*}{$n=4$} & 0.1 & 0.5432 & 0.6166 & 0.7011 & 0.7874 & 0.8363 & 0.8668 & 0.8968 & 0.9305 & 0.9694 \\
\hline & 0.2 & 0.5432 & 0.6052 & 0.6735 & 0.7515 & 0.7945 & 0.8210 & 0.8469 & 0.8715 & 0.9071 \\
\hline & 0.3 & 0.5432 & 0.5905 & 0.6448 & 0.7055 & 0.7404 & 0.7640 & 0.7842 & 0.8139 & 0.8336 \\
\hline & 0.4 & 0.5432 & 0.5771 & 0.6132 & 0.6570 & 0.6846 & 0.7065 & 0.7260 & 0.7485 & 0.7706 \\
\hline & 0.5 & 0.5432 & 0.5610 & 0.5843 & 0.6139 & 0.6351 & 0.6523 & 0.6700 & 0.6897 & 0.7126 \\
\hline & 0.6 & 0.5432 & 0.5497 & 0.5582 & 0.5769 & 0.5927 & 0.6048 & 0.6234 & 0.6407 & 0.6651 \\
\hline & 0.7 & 0.5432 & 0.5324 & 0.5376 & 0.5443 & 0.5610 & 0.5702 & 0.5826 & 0.6062 & 0.6266 \\
\hline & 0.8 & 0.5432 & 0.5313 & 0.5198 & 0.5190 & 0.5337 & 0.5401 & 0.5510 & 0.5754 & 0.5950 \\
\hline \multirow[t]{8}{*}{$n=5$} & 0.1 & 0.3803 & 0.4409 & 0.5149 & 0.5917 & 0.6412 & 0.6649 & 0.6979 & 0.7386 & 0.7806 \\
\hline & 0.2 & 0.3803 & 0.4320 & 0.4902 & 0.5564 & 0.5981 & 0.6251 & 0.6428 & 0.6697 & 0.6977 \\
\hline & 0.3 & 0.3803 & 0.4186 & 0.4637 & 0.5158 & 0.5482 & 0.5697 & 0.5912 & 0.6188 & 0.6402 \\
\hline & 0.4 & 0.3803 & 0.4087 & 0.4372 & 0.4741 & 0.4989 & 0.5178 & 0.5363 & 0.5554 & 0.5778 \\
\hline & 0.5 & 0.3803 & 0.3938 & 0.4133 & 0.4374 & 0.4543 & 0.4696 & 0.4843 & 0.5016 & 0.5226 \\
\hline & 0.6 & 0.3803 & 0.3852 & 0.3909 & 0.4013 & 0.4162 & 0.4259 & 0.4422 & 0.4566 & 0.4787 \\
\hline & 0.7 & 0.3803 & 0.3686 & 0.3687 & 0.3761 & 0.3830 & 0.3961 & 0.4059 & 0.4267 & 0.4534 \\
\hline & 0.8 & 0.3803 & 0.3685 & 0.3523 & 0.3544 & 0.3578 & 0.3701 & 0.3784 & 0.4005 & 0.4303 \\
\hline \multirow[t]{8}{*}{$n=7$} & 0.1 & 0.2489 & 0.2989 & 0.3575 & 0.4345 & 0.4760 & 0.5062 & 0.5427 & 0.5910 & 0.6405 \\
\hline & 0.2 & 0.2489 & 0.2922 & 0.3378 & 0.3963 & 0.4357 & 0.4580 & 0.4867 & 0.5095 & 0.5316 \\
\hline & 0.3 & 0.2489 & 0.2800 & 0.3170 & 0.3607 & 0.3906 & 0.4112 & 0.4320 & 0.4545 & 0.4760 \\
\hline & 0.4 & 0.2489 & 0.2682 & 0.2950 & 0.3252 & 0.3465 & 0.3631 & 0.3807 & 0.3994 & 0.4198 \\
\hline & 0.5 & 0.2489 & 0.2591 & 0.2756 & 0.2909 & 0.3073 & 0.3173 & 0.3321 & 0.3496 & 0.3673 \\
\hline & 0.6 & 0.2489 & 0.2528 & 0.2563 & 0.2623 & 0.2739 & 0.2804 & 0.2945 & 0.3123 & 0.3271 \\
\hline & 0.7 & 0.2489 & 0.2369 & 0.2355 & 0.2412 & 0.2444 & 0.2538 & 0.2617 & 0.2812 & 0.3059 \\
\hline & 0.8 & 0.2489 & 0.2371 & 0.2222 & 0.2228 & 0.2226 & 0.2314 & 0.2464 & 0.2679 & 0.2990 \\
\hline \multirow[t]{8}{*}{$n=10$} & 0.1 & 0.1838 & 0.2299 & 0.2823 & 0.3560 & 0.4020 & 0.4390 & 0.4892 & 0.5442 & 0.5816 \\
\hline & 0.2 & 0.1838 & 0.2244 & 0.2648 & 0.3210 & 0.3602 & 0.3864 & 0.4124 & 0.4462 & 0.4935 \\
\hline & 0.3 & 0.1838 & 0.2120 & 0.2463 & 0.2864 & 0.3163 & 0.3368 & 0.3583 & 0.3818 & 0.4054 \\
\hline & 0.4 & 0.1838 & 0.2008 & 0.2259 & 0.2533 & 0.2723 & 0.2888 & 0.3062 & 0.3254 & 0.3469 \\
\hline & 0.5 & 0.1838 & 0.1926 & 0.2084 & 0.2207 & 0.2357 & 0.2439 & 0.2579 & 0.2754 & 0.2958 \\
\hline & 0.6 & 0.1838 & 0.1876 & 0.1900 & 0.1937 & 0.1985 & 0.2082 & 0.2212 & 0.2383 & 0.2616 \\
\hline & 0.7 & 0.1838 & 0.1709 & 0.1694 & 0.1733 & 0.1750 & 0.1826 & 0.1882 & 0.2157 & 0.2462 \\
\hline & 0.8 & 0.1838 & 0.1715 & 0.1571 & 0.1482 & 0.1540 & 0.1611 & 0.1749 & 0.1986 & 0.2490 \\
\hline
\end{tabular}

Table 4.11 FEA results of $A$ for SECP specimens (plane strain under uniaxial tension load) 


\begin{tabular}{|c|c|c|c|c|c|c|c|c|c|c|c|c|c|}
\hline \multicolumn{14}{|c|}{$\sigma / \sigma_{L}$} \\
\hline$n$ & $a / W$ & 0.00 & 0.25 & 0.50 & 0.75 & 0.90 & 1.00 & 1.10 & 1.20 & 1.30 & 1.40 & 1.50 & 1.60 \\
\hline \multirow[t]{8}{*}{3} & 0.1 & 0.8984 & 1.0313 & 1.1930 & 1.3269 & 1.3856 & 1.4114 & 1.4583 & 1.5020 & 1.5035 & 1.5313 & 1.5549 & 1.5251 \\
\hline & 0.2 & 0.8984 & 1.0311 & 1.1731 & 1.2854 & 1.3626 & 1.3932 & 1.4405 & 1.4574 & 1.4928 & 1.5223 & 1.5503 & 1.5255 \\
\hline & 0.3 & 0.8984 & 1.0176 & 1.1500 & 1.2750 & 1.3363 & 1.3869 & 1.4128 & 1.4561 & 1.4662 & 1.4982 & 1.5237 & 1.5450 \\
\hline & 0.4 & 0.8984 & 1.0084 & 1.1246 & 1.2556 & 1.3136 & 1.3464 & 1.3939 & 1.4096 & 1.4504 & 1.4838 & 1.5111 & 1.5345 \\
\hline & 0.5 & 0.8984 & 1.0001 & 1.1173 & 1.2322 & 1.2909 & 1.3390 & 1.3659 & 1.4083 & 1.4484 & 1.4549 & 1.4878 & 1.5096 \\
\hline & 0.6 & 0.8984 & 0.9985 & 1.1000 & 1.2085 & 1.2781 & 1.3097 & 1.3543 & 1.3989 & 1.4137 & 1.4509 & 1.4812 & 1.5059 \\
\hline & 0.7 & 0.8984 & 0.9896 & 1.0920 & 1.1955 & 1.2641 & 1.2969 & 1.3412 & 1.3823 & 1.4000 & 1.4328 & 1.4663 & 1.4927 \\
\hline & 0.8 & 0.8984 & 0.9886 & 1.0808 & 1.1931 & 1.2462 & 1.2920 & 1.3381 & 1.3588 & 1.3991 & 1.4336 & 1.4637 & 1.4915 \\
\hline \multirow[t]{8}{*}{4} & 0.1 & 0.5432 & 0.6358 & 0.7799 & 0.8928 & 0.9481 & 0.9800 & 1.0032 & 1.0193 & 1.0568 & 1.0631 & 1.0891 & 1.0797 \\
\hline & 0.2 & 0.5432 & 0.6487 & 0.7603 & 0.8722 & 0.9289 & 0.9621 & 0.9879 & 1.0066 & 1.0443 & 1.0545 & 1.0799 & 1.0730 \\
\hline & 0.3 & 0.5432 & 0.6404 & 0.7417 & 0.8495 & 0.9066 & 0.9380 & 0.9827 & 1.0070 & 1.0222 & 1.0568 & 1.0583 & 1.0836 \\
\hline & 0.4 & 0.5432 & 0.6308 & 0.7280 & 0.8317 & 0.8894 & 0.9214 & 0.9657 & 0.9911 & 1.0080 & 1.0430 & 1.0467 & 1.0759 \\
\hline & 0.5 & 0.5432 & 0.6244 & 0.7140 & 0.8134 & 0.8802 & 0.9136 & 0.9441 & 0.9675 & 1.0062 & 1.0226 & 1.0539 & 1.0522 \\
\hline & 0.6 & 0.5432 & 0.6189 & 0.7049 & 0.8029 & 0.8582 & 0.8900 & 0.9338 & 0.9615 & 0.9993 & 1.0157 & 1.0482 & 1.0791 \\
\hline & 0.7 & 0.5432 & 0.6143 & 0.6937 & 0.7924 & 0.8453 & 0.8790 & 0.9209 & 0.9467 & 0.9857 & 1.0242 & 1.0366 & 1.0664 \\
\hline & 0.8 & 0.5432 & 0.6056 & 0.6893 & 0.7799 & 0.8433 & 0.8751 & 0.9199 & 0.9461 & 0.9851 & 1.0039 & 1.0371 & 1.0420 \\
\hline \multirow[t]{8}{*}{$\overline{5}$} & 0.1 & 0.3803 & 0.4627 & 0.5773 & 0.6732 & 0.7241 & 0.7548 & 0.7819 & 0.8050 & 0.8234 & 0.8366 & 0.8437 & 0.8476 \\
\hline & 0.2 & 0.3803 & 0.4695 & 0.5614 & 0.6552 & 0.7070 & 0.7385 & 0.7669 & 0.7916 & 0.8116 & 0.8247 & 0.8346 & 0.8148 \\
\hline & 0.3 & 0.3803 & 0.4612 & 0.5493 & 0.6361 & 0.6880 & 0.7193 & 0.7481 & 0.7727 & 0.7932 & 0.8045 & 0.8372 & 0.8300 \\
\hline & 0.4 & 0.3803 & 0.4548 & 0.5339 & 0.6216 & 0.6733 & 0.7048 & 0.7339 & 0.7748 & 0.7980 & 0.8148 & 0.8281 & 0.8136 \\
\hline & 0.5 & 0.3803 & 0.4490 & 0.5222 & 0.6055 & 0.6647 & 0.6975 & 0.7291 & 0.7562 & 0.7795 & 0.7967 & 0.8090 & 0.8369 \\
\hline & 0.6 & 0.3803 & 0.4428 & 0.5142 & 0.5960 & 0.6466 & 0.6781 & 0.7203 & 0.7482 & 0.7746 & 0.7944 & 0.8272 & 0.8370 \\
\hline & 0.7 & 0.3803 & 0.4399 & 0.5051 & 0.5868 & 0.6371 & 0.6787 & 0.7094 & 0.7383 & 0.7622 & 0.7820 & 0.8169 & 0.8287 \\
\hline & 0.8 & 0.3803 & 0.4334 & 0.5004 & 0.5771 & 0.6338 & 0.6657 & 0.6972 & 0.7369 & 0.7623 & 0.7823 & 0.8174 & 0.8341 \\
\hline \multirow[t]{8}{*}{7} & 0.1 & 0.2489 & 0.3225 & 0.4154 & 0.4966 & 0.5337 & 0.5548 & 0.5708 & 0.5992 & 0.6119 & 0.6217 & 0.6360 & 0.6399 \\
\hline & 0.2 & 0.2489 & 0.3245 & 0.4017 & 0.4801 & 0.5196 & 0.5503 & 0.5701 & 0.5884 & 0.6150 & 0.6223 & 0.6320 & 0.6624 \\
\hline & 0.3 & 0.2489 & 0.3161 & 0.3885 & 0.4635 & 0.5106 & 0.5346 & 0.5649 & 0.5848 & 0.5973 & 0.6210 & 0.6318 & 0.6620 \\
\hline & 0.4 & 0.2489 & 0.3121 & 0.3762 & 0.4505 & 0.4977 & 0.5226 & 0.5532 & 0.5739 & 0.6028 & 0.6133 & 0.6230 & 0.6534 \\
\hline & 0.5 & 0.2489 & 0.3072 & 0.3670 & 0.4369 & 0.4837 & 0.5157 & 0.5491 & 0.5721 & 0.5883 & 0.6149 & 0.6311 & 0.6379 \\
\hline & 0.6 & 0.2489 & 0.3004 & 0.3587 & 0.4248 & 0.4690 & 0.5008 & 0.5335 & 0.5663 & 0.5972 & 0.6118 & 0.6365 & 0.6490 \\
\hline & 0.7 & 0.2489 & 0.2989 & 0.3524 & 0.4163 & 0.4662 & 0.4997 & 0.5342 & 0.5585 & 0.5883 & 0.6051 & 0.6313 & 0.6500 \\
\hline & 0.8 & 0.2489 & 0.2940 & 0.3472 & 0.4127 & 0.4582 & 0.4910 & 0.5260 & 0.5573 & 0.5890 & 0.6065 & 0.6335 & 0.6575 \\
\hline \multirow[t]{8}{*}{10} & 0.1 & 0.1838 & 0.2550 & 0.3382 & 0.4060 & 0.4421 & 0.4660 & 0.4856 & 0.5059 & 0.5257 & 0.5348 & 0.5771 & 0.5775 \\
\hline & 0.2 & 0.1838 & 0.2552 & 0.3249 & 0.3916 & 0.4287 & 0.4603 & 0.4814 & 0.5021 & 0.5127 & 0.5458 & 0.5696 & 0.5917 \\
\hline & 0.3 & 0.1838 & 0.2459 & 0.3130 & 0.3775 & 0.4203 & 0.4470 & 0.4757 & 0.4976 & 0.5071 & 0.5421 & 0.5687 & 0.5969 \\
\hline & 0.4 & 0.1838 & 0.2435 & 0.3023 & 0.3657 & 0.4088 & 0.4422 & 0.4663 & 0.4977 & 0.5188 & 0.5297 & 0.5750 & 0.5977 \\
\hline & 0.5 & 0.1838 & 0.2389 & 0.2929 & 0.3538 & 0.3963 & 0.4305 & 0.4618 & 0.4957 & 0.5186 & 0.5407 & 0.5664 & 0.6002 \\
\hline & 0.6 & 0.1838 & 0.2311 & 0.2855 & 0.3449 & 0.3875 & 0.4228 & 0.4564 & 0.4840 & 0.5147 & 0.5439 & 0.5672 & 0.5974 \\
\hline & 0.7 & 0.1838 & 0.2307 & 0.2786 & 0.3360 & 0.3795 & 0.4150 & 0.4494 & 0.4849 & 0.5113 & 0.5370 & 0.5596 & 0.6016 \\
\hline & 0.8 & 0.1838 & 0.2261 & 0.2745 & 0.3313 & 0.3739 & 0.4103 & 0.4501 & 0.4851 & 0.5120 & 0.5345 & 0.5750 & 0.6014 \\
\hline
\end{tabular}

Table 4.12 FEA results of $A$ for CCP specimens (plane strain under uniaxial tension load) 


\begin{tabular}{|c|c|c|c|c|c|c|c|c|c|c|c|c|c|}
\hline \multirow[b]{2}{*}{$n$} & \multicolumn{13}{|c|}{$\sigma / \sigma_{L}$} \\
\hline & $a / W$ & 0.00 & 0.25 & 0.50 & 0.75 & 0.90 & 1.00 & 1.10 & 1.20 & 1.30 & 1.40 & 1.50 & 1.60 \\
\hline \multirow[t]{8}{*}{3} & 0.1 & 0.8984 & 0.9835 & 1.0705 & 1.1572 & 1.1964 & 1.2196 & 1.2530 & 1.2682 & 1.3034 & 1.3324 & 1.3655 & 1.4006 \\
\hline & 0.2 & 0.8984 & 0.9665 & 1.0404 & 1.1118 & 1.1470 & .1 .1797 & 1.1963 & 1.2254 & 1.2521 & 1.2783 & 1.2829 & 1.3082 \\
\hline & 0.3 & 0.8984 & 0.9533 & 1.0105 & 1.0741 & 1.1037 & 1.1305 & 1.1447 & 1.1707 & 1.1944 & 1.2160 & 1.2368 & 1.2560 \\
\hline & 0.4 & 0.8984 & 0.9469 & 0.9862 & 1.0336 & 1.0636 & 1.0780 & 1.0993 & 1.1192 & 1.1393 & 1.1583 & 1.1774 & 1.1950 \\
\hline & 0.5 & 0.8984 & 0.9367 & 0.9709 & 1.0058 & 1.0221 & 1.0441 & 1.0550 & 1.0691 & 1.0808 & 1.1055 & 1.1132 & 1.1398 \\
\hline & 0.6 & 0.8984 & 0.9300 & 0.9526 & 0.9730 & 0.9916 & 0.9995 & 1.0089 & 1.0250 & 1.0309 & 1.0413 & 1.0586 & 1.0655 \\
\hline & 0.7 & 0.8984 & 0.9225 & 0.9388 & 0.9511 & 0.9631 & 0.9666 & 0.9735 & 0.9843 & 0.9881 & 0.9936 & 1.0046 & 1.0071 \\
\hline & 0.8 & 0.8984 & 0.9133 & 0.9258 & 0.9312 & 0.9388 & 0.9397 & 0.9453 & 0.9493 & 0.9498 & 0.9558 & 0.9600 & 0.9663 \\
\hline \multirow[t]{8}{*}{4} & 0.1 & 0.5432 & 0.6009 & 0.6767 & 0.7548 & 0.7973 & 0.8245 & 0.8467 & 0.8811 & 0.9034 & 0.9276 & 0.9669 & 0.9974 \\
\hline & 0.2 & 0.5432 & 0.5953 & 0.6516 & 0.7138 & 0.7564 & 0.7788 & 0.7998 & 0.8287 & 0.8453 & 0.8728 & 0.8887 & 0.9159 \\
\hline & 0.3 & 0.5432 & 0.5858 & 0.6295 & 0.6787 & 0.7122 & 0.7310 & 0.7557 & 0.7725 & 0.7959 & 0.8086 & 0.8323 & 0.8551 \\
\hline & 0.4 & 0.5432 & 0.5801 & 0.6107 & 0.6465 & 0.6709 & 0.6893 & 0.7034 & 0.7228 & 0.7419 & 0.7608 & 0.7809 & 0.7898 \\
\hline & 0.5 & 0.5432 & 0.5715 & 0.5946 & 0.6223 & 0.6392 & 0.6523 & 0.6629 & 0.6769 & 0.6948 & 0.7071 & 0.7188 & 0.7412 \\
\hline & 0.6 & 0.5432 & 0.5618 & 0.5826 & 0.5990 & 0.6115 & 0.6184 & 0.6278 & 0.6373 & 0.6446 & 0.6563 & 0.6671 & 0.6813 \\
\hline & 0.7 & 0.5432 & 0.5597 & 0.5698 & 0.5805 & 0.5883 & 0.5922 & 0.5992 & 0.6037 & 0.6084 & 0.6147 & 0.6219 & 0.6291 \\
\hline & 0.8 & 0.5432 & 0.5531 & 0.5608 & 0.5666 & 0.5714 & 0.5702 & 0.5736 & 0.5791 & 0.5802 & 0.5829 & 0.5846 & 0.5910 \\
\hline \multirow[t]{8}{*}{5} & 0.1 & 0.3803 & 0.4292 & 0.4901 & 0.5592 & 0.6005 & 0.6273 & 0.6527 & 0.6782 & 0.7048 & 0.7366 & 0.7707 & 0.8146 \\
\hline & 0.2 & 0.3803 & 0.4238 & 0.4697 & 0.5251 & 0.5593 & 0.5829 & 0.6041 & 0.6333 & 0.6530 & 0.6733 & 0.6921 & 0.7111 \\
\hline & 0.3 & 0.3803 & 0.4162 & 0.4508 & 0.4917 & 0.5194 & 0.5371 & 0.5595 & 0.5774 & 0.6009 & 0.6170 & 0.6405 & 0.6563 \\
\hline & 0.4 & 0.3803 & 0.4104 & 0.4353 & 0.4657 & 0.4866 & 0.5002 & 0.5161 & 0.5341 & 0.5484 & 0.5670 & 0.5872 & 0.5997 \\
\hline & 0.5 & 0.3803 & 0.4036 & 0.4218 & 0.4447 & 0.4577 & 0.4685 & 0.4780 & 0.4901 & 0.5041 & 0.5159 & 0.5292 & 0.5481 \\
\hline & 0.6 & 0.3803 & 0.3937 & 0.4125 & 0.4265 & 0.4360 & 0.4418 & 0.4477 & 0.4562 & 0.4639 & 0.4714 & 0.4816 & 0.4927 \\
\hline & 0.7 & 0.3803 & 0.3934 & 0.4010 & 0.4107 & 0.4165 & 0.4200 & 0.4265 & 0.4286 & 0.4331 & 0.4368 & 0.4438 & 0.4484 \\
\hline & 0.8 & 0.3803 & 0.3882 & 0.3937 & 0.3995 & 0.4032 & 0.4015 & 0.4041 & 0.4096 & 0.4090 & 0.4119 & 0.4124 & 0.4186 \\
\hline \multirow[t]{8}{*}{7} & 0.1 & 0.2489 & 0.2901 & 0.3387 & 0.3999 & 0.4383 & 0.4668 & 0.4902 & 0.5152 & 0.5467 & 0.5910 & 0.6393 & 0.6663 \\
\hline & 0.2 & 0.2489 & 0.2851 & 0.3226 & 0.3657 & 0.3993 & 0.4229 & 0.4424 & 0.4669 & 0.4910 & 0.5113 & 0.5387 & 0.5652 \\
\hline & 0.3 & 0.2489 & 0.2790 & 0.3063 & 0.3391 & 0.3616 & 0.3792 & 0.3975 & 0.4200 & 0.4408 & 0.4613 & 0.4824 & 0.5033 \\
\hline & 0.4 & 0.2489 & 0.2729 & 0.2937 & 0.3180 & 0.3339 & 0.3458 & 0.3586 & 0.3737 & 0.3909 & 0.4076 & 0.4280 & 0.4451 \\
\hline & 0.5 & 0.2489 & 0.2683 & 0.2825 & 0.3018 & 0.3110 & 0.3200 & 0.3270 & 0.3359 & 0.3476 & 0.3586 & 0.3733 & 0.3885 \\
\hline & 0.6 & 0.2489 & 0.2583 & 0.2754 & 0.2837 & 0.2949 & 0.2969 & 0.3026 & 0.3102 & 0.3142 & 0.3210 & 0.3303 & 0.3379 \\
\hline & 0.7 & 0.2489 & 0.2594 & 0.2648 & 0.2739 & 0.2782 & 0.2817 & 0.2818 & 0.2881 & 0.2890 & 0.2937 & 0.3002 & 0.3024 \\
\hline & 0.8 & 0.2489 & 0.2554 & 0.2592 & 0.2650 & 0.2678 & 0.2648 & 0.2679 & 0.2734 & 0.2716 & 0.2750 & 0.2743 & 0.2803 \\
\hline \multirow[t]{8}{*}{10} & 0.1 & 0.1838 & 0.2223 & 0.2651 & 0.3201 & 0.3636 & 0.3895 & 0.4208 & 0.4545 & 0.5010 & 0.5445 & 0.5806 & 0.5756 \\
\hline & 0.2 & 0.1838 & 0.2175 & 0.2491 & 0.2894 & 0.3186 & 0.3428 & 0.3703 & 0.3969 & 0.4264 & 0.4531 & 0.4837 & 0.5361 \\
\hline & 0.3 & 0.1838 & 0.2120 & 0.2365 & 0.2653 & 0.2849 & 0.3011 & 0.3201 & 0.3421 & 0.3669 & 0.3935 & 0.4177 & 0.4422 \\
\hline & 0.4 & 0.1838 & 0.2053 & 0.2250 & 0.2469 & 0.2607 & 0.2700 & 0.2814 & 0.2958 & 0.3122 & 0.3326 & 0.3544 & 0.3768 \\
\hline & 0.5 & 0.1838 & 0.2020 & 0.2145 & 0.2332 & 0.2404 & 0.2490 & 0.2530 & 0.2611 & 0.2721 & 0.2813 & 0.2944 & 0.3113 \\
\hline & 0.6 & 0.1838 & 0.1913 & 0.2084 & 0.2149 & 0.2272 & 0.2272 & 0.2328 & 0.2400 & 0.2422 & 0.2484 & 0.2524 & 0.2625 \\
\hline & 0.7 & 0.1838 & 0.1935 & 0.1979 & 0.2071 & 0.2108 & 0.2148 & 0.2137 & 0.2204 & 0.2198 & 0.2249 & 0.2316 & 0.2323 \\
\hline & 0.8 & 0.1838 & 0.1900 & 0.1930 & 0.1991 & 0.2015 & 0.1975 & 0.2010 & 0.2064 & 0.2046 & 0.2087 & 0.2073 & 0.2135 \\
\hline
\end{tabular}

Table 4.13 FEA results of $A$ for DECP specimens (plane strain under uniaxial tension load) 


\begin{tabular}{ccccc}
\hline$i$ & $e_{i 0}$ & $e_{i 1}$ & $e_{i 2}$ & $e_{i 3}$ \\
\hline 1 & 0.1898 & -0.1672 & -0.4514 & 0.4934 \\
2 & 0.0259 & 0.0668 & -0.2736 & -0.2881 \\
3 & 0.1024 & -0.5041 & 0.8484 & -0.0770 \\
\hline
\end{tabular}

Table 4.14 The polynomial coefficients $e_{i j}$ for SECP specimens (plane strain under uniaxial tension load)

\begin{tabular}{ccccc}
\hline$i$ & $e_{i 0}$ & $e_{i 1}$ & $e_{i 2}$ & $e_{i 3}$ \\
\hline 1 & 0.3598 & -0.1283 & -0.9197 & 0.8240 \\
2 & -0.0545 & -0.1793 & 1.6996 & -1.3808 \\
3 & -0.0123 & 0.2029 & -0.7885 & 0.5987 \\
\hline
\end{tabular}

Table 4.15 The polynomial coefficients $e_{i j}$ for CCP specimens (plane strain under uniaxial tension load)

\begin{tabular}{ccccc}
\hline$i$ & $e_{i 0}$ & $e_{i 1}$ & $e_{i 2}$ & $e_{i 3}$ \\
\hline 1 & -0.0229 & 1.0829 & -2.5483 & 1.5975 \\
2 & 0.4469 & -3.1960 & 6.3474 & -3.8619 \\
3 & -0.1541 & 1.4271 & -3.1123 & 1.9833 \\
\hline
\end{tabular}

Table 4.16 The polynomial coefficients $e_{i j}$ for DECP specimens (plane strain under uniaxial tension load) 


\begin{tabular}{cccc}
\hline & SECP & CCP & DECP \\
\hline$h$ & 0.0067 & 0.0182 & 0.0089 \\
\hline
\end{tabular}

Table 4.17 The values of parameter $h$ for SECP, CCP and DECP specimens (plane strain under uniaxial tension load)

\begin{tabular}{cccccc}
\hline & $\mathrm{n}=3$ & $\mathrm{n}=4$ & $\mathrm{n}=5$ & $\mathrm{n}=7$ & $\mathrm{n}=10$ \\
\hline$A_{\text {SSY }}$ & 0.8984 & 0.5432 & 0.3803 & 0.2489 & 0.1838 \\
\hline $\mathrm{m}_{1}$ & -0.4588 & -0.4064 & -0.3581 & -0.3039 & -0.2808 \\
\hline $\mathrm{m}_{2}$ & 0.0443 & 0.0398 & 0.0412 & 0.0415 & 0.0570 \\
\hline $\mathrm{m}_{3}$ & 0.1300 & 0.1124 & 0.0972 & 0.0643 & 0.0509 \\
\hline
\end{tabular}

Table 4.18 Values of coefficients for one-to-one $A-T$ relationship polynomials (plane strain under uniaxial tension load) 


\begin{tabular}{cccccc}
\hline$a / W$ & $\mathrm{n}=3$ & $\mathrm{n}=4$ & $\mathrm{n}=5$ & $\mathrm{n}=7$ & $\mathrm{n}=10$ \\
\hline 0.1 & 0.0787 & 0.0694 & 0.0670 & 0.0708 & 0.0773 \\
\hline 0.2 & 0.0830 & 0.0655 & 0.0513 & 0.0469 & 0.0521 \\
\hline 0.3 & 0.0846 & 0.0609 & 0.0513 & 0.0426 & 0.0399 \\
\hline 0.4 & 0.0845 & 0.0596 & 0.0468 & 0.0381 & 0.0341 \\
\hline 0.5 & 0.0934 & 0.0649 & 0.0494 & 0.0374 & 0.0322 \\
\hline 0.6 & 0.1164 & 0.0830 & 0.0637 & 0.0483 & 0.0417 \\
\hline 0.7 & 0.1432 & 0.1045 & 0.0840 & 0.0628 & 0.0558 \\
\hline 0.8 & 0.1406 & 0.1027 & 0.0838 & 0.0699 & 0.0627 \\
\hline
\end{tabular}

Table 4.19 Coefficient $a_{l}$ values for SECP specimen (plane strain under uniaxial tension load)

\begin{tabular}{cccccc}
\hline$a / W$ & $\mathrm{n}=3$ & $\mathrm{n}=4$ & $\mathrm{n}=5$ & $\mathrm{n}=7$ & $\mathrm{n}=10$ \\
\hline 0.1 & 0.2149 & 0.1467 & 0.1032 & 0.0289 & -0.0023 \\
\hline 0.2 & 0.1836 & 0.1207 & 0.0784 & 0.0316 & 0.0082 \\
\hline 0.3 & 0.1631 & 0.1098 & 0.0720 & 0.0353 & 0.0177 \\
\hline 0.4 & 0.1519 & 0.1016 & 0.0702 & 0.0374 & 0.0270 \\
\hline 0.5 & 0.1380 & 0.0977 & 0.0711 & 0.0445 & 0.0382 \\
\hline 0.6 & 0.1417 & 0.1064 & 0.0802 & 0.0545 & 0.0479 \\
\hline 0.7 & 0.1388 & 0.1101 & 0.0801 & 0.0594 & 0.0538 \\
\hline 0.8 & 0.1453 & 0.1069 & 0.0867 & 0.0669 & 0.0620 \\
\hline
\end{tabular}

Table 4.20 Coefficient $a_{l}$ values for CCP specimen (plane strain under uniaxial tension load)

\begin{tabular}{cccccc}
\hline$a / W$ & $\mathrm{n}=3$ & $\mathrm{n}=4$ & $\mathrm{n}=5$ & $\mathrm{n}=7$ & $\mathrm{n}=10$ \\
\hline 0.1 & 0.0872 & 0.0734 & 0.0705 & 0.0724 & 0.0674 \\
\hline 0.2 & 0.0653 & 0.0537 & 0.0446 & 0.0425 & 0.0491 \\
\hline 0.3 & 0.0609 & 0.0424 & 0.0355 & 0.0324 & 0.0327 \\
\hline 0.4 & 0.0530 & 0.0349 & 0.0268 & 0.0215 & 0.0190 \\
\hline 0.5 & 0.0448 & 0.0258 & 0.0166 & 0.0102 & 0.0058 \\
\hline 0.6 & 0.0294 & 0.0151 & 0.0072 & 0.0024 & -0.0006 \\
\hline 0.7 & 0.0182 & 0.0067 & 0.0015 & -0.0007 & -0.0010 \\
\hline 0.8 & 0.0117 & 0.0019 & -0.0007 & -0.0013 & -0.0009 \\
\hline
\end{tabular}

Table 4.21 Coefficient $a_{l}$ values for DECP specimen (plane strain under uniaxial tension load) 


\begin{tabular}{|c|c|c|c|c|c|c|c|c|}
\hline \multicolumn{3}{|c|}{$n=4$} & \multicolumn{3}{|c|}{$n=7$} & \multicolumn{3}{|c|}{$n=7$} \\
\hline \multicolumn{3}{|c|}{$\sigma / \sigma_{L}=1.3$} & \multicolumn{3}{|c|}{$\sigma / \sigma_{L}=1.2$} & \multicolumn{3}{|c|}{$\sigma / \sigma_{L}=1.3$} \\
\hline$A_{2}$ & $A_{2}$ [Ref.] & Diff.(\%) & $A_{2}$ & $A_{2}$ [Ref.] & Diff.(\%) & $A_{2}$ & $A_{2}$ [Ref.] & Diff.(\%) \\
\hline-0.8542 & -0.8122 & 5.1730 & -0.5230 & -0.5207 & 0.4445 & -0.5176 & -0.5207 & -0.5987 \\
\hline
\end{tabular}

Table 4.22 The prediction of parameter $A_{2}$ for SECP specimen, $a / W=0.5$, compared with the results from Yang (1993)

\begin{tabular}{cccccccccc}
\hline$n$ & \multicolumn{3}{c}{$\sigma / \sigma_{L}=1.3$} & \multicolumn{3}{c}{$\sigma / \sigma_{L}=1.4$} & \multicolumn{3}{c}{$\sigma / \sigma_{L}=1.6$} \\
\cline { 2 - 9 } & $Q_{S S Y}$ & $Q_{S S Y}[$ Ref.] & Diff.(\%) & $Q_{S S Y}$ & $Q_{S S Y}[$ Ref.] & Diff.(\%) & $Q_{S S Y}$ & $Q_{S S Y}[$ Ref.] & Diff.(\%) \\
\hline 10 & -0.2227 & -0.2330 & -4.4087 & -0.2571 & -0.2770 & -7.1766 & -0.3453 & -0.3750 & -7.9186 \\
\hline
\end{tabular}

Table 4.23 The prediction of parameter $Q$ for DECP specimen, $a / W=0.5$, compared with the results from O'Dowd and Shih (1994) 


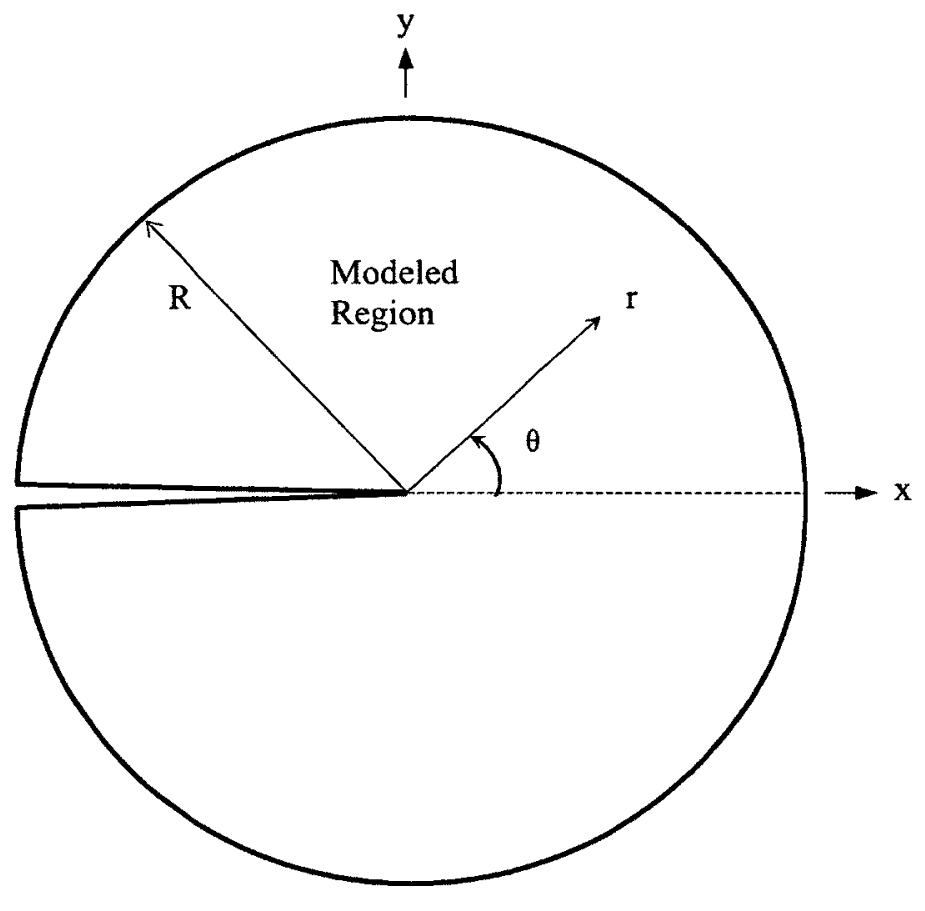

Figure 4.1 The modified boundary layer model 


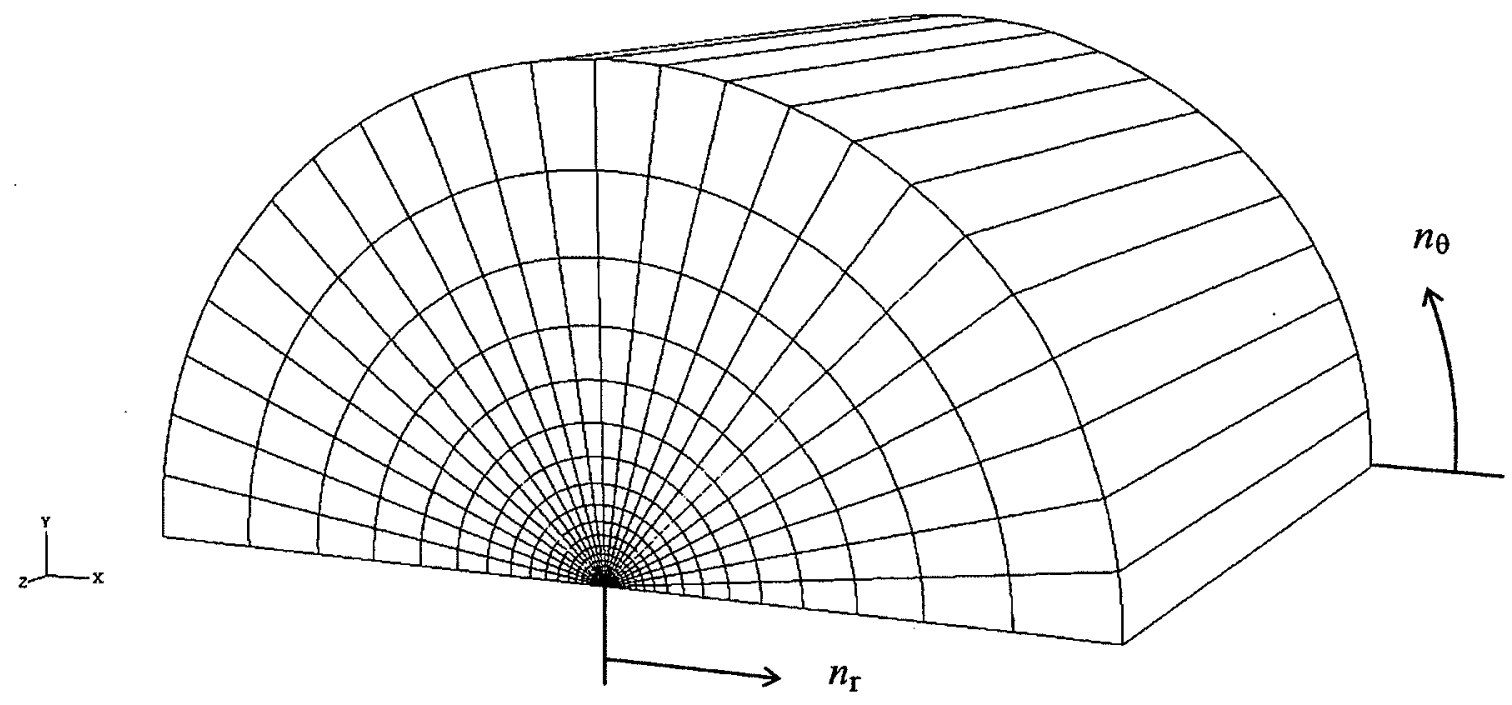

Figure 4.2 FEA mesh for the modified boundary layer problem 


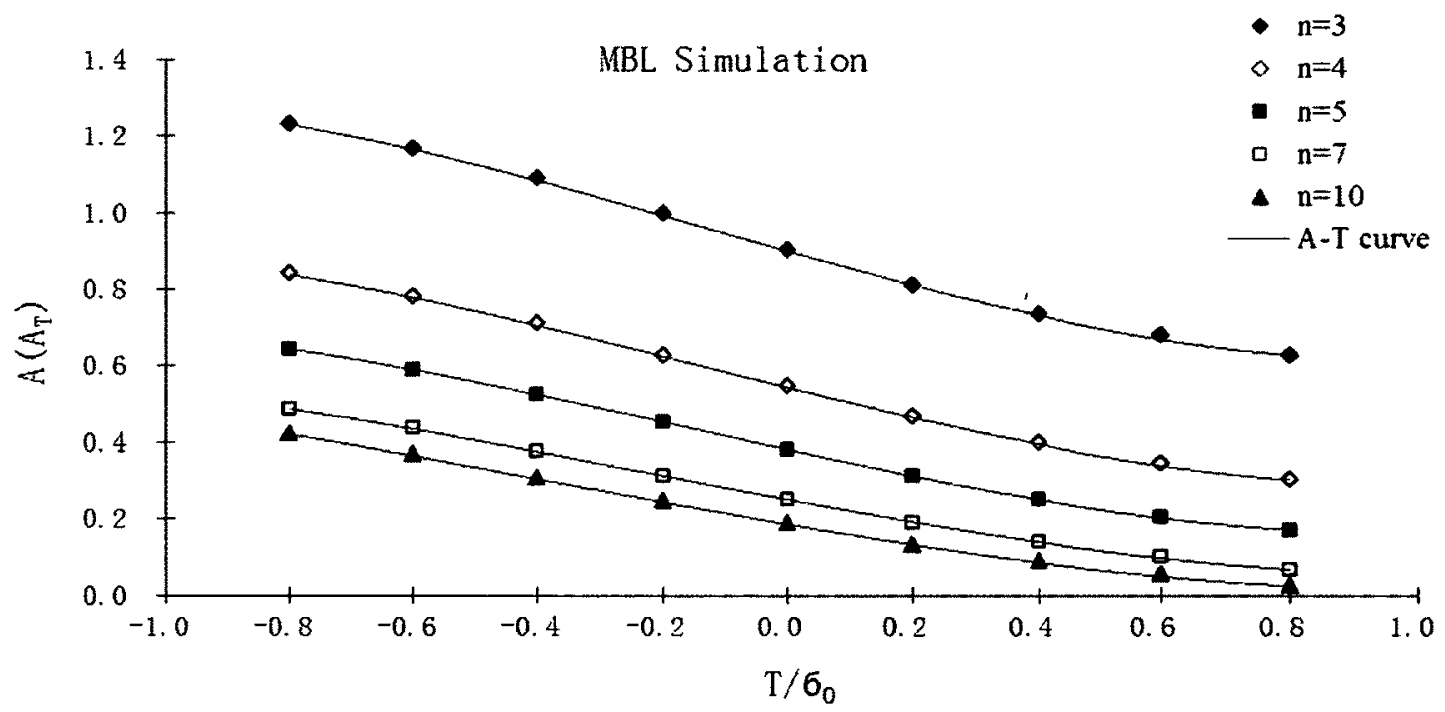

Figure 4.3 Curves of $A$ values from FEA data vs. normalized $T$-stress, $T / \sigma_{0}$, for MBL, $E / \sigma_{0}=500$, plain strain

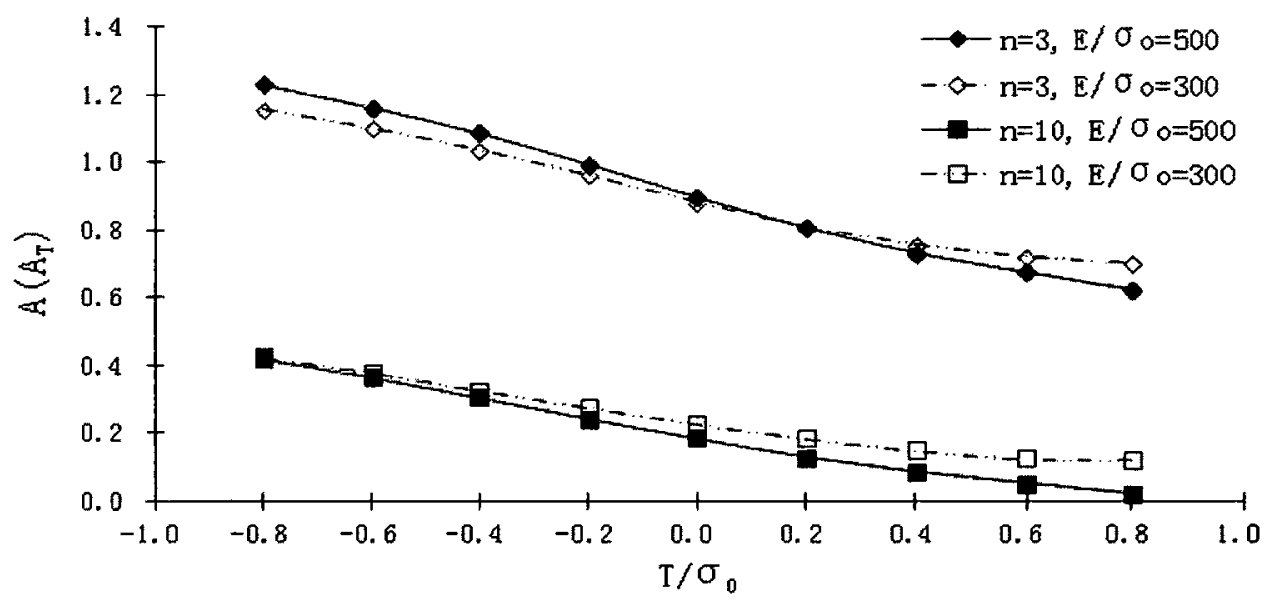

Figure 4.4 Comparison of $A_{T}\left(T / \sigma_{0}, n\right)$ for two different $E / \sigma_{0}$ ratios 


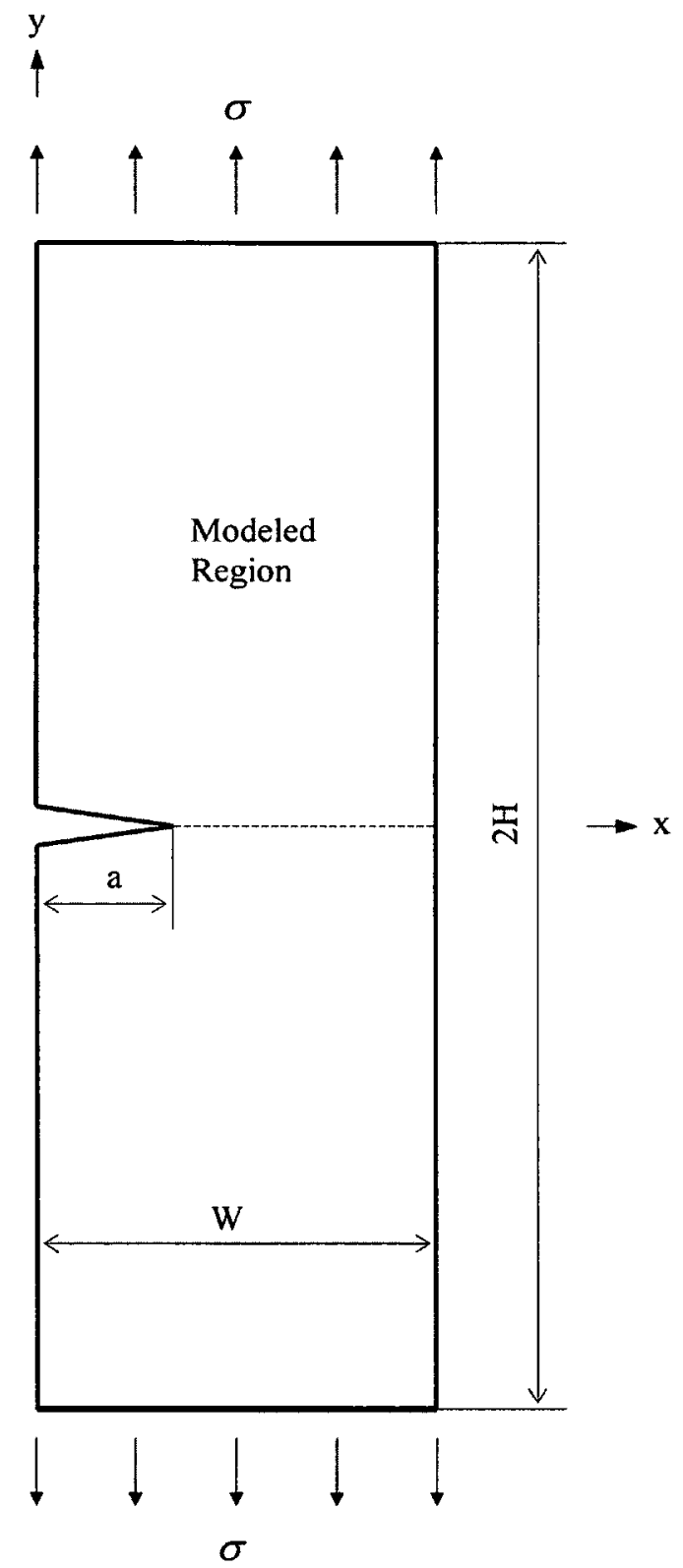

Figure 4.5 Single edge cracked plate (SECP) model 


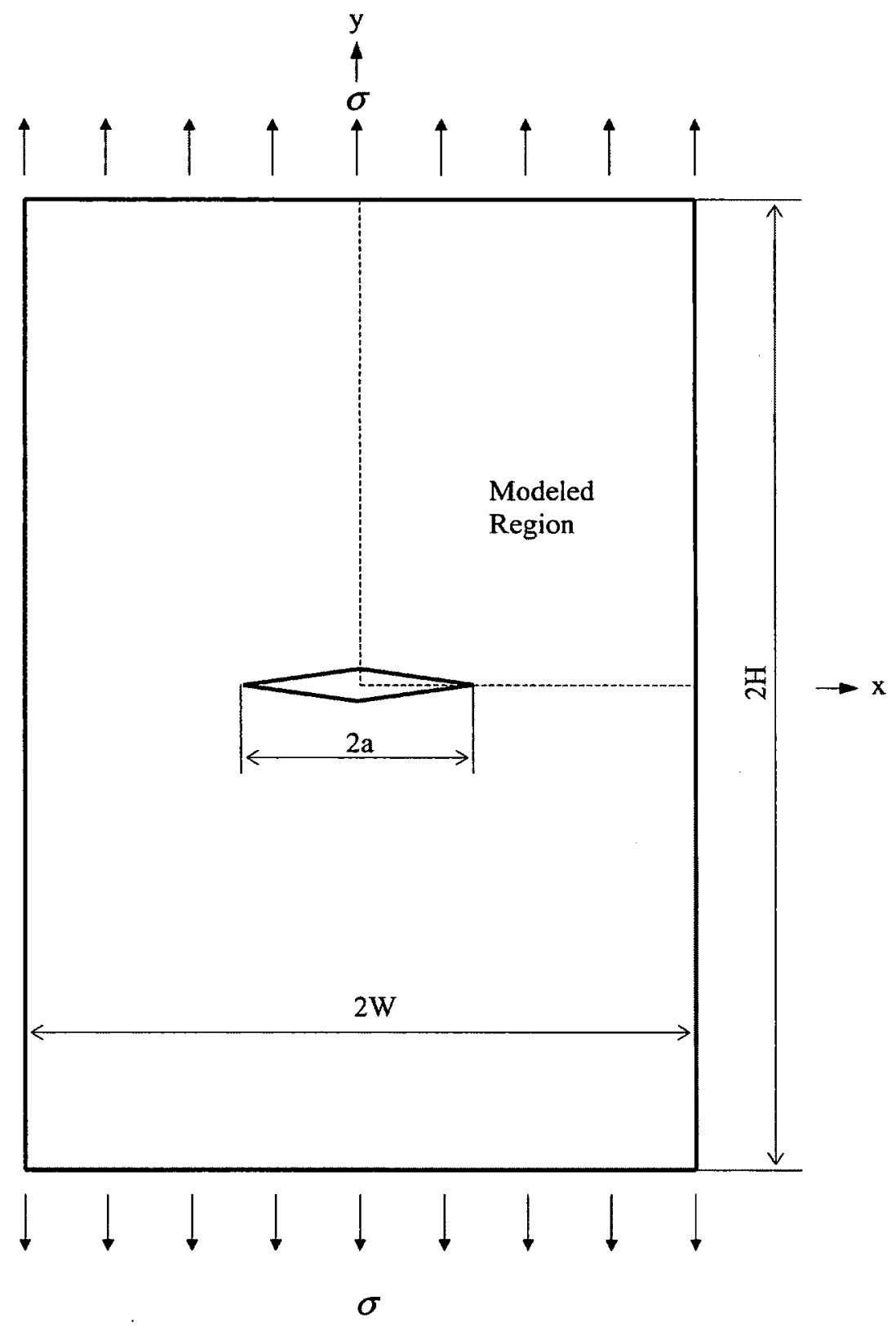

Figure 4.6 Centre cracked plate (CCP) model 


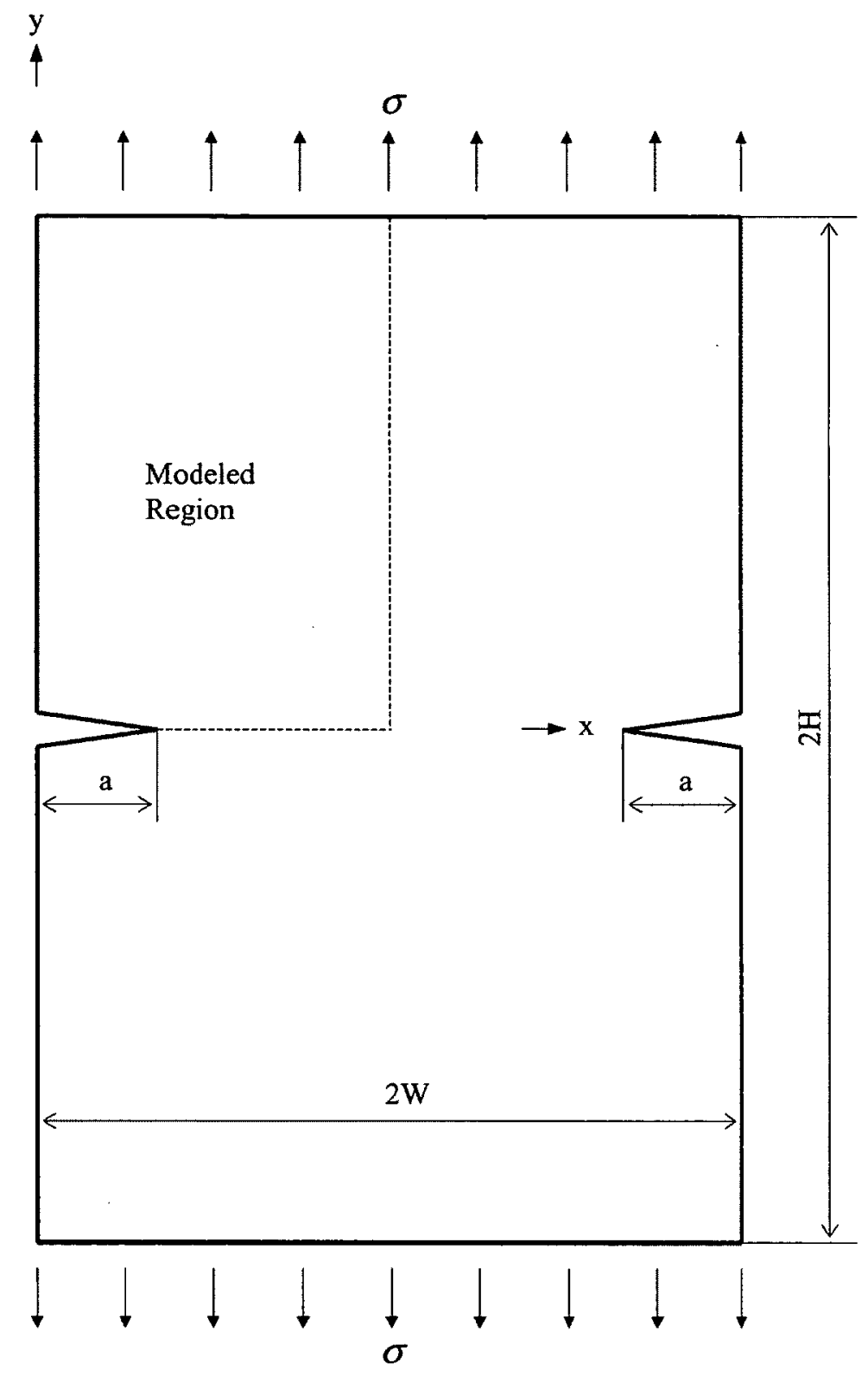

Figure 4.7 Double edge cracked plate (DECP) model 

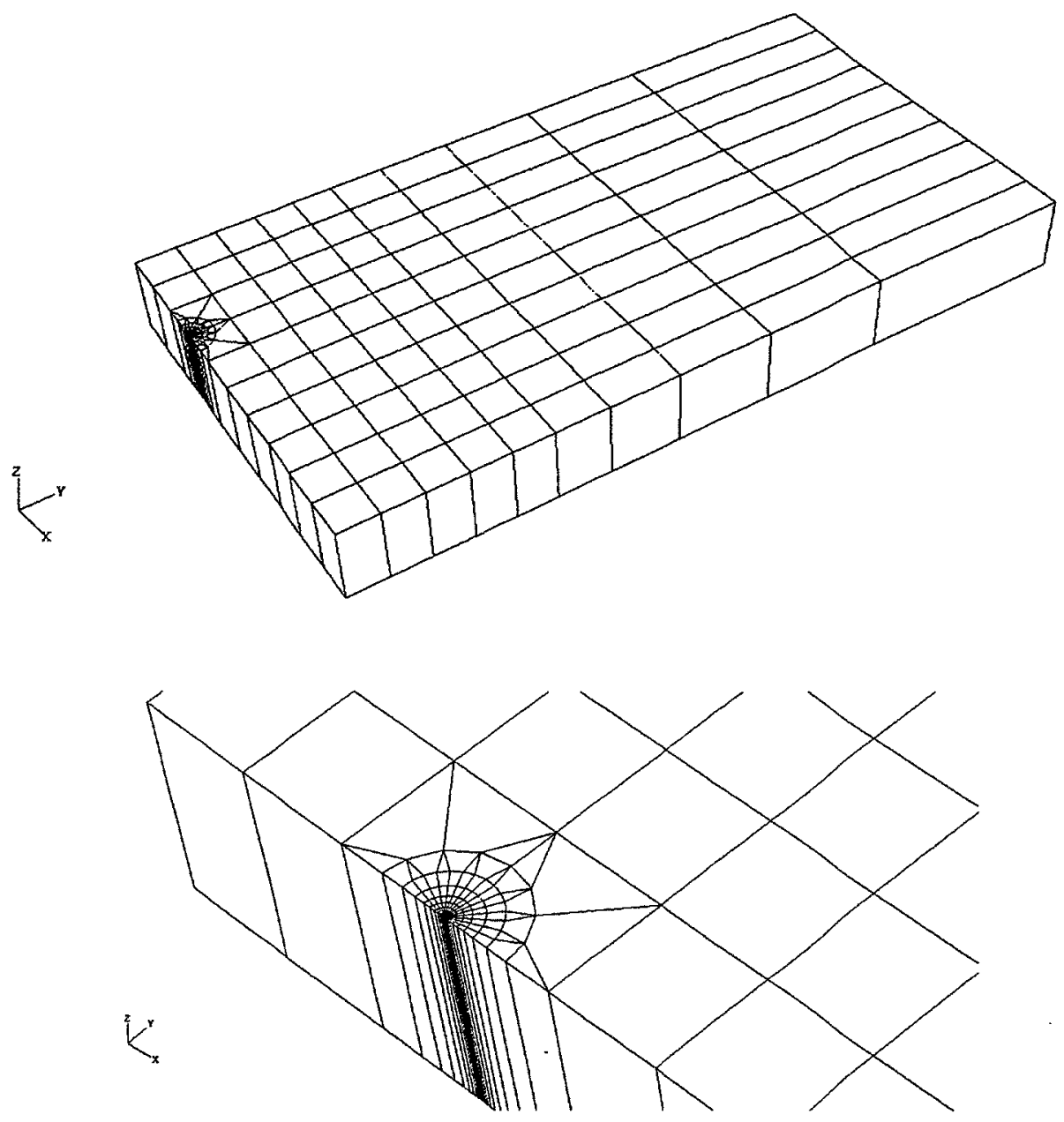

Figure 4.8 Typical FEA mesh for plane strain models under uniaxial load, $a / W=0.3$ 


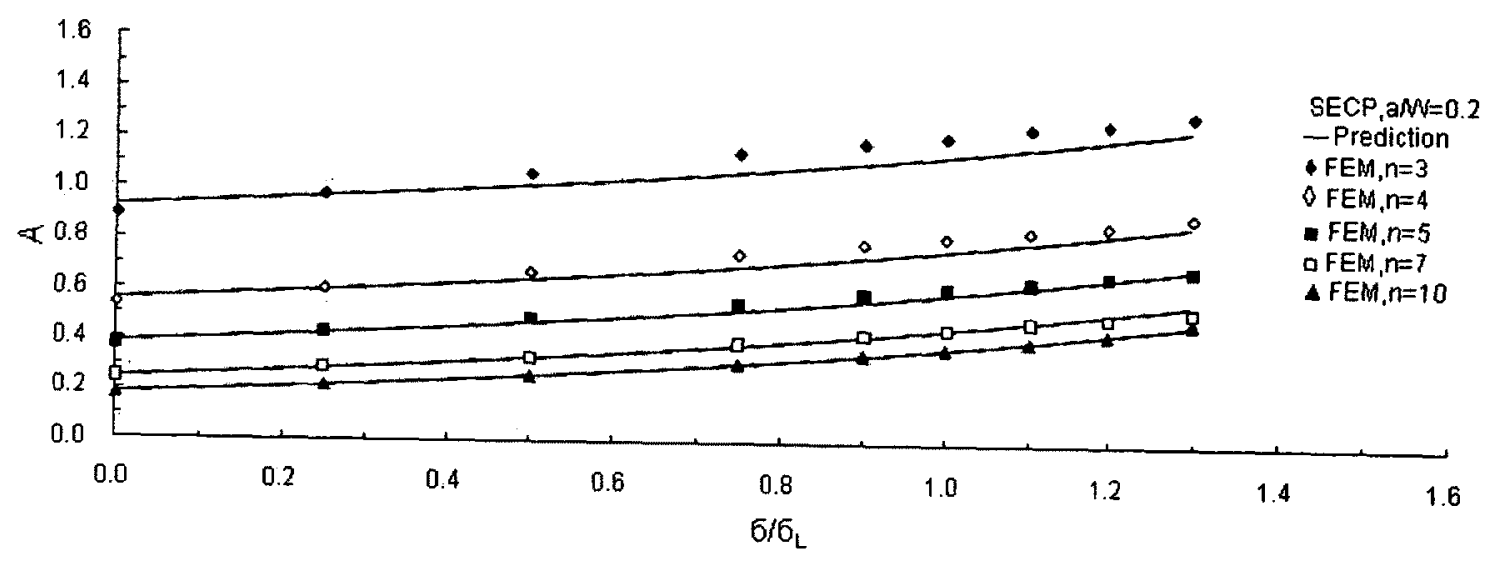

Figure 4.9 Comparisons of predicted $A$ values by curve shape similarity with FEA data for SECP, $a / W=0.2$, plane strain under uniaxial tension load.

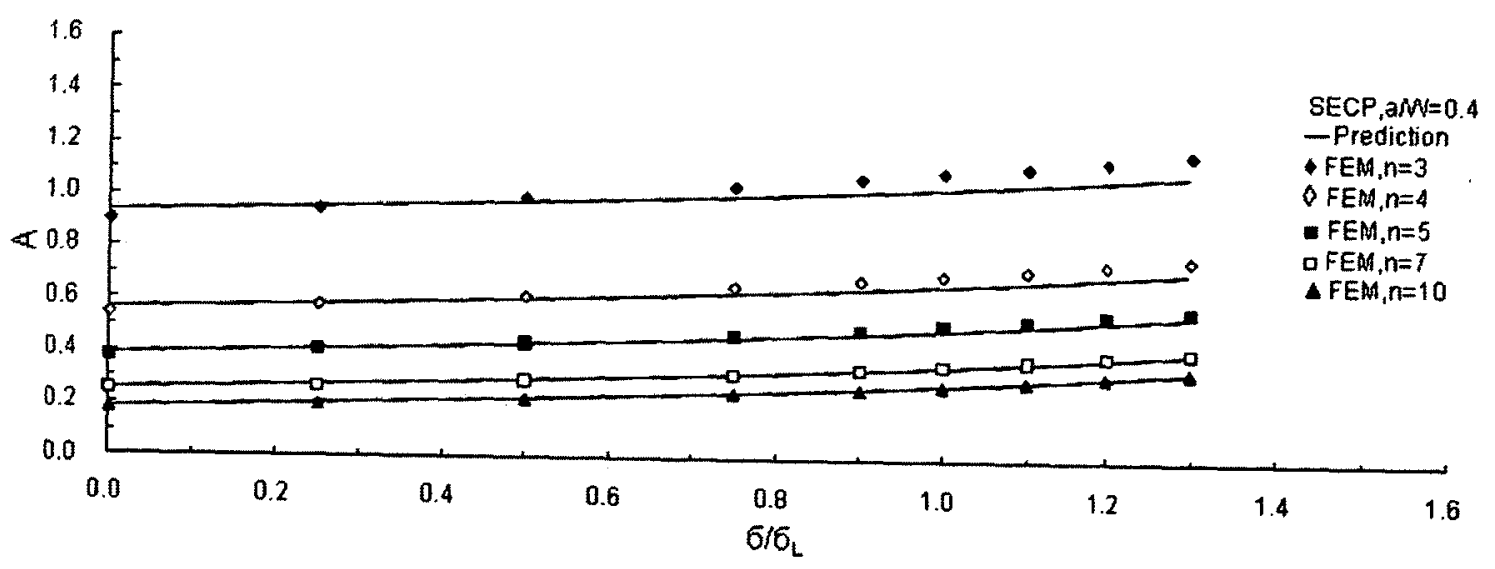

Figure 4.10 Comparisons of predicted $A$ values by curve shape similarity with FEA data for SECP, $a / W=0.4$, plane strain under uniaxial tension load. 


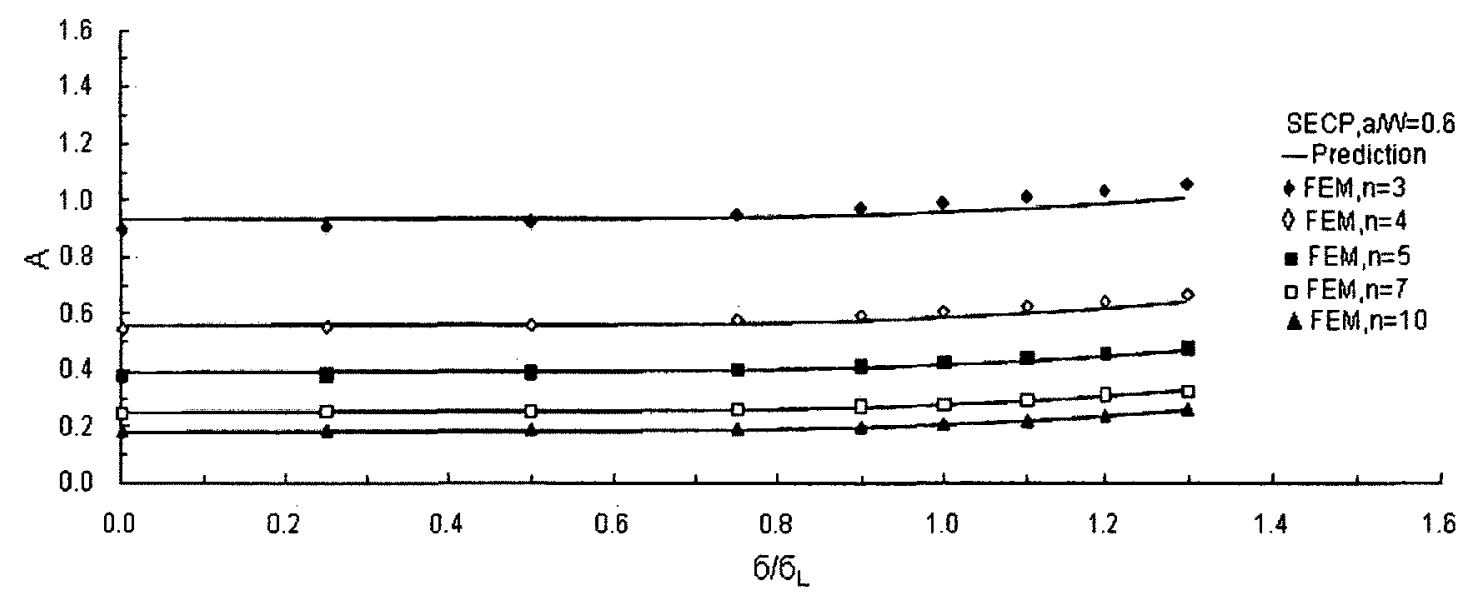

Figure 4.11 Comparisons of predicted $A$ values by curve shape similarity with FEA data for SECP, $a / W=0.6$, plane strain under uniaxial tension load.

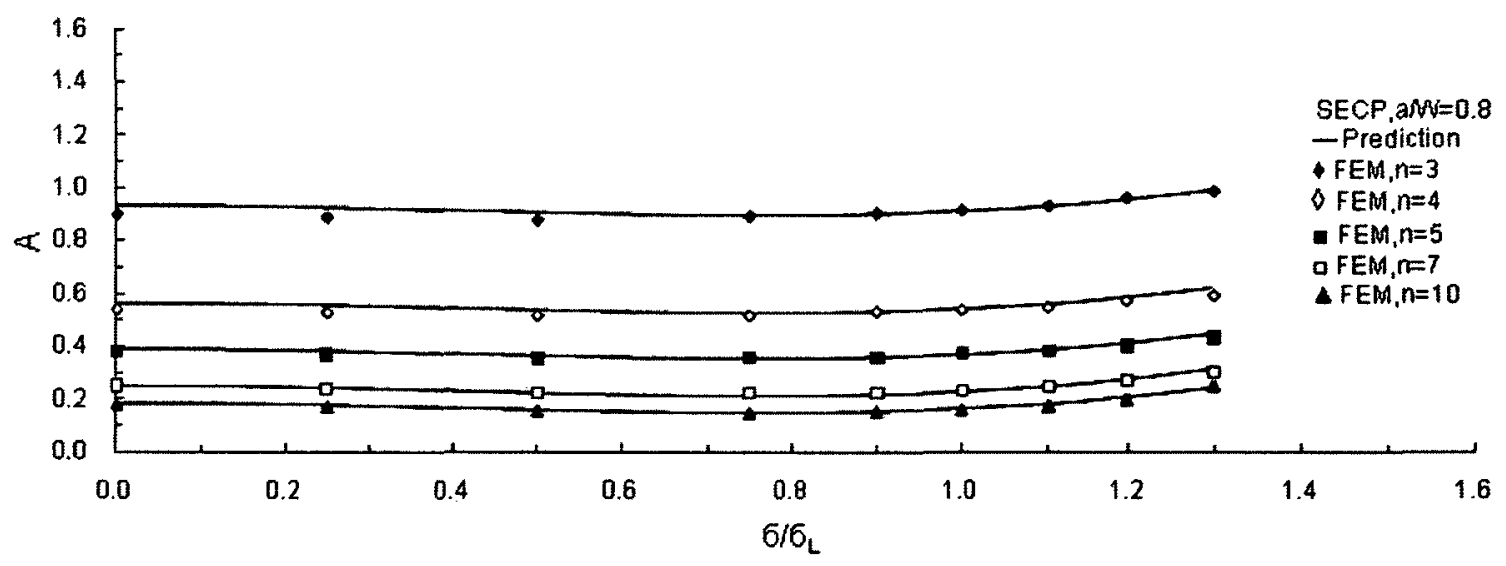

Figure 4.12 Comparisons of predicted $A$ values by curve shape similarity with FEA data for SECP, $a / W=0.8$, plane strain under uniaxial tension load. 


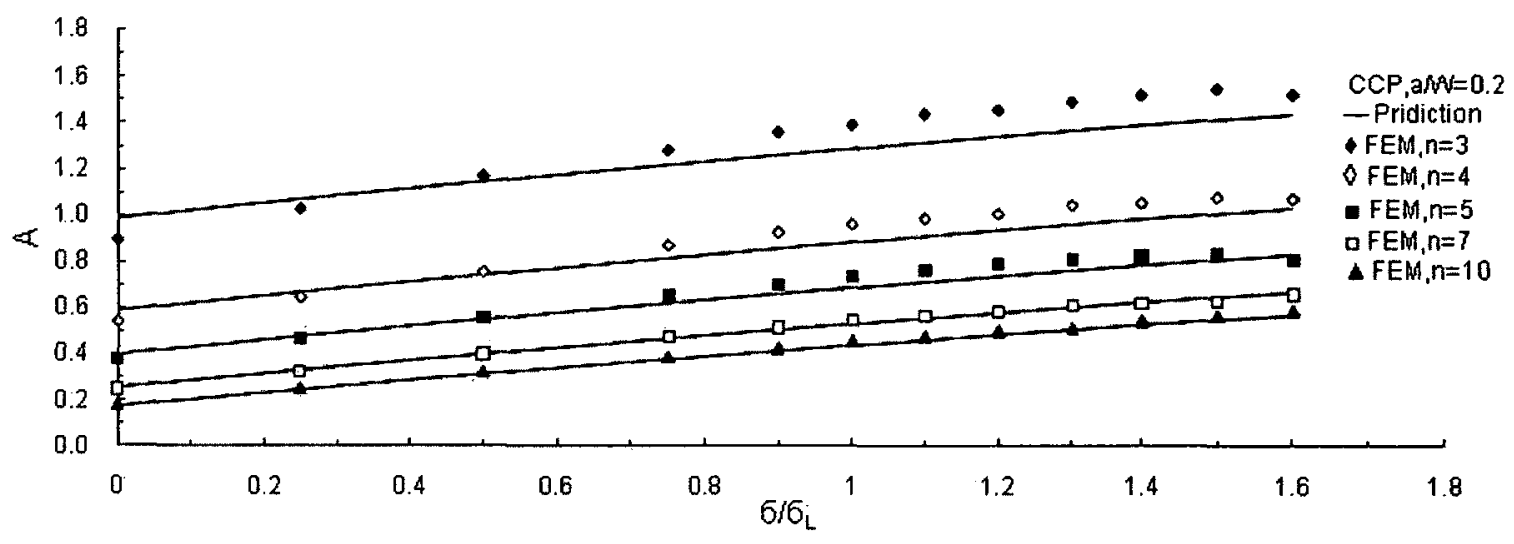

Figure 4.13 Comparisons of predicted $A$ values by curve shape similarity with FEA data for CCP, $a / W=0.2$, plane strain under uniaxial tension load.

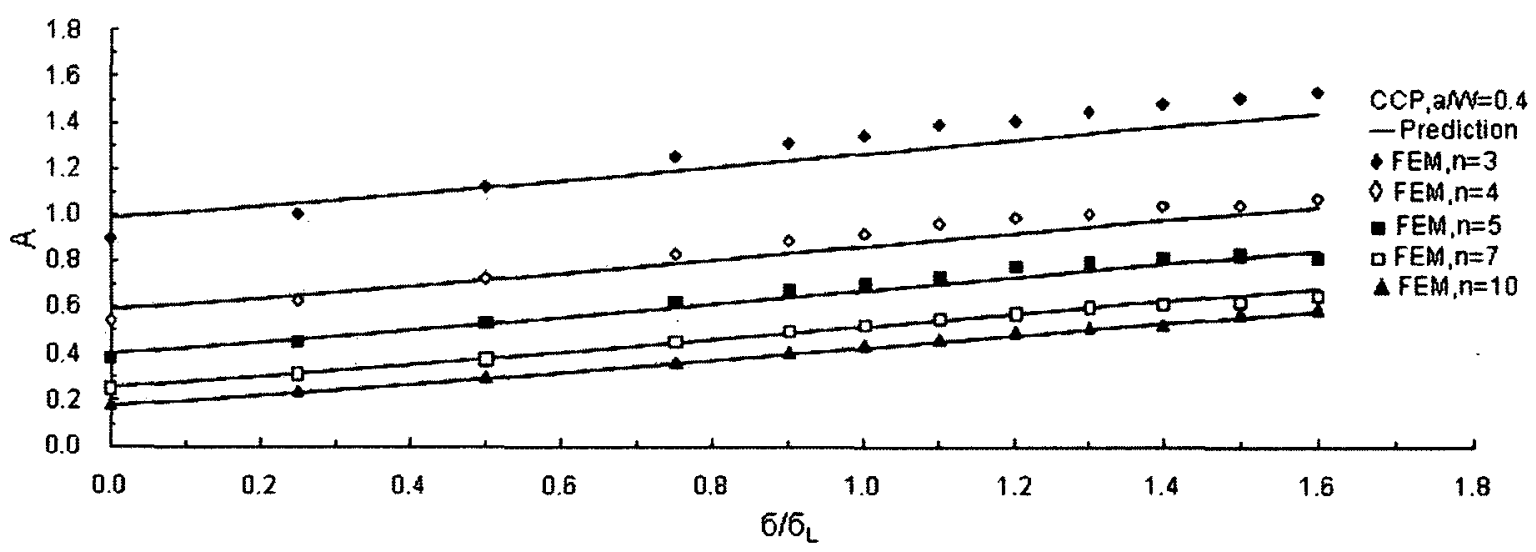

Figure 4.14 Comparisons of predicted $A$ values by curve shape similarity with FEA data for $\mathrm{CCP}, a / W=0.4$, plane strain under uniaxial tension load. 


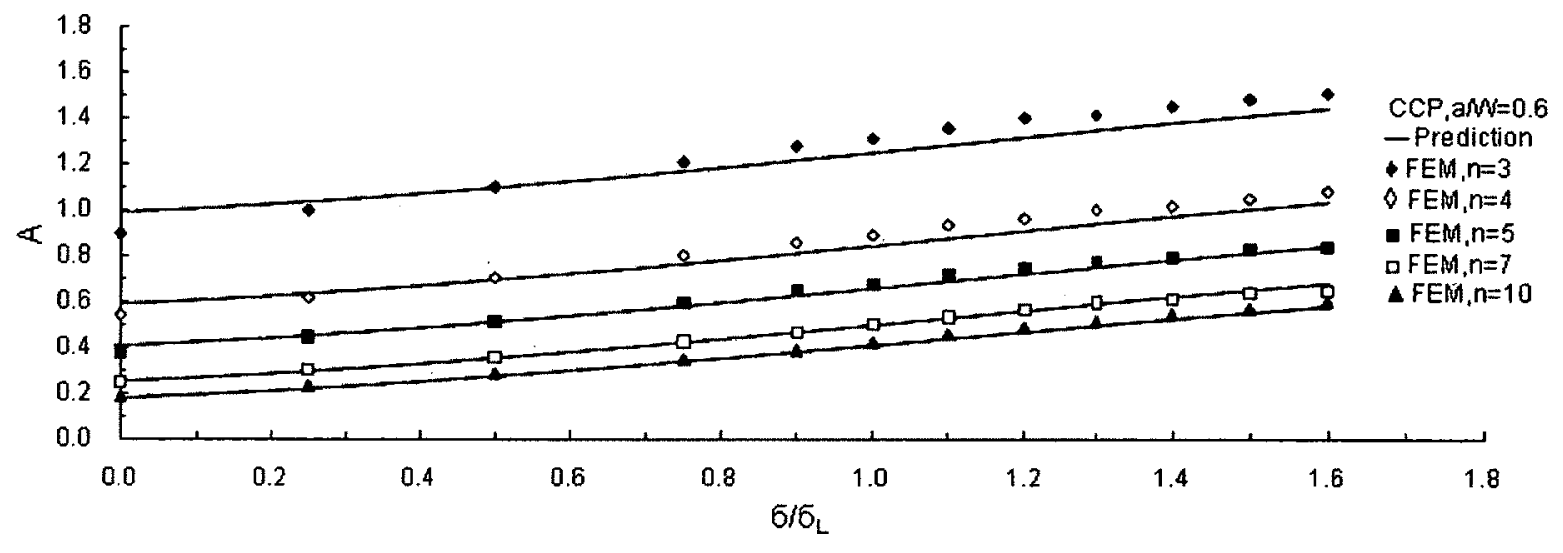

Figure 4.15 Comparisons of predicted $A$ values by curve shape similarity with FEA data for CCP, $a / W=0.6$, plane strain under uniaxial tension load.

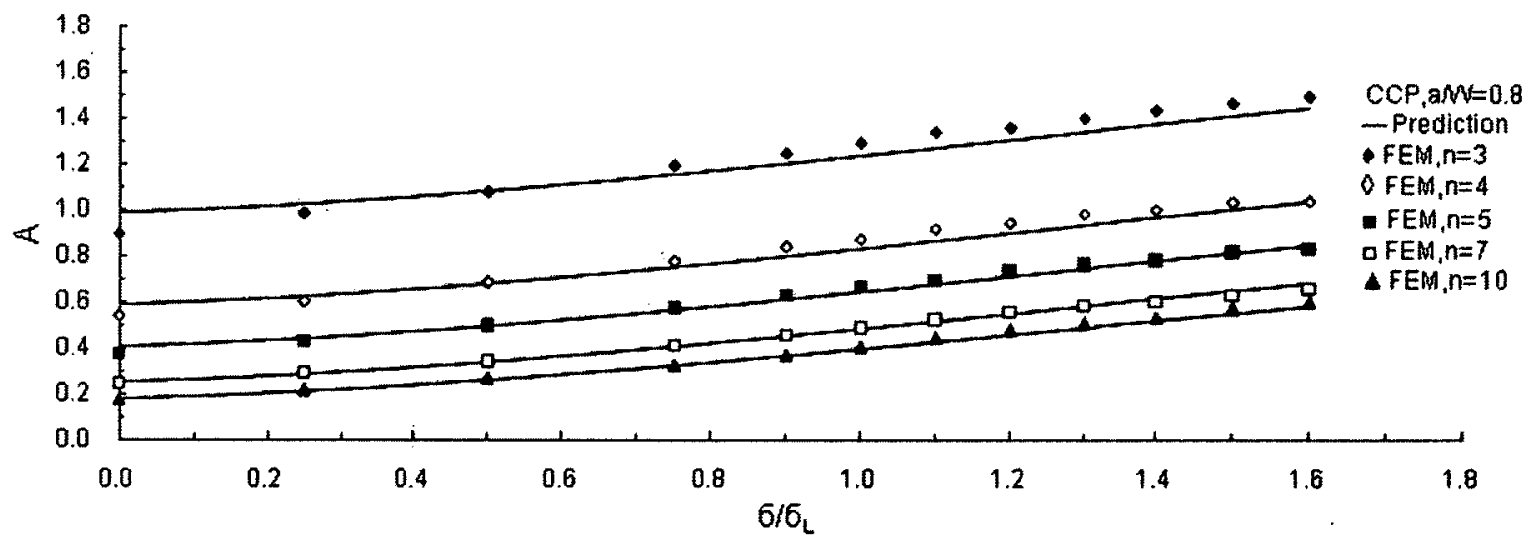

Figure 4.16 Comparisons of predicted $A$ values by curve shape similarity with FEA data for CCP, $a / W=0.8$, plane strain under uniaxial tension load. 


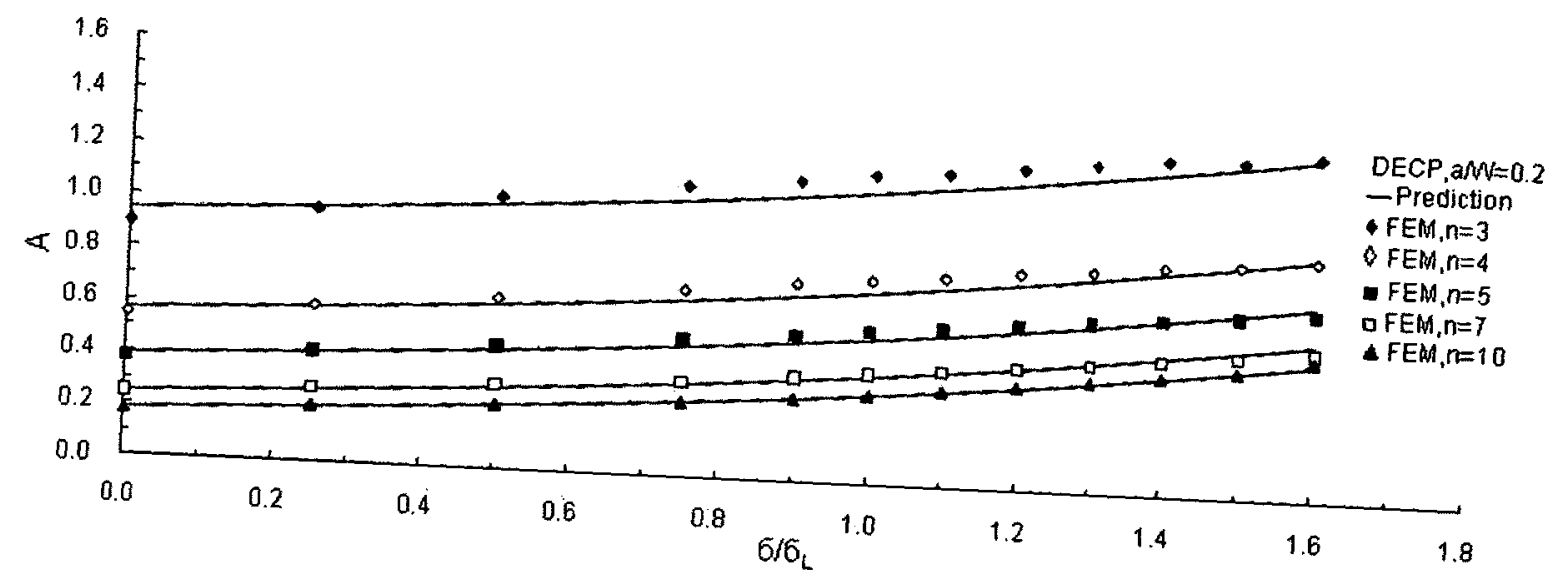

Figure 4.17 Comparisons of predicted $A$ values by curve shape similarity with FEA data for DECP, $a / W=0.2$, plane strain under uniaxial tension load.

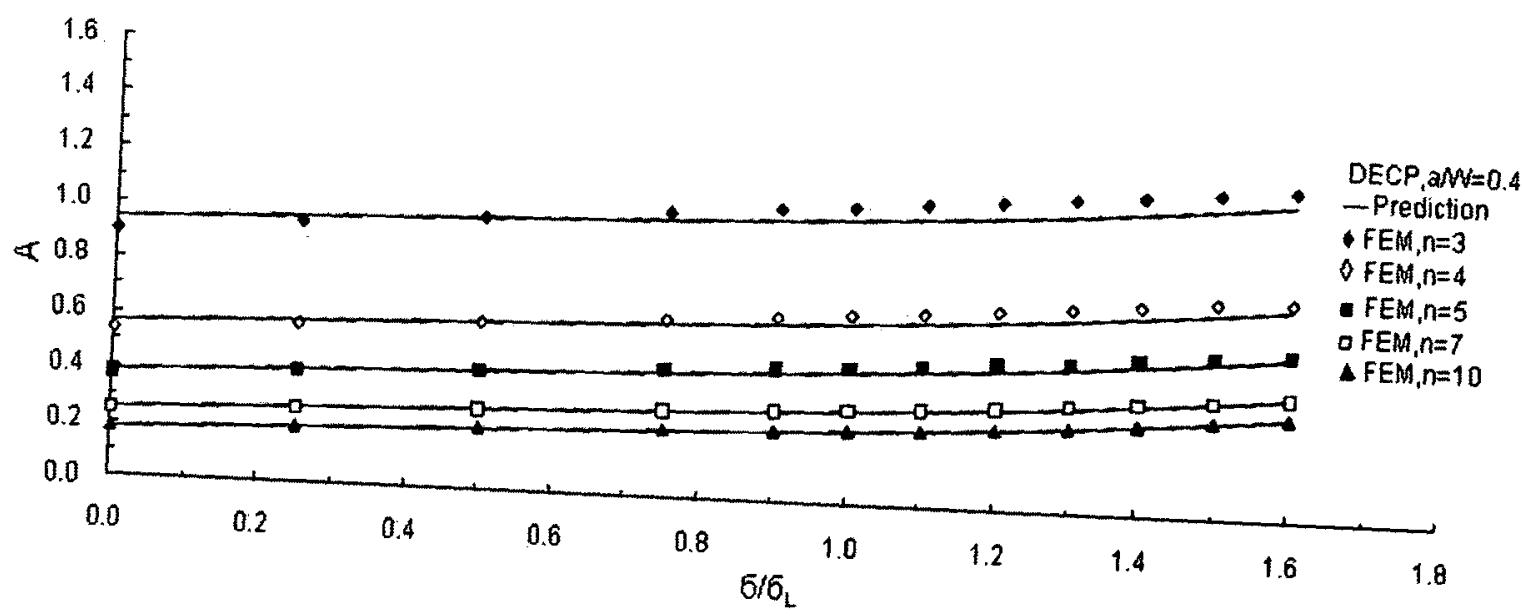

Figure 4.18 Comparisons of predicted $A$ values by curve shape similarity with FEA data for DECP, $a / W=0.4$, plane strain under uniaxial tension load. 


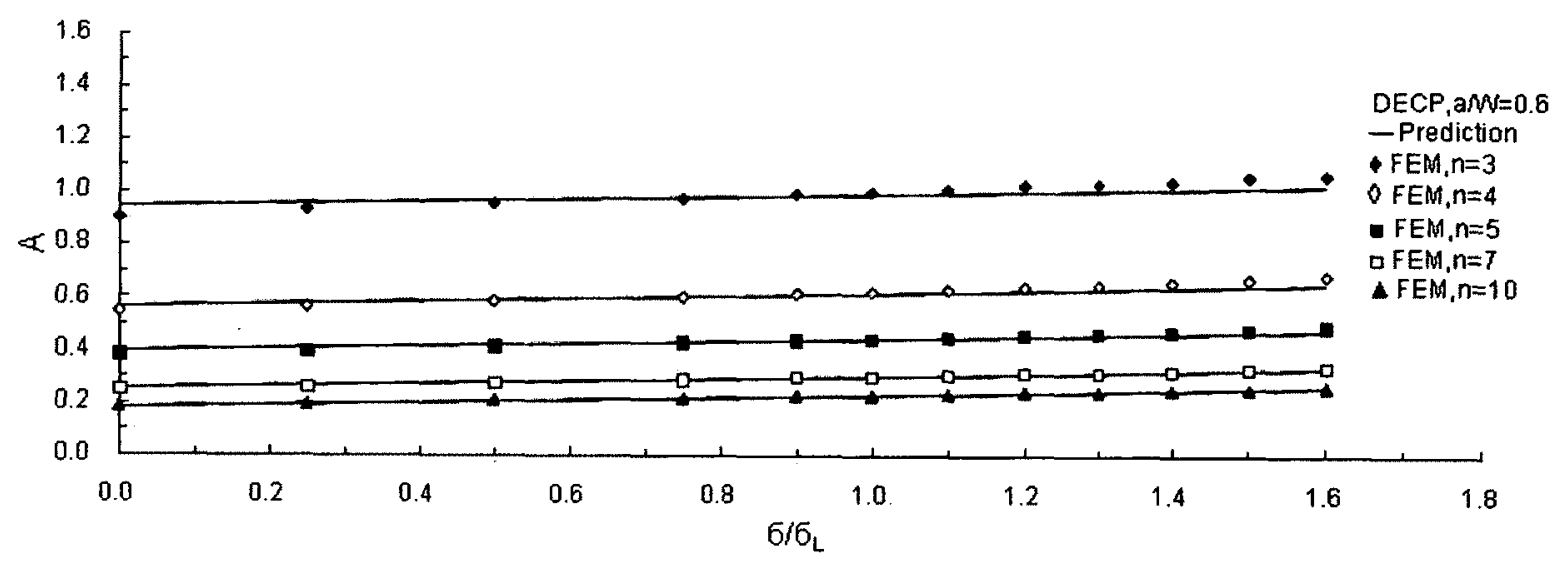

Figure 4.19 Comparisons of predicted $A$ values by curve shape similarity with FEA data for DECP, $a / W=0.6$, plane strain under uniaxial tension load.

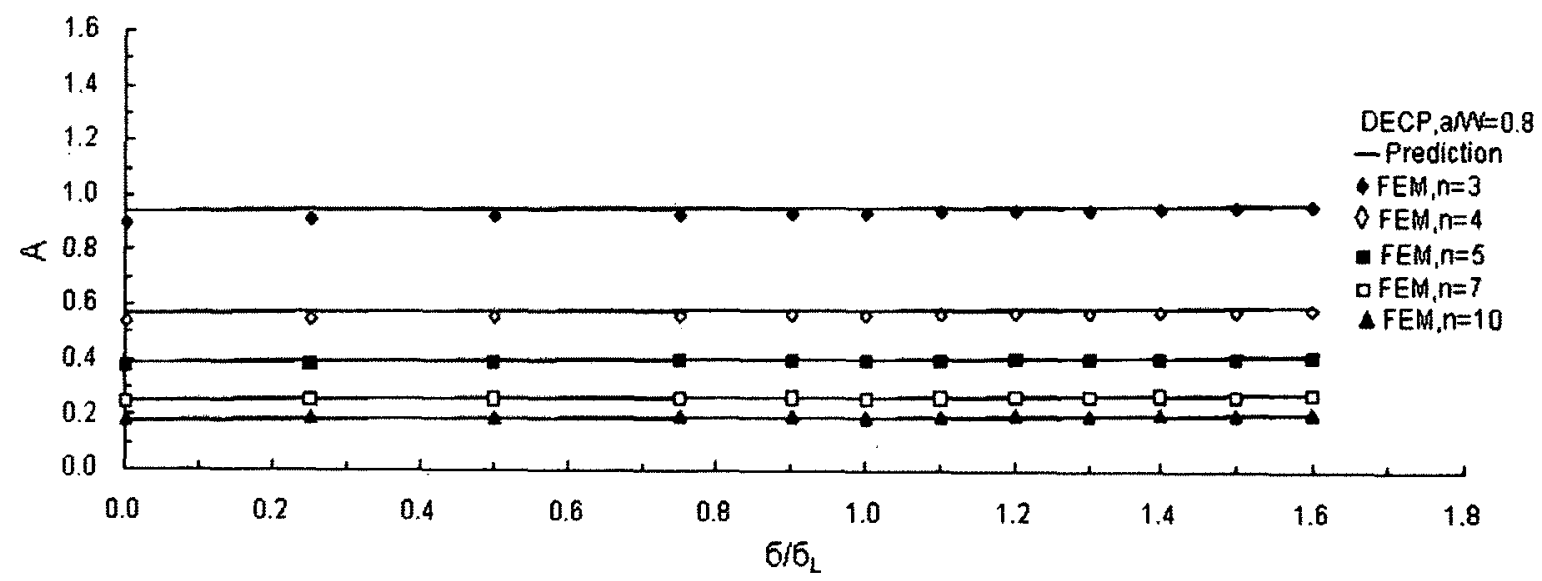

Figure 4.20 Comparisons of predicted $A$ values by curve shape similarity with FEA data for DECP, $a / W=0.8$, plane strain under uniaxial tension load. 


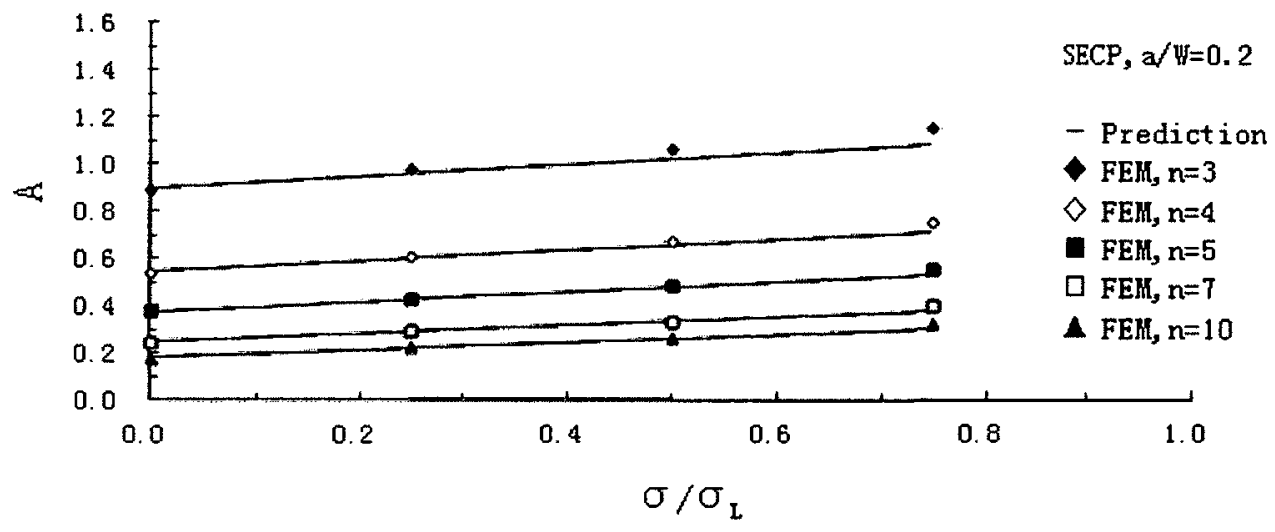

Figure 4.21 Comparisons of predicted $A$ values from $T$-stress with FEA data for SECP, $a / W=0.2$

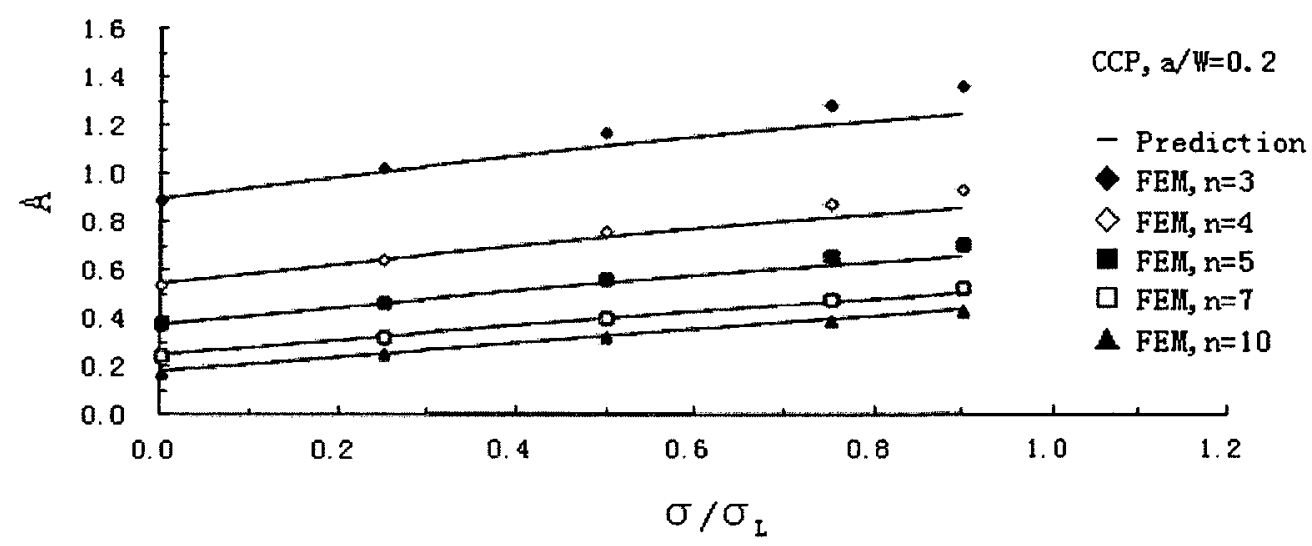

Figure 4.22 Comparisons of predicted $A$ values from $T$-stress with FEA data for CCP, $a / W=0.2$ 


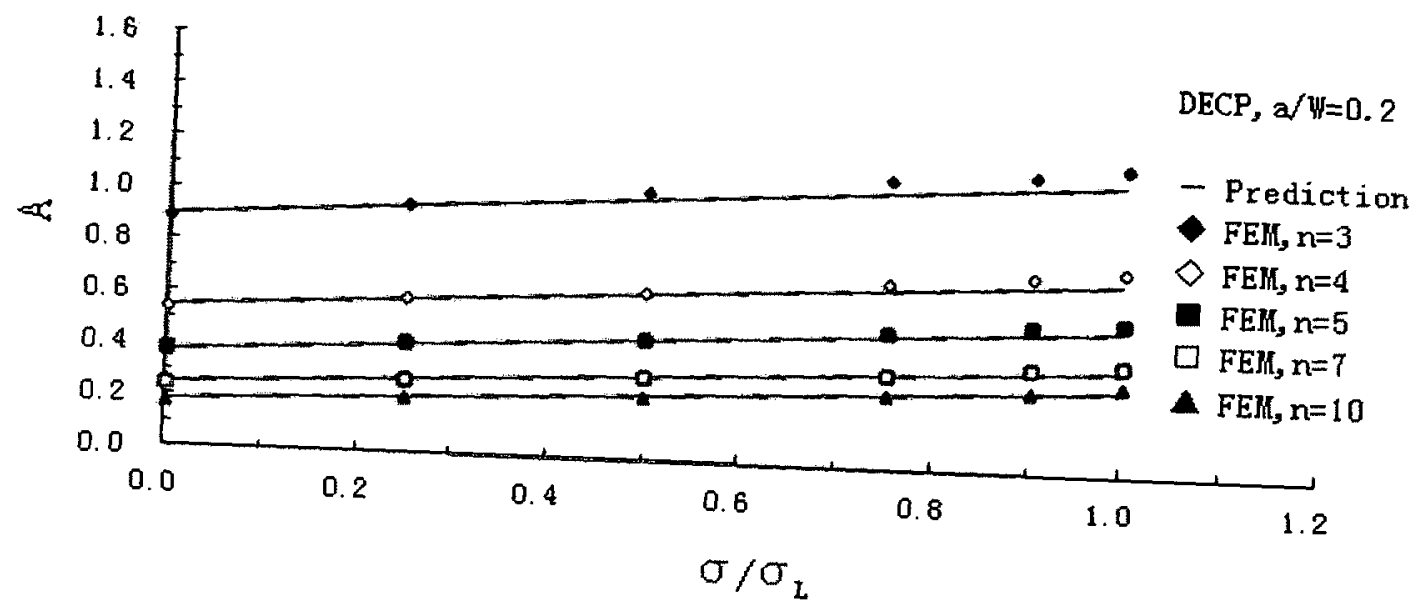

Figure 4.23 Comparisons of predicted $A$ values from $T$-stress with FEA data for DECP,
$a / W=0.2$ 


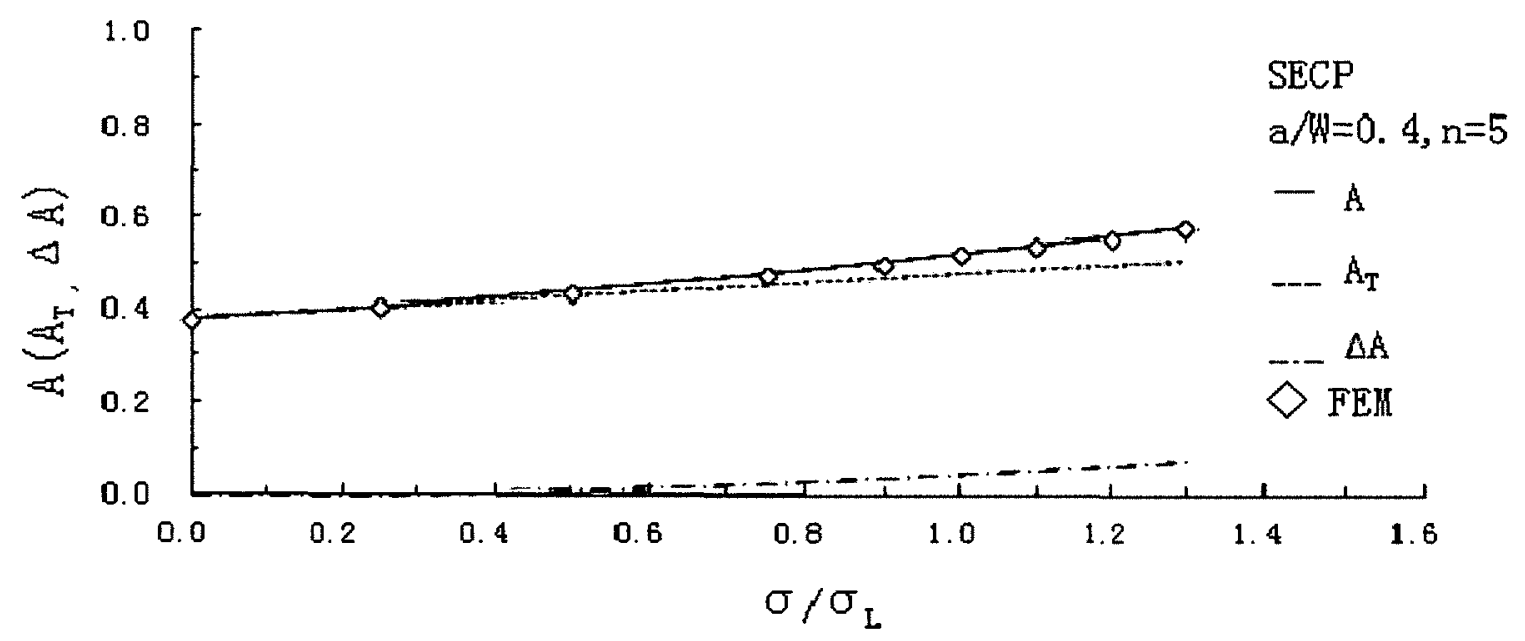

Figure 4.24 Results of $A, A_{T}$ and $\Delta A$ for SECP, $n=5$ and $a / W=0.4$, plane strain under uniaxial load

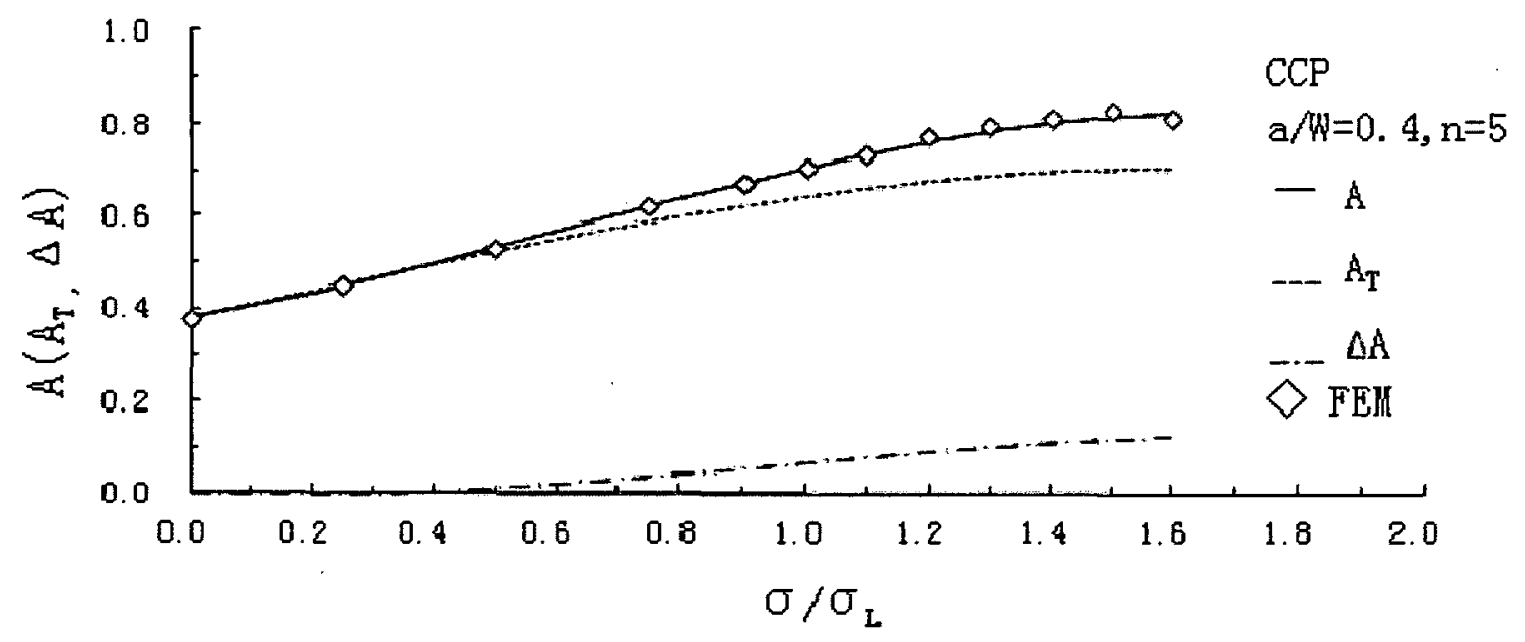

Figure 4.25 Results of $A, A_{T}$ and $\Delta A$ for CCP, $n=5$ and $a / W=0.4$, plane strain under uniaxial load 


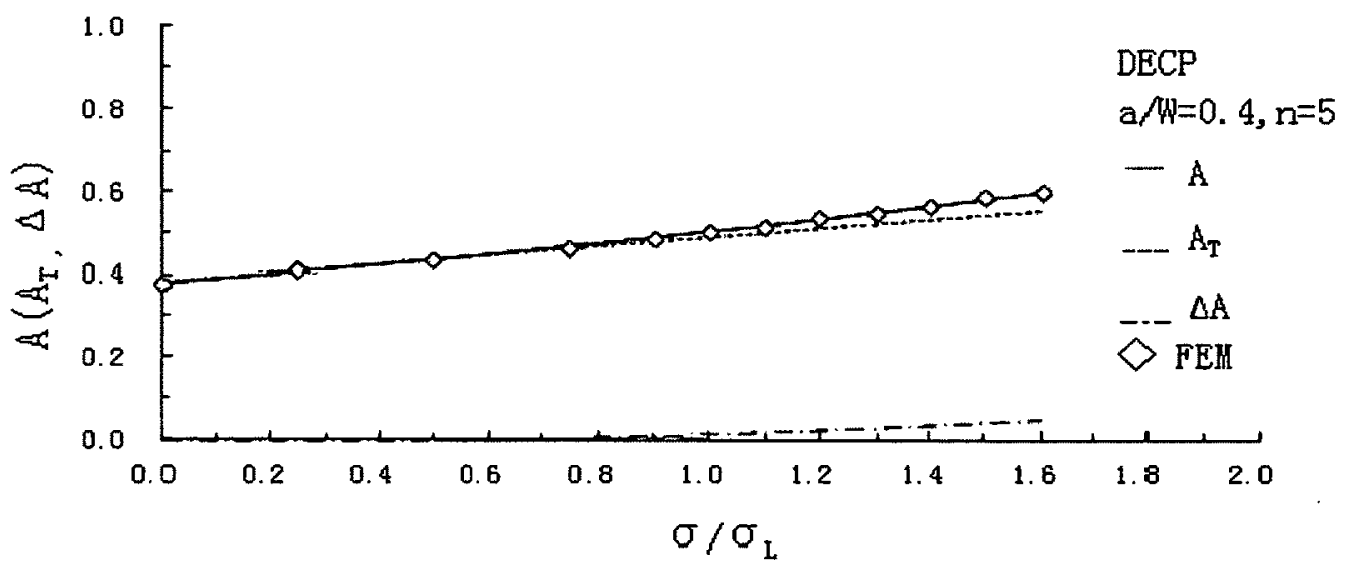

Figure 4.26 Results of $A, A_{T}$ and $\Delta A$ for DECP, $n=5$ and $a / W=0.4$, plane strain under uniaxial load 


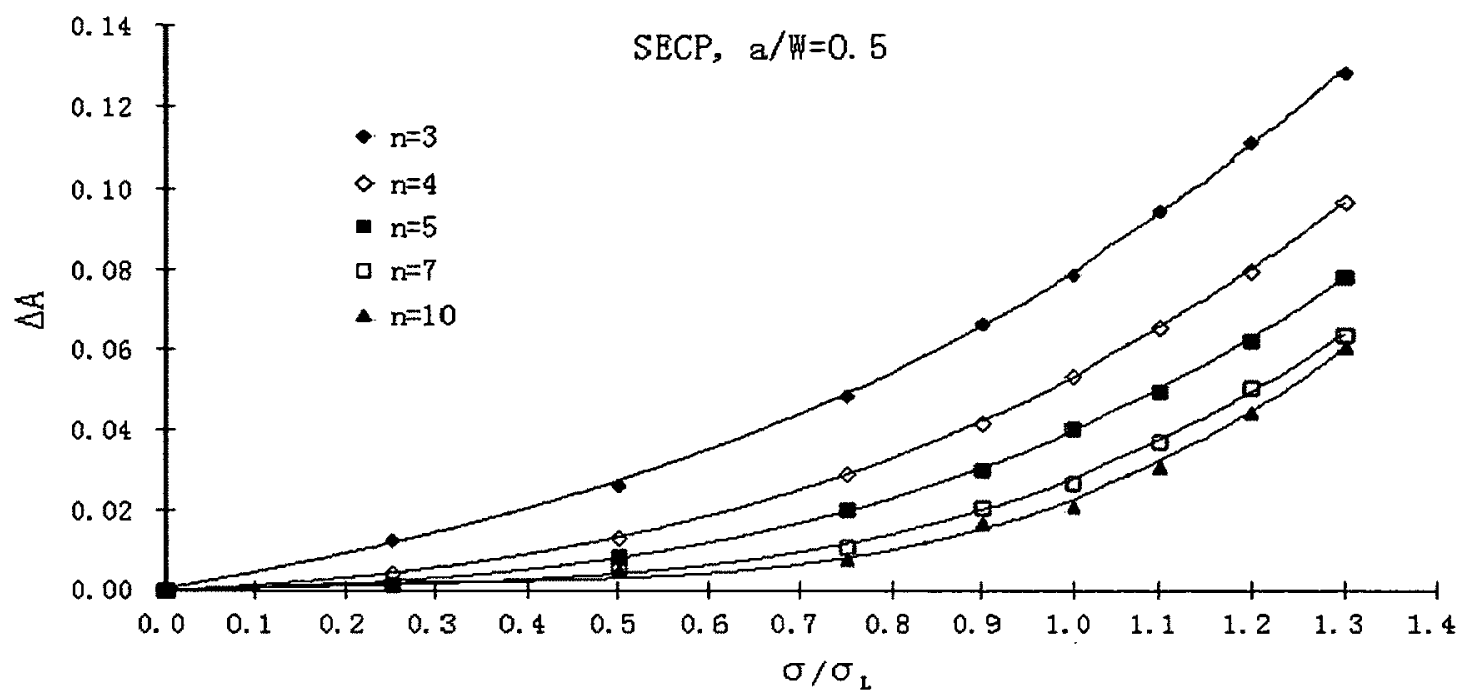

Figure 4.27 Differences between $A$ solutions from $T$-stress and from FEA, $\Delta A$, for SECP, $a / W=0.5$ with varying $n$ 


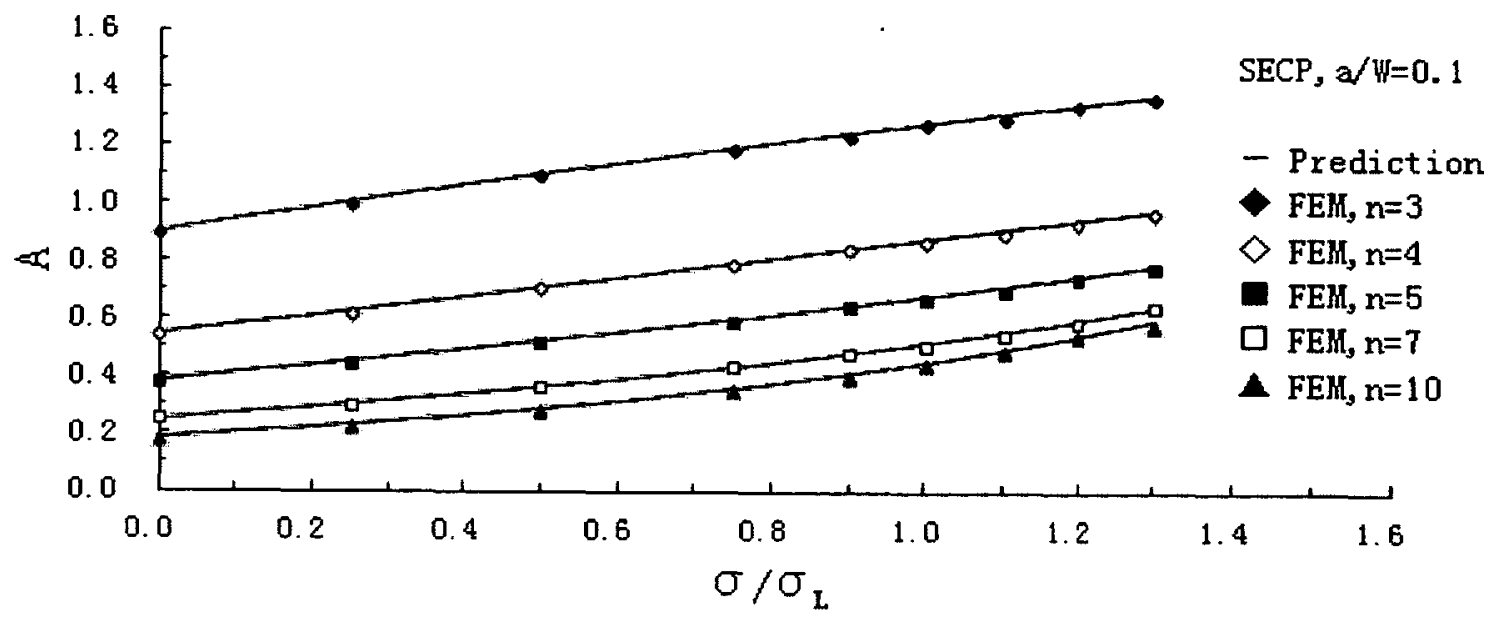

Figure 4.28 Comparisons of predicted $A$ values with FEA data for SECP, $a / W=0.1$, plane strain under uniaxial load

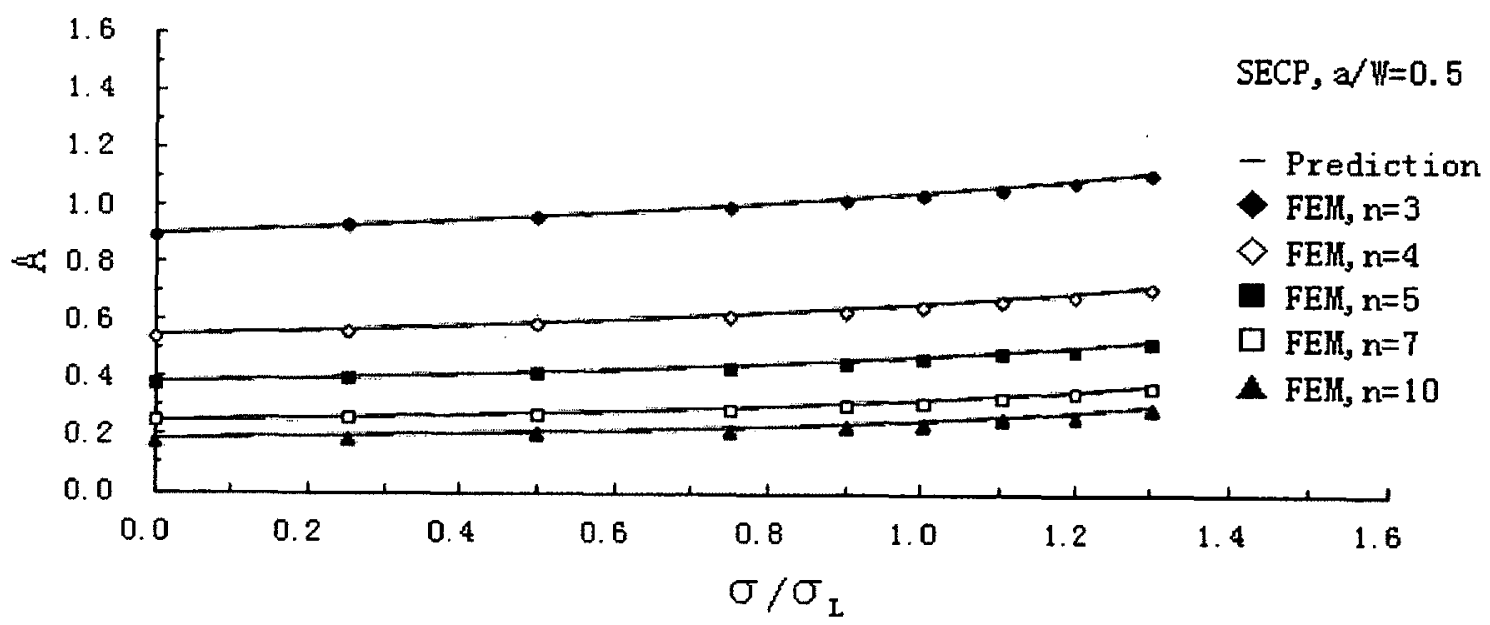

Figure 4.29 Comparisons of predicted $A$ values with FEA data for SECP, $a / W=0.5$, plane strain under uniaxial load 


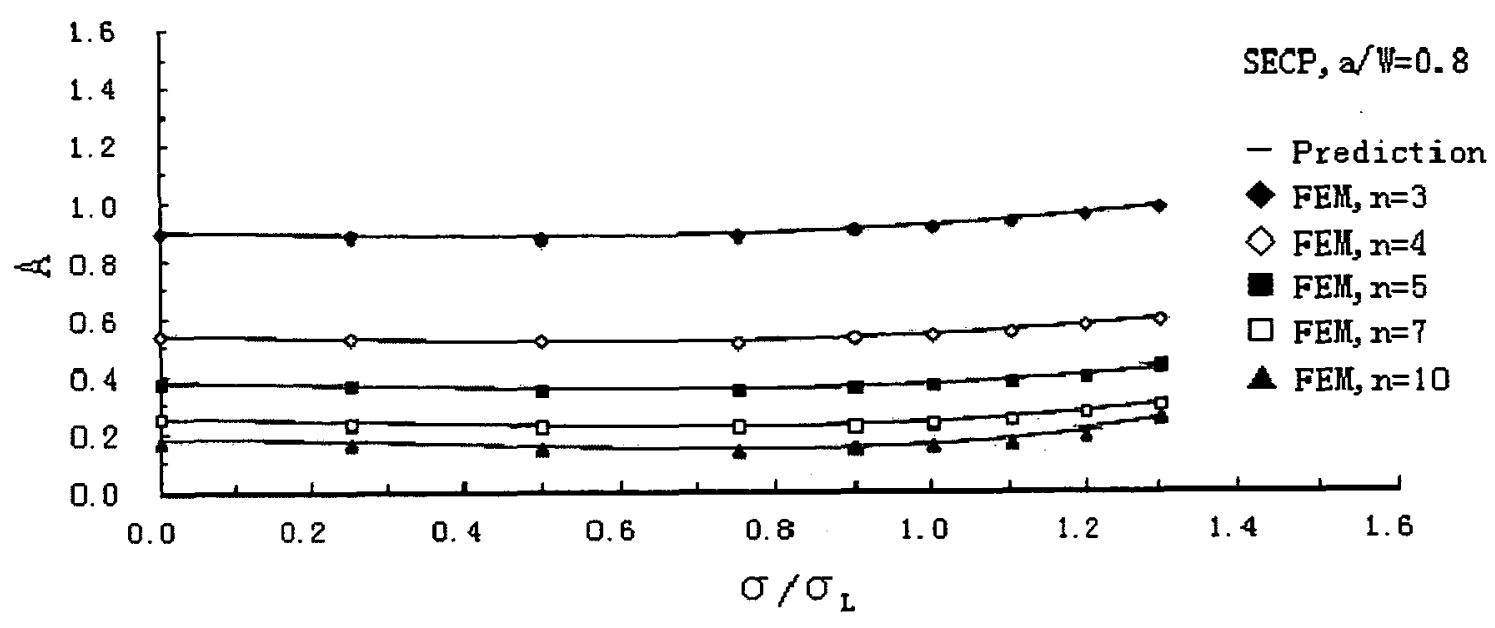

Figure 4.30 Comparisons of predicted $A$ values with FEA data for SECP, $a / W=0.8$, plane strain under uniaxial load 


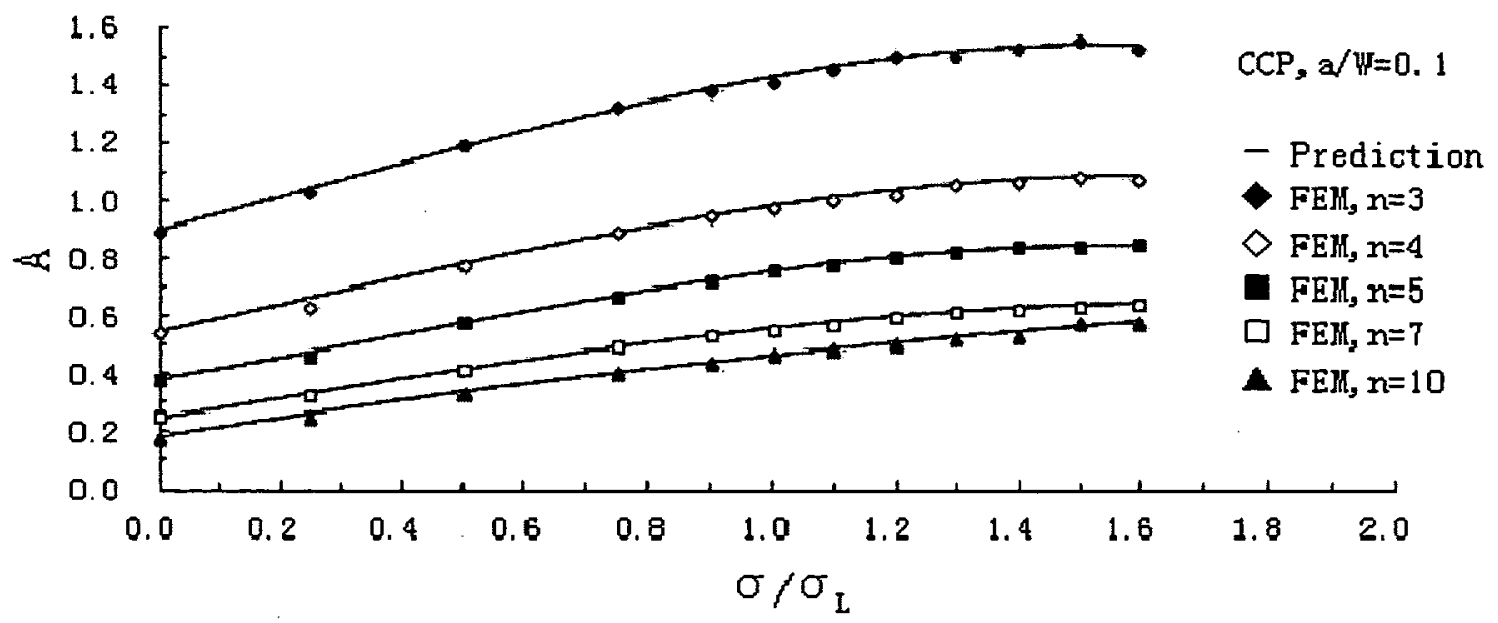

Figure 4.31 Comparisons of predicted $A$ values with FEA data for CCP, $a / W=0.1$, plane strain under uniaxial load

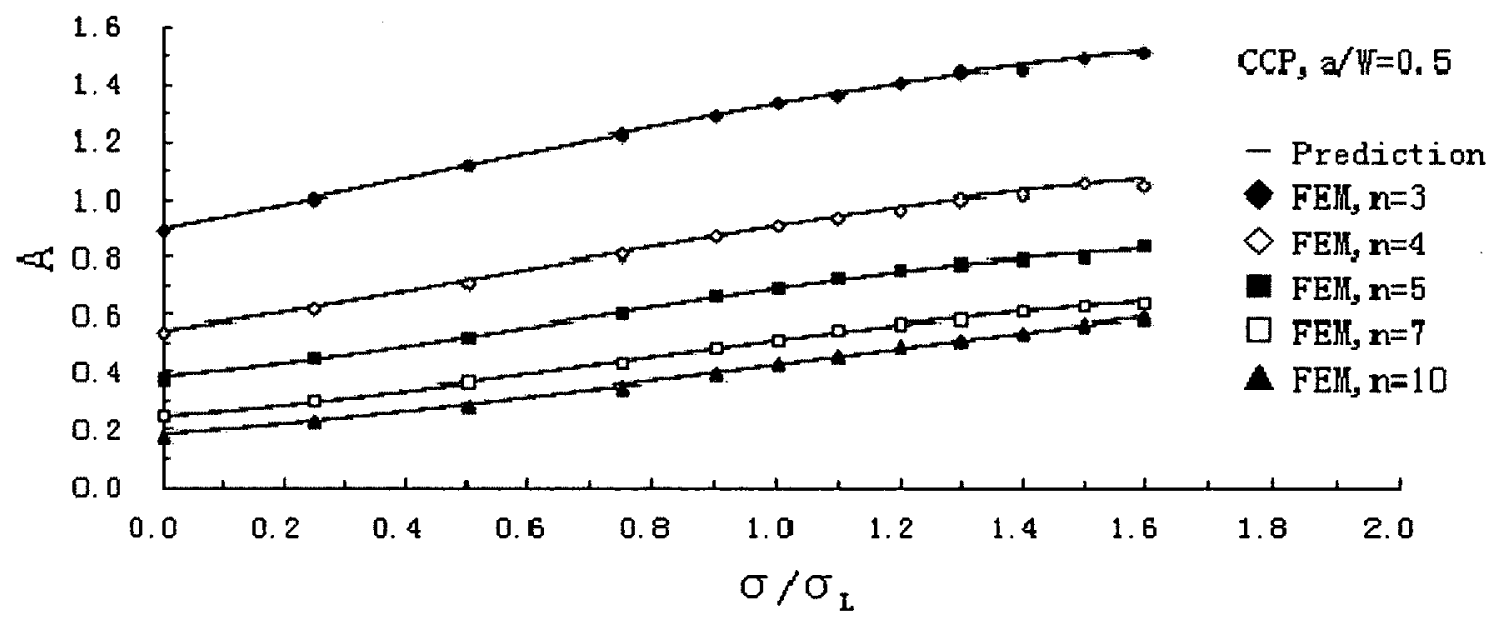

Figure 4.32 Comparisons of predicted $A$ values with FEA data for CCP, $a / W=0.5$, plane strain under uniaxial load 


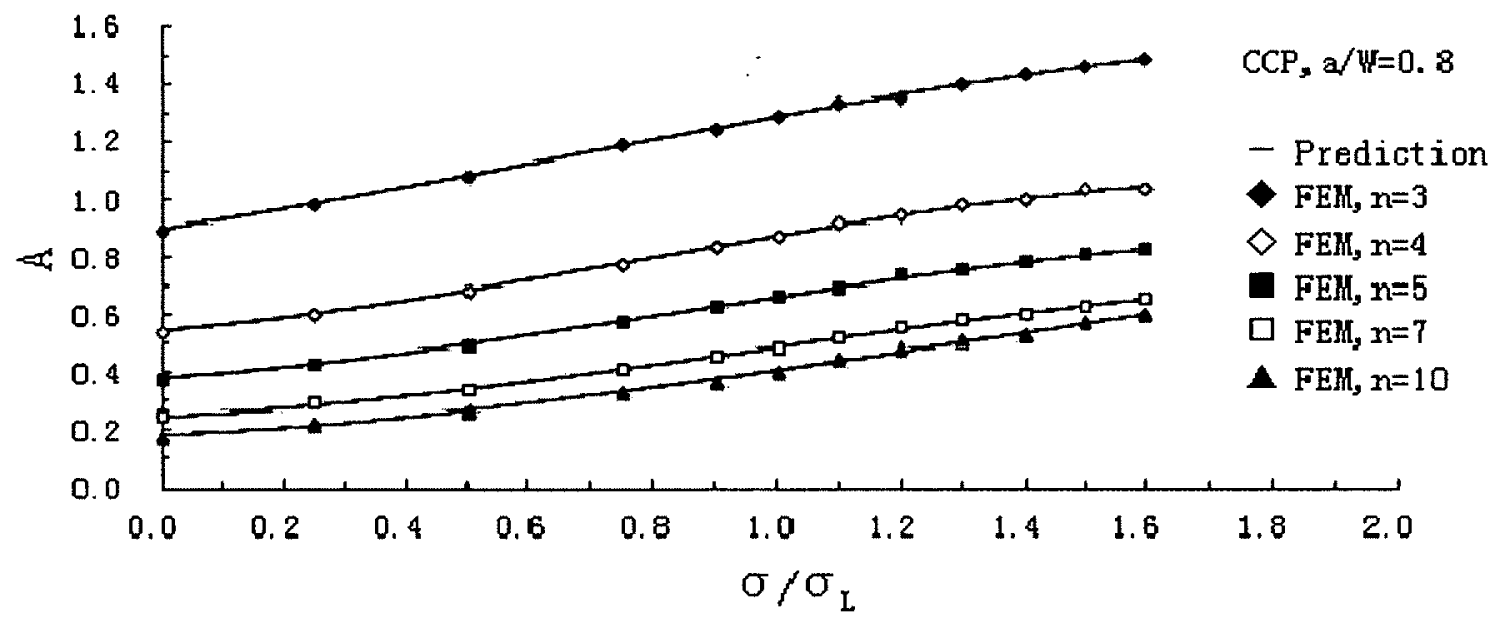

Figure 4.33 Comparisons of predicted $A$ values with FEA data for CCP, $a / W=0.8$, plane strain under uniaxial load 


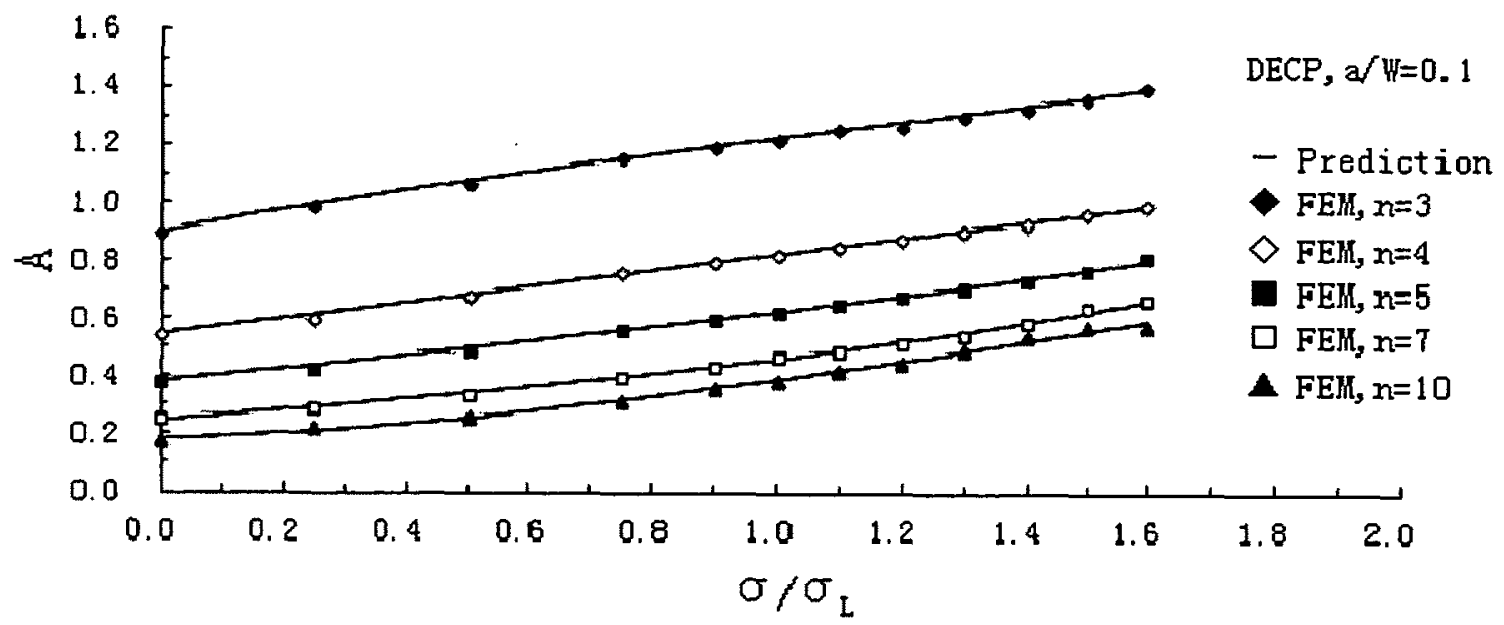

Figure 4.34 Comparisons of predicted $A$ values with FEA data for DECP, $a / W=0.1$, plane strain under uniaxial load

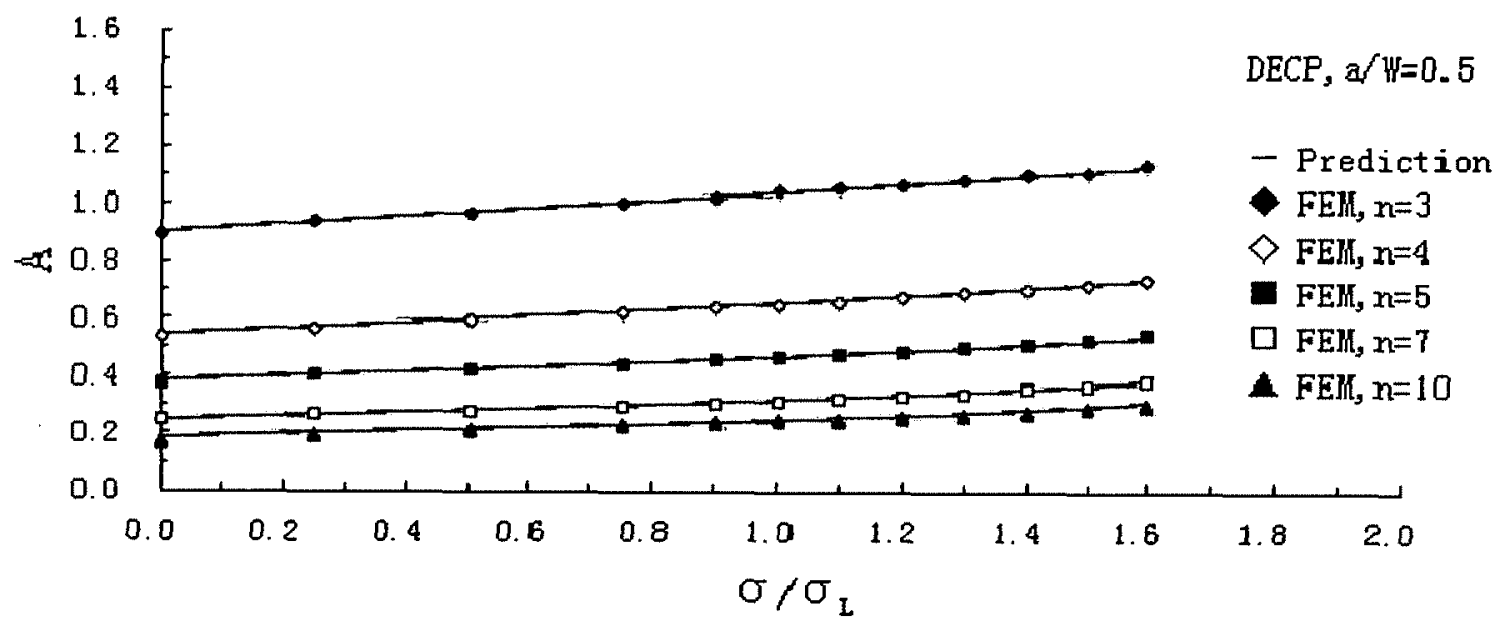

Figure 4.35 Comparisons of predicted $A$ values with FEA data for DECP, $a / W=0.5$, plane strain under uniaxial load 


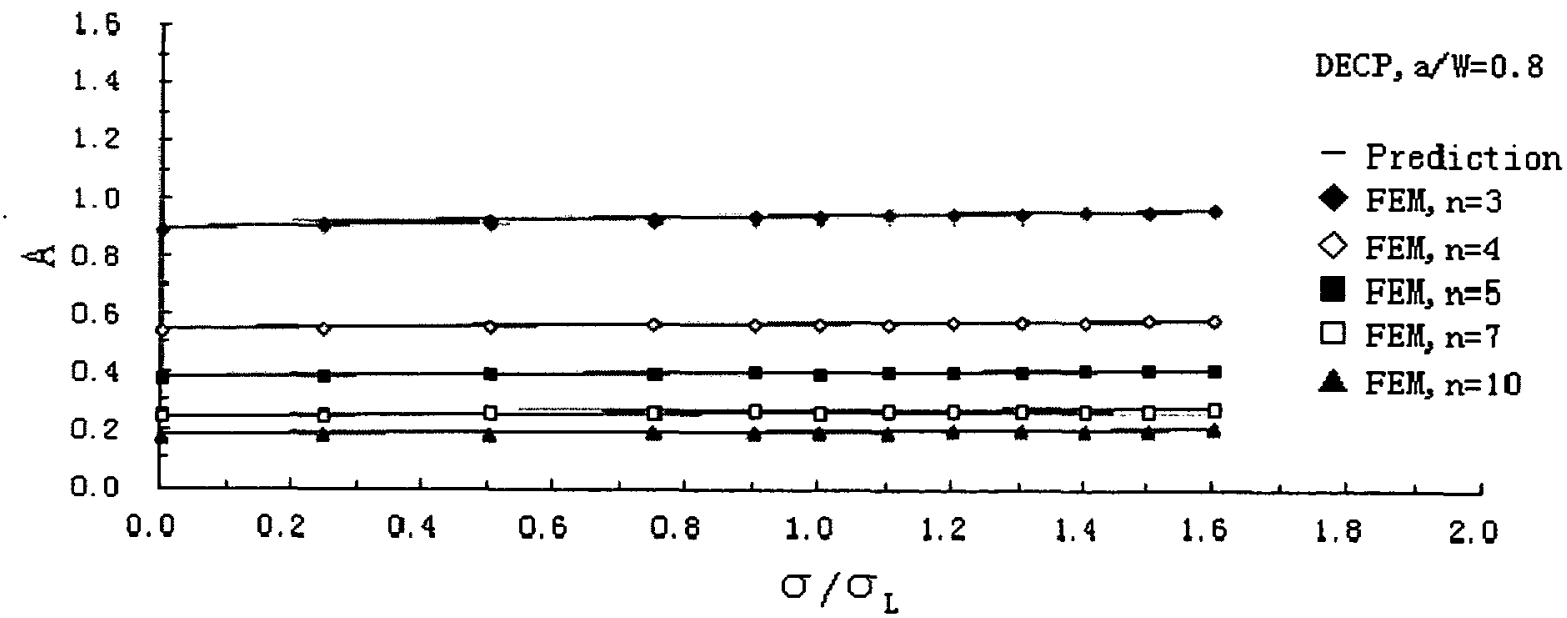

Figure 4.36 Comparisons of predicted $A$ values with FEA data for DECP, $a / W=0.8$, plane strain under uniaxial load 


\section{CHAPTER 5 SOLUTION OF CONSTRAINT PARAMETER $\boldsymbol{A}$}

\section{FOR 2D SPECIMENS UNDER BIAXIAL LOADING}

\subsection{Introduction}

Numerical solutions of constraint parameter $A$ for three plane strain specimen models (SECP, CCP and DECP) under biaxial load are obtained through extensive finite element analyses (FEA) in this chapter. The details of the finite element models are illustrated together with a presentation of material properties. With FEA results, numerical solutions of constraint parameter $A$ for SECP, CCP and DECP under biaxial load are obtained by the fitting method proposed by Nikishkov et al. (1995a).

Based on the obtained numerical solutions of constraint parameter $A$, constraint effects on 2D cracked specimens under biaxial loading are discussed.

In chapter 4 , three sets of estimate methods for parameter $A$ proposed in chapter 3 are applied for specimens under uniaxial loading conditions. In this chapter, those methods are utilized for biaxial loading conditions for three same 2D specimens (SECP, $\mathrm{CCP}$ and DECP) as those investigated in chapter 4.

Empirical formulas for parameter $A$ values by curve shape similarity of $A$ are obtained based on the numerical solutions for parameter $A$. With the detailed $A-T$ relationship determined in chapter 4 , formulas for predicting parameter $A$ from $T$-stress under small scale yielding are applied to estimate $A$ solutions for SECP, CCP and DECP under biaxial load. Based on $A$ values from $T$-stress and numerical solutions of parameter $A$, values of the coefficients $a_{l}$ in the approximate formula for $A$ under biaxial load are obtained. With the determination of the coefficients $a_{l}$, approximation formulas for parameter $A$ based on fully plastic analysis are developed to predict the parameter $A$ from low to high load (small-scale to large-scale yielding) for SECP, CCP and DECP specimens under biaxial loading.

For each of the three sets of approximation formulas, the predicted parameter $A$ 
solutions are compared with those determined from FEA results to discuss the applicability of suggested estimate formulas on 2D cracked specimens under biaxial loading.

\subsection{Finite Element Analysis of Test Specimens}

\subsubsection{Problem definition}

In chapter 4, three typical mode I plane strain test specimens, i.e. single edge cracked plate (SECP), center cracked plate (CCP) and double edge cracked plate (DECP) are studied by finite element method under uniaxial load condition. In this chapter, finite element analyses are implemented for the same three specimen models under biaxial load condition. Figure 5.1, Figure 5.2 and Figure 5.3 illustrate geometry, coordinate systems and load condition for three two-dimensional models, SECP, CCP and DECP, respectively. The geometrical sizes of three investigated specimen models are defined as: ratio of height to width, $H / W=3.0$; and relative crack length $a / W=0.1,0.3,0.5$ or 0.7 . Here $\lambda$ is defined as the ratio of the stress parallel to the crack over the stress perpendicular to the crack.

As in chapter 4, the finite element analyses (FEA) utilize deformation theory of plasticity and a Ramberg-Osgood strain hardening relation through the commercial code ABAQUS (2006). Eq. (2.4) describes the biaxial tension stress-strain curve based on Ramberg-Osgood relation.

The same material properties as for the FEA of SECP, CCP and DECP under uniaxial load are used for the biaxial load cases: yield stress $\sigma_{0}=4.0 \times 10^{8} \mathrm{~Pa}$; elasticity modulus $E=2.0 \times 10^{11} \mathrm{~Pa}$; Poisson ratio $v=0.3$; material coefficient $\alpha=1$; and hardening exponent $n=3,4,5,7$, and 10 . A wide range of both high and low strain hardening levels is covered.

The finite element analyses for all three specimens (SECP, CCP and DECP) are carried out with two different biaxial loading ratios $\lambda=0.5$ and $\lambda=1.0$ (see Figures 5.15.3). In all finite element analyses of the present biaxial loading cases, the external loads applied at the remote end of the specimens are normalized by yield stress $\sigma_{0}$, i.e. $\sigma / \sigma_{0}$. 
In the literature, several estimation methods for the $J$-integral can be found. For the purpose of comparison and verification, $J$-integral solutions from EPRI estimation formulas are summarized here.

According to the investigation of Kumar et al. (1981), the $J$-integral can be expressed as the sum of the elastic solution of $J$-integral, $J_{E}$, and the fully plastic solution, $J_{P}$ (see Eq. (2.22)). As the stress parallel to the crack plane does not affect the stress intensity factor $K$ or the $J$-integral for linear elastic conditions (see the paper of O'Dowd et al. (1999)), $J_{E}$ under biaxial loading is same as that under uniaxial loading. The elastic $J$-integral $J_{E}$ can be obtained from $K$. For fracture mode I under plane strain, the stress intensity factor for mode I, $K_{I}$, and the elastic $J$-integral are related by Eq. (2.5). And the values of $K_{I}$ under uniaxial loading can be obtained by (Tada et al. (2000)):

$$
K_{I}=\sigma \sqrt{\pi a} F
$$

where the coefficient $F$ is a function of the relative crack length a/W, i.e. $F(a / W)$. There are several empirical formulas in the literature to determine $F$. In the present work, the modified Feddersen formula for CCP suggested by Tada et al. (1973) is chosen:

$$
F\left(\frac{a}{W}\right)=\left[1-0.025\left(\frac{a}{W}\right)^{2}+0.06\left(\frac{a}{W}\right)^{4}\right] \sqrt{\sec \frac{\pi a}{2 W}}
$$

For the fully plastic solution of $J$-integral, $J_{P}$, O'Dowd et al. (1999) have proposed a normalization equation for the CCP specimen under biaxial loading, see Eq. (2.24). In Eq. (2.24), the normalization function of $J$-integral, $h_{l}$, depends on the hardening exponent $n$, the geometry ratio $a / W$ and the biaxial loading ratio $\lambda$, i.e. $h_{1}(n, a / W, \lambda)$. Values of $h_{1}$ are tabulated in the paper of O'Dowd et al. (1999). The expression of limit stress used in Eq. (2.24) for the CCP specimen under biaxial loading is also given by O’Dowd et al. (1999):

$$
\sigma_{L}=\frac{2}{\sqrt{3}} \sigma_{0}\left(1-\frac{a}{W}\right)\left[1-\lambda\left(1-\frac{a}{W}\right)\right]^{-1}
$$

Substituting Eqs. (2.5) and (2.24) into Eq. (2.22), the predicted $J$-integral values for the CCP specimen under biaxial loading can be obtained. 
Theses equations will be utilized when comparing with literature results.

\subsubsection{Finite element analysis and parameter $A$ determination}

\subsubsection{Finite element modeling}

For the meshing of the three two-dimensional (2D) specimen models, only a half of the SECP or a quarter of the CCP or DECP geometry need to be modeled in the finite element analysis because of the component symmetries (see Figures 5.1, 5.2 and 5.3).

A typical three-dimensional (3D) finite element mesh that was used here is illustrated in Figure 5.4. In total, 1196 elements are included in the mesh and the element type is a 20-node quadratic hybrid brick with linear pressure and reduced integration (ABAQUS (2006)). Note that there are a total of 11 wedge shaped elements that are used in the area of transition from crack tip core elements to the surrounding elements. The wedge shaped elements are in fact degenerated 20-node elements.

The boundary conditions, except for the loading in the $x$ direction, are exactly same as those of the uniaxial load cases (section 4.2.2.1) for all three specimen models, SECP, CCP and DECP, including the out-of-plane boundary condition: $u_{z}=0$.

\subsubsection{Determining parameter $A$ based on finite element analysis}

As the uniaxial load cases, numerical solutions of the constraint parameter $A$ for the plane strain SECP, CCP and DECP models under biaxial load conditions are determined from FEA results using the fitting method proposed by Nikishkov et al. (1995a) (Eqs. (2.29-2.32)). The fitting area to calculate the $A$ values based on FEA data is within $1.5 \leq \bar{r} \leq 3$ and $0^{\circ} \leq \theta \leq 45^{\circ}$, and the opening stress $\sigma_{\theta}$ is the stress component used for the fitting procedure.

\subsubsection{Results and discussion}

\subsubsection{Verification of FEA model and analysis procedure}

The utilized finite element model (mesh) and subsequent fitting procedure for determining $A$ values are verified by comparison of two fracture parameters, the dominant parameter $J$-integral and the constraint parameter $Q$-stress ( $J$ - $Q$ approach), with 
literature results. The comparison is based on the $J$-integral estimation formulas found in the literature and the results for CCP specimens under biaxial loading given by O'Dowd et al. (1999). The utilized $J$-integral estimation formulas are described in section 5.1.1.

Finite element analyses for a CCP specimen with hardening exponent $n=10$, biaxial loading ratio $\lambda=0.5$ and geometry ratio $a / W=0.1$ and 0.5 have been completed to verify the finite element meshes utilized in the current investigation (Figure 5.4) and the corresponding procedure for determining the parameter $A$. Predicted $J$ values from the EPRI estimation formulas (section 5.1.1) are compared with those obtained from current finite element analyses. Figure 5.5 shows the comparison results for both $a / W=0.1$ and 0.5. In Figure 5.5, it can be found that the $J$-integral values from FEA are very close to those from the estimation formulas.

With the same geometry, material properties and applied load, comparisons are made for the constraint parameter $Q$, which is the second parameter in the $J-Q$ twoparameter approach. Based on the results of finite element analyses, solutions of constraint parameter $A$ are obtained through the fitting method proposed by Nikishkov $e t$ al. (1995a) (see section 2.6.3.1). By the relationship between $A$ and $Q$ presented in section 2.6.4.3 (Eq. (2.35b)), the obtained $A$ solutions are converted to $Q$ values. The $Q$ values from the present FEA results are compared with those presented by O'Dowd et al. (1999), as shown in Figure 5.6. From Figure 5.6, it is found, for both crack length ratios $a / W=0.1$ and 0.5 , that the $Q$ solutions from the present FEA results are close to those obtained by O'Dowd et al. (1999). A numerical comparison between $Q$ values from FEA as well as the $A-Q$ relationship and those presented by O'Dowd et al. (1999) for the CCP specimen under $n=10, a / W=0.5$ and $\lambda=0.5$ is shown in Table 5.1. The maximum difference is $6.27 \%$.

\subsubsection{Numerical solution of parameter $A$ and discussion for constraint effect}

A wide range of finite element analyses for three plane-strain typical specimens (SECP, CCP and DECP) under biaxial loading are completed. Constraint parameter $A$ solutions are obtained by a fitting method (Nikishkov et al. (1995a)) based on the FEA results.

The research of Chao and Zhu (1998) on 2D plane strain models shows that the size 
requirement for ductile fracture initiation under $J-A_{2}$ dominant conditions is $a \sigma_{0} / J>6$ for the shallow cracked cases or $b \sigma_{0} J J>11$ for deep cracked ones, where $a$ is the crack length and $b$ the remaining ligament. In the present finite element analysis (FEA), the highest load levels are those corresponding to the values mentioned above. In addition, Chao and Zhu (1998) also argue that, for deep crack cases, a value of $b \sigma_{0} J J$ around 30 corresponds to fully plastic condition, while for shallow cracks, values of $a \sigma_{0} / J \leq 50$ already nearly correspond to the fully plastic deformation. According to this estimate, the external load ratios $\left(\sigma / \sigma_{0}\right)$ used here to determine $A$ numerical solutions from FEA for the three specimens cover the range from small-scale yielding (SSY) to large-scale yielding (LSY). For example, for SECP with $a / W=0.7$ (deep crack), the $b \sigma_{0} / J$ values at maximum loads are between 54-64 and 38-62 for $\lambda=0.5$ and $\lambda=1.0$, respectively. For the SECP with $a / W=0.3$ (shallow crack), the $a \sigma_{0} / J$ values at maximum loads are around 4 and between 4 8 for $\lambda=0.5$ and 1.0 , respectively.

The determined $A$ solutions from FEA results with biaxial loading ratios $\lambda=0.5,1.0$ and relative crack length $a / W=0.1,0.3,0.5,0.7$ are listed in Tables 5.2-5.3, 5.4-5.5 and 5.6-5.7 for SECP, CCP and DECP respectively. Typical numerical solutions of parameter $A$ for SECP, CCP and DECP with $a / W=0.3,0.7$ are presented in Figures 5.7-5.12 $(\lambda=0.5)$ and Figures 5.13-5.18 $(\lambda=1.0)$.

By analyzing the numerical solutions of parameter $A$ for three cracked specimens (see Tables 5.2-5.7, Figures 5.7-5.18), several dependencies have been found. First, it is found that, for any specific specimen geometry $(a / W)$ of some cracked body (SECP, CCP or DECP), generally the maximum external loading ratio $\left(\sigma / \sigma_{0}\right)$, which is determined by the above criteria presented in the research of Chao and Zhu (1998), increases with decreasing of hardening coefficient $n$.

In addition, the effects of crack geometry, hardening exponent $(n)$ and biaxial loading ratio $(\lambda)$ on parameter $A$ (constraint level) are also observed. For uniaxial loading cases $(\lambda=0.0)$ investigated in chapter 4 , it has been shown that $A$ values gradually increase with external loading. The results in the current chapter show that, with smaller biaxial loading ratio $(\lambda=0.5)$, the parameter $A$ follows a general decreasing trend with increasing 
external loading for shallow crack geometries (e.g. $a / W=0.1$ for SECP and CCP, $a / W=0.1$, 0.3 for DECP). Decreased parameter $A$ values indicate the increasing constraint level. For the cases with bigger biaxial loading ratio $(\lambda=1.0)$, a decreasing trend of parameter $A$ appears not only for those shallowest cracks but also for cracks with larger depth and even fairly deep cracks (e.g. $a / W=0.3$ for SECP, $0.3,0.5$ for CCP, $0.5,0.7$ for DECP). On the other hand, generally, a deep crack is not sensitive to the variation of the $\lambda$ value, except in the case of DECP. For example, for SECP, with $\lambda$ value increasing from 0.5 to 1.0 , the trend of $A$ curves for $a / W=0.3$ (shallow crack) changes from increasing to decreasing (see Figure 5.7 and Figure 5.13), while that for $a / W=0.7$ (deep crack) almost keeps no change (see Figure 5.8 and Figure 5.14).

As for the effect of hardening exponent $(n)$, with the increase of the biaxial loading ratio $\lambda$, decreasing trend of $A$ curves appears earlier (under smaller external loading) or is more significant for cases with smaller $n$ values than that for cases with bigger $n$ values. For example, for DECP, the trend of the $A$ curves for $a / W=0.3$ changes from increasing to decreasing as $\lambda$ increases from 0.5 to 1.0 (see Figure 5.11 and Figure 5.17), but the curve for $n=3$ decreases more quickly than that for $n=10$ (Figure 5.17).

Based on the observations represented above, it can be concluded that, (1) in general, constraint level in crack-tip fields increases with the increase of biaxial loading ratio, $\lambda$; (2) the constraint level for materials with smaller values of hardening exponent $n$ increases more with higher increased biaxial loading ratio; (3) the constraint level for shallow cracks rises more with increase of biaxial loading ratio; (4) comparing SECP and $\mathrm{CCP}$ specimens, an increase of biaxial loading ratio raises the constraint level of DECP more.

\subsection{Solution of Parameter $\boldsymbol{A}$ by Curve-Shape Similarity}

As discussed in section 3.1.1, it is shown that curve-shape similarity also can be utilized under biaxial loading with external loading ratio $\sigma / \sigma_{0}$. As a result, empirical formulas for predicting $A$ solutions of specimens under biaxial loading can be developed. For cases with external loading ratio $\sigma / \sigma_{0}$, Eq. (3.1) can be rewritten as: 


$$
A\left(\frac{a}{W}, \frac{\sigma}{\sigma_{0}}, n\right)=A_{S S Y}(n)+\left\{A\left(\frac{a}{W}, \frac{\sigma}{\sigma_{0}}, n=10\right)-A_{S S Y}(n=10)\right\}
$$

Parameter $A$ solutions from FEA results for SECP, CCP and DECP specimens with geometry ratios $a / W=0.1,0.3,0.5,0.7$ under biaxial loading (biaxial ratios $\lambda=0.5,1.0$ ) have been obtained in the present work (see section 5.1). A third-order polynomial expression of the second term in Eq. (5.4) can be determined based on $A$ solutions from FEA results using the least square fitting method. In the present work, except a special case of DECP with $\lambda=1.0$, the $A$ solutions for $n=10$ are chosen as reference for predicting $A$ solutions at other $n$ values (see Eq. (5.4)). Therefore, the FEA values of parameters $A, A\left(a / W, \sigma / \sigma_{0}, n=10\right)$, for each of the three specimens (Tables 5.2-5.7) and a numerical $A$ solution for standard small-scale yielding $(T=0)$ at $n=10\left(A_{S S Y}(n=10)\right)$ are used to carry out the curve fitting. Here the $A$ solutions of standard SSY, which have been obtained from the MBL formulation in chapter 4 and are listed in Table 4.3, are used to represent zero load solutions of parameter $A$. With the detailed expression for the second term determined in this manner, Eq. (5.4) can be given specifically as:

$$
A\left(\frac{a}{W}, \frac{\sigma}{\sigma_{0}}, n\right)=A_{S S Y}(n)+d_{1}\left(\frac{\sigma}{\sigma_{0}}\right)+d_{2}\left(\frac{\sigma}{\sigma_{0}}\right)^{2}+d_{3}\left(\frac{\sigma}{\sigma_{0}}\right)^{3}
$$

where

$$
\begin{aligned}
& d_{1}=e_{10}+e_{11}\left(\frac{a}{W}\right)+e_{12}\left(\frac{a}{W}\right)^{2}+e_{13}\left(\frac{a}{W}\right)^{3} \\
& d_{2}=e_{20}+e_{21}\left(\frac{a}{W}\right)+e_{22}\left(\frac{a}{W}\right)^{2}+e_{23}\left(\frac{a}{W}\right)^{3} \\
& d_{3}=e_{30}+e_{31}\left(\frac{a}{W}\right)+e_{32}\left(\frac{a}{W}\right)^{2}+e_{33}\left(\frac{a}{W}\right)^{3}
\end{aligned}
$$

The values of the constant $A_{S S Y}(n)$, which in fact is the standard small-scale yielding FEA solution ( $T=0)$ of parameter $A$, are available in Table 4.3. Note that Eq. (5.5) is in a similar form as Eq. (4.2), except that the external load ratio $\sigma / \sigma_{0}$ is used here instead of $\sigma / \sigma_{L}$. In chapter 4 , the polynomial coefficients for uniaxial loading with external 
loading ratio $\sigma / \sigma_{L}$ were also denoted as $e_{i j}$.

Under biaxial loading ratio $\lambda=0.5$, the determined values of the polynomial coefficients $e_{i j}$ for biaxial loading with external loading ratio $\sigma / \sigma_{0}$ are listed in Tables 5.8, 5.9 and 5.10 for SECP, CCP and DECP respectively. FEA solutions of the parameter $A$ for $\lambda=0.5$ (Tables 5.2, 5.4, 5.6) are used to compare with the predicted $A$ solutions by the proposed approximation formula (Eq. (5.5)). Good agreement is found.

For SECP and CCP, all FEA results can be approximated closely. For the SECP, with $9.50 \%$ maximum difference, all deviations are less than $10 \%$ and most are less than $5 \%$. For the $\mathrm{CCP}$, the maximum difference is $12.28 \%$; almost half of the deviations are less than $5 \%$ and most are less than $10 \%$. For the DECP specimen, the agreement between FEA results and the approximation is only fair (with a maximum difference of around $20 \%$ ). The largest deviation occurs under fully plastic conditions. At lower load levels, for DECP, the maximum difference is $12.07 \%$, and almost all difference are less than $10 \%$, while, most of them are less than $5 \%$. Comparisons of the predicted $A$ values by curve shape similarity (Eq. (5.5)) with FEA results for SECP, CCP, DECP under $a / W=0.3$ and 0.7 are shown in Figures 5.7-5.8, 5.9-5.10 and 5.11-5.12, respectively.

At a higher biaxial loading ratio, FEA solutions for the parameter $A$ at $\lambda=1.0$ (Tables $5.3,5.5,5.7)$ are also compared with those obtained from Eq. (5.5). It is found that, with a set of specified $e_{i j}$ values, which is obtained by fitting based on numerical solutions of $A$ for $a / W=0.1,0.3,0.5,0.7$ with $n=10$, Eq. (5.5) cannot accurately predict all corresponding cases (various $n$ and $a / W$ values). For example, for the SECP specimen, the obtained set of $e_{i j}$ values cannot be utilized to accurately estimate the parameter $A$ for $a / W=0.1$ (with a few differences being than $20 \%$ or more), although numerical solutions of $A$ for $a / W=0.1$ with $n=10$ have been utilized in the fitting procedure to obtain those $e_{i j}$ values. With a set of fixed $e_{i j}$ values, the accuracy of the approximation formula (Eq. (5.5)) will be reduced at higher biaxial loading ratio $\lambda$. In other words, for a specific cracked body under high biaxial loading ratio, more than one set of values of polynomial coefficients $e_{i j}$ are needed to cover all cases of relative crack depth $(a / W)$ and hardening exponent ( $n$ ). As several examples, for each specimen (SECP, CCP or DECP), one set of 
values of coefficients $e_{i j}$, which only works well for some specific values of $a / W$ and $n$, is presented in the following paragraphs.

A set of values of coefficients $e_{i j}$ for SECP specimen are determined by fitting based on numerical (FEA) solutions of parameter $A$ under $n=10$ for $a / W=0.3,0.5,0.7$ (excluding 0.1). The obtained values of $e_{i j}$, which are listed in Table 5.11, work well for all $n$ values but do not work for case of $a / W=0.1$. Same work is completed for CCP specimen, and obtained values of $e_{i j}$ are shown in Table 5.12. Same, these $e_{i j}$ values for $\mathrm{CCP}$ will not work well for $a / W=0.1$ case. For DECP specimen, a set of values of coefficients $e_{i j}$ are obtained based on FEA solutions of $A$ under $n=4$ for $a / W=0.1,0.3,0.5$, 0.7 , which are given in Table 5.13. These $e_{i j}$ values will work well for cases of all $a / W$ values and $n=3,4$, but not for $n=5,7,10$.

For SECP and CCP, FEA results of parameter $A$ (for $a / W=0.3,0.5,0.7$ under $n=3,4$, $5,7,10)$ in Table 5.3 and 5.5 respectively are compared with those from estimation of Eq. (5.5) with $e_{i j}$ values given in Table 5.11 and 5.12. For DECP, FEA solutions of $A$ (for $a / W=0.1,0.3,0.5,0.7$ under $n=3,4)$ in Table 5.7 are compared with those from approximation of Eq. (5.5) with $e_{i j}$ values in Table 5.13. Good agreement is found. For SECP and CCP, almost all differences are less than $10 \%$ and most differences are less than $5 \%$. The maximum deviation for the CCP is $12.40 \%$. For the DECP, all differences are less than $10 \%$ and most differences are less than $5 \%$; the maximum difference for the DECP is $9.44 \%$.

Comparisons of $A$ values predicted by curve shape similarity (Eq. (5.5)) with FEA results for SECP, CCP, DECP under $a / W=0.3$ and 0.7 with $\lambda=1.0$ are shown in Figures 5.13-5.14, 5.15-5.16, and 5.17-5.18, respectively.

Through above comparisons, it is determined that, under biaxial load ration $\lambda=0.5$, several maximum deviations of the approximation for the DECP under conditions of large scale yielding are somewhat bigger than $10 \%$ (maximum around $20 \%$ ); for $\lambda=1.0$, more than one set of coefficients $e_{i j}$ values in Eq. (5.6) are needed for all three specimens (SECP, CCP and DECP) to cover the whole estimating range of the parameter $A$ solutions. It means that, for 2D (plane strain) cracked specimens under biaxial loading, 
with the increase of biaxial loading ratio $\lambda$, the range over which the curve shape similarity method is accurate becomes smaller. The reason for this phenomenon can be explained as follows.

The curve shape similarity method estimates the parameter $A$ based on the similarity in the shapes of the $A$ vs. $\sigma / \sigma_{0}\left(\sigma / \sigma_{\mathrm{L}}\right)$ curves for different hardening exponents $n$ values as well as the same general trends (increase or decrease) of those curves as a function of relative crack depth $(a / W)$ (see chapters 3 and 4 for details). Under biaxial loading, as shown in this work (see section 5.1.3), the constraint level (parameter $A$ ) in the crack-tip field changes with increasing biaxial loading ratio $\lambda$. The parameter $A$ for materials with smaller values of the hardening exponent $n$ is easier to be decreased by increased biaxial loading ratio; and $A$ values for shallower cracks is easier to be decreased with the increase of $\lambda$ value than those for deeper cracks. It means, under biaxial loading, with the increase of biaxial loading ratio, $\lambda$, the shapes of $A$ vs. $\sigma / \sigma_{0}\left(\sigma / \sigma_{\mathrm{L}}\right)$ curves for cases with smaller $n$ values and/or shallower cracks will have a bigger change than those for other cases (bigger $n$ values and/or deeper cracks). This fact reduces the similarities (curvature or even trend) of the curves for various $n$ and/or $a / W$ values under biaxial loading, and therefore limit the range over which the curve shape similarity method works well.

\subsection{Solution of Parameter $\boldsymbol{A}$ by $\boldsymbol{T}$-stress}

It has been shown in section 3.2 and section 4.4 that, under small-scale yielding, $A$ solutions can be obtained directly from the available solutions of $T$-stress in the literature through the one-to-one $A-T$ relationship without finite element analysis of specimens. Since it was obtained from the modified boundary layer (MBL) formulation, theoretically the $A-T$ relationship is appropriate for any arbitrary cracked body geometry and loading type (e.g. uniaxial or biaxial load), see section 3.2.2.3. In section 4.4, the applicability of

the determined $A-T$ relationship under uniaxial loading has been demonstrated. In this section, the $A-T$ relationship will be utilized for biaxial loading conditions. 


\subsection{1 $T$-stress solution by weight function method}

To predict the constraint parameter $A$ solution from the $A-T$ relationship (Eqs. (3.6) or (3.8)), $T$-stress values have to be obtained first. In chapter 4 , the weight function method suggested by Wang (2002) for $T$-stress determination (Eqs. (2.25) and (2.26)) was utilized for uniaxial load cases. It is also appropriate for biaxial load cases. The coefficients $D_{1}$ and $D_{2}$ in Eq. (2.26) can be determined by Eq. (4.6) and Eqs. (4.7)-(4.9). In Eq. (2.25), for the present plane strain models under biaxial load, the stress distribution and stress components are determined as: (a) under uniform loading condition: $\sigma(x)=\sigma$, $\sigma$ is the applied far field load, and (b) biaxial loading: $\sigma_{x}=\lambda \sigma, \sigma_{y}=\sigma$. For biaxial loading, Eq. (2.25) can be rewritten as:

$$
T=\int_{0}^{a} \sigma(x) w(x, a) d x+\left.\sigma(\lambda-1)\right|_{x=a}
$$

Meanwhile, the normalized $T$-stress, $V$, can be determined by $V=T / \sigma$.

\subsubsection{Parameter $\boldsymbol{A}$ solution by $\boldsymbol{T}$-stress}

The polynomial approximate of the $A-T$ relationship, Eq. (3.11), can be used to predict the parameter $A$ values from $T$-stress for specimens under biaxial loading with external load ratio $\sigma / \sigma_{0}$ :

$$
A_{T}\left(\frac{\sigma}{\sigma_{0}}, \frac{a}{W}, n\right)=A_{S S Y}(n)+\left(\frac{\sigma}{\sigma_{0}}\right) g_{1}\left(\frac{a}{W}, n\right)+\left(\frac{\sigma}{\sigma_{0}}\right)^{2} g_{2}\left(\frac{a}{W}, n\right)+\left(\frac{\sigma}{\sigma_{0}}\right)^{3} g_{3}\left(\frac{a}{W}, n\right)
$$

where $g_{i}(a / W, n)=[V(a / W)]^{i} m_{i}(n), i=1,2,3 ; V=T / \sigma$ (normalized $T$-stress). In Eq. (3.11), Parameter $A$ solutions under zero load $\left(A_{S S Y}(n)\right)$ and the values of coefficients $m_{i}(n)$ have been obtained in chapter 4 , see Table 4.3 and Table 4.18 respectively. With the determination of $T$-stress, parameter $A$ solutions for SECP, CCP and DECP under low load conditions are estimated from $T$-stress by Eq. (3.11).

The solutions of constraint parameter $A$ estimated directly from $T$-stress (or $V$ ) are compared with those obtained from FEA results for SECP, CCP and DECP specimens (Tables 5.2-5.7). For all three specimens, with biaxial loading ratios $\lambda=0.5,1.0$ and ratios of crack length to specimen width $a / W=0.1,0.3,0.5,0.7$, an extensive 
comparison between the $A$ solutions from $T$-stress and those from FEA results has been made with hardening exponents $n=3,4,5,7$ and 10 . Good agreement is shown.

Based on the criterion that the maximum difference between Eq. (3.11) and FEA results should be less than around $10 \%$, the ranges of applicability (maximum $\sigma / \sigma_{0}$ value) of the $A-T$ relationship (Eq. (3.11)) are determined for the three specimens, and are listed in Table 5.14. With the determined ranges of applicability of Eq. (3.11), for SECP under $\lambda=0.5$, almost all deviations of the approximate relation are less than $10 \%$ and almost half of the deviations are less than $5 \%$. For SECP at $\lambda=1.0$ as well as CCP and DECP at $\lambda=0.5$ and 1.0, almost all deviations are less than $10 \%$ and most are less than $5 \%$. For SECP with $\lambda=0.5$ and 1.0 , the maximum differences are $10.84 \%$ and $10.85 \%$, respectively; for CCP they are $10.89 \%$ and $10.92 \%$; and for DECP, they are $10.99 \%$ and $10.99 \%$ respectively. The maximum difference overall for all three specimens is $10.99 \%$.

As examples, comparisons of the $A$ values predicted by $T$-stress (Eq. (3.11)) with FEA data for the CCP for $a / W=0.1$ and 0.5 are shown in Figures 5.19 and 5.20 for $\lambda=0.5$ and in Figures 5.21 and 5.22 for $\lambda=1.0$.

In section 5.1.3.2 (numerical solutions of $A$ ), it has been found that, for any specified specimen geometry $(a / W)$ of a cracked body, the maximum external loading ratio $\left(\sigma / \sigma_{0}\right)$ at the limit of applicability increases with decreasing hardening coefficient $n$. Through Table 5.14 and Figures 19-22, it is also found that the limit of applicability of Eq. (3.11) increases with decreasing $n$. In other words, for a specified specimen geometry $(a / W)$, the maximum predictable remote loads $\left(\sigma / \sigma_{0}\right)$ under $n=3,4,5$ are 7 are always larger than (or at least equal to) those under $n=10$.

In the present work, $A$ solutions for specimens under biaxial loading are also predicted through the simplified format of the $T$-stress-based estimation formula which comes from the simplified format of the one-to-one $A-T$ relationship (Eq. (3.8) or (3.7)). Substituting Eq. (3.9) into Eq. (3.8), a simplified form of Eq. (3.11) is obtained which can be expressed as:

$$
A_{T}\left(\frac{\sigma}{\sigma_{0}}, \frac{a}{W}, n\right)=A_{S S Y}(n)
$$




$$
+\left(\frac{\sigma}{\sigma_{0}}\right) g_{1}\left(\frac{a}{W}, n=10\right)+\left(\frac{\sigma}{\sigma_{0}}\right)^{2} g_{2}\left(\frac{a}{W}, n=10\right)+\left(\frac{\sigma}{\sigma_{0}}\right)^{3} g_{3}\left(\frac{a}{W}, n=10\right)
$$

where $g_{i}(a / W)=[V(a / W)]^{i} m_{i}(n=10), i=1,2,3$.

With the parameter $A$ solutions predicted by Eq. (5.8), the same extensive comparison as for the prediction of $A$ based on Eq. (3.11) is completed. Good agreement is also obtained. The limits of applicability in terms of load are determined by the criterion that the deviation from FEA results should be less than around $10 \%$. The resulting limits of applicability are listed in Table 5.15.

Within the determined limits of applicability, for the SECP with $\lambda=1.0$ as well as for the CCP and DECP with $\lambda=0.5$ or 1.0 , almost all differences to FEA results are less than $10 \%$ and most are less than $5 \%$. For the SECP specimen with $\lambda=0.5$, almost all differences to FEA are less than $10 \%$ and almost half of them are less than $5 \%$. Maximum differences to FEA are $10.95 \%$ and $10.98 \%$ for the SECP at $\lambda=0.5$ and 1.0 , $10.90 \%$ and $10.83 \%$ for the CCP at $\lambda=0.5$ and 1.0 and $10.99 \%, 10.69 \%$ for the DECP. The maximum difference overall is $10.99 \%$ for all three specimens.

By comparing the Table 5.14 and Table 5.15, it can be found that, for SECP, CCP and DECP specimens, generally, the range of applicability (maximum $\sigma / \sigma_{0}$ value) of the simplified format of $A-T$ relationship (Eq. (5.8) or (3.8)) is smaller than that of the original format (Eq. (3.11) or (3.6)), though the difference is not very significant. Since the simplified format (Eq. (5.8)) is based on $n=10$, a decrease of the range of applicability only occurs for $n=3,4,5$ and 7 . The same was found for cases under uniaxial loading (chapter 4). In addition, in some cases, the range of applicability for $n=3,4,5$ and 7 even decreases to less than that for $n=10$. For example, for the DECP with $a / W=0.3$ and $\lambda=1.0$, the range limit of applicability for $n=3$ is $\sigma / \sigma_{0}=0.650$ while that for $n=10$ is $\sigma / \sigma_{0}=$ 0.900. This phenomenon does not appear under uniaxial loading conditions.

The reason for the reduced range of applicability has been given in section 4.7: curves of $A$ values vs. normalized $T$-stress, $T / \sigma_{0}$ (or normalized external remote stress, $\sigma / \sigma_{0}$ or $\left.\sigma / \sigma_{L}\right)$ for various values of the hardening exponent $n$, see Figures 5.19-5.22 
for example, only have "similar" shapes rather than identical shapes. The effect of biaxial loading should be related to the behavior observed earlier in this chapter (section 5.1.3): under biaxial loading, with increasing of biaxial loading ratio, $\lambda$, the shapes of $A$ vs. $\sigma / \sigma_{0}\left(\sigma / \sigma_{L}\right)$ curves for smaller $n$ values change more than those with bigger $n$ values. The increase of $\lambda$ reduces the similarities of curves for various $n$ values, and therefore the range of applicability of the simplified $A-T$ approximation formula is reduced for smaller $n$ values, since the present methodology carries out the fits to the curve with the largest $n$ $(n=10)$.

Even with the reduced range of applicability, the simplified $A-T$ approximation formula is still conditionally useful to predict parameter $A$ solutions for other various $n$ values only based on the finite element analysis of the MBL problem for one specific value of $n$.

While $A$ solutions estimation equations based on $T$-stress (Eqs. (3.6) and (3.8) or Eqs. (3.10) and (4.10) or Eqs. (3.11) and (5.8)) can be utilized for any of various cracked specimens and loading conditions (such as uniaxial and biaxial), they are only appropriate for SSY conditions at low levels of loading.

\subsection{Solution of Parameter $\boldsymbol{A}$ by Fully Plastic Analysis}

As that for uniaxial load cases in section 4.5, when $A$ approximation formulas for low loading based on $T$-stress are used, an additional term (difference $\Delta A$ / fully plastic solution) has to be considered together with Eq. (3.11) (or Eq. (3.10)) to accurately estimate $A$ values under high loading conditions (see Eq. (3.19)).

\subsubsection{Determination of coefficient $a_{I}$}

A coefficient $a_{1}$ has to be determined first to obtain the difference $\Delta A$ under any (arbitrary) loading condition by Eq. (3.16) (or Eq. (3.15)). For the present work with external loading ratio $\sigma / \sigma_{0}, a_{1}$ is obtained by directly solving Eq. (3.16) with known $\Delta A$ solutions for several specified high loading cases, see section 3.3.2.

The difference $\Delta A$ values for several specified high loading cases can be determined 
by Eq. (3.19), with $A$ values from FEA results for SECP, CCP and DECP obtained in this chapter (Tables 5.2-5.7) and $A_{T}$ solutions obtained from Eq. (3.11). For each specimen (SECP, CCP or DECP), the three highest loading FEA results for each case (specified $a / W$ and $n$ values) in Tables 5.2-5.7 are chosen to calculate $a_{1}$. A final proposed $a_{1}$ value for each case is obtained by averaging the three $a_{1}$ values determined from the three highest loading FEA results. The proposed values of coefficient $a_{1}$ for SECP, CCP and DECP specimens with external loading ratio $\sigma / \sigma_{0}$ are listed in Tables 5.16, 5.17 and 5.18, respectively, based on biaxial loading ratio $\lambda=0.5,1.0$, with $n=3,4,5,7,10$ and $a / W$ $=0.1,0.3,0.5,0.7$.

\subsubsection{Parameter $A$ solution by fully plastic analysis}

Based on the determined coefficient $a_{1}$, the solution of the difference term (fully plastic solution) $\Delta A$ under any arbitrary loading can be estimated accurately and effectively by Eq. (3.16). Substituting Eqs. (3.16) and (3.11) into Eq. (3.19) (or Eq. (3.11) into Eq. (3.21)), the approximate formula for predicting $A$ values of cracked specimens under low to high load is obtained as:

$$
A=A_{S S Y}(n)+\left(\frac{\sigma}{\sigma_{0}}\right) g_{1}\left(\frac{a}{W}, n\right)+\left(\frac{\sigma}{\sigma_{0}}\right)^{2} g_{2}\left(\frac{a}{W}, n\right)+\left(\frac{\sigma}{\sigma_{0}}\right)^{3} g_{3}\left(\frac{a}{W}, n\right)+a_{1}\left(\frac{\sigma}{\sigma_{0}}\right)^{(n+1)(t-s)}
$$

where $a_{1}(a / W, n)$ and $g_{i}(a / W, n)=[V(a / W)]^{i} m_{i}(n), i=1,2,3$.

By Eq. (5.9), $A$ solutions for SECP, CCP and DECP cracked specimens are approximated over the range from small-scale yielding to large-scale yielding. The approximate $A$ solutions are compared with those from FEA results in Tables 5.2-5.7. Comparisons for SECP, CCP and DECP specimens are carried out over a large parameter range $(\lambda=0.5,1.0 ; a / W=0.1,0.3,0.5,0.7$ under $n=3,4,5,7$ and 10 , respectively). Good agreement is found. For SECP under $\lambda=0.5,1.0, \mathrm{CCP}$ under $\lambda=0.5$, and DECP under $\lambda=0.5,1.0$, almost all differences are less than $10 \%$ and most differences are less than $5 \%$. For CCP under $\lambda=1.0$, most differences are less than $10 \%$ while most differences are less than 5\%. Maximum differences are $10.48 \%$ and $10.93 \%$ for the SECP 
under $\lambda=0.5,1.0$, respectively, $10.62 \%$ and $11.03 \%$ for CCP under $\lambda=0.5,1.0$, respectively, and $10.83 \%$ and $11.77 \%$ for DECP under $\lambda=0.5,1.0$, respectively. The maximum difference overall is $11.77 \%$ for all three specimens.

Comparisons of the predicted $A$ values by superposition (Eq. (5.9)) with FEA results for $\mathrm{CCP}$ with $a / W=0.1,0.5$ under $\lambda=0.5,1.0$ are shown in Figures 5.23-5.26 as examples.

\subsection{Conclusions}

In this chapter, numerical (finite element) solutions for the constraint parameter $A$ for three plane strain mode I specimens, SECP, CCP and DECP, under biaxial load have been determined based on the extensive FEA of specimen crack-tip fields.

Through numerical solutions of $A$, the constraint effect for 2D cracked models under biaxial loading is analyzed. The following characterizations are founded:

(1) generally, the maximum external loading ratio $\left(\sigma / \sigma_{0}\right)$, for which a valid solution can be obtained, increases with decreasing hardening coefficient $n$,

(2) in general, the constraint level in crack-tip fields increases with an increase of biaxial loading ratio, $\lambda$;

(3) the constraint level for materials with smaller values of hardening exponent $n$ rises more with increased biaxial loading ratio;

(4) the constraint level for shallow cracks increases more than for deep cracks with an increase of biaxial loading ratio;

(5) compared with SECP and CCP specimens, a higher biaxial loading ratio increases the constraint level of DECP more.

Three estimation methods for parameter $A$ were proposed in chapter 3, namely estimation based on curve shape similarity, on $T$-stress solution and on fully plastic analysis. These are applied for SECP, CCP and DECP specimens under biaxial loading conditions based on the numerical solutions of $A$. The predicted parameter $A$ solutions from the three estimating methods are compared with those from FEA results. The predicted ranges of the estimates for 2D specimens under biaxial loading are discussed.

Through the comparison of parameter $A$ solutions and the discussions about 
predicting ranges of estimate formulas, in general, it can be concluded that all of the three sets of developed approximation formulas are appropriate to 2D cracked specimens under biaxial loading.

Under small-scale yielding condition, as long as solutions of $T$-stress are available in the literature, approximate solutions of constraint parameter $A$ can be obtained through $A$ $T$ relationship more conveniently than through other two approximation methods. With solutions of $T$-stress being available, through determining the solutions of coefficient $a_{l}$, method based on fully plastic solution of $A$ can be used to estimate parameter $A$ for cases that external loadings vary from low to high (small-scale to large-scale yielding deformation). Curve shape similarity method can be used for cases that loading varies from low to high without the need for $T$-stress solutions. However, under high biaxial loading ratio $\lambda$, curve shape similarity method needs more than one set of coefficients for approximation formulas, $e_{i j}$, which are obtained based on FEA results, to cover the whole estimation range. It means that, for cases with high biaxial loading ratio $\lambda$, more finite element analyses are needed to enable curve shape similarity method to be appropriate for application on 2D cracked specimens. 


\begin{tabular}{ccccccccc}
\hline $\log \left(J /\left(a \sigma_{0}\right)\right)$ & -2.900 & -2.491 & -2.256 & -2.074 & -1.855 & -1.593 & -1.299 & -0.991 \\
\hline$Q_{\text {SSY }}[$ Ref.] & -0.2107 & -0.3553 & -0.4822 & -0.5711 & -0.6497 & -0.7690 & -0.8223 & -0.9315 \\
$Q_{\text {SSY }}$ & -0.2196 & -0.3714 & -0.4849 & -0.5763 & -0.6758 & -0.7790 & -0.8739 & -0.9815 \\
\hline Diff. (\%) & 4.232 & 4.526 & 0.558 & 0.923 & 4.003 & 1.299 & 6.274 & 5.376 \\
\hline
\end{tabular}

Table 5.1 Comparison of constraint parameter $Q$ for CCP under $a / W=0.5, \mathrm{n}=10, \lambda=0.5$, compared with results from O'Dowd et al. (1999) 


\begin{tabular}{|c|c|c|c|c|c|c|c|c|c|c|c|}
\hline$a / W$ & 0.1 & & & & & 0.3 & & & & & \\
\hline$\sigma / \sigma_{0}$ & $n=3$ & $n=4$ & $n=5$ & $n=7$ & $\mathrm{n}=10$ & $\sigma / \sigma_{0}$ & $\mathrm{n}=3$ & $n=4$ & $\mathrm{n}=5$ & $\mathrm{n}=7$ & $\mathrm{n}=10$ \\
\hline 0.250 & 0.91524 & 0.55153 & 0.38552 & 0.25194 & 0.18600 & 0.150 & 0.91848 & 0.55422 & 0.38785 & 0.25432 & 0.18828 \\
\hline 0.500 & 0.92583 & 0.55861 & 0.39022 & 0.25514 & 0.18820 & 0.250 & 0.92787 & 0.56120 & 0.39339 & 0.25847 & 0.19307 \\
\hline 0.750 & 0.93635 & 0.56870 & 0.39808 & 0.25894 & 0.18998 & 0.350 & 0.93984 & 0.57046 & 0.40079 & 0.26298 & 0.19567 \\
\hline 0.900 & 0.93816 & 0.57312 & 0.40230 & 0.26189 & 0.19169 & 0.450 & 0.95082 & 0.58010 & 0.40830 & 0.26851 & 0.19989 \\
\hline 1.000 & 0.93815 & 0.57465 & 0.40464 & 0.26473 & 0.19350 & 0.550 & 0.96266 & 0.59058 & 0.41728 & 0.27570 & 0.20481 \\
\hline 1.100 & 0.93624 & 0.57543 & 0.40646 & 0.26701 & 0.19586 & 0.650 & 0.97216 & 0.60094 & 0.42667 & 0.28420 & 0.21255 \\
\hline 1.200 & 0.93422 & 0.57461 & 0.40738 & 0.26909 & 0.19841 & 0.750 & 0.98224 & 0.61135 & 0.43656 & 0.29347 & 0.22260 \\
\hline 1.300 & 0.92749 & 0.57367 & 0.40708 & 0.27057 & 0.20102 & 0.760 & 0.98243 & 0.61224 & 0.43751 & 0.29452 & 0.22376 \\
\hline 1.400 & 0.92144 & 0.57114 & 0.40649 & 0.27139 & 0.20346 & 0.780 & 0.98498 & 0.61374 & 0.43951 & 0.29626 & 0.22521 \\
\hline 1.450 & 0.91770 & 0.56776 & 0.40569 & 0.27165 & 0.20471 & 0.800 & 0.98738 & 0.61595 & 0.44135 & 0.29836 & 0.22766 \\
\hline 1.500 & 0.91686 & 0.56569 & 0.40371 & 0.27184 & 0.20520 & 0.810 & 0.98750 & 0.61664 & 0.44223 & 0.29939 & 0.22884 \\
\hline 1.600 & 0.90796 & 0.56079 & 0.40124 & 0.27086 & 0.20712 & 0.850 & 0.98972 & 0.62004 & 0.44594 & 0.30331 & 0.23291 \\
\hline 1.700 & 0.89741 & 0.55511 & 0.39682 & 0.26981 & 0.20857 & 0.900 & 0.99387 & 0.62480 & 0.45027 & 0.30814 & 0.23877 \\
\hline 1.800 & 0.89022 & 0.54923 & 0.39370 & 0.26909 & 0.21073 & 0.950 & 0.99752 & 0.62919 & 0.45497 & 0.31297 & 0.24481 \\
\hline 1.900 & 0.88251 & 0.54524 & 0.39123 & 0.26969 & & 1.000 & 1.00097 & 0.63260 & 0.45915 & 0.31797 & 0.25032 \\
\hline 2.000 & 0.87144 & 0.54097 & 0.39000 & 0.27253 & & 1.050 & 1.00410 & 0.63700 & 0.46394 & 0.32345 & 0.25702 \\
\hline 2.100 & 0.86730 & 0.53830 & 0.39109 & & & 1.100 & 1.00960 & 0.64135 & 0.46831 & 0.32895 & 0.26489 \\
\hline 2.200 & 0.85850 & 0.53831 & 0.39465 & & & 1.150 & 1.01256 & 0.64566 & 0.47320 & 0.33449 & 0.27227 \\
\hline 2.300 & 0.85574 & 0.53960 & 0.40008 & & & 1.200 & 1.01744 & 0.64968 & 0.47739 & 0.34043 & \\
\hline 2.400 & 0.85089 & 0.54220 & 0.40819 & & & 1.250 & 1.02045 & 0.65409 & 0.48381 & & \\
\hline \multirow[t]{2}{*}{2.500} & 0.84605 & 0.54595 & 0.40777 & & & 1.300 & 1.02606 & 0.66051 & & & \\
\hline & & & & & & 1.400 & 1.03790 & & & & \\
\hline$a / W$ & 0.5 & & & & & 0.7 & & & & & \\
\hline$\sigma / \sigma_{0}$ & $n=3$ & $\mathrm{n}=4$ & $n=5$ & $n=7$ & $n=10$ & $\sigma / \sigma_{0}$ & $n=3$ & $n=4$ & $n=5$ & $n=7$ & $\mathrm{n}=10$ \\
\hline 0.050 & 0.90776 & 0.54631 & 0.38146 & 0.24915 & 0.18447 & 0.010 & 0.89720 & 0.53809 & 0.37467 & 0.24350 & 0.17829 \\
\hline 0.100 & 0.91152 & 0.54799 & 0.38246 & 0.25035 & 0.18470 & 0.020 & 0.89423 & 0.53474 & 0.37177 & 0.23876 & 0.17434 \\
\hline 0.150 & 0.91955 & 0.55268 & 0.38576 & 0.25173 & 0.18561 & 0.030 & 0.89173 & 0.53033 & 0.36724 & 0.23706 & 0.17199 \\
\hline 0.200 & 0.93053 & 0.56034 & 0.39127 & 0.25422 & 0.18691 & 0.040 & 0.89061 & 0.52866 & 0.36531 & 0.23293 & 0.16760 \\
\hline 0.250 & 0.94523 & 0.57130 & 0.39853 & 0.25971 & 0.18961 & 0.050 & 0.89235 & 0.52783 & 0.36371 & 0.23065 & 0.16503 \\
\hline 0.290 & 0.95976 & 0.58280 & 0.40750 & 0.26493 & 0.19437 & 0.060 & 0.89697 & 0.52928 & 0.36403 & 0.22991 & 0.16382 \\
\hline 0.320 & 0.97212 & 0.59245 & 0.41527 & 0.27163 & 0.20003 & 0.070 & 0.90474 & 0.53483 & 0.36613 & 0.23019 & 0.16289 \\
\hline 0.350 & 0.98591 & 0.60372 & 0.42555 & 0.28017 & 0.20751 & 0.080 & 0.91638 & 0.54304 & 0.37198 & 0.23524 & 0.16446 \\
\hline 0.380 & 1.00133 & 0.61660 & 0.43649 & 0.29048 & 0.21678 & 0.085 & 0.92330 & 0.54792 & 0.37719 & 0.23715 & 0.16544 \\
\hline 0.400 & 1.01265 & 0.62591 & 0.44562 & 0.29848 & 0.22604 & 0.090 & 0.93138 & 0.55575 & 0.38172 & 0.24035 & 0.16915 \\
\hline 0.420 & 1.02430 & 0.63620 & 0.45449 & 0.30771 & 0.23951 & 0.095 & 0.94057 & 0.56281 & 0.38760 & 0.24638 & 0.17440 \\
\hline 0.440 & 1.03745 & 0.64737 & 0.46606 & 0.32072 & & 0.100 & 0.95116 & 0.57116 & 0.39635 & 0.25400 & 0.18140 \\
\hline 0.450 & 1.04403 & 0.65423 & 0.47252 & 0.32716 & & 0.105 & 0.96122 & 0.58068 & 0.40441 & 0.26322 & 0.19312 \\
\hline 0.460 & 1.05195 & 0.66160 & 0.47764 & 0.33425 & & 0.110 & 0.97422 & 0.59175 & 0.41412 & 0.27427 & 0.20781 \\
\hline 0.470 & 1.05901 & 0.66752 & 0.48483 & 0.34231 & & 0.115 & 0.98931 & 0.60421 & 0.42753 & 0.28745 & \\
\hline 0.480 & 1.06637 & 0.67394 & 0.49279 & & & 0.120 & 1.00308 & 0.61856 & 0.44058 & & \\
\hline 0.490 & 1.07404 & 0.68244 & 0.50135 & & & & & & & & \\
\hline 0.500 & 1.08353 & 0.69169 & 0.50789 & & & & & & & & \\
\hline 0.510 & 1.09180 & 0.69890 & 0.51741 & & & & & & & & \\
\hline
\end{tabular}

Table 5.2 FEA results for SECP specimen under $\lambda=0.5$ 


\begin{tabular}{|c|c|c|c|c|c|c|c|c|c|c|c|}
\hline$a / W$ & 0.1 & & & & & 0.3 & & & & & \\
\hline$\overline{\sigma / \sigma_{0}}$ & $n=3$ & $n=4$ & $n=5$ & $n=7$ & $\mathrm{n}=10$ & $\sigma / \sigma_{0}$ & $n=3$ & $n=4$ & $n=5$ & $n=7$ & $n=10$ \\
\hline 0.100 & 0.87886 & 0.52283 & 0.36139 & 0.23252 & 0.16637 & 0.250 & 0.86428 & 0.50841 & 0.34846 & 0.22094 & 0.15636 \\
\hline 0.150 & 0.87193 & 0.51733 & 0.35721 & 0.22864 & 0.16388 & 0.500 & 0.82627 & 0.47641 & 0.32046 & 0.19371 & 0.12998 \\
\hline 0.200 & 0.86218 & 0.50929 & 0.35109 & 0.22374 & 0.16031 & 0.600 & 0.80984 & 0.46398 & 0.30834 & 0.18463 & 0.12093 \\
\hline 0.250 & 0.85161 & 0.49880 & 0.34068 & 0.21418 & 0.15242 & 0.750 & 0.78229 & 0.44228 & 0.29016 & 0.16935 & 0.10651 \\
\hline 0.350 & 0.82909 & 0.47963 & 0.32432 & 0.20187 & 0.13979 & 0.850 & 0.76195 & 0.42490 & 0.27680 & 0.15829 & 0.09611 \\
\hline 0.500 & 0.79407 & 0.45111 & 0.30062 & 0.18177 & 0.12125 & 0.900 & 0.75108 & 0.41559 & 0.26863 & 0.15116 & 0.08927 \\
\hline 0.600 & 0.77063 & 0.43254 & 0.28539 & 0.16720 & 0.10688 & 0.950 & 0.73967 & 0.40585 & 0.25995 & 0.14343 & 0.08173 \\
\hline 0.750 & 0.73304 & 0.40275 & 0.25903 & 0.14895 & 0.09111 & 1.000 & 0.72792 & 0.39549 & 0.25051 & 0.13646 & 0.07483 \\
\hline 0.850 & 0.70541 & 0.37904 & 0.24150 & 0.13336 & 0.08086 & 1.050 & 0.71585 & 0.38461 & 0.24033 & 0.12657 & 0.06438 \\
\hline 0.900 & 0.69224 & 0.36832 & 0.23012 & 0.12645 & 0.07315 & 1.100 & 0.70320 & 0.37306 & 0.22917 & 0.11525 & 0.05330 \\
\hline 0.950 & 0.67725 & 0.35462 & 0.22064 & 0.11689 & 0.06747 & 1.150 & 0.69053 & 0.36099 & 0.21703 & 0.10185 & \\
\hline 1.000 & 0.66360 & 0.34334 & 0.20847 & 0.10631 & 0.05870 & 1.200 & 0.67721 & 0.34673 & 0.20361 & & \\
\hline 1.050 & 0.64850 & 0.32933 & 0.19825 & 0.09724 & & 1.300 & 0.65063 & 0.31715 & 0.17182 & & \\
\hline 1.100 & 0.63293 & 0.31458 & 0.18450 & 0.08444 & & 1.400 & 0.62324 & 0.28424 & & & \\
\hline 1.150 & 0.61916 & 0.30239 & 0.16985 & 0.06920 & & 1.500 & 0.59711 & & & & \\
\hline 1.200 & 0.60332 & 0.28683 & 0.15454 & & & & & & & & \\
\hline 1.250 & 0.58730 & 0.27077 & 0.13766 & & & & & & & & \\
\hline 1.300 & 0.57114 & 0.25416 & & & & & & & & & \\
\hline 1.400 & 0.53570 & 0.21928 & & & & & & & & & \\
\hline 1.500 & 0.50197 & 0.18206 & & & & & & & & & \\
\hline 1.600 & 0.46465 & & & & & & & & & & \\
\hline$a / W$ & 0.5 & & & & & 0.7 & & & & & \\
\hline$\sigma / \sigma_{0}$ & $n=3$ & $\mathrm{n}=4$ & $n=5$ & $\mathrm{n}=7$ & $n=10$ & $\sigma / \sigma_{0}$ & $\mathrm{n}=3$ & $n=4$ & $\mathrm{n}=5$ & $n=7$ & $\mathrm{n}=10$ \\
\hline 0.050 & 0.89539 & 0.53593 & 0.37253 & 0.24163 & 0.17648 & 0.010 & 0.89473 & 0.53599 & 0.37287 & 0.24197 & 0.17685 \\
\hline 0.100 & 0.88733 & 0.52721 & 0.36488 & 0.23443 & 0.17060 & 0.020 & 0.88944 & 0.53067 & 0.36825 & 0.23572 & 0.17150 \\
\hline 0.150 & 0.88333 & 0.52205 & 0.35942 & 0.22926 & 0.16424 & 0.030 & 0.88477 & 0.52430 & 0.36202 & 0.23258 & 0.16777 \\
\hline 0.200 & 0.88231 & 0.51950 & 0.35476 & 0.22375 & 0.15827 & 0.040 & 0.88147 & 0.52076 & 0.35844 & 0.22695 & 0.16194 \\
\hline 0.250 & 0.88454 & 0.52020 & 0.35377 & 0.21954 & 0.15259 & 0.050 & 0.88117 & 0.51803 & 0.35517 & 0.22317 & 0.15793 \\
\hline 0.300 & 0.89055 & 0.52282 & 0.35431 & 0.21939 & 0.14867 & 0.060 & 0.88350 & 0.51901 & 0.35378 & 0.22090 & 0.15523 \\
\hline 0.350 & 0.89971 & 0.52851 & 0.35893 & 0.22101 & 0.14978 & 0.070 & 0.88898 & 0.52136 & 0.35405 & 0.21952 & 0.15264 \\
\hline 0.380 & 0.90671 & 0.53415 & 0.36306 & 0.22482 & 0.15137 & 0.080 & 0.89831 & 0.52735 & 0.35806 & 0.22293 & 0.15239 \\
\hline 0.400 & 0.91272 & 0.54037 & 0.36806 & 0.22831 & 0.15571 & 0.090 & 0.91089 & 0.53800 & 0.36607 & 0.22799 & 0.15500 \\
\hline 0.420 & 0.91989 & 0.54386 & 0.37220 & 0.23301 & 0.16182 & 0.095 & 0.91888 & 0.54394 & 0.37266 & 0.23134 & 0.15918 \\
\hline 0.440 & 0.92651 & 0.55243 & 0.37959 & 0.23953 & & 0.100 & 0.92830 & 0.55118 & 0.37852 & 0.23782 & 0.16529 \\
\hline 0.450 & 0.93211 & 0.55492 & 0.38148 & 0.24372 & & 0.105 & 0.93908 & 0.55960 & 0.38576 & 0.24612 & 0.17609 \\
\hline 0.460 & 0.93605 & 0.56045 & 0.38644 & 0.24899 & & 0.110 & 0.94889 & 0.56956 & 0.39663 & 0.25629 & 0.19288 \\
\hline 0.470 & 0.94035 & 0.56369 & 0.39208 & 0.25838 & & 0.115 & 0.96294 & 0.58105 & 0.40683 & 0.27180 & \\
\hline 0.480 & 0.94748 & 0.57034 & 0.39848 & 0.26546 & & 0.120 & 0.97548 & 0.59448 & 0.42219 & & \\
\hline 0.490 & 0.95240 & 0.57420 & 0.40189 & & & 0.125 & 0.99333 & 0.60988 & 0.43656 & & \\
\hline \multirow[t]{2}{*}{0.500} & 0.95775 & 0.57854 & 0.40964 & & & 0.130 & 1.00926 & 0.62795 & & & \\
\hline & & & & & & 0.135 & 1.02745 & 0.64357 & & & \\
\hline
\end{tabular}

Table 5.3 FEA results for SECP specimen under $\lambda=1.0$ 


\begin{tabular}{|c|c|c|c|c|c|c|c|c|c|c|c|}
\hline$a / W$ & 0.1 & & & & & 0.3 & & & & & \\
\hline$\sigma / \sigma_{0}$ & $\mathrm{n}=3$ & $n=4$ & $n=5$ & $\mathrm{n}=7$ & $n=10$ & $\sigma / \sigma_{0}$ & $n=3$ & $n=4$ & $n=5$ & $\mathrm{n}=7$ & $\mathrm{n}=10$ \\
\hline 0.500 & 1.03293 & 0.64948 & 0.46887 & 0.32352 & 0.25390 & 0.250 & 0.98134 & 0.60644 & 0.43267 & 0.29277 & 0.22469 \\
\hline 0.700 & 1.07906 & 0.68806 & 0.50129 & 0.34936 & 0.27751 & 0.500 & 1.05796 & 0.66917 & 0.48450 & 0.33539 & 0.26464 \\
\hline 0.900 & 1.11904 & 0.71974 & 0.52744 & 0.36991 & 0.29557 & 0.750 & 1.12909 & 0.73037 & 0.53563 & 0.37581 & 0.30014 \\
\hline 1.000 & 1.13527 & 0.73370 & 0.53894 & 0.37836 & 0.30237 & 0.900 & 1.16595 & 0.76170 & 0.56335 & 0.39798 & 0.31924 \\
\hline 1.200 & 1.15529 & 0.75361 & 0.55608 & 0.39210 & 0.31170 & 1.000 & 1.18836 & 0.77990 & 0.58012 & 0.41203 & 0.33108 \\
\hline 1.500 & 1.16895 & 0.76686 & 0.56621 & 0.39975 & 0.31748 & 1.100 & 1.20084 & 0.79773 & 0.59419 & 0.42491 & 0.34168 \\
\hline 1.800 & 1.16139 & 0.75712 & 0.56246 & 0.39239 & 0.31165 & 1.200 & 1.21286 & 0.80829 & 0.60522 & 0.43449 & 0.35199 \\
\hline 2.000 & 1.13246 & 0.74272 & 0.54581 & 0.38135 & 0.30277 & 1.300 & 1.22667 & 0.81872 & 0.61313 & 0.44234 & 0.36159 \\
\hline 2.100 & 1.11699 & 0.73432 & 0.53681 & 0.37501 & 0.29808 & 1.400 & 1.22663 & 0.82549 & 0.62225 & 0.44924 & 0.37116 \\
\hline 2.200 & 1.09825 & 0.71748 & 0.52619 & 0.36777 & 0.29321 & 1.450 & 1.22873 & 0.82394 & 0.62381 & 0.45351 & 0.37509 \\
\hline 2.300 & 1.08745 & 0.70466 & 0.51373 & 0.35901 & 0.28309 & 1.500 & 1.22943 & 0.82773 & 0.62419 & 0.45429 & 0.37893 \\
\hline 2.400 & 1.06267 & 0.68221 & 0.49896 & 0.34764 & 0.27633 & 1.550 & 1.22865 & 0.83059 & 0.62359 & 0.45407 & 0.37497 \\
\hline 2.450 & 1.04035 & 0.67860 & 0.49075 & 0.34698 & & 1.600 & 1.22625 & 0.82498 & 0.62134 & 0.45371 & 0.38314 \\
\hline 2.500 & 1.03254 & 0.66464 & 0.48187 & 0.33447 & & 1.650 & 1.22214 & 0.82517 & 0.62323 & 0.45807 & 0.39281 \\
\hline 2.600 & 1.01409 & 0.64474 & 0.46421 & & & 1.700 & 1.21616 & 0.82321 & 0.62016 & 0.44720 & 0.39826 \\
\hline \multirow[t]{2}{*}{2.700} & 0.97462 & 0.62172 & & & & 1.750 & 1.20833 & 0.81313 & 0.61771 & 0.46113 & 0.40449 \\
\hline & & & & & & 1.800 & 1.21234 & 0.81012 & 0.59436 & 0.45637 & 0.41530 \\
\hline$a / W$ & 0.5 & & & & & 0.7 & & & & & \\
\hline$\sigma / \sigma_{0}$ & $n=3$ & $n=4$ & $n=5$ & $n=7$ & $n=10$ & $\sigma / \sigma_{0}$ & $n=3$ & $n=4$ & $\mathrm{n}=5$ & $n=7$ & $\mathrm{n}=10$ \\
\hline 0.100 & 0.94657 & 0.57838 & 0.40893 & 0.27252 & 0.20548 & 0.050 & 0.94003 & 0.57410 & 0.40565 & 0.27004 & 0.20336 \\
\hline 0.150 & 0.96767 & 0.59526 & 0.42335 & 0.28498 & 0.21745 & 0.100 & 0.97886 & 0.60394 & 0.43006 & 0.28991 & 0.22255 \\
\hline 0.250 & 1.01066 & 0.63010 & 0.45229 & 0.30762 & 0.23933 & 0.150 & 1.01980 & 0.63643 & 0.45724 & 0.31137 & 0.24264 \\
\hline 0.350 & 1.05770 & 0.66699 & 0.48260 & 0.33321 & 0.26171 & 0.250 & 1.11106 & 0.71061 & 0.51835 & 0.36082 & 0.28602 \\
\hline 0.450 & 1.10582 & 0.70680 & 0.51559 & 0.35931 & 0.28520 & 0.350 & 1.19965 & 0.79269 & 0.59128 & 0.42146 & 0.34039 \\
\hline 0.550 & 1.15103 & 0.74722 & 0.55041 & 0.38757 & 0.31005 & 0.400 & 1.24116 & 0.83100 & 0.62637 & 0.45712 & 0.37568 \\
\hline 0.650 & 1.19119 & 0.78594 & 0.58557 & 0.41778 & 0.33689 & 0.450 & 1.28136 & 0.86873 & 0.66098 & 0.49110 & 0.41489 \\
\hline 0.750 & 1.23122 & 0.82265 & 0.61827 & 0.44734 & 0.36587 & 0.460 & 1.28455 & 0.87745 & 0.66976 & 0.49773 & 0.42362 \\
\hline 0.850 & 1.25716 & 0.85287 & 0.64612 & 0.47604 & 0.39809 & 0.470 & 1.29303 & 0.88141 & 0.67470 & 0.50429 & 0.43117 \\
\hline 0.900 & 1.27272 & 0.86516 & 0.65953 & 0.48757 & 0.41343 & 0.480 & 1.30103 & 0.89001 & 0.68317 & 0.51077 & 0.43860 \\
\hline 0.950 & 1.28575 & 0.87540 & 0.66842 & 0.49850 & 0.42643 & 0.490 & 1.30828 & 0.89773 & 0.68769 & 0.51719 & 0.44600 \\
\hline 1.000 & 1.29677 & 0.88340 & 0.67961 & 0.50783 & 0.44074 & 0.500 & 1.31593 & 0.90135 & 0.69194 & 0.52353 & 0.45333 \\
\hline 1.050 & 1.30484 & 0.89560 & 0.68895 & 0.51551 & 0.45175 & 0.510 & 1.31719 & 0.90949 & 0.70028 & 0.52982 & 0.45925 \\
\hline 1.100 & 1.31050 & 0.89834 & 0.69041 & 0.51172 & 0.46758 & 0.550 & 1.34438 & 0.93362 & 0.72286 & 0.55120 & 0.48569 \\
\hline 1.150 & 1.31258 & 0.90536 & 0.69556 & 0.53219 & 0.48279 & 0.580 & 1.36147 & 0.94855 & 0.73659 & 0.56478 & 0.50540 \\
\hline \multirow[t]{4}{*}{1.200} & 1.32147 & 0.90225 & 0.70169 & 0.54275 & & 0.600 & 1.37217 & 0.95601 & 0.74623 & 0.57506 & 0.50833 \\
\hline & & & & & & 0.620 & 1.37384 & 0.96127 & 0.75475 & 0.58194 & \\
\hline & & & & & & 0.640 & 1.38217 & 0.97261 & 0.75650 & 0.57980 & \\
\hline & & & & & & 0.660 & 1.39806 & 0.98232 & 0.76824 & 0.59876 & \\
\hline
\end{tabular}

Table 5.4 FEA results for CCP specimen under $\lambda=0.5$ 


\begin{tabular}{|c|c|c|c|c|c|c|c|c|c|c|c|}
\hline$a / W$ & 0.1 & & & & & 0.3 & & & & & \\
\hline$\sigma / \sigma_{0}$ & $\mathrm{n}=3$ & $n=4$ & $\mathrm{n}=5$ & $\mathrm{n}=7$ & $n=10$ & $\sigma / \sigma_{0}$ & $\mathrm{n}=3$ & $n=4$ & $n=5$ & $\mathrm{n}=7$ & $\mathrm{n}=10$ \\
\hline 0.500 & 0.89279 & 0.53665 & 0.37503 & 0.24536 & 0.18130 & 0.500 & 0.91158 & 0.55164 & 0.38764 & 0.25670 & 0.19110 \\
\hline 0.750 & 0.87398 & 0.52211 & 0.36309 & 0.23732 & 0.17650 & 0.750 & 0.90119 & 0.54687 & 0.38453 & 0.25403 & 0.19075 \\
\hline 0.900 & 0.85974 & 0.51059 & 0.35378 & 0.23024 & 0.17073 & 0.900 & 0.89260 & 0.53934 & 0.37876 & 0.25124 & 0.18810 \\
\hline 1.000 & 0.84974 & 0.50165 & 0.34734 & 0.22645 & 0.16867 & 1.000 & 0.88388 & 0.53271 & 0.37344 & 0.24757 & 0.18544 \\
\hline 1.200 & 0.82588 & 0.48414 & 0.33109 & 0.21159 & 0.15611 & 1.200 & 0.86152 & 0.51500 & 0.35847 & 0.23465 & 0.17641 \\
\hline 1.400 & 0.79886 & 0.46125 & 0.31191 & 0.19430 & 0.13836 & 1.300 & 0.84798 & 0.50381 & 0.34851 & 0.22595 & 0.16850 \\
\hline 1.500 & 0.78414 & 0.44974 & 0.30024 & 0.18333 & 0.12928 & 1.400 & 0.83435 & 0.49110 & 0.33705 & 0.21513 & 0.15796 \\
\hline 1.700 & 0.75316 & 0.42327 & 0.27812 & 0.16285 & 0.10628 & 1.500 & 0.81667 & 0.47705 & 0.32418 & 0.20261 & 0.14473 \\
\hline 1.900 & 0.71719 & 0.39497 & 0.25383 & 0.14332 & 0.08701 & 1.600 & 0.79928 & 0.46145 & 0.30956 & 0.18870 & 0.12943 \\
\hline 2.100 & 0.67310 & 0.36392 & 0.22880 & 0.12295 & 0.07116 & 1.700 & 0.77977 & 0.44471 & 0.29429 & 0.17382 & \\
\hline 2.250 & 0.63545 & 0.33786 & 0.20833 & 0.10738 & 0.06048 & 1.800 & 0.75775 & 0.42657 & 0.27752 & 0.15722 & \\
\hline 2.300 & 0.62310 & 0.32864 & 0.20185 & 0.10326 & 0.05819 & 1.900 & 0.73375 & 0.40695 & 0.25987 & & \\
\hline 2.400 & 0.59669 & 0.30860 & 0.18622 & 0.09313 & & 2.000 & 0.70638 & 0.38399 & 0.24039 & & \\
\hline \multirow[t]{2}{*}{2.500} & 0.56789 & 0.28749 & 0.17001 & 0.08243 & & 2.100 & 0.67493 & 0.36126 & & & \\
\hline & & & & & & 2.200 & 0.64552 & & & & \\
\hline$a / W$ & 0.5 & & & & & 0.7 & & & & & \\
\hline$\sigma / \sigma_{0}$ & $n=3$ & $\mathrm{n}=4$ & $\mathrm{n}=5$ & $\mathrm{n}=7$ & $\mathrm{n}=10$ & $\sigma / \sigma_{0}$ & $n=3$ & $n=4$ & $n=5$ & $\mathrm{n}=7$ & $\mathrm{n}=10$ \\
\hline 0.150 & 0.92731 & 0.56293 & 0.39591 & 0.26173 & 0.19560 & 0.020 & 0.91750 & 0.55434 & 0.38870 & 0.25562 & 0.19002 \\
\hline 0.250 & 0.94008 & 0.57296 & 0.40354 & 0.26788 & 0.20128 & 0.050 & 0.92701 & 0.56345 & 0.39655 & 0.26195 & 0.19615 \\
\hline 0.350 & 0.95237 & 0.58435 & 0.41347 & 0.27599 & 0.20909 & 0.080 & 0.94209 & 0.57458 & 0.40569 & 0.26987 & 0.20313 \\
\hline 0.450 & 0.96587 & 0.59477 & 0.42278 & 0.28397 & 0.21564 & 0.100 & 0.95152 & 0.58162 & 0.41110 & 0.27409 & 0.20682 \\
\hline 0.550 & 0.97609 & 0.60460 & 0.43141 & 0.29068 & 0.22245 & 0.120 & $0.961^{\prime} 33$ & 0.58941 & 0.41727 & 0.27917 & 0.21165 \\
\hline 0.650 & 0.98365 & 0.61287 & 0.43900 & 0.29739 & 0.22770 & 0.150 & 0.97688 & 0.60129 & 0.42727 & 0.28709 & 0.21988 \\
\hline 0.750 & 0.98812 & 0.61906 & 0.44531 & 0.30295 & 0.23276 & 0.180 & 0.99285 & 0.61442 & 0.43815 & 0.29533 & 0.22757 \\
\hline 0.800 & 0.98985 & 0.62150 & 0.44737 & 0.30518 & 0.23472 & 0.200 & 1.00380 & 0.62312 & 0.44522 & 0.30168 & 0.23244 \\
\hline 0.850 & 0.99025 & 0.62319 & 0.44922 & 0.30709 & 0.23698 & 0.220 & 1.01481 & 0.63209 & 0.45263 & 0.30755 & 0.23784 \\
\hline 0.900 & 0.98949 & 0.62396 & 0.45041 & 0.30859 & 0.23838 & 0.250 & 1.03259 & 0.64629 & 0.46423 & 0.31652 & 0.24565 \\
\hline 0.920 & 0.98942 & 0.62418 & 0.45089 & 0.30906 & 0.23877 & 0.270 & 1.04286 & 0.65555 & 0.47222 & 0.32281 & 0.25105 \\
\hline 0.930 & 0.98763 & 0.62363 & 0.45059 & 0.30919 & 0.23914 & 0.280 & 1.04963 & 0.66082 & 0.47647 & 0.32620 & 0.25416 \\
\hline 0.940 & 0.98931 & 0.62443 & 0.45123 & 0.30928 & 0.23916 & 0.290 & 1.05454 & 0.66546 & 0.48050 & 0.32960 & 0.25676 \\
\hline 0.950 & 0.98723 & 0.62383 & 0.45089 & 0.30952 & 0.23905 & 0.300 & 1.06127 & 0.67082 & 0.48454 & 0.33284 & 0.25996 \\
\hline 1.100 & 0.98060 & 0.61937 & 0.44883 & 0.30853 & 0.24025 & 0.350 & 1.08686 & 0.69490 & 0.50622 & 0.35042 & 0.27532 \\
\hline 1.200 & 0.96946 & 0.61318 & 0.44308 & 0.30531 & 0.23882 & 0.400 & 1.11401 & 0.72094 & 0.52818 & 0.36947 & 0.29217 \\
\hline 1.300 & 0.95729 & 0.60259 & 0.43585 & 0.29999 & 0.23579 & 0.450 & 1.13808 & 0.74526 & 0.55133 & 0.38980 & 0.31163 \\
\hline 1.400 & 0.93431 & 0.58766 & 0.42178 & 0.28988 & 0.23386 & 0.500 & 1.15667 & 0.76722 & 0.57125 & 0.41117 & 0.33520 \\
\hline 1.450 & 0.92294 & 0.57975 & 0.41574 & 0.28653 & 0.22748 & 0.540 & 1.17207 & 0.78207 & 0.58696 & 0.42791 & 0.35614 \\
\hline 1.500 & 0.90961 & 0.56595 & 0.40578 & 0.28015 & 0.22326 & 0.570 & 1.18153 & 0.79379 & 0.59996 & 0.44119 & 0.37169 \\
\hline 1.550 & 0.89519 & 0.55579 & 0.39827 & 0.27265 & & 0.600 & 1.18951 & 0.80087 & 0.60980 & 0.45302 & 0.38818 \\
\hline 1.600 & 0.88749 & 0.54476 & 0.39138 & 0.25768 & & 0.620 & 1.19964 & 0.80759 & 0.61426 & 0.45920 & 0.40008 \\
\hline \multirow[t]{3}{*}{1.650} & 0.87294 & & & & & 0.650 & 1.20579 & 0.81690 & 0.62560 & 0.47051 & 0.41449 \\
\hline & & & & & & 0.700 & 1.21008 & 0.82964 & 0.63574 & 0.48499 & \\
\hline & & & & & & 0.750 & 1.21750 & 0.83818 & 0.64569 & 0.49148 & \\
\hline
\end{tabular}

Table 5.5 FEA results for CCP specimen under $\lambda=1.0$ 


\begin{tabular}{|c|c|c|c|c|c|c|c|c|c|c|c|}
\hline$a / W$ & 0.1 & & & & & 0.3 & & & & & \\
\hline$\sigma / \sigma_{0}$ & $n=3$ & $\mathrm{n}=4$ & $n=5$ & $n=7$ & $n=10$ & $\sigma / \sigma_{0}$ & $n=3$ & $n=4$ & $n=5$ & $n=7$ & $\mathrm{n}=10$ \\
\hline 0.150 & 0.90737 & 0.54685 & 0.38224 & 0.24968 & 0.18406 & 0.100 & 0.90818 & 0.54679 & 0.38187 & 0.24907 & 0.18328 \\
\hline 0.250 & 0.91158 & 0.54958 & 0.38434 & 0.24954 & 0.18362 & 0.150 & 0.91077 & 0.54809 & 0.38272 & 0.24964 & 0.18426 \\
\hline 0.450 & 0.91661 & 0.55176 & 0.38502 & 0.25097 & 0.18526 & 0.250 & 0.91332 & 0.54994 & 0.38431 & 0.25151 & 0.18576 \\
\hline 0.650 & 0.92183 & 0.55453 & 0.38650 & 0.25036 & 0.18286 & 0.350 & 0.91581 & 0.55120 & 0.38497 & 0.25171 & 0.18676 \\
\hline 0.800 & 0.92350 & 0.55846 & 0.38904 & 0.25207 & 0.18261 & 0.450 & 0.91898 & 0.55328 & 0.38644 & 0.25232 & 0.18717 \\
\hline 0.900 & 0.92404 & 0.55951 & 0.39042 & 0.25231 & 0.18207 & 0.550 & 0.92276 & 0.55630 & 0.38841 & 0.25332 & 0.18683 \\
\hline 1.000 & 0.92281 & 0.55986 & 0.39114 & 0.25285 & 0.18237 & 0.650 & 0.92587 & 0.55910 & 0.38993 & 0.25278 & 0.18533 \\
\hline 1.100 & 0.91969 & 0.55978 & 0.39137 & 0.25287 & 0.18183 & 0.750 & 0.92773 & 0.56158 & 0.39163 & 0.25428 & 0.18495 \\
\hline 1.200 & 0.91664 & 0.55808 & 0.39106 & 0.25291 & 0.18259 & 0.900 & 0.92889 & 0.56526 & 0.39526 & 0.25590 & 0.18511 \\
\hline 1.300 & 0.91133 & 0.55569 & 0.39007 & 0.25310 & 0.18236 & 1.000 & 0.92894 & 0.56735 & 0.39783 & 0.25800 & 0.18644 \\
\hline 1.400 & 0.90467 & 0.55211 & 0.38811 & 0.25245 & 0.18210 & 1.100 & 0.92694 & 0.56796 & 0.40010 & 0.26100 & 0.18924 \\
\hline 1.500 & 0.89569 & 0.54713 & 0.38449 & 0.25125 & 0.18167 & 1.200 & 0.92502 & 0.56908 & 0.40195 & 0.26447 & 0.19433 \\
\hline 1.600 & 0.88816 & 0.54103 & 0.38036 & 0.24910 & 0.18155 & 1.300 & 0.91854 & 0.56778 & 0.40363 & 0.26817 & 0.19935 \\
\hline 1.700 & 0.87634 & 0.53350 & 0.37532 & 0.24657 & 0.18074 & 1.400 & 0.91268 & 0.56469 & 0.40386 & 0.27134 & 0.20534 \\
\hline 1.800 & 0.86651 & 0.52693 & 0.37039 & 0.24319 & 0.17890 & 1.500 & 0.90479 & 0.56190 & 0.40302 & 0.27421 & 0.21158 \\
\hline 1.900 & 0.85595 & 0.51778 & 0.36420 & 0.23931 & 0.17728 & 1.600 & 0.89916 & 0.55791 & 0.40105 & 0.27645 & \\
\hline 2.000 & 0.84454 & 0.51006 & 0.35776 & 0.23568 & 0.17673 & 1.700 & 0.88761 & 0.55272 & 0.39962 & 0.27887 & \\
\hline 2.100 & 0.83298 & 0.50128 & 0.35187 & 0.23310 & & 1.800 & 0.87853 & 0.54614 & 0.39536 & & \\
\hline 2.200 & 0.82121 & 0.49443 & 0.34718 & 0.23264 & & 1.900 & 0.86295 & 0.53798 & 0.39186 & & \\
\hline 2.300 & 0.81263 & 0.48848 & 0.34429 & & & 2.000 & 0.85140 & 0.53106 & & & \\
\hline 2.400 & 0.80199 & 0.48358 & 0.34293 & & & 2.100 & 0.84044 & 0.52425 & & & \\
\hline 2.500 & 0.79129 & 0.47979 & & & & & & & & & \\
\hline 2.600 & 0.78375 & 0.47724 & & & & & & & & & \\
\hline$a / W$ & 0.5 & & & & & 0.7 & & & & & \\
\hline$\sigma / \sigma_{0}$ & $n=3$ & $\mathrm{n}=4$ & $\mathrm{n}=5$ & $\mathrm{n}=7$ & $\mathrm{n}=10$ & $\sigma / \sigma_{0}$ & $n=3$ & $n=4$ & $\mathrm{n}=5$ & $\mathrm{n}=7$ & $\mathrm{n}=10$ \\
\hline 0.150 & 0.91213 & 0.54967 & 0.38424 & 0.25152 & 0.18575 & 0.050 & 0.90756 & 0.54713 & 0.38246 & 0.24982 & 0.18460 \\
\hline 0.250 & 0.91537 & 0.55176 & 0.38630 & 0.25296 & 0.18823 & 0.100 & 0.91131 & 0.54896 & 0.38365 & 0.25101 & 0.18524 \\
\hline 0.350 & 0.92087 & 0.55515 & 0.38849 & 0.25473 & 0.18914 & 0.150 & 0.91350 & 0.55048 & 0.38552 & 0.25269 & 0.18811 \\
\hline 0.450 & 0.92621 & 0.55914 & 0.39048 & 0.25557 & 0.18958 & 0.250 & 0.92076 & 0.55507 & 0.38757 & 0.25433 & 0.18911 \\
\hline 0.550 & 0.93263 & 0.56450 & 0.39434 & 0.25664 & 0.18947 & 0.350 & 0.93280 & 0.56356 & 0.39414 & 0.25718 & 0.18980 \\
\hline 0.650 & 0.93932 & 0.57156 & 0.40038 & 0.26070 & 0.19044 & 0.400 & 0.94105 & 0.57144 & 0.40002 & 0.26099 & 0.19214 \\
\hline 0.750 & 0.94625 & 0.58013 & 0.40872 & 0.26762 & 0.19572 & 0.450 & 0.95005 & 0.58115 & 0.40893 & 0.26817 & 0.19751 \\
\hline 0.800 & 0.94794 & 0.58492 & 0.41389 & 0.27244 & 0.20114 & 0.500 & 0.95887 & 0.59267 & 0.42054 & 0.27911 & 0.20770 \\
\hline 0.850 & 0.95095 & 0.58934 & 0.41932 & 0.27876 & 0.20698 & 0.510 & 0.96088 & 0.59506 & 0.42304 & 0.28158 & 0.21009 \\
\hline 0.900 & 0.95329 & 0.59420 & 0.42516 & 0.28572 & 0.21520 & 0.520 & 0.96283 & 0.59756 & 0.42566 & 0.28417 & 0.21276 \\
\hline 0.920 & 0.95461 & 0.59628 & 0.42726 & 0.28844 & 0.21833 & 0.530 & 0.96481 & 0.60010 & 0.42834 & 0.28693 & 0.21570 \\
\hline 0.940 & 0.95575 & 0.59727 & 0.42972 & 0.29143 & 0.22230 & 0.540 & 0.96671 & 0.60269 & 0.43112 & 0.29050 & 0.21966 \\
\hline 0.950 & 0.95501 & 0.59886 & 0.43069 & 0.29296 & 0.22428 & 0.550 & 0.96864 & 0.60531 & 0.43396 & 0.29343 & 0.22287 \\
\hline 1.050 & 0.95901 & 0.60715 & 0.44230 & 0.30817 & & 0.600 & 0.97945 & 0.61862 & 0.44863 & 0.30978 & 0.24169 \\
\hline 1.100 & 0.95830 & 0.61053 & 0.44644 & 0.31548 & & 0.650 & 0.98736 & 0.63150 & 0.46353 & 0.32651 & \\
\hline 1.150 & 0.95977 & 0.61315 & 0.45130 & 0.32235 & & 0.680 & 0.99393 & 0.63871 & 0.47106 & 0.33586 & \\
\hline 1.200 & 0.95740 & 0.61509 & 0.45563 & 0.32872 & & 0.700 & 0.99604 & 0.64316 & 0.47639 & 0.34215 & \\
\hline 1.250 & 0.95773 & 0.61597 & 0.45938 & 0.33408 & & & & & & & \\
\hline
\end{tabular}

Table 5.6 FEA results for DECP specimens under $\lambda=0.5$ 


\begin{tabular}{|c|c|c|c|c|c|c|c|c|c|c|c|}
\hline$a / W$ & 0.1 & & & & & 0.3 & & & & & \\
\hline$\sigma / \sigma_{0}$ & $n=3$ & $n=4$ & $n=5$ & $n=7$ & $\mathrm{n}=10$ & $\sigma / \sigma_{0}$ & $n=3$ & $n=4$ & $n=5$ & $n=7$ & $n=10$ \\
\hline 0.150 & 0.86925 & 0.51533 & 0.35529 & 0.22685 & 0.16266 & 0.150 & 0.87254 & 0.51647 & 0.35567 & 0.22672 & 0.16228 \\
\hline 0.250 & 0.84806 & 0.49772 & 0.34026 & 0.21168 & 0.14827 & 0.250 & 0.84930 & 0.49767 & 0.33986 & 0.21364 & 0.15104 \\
\hline 0.500 & 0.78679 & 0.44669 & 0.29733 & 0.17643 & 0.11728 & 0.500 & 0.78991 & 0.44755 & 0.29760 & 0.17940 & 0.12105 \\
\hline 0.650 & 0.74962 & 0.41600 & 0.27248 & 0.15509 & 0.09888 & 0.650 & 0.75172 & 0.41771 & 0.27370 & 0.15879 & 0.10214 \\
\hline 0.750 & 0.72289 & 0.39416 & 0.25184 & 0.14370 & 0.08746 & 0.750 & 0.72614 & 0.39540 & 0.25541 & 0.14475 & 0.09047 \\
\hline 0.900 & 0.68110 & 0.35892 & 0.22231 & 0.12096 & 0.06931 & 0.900 & 0.68443 & 0.36244 & 0.22572 & 0.12207 & 0.07298 \\
\hline 1.000 & 0.65233 & 0.33357 & 0.20057 & 0.10054 & 0.05484 & 1.000 & 0.65589 & 0.33742 & 0.20404 & 0.10436 & 0.05877 \\
\hline 1.100 & 0.62156 & 0.30469 & 0.17667 & 0.07855 & & 1.100 & 0.62717 & 0.31193 & 0.18046 & 0.08248 & \\
\hline 1.150 & 0.60835 & 0.29324 & 0.16211 & & & 1.150 & 0.61160 & 0.29698 & 0.16595 & & \\
\hline 1.200 & 0.59283 & 0.27797 & 0.14712 & & & 1.200 & 0.59593 & 0.28166 & 0.15098 & & \\
\hline 1.250 & 0.57732 & 0.26237 & 0.13066 & & & 1.250 & 0.58020 & 0.26597 & 0.13868 & & \\
\hline 1.300 & 0.56184 & 0.24644 & & & & 1.300 & 0.56443 & 0.24989 & 0.12144 & & \\
\hline 1.350 & 0.54324 & 0.23021 & & & & 1.350 & 0.54865 & 0.23344 & & & \\
\hline 1.400 & 0.52774 & 0.21371 & & & & 1.400 & 0.53291 & 0.21662 & & & \\
\hline 1.450 & 0.51192 & 0.19698 & & & & 1.450 & 0.51725 & & & & \\
\hline 1.500 & 0.49678 & & & & & 1.500 & 0.49766 & & & & \\
\hline 1.800 & 0.40071 & & & & & 1.600 & 0.46654 & & & & \\
\hline \multirow[t]{2}{*}{1.900} & 0.36851 & & & & & 1.700 & 0.43085 & & & & \\
\hline & & & & & & 1.800 & 0.39539 & & & & \\
\hline$a / W$ & 0.5 & & & & & 0.7 & & & & & \\
\hline$\sigma / \sigma_{0}$ & $\mathrm{n}=3$ & $n=4$ & $\mathrm{n}=5$ & $\mathrm{n}=7$ & $n=10$ & $\sigma / \sigma_{0}$ & $\mathrm{n}=3$ & $\mathrm{n}=4$ & $n=5$ & $\mathrm{n}=7$ & $\mathrm{n}=10$ \\
\hline 0.150 & 0.87424 & 0.51821 & 0.35734 & 0.22834 & 0.16451 & 0.100 & 0.88853 & 0.52789 & 0.36562 & 0.23577 & 0.17097 \\
\hline 0.250 & 0.85134 & 0.49955 & 0.34149 & 0.21579 & 0.15266 & 0.150 & 0.87517 & 0.51917 & 0.35853 & 0.23019 & 0.16620 \\
\hline 0.350 & 0.82888 & 0.48056 & 0.32587 & 0.20241 & 0.14183 & 0.250 & 0.85440 & 0.49999 & 0.34298 & 0.21630 & 0.15487 \\
\hline 0.500 & 0.79558 & 0.45179 & 0.30169 & 0.18284 & 0.12336 & 0.350 & 0.83843 & 0.48690 & 0.32904 & 0.20342 & 0.14223 \\
\hline 0.650 & 0.76040 & 0.42457 & 0.27963 & 0.16356 & 0.10690 & 0.450 & 0.82472 & 0.47563 & 0.31904 & 0.19605 & 0.13322 \\
\hline 0.750 & 0.73700 & 0.40661 & 0.26290 & 0.15092 & 0.09652 & 0.550 & 0.81355 & 0.47025 & 0.31567 & 0.19398 & 0.13129 \\
\hline 0.850 & 0.71251 & 0.38709 & 0.24703 & 0.13889 & 0.08753 & 0.650 & 0.80264 & 0.46755 & 0.31802 & 0.19668 & 0.13503 \\
\hline 0.950 & 0.68765 & 0.36495 & 0.23096 & 0.12676 & 0.07590 & 0.750 & 0.79021 & 0.46407 & 0.31850 & 0.20183 & 0.14366 \\
\hline 1.000 & 0.67356 & 0.35483 & 0.21976 & 0.12006 & 0.07036 & 0.850 & 0.77393 & 0.45752 & 0.31708 & 0.20500 & 0.14922 \\
\hline 1.050 & 0.65937 & 0.34459 & 0.21074 & 0.10979 & & 0.860 & 0.77172 & 0.45652 & 0.31663 & 0.20504 & 0.15020 \\
\hline 1.100 & 0.64599 & 0.33115 & 0.20129 & 0.10092 & & 0.870 & 0.76927 & 0.45543 & 0.31606 & 0.20495 & 0.15023 \\
\hline 1.150 & 0.63102 & 0.32000 & 0.18807 & 0.09070 & & 0.880 & 0.76820 & 0.45447 & 0.31541 & 0.20483 & 0.15019 \\
\hline 1.200 & 0.61733 & 0.30549 & 0.17686 & 0.07515 & & 0.890 & 0.76556 & 0.45323 & 0.31467 & 0.20457 & 0.15003 \\
\hline 1.300 & 0.58549 & 0.27751 & 0.14798 & & & 0.900 & 0.76281 & 0.45187 & 0.31387 & 0.20437 & 0.15008 \\
\hline 1.400 & 0.55166 & 0.24273 & & & & 0.950 & 0.75103 & 0.44386 & 0.30871 & 0.19984 & 0.14562 \\
\hline 1.500 & 0.51534 & 0.20277 & & & & 1.000 & 0.73702 & 0.43410 & 0.29979 & 0.19153 & 0.13142 \\
\hline 1.600 & 0.47414 & & & & & 1.050 & 0.72000 & 0.42111 & 0.28561 & 0.17653 & \\
\hline 1.700 & 0.43161 & & & & & 1.100 & 0.70436 & 0.40281 & & & \\
\hline
\end{tabular}

Table 5.7 FEA results for DECP specimens under $\lambda=1.0$ - 


\begin{tabular}{ccccc}
\hline$i$ & $e_{i 0}$ & $e_{i 1}$ & $e_{i 2}$ & $e_{i 3}$ \\
\hline 1 & 0.0371 & -0.6207 & 2.5556 & -2.2771 \\
2 & 4.1907 & -64.6390 & 258.6200 & -298.0900 \\
3 & -47.9050 & 735.9100 & -2893.2000 & 3242.1000 \\
\hline
\end{tabular}

Table 5.8 The polynomial coefficients $e_{i j}$ for SECP specimens under $\lambda=0.5$

\begin{tabular}{ccccc}
\hline$i$ & $e_{i 0}$ & $e_{i 1}$ & $e_{i 2}$ & $e_{i 3}$ \\
\hline 1 & 0.1195 & 0.7890 & -2.2288 & 1.8042 \\
2 & -0.1384 & 1.3655 & -6.9337 & 10.3540 \\
3 & 0.1253 & -1.9955 & 8.5781 & -10.4480 \\
\hline
\end{tabular}

Table 5.9 The polynomial coefficients $e_{i j}$ for CCP specimens under $\lambda=0.5$

\begin{tabular}{ccccc}
\hline$i$ & $e_{i 0}$ & $e_{i 1}$ & $e_{i 2}$ & $e_{i 3}$ \\
\hline 1 & -0.0139 & 0.0676 & 0.2556 & -0.1646 \\
2 & 0.0433 & -0.4830 & 1.3438 & -2.7000 \\
3 & -0.1216 & 1.8162 & -7.2350 & 9.6833 \\
\hline
\end{tabular}

Table 5.10 The polynomial coefficients $e_{i j}$ for DECP specimens under $\lambda=0.5$ 


\begin{tabular}{ccccc}
\hline$i$ & $e_{i 0}$ & $e_{i 1}$ & $e_{i 2}$ & $e_{i 3}$ \\
\hline 1 & -0.3268 & 0.7140 & -0.4025 & 0.0000 \\
2 & -29.4630 & 160.0000 & -204.5200 & 0.0000 \\
3 & 305.6600 & -1635.5000 & 2054.3000 & 0.0000 \\
\hline
\end{tabular}

Table 5.11 The polynomial coefficients $e_{i j}$ for SECP specimens under $\lambda=1.0$

\begin{tabular}{ccccc}
\hline$i$ & $e_{i 0}$ & $e_{i 1}$ & $e_{i 2}$ & $e_{i 3}$ \\
\hline 1 & 0.0853 & -0.6315 & 1.1963 & 0.0000 \\
2 & -0.0078 & 0.2820 & -0.4600 & 0.0000 \\
3 & 0.6750 & -3.8135 & 4.8038 & 0.0000 \\
\hline
\end{tabular}

Table 5.12 The polynomial coefficients $e_{i j}$ for CCP specimens under $\lambda=1.0$

\begin{tabular}{ccccc}
\hline$i$ & $e_{i 0}$ & $e_{i 1}$ & $e_{i 2}$ & $e_{i 3}$ \\
\hline 1 & -0.1607 & -0.3862 & 1.3744 & -1.6313 \\
2 & -0.0937 & 1.4709 & -5.4706 & 6.5104 \\
3 & 0.0339 & -0.9277 & 3.4531 & -3.9813 \\
\hline
\end{tabular}

Table 5.13 The polynomial coefficients $e_{i j}$ for DECP specimens under $\lambda=1.0$ 


\begin{tabular}{lccccccccc}
\hline & \multicolumn{1}{c}{$\lambda=0.5$} & \multicolumn{7}{c}{$\lambda=1.0$} \\
\hline model & $n$ & $\begin{array}{c}a / W \\
=0.1\end{array}$ & $\begin{array}{c}a / W \\
=0.3\end{array}$ & $\begin{array}{c}a / W \\
=0.5\end{array}$ & $\begin{array}{c}a / W \\
=0.7\end{array}$ & $\begin{array}{c}a / W \\
=0.1\end{array}$ & $\begin{array}{c}a / W \\
=0.3\end{array}$ & $\begin{array}{c}a / W \\
=0.5\end{array}$ & $\begin{array}{c}a / W \\
=0.7\end{array}$ \\
\hline SECP & 3 & 1.800 & 1.400 & 0.350 & 0.080 & 1.000 & 1.400 & 0.350 & 0.080 \\
& 4 & 1.700 & 1.300 & 0.320 & 0.070 & 0.900 & 1.200 & 0.300 & 0.070 \\
& 5 & 1.600 & 1.100 & 0.320 & 0.070 & 0.850 & 1.150 & 0.300 & 0.070 \\
& 7 & 1.600 & 0.900 & 0.320 & 0.070 & 0.750 & 1.100 & 0.300 & 0.060 \\
& 10 & 1.600 & 0.810 & 0.320 & 0.060 & 0.500 & 0.950 & 0.300 & 0.060 \\
\hline CCP & 3 & 1.800 & 1.800 & 1.200 & 0.640 & 1.200 & 1.300 & 1.300 & 0.750 \\
& 4 & 1.500 & 1.800 & 1.200 & 0.400 & 1.000 & 1.000 & 1.200 & 0.750 \\
& 5 & 1.200 & 1.750 & 1.200 & 0.400 & 1.000 & 1.000 & 1.200 & 0.750 \\
& 7 & 1.200 & 1.500 & 1.200 & 0.400 & 1.000 & 1.000 & 1.100 & 0.540 \\
& 10 & 1.000 & 1.200 & 1.150 & 0.400 & 1.000 & 1.000 & 1.100 & 0.500 \\
\hline DECP & 3 & 2.000 & 2.000 & 1.250 & 0.700 & 1.000 & 1.100 & 1.200 & 1.200 \\
& 4 & 2.000 & 2.200 & 1.250 & 0.650 & 0.900 & 1.000 & 1.100 & 0.870 \\
& 5 & 1.900 & 2.200 & 1.150 & 0.550 & 0.900 & 0.900 & 1.050 & 0.650 \\
& 7 & 1.900 & 2.000 & 0.950 & 0.550 & 0.900 & 0.900 & 1.050 & 0.550 \\
& 10 & 1.800 & 1.700 & 0.940 & 0.540 & 0.750 & 0.900 & 1.050 & 0.550 \\
\hline
\end{tabular}

Table 5.14 Limits of applicability in terms of normalized load, $\sigma / \sigma_{0}$, of the $A-T$ approximation, Eq. (3.11)

\begin{tabular}{|c|c|c|c|c|c|c|c|c|c|}
\hline & & $\lambda=0.5$ & & & & $\lambda=1.0$ & & & \\
\hline \multirow{2}{*}{ model } & $n$ & $a / W$ & $a / W$ & $a / W$ & $a / W$ & $a / W$ & $a / W$ & $a / W$ & $a / W$ \\
\hline & & $=0.1$ & $=0.3$ & $=0.5$ & $=0.7$ & $=0.1$ & $=0.3$ & $=0.5$ & $=0.7$ \\
\hline \multirow{5}{*}{ SECP } & 3 & 2.100 & 1.400 & 0.380 & 0.095 & 0.600 & 1.050 & 0.450 & 0.100 \\
\hline & 4 & 1.900 & 1.150 & 0.350 & 0.085 & 0.600 & 0.950 & 0.400 & 0.090 \\
\hline & 5 & 1.800 & 0.950 & 0.320 & 0.080 & 0.600 & 0.950 & 0.380 & 0.080 \\
\hline & 7 & 1.700 & 0.850 & 0.320 & 0.070 & 0.600 & 1.000 & 0.350 & 0.070 \\
\hline & 10 & 1.600 & 0.810 & 0.320 & 0.060 & 0.500 & 0.950 & 0.300 & 0.060 \\
\hline \multirow[t]{5}{*}{ CCP } & 3 & 2.200 & 1.800 & 0.450 & 0.250 & 1.200 & 1.300 & 1.450 & 0.400 \\
\hline & 4 & 1.800 & 1.800 & 0.550 & 0.250 & 1.000 & 1.200 & 1.400 & 0.350 \\
\hline & 5 & 1.500 & 1.750 & 0.650 & 0.250 & 1.000 & 1.000 & 1.300 & 0.400 \\
\hline & 7 & 1.200 & 1.500 & 1.200 & 0.350 & 1.000 & 1.000 & 1.200 & 0.500 \\
\hline & 10 & 1.000 & 1.200 & 1.150 & 0.400 & 1.000 & 1.000 & 1.100 & 0.500 \\
\hline \multirow[t]{5}{*}{ DECP } & 3 & 2.100 & 2.100 & 1.250 & 0.700 & 0.650 & 0.650 & 0.750 & 1.050 \\
\hline & 4 & 2.000 & 2.200 & 1.250 & 0.600 & 0.500 & 0.500 & 0.650 & 1.100 \\
\hline & 5 & 2.000 & 2.200 & 1.050 & 0.550 & 0.650 & 0.650 & 0.650 & 1.200 \\
\hline & 7 & 1.900 & 2.000 & 0.950 & 0.550 & 0.750 & 0.750 & 0.850 & 0.650 \\
\hline & 10 & 1.800 & 1.700 & 0.940 & 0.540 & 0.750 & 0.900 & 1.050 & 0.550 \\
\hline
\end{tabular}

Table 5.15 Limits of applicability in terms of normalized load, $\sigma / \sigma_{0}$, of the simplified $A-T$ approximation, Eq. (5.8) 


\begin{tabular}{ccccccc}
\hline & $\mathrm{a} / \mathrm{W}$ & $\mathrm{n}=3$ & $\mathrm{n}=4$ & $\mathrm{n}=5$ & $\mathrm{n}=7$ & $\mathrm{n}=10$ \\
\hline$\lambda=0.5$ & 0.1 & -0.06841 & -0.03530 & -0.01908 & -0.01348 & -0.00924 \\
& 0.3 & 0.05472 & 0.04799 & 0.04397 & 0.04152 & 0.04259 \\
& 0.5 & 0.37808 & 0.35340 & 0.34448 & 0.30919 & 0.24562 \\
& 0.7 & 1.53049 & 1.93042 & 2.28657 & 2.87597 & 3.66906 \\
\hline$\lambda=1.0$ & 0.1 & -0.11273 & -0.08669 & -0.05812 & -0.03833 & -0.02343 \\
& 0.3 & -0.03840 & -0.02970 & -0.02140 & -0.01112 & -0.01327 \\
& 0.5 & 0.33799 & 0.31065 & 0.29497 & 0.26894 & 0.15413 \\
& 0.7 & 1.77624 & 2.28103 & 2.43541 & 2.80894 & 3.49042 \\
\hline
\end{tabular}

Table 5.16 Coefficient $a_{1}$ values for SECP specimen

\begin{tabular}{ccccccc}
\hline & $\mathrm{a} / \mathrm{W}$ & $\mathrm{n}=3$ & $\mathrm{n}=4$ & $\mathrm{n}=5$ & $\mathrm{n}=7$ & $\mathrm{n}=10$ \\
\hline$\lambda=0.5$ & 0.1 & -0.11077 & -0.08088 & -0.06549 & -0.05559 & -0.05124 \\
& 0.3 & -0.03447 & -0.03170 & -0.03369 & -0.03126 & -0.02390 \\
& 0.5 & 0.06186 & 0.04282 & 0.03151 & 0.02188 & 0.03424 \\
& 0.7 & 0.22550 & 0.21342 & 0.19565 & 0.18498 & 0.24847 \\
\hline$\lambda=1.0$ & 0.1 & -0.13499 & -0.08711 & -0.06256 & -0.04124 & -0.03059 \\
& 0.3 & -0.14364 & -0.09643 & -0.07200 & -0.04899 & -0.03377 \\
& 0.5 & -0.12663 & -0.08796 & -0.06867 & -0.05075 & -0.03309 \\
& 0.7 & 0.08920 & 0.09632 & 0.09313 & 0.10558 & 0.15611 \\
\hline
\end{tabular}

Table 5.17 Coefficient $a_{1}$ values for CCP specimen

\begin{tabular}{ccccccc}
\hline & $\mathrm{a} / \mathrm{W}$ & $\mathrm{n}=3$ & $\mathrm{n}=4$ & $\mathrm{n}=5$ & $\mathrm{n}=7$ & $\mathrm{n}=10$ \\
\hline$\lambda=0.5$ & 0.1 & -0.05799 & -0.03155 & -0.01945 & -0.01044 & -0.00685 \\
& 0.3 & -0.04138 & -0.01847 & -0.00361 & 0.00477 & 0.00380 \\
& 0.5 & 0.02570 & 0.03683 & 0.04092 & 0.04510 & 0.02655 \\
& 0.7 & 0.09951 & 0.11363 & 0.11783 & 0.12666 & 0.08475 \\
\hline$\lambda=1.0$ & 0.1 & -0.11979 & -0.07484 & -0.04831 & -0.02080 & -0.01215 \\
& 0.3 & -0.11898 & -0.06943 & -0.05076 & -0.01976 & -0.00956 \\
& 0.5 & -0.10652 & -0.06101 & -0.03242 & -0.01868 & -0.00265 \\
& 0.7 & 0.00421 & 0.03588 & 0.05370 & 0.05619 & 0.06413 \\
\hline
\end{tabular}

Table 5.18 Coefficient $a_{1}$ values for DECP specimen 


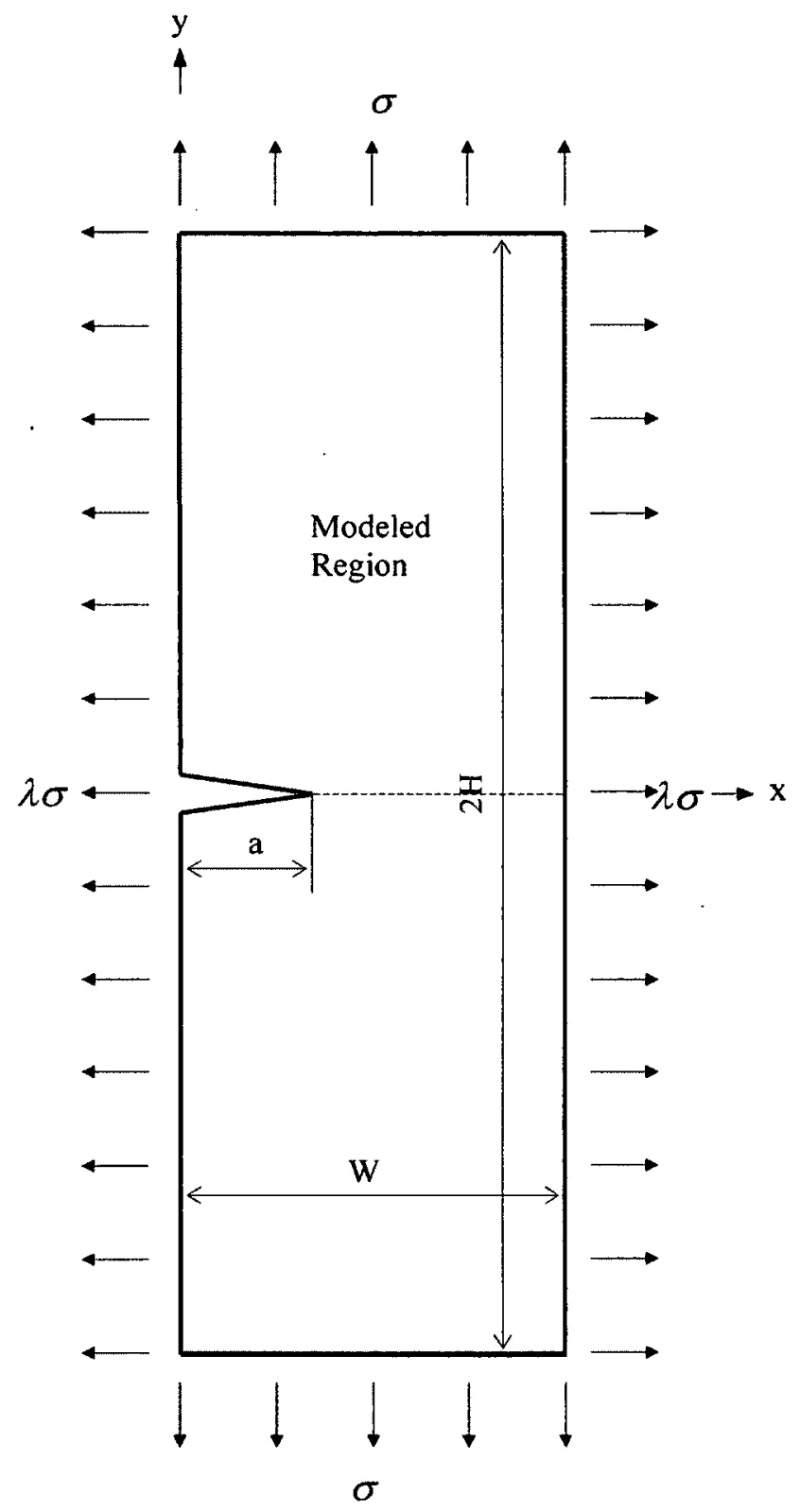

Figure 5.1 Single edge cracked plate (SECP) model 


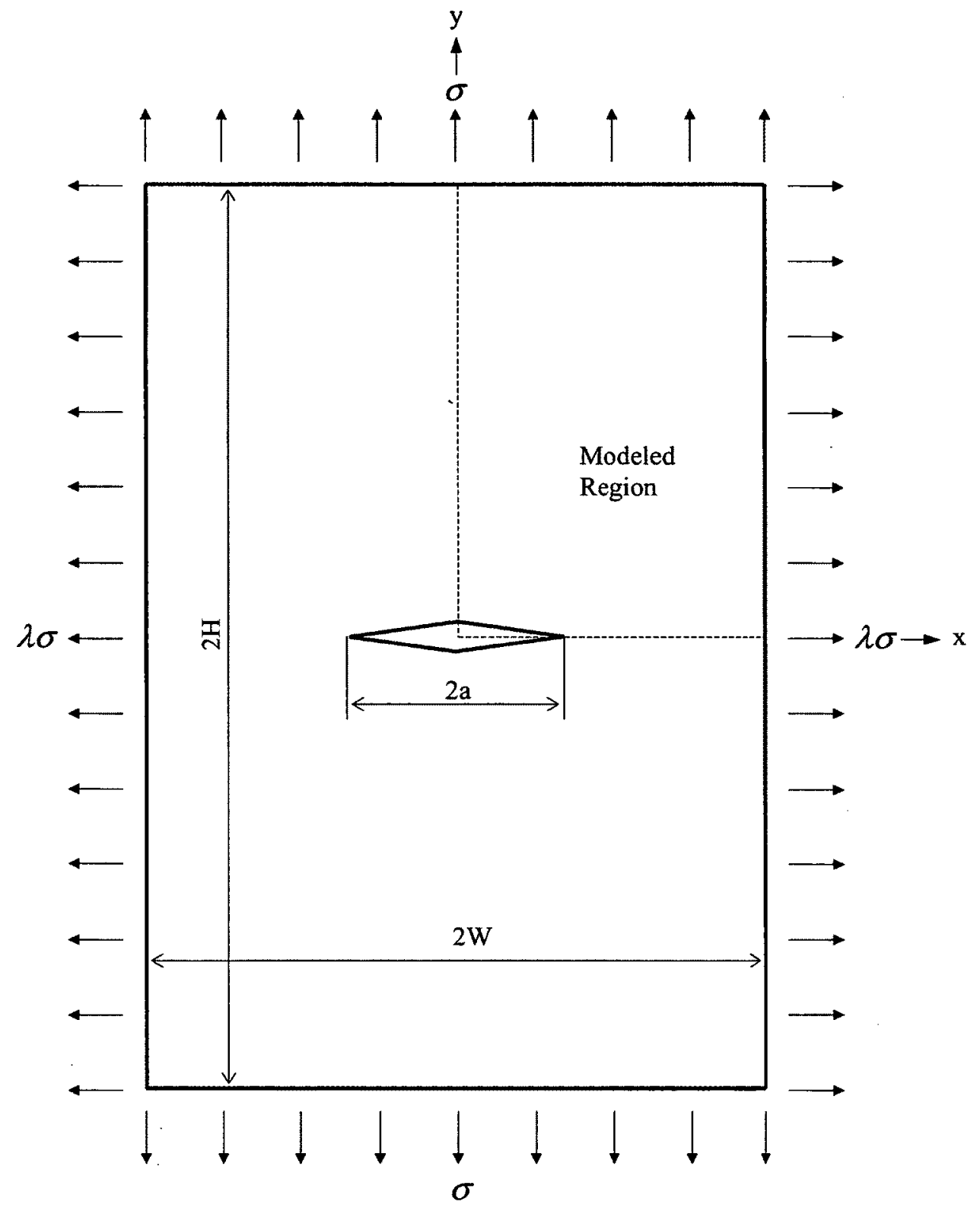

Figure 5.2 Centre cracked plate (CCP) model 


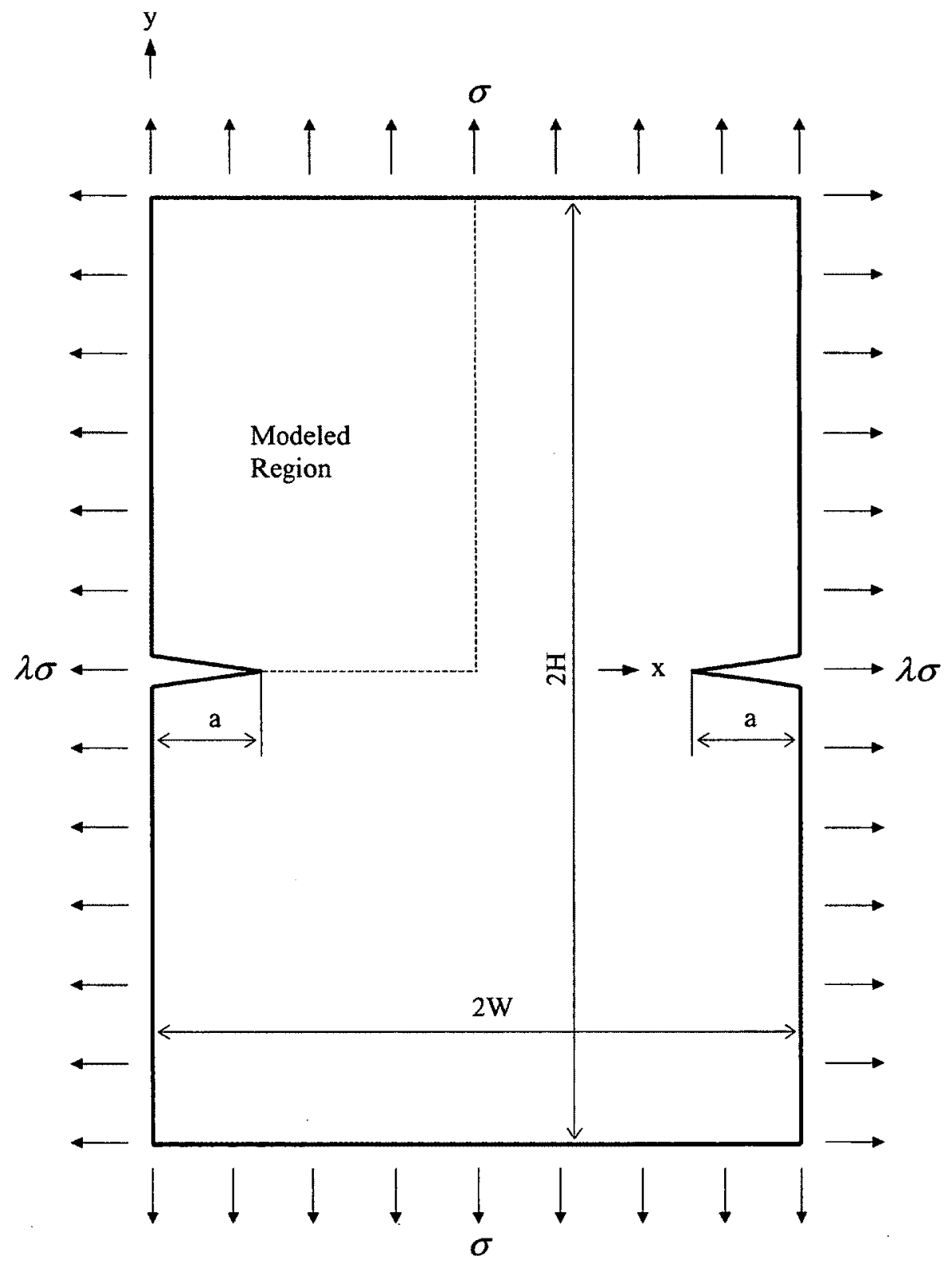

Figure 5.3 Double edge cracked plate (DECP) model 

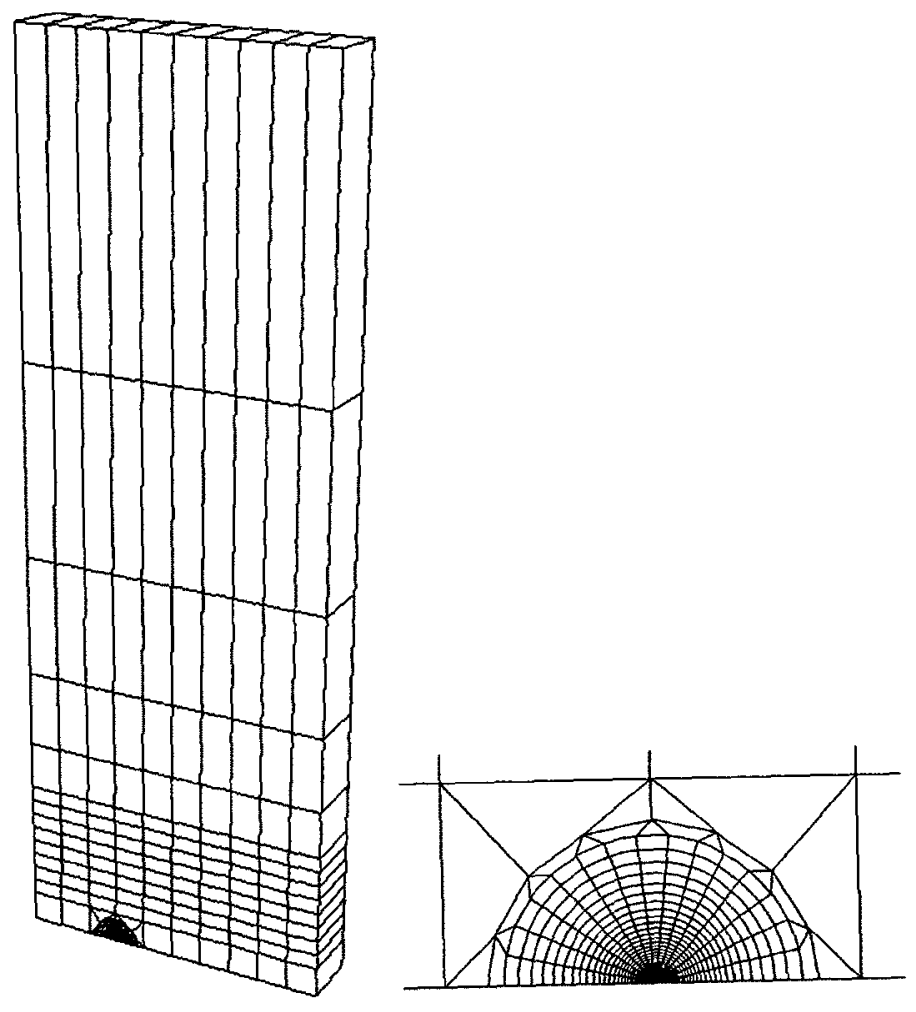

Figure 5.4 Typical FEA mesh, $a / W=0.3$ 


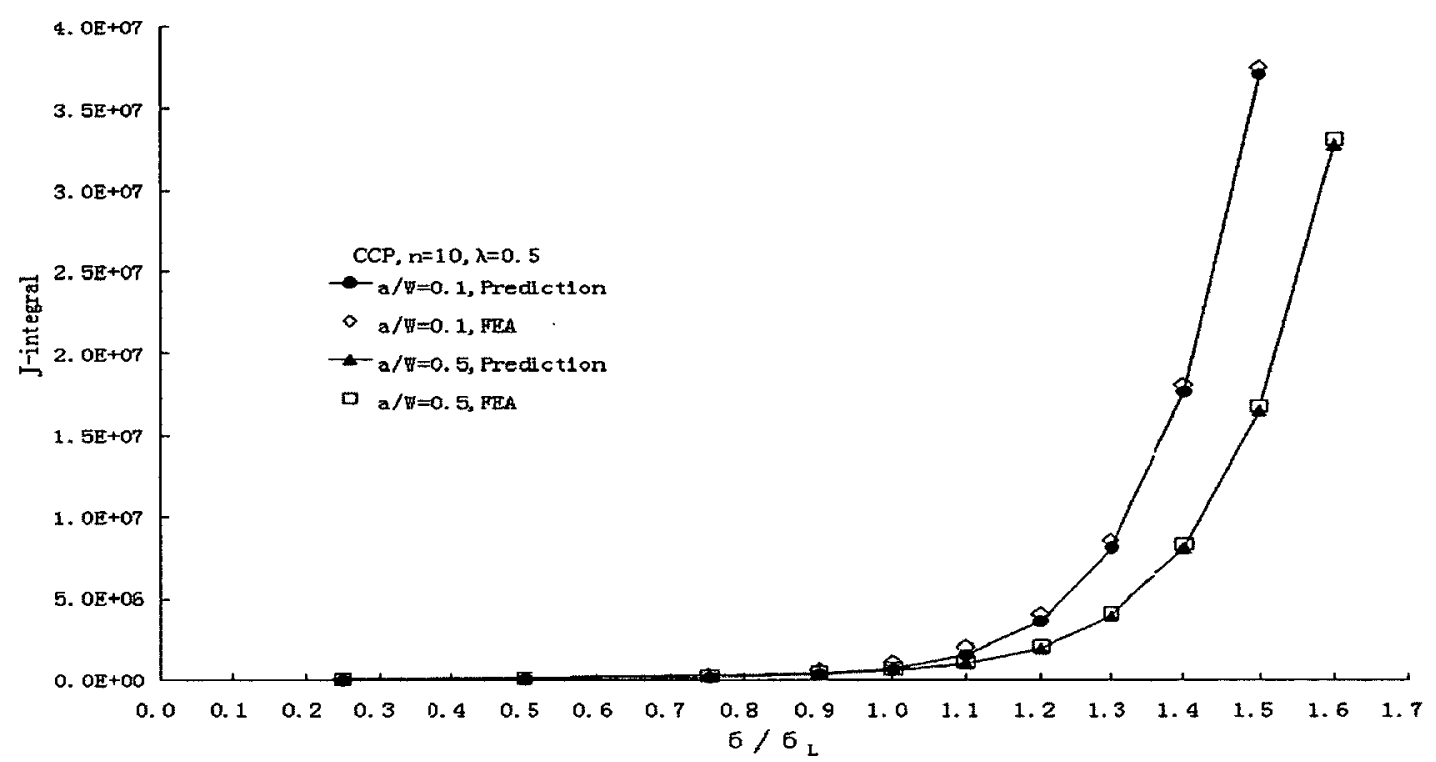

Figure 5.5 Comparisons of $J$-integral, CCP

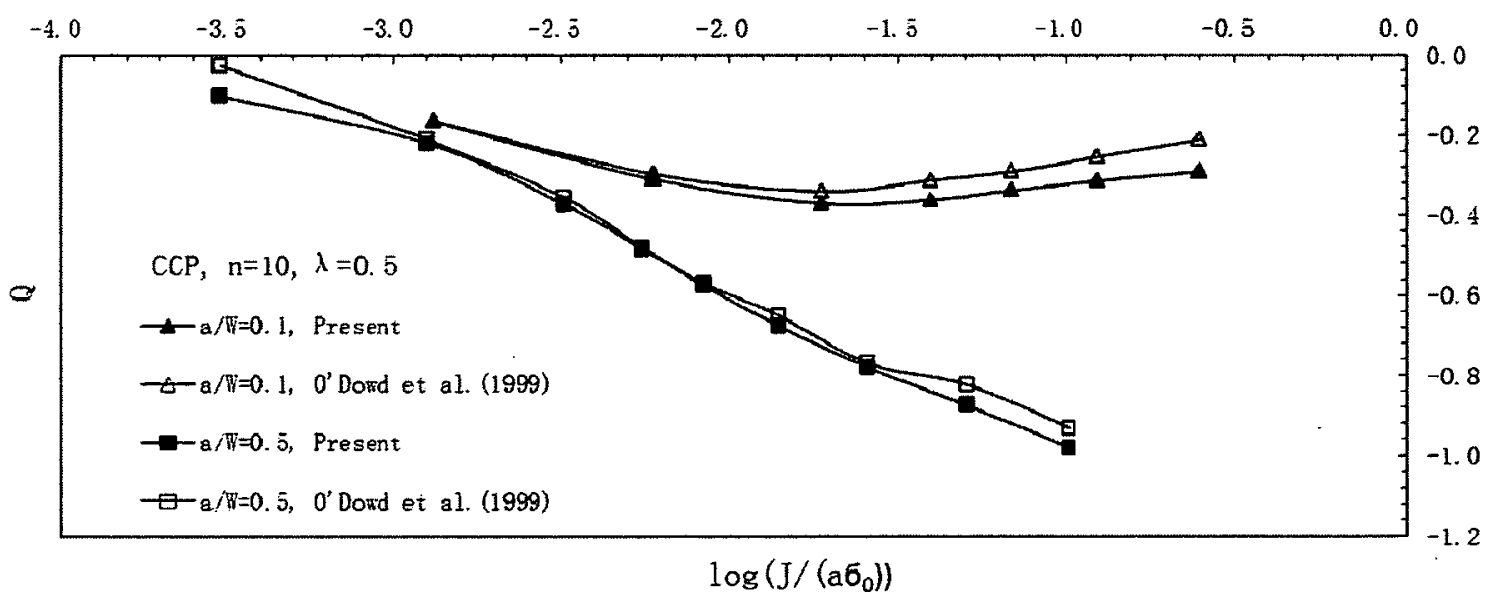

Figure 5.6 Comparisons of $Q$ values, CCP 


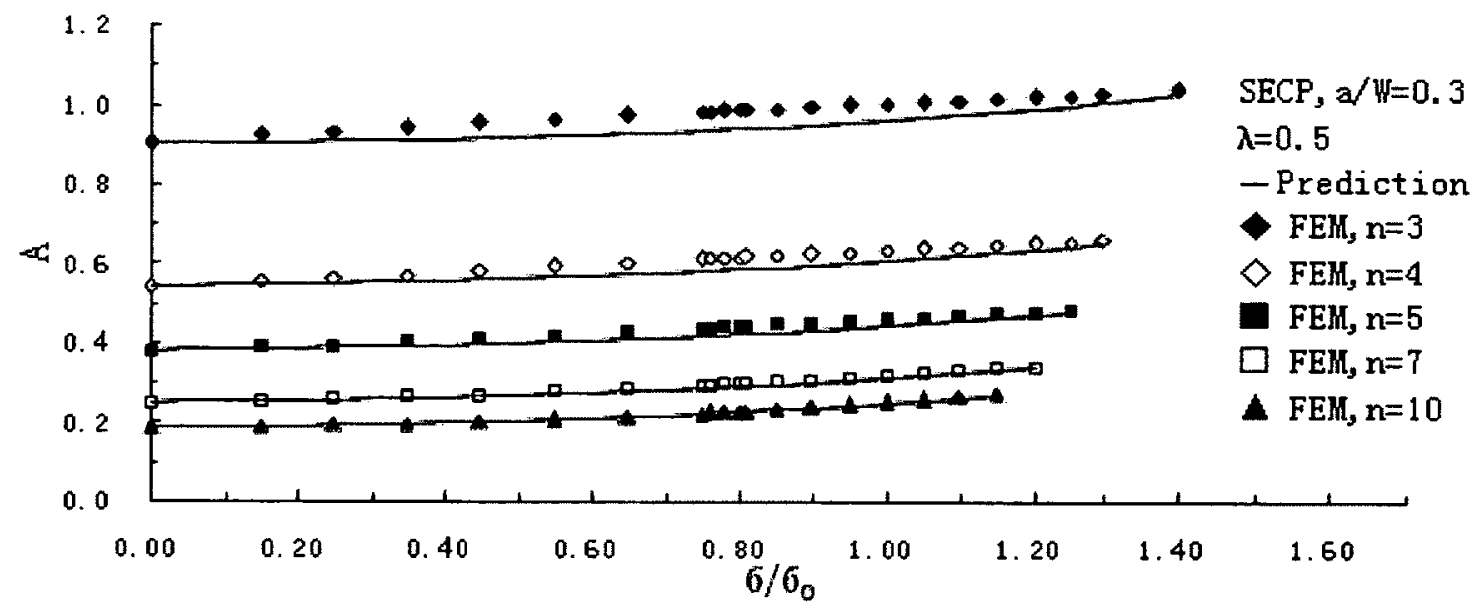

Figure 5.7 Comparisons of predicted $A$ values by curve shape similarity with FEA data for SECP, $a / W=0.3, \lambda=0.5$

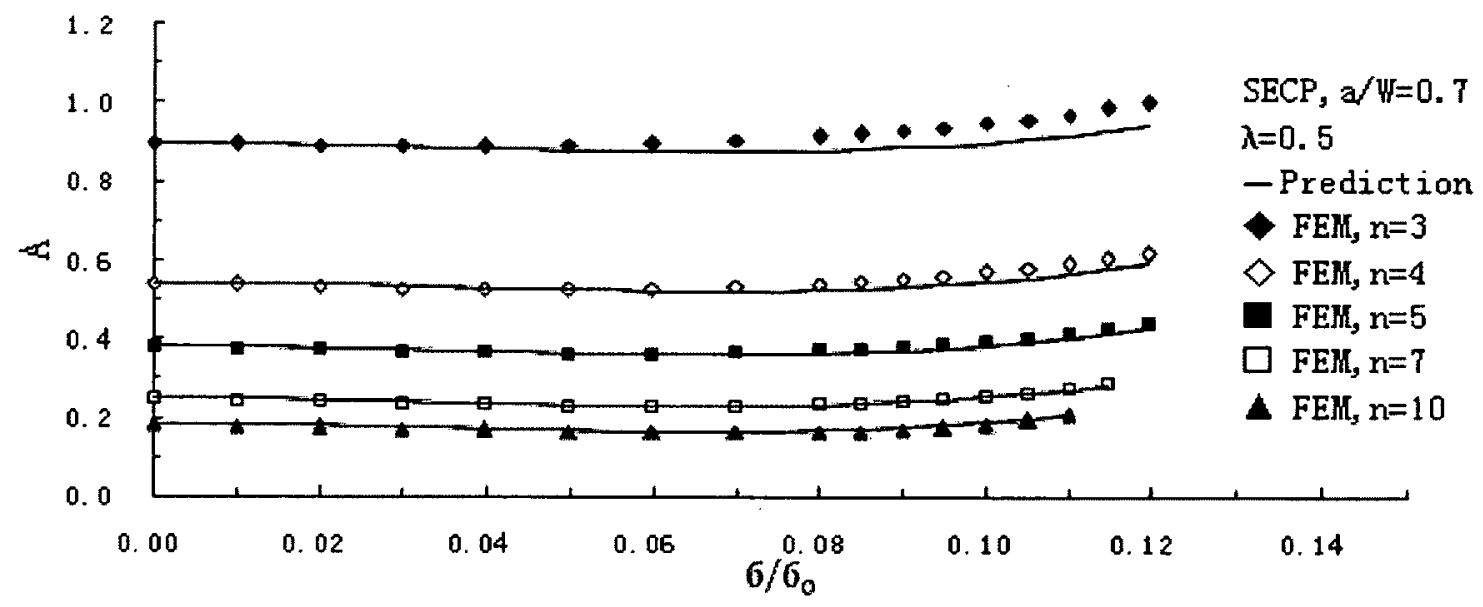

Figure 5.8 Comparisons of predicted $A$ values by curve shape similarity with FEA data for SECP, $a / W=0.7, \lambda=0.5$ 


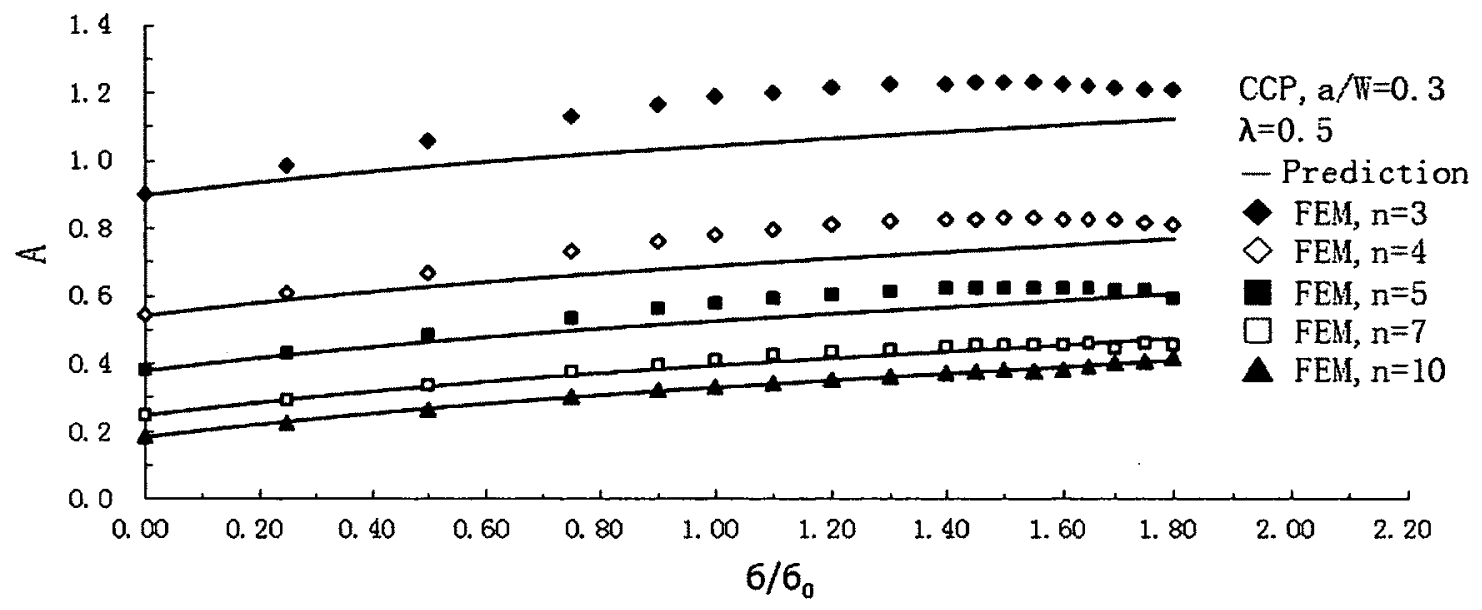

Figure 5.9 Comparisons of predicted $A$ values by curve shape similarity with FEA data for $\mathrm{CCP}, a / W=0.3, \lambda=0.5$

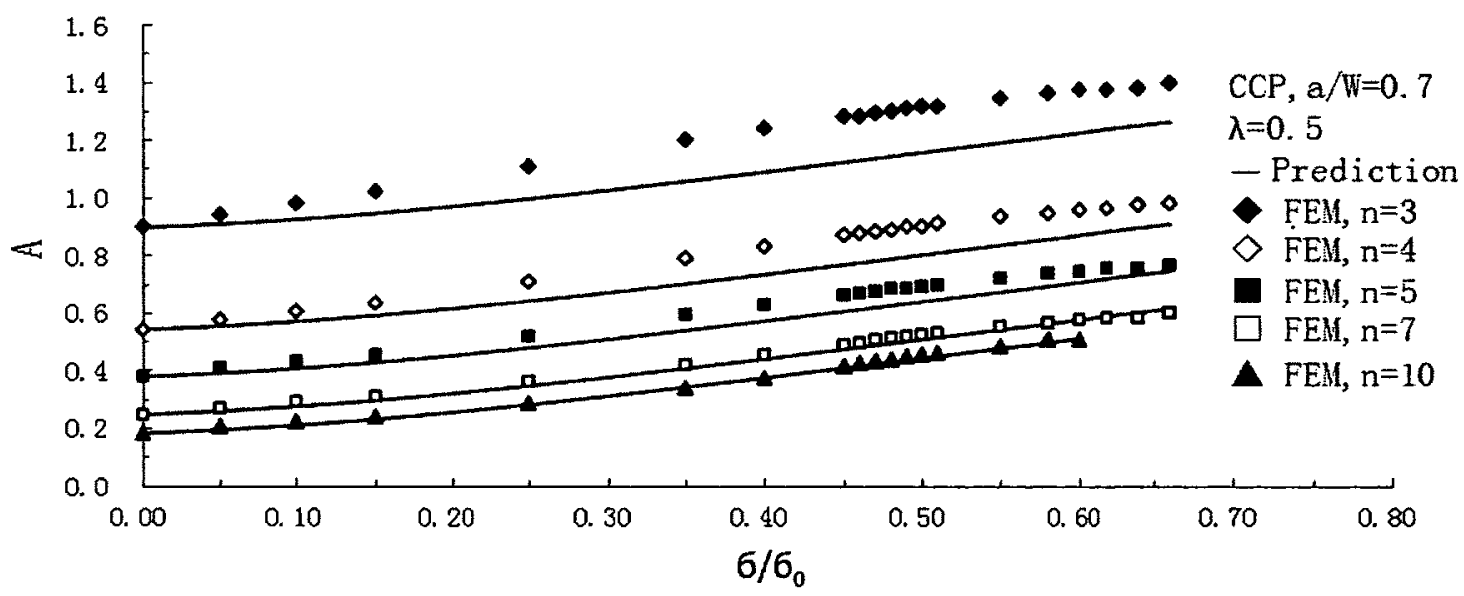

Figure 5.10 Comparisons of predicted $A$ values by curve shape similarity with FEA data for $\mathrm{CCP}, a / W=0.7, \lambda=0.5$ 


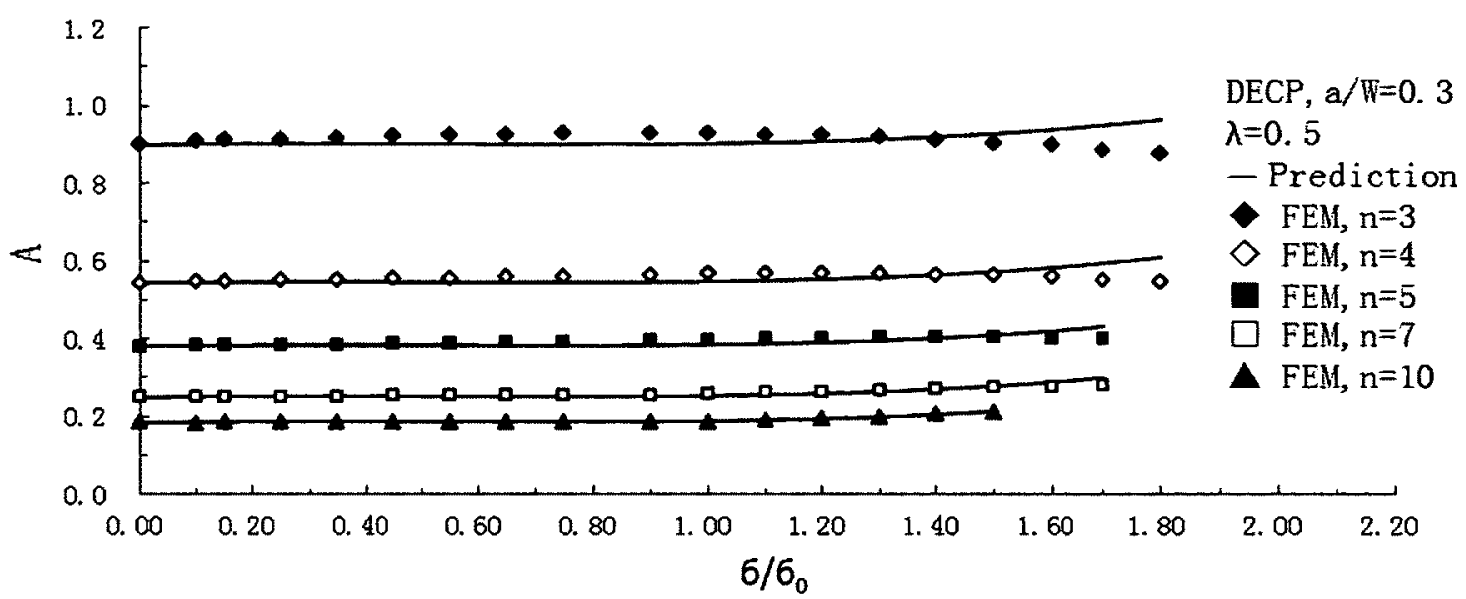

Figure 5.11 Comparisons of predicted $A$ values by curve shape similarity with FEA data for DECP, $a / W=0.3, \lambda=0.5$

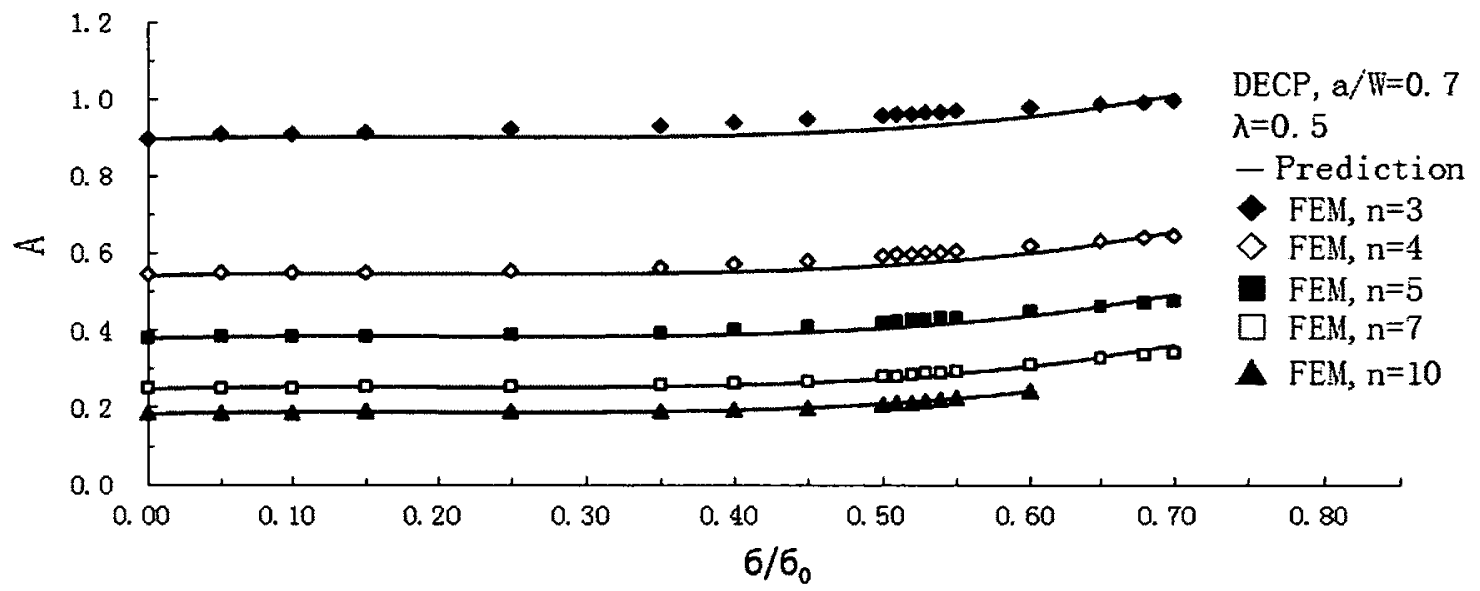

Figure 5.12 Comparisons of predicted $A$ values by curve shape similarity with FEA data for DECP, $a / W=0.7, \lambda=0.5$ 


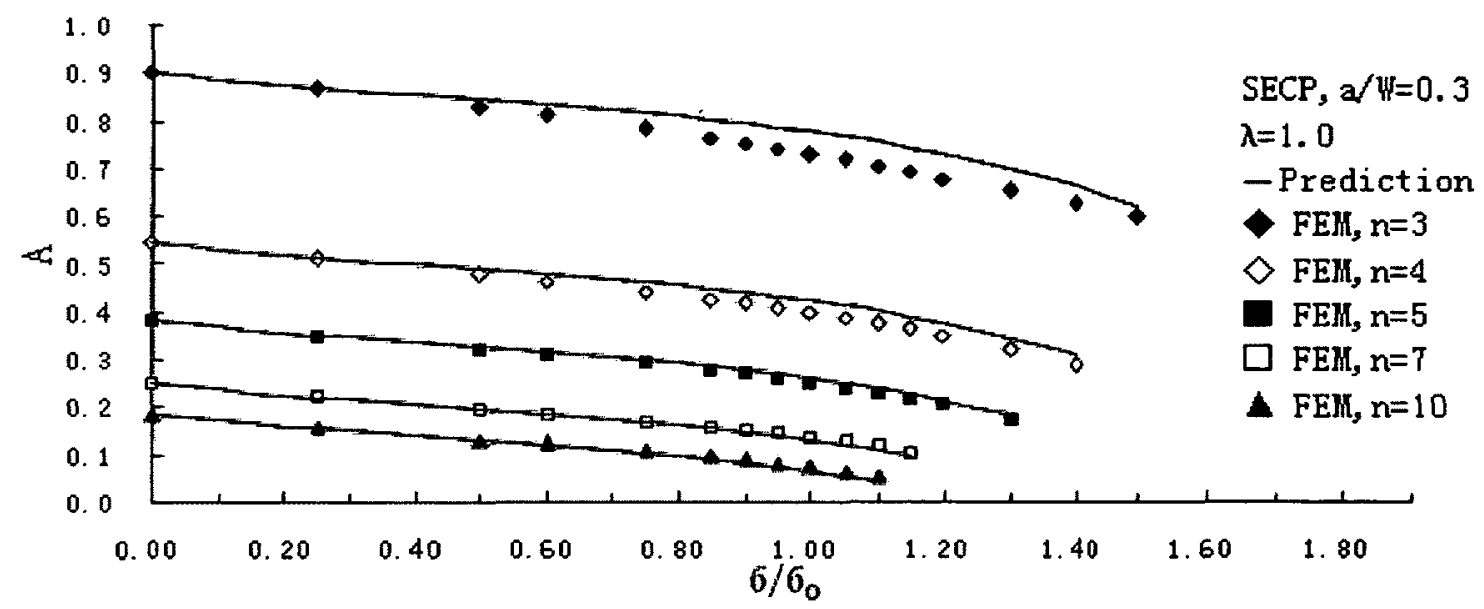

Figure 5.13 Comparisons of predicted $A$ values by curve shape similarity with FEA data for SECP, $a / W=0.3, \lambda=1.0$

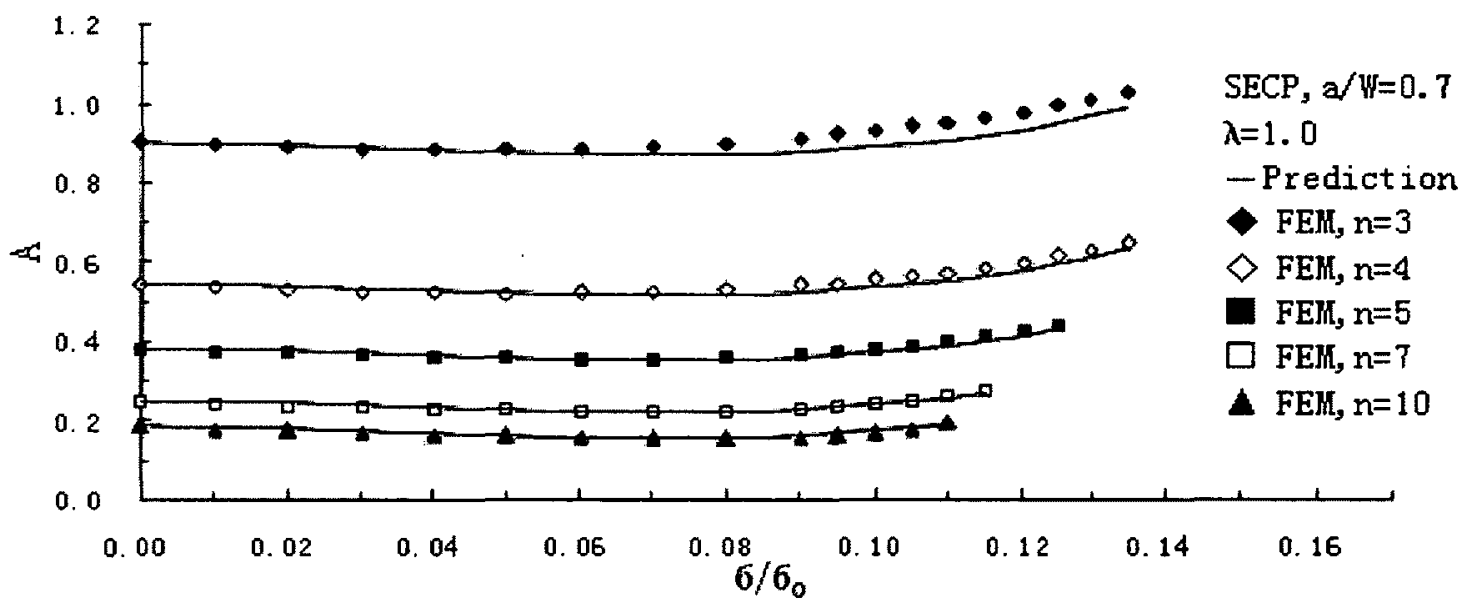

Figure 5.14 Comparisons of predicted $A$ values by curve shape similarity with FEA data for SECP $, a / W=0.7, \lambda=1.0$ 


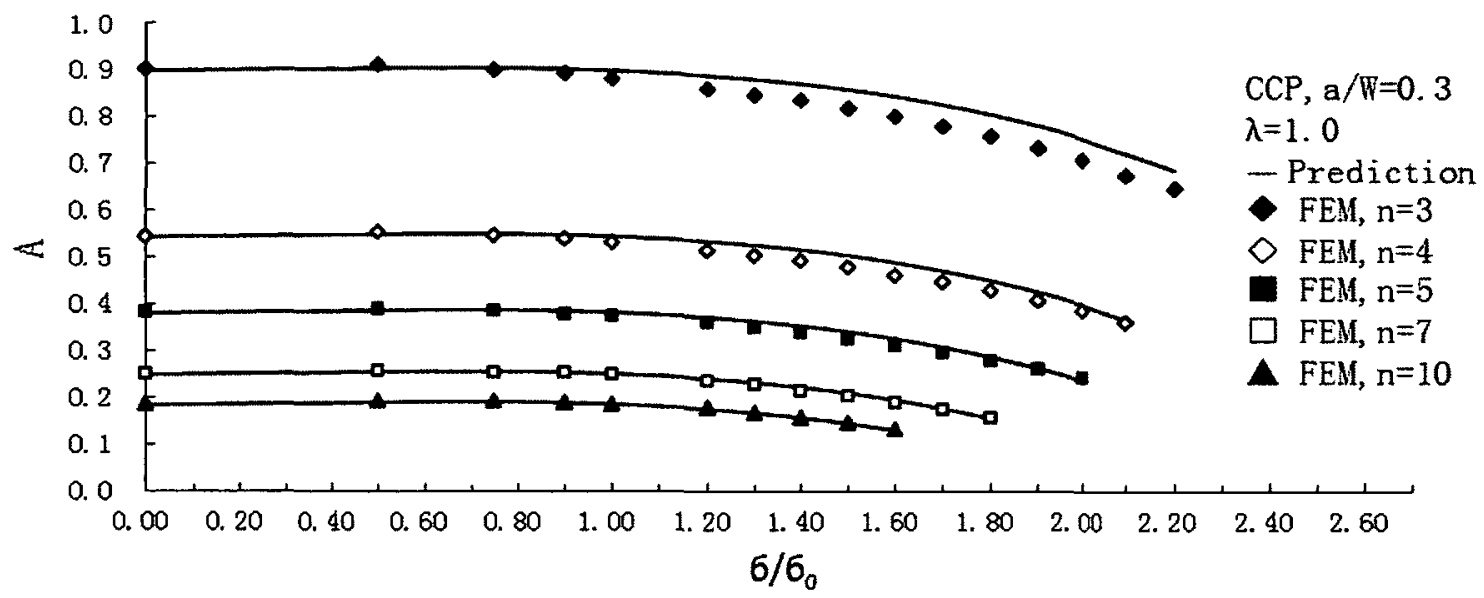

Figure 5.15 Comparisons of predicted $A$ values by curve shape similarity with FEA data for $\mathrm{CCP}, a / W=0.3, \lambda=1.0$

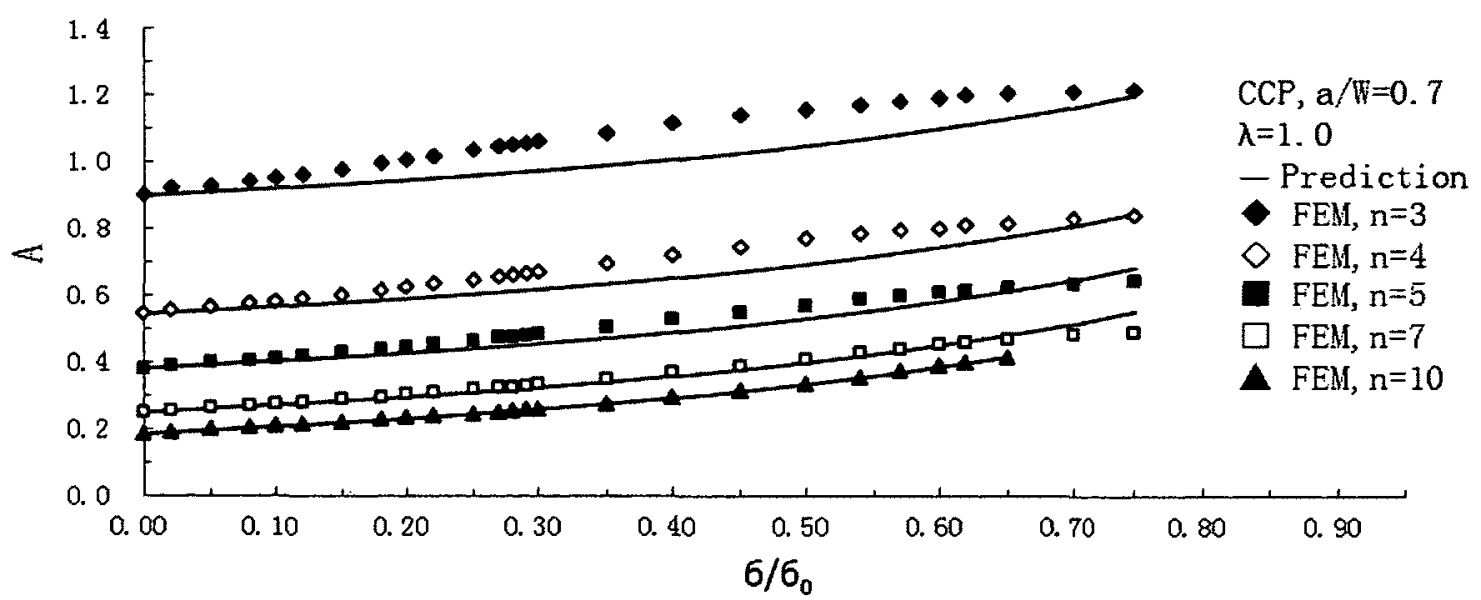

Figure 5.16 Comparisons of predicted $A$ values by curve shape similarity with FEA data for $\mathrm{CCP}, a / W=0.7, \lambda=1.0$ 


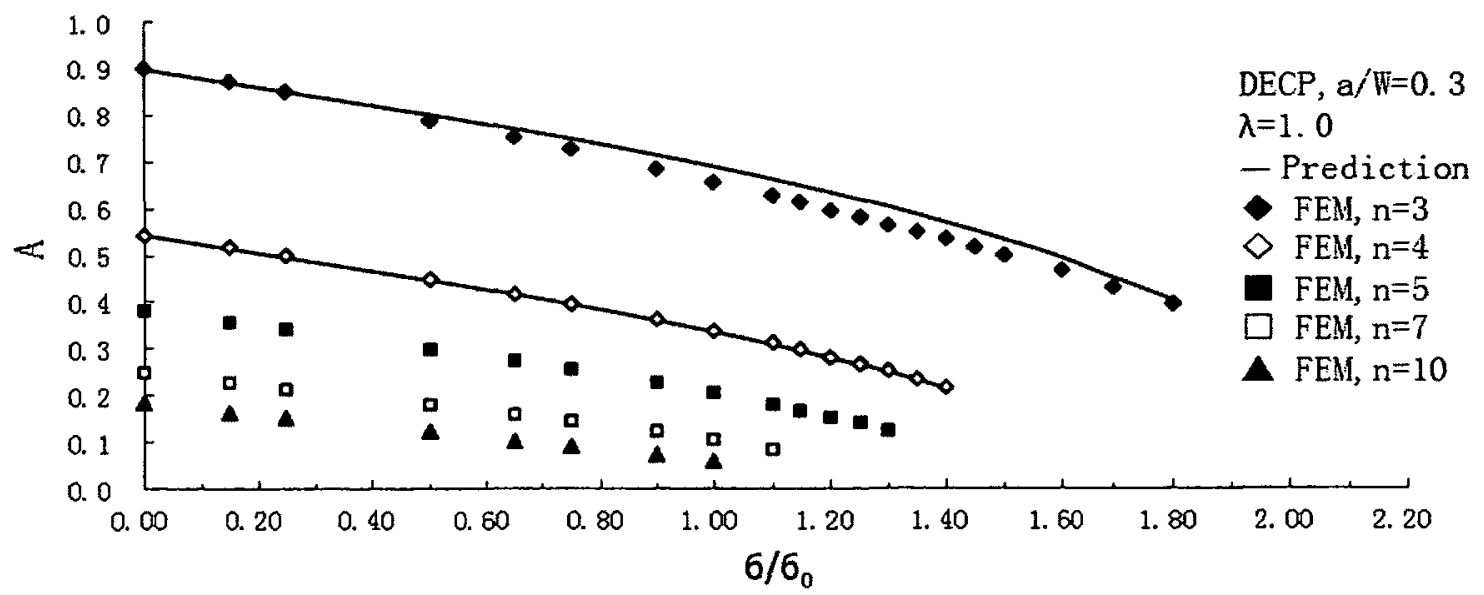

Figure 5.17 Comparisons of predicted $A$ values by curve shape similarity with FEA data for DECP $, a / W=0.3, \lambda=1.0$

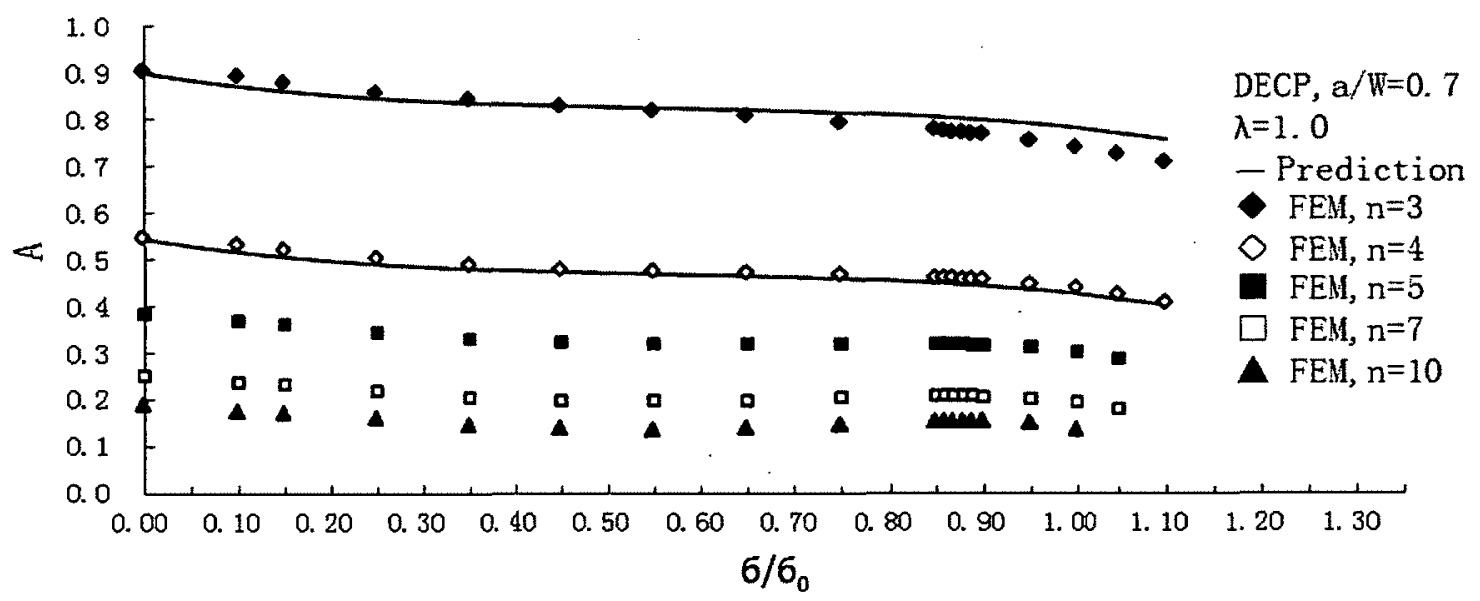

Figure 5.18 Comparisons of predicted $A$ values by curve shape similarity with FEA data for DECP, $a / W=0.7,=1.0$ 


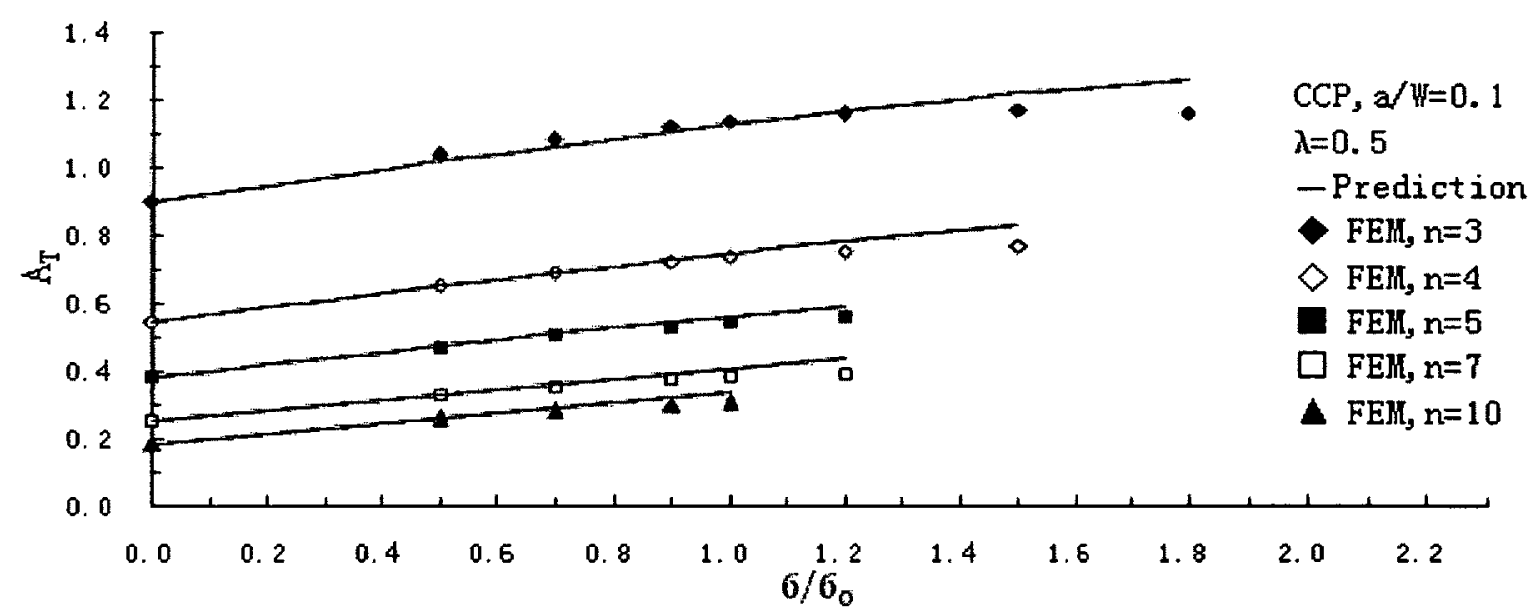

Figure 5.19 Comparisons of predicted $A$ values from $T$-stress with FEA data for CCP, $a / W=0.1, \lambda=0.5$

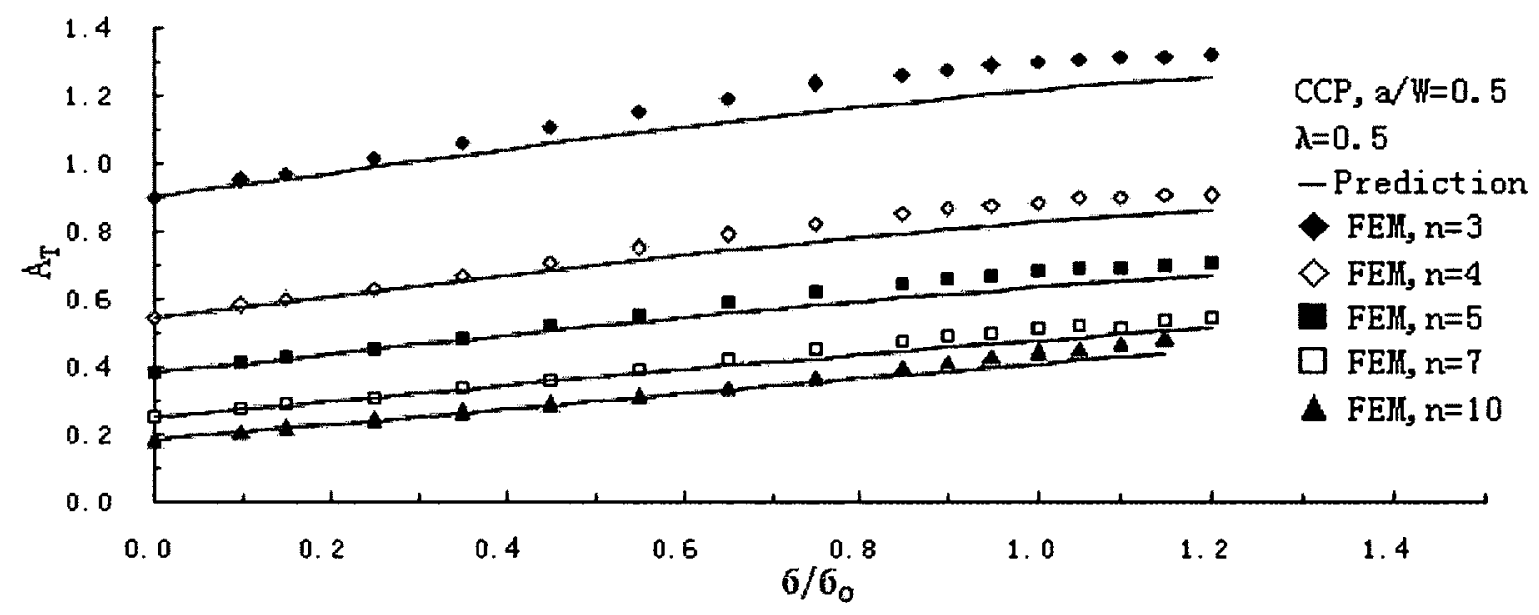

Figure 5.20 Comparisons of predicted $A$ values from $T$-stress with FEA data for CCP, $a / W=0.5, \lambda=0.5$ 


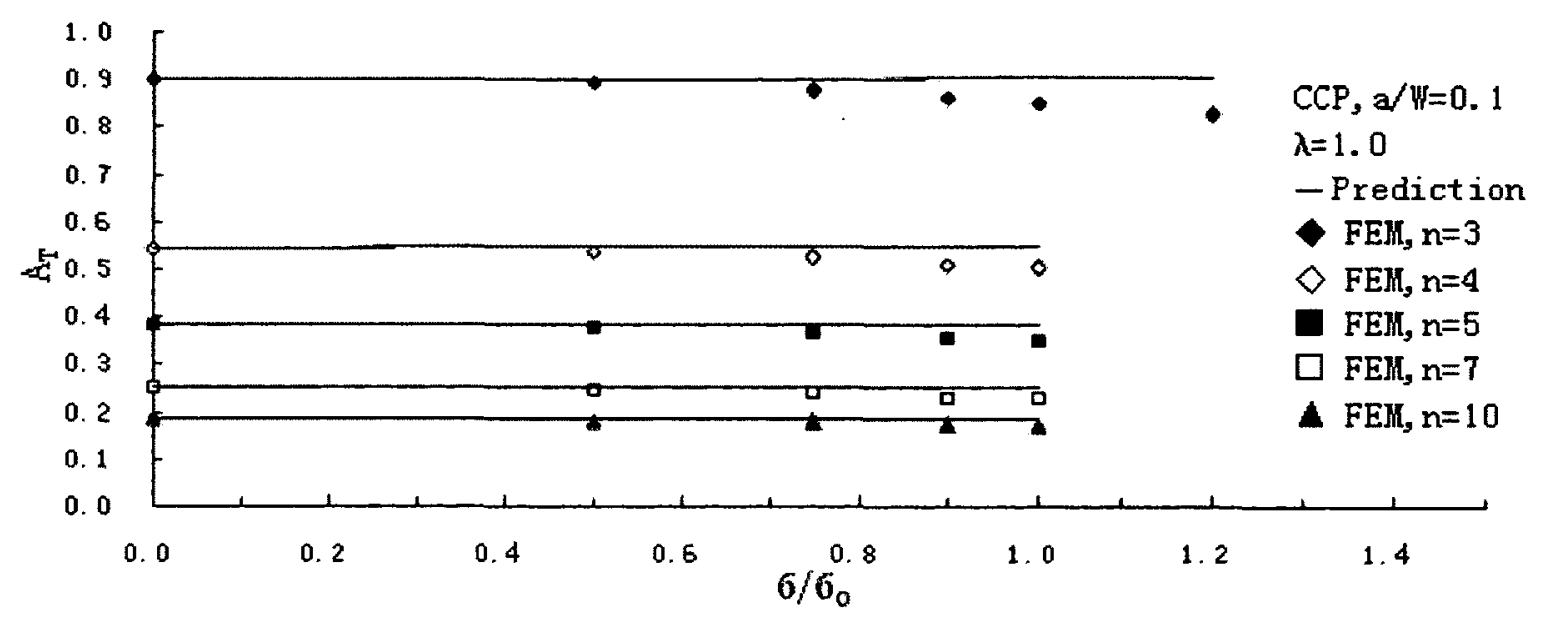

Figure 5.21 Comparisons of predicted $A$ values from $T$-stress with FEA data for CCP, $a / W=0.1, \lambda=1.0$

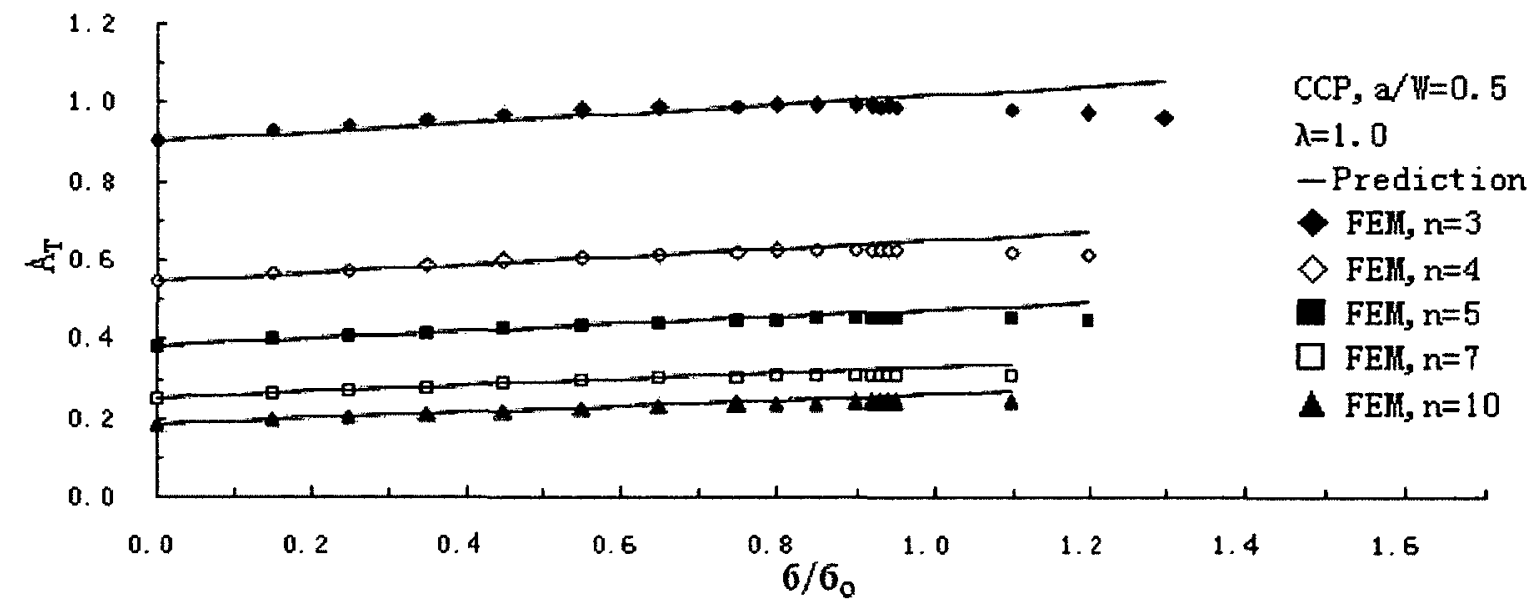

Figure 5.22 Comparisons of predicted $A$ values from $T$-stress with FEA data for CCP, $\mathrm{a} / \mathrm{W}=0.5, \lambda=1.0$ 


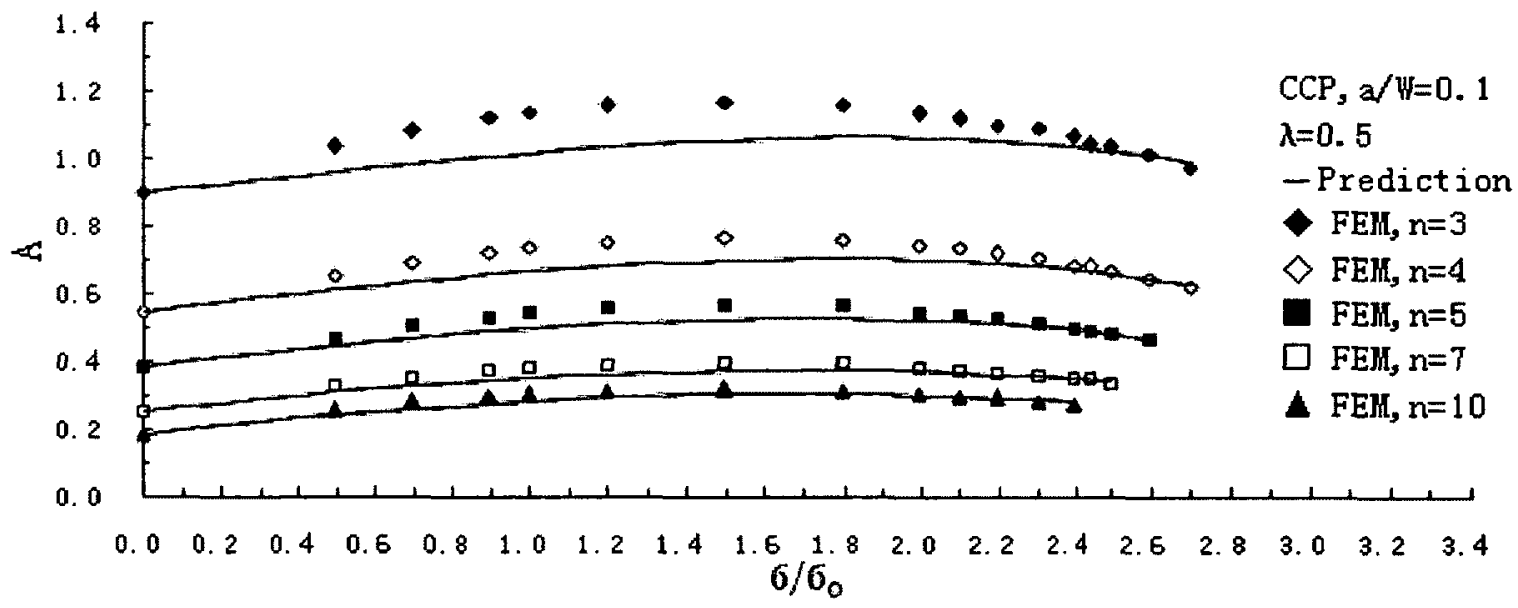

Figure 5.23 Comparisons of predicted $A$ values by superposition with FEA data for CCP, $a / W=0.1, \lambda=0.5$

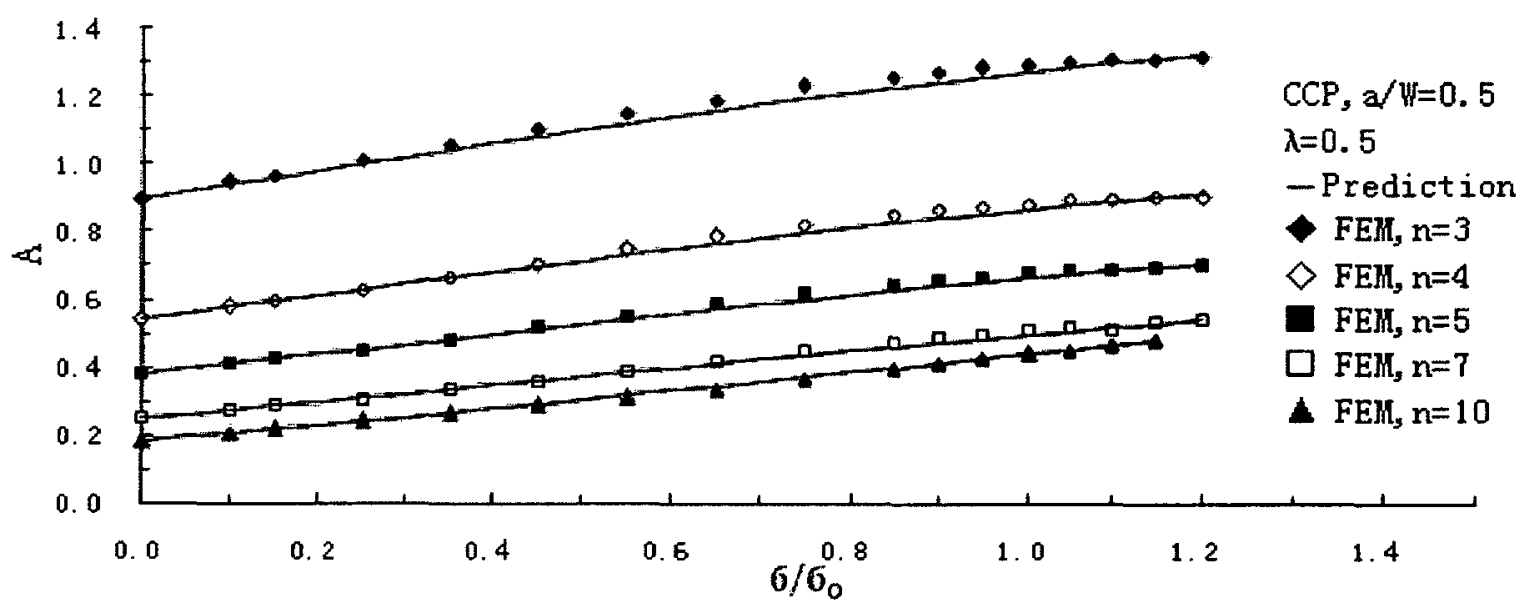

Figure 5.24 Comparisons of predicted $A$ values by superposition with FEA data for CCP, $a / W=0.5, \lambda=0.5$ 


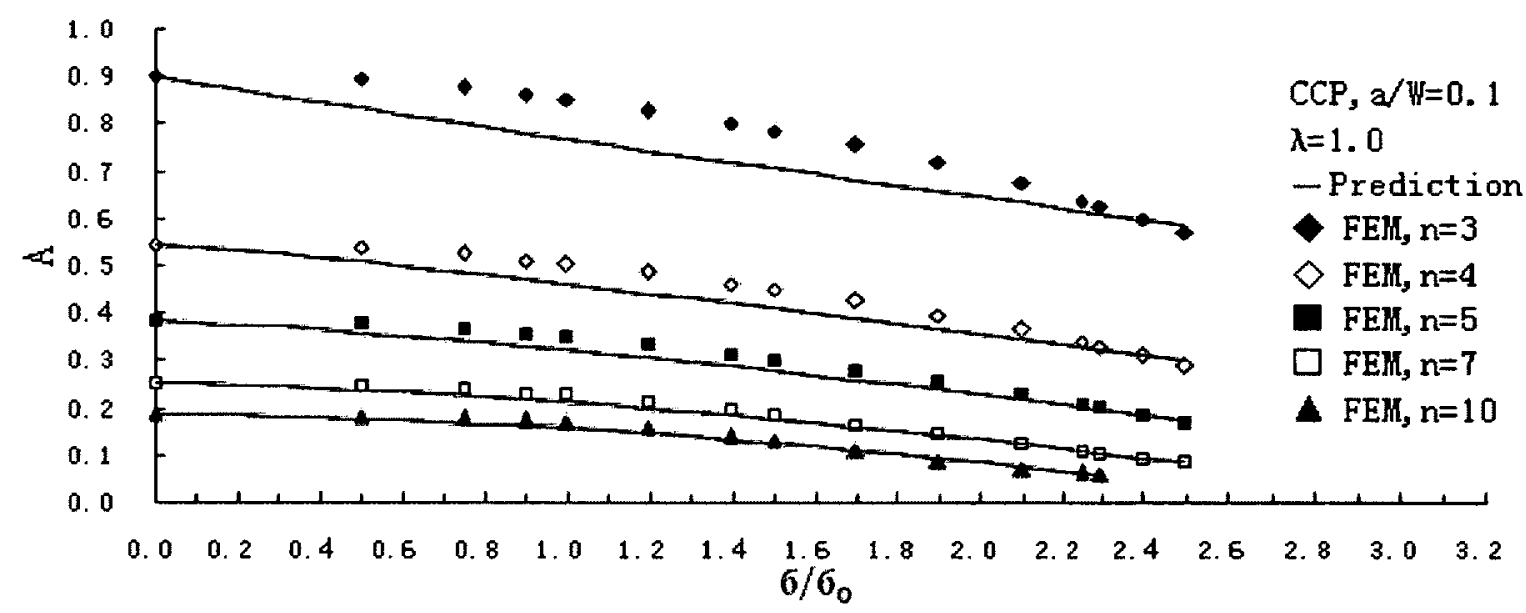

Figure 5.25 Comparisons of predicted $A$ values by superposition with FEA data for CCP, $a / W=0.1, \lambda=1.0$

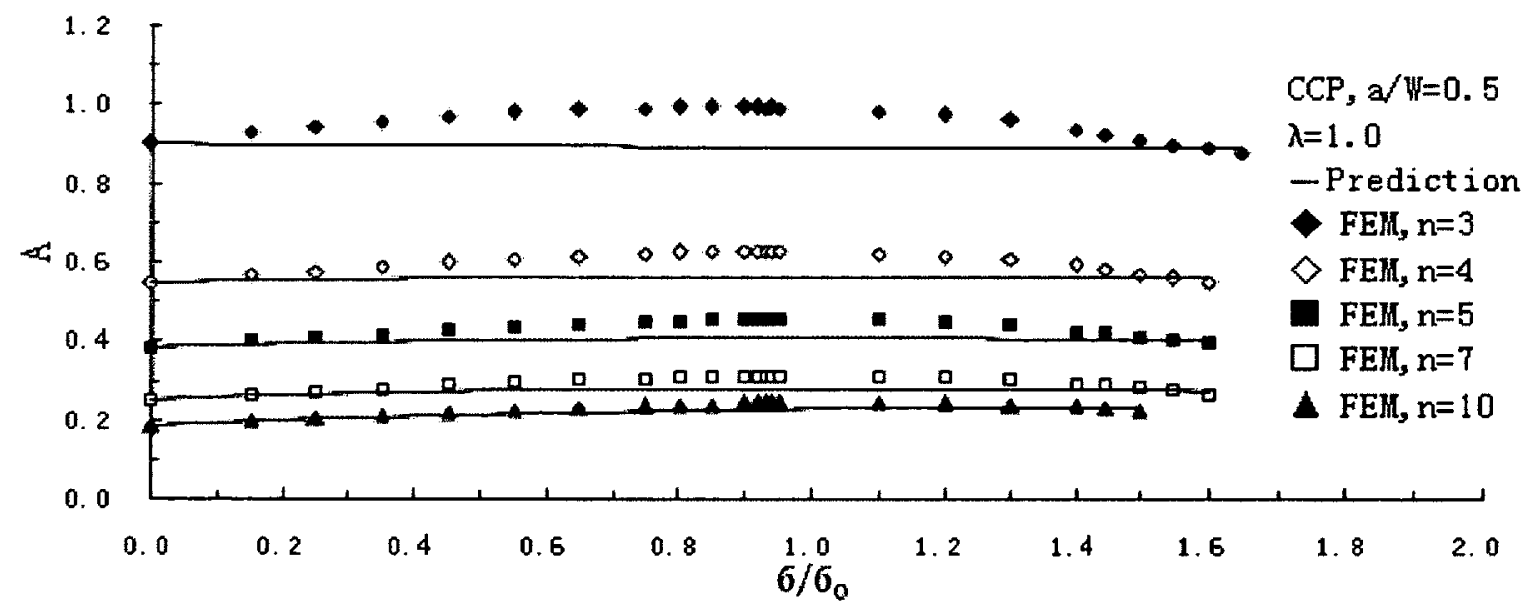

Figure 5.26 Comparisons of predicted $A$ values by superposition with FEA data for CCP, $a / W=0.5, \lambda=1.0$ 


\section{CHAPTER 6 SOLUTION OF CONSTRAINT PARAMETER $\boldsymbol{A}$}

\section{FOR 3D SPECIMENS}

\subsection{Introduction}

In this chapter, crack-front stress fields of the three dimensional (3D) modified boundary layer (MBL) problem and single edge cracked plate (SECP) specimen with small thickness are obtained through extensive finite element analyses (FEA). The utilized finite element models and material properties are described. Based on the FEA results, numerical solutions of constraint parameter $A$ for the 3D MBL and SECP are obtained by the fitting method proposed by Nikishkov et al. (1995a).

The distributions of the resulting $J$-integral and $A$ values are presented. With the obtained numerical solutions of constraint parameter $A$, the constraint effects in $3 \mathrm{D}$ models are analyzed and discussed.

Through the numerical solutions of constraint parameter $A$ for the 3D SECP model, coefficients for empirical formulas to the estimate parameter $A$ in specified planes along the model thickness are determined by $A$ curve-shape similarity. Based on numerical solutions for the parameter $A$ for the 3D MBL problem, expressions of $A-T$ relationships in each specified plane are determined. Similarly, specific formulas estimating the parameter $A$ directly from $T$-stress at specified planes of the 3D SECP model under low loads are proposed. With the determination of the coefficients $a_{l}$ for specified planes of the 3D SECP based on $A$ values from $T$-stress, and numerical solutions of parameter $A$, approximation equations predicting parameter $A$ for any specified plane of the 3D SECP from low to high load (small-scale to large-scale yielding) can be determined.

Solutions of constraint parameter $A$ predicted by each of the three kinds of estimation formulas proposed in this chapter are compared with those obtained from FEA results to discuss the applicability of the developed approximation formulas to $3 \mathrm{D}$ cracked specimens under uniaxial and biaxial loading. 


\subsection{Finite Element Analysis of 3D Modified Boundary Layer Formulation}

\subsubsection{Problem definition}

Figure 6.1 shows the geometry and coordinate systems (polar and Cartesian coordinates) of the three-dimensional (3D) modified boundary layer (MBL) problem. In the figure, $R$ presents the maximum radius of the 3D MBL model and $t$ indicates the thickness of the model. The ratio $t / R$ is fixed as 0.1 and the thickness $t$ is chosen as $0.04064 \mathrm{~m}$ for the investigation in this chapter.

Same as for the 2D modified boundary layer problem, the material behavior in the finite element analysis (FEA) for the 3D MBL is described by deformation plasticity theory. A Ramberg-Osgood power-law strain hardening relation for multi-axial cases is provided in the commercial finite element code ABAQUS (2006). As illustrated in section 2.7, the near crack-front fields in specified planes along the $3 \mathrm{D}$ model thickness (excepting planes near the free surface) can be considered as being under plane strain conditions. The stress-strain curve from the Ramberg-Osgood relation for uniaxial or biaxial load is described in Eq. (2.4).

For the FEA for the 3D MBL problem, the same material properties as those for the $2 \mathrm{D}$ case are utilized, i.e. yield stress $\sigma_{0}=4.0 \times 10^{8} \mathrm{~Pa}$; modulus of elasticity $E=2.0 \times 10^{11} \mathrm{~Pa}$; Poisson ratio $v=0.3$; hardening exponent $n=3,4,5,7$ and 10 ; and material coefficient $\alpha=1.0$. A wide range of both high and low strain hardening behaviors is covered by these material properties.

A finite element formulation of 3D MBL with small model thickness is carried out.

In the present $3 \mathrm{D} \mathrm{MBL}$ analysis, the relative thickness of the model is small (geometry ratio $t / R=0.1$ ), therefore, in far fields of the model, plane stress state is dominant, although the near-front fields are in plane strain state (see section 2.7). Since the far fields of the model are in plane stress state, taken to be the same as that used by Yuan and Brocks (1998), the loading condition of the 3D MBL model is described by the displacement boundary conditions for plane stress case. Therefore, plane stress displacement boundary conditions, Eqs. (2.9) and (2.12), are utilized as load application of the 3D MBL model. In Eqs. (2.9) and (2.12), displacement boundary conditions are 
described by stress intensity factor $K_{I}$ and $T$-stress.

According to the research of Kim et al. (2001), the FEA results in the region near the crack front of thin 3D MBL model are nearly identical with and without the application of $u_{z}$ on the boundary of the 3D model. The difference is only in the region near the far field boundary. In the present investigation, the finite element analysis carried out for 3D MBL problem does not include the application of $u_{z}$, as this work focuses on the crack-front region of 3D model.

The value of the far-field $J$-integral is fixed as $2.55 \times 10^{4} \mathrm{~J} / \mathrm{m}^{2}$, which is related to $K_{I}$ by Eq. (2.11), so as to simulate the small-sale yielding (low load) condition of the MBL problem. Various values of $T$-stress, $T / \sigma_{0}=-1.0,-0.8,-0.6,-0.4,-0.2,0.0,0.2$, $0.4,0.6,0.8,1.0$ at different hardening exponents $n=3,4,5,7,10$ are applied in a series of finite element analyses.

\subsubsection{Finite element analysis and parameter $\boldsymbol{A}$ determination}

\subsubsection{Finite element modeling}

Only a quarter model of the 3D MBL is meshed for finite element analysis due to geometrical symmetry. The origin of the coordinates is set at the crack tip in the middle plane of the 3D model. The quarter of the 3D MBL model with positive $y$ values and negative $z$ values is meshed, see Figure 6.1.

A prototypical three-dimensional (3D) finite element mesh used for the 3D MBL formulation is illustrated in Figure 6.2. There are 13440 elements in the 3D mesh in total and the element type for all of the elements is assigned as a 20-node quadratic hybrid brick with linear pressure, reduced integration (ABAQUS (2006)). The radial sizes for all elements in the finite element mesh are varied according to a geometric progression. Fourteen element layers are generated along the thickness of the 3D MBL model. From the middle plane to the surface, the thickness of first element layer is set small enough to close the middle plane, and the second layer has the largest thickness. From the second to

fourteenth layer, the thicknesses are varied according to a geometric progression. The smallest element thickness size is in the element layer at the free surface of the $3 \mathrm{D} \mathrm{MBL}$ 
model.

For the current $3 \mathrm{D}$ case, the investigation will be performed on several specified planes along the thickness of the 3D MBL model. Three planes are chosen, plane I at $z / t=1.4 \%$, plane II at $z / t=27.1 \%$ and plane III at $z / t=49.8 \%$, as shown in Figure 6.2 .

Along the uncracked ligament of model, similar as the boundary condition for the 2D MBL formulation, elements are constrained in $y$ direction but in the $x$ direction and $z$ direction they are free to displace. In the mid-plane ( $x-y$ plane), element nodes are constrained in $z$ direction but are free to displace in the $x$ and $y$ directions to simulate the symmetry of the mid-plane in the entire $3 \mathrm{D}$ geometry.

\subsubsection{Determining parameter $A$ based on finite element analysis}

To obtain numerical $A$ solutions near the $3 \mathrm{D}$ crack front, the fitting method suggested by Nikishkov et al. (1995a) (Eqs. (2.29)-(2.32)) to calculate constrain parameter $A$ value from FEA results is utilized. The fitting method is developed based on a plane strain condition. As discussed in section 2.7, except for the planes near the free surface of the 3D model, in any plane along the model thickness the near crack front stress fields are in plane strain. Therefore, for the current 3D MBL problem, the fitting method suggested by Nikishkov et al. (1995a) for determining the solutions of constrain parameter $A$ can be used in any plane along model thickness except for the planes near the model free surface.

In the 3D MBL problem model used in this chapter, the fitting area to calculate the $A$ values based on the FEA results by Eq. (2.29) is limited by $1.5 \leq \bar{r} \leq 3$ and $0^{0} \leq \theta \leq 45^{\circ}$. The opening stress, $\sigma_{\theta}$, is the stress component used for the fitting procedure following the method suggested by Nikishkov et al. (1995a).

\subsubsection{Results and discussion}

\subsubsection{Verifications}

All finite element analyses for the $3 \mathrm{D}$ modified boundary layer (MBL) problem having a far-field $J$-integral, $J=2.55 \times 10^{4} J / \mathrm{m}^{2}$, generate a plastic zone with a small 
radius, $r_{\mathrm{p}}$, in the finite element model, which is less than $5 \%$ of the maximum radius $r_{\max }$ $=R$ of the model. These plastic zones are small enough to simulate small-scale yielding (SSY) conditions for the 3D MBL problem. Therefore, the choice of far-field stress $(J$ integral) is appropriate.

For example, with specified far-field stress corresponding to a $J$-integral of $2.55 \times 10^{4} \mathrm{~J} / \mathrm{m}^{2}$, for hardening exponent $n=10$, the 3D MBL model generates a plastic zone $r_{\mathrm{p}}$ which is less than $0.78 \%(0.87 \%)$ of the outside radius $r_{\max }=R$ of the model on mid-plane (surface plane) under standard small-scale yielding status $(T=0)$, which is one of the cases of SSY conditions. The element size of the 3D MBL mesh decreases geometrically towards the crack-front $(r \rightarrow 0)$ to focus the greatest refinement near the crack-front. Under the $n=10$ and $T=0$ conditions, typically, around 230 elements are included in the plastic zone with the 960 elements in each element layer of the SSY 3D model. For all the small-scale yielding cases $\left(T / \sigma_{0}=-1.0-1.0, n=3-10\right)$, on plane I, II and III, the number of points used in the fitting area for determining the parameter $A$ is at least 24 within the specified fitting area used for calculating the $A$ values from the FEA results by Eq. (2.29).

Similar finite element mesh as for the 2D MBL formulation is utilized here for the 3D MBL problem. The difference between the mesh for the 2D MBL model and that for the 3D MBL model is the thickness of model and the number of element layers along the thickness. The finite element mesh for the $2 \mathrm{D} \mathrm{MBL}$ problem as well as the procedure for determining $A$ values were validated in section 4.1. Therefore, the current 3D MBL finite element model and corresponding analysis procedure are considered appropriate to determine the constraint parameter $A$ in the $3 \mathrm{D} \mathrm{MBL}$ problem.

\subsubsection{Numerical solutions of parameter $A$}

The numerical solutions of constraint parameter $A$ from FEA results for the 3D MBL problem are determined based on the analysis/calculation procedure illustrated above. Numerical solutions for $T / \sigma_{0}=-1.0,-0.8,-0.6,-0.4,-0.2,0.0,0.2,0.4,0.6,0.8$, 1.0 and hardening exponents $n=3,4,5,7,10$ have been obtained at all planes (fourteen layers with four planes in each layer) of the 3D model (mesh). As mentioned in section 
2.7 , the values of parameter $A$ vary with the plane location along the model thickness. In Tables $6.1,6.2$ and 6.3 , numerical solutions of parameter $A$ in the three specified planes, plane I, plane II and plane III, are shown. As discussed in section 3.1.1, the zero load status of specimens can be simulated by the standard SSY case that corresponds to the $T=0$ case of the MBL problem. The numerical solution for parameter $A$ under standard SSY status, $A_{S S Y}$, can be found in the column $T / \sigma_{0}=0$ of Tables 6.1, 6.2 and 6.3. For the 3D model, solutions of $A$ vary depending on not only the hardening exponent, $n$, but also on the location of each plane along the crack-front (thickness), therefore the $A_{S S Y}$ for the 3D model should be expressed as $A_{S S Y}(n, z)$. Results in Tables 6.1-6.3 are illustrated in Figures $6.3-6.5$, respectively.

Figures $6.3,6.4$ show that generally the $A$ vs. $T / \sigma_{0}$ curves on planes I and II for different hardening exponent $n$ have a similar shape and only differ from each other by zero load solutions of parameter $A$. This characteristic has been utilized to develop a simplified format of $A-T$ relationship, see section 3.2.2.3. For the plane near the free surface (planes III, Figure 6.5), the curve shape similarity does not apply well for curves of $A$ vs. $T / \sigma_{0}$ with various values of $n$.

\subsection{Finite Element Analysis of 3D Test Specimens}

\subsubsection{Problem definition}

Finite element analyses for 3D mode I test specimens of the single edge cracked plate (SECP) under both uniaxial $(\lambda=0.0)$ and biaxial load $(\lambda=1.0)$ are performed. The 3D SECP model is illustrated for geometry, coordinate systems and load condition in Figure 6.6. The geometry of the 3D SECP specimen model is defined by the ratio of height to width, $H / W$, the relative crack length $a / W$ and the ratio of model thickness to width, $t / W$. For the 3D SECP models, the ratio $H / W$ is fixed at 3.0 and the ratio $t / W$ at 0.1 with a thickness $t=0.04064 \mathrm{~m}$. Models with various relative crack lengths $a / W=0.1,0.3,0.7$ are investigated.

Deformation plasticity theory is used for the finite element analysis of the 3D SECP specimens under uniaxial and biaxial loading. The utilized commercial code ABAQUS 
(2006) provides a Ramberg-Osgood power-law strain hardening relation (see Eq. (2.4)).

Material properties for the finite element analysis of the 3D SECP specimens investigated in this chapter are the same as those for the FEA of the 2D SECP except for the values of hardening exponent $n$ : yield stress $\sigma_{0}=4.0 \times 10^{8} \mathrm{~Pa}$; modulus of elasticity $E=2.0 \times 10^{11} \mathrm{~Pa}$; Poisson ratio $v=0.3$; material coefficient $\alpha=1.0$; and hardening exponent $n=3,5$ and 10. The material properties cover a wide range of both high and low strain hardening behaviors.

The remote load applied to the 3D SECP specimen, $\sigma$, is normalized by the material yield stress $\sigma_{0}$. Two loading conditions, uniaxial $(\lambda=0.0)$ and biaxial $(\lambda=1.0)$, are investigated for the present 3D SECP specimen model.

In order to compare the $T$-stress values with those reported by Nakamura and Parks (1992), a 3D SECP model with a relative thickness $t / W=0.5$ is also investigated for $a / W=0.1,0.2,0.3,0.4,0.5,0.6$ and 0.7 (with $n=10$ and $\lambda=0.0$ ).

\subsubsection{Finite element analysis and parameter $\boldsymbol{A}$ determination}

\subsubsection{Finite element modeling}

Due to the component symmetry (see Figure 6.6), only a quarter of the 3D SECP structure is meshed in the finite element analysis (FEA). The three-dimensional (3D) finite element mesh used for modeling the 3D SECP models is illustrated in Figure 6.7. In the mesh, there are 9058 elements in total, The elements are 20 -node quadratic hybrid bricks with linear pressure, reduced integration (ABAQUS (2006)). Note that in the area of transition from crack-tip core elements to the global mesh, there are a total of 7 wedge shaped elements, which are degenerated 20-node elements. Same as that for 3D MBL mesh, along the thickness of the 3D SECP model, 14 element layers are used. Except for the first layer (adjacent to the middle plane), the thickness of the other thirteen element layers is varied according to a geometric progression. The smallest element thickness size is used in the element layer at the free surface of model.

For the 3D SECP model, same as for 3D MBL case, the research will be carried out mainly on three specified planes along the thickness (crack-front). In the same way as for 
3D MBL model, these three planes are chosen as: plane I at $z / t=1.4 \%$, plane II at $z / t=27.1 \%$ and plane III at $z / t=49.8 \%$, as illustrated in Figure 6.7 .

Displacement boundary conditions for the 3D SECP mesh are applied to the element nodes of the uncracked ligament, which are constrained in the $y$ direction, they are free to displace in the $x, z$ directions. For symmetry, element nodes on the middle plane ( $x-y$ plane) are constrained in the $z$ direction but are free to displace in the $x$ and $y$ directions.

\subsubsection{Determining parameter $A$ based on finite element analysis}

As mentioned in section 6.1.2.2 for the 3D MBL model, the fitting method suggested by Nikishkov et al. (1995a) to determine the numerical solutions of constraint parameter $A$ (Eqs. (2.29-2.32)) can be utilized in any plane along the thickness except for the planes near the free surface of the present 3D SECP model.

In each specified plane, the fitting area used to determine the $A$ values for the 3D SECP from the FEA results following the fitting method suggested by Nikishkov et al. (1995a) is set as $1.5 \leq \bar{r} \leq 3$ and $0^{0} \leq \theta \leq 45^{\circ}$, and the opening stress $\sigma_{\theta}$ is chosen as the stress component in the fitting procedure.

\subsubsection{Results and discussion}

\subsubsection{Verification}

For the 3D SECP, the number of integrated points within the fitting area is generally between 14 and 56 when calculating the constraint parameter $A$ values from the FEA results by Eq. (2.29) with the specified fitting area and stress component.

To verify the 3D mesh used for 3D SECP finite element analysis, the $T$-stress solutions for the 3D mesh with relative thickness $t / W=0.5$ are obtained by FEA for $a / W=0.1,0.2,0.3,0.4,0.5,0.6$ and 0.7 . To compare $T$-stress results with those reported by Nakamura and Parks (1992), the relative thickness of the 3D mesh $(t / W)$ is changed to 0.5 instead of the 0.1 which is used for current investigation, and the $T$-stress solutions in the middle plane $(z=0)$ are determined for comparison. The comparison between the obtained $T$-stress solutions and those determined by Nakamura and Parks (1992) for $z=0$ plane is shown in Figure 6.8. 
The above verification for the FEA mesh of the 3D SECP specimen is only based on the linear elastic state ( $T$-stress). The current investigation focuses on elastic-plastic analysis. Therefore, a further verification based on constraint parameter $A_{2}$ is also performed. In the research of Zhu et al. (2002), based on finite element analysis, numerical solutions of the constraint parameter $A_{2}$ under several remote loading conditions have been obtained for a thin 3D CCP specimen with $H / W=2.0, t / W=0.1$ and $a / W=0.5$. With a specified crack depth $a / W=0.5$, the present FEA mesh $(H / W=3.0, t / W$ $=0.1$ ) for the 3D SECP is used to perform finite element analysis based on the same material properties as those utilized by $\mathrm{Zhu}$ et al. (2002), namely $E=222.5 \mathrm{GPa}$, $\sigma_{0}=445 \mathrm{MPa}, v=0.3$ and $\alpha=1.0$. The FEA is performed under remote load corresponding to $J^{\text {center }} /\left(\mathrm{a}_{0}\right)=0.00571$, which is one of the load levels used in the research of Zhu et al. (2002). In the expression of load, $J^{\text {center }}$ is the $J$-integral value in the mid-plane of the mesh.

Based on the FEA results, the numerical solutions of parameter $A$ for the current mesh are determined by the fitting method suggested by Nikishkov et al. (1995a). Then the solutions for parameter $A$ are converted to parameter $A_{2}$ values through the relationship between $A$ and $A_{2}$ (Eq. (2.34)). The results of parameter $A_{2}$ are compared with those reported by Zhu et al. (2002) in several planes along the model thickness. A comparison of results is shown in Table 6.4 and in Figure 6.9. Reasonably good agreement is found with maximum difference of $7.40 \%$.

Through the calculations and comparisons above, it can be concluded that the current utilized 3D finite element mesh and corresponding procedure of determining parameter $A$ are appropriate to perform constraint analysis for the 3D SECP model.

\subsubsection{Numerical solutions of parameter $A$ and discussion for constraint effect}

To determine the constraint parameter $A$ values for the three-dimensional SECP specimen, the finite element analyses (FEA) of the 3D SECP model are performed. Then through the fitting method suggested by Nikishkov et al. (1995a), numerical solutions of constraint parameter $A$ are obtained from the FEA results. With remote tension loading increasing to cover deformation range varying from small-scale to large-scale yielding, numerical results for parameter $A$ for the whole 3D SECP model are obtained. 
Numerical solutions for parameter $A$ have been obtained on each plane (fourteen layers with four planes in each layer) along the crack-front (model thickness). As an example, the variation of $A$ along the thickness for $a / W=0.3, n=5$ under a lower load $\left(\sigma / \sigma_{0}=0.25\right)$ and a higher load $\left(\sigma / \sigma_{0}=0.55\right)$, are presented. Figure 6.10 illustrates the variations of constraint level (parameter $A$ ) along the crack-front for both the uniaxial and biaxial condition. The corresponding variations of load level ( $J$-integral) along the crackfront are presented in Figure 6.11. The data shown in Figures 6.10 and 6.11 are listed in Table 6.5 and 6.6 , respectively, for reference.

By examining Figure 6.10 (Table 6.5) for the parameter $A$, as well as Figure 6.11 (Table 6.6) for the $J$-integral, it can be found that the minimum value of the constraint parameter $A$ and the maximum value of the $J$-integral are on middle plane, i.e. both constraint and stress reach their highest levels on the middle plane. This phenomenon is consistent with the observation of experimental work that cracks always initiate at the middle plane of a plate. Except for the planes near the free surfaces, both constraint $(A)$ and stress $(J)$ levels have only a small variation along the crack-front (thickness). These findings coincide with those reported by Zhu et al. (2002) and Kim et al. (2001), implemented the $J-A_{2}$ approach.

The variation of stress level ( $J$-integral) along the crack-front is slightly affected by the biaxial load ratio $\lambda$, see Figure 6.11 (Table 6.6). Figure 6.10 (Table 6.5) shows that the level of constraint increases with the increase of biaxial loading ratio $\lambda$, except on the planes near the free surface where increase of constraint is very small. This conclusion is consistent with the findings for 2D models (see section 5.1.3.2).

Similar as for 2D models under biaxial loading (section 5.1.3.2), according to the research of Chao and Zhu (1998) on 2D plane strain models, the requirement for ductile fracture initiation in the shallow cracked specimens under $J-A_{2}$ dominant conditions is $a \sigma_{0} / J>6$, and that for deep cracks is $b \sigma_{0} / J>11$. The $a$ and $b$ are crack length and remaining ligament, respectively. The highest loads considered in the present finite element analysis (FEA) are the load levels corresponding to above criterion values. The values of $a \sigma_{0} / J$ or $b \sigma_{0} / J$ for the highest loading level of each case in both plane I and 
plane II are within the ranges $6-18,6-25,22-45$ (under $\lambda=0.0$ ) or $13-28,8-23,21-45$ (under $\lambda=1.0$ ) for $a / W=0.1,0.30 .7$, respectively. Research of Chao and Zhu (1998) shows that, for shallow cracks, the values of $a \sigma_{0} / J \leq 50$ already nearly correspond to fully plastic deformation; for deep ones, the values of $b \sigma_{0} J J$ around 30 correspond to the fully plastic condition. It means the current FEA calculations for 3D SECP have reached the fully plastic condition for almost all of the investigated cases.

The determined numerical solutions of parameter $A$ for the three specified planes of the 3D SECP are listed in Tables 6.7-6.12. For plane I, plane II and plane III respectively, Tables 6.7-6.9 show the results under load ratio $\lambda=0$, and Tables 6.10-6.12 list the results under $\lambda=1.0$. The numerical results of $A$ for planes I and II are shown in Figures 6.12-6.17. For $a / W=0.1,0.3,0.7$, Figures $6.12-6.14$ show the results under a load ratio $\lambda=0$, and those under $\lambda=1.0$ are illustrated in Figures 6.15-6.17. In these tables and figures for the 3D SECP, the parameter $A$ solutions under zero load are in fact the $A$ values obtained from the standard SSY case of the 3D MBL problem (column $T / \sigma_{0}=0$ of Tables 6.1, 6.2 and 6.3), see section 6.1.3.2.

By analyzing the numerical solutions of parameter $A$ for the 3D SECP specimen (Tables 6.7-6.12), it is found that, in general, the maximum external loading ratios $\left(\sigma / \sigma_{0}\right)$, which are determined based on the criteria suggested by Chao and Zhu (1998), increase with the decrease of hardening coefficient $n$. In addition, it is observed that, generally, with the increase of biaxial loading ratio $\lambda$ from 0.0 to 1.0 , the constraint level of the 3D SECP specimen increases (i.e. the parameter $A$ values decrease). This is consistent with the finding shown in previous paragraphs. For shallow cracks and/or cases with smaller hardening exponent $n$, the effect from the change in biaxiality is significant, while for deep cracks and/or larger values of $n$, the effect is small or even negligible. For example, when $\lambda$ increases from 0.0 to 1.0 , for $a / W=0.1$ (shallow crack) with $n=3$, the parameter $A$ values show a greater reduction than for the same crack with $n=10$. For the latter, there is almost no change of the $A$ values. For $a / W=0.7$ (deep crack), both with $n=3$ and $n=10$, the variation of $A$ is quite small when the biaxiality $\lambda$ increases.

The above observations are consistent with those made for the $2 \mathrm{D}$ case (see section 
5.1.3.2). However, the change of constraint caused by an increase in $\lambda$ for the $3 D$ cracked specimen is much smaller than that for $2 \mathrm{D}$ ones, especially for the shallow crack cases. For example, for the 2D SECP with $a / W=0.3$, with an increase in $\lambda$ from 0.0 to 1.0 , the trend of the curves for the constraint parameter $A$ varies from ascending to descending when plotted against the far field stress (see Table 4.11 and Table 5.3). Meanwhile, for the 3D SECP case, the curves' slope remains almost unaffected with only a small reduction in the $A$ values (see Tables 6.8 and 6.11). Therefore, it can be concluded that, a change of biaxial loading ratio $\lambda$ will usually introduce smaller effect on the variation of constraint level in $3 \mathrm{D}$ models than that for $2 \mathrm{D}$ (plane strain) ones.

As for 2D specimen models, although the numerical solutions of parameter $A$ that were obtained for 3D specimens (for example, in Tables 6.7-6.8 under $\lambda=0.0$ or in Tables 6.10-6.11 under $\lambda=1.0$ for planes I and II) are appropriate to direct application, for engineering analysis purpose, it is more desirable to obtain empirical equations of $A$ solutions for 3D specimen with different material properties, crack depths and load levels. In the following sections, approximation formulas for the constraint parameter $A$ will be proposed for two planes, planes I and II, respectively.

\subsection{Solution of Parameter $\boldsymbol{A}$ by Curve Shape Similarity}

As for two-dimensional (2D) specimens (chapter 4 and 5), the constraint parameter $A$ solutions for a 3D specimen can be predicted through curve shape similarity by Eq. (3.1) or Eq. (5.4). For the present 3D SECP model, the curve shape similarity is utilized in two planes, plane I and plane II. To utilize Eq. (3.1) or Eq. (5.4), parameter $A$ solutions under zero load have to be obtained first. In section 6.1.3.2, finite element analyses (FEA) for the 3D modified boundary layer (MBL) problem have been carried out to obtain the $A$ values for various $T$-stress values in any plane. Numerical $A$ solutions under zero load, $A_{S S Y}(n, z)$, have been tabulated in the $T / \sigma_{0}=0$ column of Tables 6.1 and 6.2 for plane I and plane II, respectively.

In a specified plane perpendicular to the thickness direction, constraint parameter $A$ solutions for other $n$ values can be predicted based on the $A$ numerical solutions for a 
specified $n$ value by equation (3.1) or (5.4) and the fitting procedure developed in section 3.1.2. Here numerical solutions of parameter $A$ for $n=10$ are chosen for the development of an approximation formula. Based on the numerical solutions for $n=10$ (Tables 6.1, 6.2 and Tables $6.7,6.8$ or $6.10,6.11$ for planes I and II) and the fitting procedure developed in section 3.1.2, the second term in the right side of Eq. (3.1) (or Eq. (5.4)) is determined. Then a set of empirical formulas, which has a similar format to Eq. (5.5) and Eq. (5.6), is obtained from Eq. (5.4):

$$
A\left(\frac{a}{W}, \frac{\sigma}{\sigma_{0}}, n, z\right)=A_{S S Y}(n, z)+d_{1}(z)\left(\frac{\sigma}{\sigma_{0}}\right)+d_{2}(z)\left(\frac{\sigma}{\sigma_{0}}\right)^{2}+d_{3}(z)\left(\frac{\sigma}{\sigma_{0}}\right)^{3}
$$

where

$$
\begin{aligned}
& d_{1}(z)=e_{10}(z)+e_{11}(z)\left(\frac{a}{W}\right)+e_{12}(z)\left(\frac{a}{W}\right)^{2} \\
& d_{2}(z)=e_{20}(z)+e_{21}(z)\left(\frac{a}{W}\right)+e_{22}(z)\left(\frac{a}{W}\right)^{2} \\
& d_{3}(z)=e_{30}(z)+e_{31}(z)\left(\frac{a}{W}\right)+e_{32}(z)\left(\frac{a}{W}\right)^{2}
\end{aligned}
$$

Here $A_{S S Y}(n, z)$, are the standard SSY $(T=0)$ numerical solutions of parameter $A$ (Tables $6.1,6.2)$, which are utilized to approximate the zero load solution of parameter $A$. Along the 3D model thickness, the values of polynomial coefficients $e_{i j}(z)$ for planes I and II of 3D SECP specimen are listed in Tables $6.13,6.14$ for $\lambda=0.0$ and Tables $6.15,6.16$ for $\lambda=1.0$, respectively.

Note that, all the coefficients in Eq. (6.1) and Eq. (6.2) are functions of thickness coordinate $z$, since the $A$ solutions vary depending on the location of the plane along the thickness (crack-front). In addition, as the numerical solutions of parameter $A$ used for the fitting procedure are only available for three crack depths, $a / W=0.1,0.3$ and 0.7 , the polynomials for $d_{i}$ in Eq. (6.2) need to be reduced to second-order ones instead of thirdorder ones as those presented in Eq. (5.6).

Based on the developed empirical formulae (Eqs. (6.1) and (6.2)) with the fitted 
values of coefficients $e_{i j}(z)$ for the 3D SECP, the predicted parameter $A$ values are compared with finite element results for planes I and II (Tables 6.7-6.8 for $\lambda=0.0$ and Tables 6.10-6.11 for $\lambda=1.0$ ). For both planes (plane I and plane II), good agreement is found between the predicted $A$ values and numerical solutions under uniaxial or biaxial loading. Generally, almost all of the differences are less than $10 \%$ for both planes of the 3D SECP model with $\lambda=0.0$ or $\lambda=1.0$, and most of the differences are less than $5 \%$. For the uniaxial load condition $(\lambda=0.0)$, the maximum differences between formula and FEA results are $12.47 \%$ and $16.64 \%$ for plane I and plane II, respectively; for biaxial load $(\lambda=1.0)$, they are $12.04 \%$ and $13.52 \%$ for plane I and plane II, respectively. Comparison between predicted $A$ values and those from FEA results are shown in Figure 6.12-6.14 for $\lambda=0.0$ and Figure 6.15-6.17 for $\lambda=1.0$.

In chapter 5 , it was observed for 2D models that, the similarity of curves $A$ vs. $\sigma / \sigma_{0}$ for various values of the hardening exponent $n$ was reduced at higher biaxial loading ratio $\lambda$. This induces a reduction in the range of validity for curve shape similarity approximation, see section 5.2. In this chapter, it is found that, even with a high value of biaxial loading ratio $\lambda(\lambda=1.0)$, the approximation formulas remain valid over the entire load range. This phenomenon can be explained based on the fact that the change of $\lambda$ values has a smaller effect on the variation of constraint level in the present 3D cracked structure than that in $2 \mathrm{D}$ (plane strain) ones, see section 6.2.3.2.

\subsection{Solution of Parameter $\boldsymbol{A}$ by $\boldsymbol{T}$-stress}

\subsubsection{Determination of $A-T$ relationship for $3 D$ cases}

The existence of a functional $A-T$ relationship has been demonstrated and the general expression of the relationship (Eq. (3.6)) has been proposed in chapter 3. In this section, $A-T$ relations for specified planes of 3D SECP model will be determined.

In section 6.1 , based on extensive finite element analyses of the $3 \mathrm{D}$ modified boundary layer (MBL) model, numerical solutions of the parameter $A$ under varied $T$ stress values have been obtained by the fitting method suggested by Nikishkov et al. (1995a). Tables 6.1, 6.2 and 6.3 present those solutions for the three specified planes 
along the model thickness, planes I, II and III, respectively.

Since the values of constraint parameter $A$ depend on the location of the plane along the thickness, the general expression of $A-T$ relationship (Eq. (3.6)) should be rewritten as:

$$
A_{T}\left(\frac{T}{\sigma_{0}}, n, z\right)=A_{S S Y}(n, z)+m_{1}(n, z)\left(\frac{T}{\sigma_{0}}\right)+m_{2}(n, z)\left(\frac{T}{\sigma_{0}}\right)^{2}+m_{3}(n, z)\left(\frac{T}{\sigma_{0}}\right)^{3}
$$

For two specified planes, plane I and plane II, based on the numerical solution of $A$ for the 3D MBL model (Tables 6.1, 6.2), the values of coefficients: $m_{1}(n, z), m_{2}(n, z)$ and $m_{3}(n, z)$ in Eq. (6.3) for each hardening exponent $n$, are obtained by a least square fitting procedure. Their values are listed in Tables 6.17 and 6.18 for plane I and plane II, respectively. The $A-T$ relationship curves (Eq. (6.3)) for planes I and II are presented in Figures 6.3 and 6.4 .

\subsection{2 $T$-stress solution by finite element method}

As mentioned in section 2.6.1.1, a method to numerically compute the $T$-stress solution along a three-dimensional (3D) crack fronts was suggested by Nakamura and Parks (1992), which has been adopted in the commercial code ABAQUS (2006). For the present 3D SECP investigation, the proposed numerical method is utilized to determine the $T$-stress (or normalized $T$-stress, $V(V=T / \sigma))$ in specified planes of 3D SECP specimen through ABAQUS. The obtained $V(z)$ values for $\lambda=0.0$ and 1.0 are listed in Table 6.19 for planes I and II.

\subsubsection{Parameter $A$ solution by $T$-stress}

Zero load approximate solutions of parameter $A, A_{S S Y}(n, z)$ (see Tables 6.1, 6.2), values of the coefficients $m_{i}(n, z)$ (Table 6.17 and 6.18) and solutions of normalized $T$ stress, $V$ (section 6.4.2), have been obtained at this point. With these determined data, solutions of the constraint parameter $A$ under SSY conditions in specified planes of the 3D SECP specimen can be predicted directly from $T$-stress by Eq. (3.11). With the variation of parameter $A$ solutions along the thickness, Eq. (3.11) is rewritten as: 


$$
A_{T}\left(\frac{\sigma}{\sigma_{0}}, \frac{a}{W}, n, z\right)=A_{S S Y}(n, z)+\left(\frac{\sigma}{\sigma_{0}}\right) g_{1}\left(\frac{a}{W}, n, z\right)+\left(\frac{\sigma}{\sigma_{0}}\right)^{2} g_{2}\left(\frac{a}{W}, n, z\right)+\left(\frac{\sigma}{\sigma_{0}}\right)^{3} g_{3}\left(\frac{a}{W}, n, z\right)
$$

where $g_{i}(a / W, n, z)=[V(a / W, z)]^{i} m_{i}(n, z), i=1,2,3$.

Eq. (6.4) is appropriate if the remote load applied on the specimen, $\sigma$, is normalized by the material yield stress $\sigma_{0}$. The $T$-based $A$ solutions obtained from Eq. (6.4) are compared with those determined from FEA results in specified planes of the 3D SECP in Tables 6.7-6.8 for $\lambda=0.0$ and Tables 6.10-6.11 for $\lambda=1.0$. For planes I and II, the comparison is carried out for relative cracks depth $a / W=0.1,0.3,0.7$ with hardening exponent $n=3,5$ and 10 .

Overall, good agreement is found for all compared cases. The results of the comparison for planes I and II with $\lambda=0.0$ are plotted in Figures 6.18-6.20 for $a / W=0.1$, 0.3 and 0.7 respectively; the comparison results for $\lambda=1.0$ are shown in Figures 6.216.23 for $a / W=0.1,0.3$ and 0.7 respectively. The ranges of applicability of the $A-T$ relation are summarized in Table 6.20. Note that, to show the variation trends of curves more clearly, the curves of $A$ vs. $\sigma / \sigma_{0}$ from both FEA and $A-T$ relationship in Figures 6.18-6.23 are plotted beyond the ranges of applicability of the $A-T$ relationship (Table 6.20). Within the applicability ranges, for both uniaxial load $(\lambda=0.0)$ and biaxial load $(\lambda=1.0)$ conditions, and in both plane I and plane II, most of the differences between formula and FEA results are less than $10 \%$, while about 35 percent of the differences are less than $5 \%$. The maximum difference overall is $12.95 \%$ for the compared cases. Through the above comparison made for a wide range of material and geometrical characteristics, it can be concluded that the estimation method of parameter $A$ based on $T$ stress can be used for 3D SECP to predict $A$ under SSY conditions with acceptable accuracy.

By comparing Table 6.20 and Table 5.14, the ranges of applicability of the $A-T$ relation for the present $3 \mathrm{D}$ cracked structure (SECP) are smaller than those for $2 \mathrm{D}$ (SECP) model. This implies the dominance of the elastic solution is lost earlier in the 3D model than that in the 2D case. In addition, it is also found, for the deep crack case $(a / W=0.7)$ of 
3D SECP, the range of applicability of the $A-T$ relation is almost not affected by a change in biaxial load ratio $(\lambda)$, while for shallow cracks $(a / W=0.1$ or 0.3$)$, the range of applicability gets smaller with an increase in remote stress biaxiality. This phenomenon coincides with a finding in this chapter (section 6.2.3.2) that, for shallower cracks, a change in biaxial load ratio $\lambda$ has a greater effect on the level of constraint.

\subsection{Solution of Parameter $A$ by Fully Plastic Analysis}

\subsubsection{Fully plastic solution of parameter $\boldsymbol{A}$}

In section 6.4.3, it has been shown that parameter $A$ solutions of the 3D SECP can be predicted based on $T$-stress $\left(A_{T}\right)$ under SSY conditions (small loads). Same as for 2D specimens, for the $3 \mathrm{D}$ SECP specimen, the difference term $\triangle A$, which is the difference between $A$ solution from $T$-stress $\left(A_{T}\right)$ and that from FEA results, increases with the external load. In planes I and II, for $a / W=0.1,0.3,0.7$ and $n=3,5$ and 10 , the $\Delta A$ values (fully-plastic solution of parameter $A$ ) under increasing loading (load ratio) are calculated by Eq. (3.19). According to Eq. (3.19), the calculation is based on $A_{T}$ and the finite element results for the parameter $A$ (Tables 6.7-6.8 for $\lambda=0.0$ and Tables 6.10-6.11 for $\lambda=1.0)$.

\subsubsection{Determination of coefficient $a_{1}$}

In section 6.5.1, based on the determined numerical solution of parameter $A$, the difference $\Delta A$ (fully-plastic solution of parameter $A$ ) is obtained through Eq. (3.19). In addition, Eq. (3.16) can be used to obtain the difference $\Delta A$ under any (arbitrary) loading condition, without the numerical solution of parameter $A$. To utilize Eq. (3.16), the coefficient $a_{1}$ has to be determined first. In the present work, known $\Delta A$ for several specified high loading cases are used to determine $a_{1}$ through directly solving Eq. (3.16). For the present 3D models, the values of $A$ (and also $a_{l}$ ) depend on the reference plane in the thickness direction (z), therefore Eq. (3.16) is rewritten as:

$$
A(z)=a_{1}(z)\left(\frac{\sigma}{\sigma_{0}}\right)^{(n+1)(t-s)}
$$


To determine the coefficient $a_{1}$ in Eq. (6.5), the values of the difference $\Delta A$ for several specified high loading cases are obtained through Eq. (3.19), based on the $A$ solutions from $T$-stress $\left(A_{T}\right.$, Eq. (3.6)/ (6.3) or (3.11)/ (6.4)) and finite element solutions for the 3D SECP (Tables 6.7-6.8 and Tables 6.10-6.11). For each case (with various $a / W$, $n$ and $\lambda$ values and in different planes), the values of coefficient $a_{1}(a / W, n, z)$ are calculated for the three highest load conditions. The final values of coefficient $a_{1}$ are determined by averaging the three $a_{1}$ values calculated for the three highest loading cases.

The resulting values of the coefficient $a_{1}$, which depends on the hardening exponent $n$ and the relative crack depth $a / W$ in each plane, i.e. $a_{1}(a / W, n, z)$, are tabulated following a handbook format in Tables 6.21 and 6.22 for $n=3,5,10, a / W=0.1,0.3$ and 0.7 , and $\lambda=0.0,1.0$. The coefficient $a_{1}$ values in Table 6.21 are for planes I and II under uniaxial load $(\lambda=0.0)$. Under biaxial load $(\lambda=1.0)$, the coefficient values for planes I and II are listed in Table 6.22.

\subsubsection{Parameter $\boldsymbol{A}$ solution by fully plastic analysis}

Eq. (3.21) can be utilized to estimate the constraint parameter $A$ through fully plastic solutions of the parameter $A$. With the consideration that $A$ varies through the thickness of the 3D models, Eq. (3.21) should be rewritten as:

$$
A(z)=A_{T}\left(\frac{T}{\sigma_{0}}, n, z\right)+a_{1}(z)\left(\frac{\sigma}{\sigma_{0}}\right)^{(n+1)(t-s)}
$$

With the obtained values for coefficient $a_{1}$, the second term on the right side of Eq. (6.6) can be determined for varying external load ratios. For the present external load ratio $\sigma / \sigma_{0}$, substituting the expression of $A_{T}$ (Eq. (6.4)) into Eq. (6.6), the final expression which predicts $A$ values of the 3D cracked specimen through fully plastic solutions of the parameter $A$ can be obtained. The expression has a similar format as Eq. (5.9):

$$
A\left(\frac{a}{W}, n, z\right)=A_{S S Y}(n, z)+\left(\frac{\sigma}{\sigma_{0}}\right) g_{1}\left(\frac{a}{W}, n, z\right)
$$




$$
+\left(\frac{\sigma}{\sigma_{0}}\right)^{2} g_{2}\left(\frac{a}{W}, n, z\right)+\left(\frac{\sigma}{\sigma_{0}}\right)^{3} g_{3}\left(\frac{a}{W}, n, z\right)+a_{1}\left(\frac{a}{W}, n, z\right)\left(\frac{\sigma}{\sigma_{0}}\right)^{(n+1)(t-s)}
$$

where $g_{i}(a / W, n, z)=[V(a / W, z)]^{i} m_{i}(n, z), i=1,2,3$. In the present study, the values of $a_{1}(a / W, n, z)$ are for the 3D SECP specimen, which are given in Tables 6.21 and 6.22.

In each specified plane along the thickness of 3D SECP model, Eq. (6.7) is utilized to estimate parameter $A$ solutions from small-scale yielding (SSY) to large-scale yielding (LSY) conditions. The resulting approximations are compared with numerical solutions for the parameter $A$ listed in Tables 6.7-6.8 (for $\lambda=0.0$ ) and Tables 6.10-6.11 (for $\lambda=1.0$ ). A complete range of comparisons $(a / W=0.1,0.3,0.7$ with $n=3,5$ and 10 and $\lambda=0.0$ and 1.0) on planes I and II of 3D SECP model is performed. Comparisons under varying $n$ values are illustrated in Figures 6.24-6.26 (for the $\lambda=0.0$ case), or in Figures 6.27-6.29 (for the $\lambda=1.0$ case) for $a / W=0.1,0.3,0.7$ respectively. As typical examples, using $a / W=0.1$ and $n=5$, Figures 6.30-6.31 illustrate the superposition (see Eq. (6.6), (6.7)) of the difference term $\triangle A$ and the $T$-stress-based parameter $A$ approximation, $A_{T}$, together with the total $A$ values both from prediction and FEA results. Figure 6.30 is for the case $\lambda=0.0$, and Figure 6.31 is for $\lambda=1.0$.

Under uniaxial load $(\lambda=0.0)$ condition, for both plane I and plane II, most of the differences between approximation formula and FEA results are less than $10 \%$, while almost half of the differences are less than 5\%. The maximum differences are $14.00 \%$, $11.79 \%$ and $22.23 \%$ for $a / W=0.1,0.3$ and 0.7 , respectively, where the difference $22.23 \%$ occurs in a case with low value of parameter $A(A=0.2443)$. The agreement can be considered as good.

Under biaxial loading with large biaxiality ratio $(\lambda=1.0)$, for both plane I and plane II, generally larger differences to the FEA results are obtained, although about half of them are less than $10 \%$. For $a / W=0.1,0.3$ and 0.7 , the maximum differences are $36.10 \%$, $20.55 \%$ and $26.64 \%$, respectively. In general, the big differences appear under relatively low load conditions.

It can be concluded that, although the estimate method based on fully plastic analysis does not work well enough for 3D SECP under high biaxial loading condition 
(e.g. $\lambda=1.0$ ) with reduced prediction accuracy, generally, the method is appropriate for the application in 3D cracked structure with enough accuracy for most cases.

\subsection{Conclusions}

With the consideration that near crack-front fields in any plane (except planes near the free surface) along the 3D model thickness are dominated by plane strain state, the $J$ $A$ fracture mechanics approach and fitting method determining numerical solutions of the constraint parameter $A$ from finite element results, which are proposed by Nikishkov (1995) and Nikishkov et al. (1995a) based on plane strain state, can be used for threedimensional (3D) models.

Then, through the finite element analyses, numerical solutions for the parameter $A$ for the 3D modified boundary layer (MBL) problem and a thin single edge cracked plate (SECP) specimen have been obtained. Numerical solutions for the parameter $A$ for the 3D MBL are determined for hardening exponents $n=3,4,5,7$ and 10. The numerical solutions for the 3D SECP specimen are obtained for $n=3,5,10$ and a relative crack depth $a / W=0.1,0.3,0.7$ under both uniaxial $(\lambda=0.0)$ and biaxial $(\lambda=1.0)$ loading conditions.

Based on FEA results and numerical solutions for the constraint parameter $A$, the following observations can be made regarding constraint effects for $3 \mathrm{D}$ cracked specimens under uniaxial and biaxial loading are found:

(1) both constraint and load reach their highest levels at the mid-plane. This fact is consistent with the observation in experiments that a crack always initiates at the midplane of a plate;

(2) except for planes near the free surface, both constraint $(A)$ and load $(J)$ levels have a small variation along the crack-front (thickness);

(3) generally, the maximum external loading ratio $\left(\sigma / \sigma_{0}\right)$, for which a valid solution can be obtained, increases with decreasing hardening coefficient $n$;

(4) in general, the constraint level in crack-tip fields increases with the increasing biaxial loading ratio, $\lambda$; 
(5) the constraint level for materials with smaller values of hardening exponent $n$ is increased more by the increased biaxial loading ratio;

(6) the constraint level for shallow cracks increases more with an increase of biaxial loading ratio;

(7) a change of biaxial loading ratio $\lambda$ usually introduces a smaller effect on the variation of constraint level in $3 \mathrm{D}$ models than that for $2 \mathrm{D}$ (plane strain) ones.

Items (3)-(6) in fact are consistent with those found for 2D models under biaxial loading (see section 5.5).

Approximation methods for the constraint parameter $A$, estimation based on curveshape similarity, prediction from $T$-stress and based on a fully plastic solution, which were developed based on a plane strain state in this work (chapter 3 ), also can be applied for planes (but not those near the free surface) along the 3D model thickness (crackfront).

In this chapter, these three approximation methods have been utilized for two specified planes (at a half and a quarter of the model thickness, i.e. plane I and plane II) of the 3D SECP specimen with hardening exponent $n=3,5,10$, relative crack depth $a / W=0.1,0.3,0.7$ and load ratio $\lambda=0.0,1.0$. Predicted parameter $A$ values from the three approximation methods are compared with those obtained from finite element analyses. With the specified material properties, specimen geometries and loading conditions, the applicability of the three approximation methods to 3D specimens is discussed. Both the estimate through curve-shape similarity (up to LSY) and the method of predicting from $T$-stress (for SSY) work well for 3D SECP specimen. Under uniaxial loading $(\lambda=0.0)$, approximating the parameter $A$ based on a fully plastic solution works well on the specified planes of 3D SECP; under high biaxial load ratio $(\lambda=1.0)$, the estimate has reduced predicting accuracy for constraint parameter $A$ values. Even though, based on the discussion in this chapter (section 6.5.3), it can be concluded that, generally, the estimate method based on fully plastic solution is appropriate to the application for $3 \mathrm{D}$ cracked structure under uniaxial and biaxial loading with enough accuracy in most cases.

Under small-scale yielding condition, with available solutions of $T$-stress in the literature, approximate solutions of constraint parameter $A$ for $3 \mathrm{D}$ cracked specimens can 
be conveniently determined by using $A-T$ relationship. For cases that external loadings vary from low to high (small-scale to large-scale yielding deformation), curve shape similarity method can be used to predict parameter $A$. If solutions of $T$-stress are available, method based on fully plastic solution of $A$ can be used to estimate parameter $A$ from low to high external loading with fewer finite element analyses. However, for cases under high biaxial loading ratio $\lambda$, one should use the approximate method based on fully plastic solution of $A$ with caution, since the estimating accuracy might be reduced.

Tables 6.5 and 6.6 as well as Figures 6.10 and 6.11 show that, except for planes near the free surface, the near crack-front fields in planes perpendicular to the crack-front have small variations of $J$-integral and parameter $A$ along the model thickness. Therefore, although only two planes, plane I and plane II, are studied here, it can be concluded that the applicability of the three newly-developed approximation methods applies in any plane through the thickness of 3D model except for planes near the free surface. 


\begin{tabular}{cccccccccccc}
\hline & & \multicolumn{10}{c}{$T / \sigma_{0}$} \\
\hline$n$ & -1.0 & -0.8 & -0.6 & -0.4 & -0.2 & 0.0 & 0.2 & 0.4 & 0.6 & 0.8 & 1.0 \\
\hline 3 & 1.2629 & 1.2141 & 1.1521 & 1.0801 & 1.0045 & 0.9277 & 0.8593 & 0.8080 & 0.7713 & 0.7599 & 0.7475 \\
\hline 4 & 0.8789 & 0.8334 & 0.7757 & 0.7061 & 0.6320 & 0.5591 & 0.4966 & 0.4490 & 0.4154 & 0.4146 & 0.3995 \\
\hline 5 & 0.6760 & 0.6380 & 0.5857 & 0.5240 & 0.4547 & 0.3887 & 0.3325 & 0.2898 & 0.2601 & 0.2620 & 0.2496 \\
\hline 7 & 0.5213 & 0.4865 & 0.4343 & 0.3730 & 0.3097 & 0.2508 & 0.2011 & 0.1679 & 0.1442 & 0.1452 & 0.1369 \\
\hline 10 & 0.4578 & 0.4169 & 0.3633 & 0.3004 & 0.2392 & 0.1828 & 0.1356 & 0.1062 & 0.0959 & 0.0921 & 0.0881 \\
\hline
\end{tabular}

Table 6.1 $A$ values from FEA results for the 3D MBL problem, plane I

\begin{tabular}{cccccccccccc}
\hline & & \multicolumn{10}{c}{$T / \sigma_{0}$} \\
\hline$n$ & -1.0 & -0.8 & -0.6 & -0.4 & -0.2 & 0.0 & 0.2 & 0.4 & 0.6 & 0.8 & 1.0 \\
\hline 3 & 1.2610 & 1.2128 & 1.1617 & 1.0891 & 1.0149 & 0.9435 & 0.8792 & 0.8312 & 0.7974 & 0.7730 & 0.7795 \\
\hline 4 & 0.8772 & 0.8322 & 0.7760 & 0.7099 & 0.6405 & 0.5731 & 0.5154 & 0.4713 & 0.4404 & 0.4186 & 0.4299 \\
\hline 5 & 0.6779 & 0.6439 & 0.5862 & 0.5269 & 0.4615 & 0.4006 & 0.3495 & 0.3103 & 0.2831 & 0.2647 & 0.2775 \\
\hline 7 & 0.5206 & 0.4857 & 0.4344 & 0.3750 & 0.3147 & 0.2602 & 0.2157 & 0.1830 & 0.1610 & 0.1473 & 0.1620 \\
\hline 10 & 0.4574 & 0.4174 & 0.3632 & 0.3018 & 0.2431 & 0.1903 & 0.1485 & 0.1206 & 0.1035 & 0.0944 & 0.1117 \\
\hline
\end{tabular}

Table 6.2 $A$ values from FEA results for the 3D MBL problem, plane II

\begin{tabular}{cccccccccccc}
\hline & \multicolumn{1}{c}{$T / \sigma_{0}$} \\
\hline$n$ & -1.0 & -0.8 & -0.6 & -0.4 & -0.2 & 0.0 & 0.2 & 0.4 & 0.6 & 0.8 & 1.0 \\
\hline 3 & 1.6000 & 1.6238 & 1.6461 & 1.6509 & 1.6520 & 1.6280 & 1.5904 & 1.5874 & 1.5728 & 1.5478 & 1.5563 \\
\hline 4 & 1.0819 & 1.1328 & 1.1601 & 1.1400 & 1.1257 & 1.1002 & 1.0851 & 1.0604 & 1.0455 & 1.0409 & 1.0437 \\
\hline 5 & 0.8466 & 0.8819 & 0.8957 & 0.8702 & 0.8513 & 0.8416 & 0.8195 & 0.8005 & 0.7874 & 0.7824 & 0.7837 \\
\hline 7 & 0.6589 & 0.6680 & 0.6537 & 0.6526 & 0.6308 & 0.6124 & 0.5966 & 0.5830 & 0.5438 & 0.5367 & 0.5672 \\
\hline 10 & 0.5635 & 0.5576 & 0.5574 & 0.5376 & 0.5201 & 0.5057 & 0.4728 & 0.4591 & 0.4475 & 0.4399 & 0.4375 \\
\hline
\end{tabular}

Table 6.3 $A$ values from FEA results for the 3D MBL problem, plane III 


\begin{tabular}{crrrrrr}
\hline$z / t$ & 0.132 & 0.245 & 0.337 & 0.408 & 0.457 & 0.490 \\
\hline$A_{2}[$ Ref. $]$ & -1.5200 & -1.5950 & -1.6700 & -1.8400 & -2.1150 & -2.8500 \\
$A_{2}[$ Present $]$ & -1.5334 & -1.5988 & -1.6979 & -1.8749 & -2.1176 & -2.6390 \\
\hline Diff. (\%) & 0.8845 & 0.2405 & 1.6715 & 1.8984 & 0.1213 & -7.4019 \\
\hline
\end{tabular}

Table 6.4 Comparison of parameter $A_{2}$ between present results and those from Zhu et al. (2002) 


\begin{tabular}{ccccc}
\hline & $\lambda=0.0$ & & $\lambda=1.0$ & \\
\hline$z / t$ & $\sigma / \sigma_{0}=0.25$ & $\sigma / \sigma_{0}=0.55$ & $\sigma / \sigma_{0}=0.25$ & $\sigma / \sigma_{0}=0.55$ \\
\hline 0.014 & 0.4615 & 0.6819 & 0.3992 & 0.6421 \\
0.099 & 0.4641 & 0.6902 & 0.4030 & 0.6504 \\
0.221 & 0.4759 & 0.7201 & 0.4193 & 0.6801 \\
0.306 & 0.4955 & 0.7603 & 0.4445 & 0.7281 \\
0.365 & 0.5171 & 0.7999 & 0.4698 & 0.7723 \\
0.407 & 0.5510 & 0.8451 & 0.5049 & 0.8187 \\
0.436 & 0.5871 & 0.8804 & 0.5473 & 0.8541 \\
0.457 & 0.6203 & 0.9254 & 0.5838 & 0.8981 \\
0.471 & 0.6706 & 0.9524 & 0.6363 & 0.9400 \\
0.481 & 0.7126 & 0.9915 & 0.6818 & 0.9656 \\
0.488 & 0.7745 & 1.0353 & 0.7477 & 0.9973 \\
0.493 & 0.8242 & 1.0514 & 0.7988 & 1.0359 \\
0.497 & 0.9024 & 1.0912 & 0.8765 & 1.0796 \\
0.499 & 0.9937 & 1.1329 & 0.9657 & 1.1330 \\
\hline
\end{tabular}

Table 6.5 Variation of parameter $A$ along the crack-front of the 3D SECP, $a / W=0.3, n=5$

\begin{tabular}{crrrr}
\hline \multicolumn{1}{l}{$\lambda=0.0$} & \multicolumn{3}{l}{$\lambda=1.0$} \\
\hline$z / t$ & $\sigma / \sigma_{0}=0.25$ & $\sigma / \sigma_{0}=0.55$ & $\sigma / \sigma_{0}=0.25$ & $\sigma / \sigma_{0}=0.55$ \\
\hline 0.000 & $6.7310 \mathrm{E}+04$ & $7.2538 \mathrm{E}+05$ & $6.7338 \mathrm{E}+04$ & $7.0711 \mathrm{E}+05$ \\
0.014 & $6.7292 \mathrm{E}+04$ & $7.2481 \mathrm{E}+05$ & $6.7321 \mathrm{E}+04$ & $7.0655 \mathrm{E}+05$ \\
0.099 & $6.6912 \mathrm{E}+04$ & $7.1190 \mathrm{E}+05$ & $6.6890 \mathrm{E}+04$ & $6.9315 \mathrm{E}+05$ \\
0.221 & $6.5324 \mathrm{E}+04$ & $6.6664 \mathrm{E}+05$ & $6.5131 \mathrm{E}+04$ & $6.4691 \mathrm{E}+05$ \\
0.306 & $6.2707 \mathrm{E}+04$ & $6.0954 \mathrm{E}+05$ & $6.2310 \mathrm{E}+04$ & $5.8957 \mathrm{E}+05$ \\
0.365 & $5.9304 \mathrm{E}+04$ & $5.5197 \mathrm{E}+05$ & $5.8724 \mathrm{E}+04$ & $5.3236 \mathrm{E}+05$ \\
0.407 & $5.5366 \mathrm{E}+04$ & $4.9802 \mathrm{E}+05$ & $5.4637 \mathrm{E}+04$ & $4.7881 \mathrm{E}+05$ \\
0.436 & $5.1116 \mathrm{E}+04$ & $4.4869 \mathrm{E}+05$ & $5.0264 \mathrm{E}+04$ & $4.2952 \mathrm{E}+05$ \\
0.457 & $4.6719 \mathrm{E}+04$ & $4.0346 \mathrm{E}+05$ & $4.5758 \mathrm{E}+04$ & $3.8393 \mathrm{E}+05$ \\
0.471 & $4.2279 \mathrm{E}+04$ & $3.6135 \mathrm{E}+05$ & $4.1214 \mathrm{E}+04$ & $3.4114 \mathrm{E}+05$ \\
0.481 & $3.7827 \mathrm{E}+04$ & $3.2105 \mathrm{E}+05$ & $3.6656 \mathrm{E}+04$ & $3.0006 \mathrm{E}+05$ \\
0.488 & $3.3315 \mathrm{E}+04$ & $2.8112 \mathrm{E}+05$ & $3.2040 \mathrm{E}+04$ & $2.5948 \mathrm{E}+05$ \\
0.493 & $2.8608 \mathrm{E}+04$ & $2.3983 \mathrm{E}+05$ & $2.7231 \mathrm{E}+04$ & $2.1786 \mathrm{E}+05$ \\
0.497 & $2.3048 \mathrm{E}+04$ & $1.9132 \mathrm{E}+05$ & $2.1610 \mathrm{E}+04$ & $1.7001 \mathrm{E}+05$ \\
0.499 & $1.5178 \mathrm{E}+04$ & $1.2329 \mathrm{E}+05$ & $1.3785 \mathrm{E}+04$ & $1.0454 \mathrm{E}+05$ \\
0.500 & $8.9805 \mathrm{E}+03$ & $7.3020 \mathrm{E}+04$ & $8.0122 \mathrm{E}+03$ & $6.0425 \mathrm{E}+04$ \\
\hline
\end{tabular}

Table 6.6 Variation of $J$-integral along the crack-front of the 3D SECP, $a / W=0.3, n=5$ 


\begin{tabular}{|c|c|c|c|c|c|c|c|c|c|c|c|}
\hline$n$ & 3 & & & 5 & & & & 10 & & & \\
\hline$\sigma / \sigma_{0}$ & $\begin{array}{l}\text { Plane } \\
\text { I }\end{array}$ & $\begin{array}{l}\text { Plane } \\
\text { II }\end{array}$ & $\begin{array}{l}\text { Plane } \\
\text { III }\end{array}$ & $\sigma / \sigma_{0}$ & $\begin{array}{l}\text { Plane } \\
\text { I }\end{array}$ & $\begin{array}{l}\text { Plane } \\
\text { II }\end{array}$ & $\begin{array}{l}\text { Plane } \\
\text { III }\end{array}$ & $\sigma / \sigma_{0}$ & $\begin{array}{c}\text { Plane } \\
\text { I }\end{array}$ & $\begin{array}{l}\text { Plane } \\
\text { II }\end{array}$ & $\begin{array}{l}\text { Plane } \\
\text { III }\end{array}$ \\
\hline 0.000 & 0.9277 & 0.9435 & 1.6280 & 0.000 & 0.3887 & 0.4006 & 0.8416 & 0.000 & 0.1828 & 0.1903 & 0.5057 \\
\hline 0.250 & 0.9421 & 0.9474 & 1.4576 & 0.250 & 0.4137 & 0.4161 & 0.7717 & 0.250 & 0.2159 & 0.2173 & 0.0000 \\
\hline 0.350 & 1.0190 & 1.0284 & 1.6943 & 0.350 & 0.4553 & 0.4601 & 0.8328 & 0.350 & 0.2382 & 0.2407 & 0.4975 \\
\hline 0.450 & 1.0635 & 1.0800 & 1.8594 & 0.450 & 0.4832 & 0.4918 & 0.9176 & 0.450 & 0.2566 & 0.2622 & 0.5433 \\
\hline 0.550 & 1.1025 & 1.1281 & 1.9616 & 0.550 & 0.5124 & 0.5295 & 0.9760 & 0.550 & 0.2765 & 0.2870 & 0.5608 \\
\hline 0.650 & 1.1475 & 1.1838 & 2.0564 & 0.650 & 0.5442 & 0.5695 & 1.0209 & 0.650 & 0.3014 & 0.3191 & 0.5987 \\
\hline 0.750 & 1.1936 & 1.2419 & 2.1085 & 0.750 & 0.5807 & 0.6191 & 1.0685 & 0.750 & 0.3306 & 0.3598 & 0.6300 \\
\hline 0.850 & 1.2420 & 1.3030 & 2.2190 & 0.850 & 0.6223 & 0.6703 & 1.1075 & 0.850 & 0.3738 & 0.4128 & 0.6595 \\
\hline 0.950 & 1.2943 & 1.3662 & 2.2078 & 0.950 & 0.6755 & 0.7241 & 1.1393 & 0.950 & 0.4417 & 0.4752 & 0.6754 \\
\hline 1.150 & 1.4149 & 1.4917 & 2.2196 & 1.050 & 0.7401 & 0.7897 & 1.1129 & 1.050 & --- & 0.5441 & 0.6675 \\
\hline 1.250 & 1.4853 & 1.5680 & 2.1576 & 1.150 & 0.8098 & 0.8477 & 1.1222 & & & & \\
\hline 1.400 & 1.5762 & 1.6634 & 2.1214 & 1.200 & 0.8446 & 0.8736 & 1.1196 & & & & \\
\hline 1.500 & 1.6434 & 1.7241 & 2.1110 & 1.250 & 0.8709 & 0.8993 & 1.1139 & & & & \\
\hline 1.600 & $\ldots$ & 1.7392 & 2.0937 & 1.300 & 0.9006 & 0.9206 & 1.0639 & & & & \\
\hline
\end{tabular}

Table 6.7 $A$ values from FEA results for the 3D SECP specimen, $\lambda=0.0, a / W=0.1$

\begin{tabular}{ccccccccccccc}
\hline$n$ & 3 & \multicolumn{1}{c}{5} & \multicolumn{1}{c}{ 10 } & \multicolumn{1}{c}{ 10 } \\
\hline$\sigma / \sigma_{0}$ & Plane & Plane & Plane & $\sigma / \sigma_{0}$ & Plane & Plane & Plane & $\sigma / \sigma_{0}$ & Plane & Plane & Plane \\
& II & III & I & II & III & II & III \\
\hline 0.000 & 0.9277 & 0.9435 & 1.6280 & 0.000 & 0.3887 & 0.4006 & 0.8416 & 0.000 & 0.1828 & 0.1903 & 0.5057 \\
0.250 & 1.0372 & 1.0683 & 1.9102 & 0.250 & 0.4615 & 0.4845 & 0.9763 & 0.250 & 0.2377 & 0.2573 & 0.5582 \\
0.350 & 1.1169 & 1.1723 & 2.1085 & 0.350 & 0.5188 & 0.5588 & 1.0275 & 0.350 & 0.2768 & 0.3176 & 0.6064 \\
0.450 & 1.2134 & 1.2922 & 2.1761 & 0.450 & 0.5908 & 0.6446 & 1.0879 & 0.450 & 0.3398 & 0.3803 & 0.6498 \\
0.550 & 1.3288 & 1.4216 & 2.1883 & 0.550 & 0.6819 & 0.7364 & 1.1216 & 0.550 & 0.4230 & 0.4590 & 0.6550 \\
0.600 & 1.3908 & 1.4799 & 2.2030 & 0.600 & 0.7346 & 0.7875 & 1.1072 & 0.600 & 0.4776 & 0.5014 & 0.6649 \\
0.700 & 1.5066 & 1.6020 & 2.2112 & 0.700 & 0.8315 & 0.8673 & 1.1033 & 0.700 & 0.5633 & $-\ldots$ & 0.6467 \\
0.800 & 1.6155 & 1.6820 & 2.0791 & 0.800 & 0.9058 & 0.9209 & 1.0776 & & & & \\
0.900 & 1.7019 & 1.7234 & 2.0090 & 0.900 & 0.9120 & 0.9058 & 0.9838 & & & & \\
1.000 & 1.7513 & 1.7327 & 1.9053 & & & & & & & & \\
1.100 & 1.7558 & 1.7476 & 1.8865 & & & & & & & & \\
1.200 & --- & 1.7530 & 1.7427 & & & & & & & & \\
\hline
\end{tabular}

Table 6.8 $A$ values from FEA results for the 3D SECP specimen, $\lambda=0.0, a / W=0.3$ 


\begin{tabular}{cccccccccccc}
\hline$n$ & 3 & \multicolumn{1}{c}{5} & \multicolumn{1}{c}{5} & \multicolumn{1}{c}{10} \\
\hline$\sigma / \sigma_{0}$ & $\begin{array}{c}\text { Plane } \\
\text { I }\end{array}$ & Plane & II & Plane & III & $\sigma / \sigma_{0}$ & Plane & Plane & Plane & \multicolumn{3}{c}{ Plane } & Plane & Plane \\
& II & III & $\sigma / \sigma_{0}$ & I & II & III \\
\hline 0.000 & 0.9277 & 0.9435 & 1.6280 & 0.000 & 0.3887 & 0.4006 & 0.8416 & 0.000 & 0.1828 & 0.1903 & 0.5057 \\
0.020 & 0.8981 & 0.9076 & 1.4510 & 0.020 & 0.3743 & 0.3798 & 0.7316 & 0.020 & 0.1785 & 0.1817 & 0.4577 \\
0.030 & 0.9286 & 0.9483 & 1.6858 & 0.030 & 0.3851 & 0.3994 & 0.8573 & 0.030 & 0.1819 & 0.1909 & 0.4867 \\
0.040 & 0.9565 & 0.9905 & 1.8671 & 0.040 & 0.3955 & 0.4219 & 0.9137 & 0.040 & 0.1823 & 0.2033 & 0.5443 \\
0.050 & 0.9959 & 1.0413 & 1.9724 & 0.050 & 0.4211 & 0.4636 & 0.9654 & 0.050 & 0.1999 & 0.2359 & 0.5779 \\
0.060 & 1.0415 & 1.1096 & 2.0870 & 0.060 & 0.4591 & 0.5082 & 1.0374 & 0.060 & 0.2305 & 0.2795 & 0.5937 \\
0.070 & 1.0994 & 1.1835 & 2.1558 & 0.070 & 0.5011 & 0.5709 & 1.0727 & 0.070 & 0.2635 & 0.3334 & 0.6174 \\
0.080 & 1.1688 & 1.2664 & 2.1701 & 0.080 & 0.5677 & 0.6346 & 1.1013 & 0.080 & 0.3422 & 0.3968 & 0.6536 \\
0.090 & 1.2512 & 1.3538 & 2.1746 & 0.090 & 0.6414 & 0.7064 & 1.1257 & 0.090 & 0.4572 & 0.4783 & 0.6718 \\
0.100 & 1.3408 & 1.4385 & 2.2196 & 0.100 & 0.7310 & 0.7814 & 1.1019 & 0.095 & --- & 0.5200 & 0.6637 \\
0.110 & 1.4240 & 1.5220 & 2.2155 & 0.110 & --- & 0.8429 & 1.1099 & & & & \\
0.120 & --- & 1.6039 & 2.1968 & & & & & & & & \\
0.130 & --- & 1.6672 & 2.2033 & & & & & & & & \\
0.140 & --- & 1.7339 & 2.1051 & & & & & & & & \\
\hline
\end{tabular}

Table $6.9 A$ values from FEA results for the 3 D SECP specimen, $\lambda=0.0, a / W=0.7$

\begin{tabular}{|c|c|c|c|c|c|c|c|c|c|c|c|}
\hline$n$ & 3 & & & 5 & & & & 10 & & & \\
\hline$\sigma / \sigma_{0}$ & $\begin{array}{c}\text { Plane } \\
\text { I }\end{array}$ & $\begin{array}{l}\text { Plane } \\
\text { II }\end{array}$ & $\begin{array}{l}\text { Plane } \\
\text { III }\end{array}$ & $\sigma / \sigma_{0}$ & $\begin{array}{c}\text { Plane } \\
\text { I }\end{array}$ & $\begin{array}{l}\text { Plane } \\
\text { II }\end{array}$ & $\begin{array}{l}\text { Plane } \\
\text { III }\end{array}$ & $\sigma / \sigma_{0}$ & $\begin{array}{c}\text { Plane } \\
\text { I }\end{array}$ & $\begin{array}{l}\text { Plane } \\
\text { II }\end{array}$ & $\begin{array}{c}\text { Plane } \\
\text { III }\end{array}$ \\
\hline 0.000 & 0.9277 & 0.9435 & 1.6280 & 0.000 & 0.3887 & 0.4006 & 0.8416 & 0.000 & 0.1828 & 0.1903 & 0.5057 \\
\hline 0.250 & 0.8219 & 0.8324 & 1.3948 & 0.250 & 0.3205 & 0.3264 & 0.7396 & 0.250 & 0.1454 & 0.1481 & 0.0000 \\
\hline 0.350 & 0.8756 & 0.8928 & 1.6131 & 0.350 & 0.3403 & 0.3526 & 0.7574 & 0.350 & 0.1454 & 0.1530 & 0.4660 \\
\hline 0.450 & 0.8868 & 0.9154 & 1.7284 & 0.450 & 0.3554 & 0.3671 & 0.8624 & 0.450 & 0.1458 & 0.1641 & 0.5100 \\
\hline 0.550 & 0.9167 & 0.9531 & 1.8912 & 0.550 & 0.3685 & 0.4028 & 0.9328 & 0.550 & 0.1563 & 0.1858 & 0.5358 \\
\hline 0.650 & 0.9488 & 1.0060 & 1.9826 & 0.650 & 0.4037 & 0.4391 & 0.9679 & 0.650 & 0.1864 & 0.2252 & 0.5690 \\
\hline 0.750 & 0.9934 & 1.0671 & 2.0265 & 0.750 & 0.4398 & 0.5009 & 1.0037 & 0.750 & 0.2272 & 0.2782 & 0.5914 \\
\hline 0.900 & 1.0836 & 1.1797 & 2.1152 & 0.900 & 0.5383 & 0.6147 & 1.0536 & 0.900 & 0.3482 & 0.3969 & 0.6267 \\
\hline 1.000 & 1.1607 & 1.2560 & 2.1007 & 1.000 & 0.6329 & 0.6946 & 1.0763 & 1.000 & -- & 0.4993 & 0.6396 \\
\hline 1.100 & 1.2337 & 1.3408 & 2.1344 & 1.100 & 0.7205 & 0.7760 & 1.0899 & & & & \\
\hline 1.200 & -- & 1.4163 & 2.0908 & & & & & & & & \\
\hline
\end{tabular}

Table 6.10 $A$ values from FEA results for the 3D SECP specimen, $\lambda=1.0, a / W=0.1$ 


\begin{tabular}{|c|c|c|c|c|c|c|c|c|c|c|c|}
\hline$n$ & 3 & & & 5 & & & & 10 & & & \\
\hline$\sigma / \sigma_{0}$ & $\begin{array}{c}\text { Plane } \\
\text { I }\end{array}$ & $\begin{array}{c}\text { Plane } \\
\text { II }\end{array}$ & $\begin{array}{c}\text { Plane } \\
\text { III }\end{array}$ & $\sigma / \sigma_{0}$ & $\begin{array}{c}\text { Plane } \\
\text { I }\end{array}$ & $\begin{array}{c}\text { Plane } \\
\text { II }\end{array}$ & $\begin{array}{c}\text { Plane } \\
\text { III }\end{array}$ & $\sigma / \sigma_{0}$ & $\begin{array}{c}\text { Plane } \\
\text { I }\end{array}$ & $\begin{array}{c}\text { Plane } \\
\text { II }\end{array}$ & $\begin{array}{c}\text { Plane } \\
\text { III }\end{array}$ \\
\hline 0.000 & 0.9277 & 0.9435 & 1.6280 & 0.000 & 0.3887 & 0.4006 & 0.8416 & 0.000 & 0.1828 & 0.1903 & 0.5057 \\
\hline 0.200 & 0.9291 & 0.9547 & 1.7422 & 0.200 & 0.3837 & 0.4040 & 0.8814 & 0.200 & 0.1767 & 0.1885 & 0.5250 \\
\hline 0.250 & 0.9579 & 0.9915 & 1.9092 & 0.250 & 0.3992 & 0.4307 & 0.9494 & 0.250 & 0.1851 & 0.2156 & 0.5558 \\
\hline 0.350 & 1.0275 & 1.0884 & 2.0756 & 0.350 & 0.4512 & 0.5057 & 1.0283 & 0.350 & 0.2289 & 0.2833 & 0.5885 \\
\hline 0.450 & 1.1217 & 1.2151 & 2.1077 & 0.450 & 0.5302 & 0.6003 & 1.0799 & 0.450 & 0.3081 & 0.3549 & 0.6267 \\
\hline 0.550 & 1.2451 & 1.3503 & 2.1787 & 0.550 & 0.6421 & 0.7042 & 1.1189 & 0.550 & 0.4071 & 0.4473 & 0.6519 \\
\hline 0.650 & 1.3776 & 1.4752 & 2.1234 & 0.650 & 0.7612 & 0.8070 & 1.0854 & 0.650 & 0.5314 & 0.5378 & 0.6451 \\
\hline 0.750 & 1.4888 & 1.5858 & 2.1102 & 0.750 & 0.8602 & 0.8726 & 1.0725 & & & & \\
\hline 0.850 & 1.5845 & 1.6327 & 2.0587 & 0.800 & 0.8899 & 0.8995 & 1.0377 & & & & \\
\hline 0.950 & 1.6507 & 1.6587 & 1.9711 & 0.850 & -.. & 0.8922 & 1.0363 & & & & \\
\hline 1.100 & --- & 1.6352 & 1.7944 & 0.900 & -- & 0.8828 & 0.9880 & & & & \\
\hline
\end{tabular}

Table 6.11 $A$ values from FEA results for the 3D SECP specimen, $\lambda=1.0, a / W=0.3$

\begin{tabular}{|c|c|c|c|c|c|c|c|c|c|c|c|}
\hline$n$ & 3 & & & 5 & & & & 10 & & & \\
\hline$\sigma / \sigma_{0}$ & $\begin{array}{c}\text { Plane } \\
\text { I }\end{array}$ & $\begin{array}{c}\text { Plane } \\
\text { II }\end{array}$ & $\begin{array}{c}\text { Plane } \\
\text { III }\end{array}$ & $\sigma / \sigma_{0}$ & $\begin{array}{c}\text { Plane } \\
\text { I }\end{array}$ & $\begin{array}{l}\text { Plane } \\
\text { II }\end{array}$ & $\begin{array}{l}\text { Plane } \\
\text { III }\end{array}$ & $\sigma / \sigma_{0}$ & $\begin{array}{c}\text { Plane } \\
\text { I }\end{array}$ & $\begin{array}{l}\text { Plane } \\
\text { II }\end{array}$ & $\begin{array}{c}\text { Plane } \\
\text { III }\end{array}$ \\
\hline 0.000 & 0.9277 & 0.9435 & 1.6280 & 0.000 & 0.3887 & 0.4006 & 0.8416 & 0.000 & 0.1828 & 0.1903 & 0.5057 \\
\hline 0.020 & 0.8901 & 0.9000 & 1.4470 & 0.020 & 0.3679 & 0.3737 & 0.7290 & 0.020 & 0.1732 & 0.1766 & 0.4562 \\
\hline 0.030 & 0.9185 & 0.9389 & 1.6816 & 0.030 & 0.3767 & 0.3918 & 0.8542 & 0.030 & 0.1746 & 0.1844 & 0.4844 \\
\hline 0.040 & 0.9451 & 0.9804 & 1.8627 & 0.040 & 0.3856 & 0.4134 & 0.9104 & 0.040 & 0.1738 & 0.1964 & 0.5418 \\
\hline 0.050 & 0.9844 & 1.0307 & 1.9681 & 0.050 & 0.4115 & 0.4559 & 0.9857 & 0.050 & 0.1917 & 0.2298 & 0.5755 \\
\hline 0.060 & 1.0303 & 1.1005 & 2.0830 & 0.060 & 0.4508 & 0.5016 & 1.0347 & 0.060 & 0.2238 & 0.2750 & 0.5916 \\
\hline 0.070 & 1.0887 & 1.1756 & 2.1525 & 0.070 & 0.4944 & 0.5668 & 1.0713 & 0.070 & 0.2707 & 0.3314 & 0.6166 \\
\hline 0.080 & 1.1608 & 1.2603 & 2.1678 & 0.080 & 0.5640 & 0.6335 & 1.1019 & 0.080 & 0.3434 & 0.3984 & 0.6567 \\
\hline 0.090 & 1.2437 & 1.3496 & 2.1732 & 0.090 & 0.6531 & 0.7076 & 1.1285 & 0.090 & 0.4654 & 0.4882 & 0.6628 \\
\hline 0.100 & 1.3353 & 1.4302 & 2.2188 & 0.100 & 0.7337 & 0.7857 & 1.1065 & 0.095 & --- & 0.5285 & 0.6717 \\
\hline 0.110 & 1.4249 & 1.5199 & 2.1706 & 0.110 & -.. & 0.8481 & 1.1091 & & & & \\
\hline 0.120 & --- & 1.5937 & 2.1969 & & & & & & & & \\
\hline 0.130 & $\ldots$ & 1.6663 & 2.2036 & & & & & & & & \\
\hline 0.140 & --- & 1.7311 & 2.1051 & & & & & & & & \\
\hline
\end{tabular}

Table 6.12 $A$ values from FEA results for the 3D SECP specimen, $\lambda=1.0, a / W=0.7$ 


\begin{tabular}{rrrr}
\hline$i$ & $e_{i 0}$ & $e_{i 1}$ & $e_{i 2}$ \\
\hline 1 & 0.5235 & -3.7055 & 6.0913 \\
2 & -8.5333 & 107.9300 & -254.2400 \\
3 & 113.2600 & -1504.4000 & 3754.5000 \\
\hline
\end{tabular}

Table 6.13 The polynomial coefficients $e_{i j}$ for the 3D SECP specimen, $\lambda=0.0$, plane I

\begin{tabular}{rrrr}
\hline$i$ & $e_{i 0}$ & $e_{i 1}$ & $e_{i 2}$ \\
\hline 1 & -0.0072 & 1.6955 & -5.3575 \\
2 & 4.3289 & -59.3700 & 162.5600 \\
3 & 13.1640 & -171.7600 & 420.3000 \\
\hline
\end{tabular}

Table 6.14 The polynomial coefficients $e_{i j}$ for the 3D SECP specimen, $\lambda=0.0$, plane II 


\begin{tabular}{rrrr}
\hline$i$ & $e_{i 0}$ & $e_{i 1}$ & $e_{i 2}$ \\
\hline 1 & 0.1124 & -2.6837 & 4.3042 \\
2 & -7.3460 & 92.7440 & -213.5400 \\
3 & 109.0300 & -1444.9000 & 3605.6000 \\
\hline
\end{tabular}

Table 6.15 The polynomial coefficients $e_{i j}$ for the 3D SECP specimen, $\lambda=1.0$, plane I

\begin{tabular}{rrrr}
\hline$i$ & $e_{i 0}$ & $e_{i 1}$ & $e_{i 2}$ \\
\hline 1 & -0.2866 & 0.9105 & -4.4062 \\
2 & 4.2481 & -56.5710 & 168.0400 \\
3 & 14.6130 & -188.2400 & 448.9100 \\
\hline
\end{tabular}

Table 6.16 The polynomial coefficients $e_{i j}$ for the 3D SECP specimen, $\lambda=1.0$, plane II 


\begin{tabular}{rrrrrr}
\hline & $n=3$ & $n=4$ & $n=5$ & $n=7$ & $n=10$ \\
\hline$A_{S S Y}$ & 0.9277 & 0.5591 & 0.3887 & 0.2508 & 0.1828 \\
\hline$m_{1}$ & -0.3509 & -0.3306 & -0.3009 & -0.2668 & -0.2478 \\
\hline$m_{2}$ & 0.0786 & 0.0814 & 0.0753 & 0.0797 & 0.0915 \\
\hline$m_{3}$ & 0.0954 & 0.0938 & 0.0904 & 0.0761 & 0.0645 \\
\hline
\end{tabular}

Table 6.17 Values of coefficients for polynomial $A-T$ relationship, plane I

\begin{tabular}{rrrrrr}
\hline & $n=3$ & $n=4$ & $n=5$ & $n=7$ & $n=10$ \\
\hline$A_{S S Y}$ & 0.9435 & 0.5731 & 0.4006 & 0.2602 & 0.1903 \\
\hline$m_{1}$ & -0.3385 & -0.3138 & -0.2863 & -0.2557 & -0.2414 \\
\hline$m_{2}$ & 0.0746 & 0.0779 & 0.0753 & 0.0792 & 0.0927 \\
\hline$m_{3}$ & 0.0981 & 0.0895 & 0.0846 & 0.0750 & 0.0673 \\
\hline
\end{tabular}

Table 6.18 Values of coefficients for polynomial $A-T$ relationship, plane II 


\begin{tabular}{ccccccc}
\hline & $\lambda=0.0$ & \multicolumn{5}{c}{$\lambda=1.0$} \\
\hline$z / t$ & $a / W=0.1$ & $a / W=0.3$ & $a / W=0.7$ & $a / W=0.1$ & $a / W=0.3$ & $a / W=0.7$ \\
\hline $\begin{array}{c}\text { Plane } \\
\text { I }\end{array}$ & 0.5098 & 0.5513 & 3.4003 & -0.4902 & -0.4487 & 2.4003 \\
\hline $\begin{array}{c}\text { Plane } \\
\text { II }\end{array}$ & 0.5179 & 0.5693 & 3.4693 & -0.4821 & -0.4307 & 2.4693 \\
\hline
\end{tabular}

Table 6.19 Numerical solutions of normalized $T$-stress $(V)$ for the 3D SECP specimen, $\lambda=0.0,1.0$, planes I and II

\begin{tabular}{|c|c|c|c|c|c|c|c|}
\hline & & $\lambda=0.0$ & & & $\lambda=1.0$ & & \\
\hline$a / W$ & & $n=3$ & $n=5$ & $n=10$ & $n=3$ & $n=5$ & $n=10$ \\
\hline \multirow[t]{2}{*}{0.1} & Plane I & 0.750 & 0.650 & 0.650 & 0.550 & 0.450 & 0.450 \\
\hline & Plane II & 0.650 & 0.550 & 0.550 & 0.550 & 0.450 & 0.400 \\
\hline \multirow[t]{2}{*}{0.3} & Plane I & 0.350 & 0.250 & 0.250 & 0.250 & 0.200 & 0.200 \\
\hline & Plane II & 0.250 & 0.250 & 0.200 & 0.250 & 0.200 & 0.200 \\
\hline \multirow[t]{2}{*}{0.7} & Plane I & 0.050 & 0.040 & 0.030 & 0.050 & 0.040 & 0.030 \\
\hline & Plane II & 0.040 & 0.035 & 0.030 & 0.040 & 0.035 & 0.030 \\
\hline
\end{tabular}

Table 6.20 Maximum load range of applicability of $A-T$ relation, $\sigma / \sigma_{0}$, for the 3D SECP 


\begin{tabular}{rrrrrrr}
\hline \multicolumn{7}{c}{ Plane I } \\
\hline$a / W$ & $n=3$ & $n=5$ & $n=10$ & $n=3$ & $n=5$ & $n=10$ \\
\hline 0.1 & 0.2904 & 0.1906 & 0.1078 & 0.3535 & 0.2105 & 0.1710 \\
\hline 0.3 & 0.6473 & 0.4836 & 0.5455 & 0.5828 & 0.4944 & 0.5908 \\
\hline 0.7 & 4.3209 & 7.6728 & 17.6606 & 5.6944 & 9.3293 & 23.0759 \\
\hline
\end{tabular}

Table 6.21 Coefficient $a_{1}$ values for the 3D SECP specimen, $\lambda=0.0$, planes I and II

\begin{tabular}{rrrrrrr}
\hline \multicolumn{1}{c}{ Plane I } & \multicolumn{5}{c}{ Plane II } \\
\hline$a / W$ & $n=3$ & $n=5$ & $n=10$ & $n=3$ & $n=5$ & $n=10$ \\
\hline 0.1 & 0.3740 & 0.3583 & 0.2217 & 0.4951 & 0.4069 & 0.3412 \\
\hline 0.3 & 0.9142 & 0.8334 & 0.8232 & 0.8809 & 0.7606 & 0.9059 \\
\hline 0.7 & 4.5320 & 8.2872 & 19.4928 & 5.8738 & 9.8680 & 24.6839 \\
\hline
\end{tabular}

Table 6.22 Coefficient $a_{1}$ values for the 3D SECP specimen, $\lambda=1.0$, planes I and II 


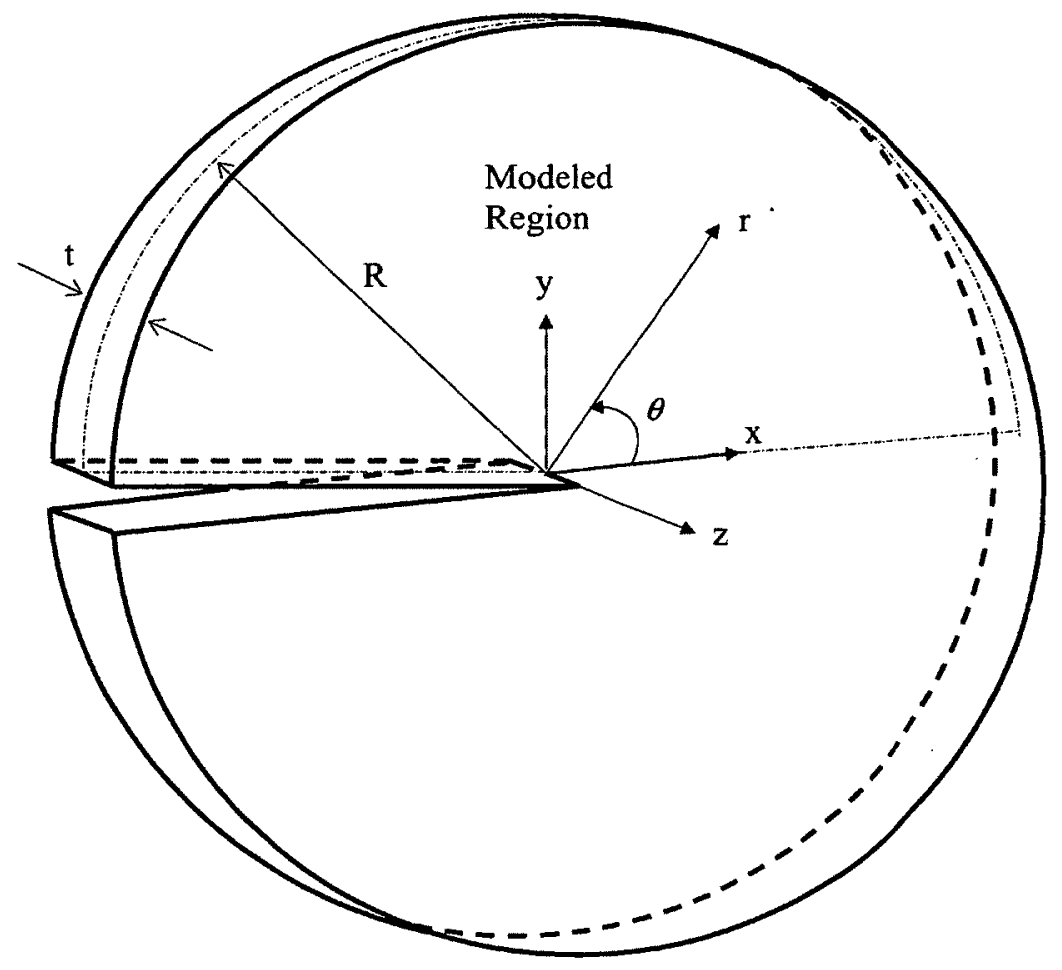

Figure 6.1 The modified boundary layer model 

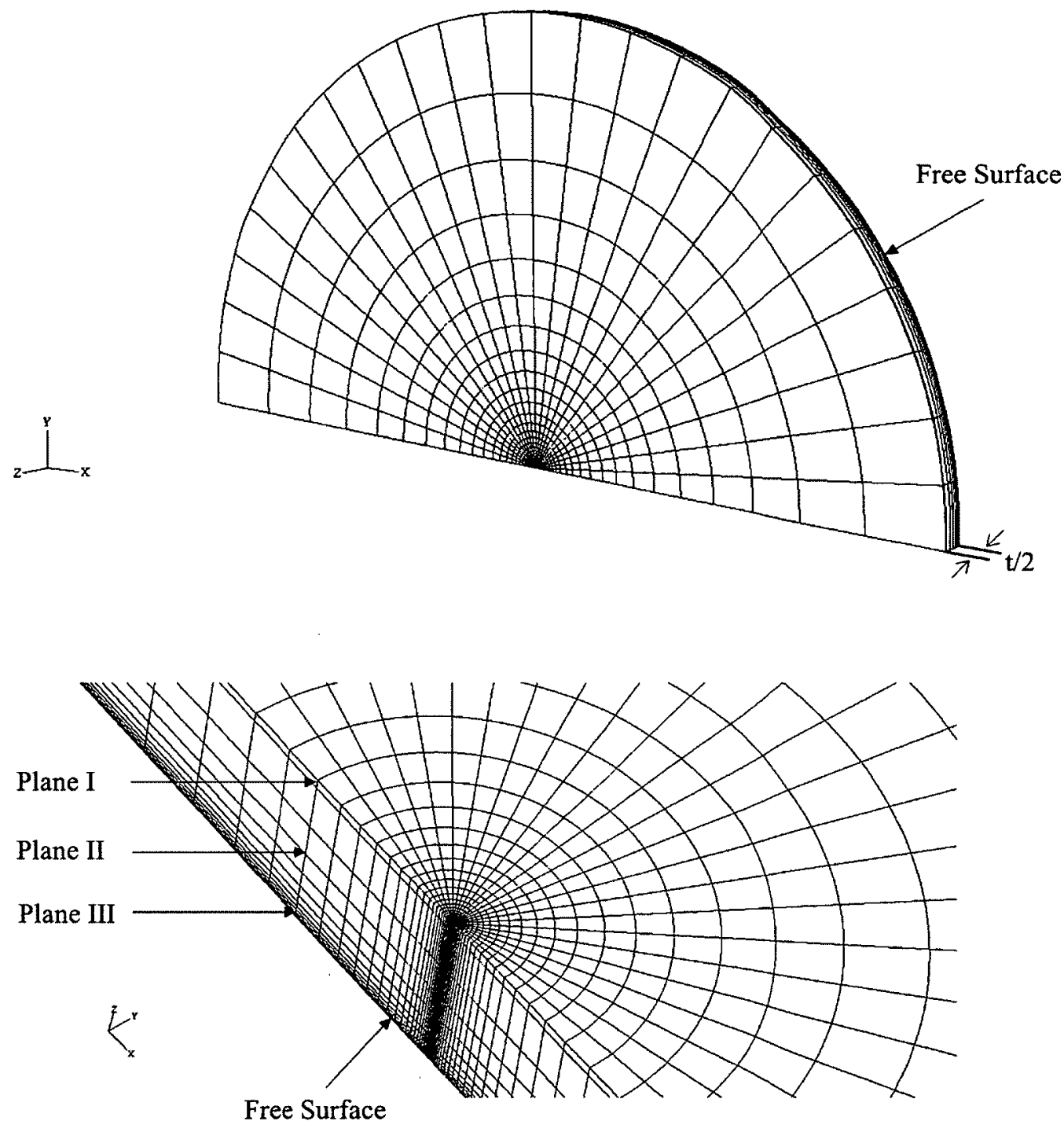

Figure 6.2 FEA mesh for the modified boundary layer problem 


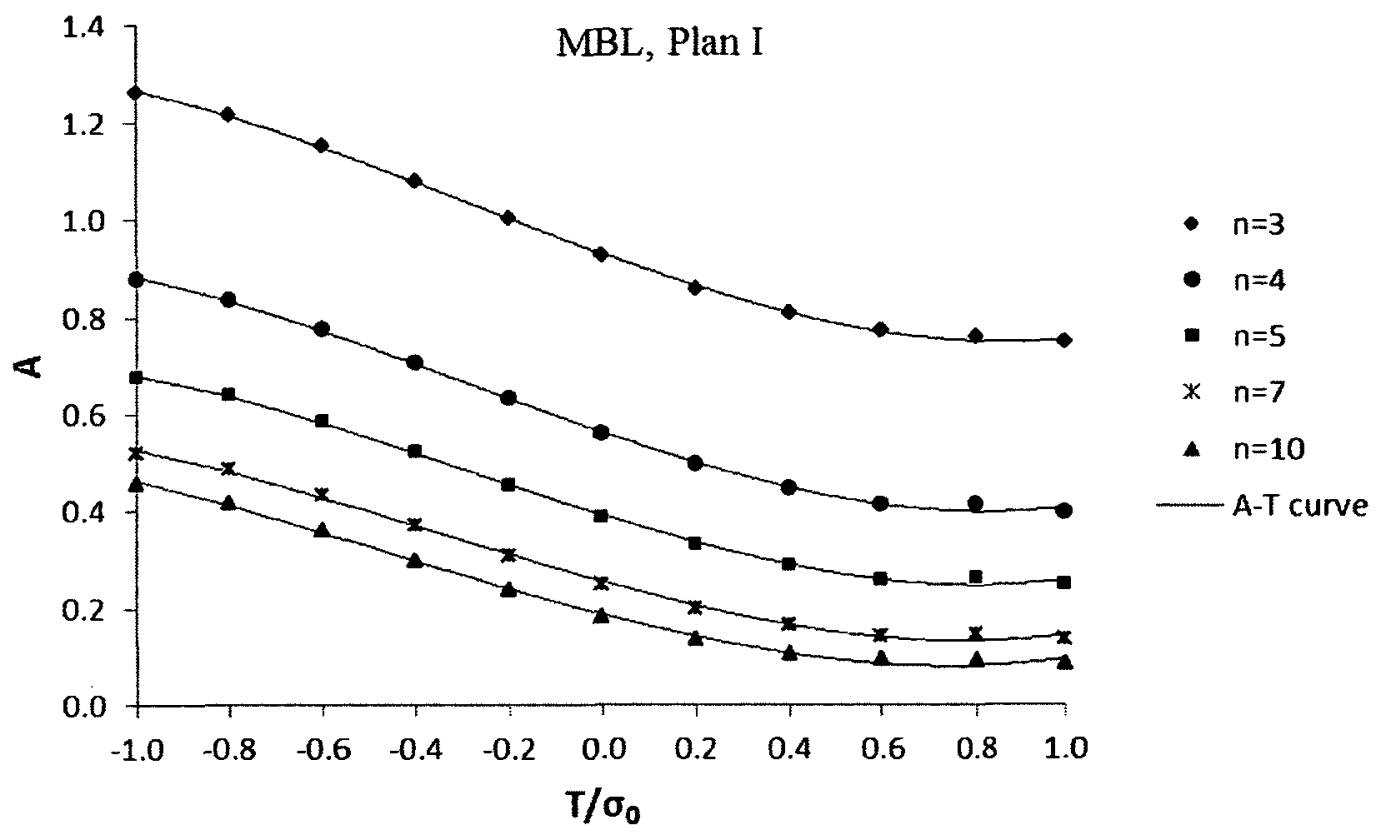

Figure 6.3 $A-T$ relationship curves from 3D MBL formulation, plane I

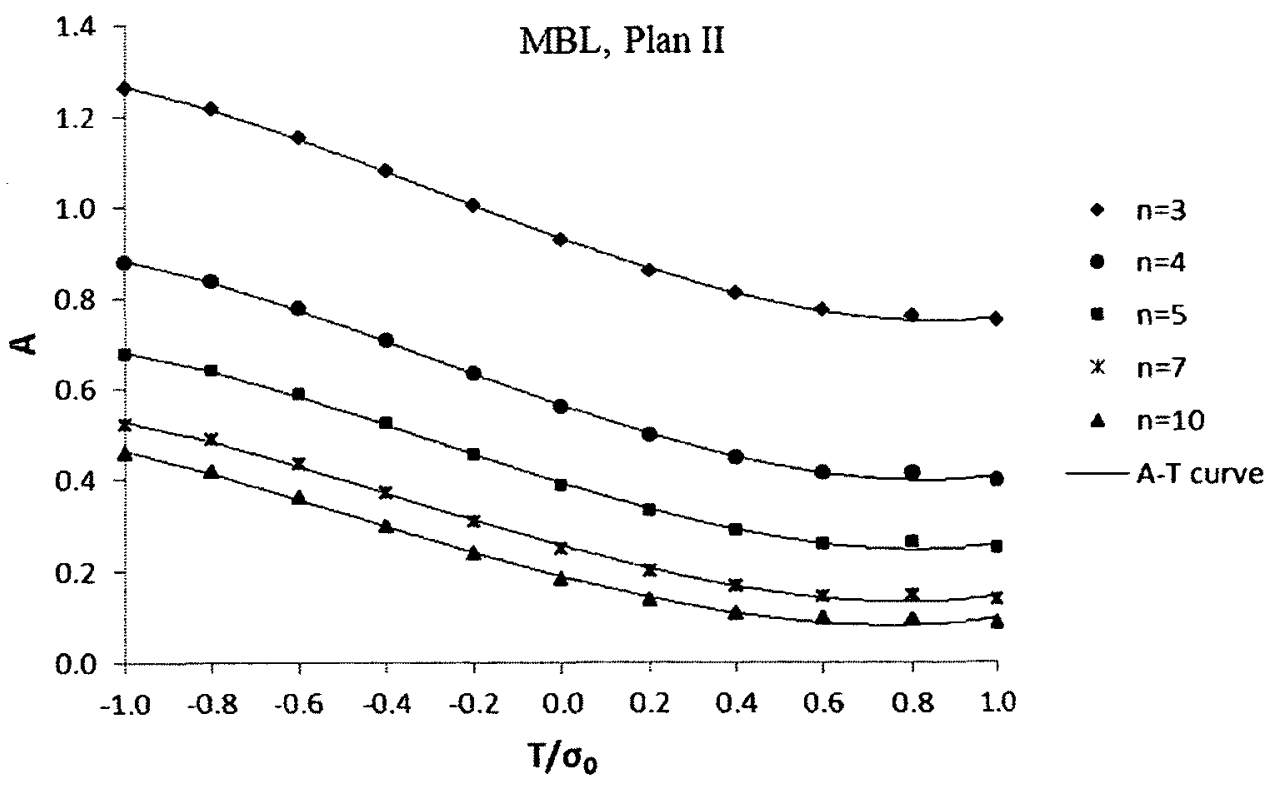

Figure 6.4 $A-T$ relationship curves from 3D MBL formulation, plane II 


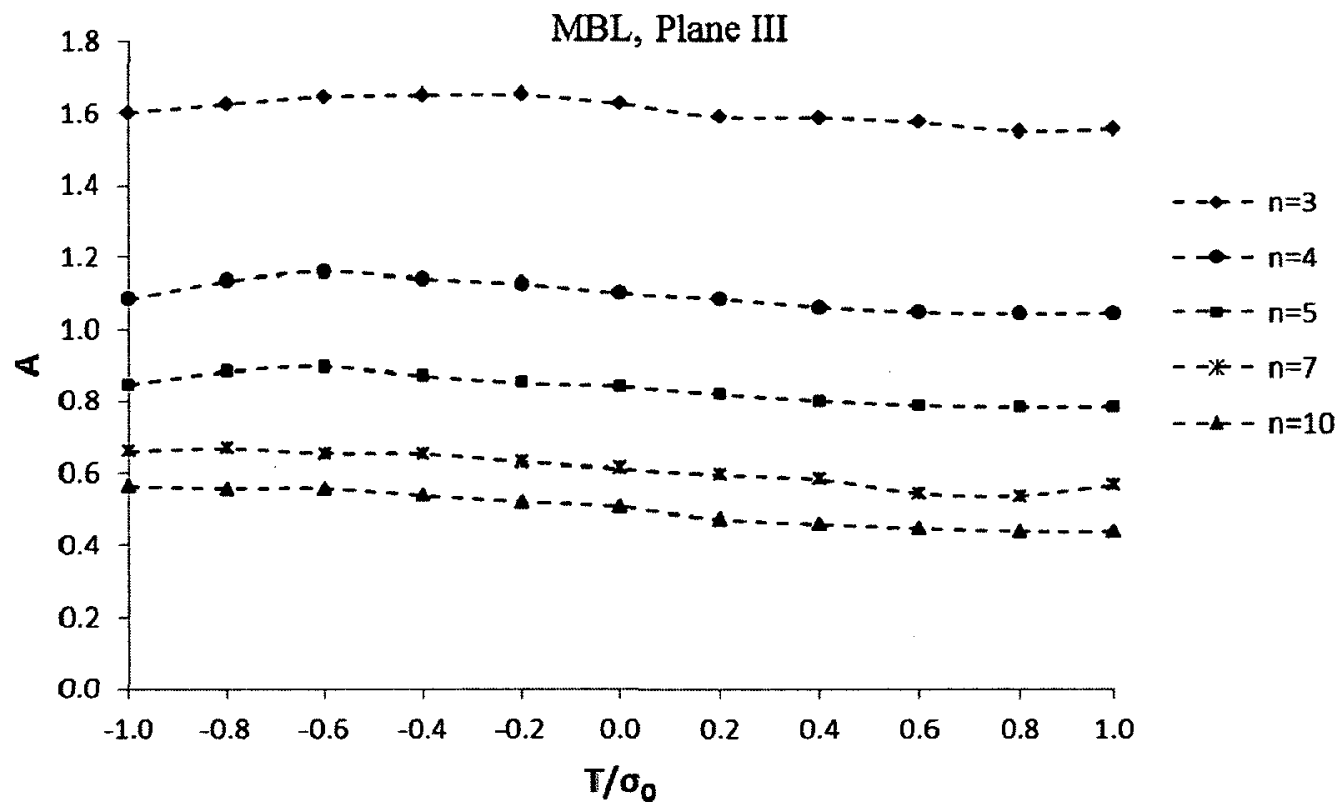

Figure 6.5 $A-T$ relationship curves from 3D MBL formulation, plane III 


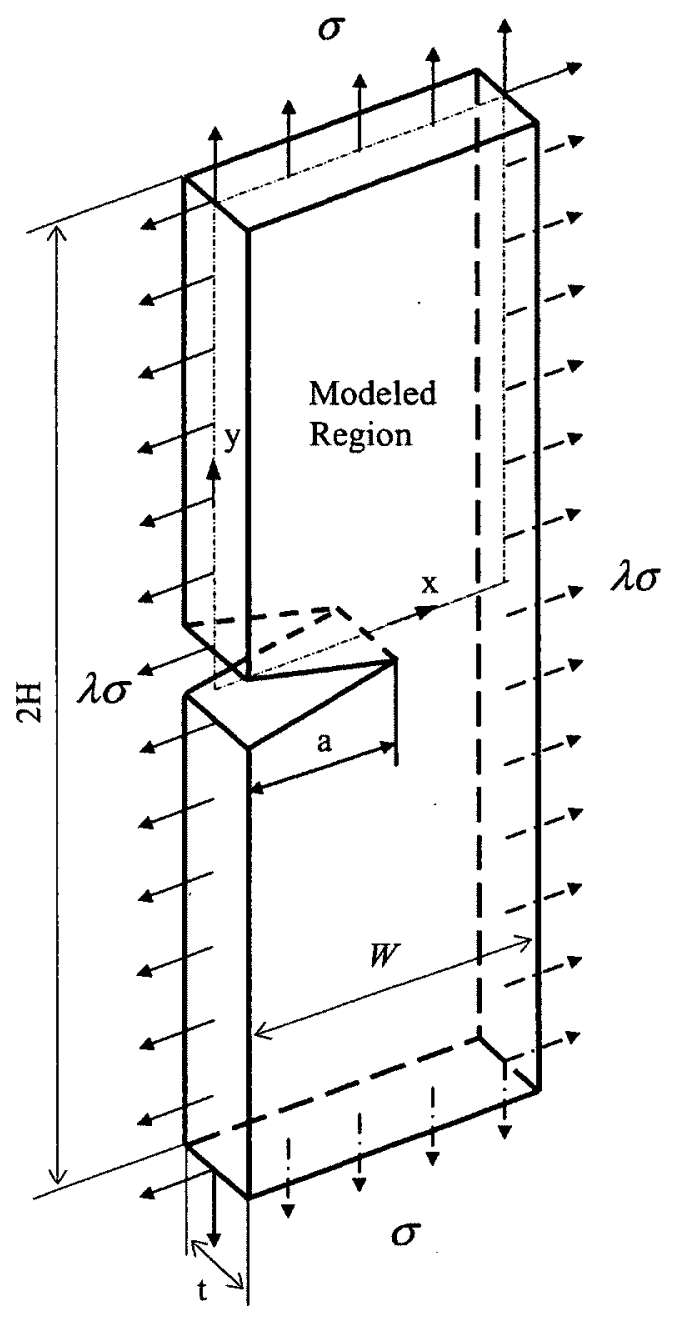

Figure 6.6 3D single edge cracked plate (SECP) model 


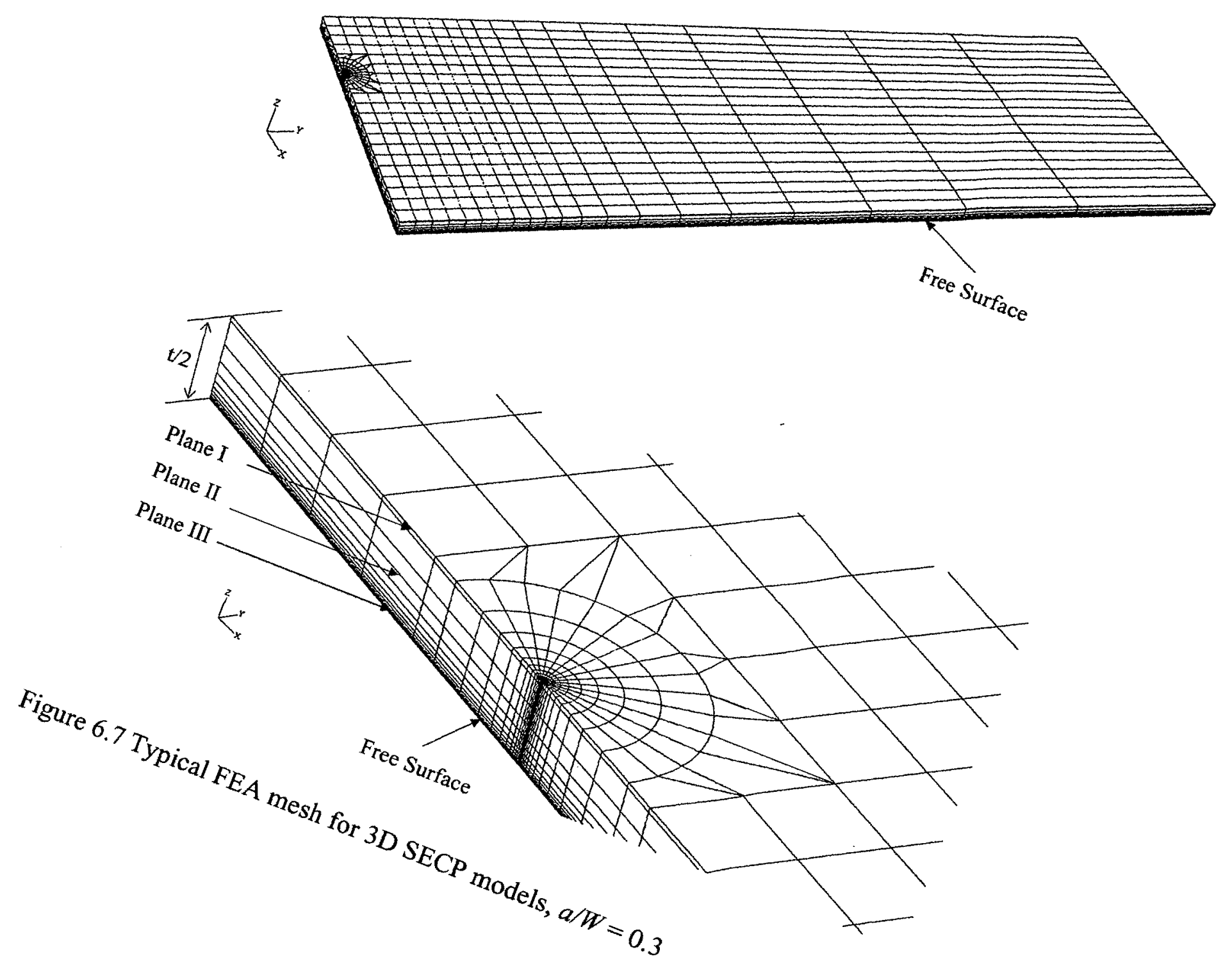

198 


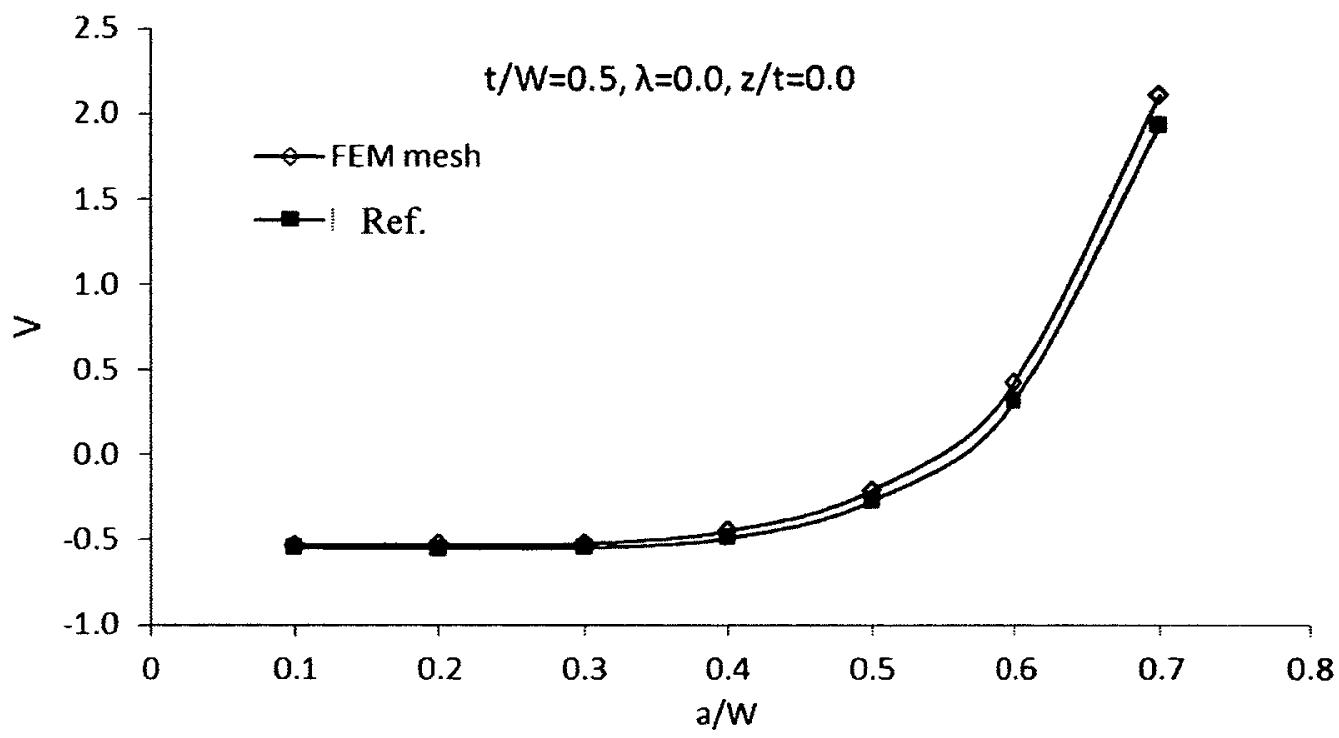

Figure 6.8 Comparison of normalized $T$-stress $(V)$ between FEA results and those from Nakamura and Parks (1992)

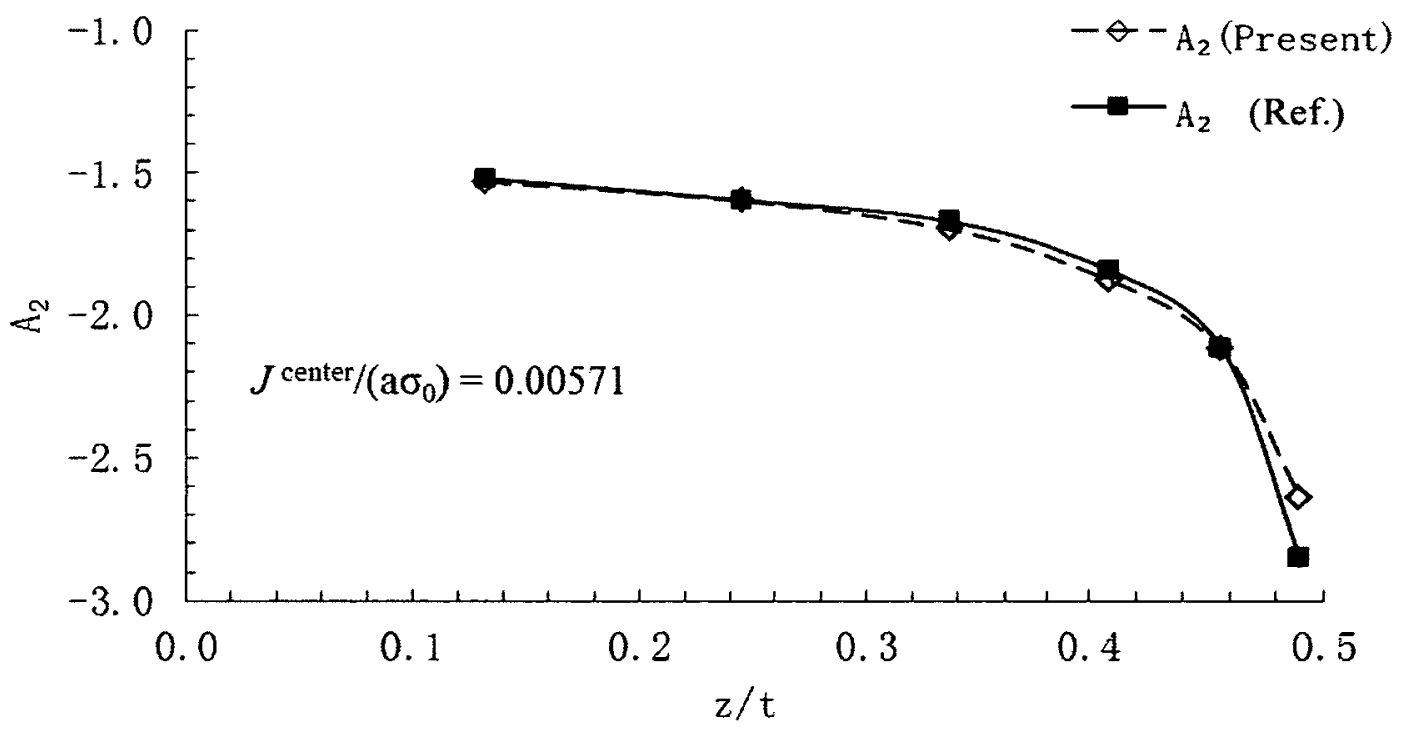

Figure 6.9 Comparison of parameter $A_{2}$ between present results and those from Zhu et al. (2002) 

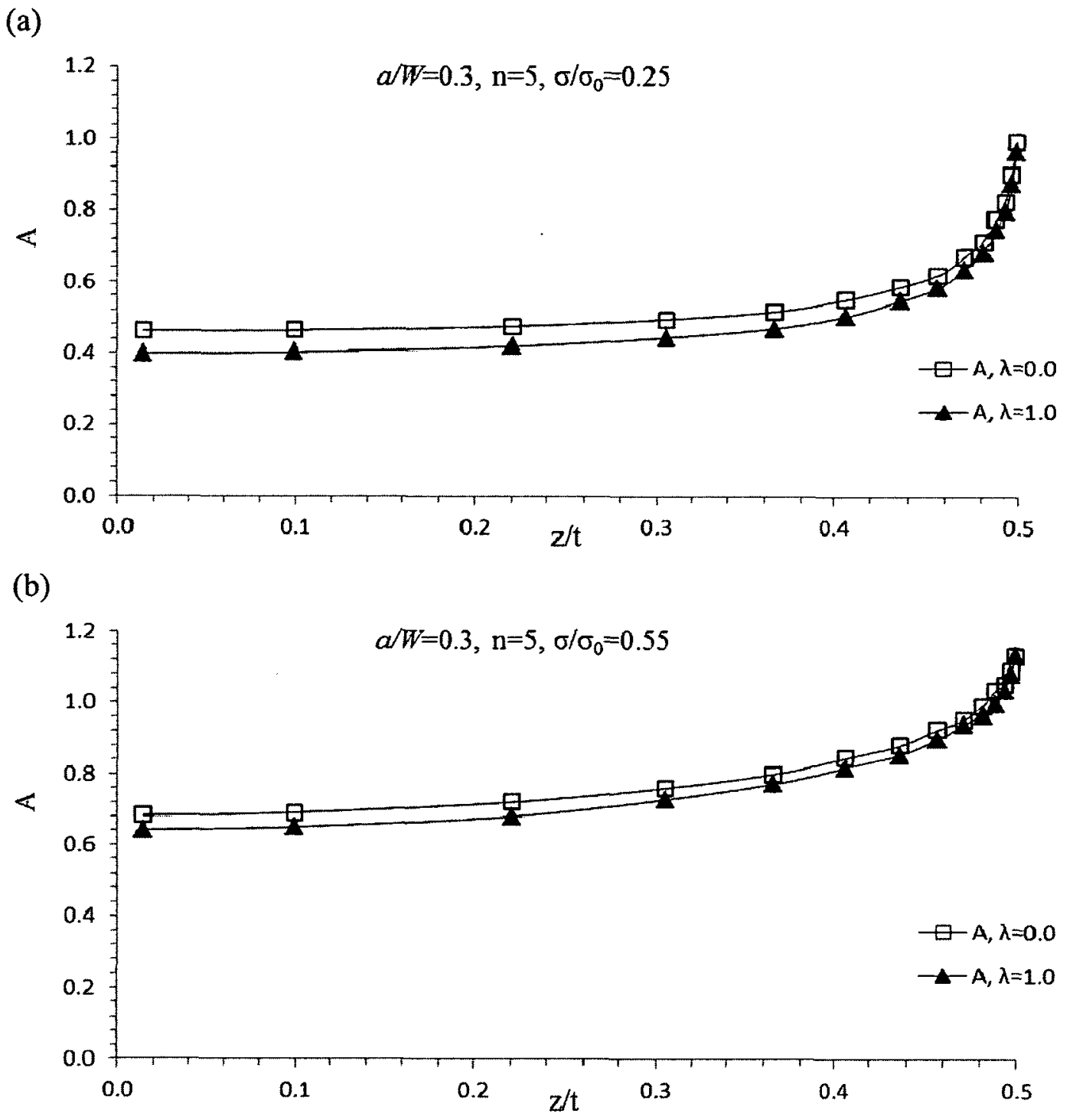

Figure 6.10 Variation of parameter $A$ along the crack-front of the 3D SECP, $a / W=0.3$, $n=5$ 
(a)

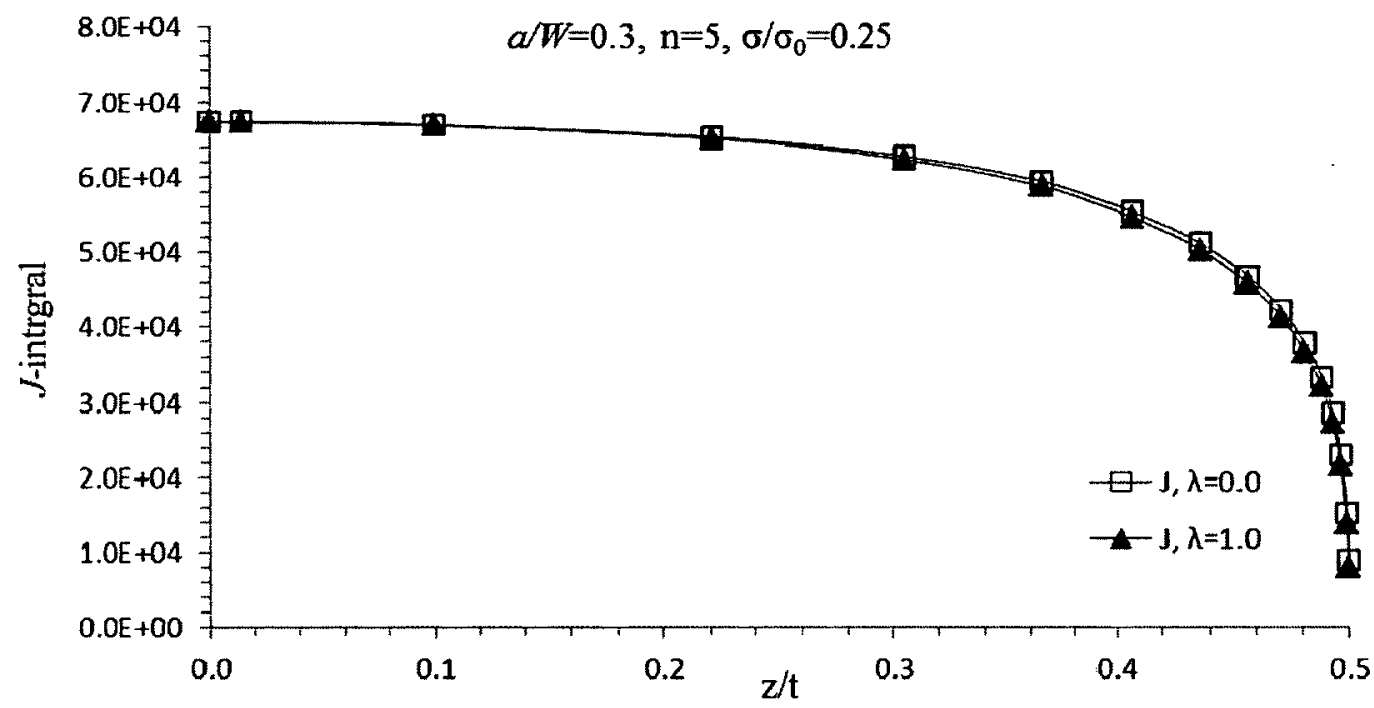

(b)

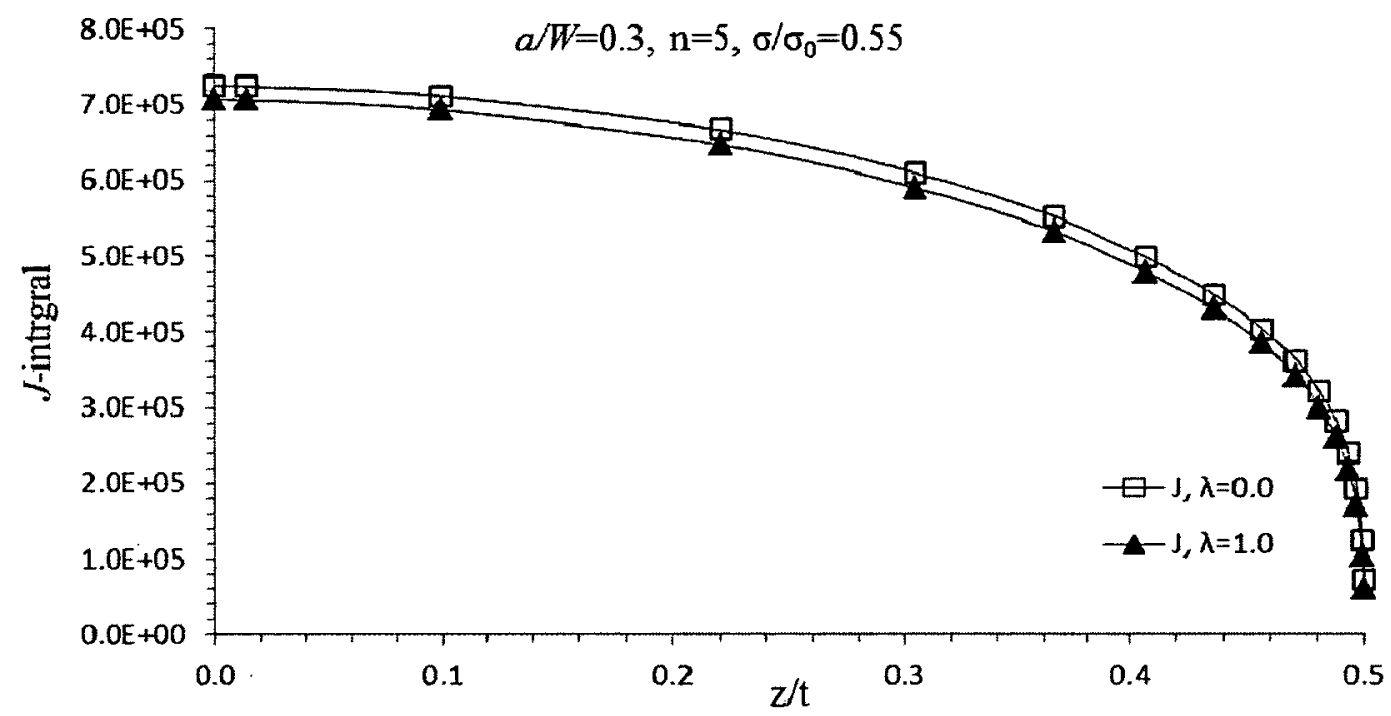

Figure 6.11 Variation of $J$-integral along the crack-front of the 3D SECP, $a / W=0.3, n=5$ 
(a)

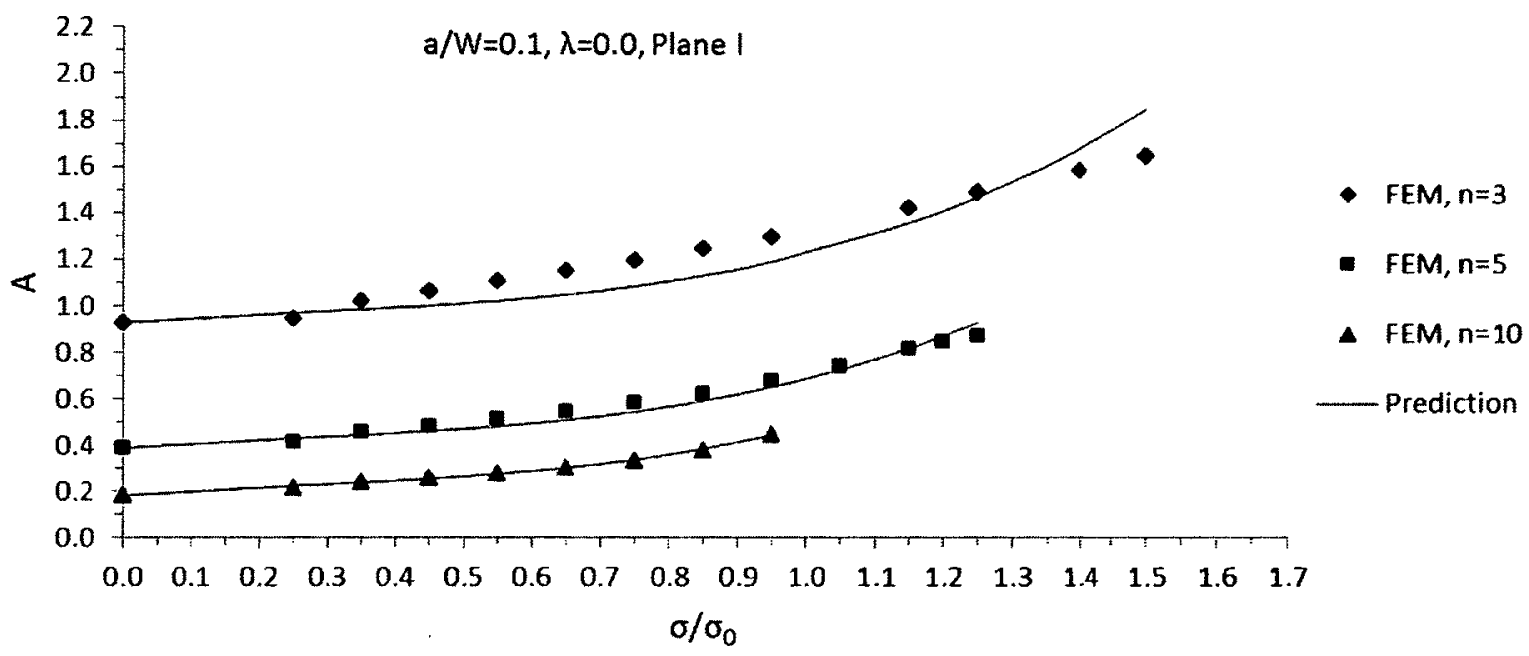

(b)

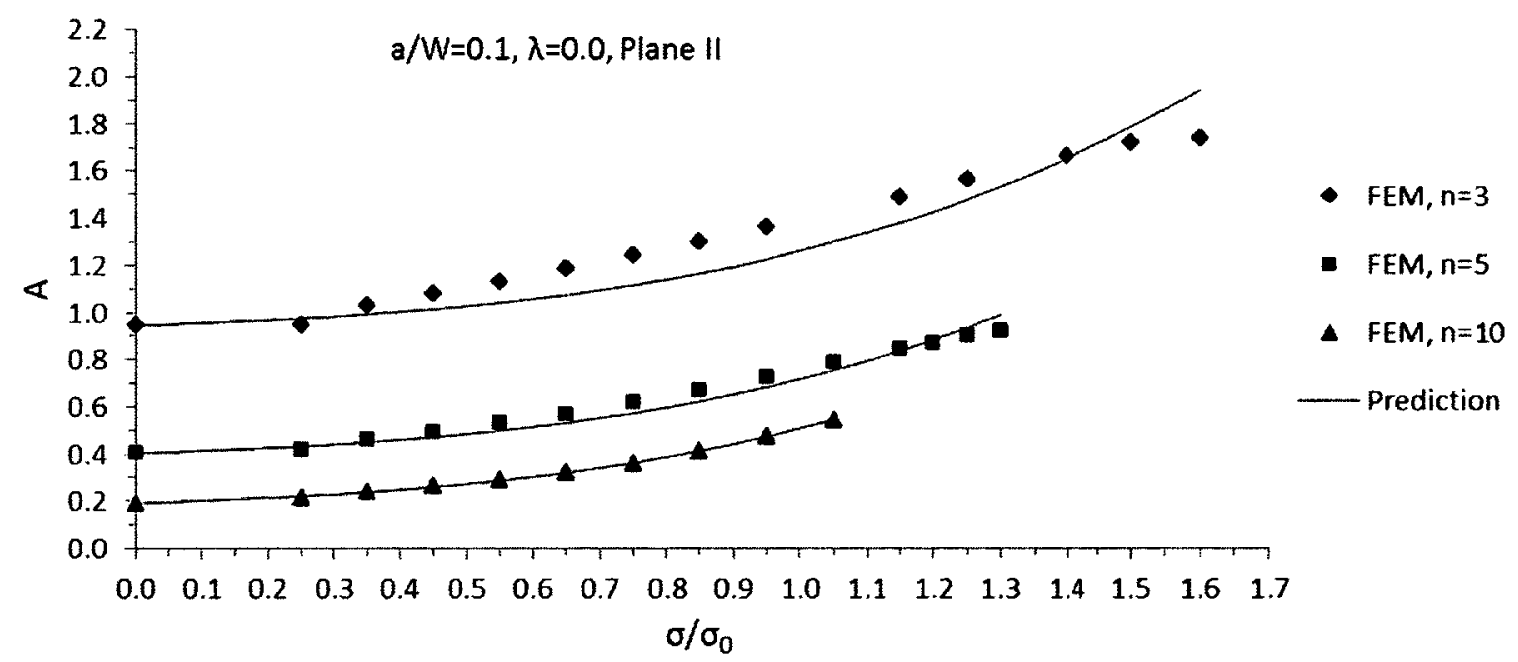

Figure 6.12 Comparisons of $A$ values predicted by curve shape similarity with FEA data for the 3D SECP, $\lambda=0.0, a / W=0.1$, (a) plane I and (b) plane II. 
(a)

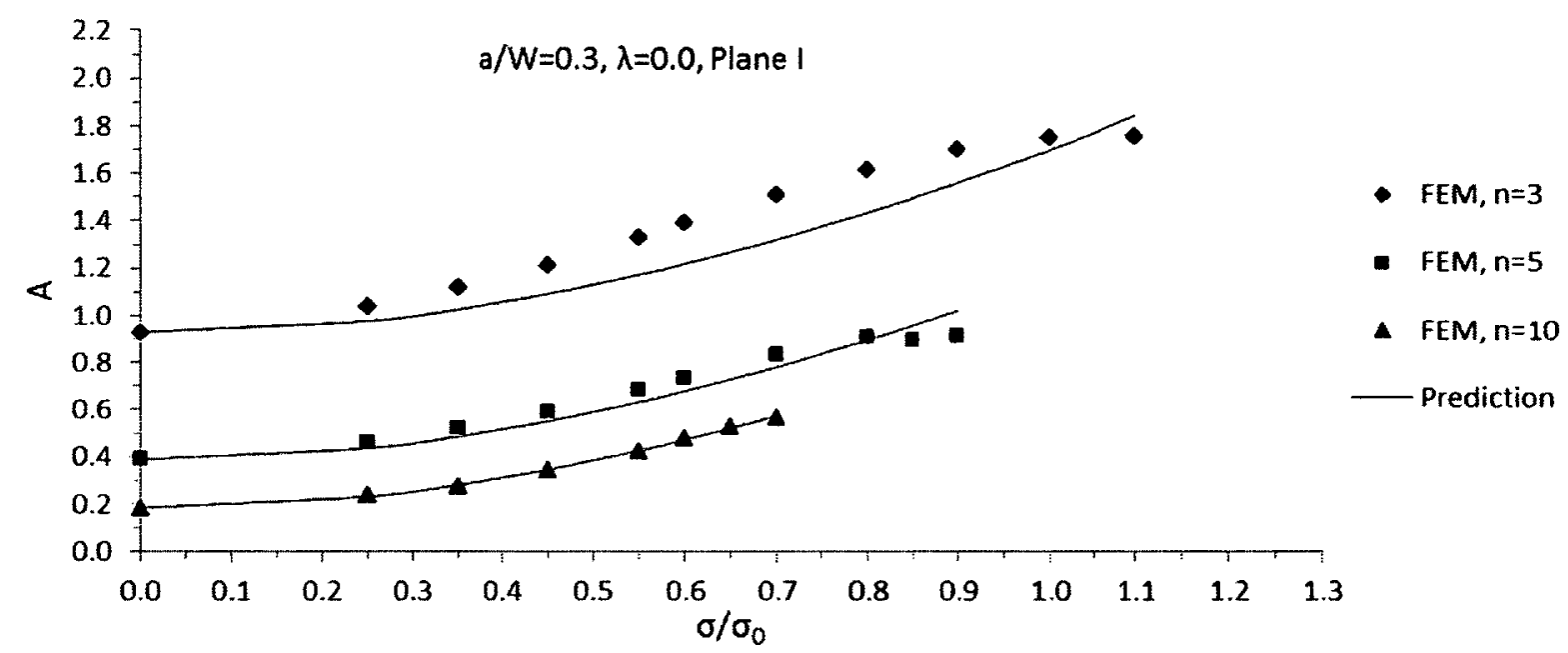

(b)

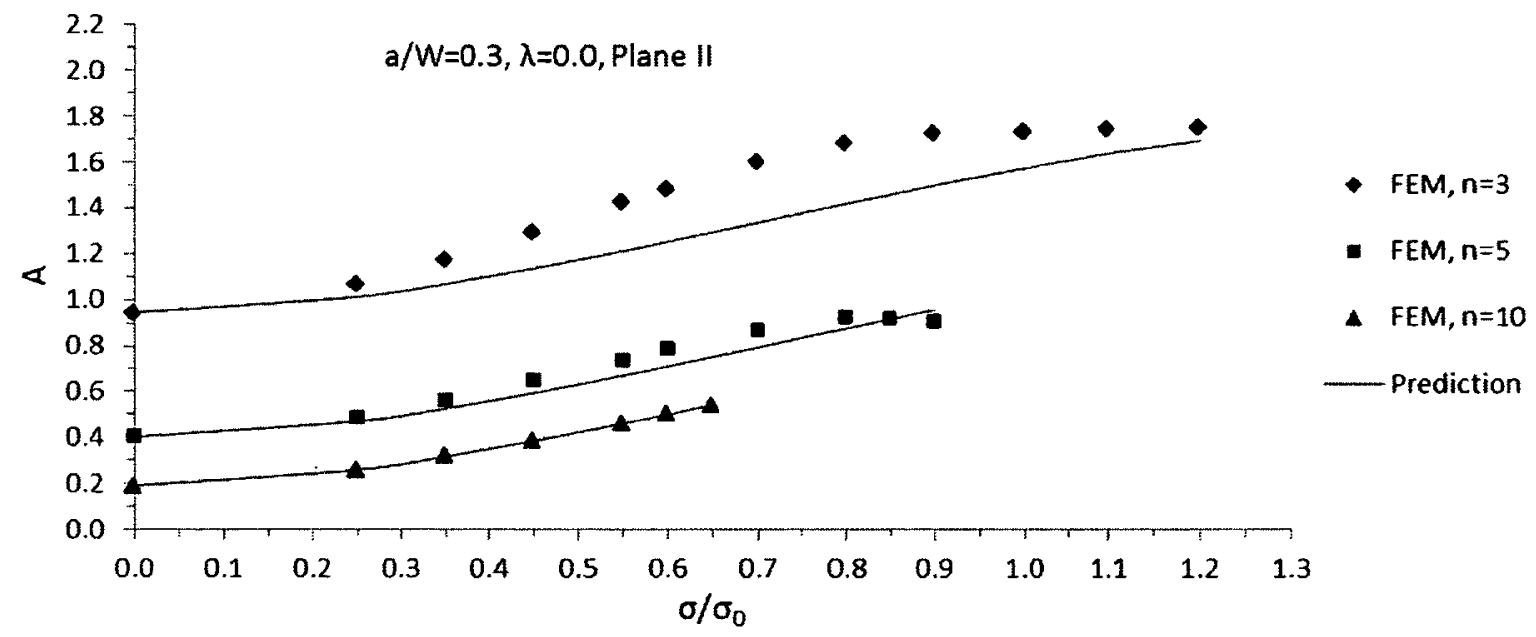

Figure 6.13 Comparisons of $A$ values predicted by curve shape similarity with FEA data for the 3D SECP, $\lambda=0.0, a / W=0.3$, (a) plane I and (b) plane II. 
(a)

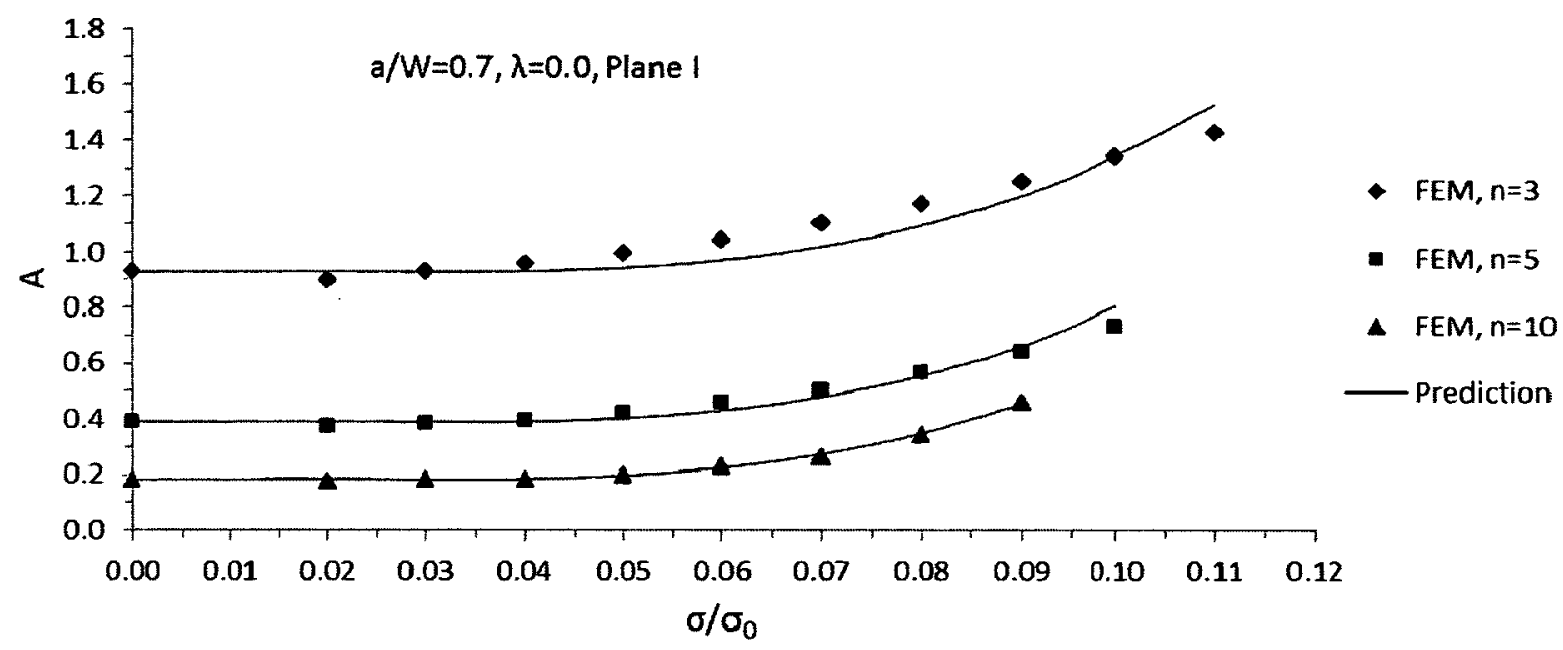

(b)

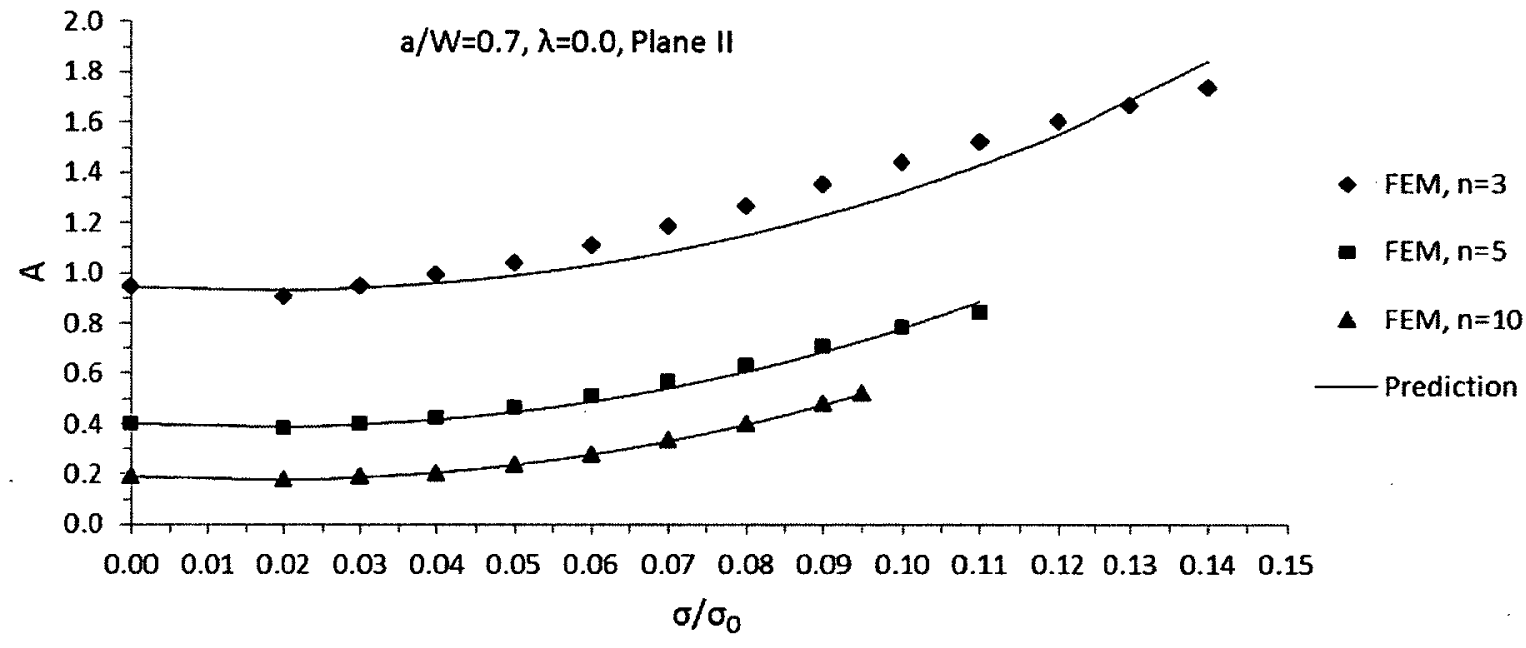

Figure 6.14 Comparisons of $A$ values predicted by curve shape similarity with FEA data for the 3D SECP, $\lambda=0.0, a / W=0.7$, (a) plane I and (b) plane II. 
(a)

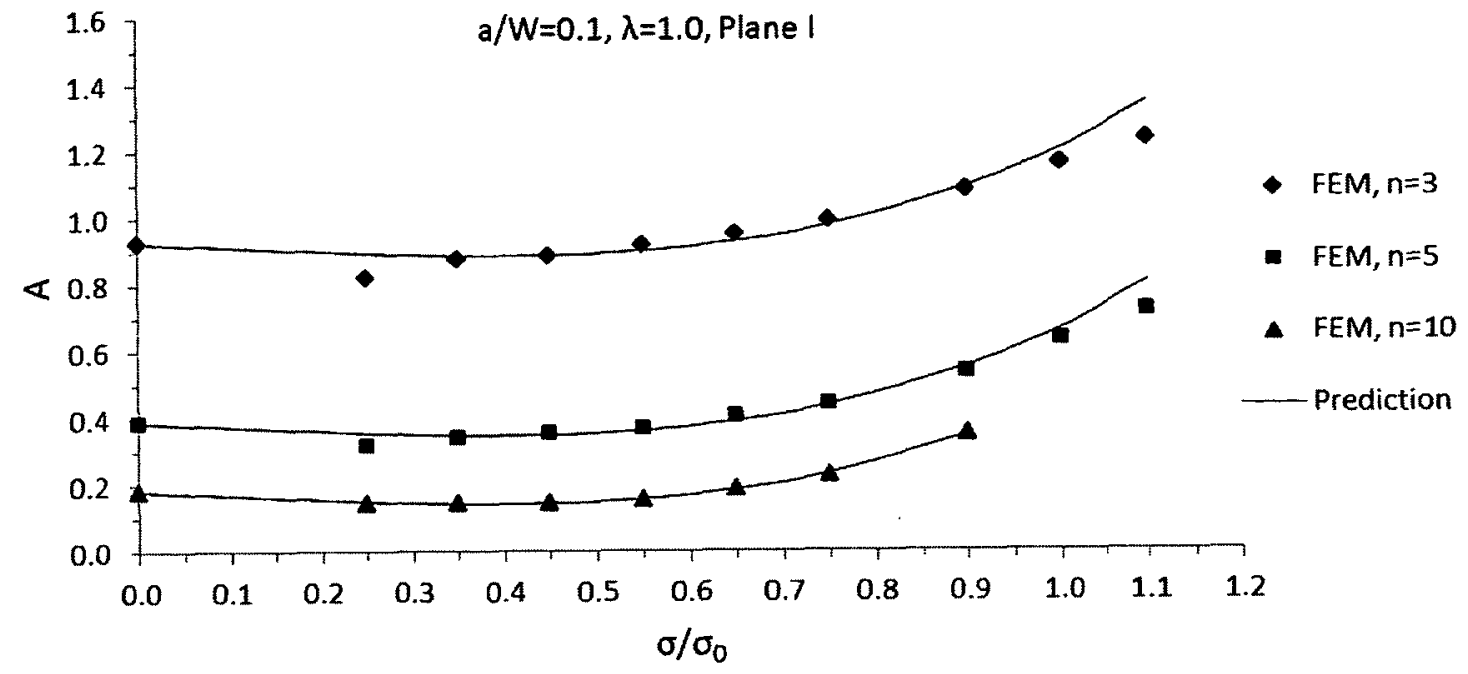

(b)

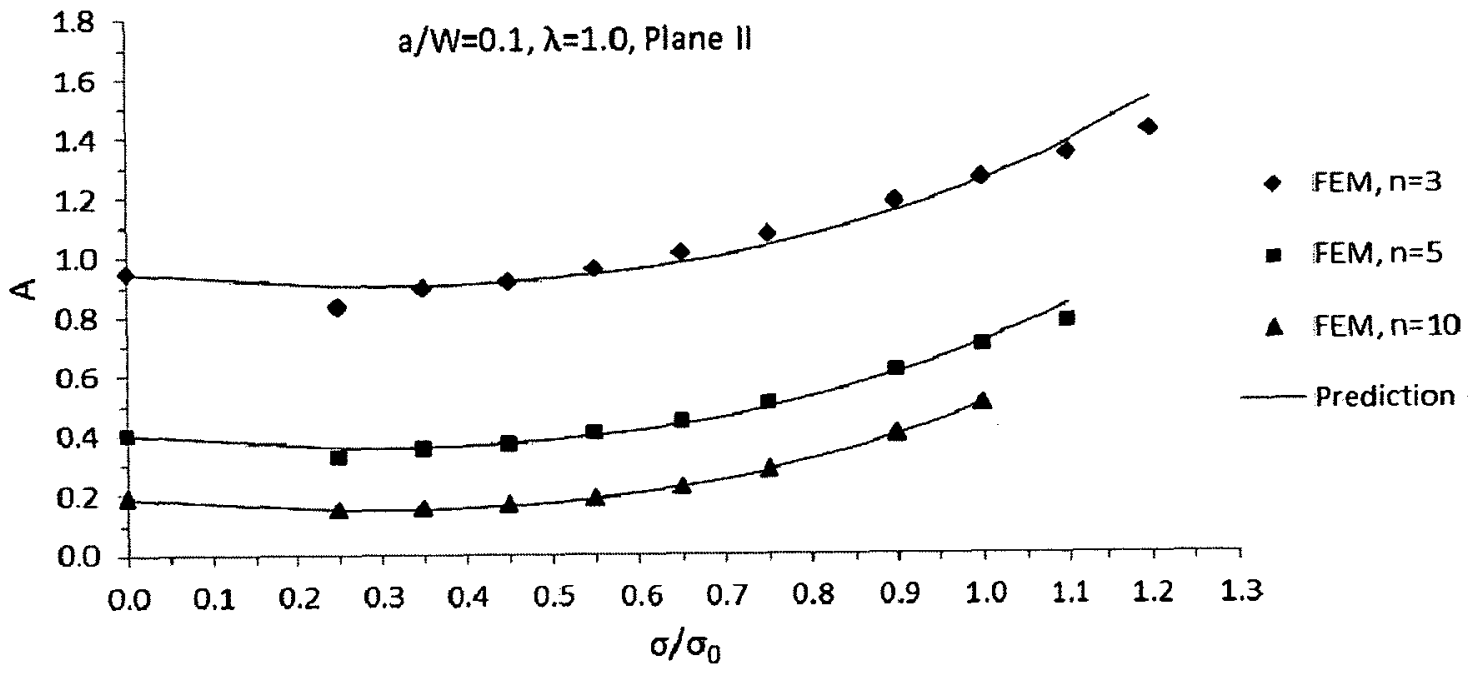

Figure 6.15 Comparisons of $A$ values predicted by curve shape similarity with FEA data for the 3D SECP, $\lambda=1.0, a / W=0.1$, (a) plane I and (b) plane II. 
(a)

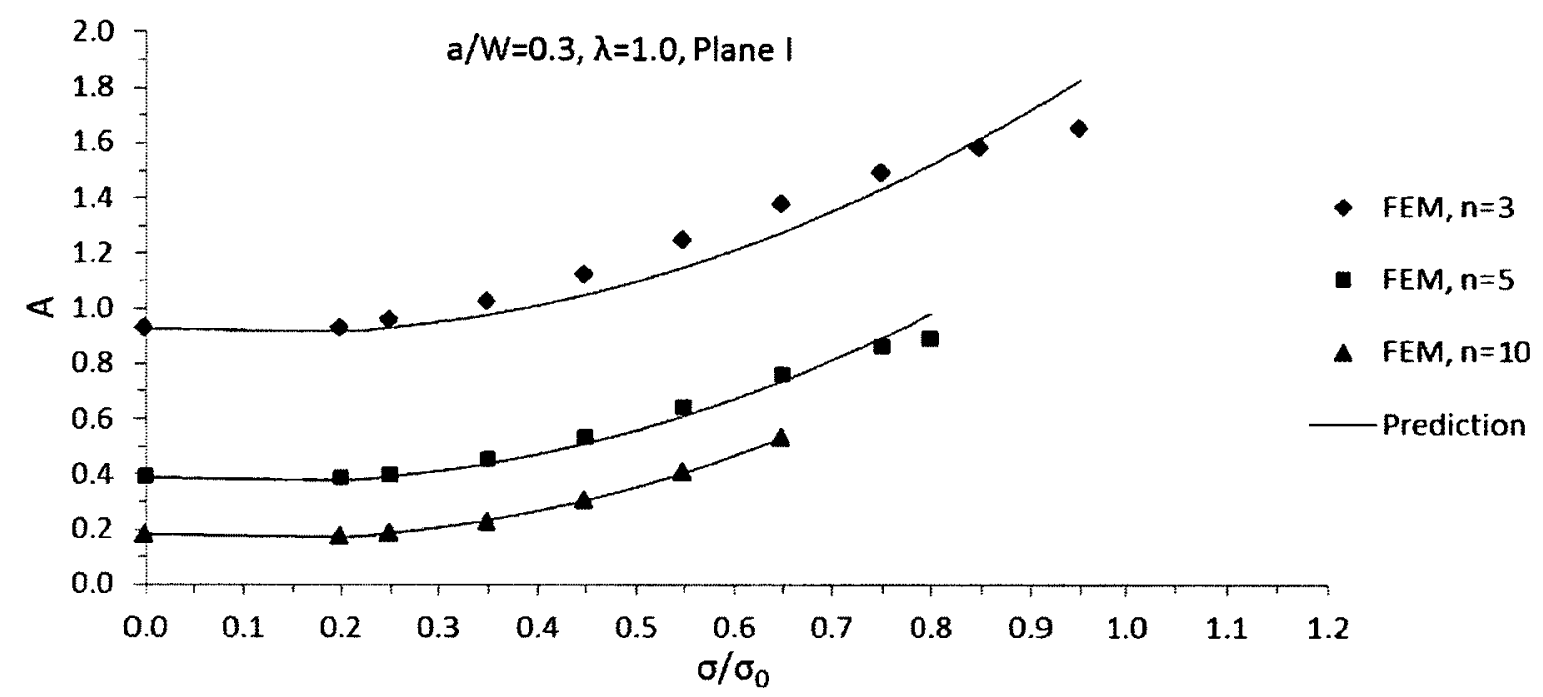

(b)

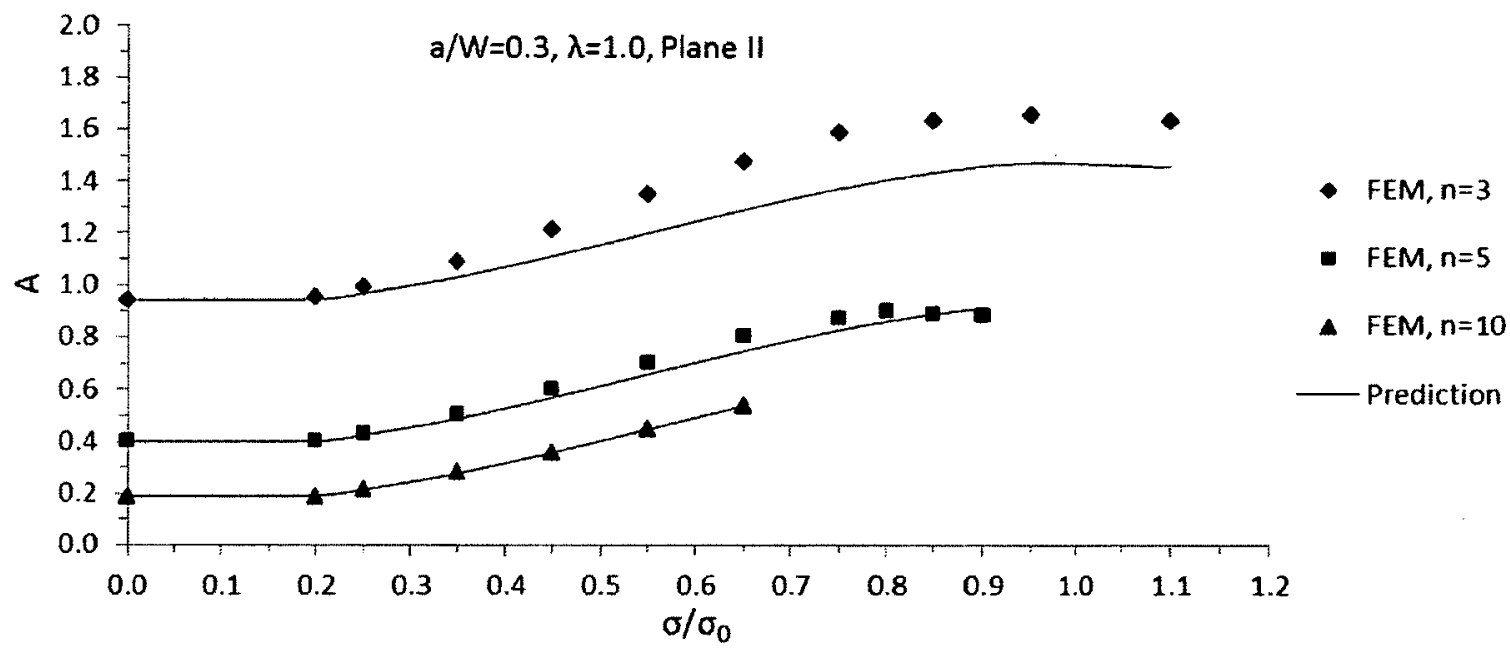

Figure 6.16 Comparisons of $A$ values predicted by curve shape similarity with FEA data for the 3D SECP, $\lambda=1.0, a / W=0.3$, (a) plane I and (b) plane II. 
(a)

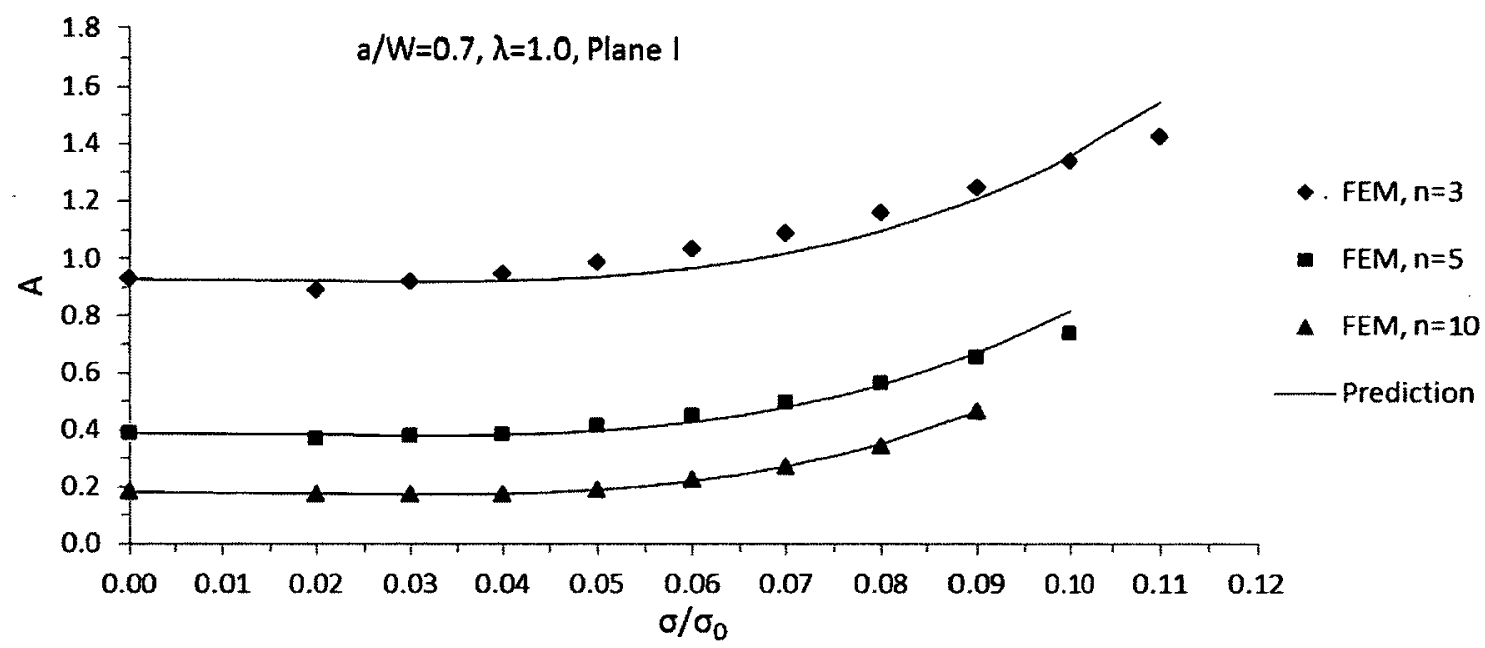

(b)

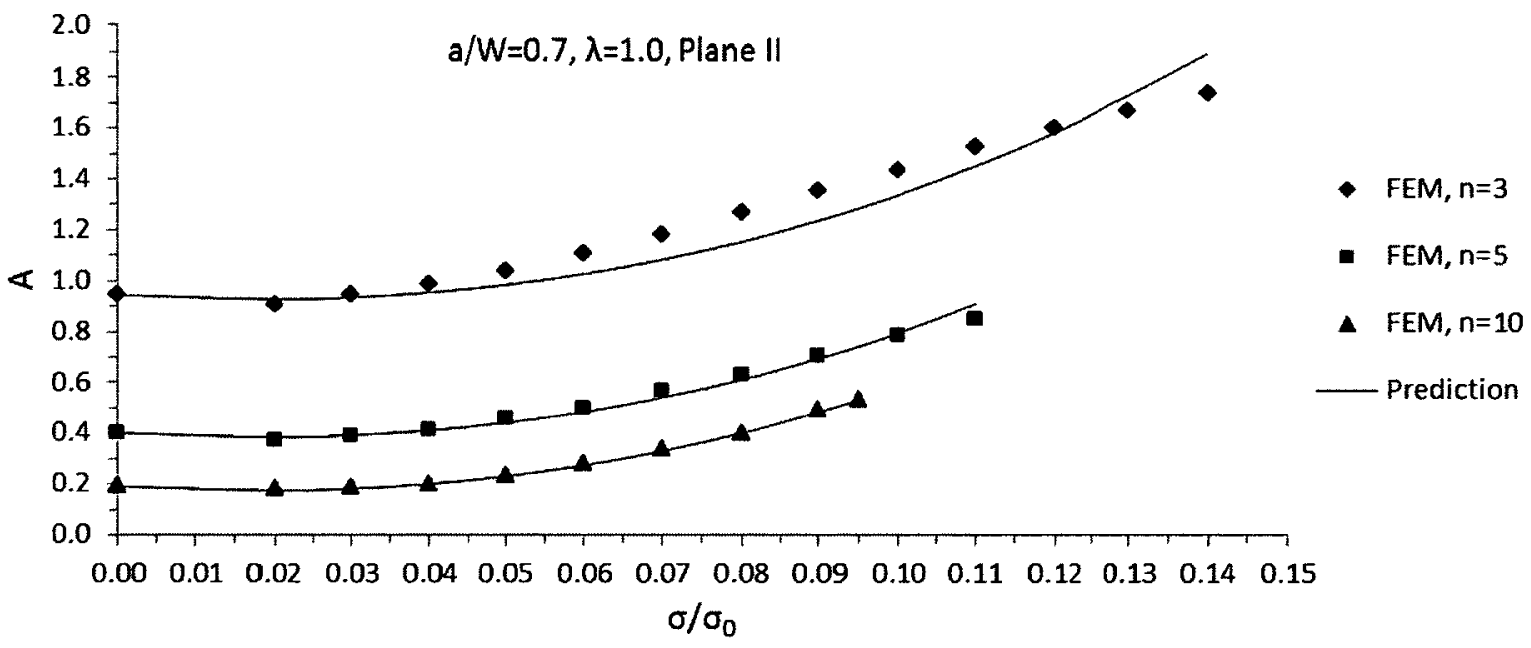

Figure 6.17 Comparisons of $A$ values predicted by curve shape similarity with FEA data for the 3D SECP, $\lambda=1.0, a / W=0.7$, (a) plane I and (b) plane II. 
(a)

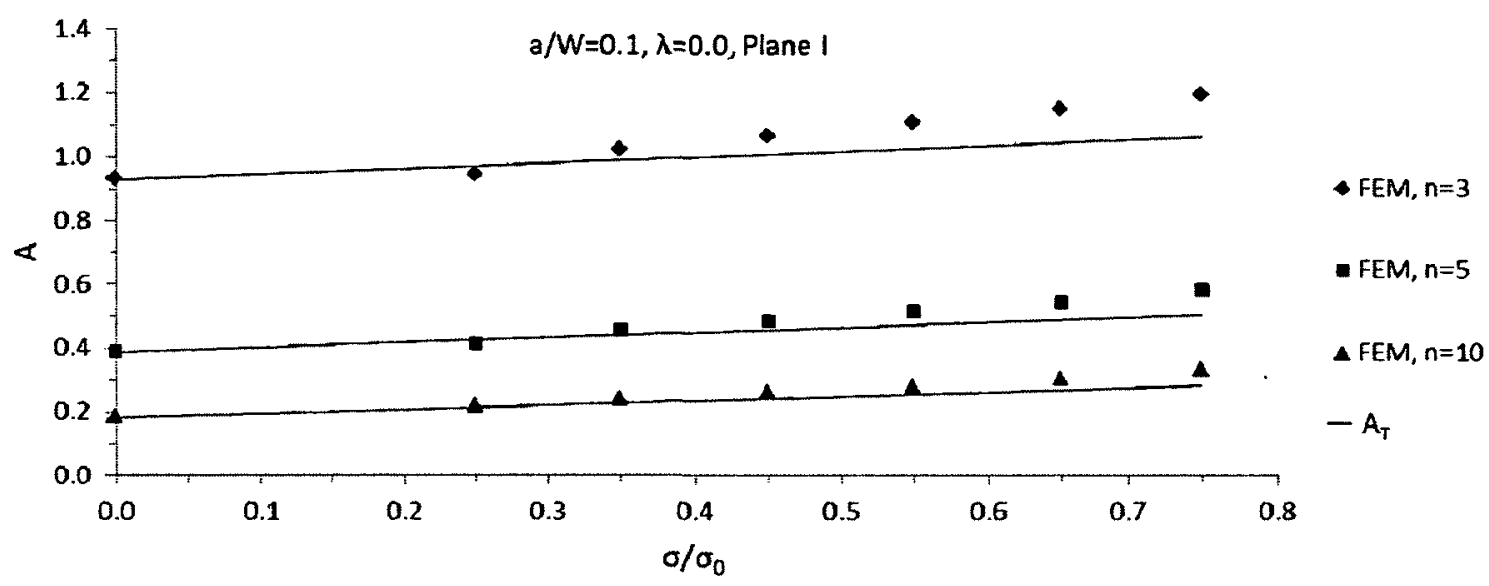

(b)

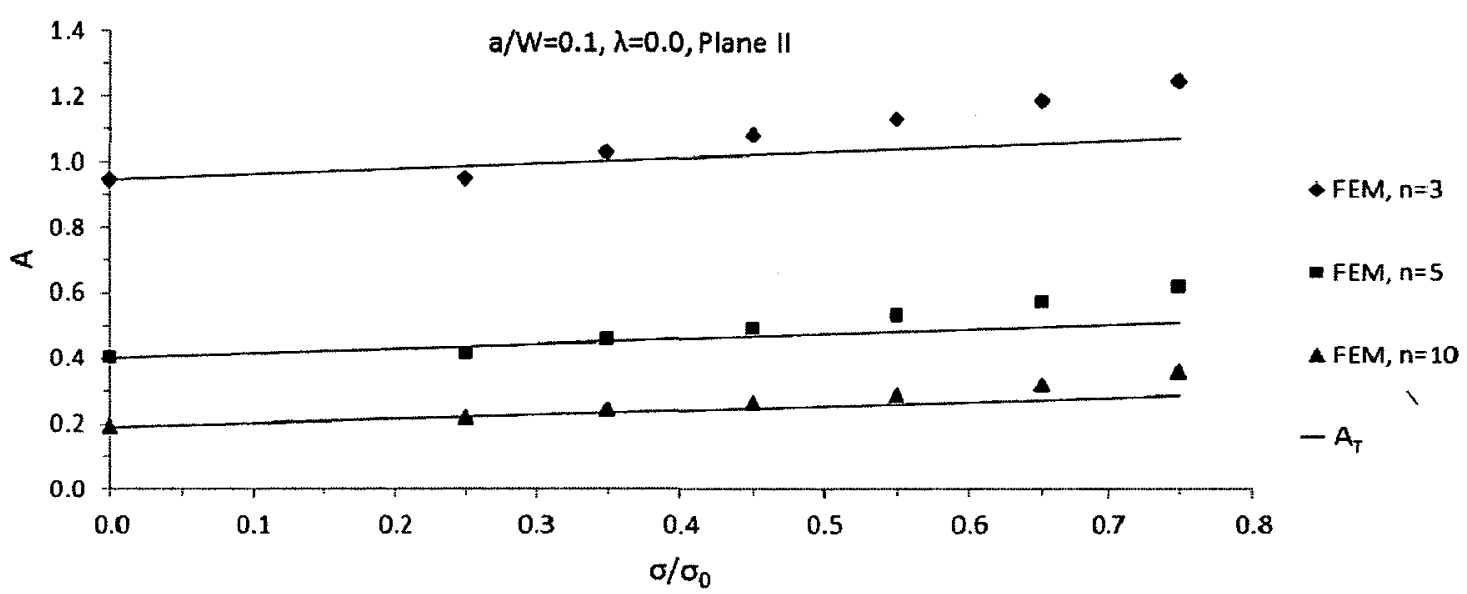

Figure 6.18 Comparisons of $A$ values predicted from $T$-stress with FEA data for the 3D SECP, $\lambda=0.0, a / W=0.1$, (a) plane I and (b) plane II. 
(a)

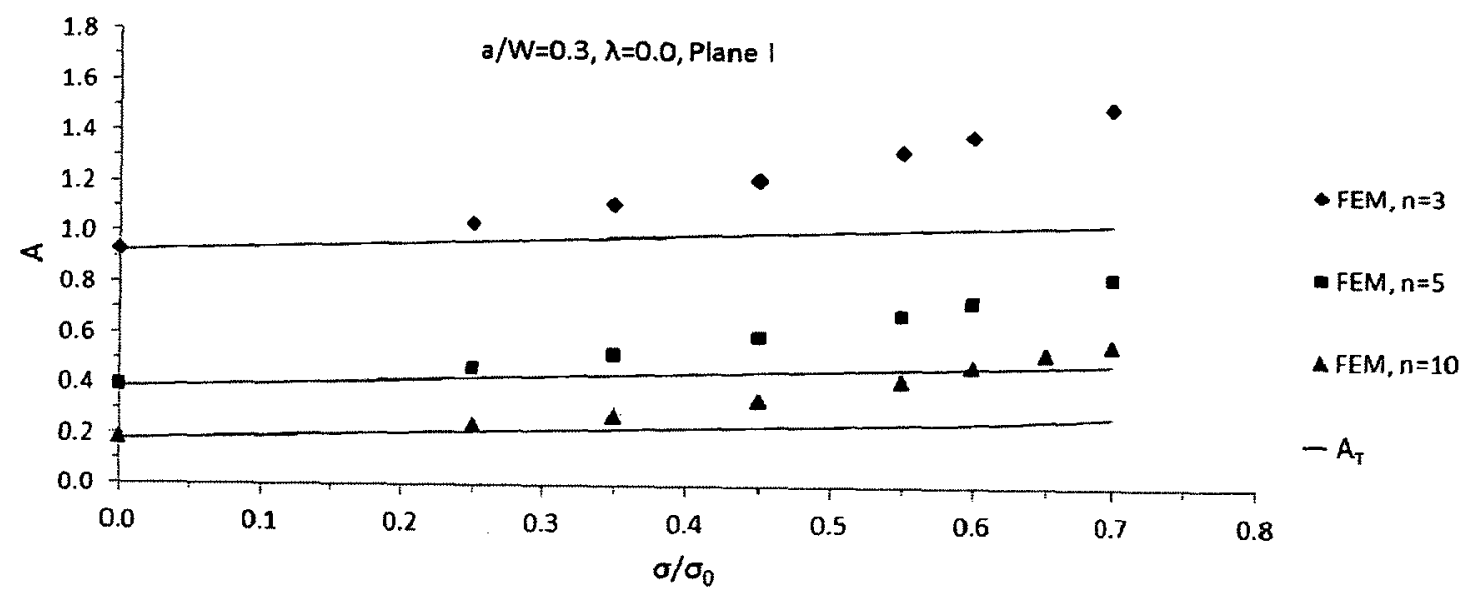

(b)

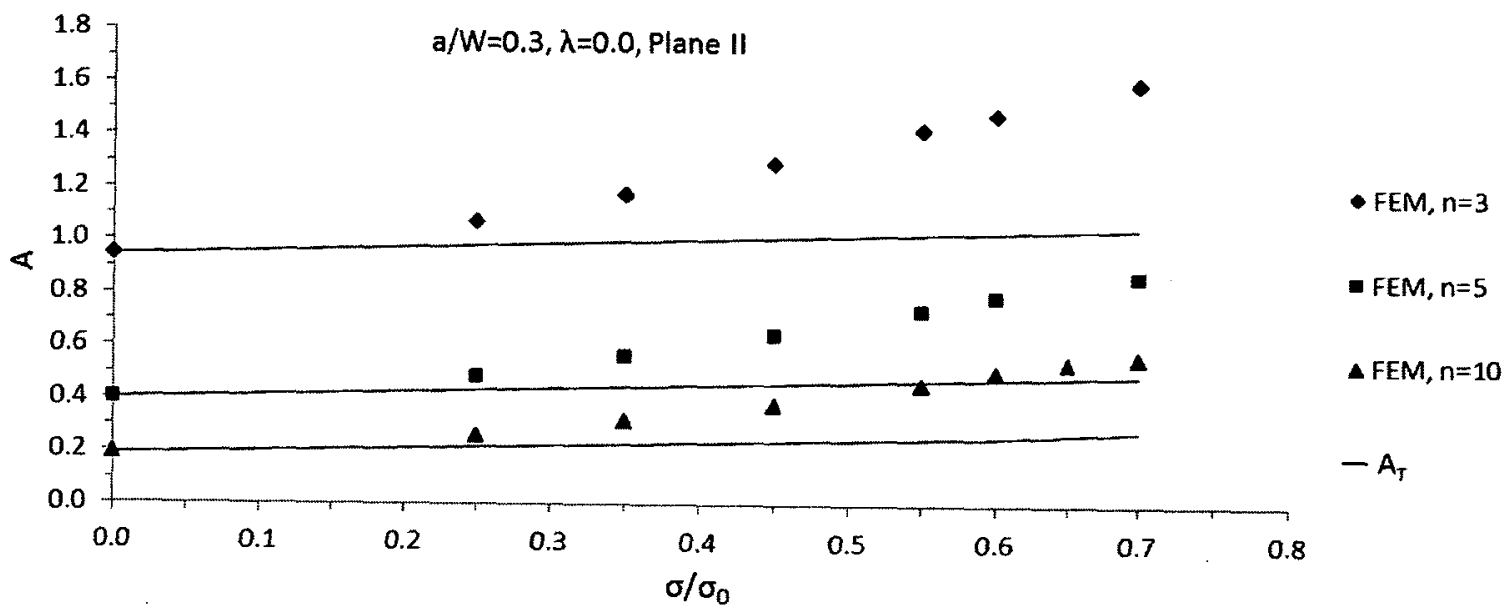

Figure 6.19 Comparisons of $A$ values predicted from $T$-stress with FEA data for the 3D SECP, $\lambda=0.0, a / W=0.3$, (a) plane I and (b) plane II. 
(a)

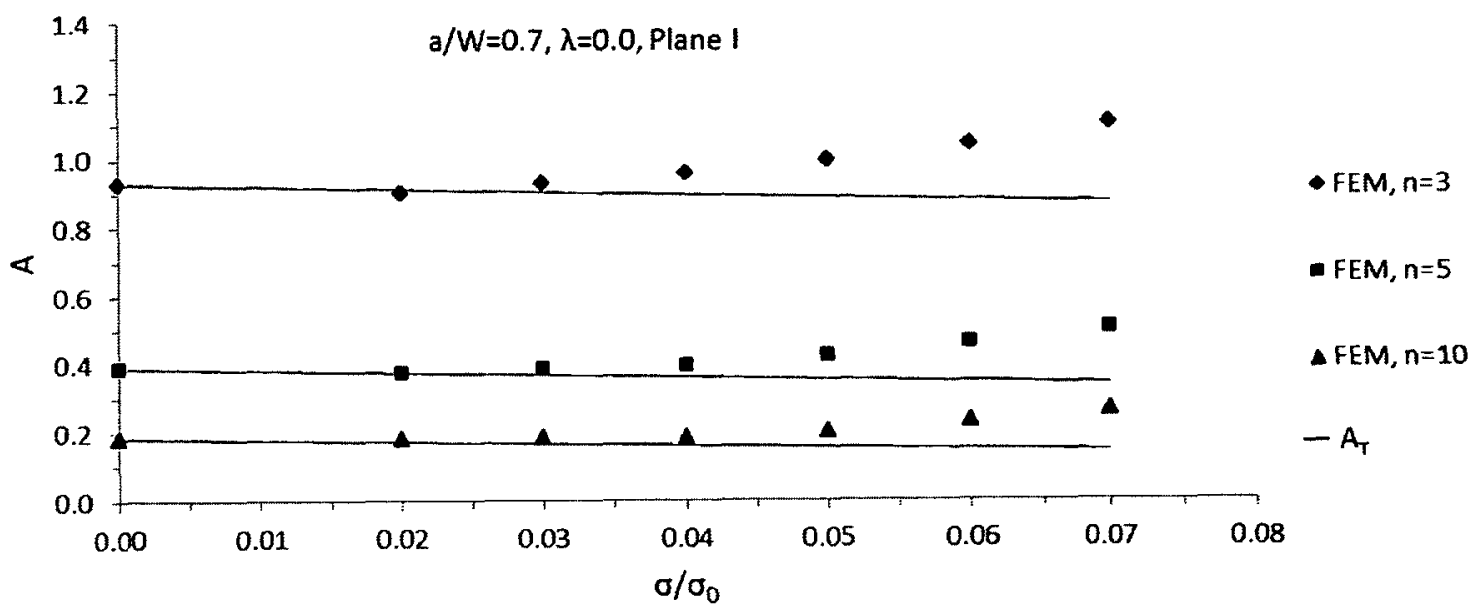

(b)

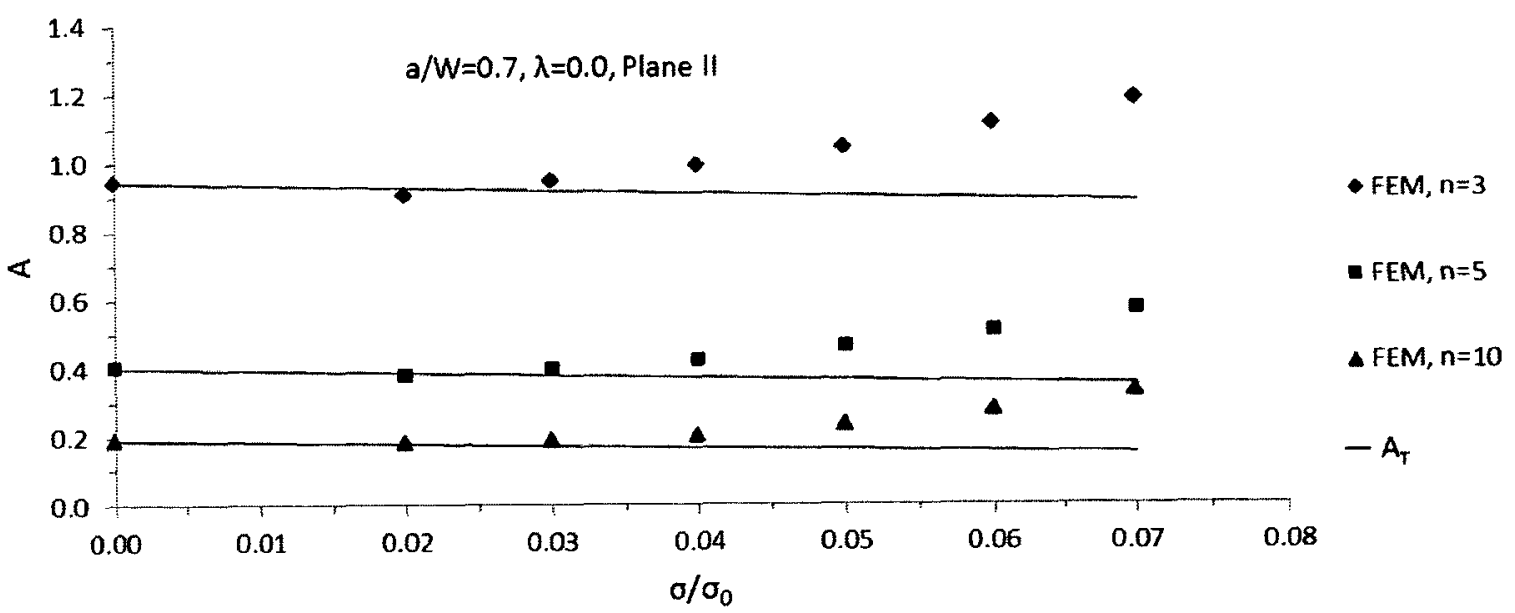

Figure 6.20 Comparisons of $A$ values predicted from $T$-stress with FEA data for the 3D SECP, $\lambda=0.0, a / W=0.7$, (a) plane I and (b) plane II. 
(a)

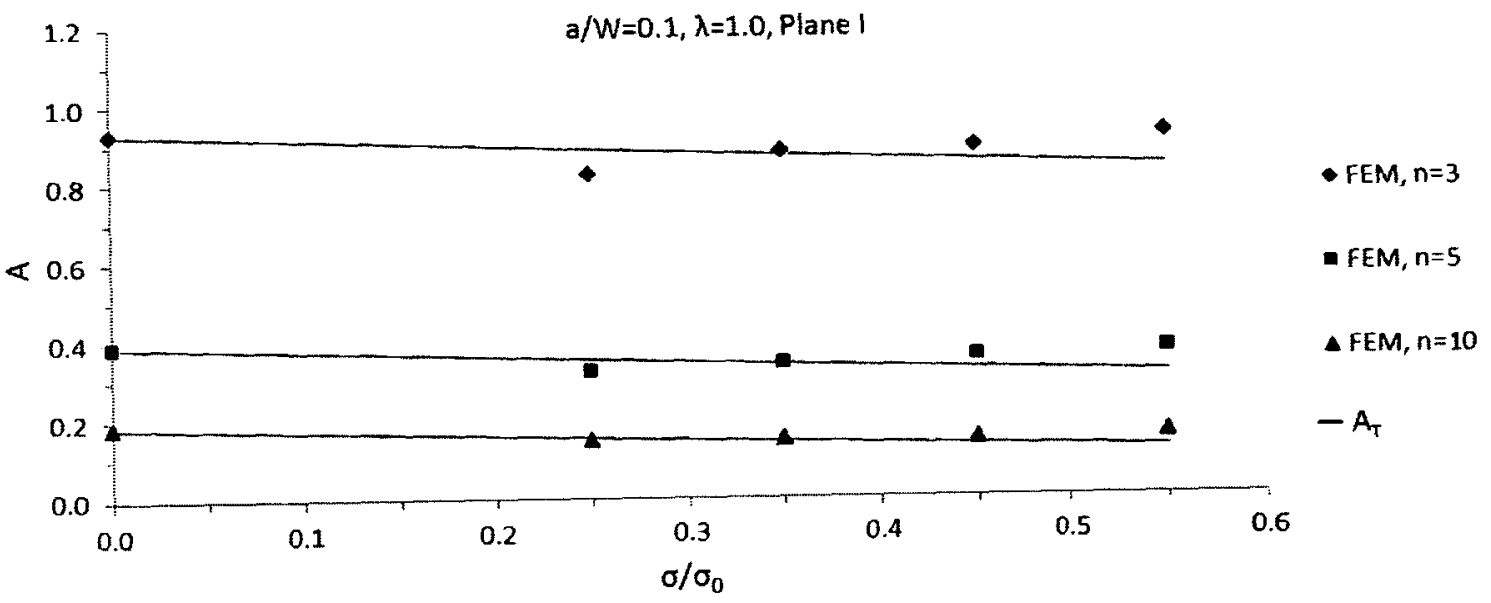

(b)

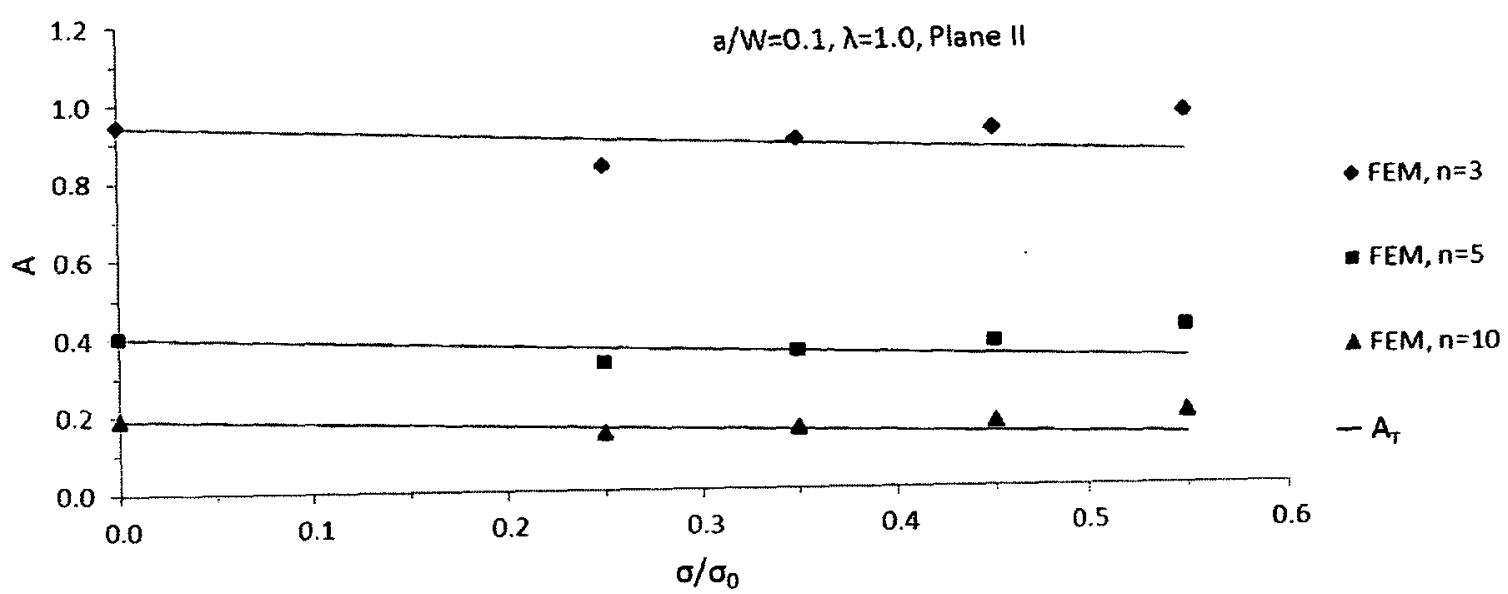

Figure 6.21 Comparisons of $A$ values predicted from $T$-stress with FEA data for the 3D SECP, $\lambda=1.0, a / W=0.1$, (a) plane I and (b) plane II. 
(a)

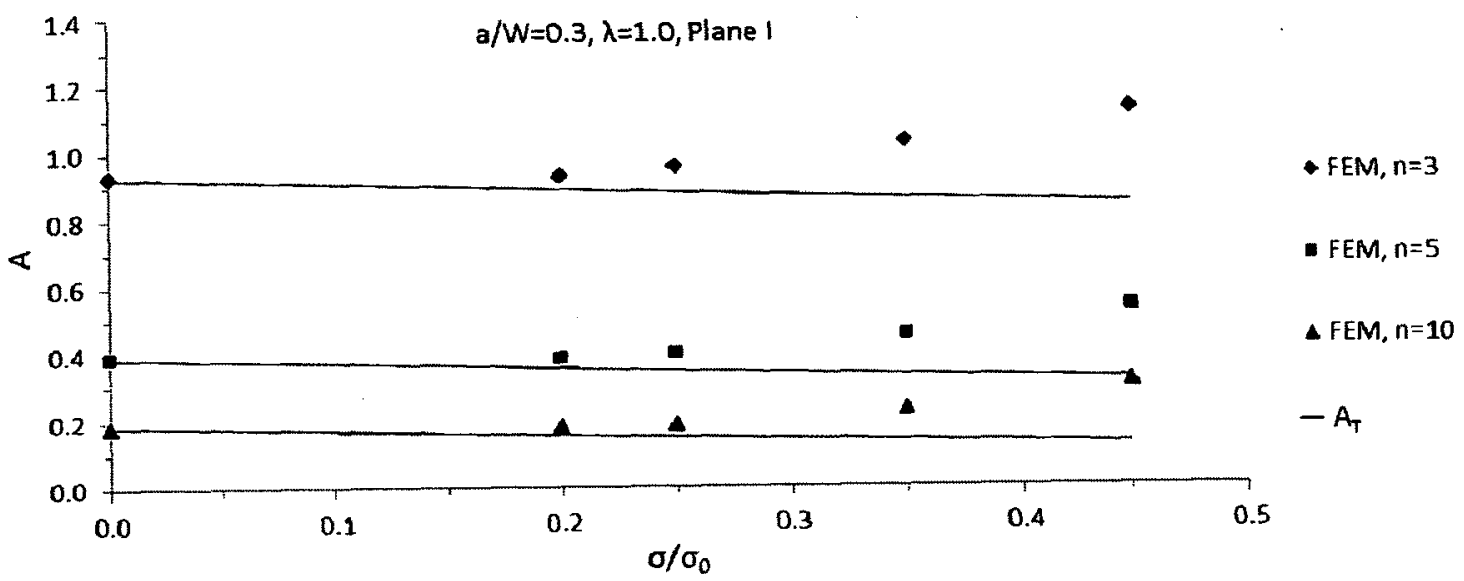

(b)

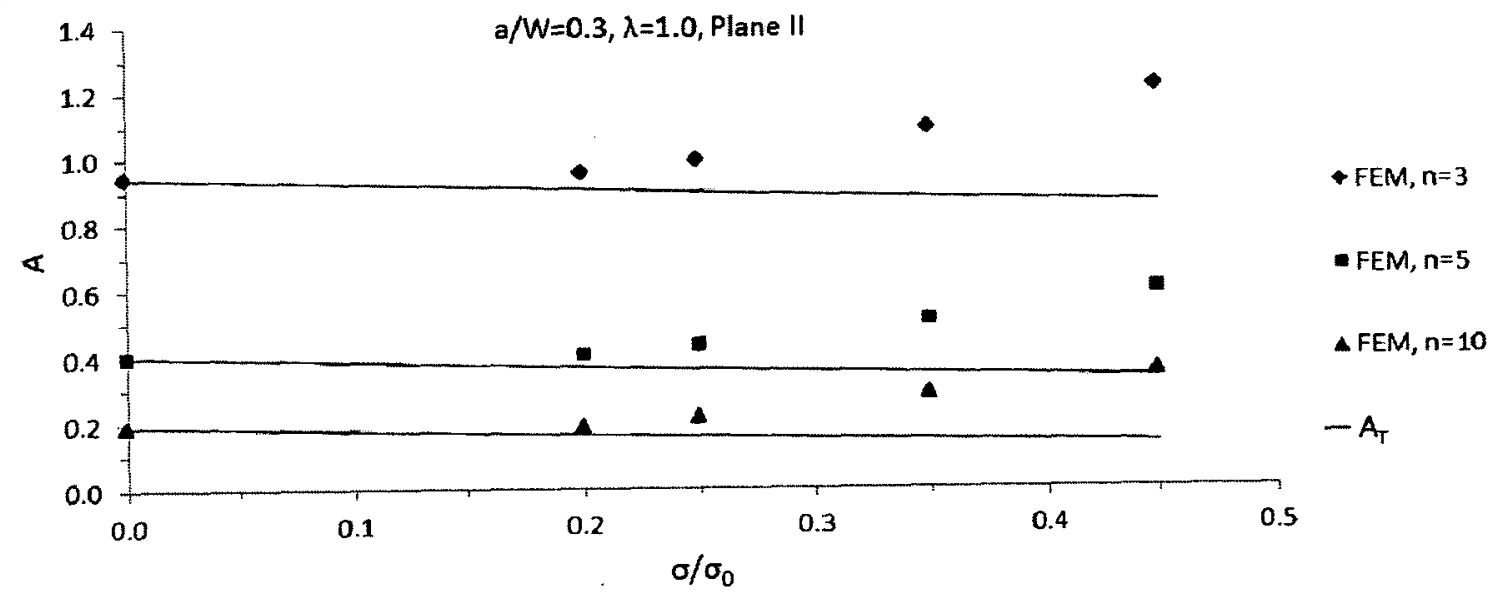

Figure 6.22 Comparisons of $A$ values predicted from $T$-stress with FEA data for the 3D SECP, $\lambda=1.0, a / W=0.3$, (a) plane I and (b) plane II. 
(a)

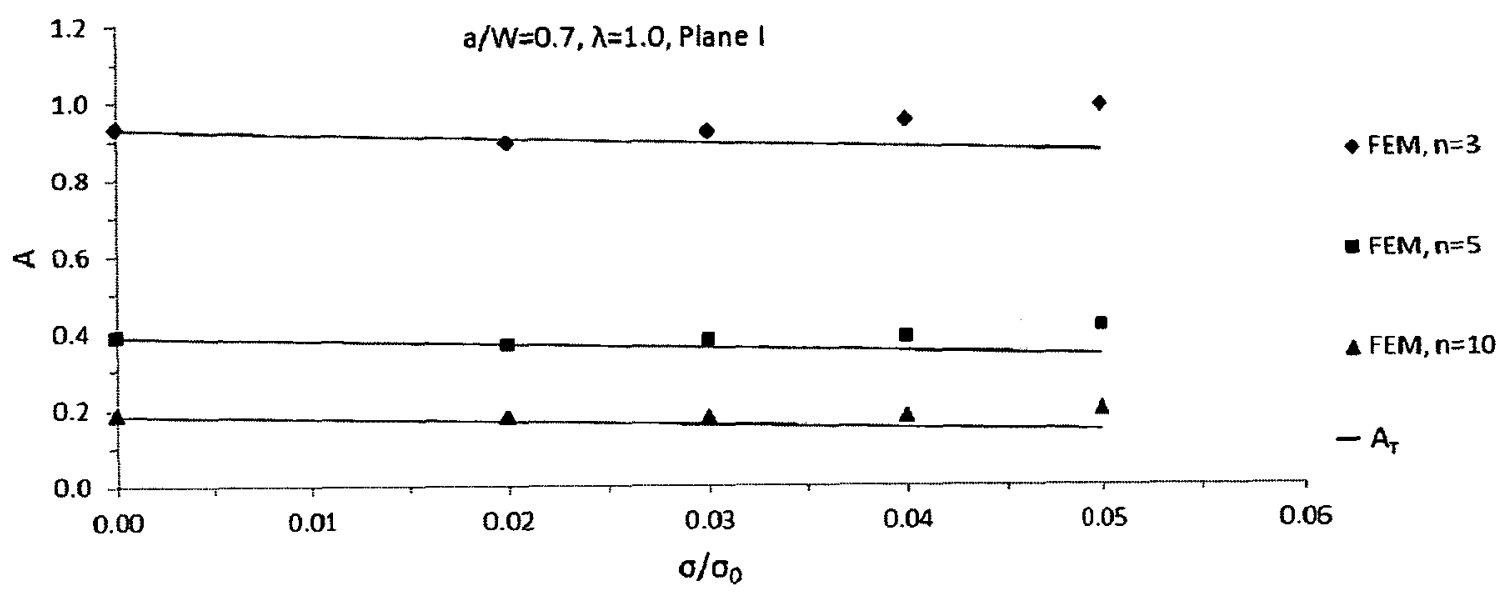

(b)

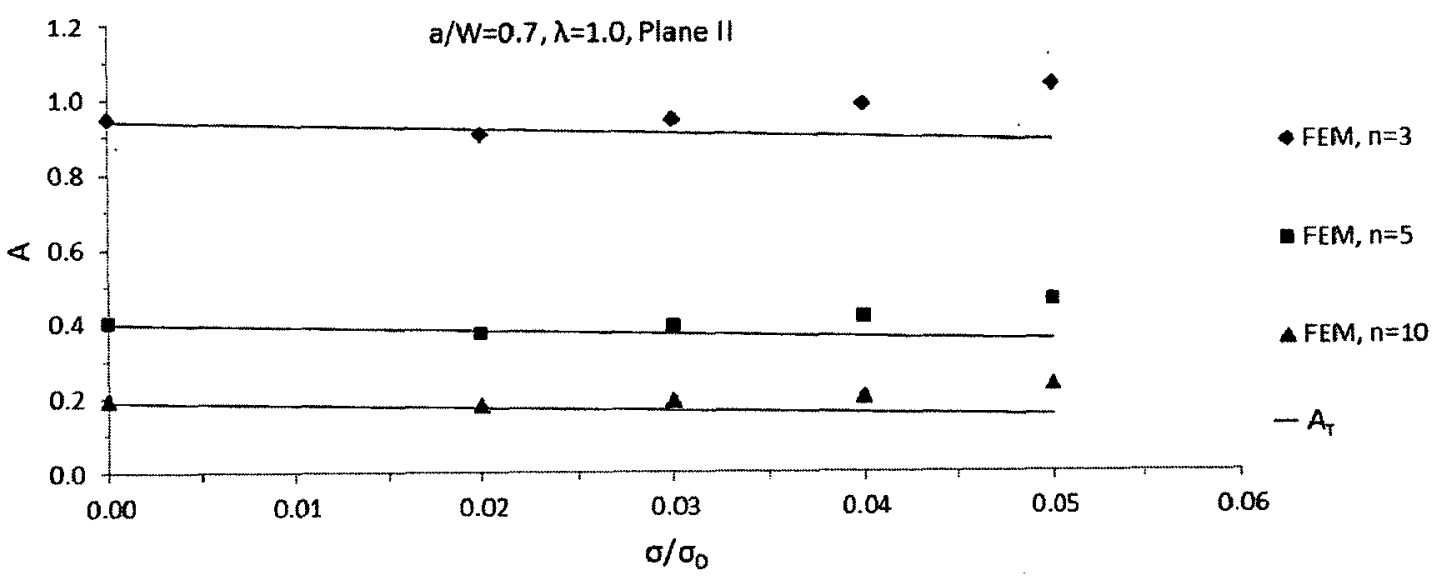

Figure 6.23 Comparisons of $A$ values predicted from $T$-stress with FEA data for the 3D SECP, $\lambda=1.0, a / W=0.7$, (a) plane I and (b) plane II. 
(a)

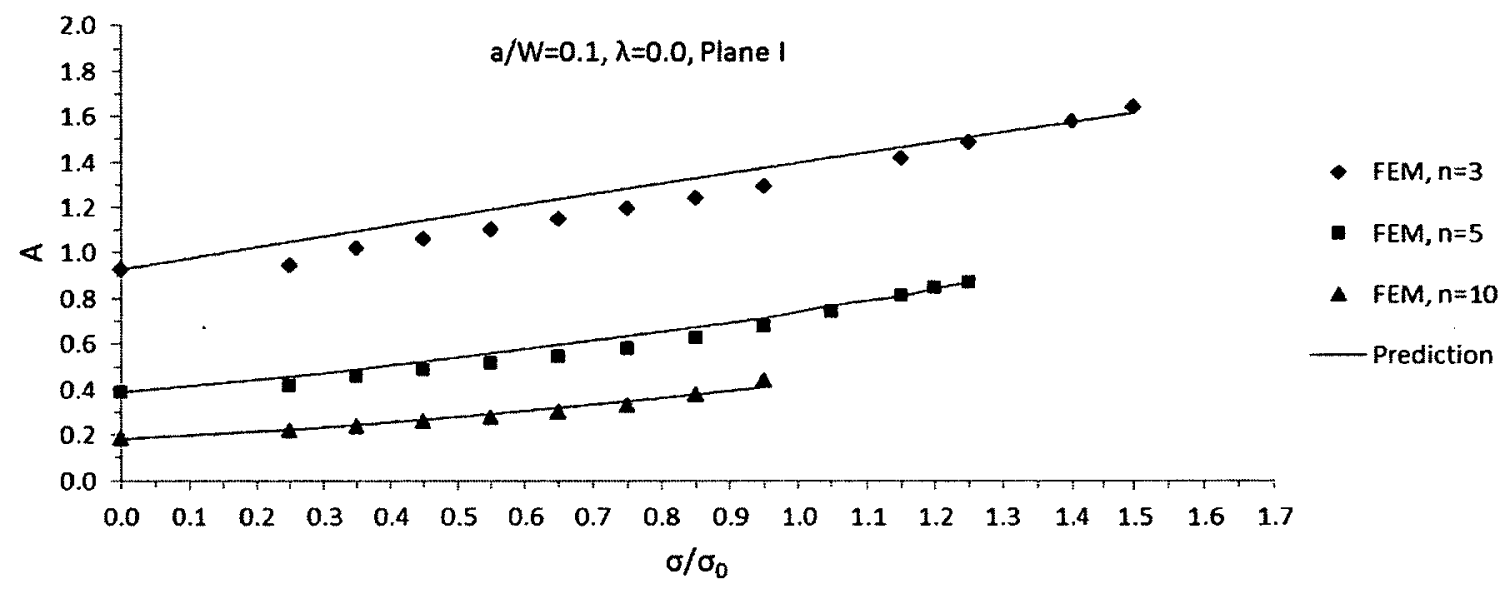

(b)

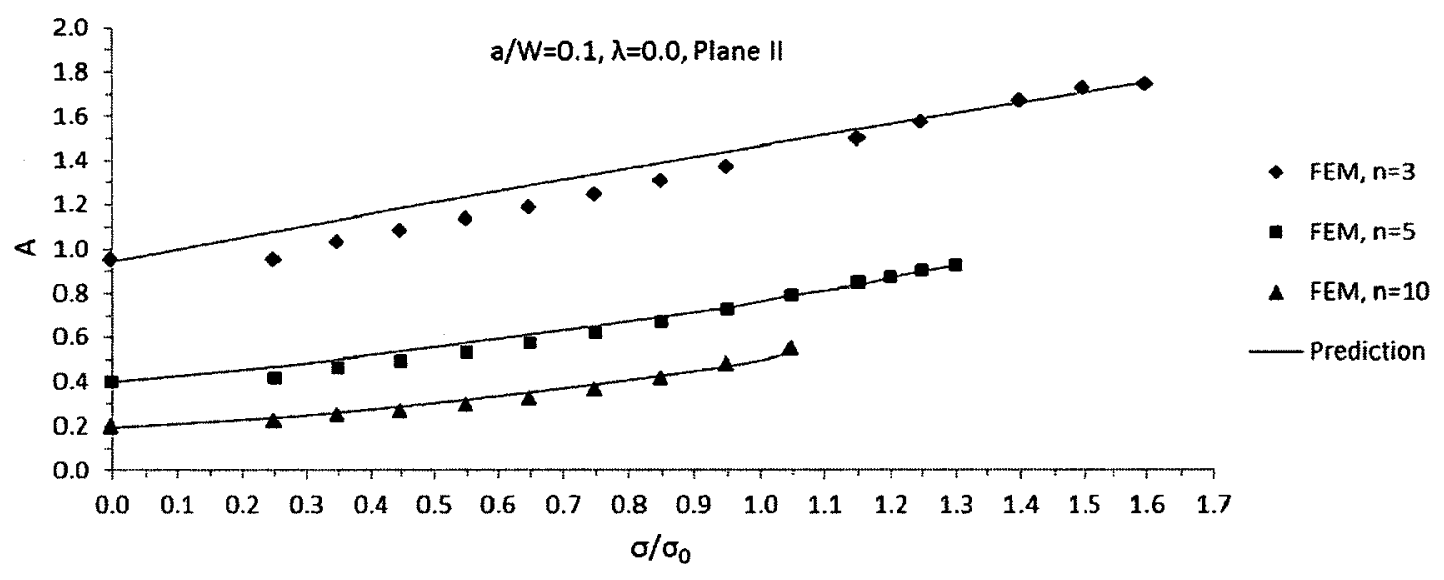

Figure 6.24 Comparisons of $A$ values predicted by superposition with FEA data for the 3D SECP, $\lambda=0.0, a / W=0.1$, (a) plane I and (b) plane II. 
(a)

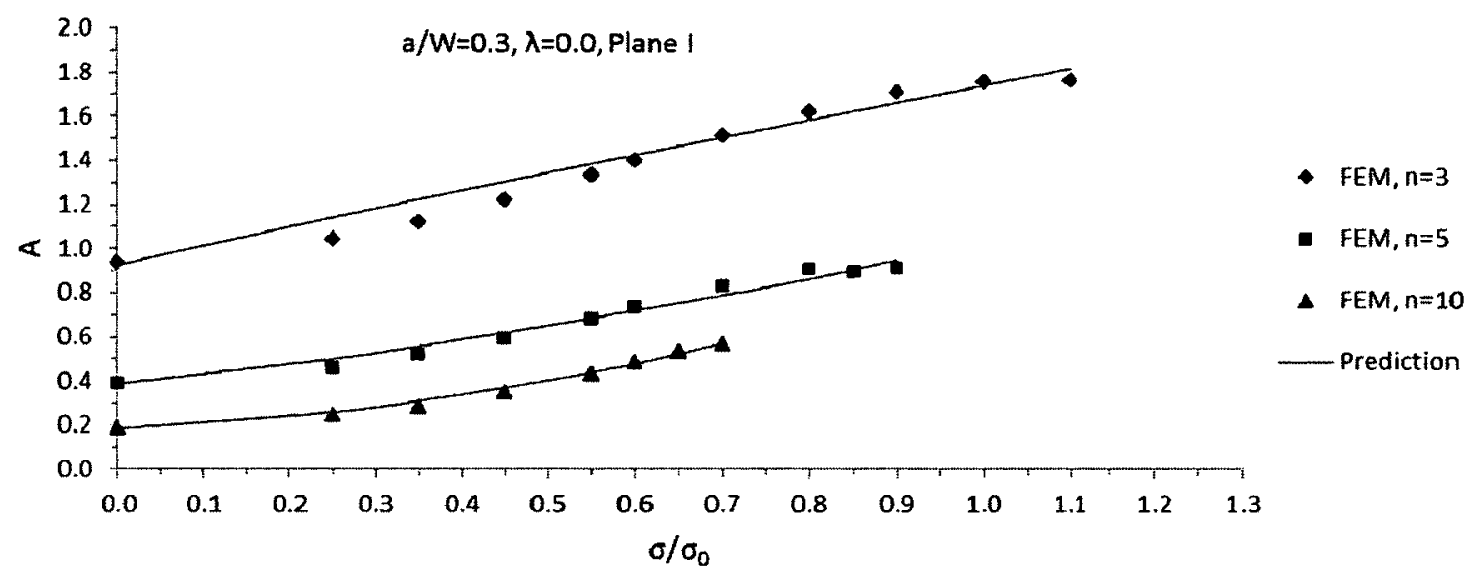

(b)

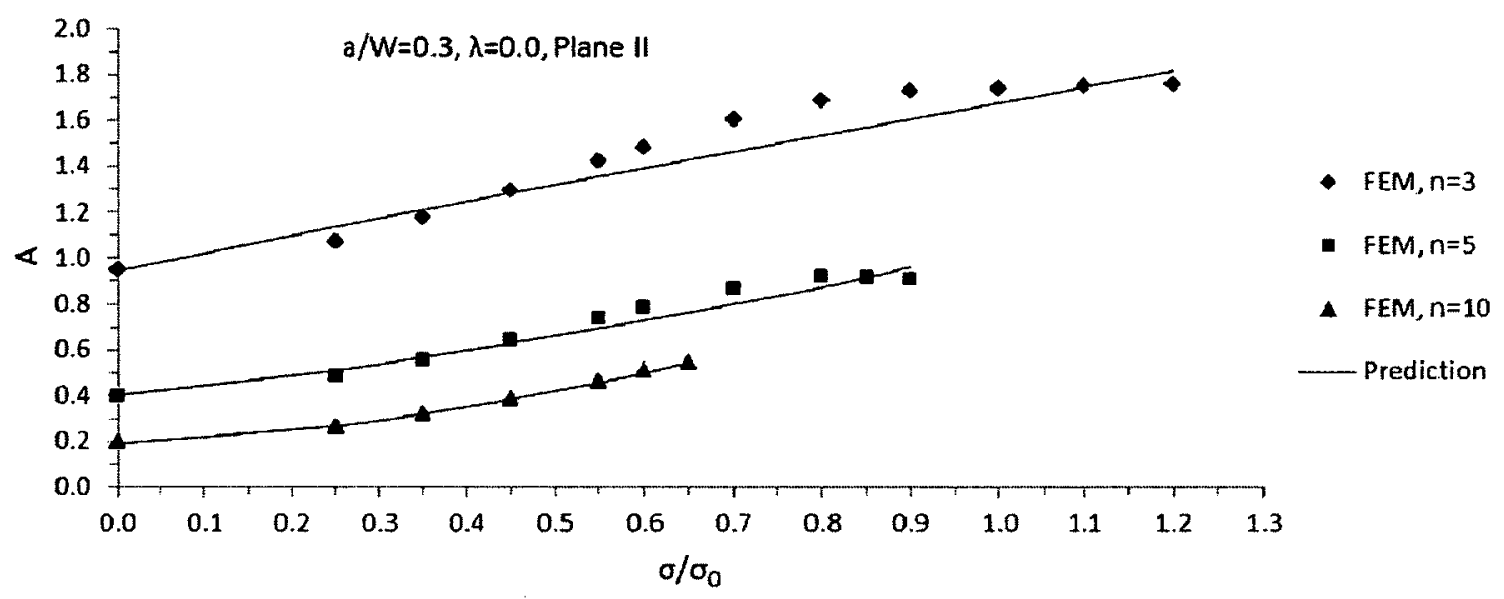

Figure 6.25 Comparisons of $A$ values predicted by superposition with FEA data for the 3D SECP, $\lambda=0.0, a / W=0.3$, (a) plane I and (b) plane II. 
(a)

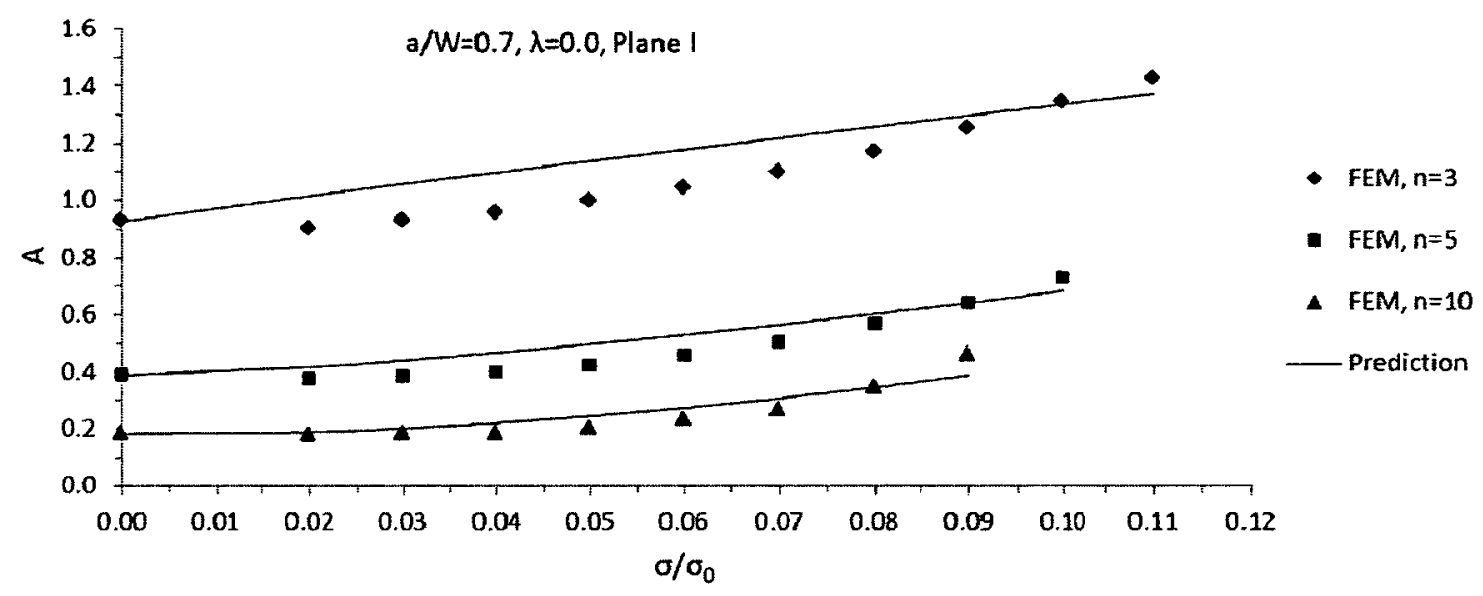

(b)

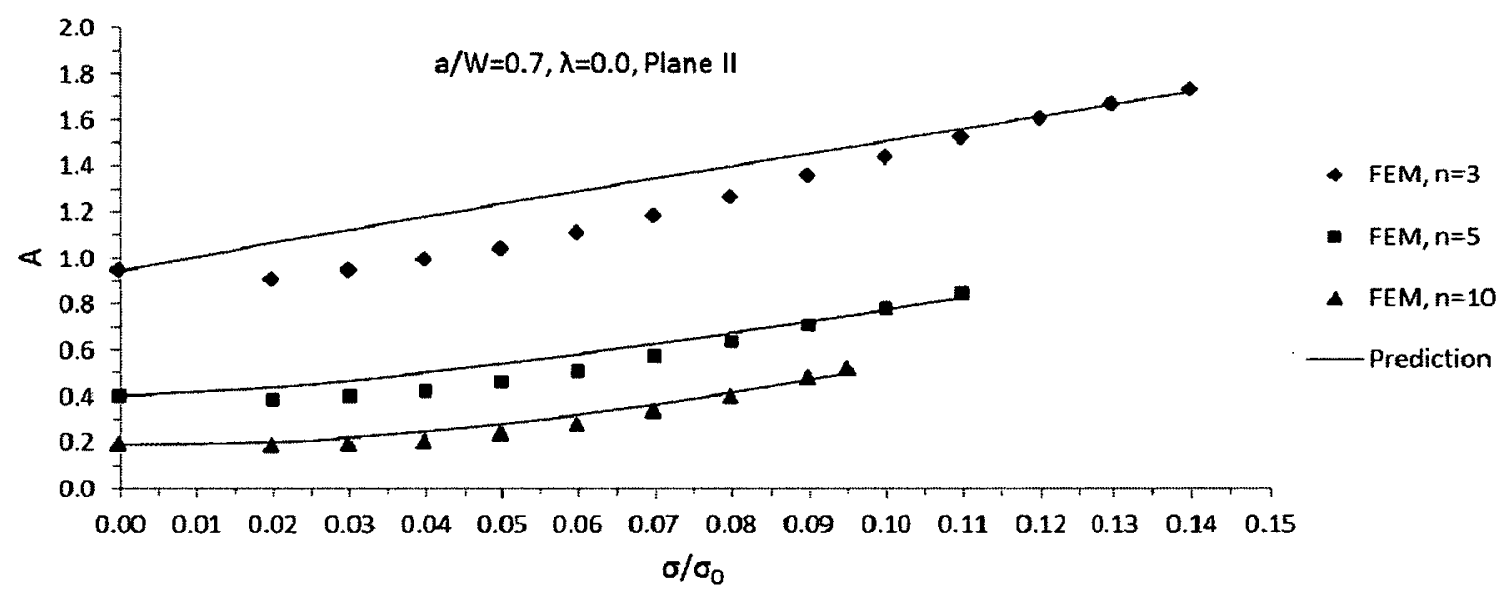

Figure 6.26 Comparisons of $A$ values predicted by superposition with FEA data for the 3D SECP, $\lambda=0.0, a / W=0.7$, (a) plane I and (b) plane II. 
(a)

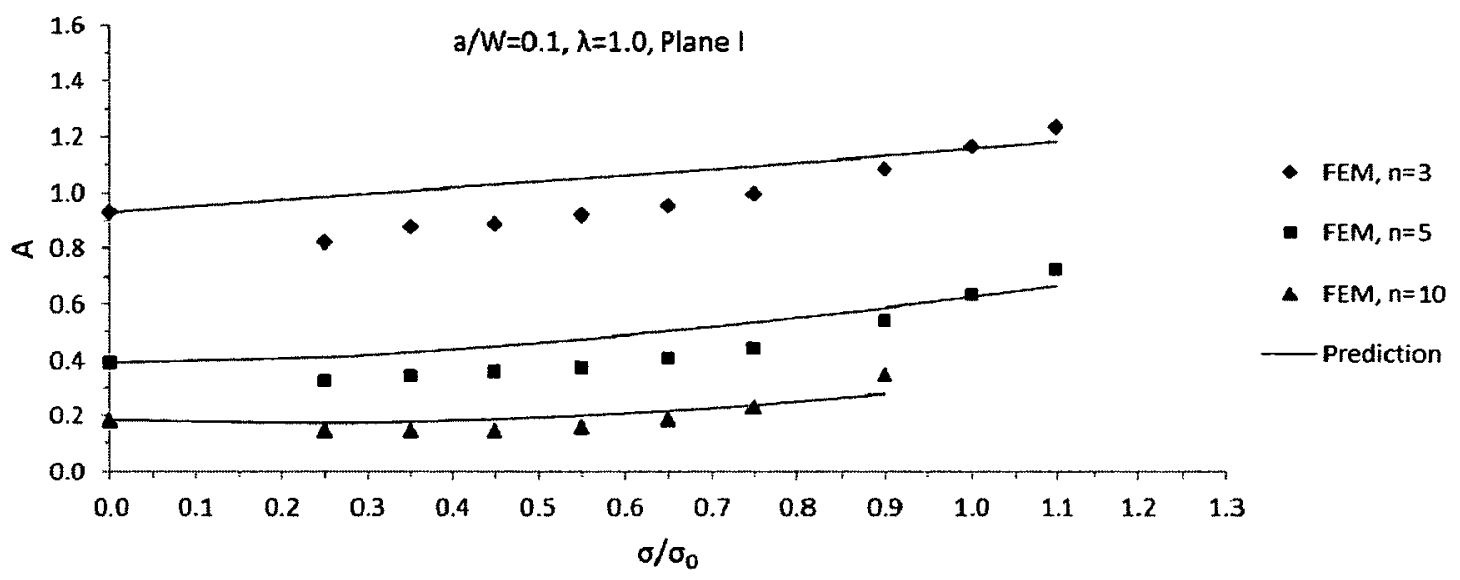

(b)

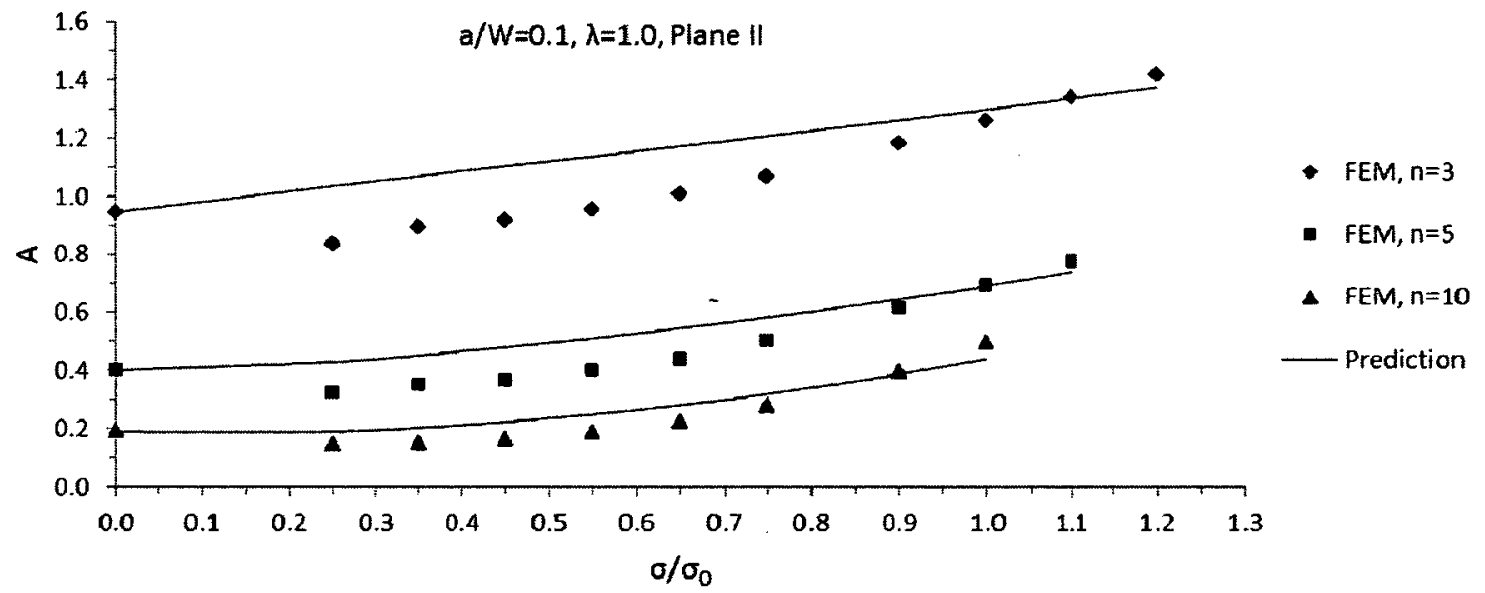

Figure 6.27 Comparisons of $A$ values predicted by superposition with FEA data for the 3D SECP, $\lambda=1.0, a / W=0.1$, (a) plane I and (b) plane II. 
(a)

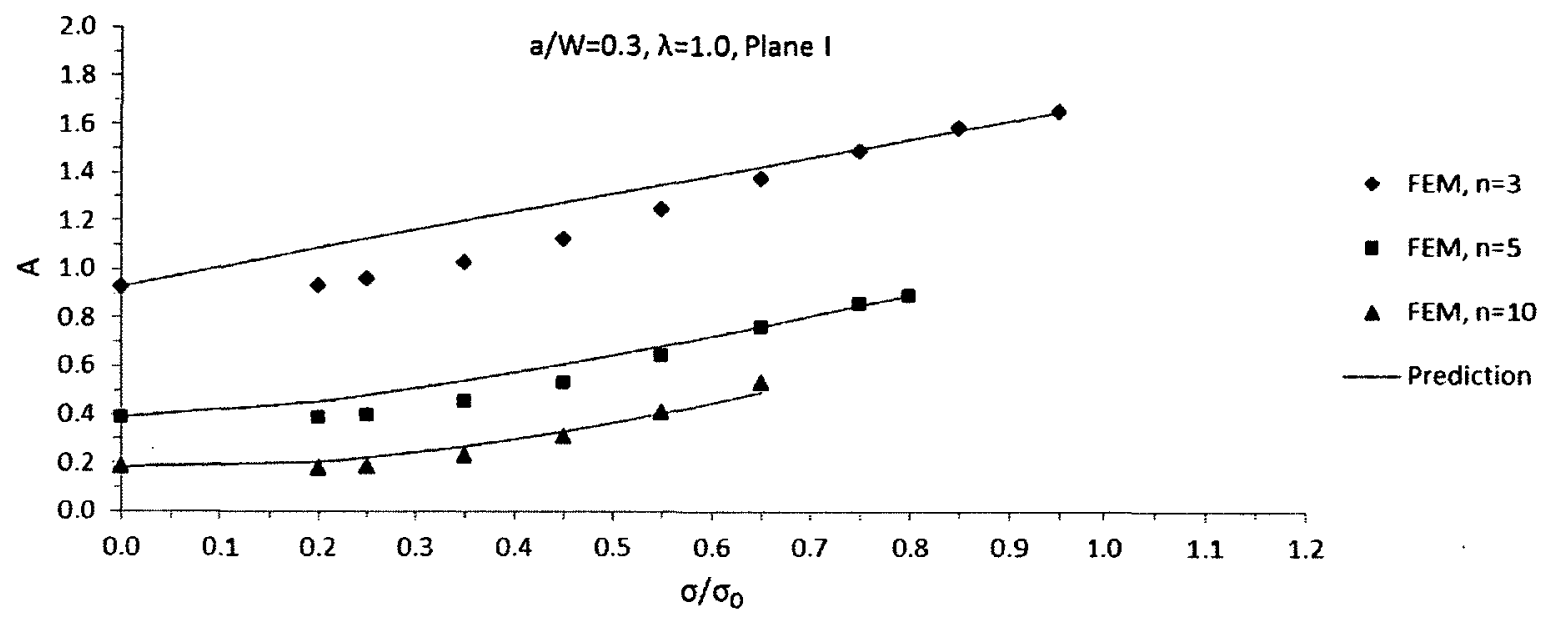

(b)

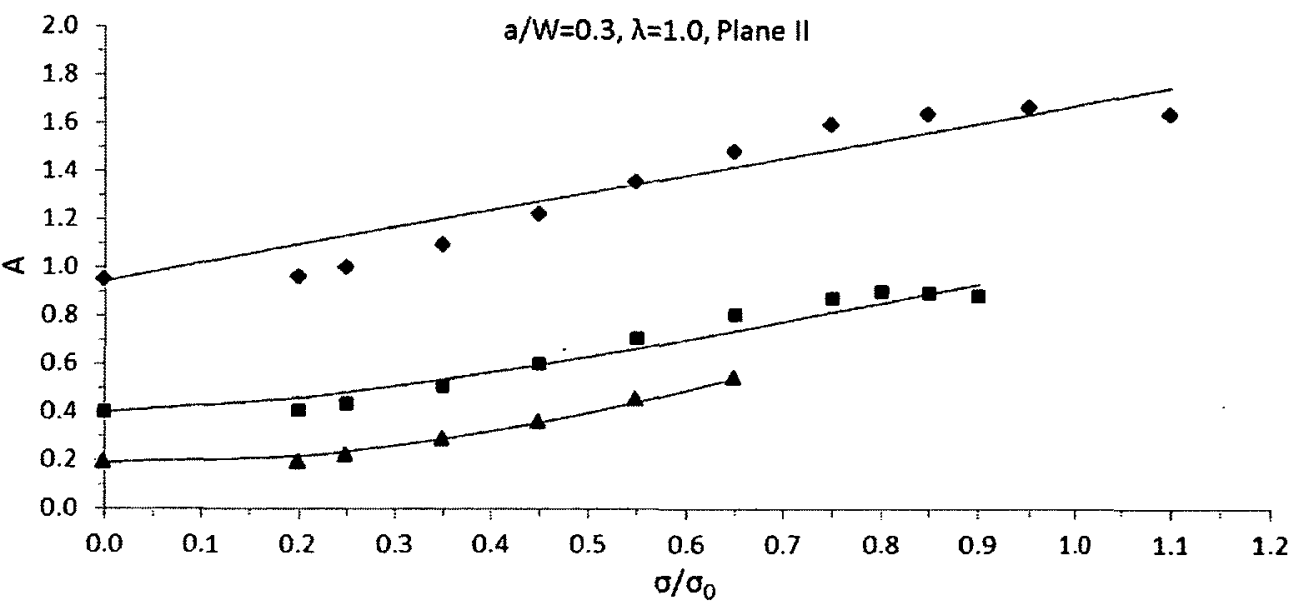

Figure 6.28 Comparisons of $A$ values predicted by superposition with FEA data for the 3D SECP, $\lambda=1.0, a / W=0.3$, (a) plane I and (b) plane II. 
(a)

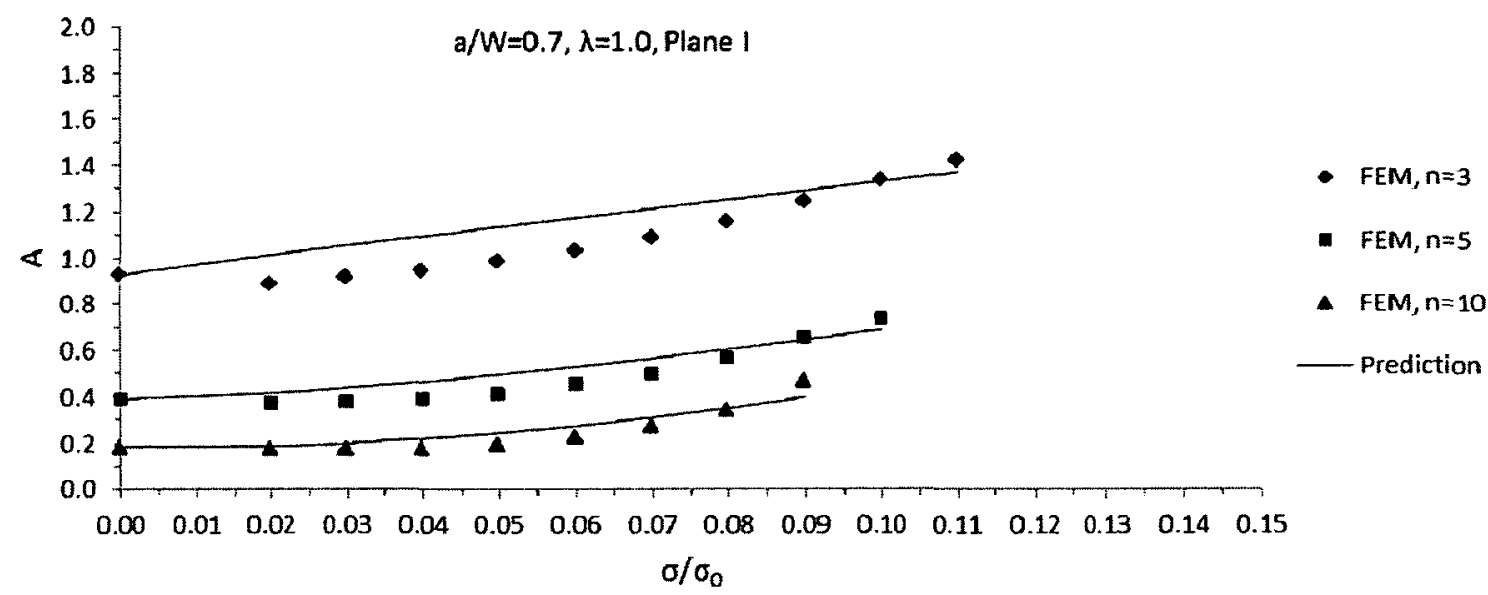

(b)

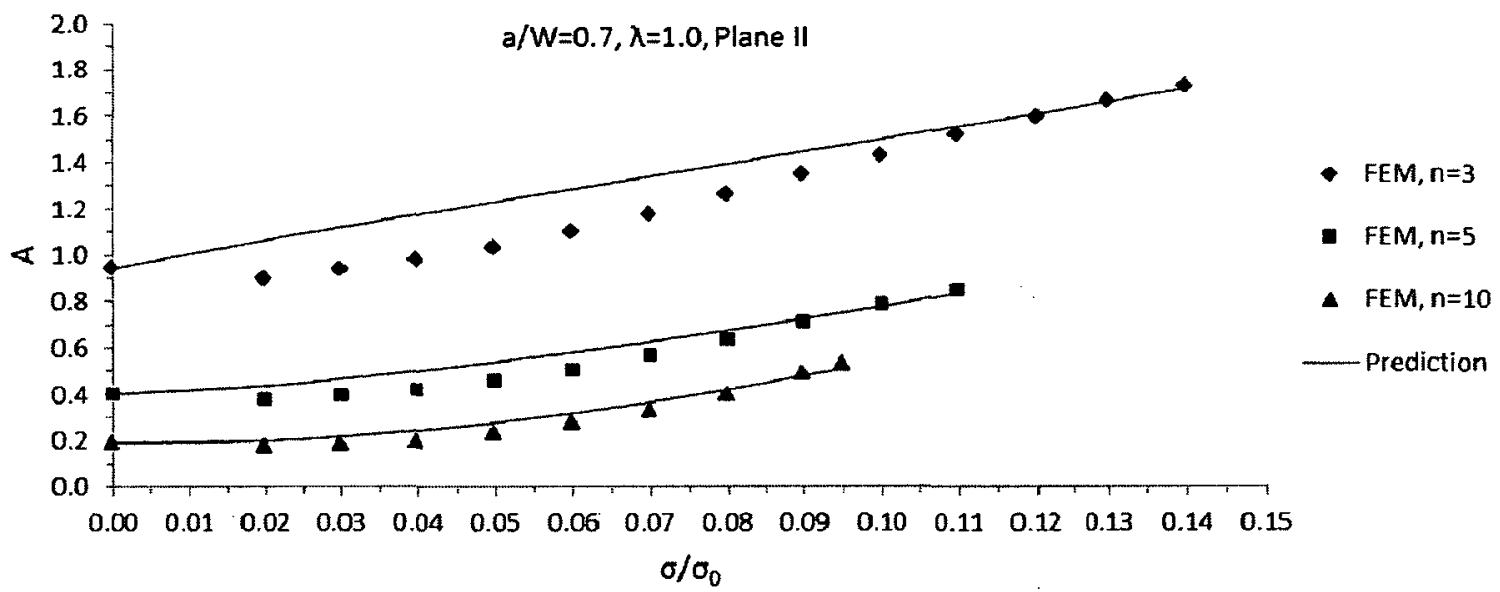

Figure 6.29 Comparisons of $A$ values predicted by superposition with FEA data for the 3D SECP, $\lambda=1.0, a / W=0.7$, (a) plane I and (b) plane II. 
(a)

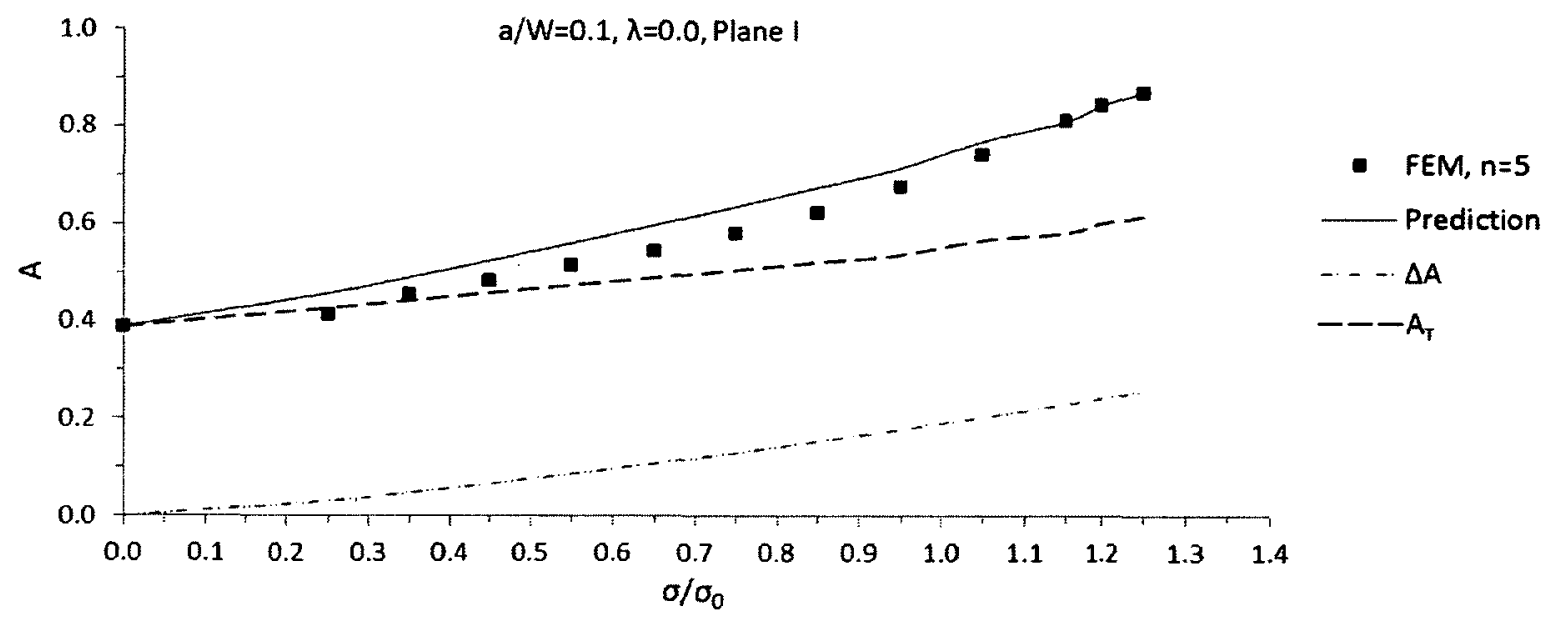

(b)

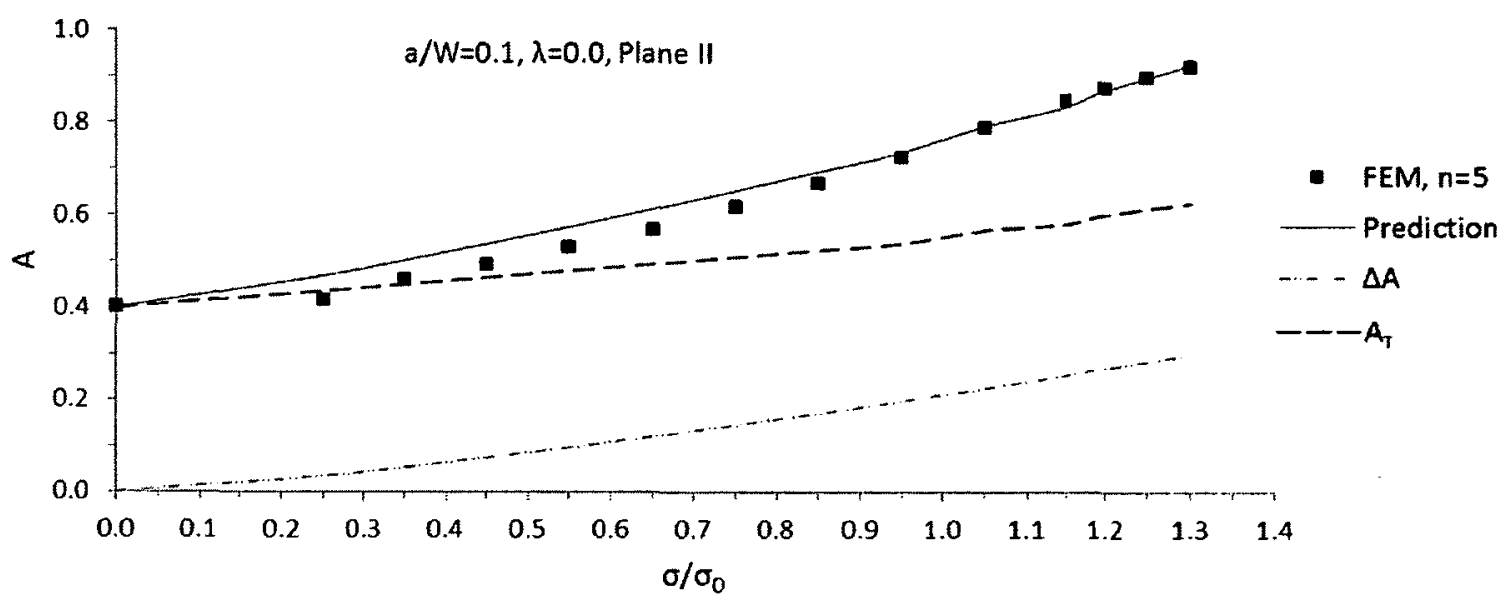

Figure 6.30 Results of $A, A_{T}$ and $\Delta A$ for the 3D SECP, $n=5, \lambda=0.0$, and $a / W=0.1$ 
(a)

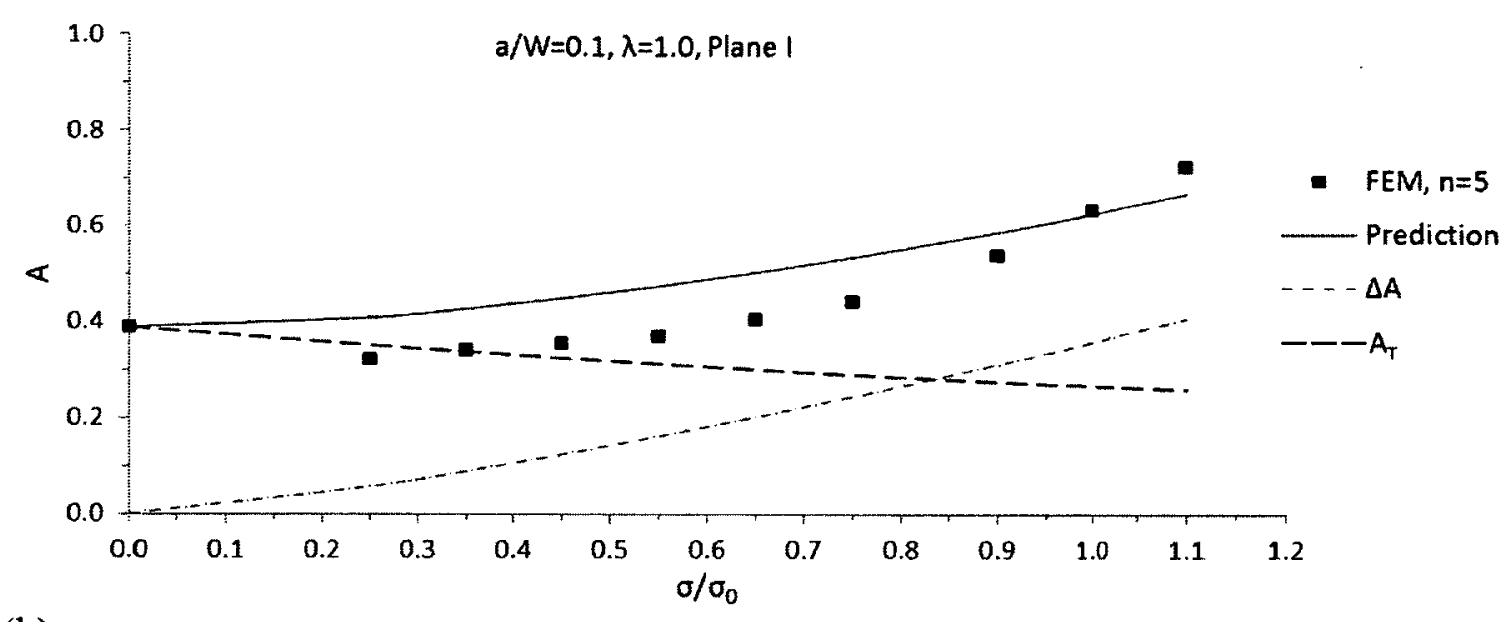

(b)

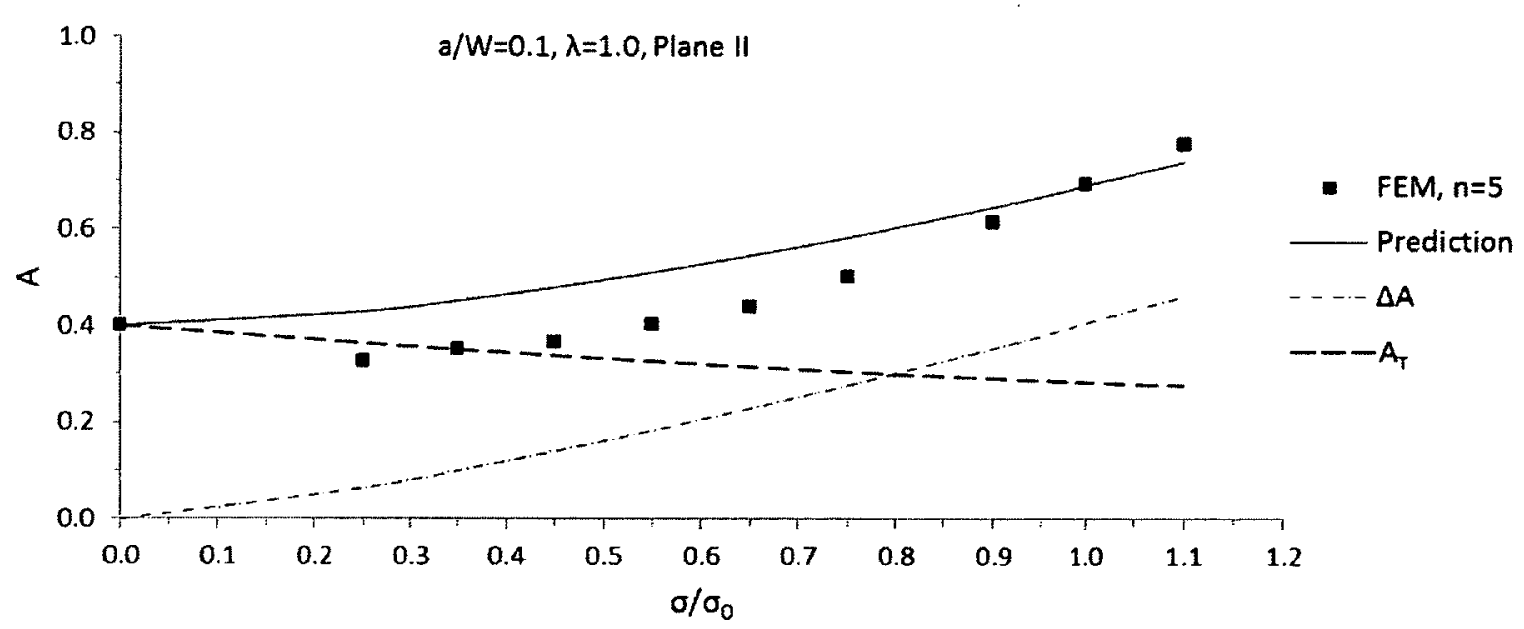

Figure 6.31 Results of $A, A_{T}$ and $\triangle A$ for the 3D SECP, $n=5, \lambda=1.0$, and $a / W=0.1$ 


\section{CHAPTER 7 CONCLUSIONS AND RECOMMENDATIONS}

\subsection{Conclusions}

In chapter 3, three sets of methodologies, which can be used to approximate solutions of the constraint parameter $A$ conveniently, have been developed based on the theoretical background and literature review represented in chapter 2. These approximation methodologies are: (1) estimating parameter $A$ by curve shape similarity, (2) predicting parameter $A$ values from $T$-stress directly, and (3) determining parameter $A$ based on fully plastic solutions of $A$.

In chapter 4 , for a wide range of geometry, material properties and loading conditions (uniaxial tension loading covering the range from small-scale to large-scale yielding), extensive finite element analyses (FEA) have been carried out to obtain numerical solutions for the constraint parameter $A$ for three mode I crack plane-strain specimens, the single edge cracked plate (SECP), the center cracked plate (CCP) and the double edge cracked plate (DECP). Numerical solutions for parameter $A$ are determined from FEA results by the fitting method suggested by Nikishkov et al (1995a). The constraint level on 2D cracked bodies under uniaxial loading is discussed based on the obtained numerical solutions of parameter $A$. With the numerical solutions for $A$ and the three approximation methodologies for parameter $A$ developed in chapter 3 , detailed approximation formulas for parameter $A$ corresponding to the three estimation methodologies are fitted for the three $2 \mathrm{D}$ crack geometries under uniaxial loading. The suggested approximation formulas are verified by comparing parameter $A$ values from predictions with their numerical solutions, and the applicability of the approximation formulas for 2D specimens under uniaxial load is discussed. For all three 2D specimens, the maximum difference between approximation and FEA result is $10.19 \%$ for the estimate by curve shape similarity with adjusting factor $f_{a d j}(n), 10.96 \%$ for the estimate based on the $A-T$ relationship and generally around $15 \%$ for the prediction from the fully plastic solution of $A$. It is shown that the developed three approximation methods can be 
used to predict the constraint parameter $A$ for 2D specimens under uniaxial loading. Using the relationships between the $A$ and the other two commonly-used constraint parameters $Q$ and $A_{2}$ summarized in chapter 2, the predicted parameter $A$ values from developed approximation formulas are used to determine corresponding values of parameter $Q$ and $A_{2}$ for several cases. Results are presented and verified, demonstrating the feasibility of determining the other two commonly-used constraint parameters $Q$ and $A_{2}$ from the obtained $A$ solutions directly.

In chapter 5, the three 2D specimens (SECP, CCP and DECP) are investigated under biaxial loading. As that for uniaxial loading (chapter 4), extensive finite element analysis is carried out for the SECP, CCP and DECP under biaxial loading condition (biaxial loading ratios, $\lambda=0.5$ and 1.0) to determine the numerical solutions for the constraint parameter $A$. Based on the numerical solutions of $A$, the constraint effects for $2 \mathrm{D}$ cracked specimens under biaxial loading are discussed, and several properties are found (see section 5.1.3.2 or 5.5). With the obtained numerical solutions of parameter $A$, three sets of are investigated formulas for $A$ of SECP, CCP and DECP under biaxial loading are proposed based on the three methodologies developed in chapter 3. Approximate solutions for parameter $A$ are compared with corresponding numerical solutions. The maximum differences between approximation and FEA results for all three specimens are generally around $17 \%(\lambda=0.5)$ and $15 \%(\lambda=1.0)$ for the prediction by curve shape similarity, $10.99 \%(\lambda=0.5)$ and $10.99 \%(\lambda=1.0)$ for estimate based on $A-T$ relationship and $10.83 \%(\lambda=0.5)$ and $11.77 \%(\lambda=1.0)$ for the prediction from fully plastic solution of $A$. It can be concluded that for $2 \mathrm{D}$ specimens under biaxial loading, the three sets of estimate methods are appropriate.

In chapter 6, extensive finite element analysis is performed for a 3D SECP cracked specimen with relative thickness $t / W=0.1$ under both uniaxial $(\lambda=0.0)$ and biaxial $(\lambda=1.0)$ loading, to obtain the constraint parameter $A$. Numerical solutions of the parameter $A$ for the 3D SECP are obtained for typical planes at three locations along the model thickness (crack-front), plane I at $z / t=1.4 \%$, plane II at $z / t=27.1 \%$ and plane III at $z / t=49.8 \%$. Using the numerical solutions of constraint parameter $A$, constraint effects in $3 \mathrm{D}$ cracked specimen are discussed, and several properties are observed (see section 6.2.3.2 or 6.6). 
With the numerical solutions of parameter $A$, the three approximation methodologies for $A$ are applied to plane I and plane II of the 3D SECP. The coefficients in the suggested approximation formulas from each methodology depend on the locations ( $z$ values) of planes along the 3D model thickness. The $A$ solutions from the suggested approximation formulas are compared with the corresponding numerical solutions. For both planes I and II, the maximum estimation differences are: $16.64 \%(\lambda=0.0) / 13.52 \%(\lambda=1.0)$ for the approximation by curve shape similarity, $12.95 \%$ for prediction from $T$-stress, and $22.23 \%(\lambda=0.0) / 36.10 \%(\lambda=1.0)$ for approximation by fully plastic analysis. While under the biaxial loading with high biaxial loading ratio $(\lambda=1.0)$, the estimation method based on fully plastic analysis introduces larger deviation of the approximation (maximum difference, $36.10 \%$ ), for uniaxial loading, the chosen form of the method based on fully plastic solution fits the curve trends of $A$ vs. load better. In the two planes (planes I and II), it is shown that the three sets of estimate methods are appropriate for 3D cracked structure. It therefore can be concluded that, the three sets of developed methodologies for the prediction of constraint parameter $A$ can be utilized for 3D cracked structures, except the planes near the free surface.

In summary, mainly five aspects of works have been accomplished in the research presented in the present thesis.

Firstly, extensive finite element analysis has been conducted in current research for both two-dimensional (2D) and three-dimensional (3D) cracked specimen geometries under uniaxial and biaxial loading conditions. Based on the finite element analysis, numerical solutions for the constraint parameter $A$ are obtained for 2D and 3D cracked models under uniaxial and biaxial loading conditions through the least-square fitting method proposed by Nikishkov et al. (1995a). The resulted numerical solutions have been tabulated systematically in the thesis for further theoretical analyses and engineering applications.

Secondly, the constraint effect at the crack-tip (-front) of cracked structures has been analyzed and summarized for $2 \mathrm{D}$ as well as $3 \mathrm{D}$ specimens under uniaxial and/or biaxial loading based on the obtained numerical solutions of constraint parameter $A$.

Thirdly, for the convenience of engineering applications and theoretical research, 
three different approximation methodologies for predicting the constraint parameter $A$ are developed.

Fourthly, supplemented with the determined numerical solutions of the constraint parameter $A$, approximation equations of $A$ values corresponding to the three newlydeveloped estimation methodologies are fitted for the specified geometries (2D/3D) analysed in this thesis and different loading conditions (uniaxial/biaxial loading). By comparing the predicted $A$ values with the corresponding numerical solutions, the applicability of three approximate methods for various geometrical configurations and loading conditions is verified and discussed. Although the three methods are developed based on two-dimensional (2D) plane strain cases, the three sets of newly-developed estimation methods have also been successfully applied for three-dimensional (3D) cracked structures in the present work.

Fifthly, the relationships between the constraint parameter $A$ and other two commonly-used parameters $Q$ and $A_{2}\left(A_{2}\right.$ is in fact a different normalizing format of $A$ ) are summarized and utilized in the current investigation. Through the relationships among $A, Q$ and $A_{2}$, solutions for the constraint parameter $Q$ and $A_{2}$ can be determined from the available parameter $A$ values. Several practical examples of obtaining $Q$ and $A_{2}$ directly from the parameter $A$ by these relationships have been implemented in the current research to demonstrate and verify the validity of the conversion procedure. The work greatly increases the availability of solutions for various constraint parameters. All the solutions for the constraint parameter $A$ obtained in this research can be directly utilized to determine the other two constraint parameters $Q$ and $A_{2}$.

Numerical solutions for the parameter $A$ obtained in the present work are very valuable due to the scarcity of elastic-plastic constraint parameter solutions for $2 \mathrm{D}$ or $3 \mathrm{D}$ cracked structures under uniaxial or biaxial loading. The present work contributes solutions not only for the parameter $A$ but also for $A_{2}$ and $Q$ (by the relationships among them). In fact, this thesis is among the first attempts to obtain numerical solutions of the constraint parameter $A$ (or $A_{2}$ ) for 2D and/or 3D cracked specimens with uniaxial and/or biaxial loading conditions. Analyses of the constraint effect completed in this research advance the state of the art in constraint effect characterization for elastic-plastic fracture. 
In particular, the constraint effect analysis completed in this work includes the constraint characterization for 3D cracked body under biaxial loading through constraint parameter $A$ (or $A_{2}$ ) which has never been carried out before. Three approximation methodologies are developed in this research, and fill the gap of parameter $A$ (and $A_{2}$ ) prediction for whether $2 \mathrm{D}$ or $3 \mathrm{D}$ cracked geometries under whether uniaxial or biaxial loading condition. Through the relationship between the $A$ and $Q$, these methodologies can be extended into parameter $Q$ approximation processes for both 2D and 3D cracked specimens with uniaxial and/or biaxial loading. In fact, currently, only one example of an approximation formula for $Q$ parameter, suggested by O'Dowd (1995), can be found in the literature, which is for 2D cracked models.

The results of present developments and analysis will be very useful for conducting constraint-based elastic-plastic fracture mechanics analyses for both $2 \mathrm{D}$ and $3 \mathrm{D}$ test specimens under various loading conditions.

\subsection{Recommendations}

In the present research, three sets of newly-developed approximation methodologies for constraint parameter $A$ have been applied to three-dimensional (3D) cracked structures. The applicability of the three approximation methods for 3D cracked specimens has been verified for two specified planes (at one half and a quarter of the model thickness) along the crack-front. Since the fields in planes near the free surface of the model deviate from the plane strain state, the three approximation methods which is developed based on plane strain should not be appropriate to the planes near the free surface. The determination of the location (plane location), where the present approximation methods are no longer appropriate, is recommended for future investigation.

A thin plate with fixed thickness is investigated as a 3D cracked structure in current work. Other $3 \mathrm{D}$ cracked specimens with various thicknesses needed to be studied in the future to understand the effect of thickness on crack-front constraint and the applicability of the three sets of estimated methods for constraint parameter $A$. 
In addition, in addition to the through-cracks studied in the present work, the investigation of surface-cracked cases using the $J-A$ two parameter approach is also worth studying to promote the understanding of constraint on the crack-front described by constraint parameter $A$ and the applicability of the three approximation methods. 


\section{APPENDLX}

O'Dowd and Shih (1991, 1992 and 1994) proved that, in the formulation of the modified boundary layer (MBL) problem, the constraint parameter $Q$ is related to $T$-stress directly, as illustrated by Eq. (3.2).

Through least square fitting and with the HRR stress/strain field as a reference field, O'Dowd and Shih (1992) show that the relationships between parameter $Q$ and $T$-stress can be closely approximated described by:

$$
Q=b_{0}(n)+b_{1}(n)\left(\frac{T}{\sigma_{0}}\right)+b_{2}(n)\left(\frac{T}{\sigma_{0}}\right)^{2}+b_{3}(n)\left(\frac{T}{\sigma_{0}}\right)^{3}
$$

Meanwhile, again with the HRR field as a reference field, the relationship between constraint parameters $Q$ and $A$ is as shown in Eq. (2.35a). Comparing Eq. (A1) and Eq. (2.35a), one can get following equation at $\theta=0, r=2 J / \sigma_{0}$ :

$$
\frac{A^{2}}{A_{0}} \bar{r}^{2 t-s} \bar{\sigma}_{i j}^{(2)}(\theta)-A \bar{r}^{t} \bar{\sigma}_{i j}^{(1)}(\theta)-\left[b_{0}(n)+b_{1}(n)\left(\frac{T}{\sigma_{0}}\right)+b_{2}(n)\left(\frac{T}{\sigma_{0}}\right)^{2}+b_{3}(n)\left(\frac{T}{\sigma_{0}}\right)^{3}\right]=0
$$

Under standard small-scale yielding (SSY, $T=0$ ) conditions, Eq. (2.21) can be rewritten as:

$$
\frac{\left(\sigma_{i j}\right)_{S S Y}}{\sigma_{0}}=A_{0} \bar{r}^{s} \bar{\sigma}_{i j}^{(0)}(\theta, n)-A_{S S Y} \bar{r}^{t} \bar{\sigma}_{i j}^{(1)}(\theta, n)+\frac{A_{S S Y}^{2}}{A_{0}} \bar{r}^{2 t-s} \bar{\sigma}_{i j}^{(2)}(\theta, n)
$$

According to the research of O'Dowd (1995), with the HRR field as a reference field, the stress field at the near crack-tip region can also be expressed as:

$$
\frac{\sigma_{i j}}{\sigma_{0}}=\frac{\left(\sigma_{i j}\right)_{H R R}}{\sigma_{0}}+b_{0}(n)+Q
$$

where $i=j$, and the first two terms correspond to the standard SSY condition ( $T=0)$. Therefore, under standard SSY conditions, the following equation can be obtained:

$$
\frac{\left(\sigma_{i j}\right)_{S S Y}}{\sigma_{0}}=\frac{\left(\sigma_{i j}\right)_{H R R}}{\sigma_{0}}+b_{0}(n)
$$

Comparing the Eqs. (A3) and (A5), and considering the condition, $A_{0} \bar{r}^{s} \bar{\sigma}_{i j}^{(0)}(\theta, n)$ 
$=\left(\sigma_{i j}\right)_{H R R} / \sigma_{0}$ (see the paper of Nikishkov et al. $\left.(1995 \mathrm{a})\right)$, one gets:

$$
b_{0}(n)=-A_{S S Y} \bar{r}^{t} \bar{\sigma}_{i j}^{(1)}(\theta, n)+\frac{A_{S S Y}^{2}}{A_{0}} \bar{r}^{2 t-s} \bar{\sigma}_{i j}^{(2)}(\theta, n)
$$

where $i=j$.

Substituting Eq. (A6) into Eq. (A2), it can be found that Eqs. (A2) and (3.4) are essentially equivalent. In other words, based on either the HRR or SSY reference fields for determining $Q$ values, a virtually identical equation including both fracture parameter $A$ and $T$-stress can be obtained. 


\section{REFERENCES}

ABAQUS Manual (2006), Version 6.6, Hibbitt, Karlsson \& Sorensen, Inc., Pawtucket, RI.

Ainsworth, R. A., Sattari-Far, I., Sherry, A. H., Hooton, D. G., and Hadley, I. (2000). "Methods for including constraint effects within SINTAP procedures," Engineering Fracture Mechanics, Vol. 67, pp. 563-571.

Al-Ani, A. M. and Hancock, S. W. (1991). " $J$-dominance of short cracks in tension and bending," Journal of the Mechanics and Physics of Solids, Vol. 39, pp. 23-43.

Anderson, T. L. (2005). Fracture Mechanics: Fundamentals and Applications, CRC Press, Boca Raton.

Begley, J. A. and Landes, J. D. (1972). "The $J$-integral as a fracture criterion," Fracture Toughness, Part II, ASTM STP 514, American Society for Testing and Materials, Philadelphia, pp. 1-20.

Betegon, C. and Hancock, J. W. (1991). "Two parameter characterization of elasticplastic crack-tip fields," Journal of Applied Mechanics, Vol. 58, pp. 104-110.

Chao, Y. J., Yang, S. and Sutton, M. A. (1994). "On the fracture of solids characterized by one or two parameters: theory and practice," Journal of the Mechanics and Physics of Solids, Vol. 42, pp. 629-647.

Chao, Y. J. and Zhu, X. K. (1998). " $J-A_{2}$ characterization of crack-tip fields: Extent of $J-A_{2}$ dominance and size requirements," International Journal of Fracture, Vol. 89, pp. 285-307. 
Dodds, R. H., Shih, C. F., and Anderson, T. L. (1993). "Continuum and micromechanics treatment of constraint in fracture," International Journal of Fracture, Vol. 64, pp. 101-133.

Gullerud, A. S. and Dodds, R. H. Jr. (1995). " $J-Q$ and toughness scaling model solutions for $\mathrm{M}(\mathrm{T}), \mathrm{DE}(\mathrm{T}), \mathrm{SE}(\mathrm{B})$ and $\mathrm{C}(\mathrm{T})$ specimens", International Journal of Fracture, Vol. 72, pp. R11-R21.

Hutchinson, J. W. (1968). "Singular behavior at the end of a tensile crack in a hardening material," Journal of the Mechanics and Physics of Solids, Vol. 16, pp. 13-31.

Irwin, G. R. (1957). "Analysis of stress and strains near the end of a crack traversing a plate," Journal of Applied Mechanics, Vol. 24, pp. 361-364.

Kim, Y., Zhu, X. K. and Chao, Y. J. (2001). "Quantification of constraint elastic-plastic 3D crack front by $J-A_{2}$ three-term solution," Engineering Fracture Mechanics, Vol. 68 , pp. 895-914.

Kim, Y., Chao, Y. J. and Zhu, X. K. (2003). "Effect of specimen size and crack depth on 3D crack-front constraint for SENB specimens," International Journal of Solids and Structures, Vol. 40, pp.6267- 6284 .

Kfouri, A. P. (1986). "Some evaluation of the elastic T-term using Eshelby's method", International Journal of Fracture, Vol. 30, pp. 301-315.

Kumar, V., German, M. D. and Shih, C. F. (1981). "An engineering approach for elasticplastic fracture analysis," EPRI Report NP-1931, Electric Power Research Institute, Palo Alto, CA. 
Landes, J. D. and Begley, J. A. (1972). "The Effect of Specimen Geometry on $J_{I C}$," Fracture Toughness, Part II, ASTM STP 514, American Society for Testing and Material, Philadelphia, pp.24-29.

Li, Y. C. and Wang, T. C. (1986). "Higher-order asymptotic field of tensile plane-strain nonlinear crack problem," Scientia Sinica (Series A), Vol. 29, pp. 941-955.

McMeeking, R. M. and Parks, D. M. (1979). "On criteria for $J$-dominance of crack-tip fields in large-scale yielding," Elastic-Plastic Fracture, ASTM STP 668, American Society for Testing and Materials, Philadelphia, pp. 175-194.

Moran, B. and Shih, C. F. (1987). "A general treatment of crack tip contour integrals," International Journal of Fracture, Vol.35, pp. 295-310

Nakamura, T. and Parks, D. M. (1992). "Determination of elastic T-stress along threedimensional crack fronts using an interaction integral," International Journal of Solids and Structures, Vol. 29, pp. 1597-1611.

Nevalainen, M. and Dodds, R. H. (1995), "Numerical investigation of 3-D constraint effects on brittle fracture in $\mathrm{SE}(\mathrm{B})$ and $\mathrm{C}(\mathrm{T})$ specimens," International Journal of Fracture, Vol. 74, pp. 131-161.

Nikishkov, G. P. (1995). "An algorithm and a computer program for the three-term asymptotic expansion of elastic-plastic crack tip stress and displacement fields," Engineering Fracture Mechanics, Vol. 50, pp. 65-83.

Nikishkov, G. P., Bruckner-Foit, A. and Munz, D. (1995a). "Calculation of the second fracture parameter for finite cracked bodies using a three-term elastic-plastic asymptotic expansion," Engineering Fracture Mechanics, Vol. 52, pp. 685-701. 
Nikishkov, G. P., Bruckner-Foit, A. and Munz, D. (1995b). "Application of the threeterm elastic-plastic asymptotic expansion for the characterization of stress fields near a front of a semi-elliptical crack," International Journal of Fracture, Vol. 70, pp. R91R97.

O'Dowd, N. P. and Shih, C. F. (1991). "Family of crack-tip fields characterized by a triaxiality parameter - I. Structure of fields," Journal of the Mechanics and Physics of Solids, Vol. 39, pp. 989-1015.

O'Dowd, N. P. and Shih, C. F. (1992). "Family of crack-tip fields characterized by a triaxiality parameter - II. Fracture applications," Journal of the Mechanics and Physics of Solids, Vol. 40, pp. 939-963.

O'Dowd, N. P. and Shih, C. F. (1994). "Two-parameter fracture mechanics: theory and applications," Fracture Mechanics: 24th Volume, ASTM STP 1207, pp. 21-47.

O'Dowd, N. P. (1995). "Applications of two parameter approaches in elastic-plastic fracture mechanics," Engineering Fracture Mechanics, Vol. 52, pp. 445-465.

O'Dowd, N. P., Kolednik, O. and Naumenko V. P. (1999). "Elastic-plastic analysis of biaxially loaded center-cracked plates," International Journal of Solids and Structures, Vol. 36, pp. 5639-5661.

Rice, J. R. (1968). "A path independent integral and the approximate analysis of strain concentration by notches and cracks," Journal of Applied Mechanics, Vol. 35, pp. 379-386.

Rice, J. R. and Rosengren, G. F. (1968). "Plane strain deformation near a crack tip in a power law hardening material," Journal of the Mechanics and Physics of Solids, Vol. 
16 , pp. $1-12$.

Sharma, S. M. and Aravas, N. (1991). "Determination of higher-order terms in asymptotic elastoplastic crack tip solutions," Journal of the Mechanics and Physics of Solids, Vol. 39, pp. 1043-1072.

Shih, C. F. and German, M. D. (1981). "Requirements for a one parameter characterization of crack tip fields by HRR singularity," International Journal of Fracture, Vol. 17, pp. 27-43.

Shih, C. F. and O'Dowd, N. P. (1992). "A fracture mechanics approach based on a toughness locus," Shallow Crack Fracture Mechanics, Toughness Tests and Application. Proceedings of International Conference, Cambridge, U.K., 23-24 September 1992.

Shih, C. F., O'Dowd, N. P. and Kirk, M. T. (1993). "A framework for quantifying crack tip constraint," Constraint effects in fracture, editors: Hackete, E. M., Schwalbe, K. H., ASTM STP 1171, pp. 2-20. American Society for Testing and Materials.

Tada, H., Paris, P. C. and Irwin, G. R. (2000). The Stress Analysis of Cracks Handbook, ASME International, 3rd edition.

Wang, X. (2002). "Elastic $T$-stress for cracks in test specimens subjected to non-uniform stress distributions," Engineering Fracture Mechanics, Vol. 69, pp. 1339 - 1352.

Wang, X. (2003). "Elastic T-stress solutions for semi-elliptical surface cracks in finite thickness plates," Engineering Fracture Mechanics, Vol. 70, pp. 731-756.

Wang, X. and Bell, R. (2004) "Elastic $T$-stress solutions for semi-elliptical surface cracks in finite thickness plates subject to non-uniform stress distributions," Engineering 
Fracture Mechanics, Vol. 71, pp. 1477-1496.

Wang, X. and Yu, X. (2005). "On the constraint-based failure assessment of surface cracked plates under biaxial loading," Journal of ASTM International, Vol. 2, ID JAI12038. Also in Fatigue and Fracture $34^{\text {th }}$ Volume, ASTM STP 1461, S. R. Daniewicz, J. C. Newman, K. H. Schwalbe, Eds., American Society for Testing and Materials, West Conshohocken, PA, 2003, pp. 245-259.

Wang, X. (2008). "On the quantification of the constraint effect along a threedimensional crack front," Journal of ASTM International, Vol. 5, paper ID: JAI101550.

Wang, X. (2009). "Two-parameter characterization of elastic-plastic crack front fields: surface cracked plates under tensile loading", Engineering Fracture Mechanics, Vol. 76, pp. 958-982.

Wang, Y. Y. (1993). “On the two-parameter characterization of elastic-plastic crack-front fields in surface-cracked plates," Constraint Effects in Fracture, ASTM STP 1171, E. M. Hackett, K. H. Schwalbe and R. H. Dodds, Eds., American Society for Testing and Materials, West Conshohocken, PA, pp. 120-138.

Wang, Y. Y. and Parks, D. M. (1995), "Limits of $J-T$ characterization of elastic-plastic crack-tip fields," In: Kirk M. and Bakker, A. editors: Constraint Effects in Fracture Theory and Applications, ASTM STP 1244. American Society for Testing and Materials; pp. 43-67.

Williams, M. L. (1957). "On the stress distribution at the base of a stationary crack," Journal of Applied Mechanics, Vol. 24, pp. 109-114.

Wu, S., Mai, Y. and Cotterell, B. (1995). “ $Q$ Solutions for compact tension and singleedge cracked tension specimens", International Journal of Fracture, Vol. 68, pp. R97- 
R103.

Yang, S. (1993). "Higher order asymptotic crack tip fields in a power-law hardening material," Ph.D. Dissertation, University of South Carolina, USA.

Yang, S., Chao, Y. J. and Sutton, M. A. (1993). "Higher-order asymptotic fields in a power-law hardening material," Engineering Fracture Mechanics, Vol. 45, pp. 1-20.

Yuan, H. and Brocks, W. (1998). "Quantification of constraint effects in elastic-plastic crack front fields," Journal of the Mechanics and Physics of Solids, Vol. 46, No. 2, pp. 219-241.

Zhu, X. K., Kim, Y., Chao, Y. J. and Lam, P. S. (2002). "Constraint effect on 3-D crackfront stress fields in elastic-plastic thin plates," Fatigue and Fracture Mechanics: $33^{\text {rd }}$ Volume, ASTM STP 1417, R. S. Piascik, and W. G. Reuter Eds., ASTM International, West Conshohocken, PA, pp. 270-287. 


\section{LIST OF PUBLICATIONS}

Ding, P. and Wang, X. (2010). "Solutions of the second elastic-plastic fracture mechanics parameter in test specimens," Engineering Fracture Mechanics, Vol. 77, pp. 34623480.

Ding, P. and Wang, X. (2012). "An estimation method for the determination of the second elastic-plastic fracture mechanics parameters," Engineering Fracture Mechanics, Vol. 79, pp. 295-311.

Ding, P. and Wang, X. (to appear). "Numerical solutions of second elastic-plastic fracture mechanics parameter in test specimens under biaxial loading," 13th International Conference on Fracture (ICF13), Beijing, China, 16-21 June, 2013. 